\title{
European Nutrition and Health Report 2009
}

Elmadfa, Ibrahim; Meyer, A.; Nowak, V.; Hasenegger, V.; Putz, P.; Verstraeten, R.; Remaut-DeWinter A, A. M.; Kolsteren, P.; Dostálová, J.; Dlouhý , P.

Total number of authors:

17

Publication date:

2009

Document Version

Publisher's PDF, also known as Version of record

Link back to DTU Orbit

Citation $(A P A)$ :

Elmadfa, I., Meyer, A., Nowak, V., Hasenegger, V., Putz, P., Verstraeten, R., Remaut-DeWinter A, A. M., Kolsteren, P., Dostálová, J., Dlouhý, P., Trolle, E., Fagt, S., Biltoft-Jensen, A. P., Mathiessen, J., Groth, M. V., Kambek, L., \& Gluškova, N. (2009). European Nutrition and Health Report 2009. Karger. Forum of Nutrition Vol. $62 \mathrm{http}: / /$ www.univie.ac.at/enhr/downloads/enhrii_book.pdf

\section{General rights}

Copyright and moral rights for the publications made accessible in the public portal are retained by the authors and/or other copyright owners and it is a condition of accessing publications that users recognise and abide by the legal requirements associated with these rights.

- Users may download and print one copy of any publication from the public portal for the purpose of private study or research.

- You may not further distribute the material or use it for any profit-making activity or commercial gain

- You may freely distribute the URL identifying the publication in the public portal 
European Nutrition and Health Report 2009 


\section{Forum of Nutrition}

\section{Vol. 62}

Series Editor

Ibrahim Elmadfa Vienna

The European Nutrition and Health Report 2009 was supported by funding under the Public Health programme 2006 (Agreement Number 2006128) of the European Union. The responsibility of the information contained in this document lies with the authors. The Executive Agency for Health and Consumers is not responsible for any use that may be made of the information contained therein. 


\section{European Nutrition and Health Report 2009}

Volume Editor

\section{Ibrahim Elmadfa Vienna}

87 figures and 162 tables, 2009

\section{Authors}

Elmadfa I, Meyer A, Nowak V, Hasenegger V,

Holm Totland T, Andersen LF NORWAY

Putz P AUSTRIA

Verstraeten R, Remaut-DeWinter AM,

Halicka E, Rejman K, Kowrygo B POLAND

Kolsteren P BELGIUM

Dostálová J, Dlouhý P CZECH REPUBLIC

Trolle E, Fagt S, Biltoft-Jensen A, Mathiessen J,

Rodrigues S, Pinhão S, Ferreira LS, Lopes C,

Ramos E, Vaz Almeida MD PORTUGAL

Vlad M ROMANIA

Velsing Groth M DENMARK

Simčič $M$, Podgrajšek K SLOVENIA

Serra Majem L, Román Viñas B, Ngo J,

Kambek L, Gluškova N ESTONIA

Ribas Barba L SPAIN

Voutilainen S, Erkkilä A FINLAND

Becker W SWEDEN

Vernay M FRANCE

Fransen H, Van Rossum C,

Krems C, Straßburg A, Vasquez-Caicedo AL,

Urban C GERMANY

Ocké M THE NETHERLANDS

Margetts B UNITED KINGDOM

Naska A, Efstathopoulou E, Oikonomou E,

Tsiotas K, Bountziouka V, Benetou V,

Trichopoulou A GREECE

Collaborating partners

Zajkás G, Kovács V, Martos E HUNGARY

Rütten A, Abu-Omar K, Gelius P

Heavey P, Kelleher C, Kennedy J IRELAND

Chapter 8.6 Physical Activity

Turrini A ITALY

Selga G, Sauka M LATVIA

Cattaneo A

Petkeviciene J, Klumbiene J LITHUANIA

Chapter 8.7 Breastfeeding

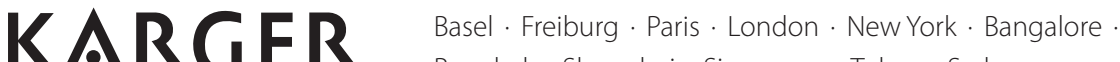 \\ KARGER}


Ibrahim Elmadfa

Institute of Nutritional Sciences

University of Vienna

Vienna (Austria)

Library of Congress Cataloging-in-Publication Data

European nutrition and health report 2009 / volume editor, Ibrahim Elmadfa.

p. ; cm. -- (Forum of nutrition, ISSN 1660-0347; v. 62)

Includes bibliographical references and index.

ISBN 978-3-8055-9296-3 (hard cover : alk. paper)

1. Nutrition--Europe. 2. Public health--Europe. I. Elmadfa, I. II. Series:

Forum of nutrition, v. 62. 1660-0347;

[DNLM: 1. Nutrition Surveys--Europe--Statistics. 2. Food Supply--statistics

\& numerical data--Europe. 3. Health Status--Europe--Statistics. W1 B1422 v.62

2009 / QU 146 GA1 E89 2009]

RA601.E977 2009

363.8094--dc22

2009033400

Bibliographic Indices. This publication is listed in bibliographic services, including Current Contents ${ }^{\oplus}$ and Index Medicus.

Disclaimer. The statements, opinions and data contained in this publication are solely those of the individual authors and contributors and not of the publisher and the editor(s). The appearance of advertisements in the book is not a warranty, endorsement, or approval of the products or services advertised or of their effectiveness, quality or safety. The publisher and the editor(s) disclaim responsibility for any injury to persons or property resulting from any ideas, methods, instructions or products referred to in the content or advertisements.

Drug Dosage. The authors and the publisher have exerted every effort to ensure that drug selection and dosage set forth in this text are in accord with current recommendations and practice at the time of publication. However, in view of ongoing research, changes in government regulations, and the constant flow of information relating to drug therapy and drug reactions, the reader is urged to check the package insert for each drug for any change in indications and dosage and for added warnings and precautions. This is particularly important when the recommended agent is a new and/or infrequently employed drug.

All rights reserved. No part of this publication may be translated into other languages, reproduced or utilized in any form or by any means electronic or mechanical, including photocopying, recording, microcopying, or by any information storage and retrieval system, without permission in writing from the publisher.

(c) Copyright 2009 by European Commission, Health and Consumer Protection, Directorate-General www.karger.com

Printed in Switzerland on acid-free and non-aging paper (ISO 9706) by Reinhardt Druck, Basel

ISSN 1660-0347

ISBN 978-3-8055-9296-3

e-ISBN 978-3-8055-9297-0 


\section{Contents}

Preface VII

List of Abbreviations $\quad$ IX

List of Participants $\quad$ XI

1. Executive Summary 1

2. Outlook 12

3. Project Background and Methodological Aspects 14

4. Trends of Average Food Supply in the European Union 17

5. Food Availability at the Household Level in the European Union 43

6. Food Consumption in Adults on the Basis of Dietary Surveys in 60 European Countries

7. Energy and Nutrient Intake in the European Union Based on National Data 68

$\begin{array}{lll}7.1 & \text { Methodology } & \mathbf{6 8}\end{array}$

7.2 Energy and Nutrient Intake in European Children $\quad 69$

7.3 Energy and Nutrient Intake in European Adolescents $\quad \mathbf{9 8}$

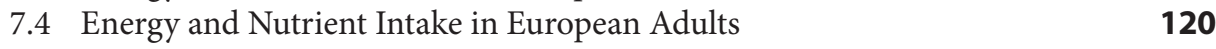

7.5 Energy and Nutrient Intake in European Elderly 135

8. Health and Lifestyle Indicators in the European Union 157

$\begin{array}{lll}8.1 & \text { Prevalence of Overweight and Obesity } & 157\end{array}$

$\begin{array}{ll}8.2 \text { Blood Lipids } & 171\end{array}$

$\begin{array}{lll}8.3 \text { Mortality/Morbidity } & 171\end{array}$

$\begin{array}{ll}8.4 \text { Smoking Behavior } & 182\end{array}$

8.5 Alcohol Consumption $\quad \mathbf{1 8 2}$

8.6 Overview of European Cross-National Data on Physical Activity $\quad 186$

$\begin{array}{ll}8.7 \text { Breastfeeding } & 196\end{array}$

9. Food and Nutrition Policies in European Countries 202

10. General Discussion 230 
11. National Reports

$\begin{array}{llr}11.1 & \text { Austria } & \mathbf{2 5 0}\end{array}$

$\begin{array}{ll}11.2 & \text { Belgium } \\ 11.3 & \mathbf{2 5 8}\end{array}$

11.3 Czech Republic 263

$\begin{array}{lll}11.4 & \text { Denmark } & \mathbf{2 6 9}\end{array}$

$\begin{array}{lll}11.5 & \text { Estonia } & \mathbf{2 7 7}\end{array}$

$\begin{array}{lll}11.6 & \text { Finland } & \mathbf{2 8 5}\end{array}$

11.7 France $\quad 294$

11.8 Germany $\quad 299$

$\begin{array}{lll}11.9 & \text { Greece } & 304\end{array}$

11.10 Hungary $\quad 309$

$\begin{array}{lll}11.11 & \text { Ireland } & 319\end{array}$

$\begin{array}{lll}11.12 & \text { Italy } & 323\end{array}$

$\begin{array}{lll}11.13 & \text { Latvia } & \mathbf{3 3 0}\end{array}$

11.14 Lithuania $\quad 334$

11.15 Norway $\quad 339$

$\begin{array}{lll}11.16 & \text { Poland } & \mathbf{3 4 5}\end{array}$

$\begin{array}{lll}11.17 & \text { Portugal } & 351\end{array}$

11.18 Romania 356

11.19 Slovenia $\quad 362$

11.20 Spain 366

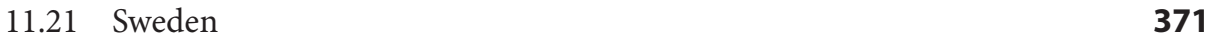

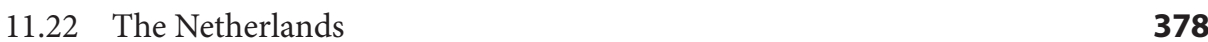

11.23 United Kingdom $\quad 384$

References

$\begin{array}{ll}\text { Subject Index } & 406\end{array}$ 


\section{Preface}

The variety and complexity of data sampling and collection, documentation and interpretation in the field of diet, health promotion, and the prevention of nutritionrelated diseases require regular survey and analysis of relevant and available information. This facilitates the early detection of changes in nutritional behavior in a favorable and/or negative sense and allows the establishment of according measures of food, nutrition, and health policies.

The main objective of the project Report on Nutrition and Health, Aggreement number 2006128, was not to generate new data, but rather to collect available and authorized data, published or unpublished, on the nutrition and health situation in the countries of the European Union (EU). Furthermore, the broad scope of the subjects covered and the improved quality of the collected data make the European Nutrition and Health Report (ENHR) 2009 an accurate documentation and sound source of information suitable for being used in international comparisons on the nutrition and health situation.

The first ENHR was released in 2004. Thirteen EU member states and Norway participated, allowing comparisons between these countries as well as the northern, southern, and western EU regions.

In the ENHR 2009, an additional eleven countries joined the original contributors, resulting in the active participation of $24 \mathrm{EU}$ member states and Norway. In all main chapters of the ENHR 2009, the impact of age and gender was emphasized and special attention paid to the different regions of the EU. For a better and easier comparison of the participating countries, four regions were defined: North (Denmark, Estonia, Finland, Latvia, Lithuania, Norway, Sweden), West (Belgium-Luxembourg, France, Ireland, The Netherlands, the UK), Central-East (Austria, the Czech Republic, Germany, Hungary, Poland, Romania, Slovenia) and South (Cyprus, Greece, Italy, Portugal, Spain). 
Specific objectives of the ENHR 2009 were

- to describe trends in food supply in the EU focusing also on the different regions,

- to compare average daily individual food availability at household level,

- to evaluate individual food consumption and energy and nutrient intake,

- to describe data on diet-related health indicators and status, and

- to analyze food and nutrition policies in countries of the EU.

Different methods of nutrition surveillance were considered in this report, taking into account their potential and limitations. Food Balance Sheets (FBS) of the Food and Agriculture Organization (FAO) of the United Nations were used to calculate the trends of average food supply (chapter 4). The food availability at household level was derived from household budget survey (HBS) data of the DAFNE (Data Food Networking) databank (chapter 5). Information on individual food consumption of adults in European countries came from national and regional dietary surveys (chapter 6). Nutrition surveys at the national level considering demographic and socioeconomic characteristics such as age, gender, and education also provided data on energy and nutrient intake and allowed the description of the nutritional situation by age and gender (chapter 7). In addition to national health surveys, data from different sources (WHO, Eurobarometer, GLOBOCAN) served to describe and comment on health indicators and status including overweight, blood lipids, total, cardiovascular, and cancer-related mortality, the prevalence of different neoplasms and diabetes mellitus, as well as the health-related lifestyle parameters smoking, alcohol consumption, physical activity, and breastfeeding (chapter 8).

Chapter 9 of the ENHR 2009 deals with Food and Nutrition Policies (FNPs) from the standpoint of the participating countries. A specially conducted survey considered types and implementing actions of FNPs, their role in ensuring diet quality, and their impact on health promotion and prevention of nutrition-related chronic diseases.

More detailed information related to the nutrition and health situation in the participating countries is documented in the corresponding national reports (chapter 11).

The European Nutrition and Health Report 2009 is intended to provide a documentation of and a basis for the work of those institutions responsible for health and nutrition policy.

As the coordinator of the ENHR 2009, I acknowledge all the participating countries and external collaborating partners who brought in their scientific expertise. My gratitude goes to the outstanding authors, the excellent proofreader Ms. Johanna Fally, and everybody who contributed to the successful finalization of this report.

Professor Dr. I. Elmadfa University of Vienna, Austria

Vienna, October 2009 


\section{List of Abbreviations}

Countries of European Nutrition and Health Report 2009

$\begin{array}{ll}\text { AT } & \text { Austria } \\ \text { BE } & \text { Belgium } \\ \text { CY } & \text { Cyprus } \\ \text { CZ } & \text { Czech Republic } \\ \text { DE } & \text { Germany } \\ \text { DK } & \text { Denmark } \\ \text { EE } & \text { Estonia } \\ \text { ES } & \text { Spain } \\ \text { FI } & \text { Finland } \\ \text { FR } & \text { France } \\ \text { GR } & \text { Greece } \\ \text { HU } & \text { Hungary } \\ \text { IE } & \text { Ireland } \\ \text { IT } & \text { Italy } \\ \text { LT } & \text { Lithuania } \\ \text { LU } & \text { Luxembourg } \\ \text { LV } & \text { Latvia } \\ \text { NL } & \text { Netherlands } \\ \text { NO } & \text { Norway } \\ \text { PL } & \text { Poland } \\ \text { PT } & \text { Portugal } \\ \text { RO } & \text { Romania } \\ \text { SE } & \text { Sweden } \\ \text { SI } & \text { Slovenia } \\ \text { UK } & \text { United Kingdom }\end{array}$


Other abbreviations

BMI

CAP

Body mass index (body weight in $\mathrm{kg} /$ body height in $\mathrm{m}^{2}$ )

CE

Common Agriculture Policy

CFP

Central and East

$\mathrm{CHO}$

Common Fisheries Policy

DAFNE

Carbohydrates

EC

Data Food Networking Project

$\% \mathrm{E}$

European Commission

FA

$\%$ of total energy

FAO

Fatty acids

FBDG

Food and Agriculture Organization of the United Nations

FBS

Food-Based Dietary Guidelines

FFQ

Food Balance Sheets

FNP

Food Frequency Questionnaire

HBS

Food and Nutrition Policy

HS

Household Budget Survey

INS

Highest supply

LS

Individual Nutrition Surveys

Max

Lowest supply

Min

Maximum

MUFA

Minimum

NGOs

$\mathrm{P}$

Monounsaturated fatty acids

Nongovernmental organizations

PA(L) Physical activity (level)

PUFA Polyunsaturated fatty acids

SD

Standard deviation

SFA

Saturated fatty acids

UK

United Kingdom

WHO

World Health Organization 


\section{List of Participants}

\section{Coordinator of the European Nutrition and Health Report}

Ibrahim Elmadfa

\section{Coordinating Center}

Ibrahim Elmadfa, Verena Nowak

Institute of Nutritional Sciences, University of Vienna, Austria

\section{Partners}

Anne-Marie Remaut-De Winter

Research Group Food Chemistry and Human Nutrition, University of Gent, Belgium

Jana Dostálová

Department of Food Chemistry and Analysis, Institute of Chemical

Technology Prague, Czech Republic

Carolin Krems

Max Rubner-Institut, Federal Research Institute of Nutrition and Food,

Germany

Ellen Trolle

Technical University of Denmark, Denmark

Luís Serra-Majem, Blance Román-Viñas

Fundación para la Investigación Nutricional, Spain 
Antonia Trichopoulou

Hellenic Health Foundation, Greece

Michel Vernay

Unité de surveillance et dépidemiologie nutritionelle, Université Paris, France

Arja Erkkilä

School of Public and Clinical Nutrition, University of Kupio (UKU), Finland Éva Martos, Gábor Zajkás

National Institute of Food Safety and Nutrition, Hungary

Aida Turrini

National Institute of Research for Food and Nutrition, Italy

Jean Kennedy

School of Public Health and Population Science, University Collage Dublin,

Ireland

Janina Petkeviciene

Institute for Biomedical Research of Kaunas, University of Medicine,

Lithuania

Guntars Selga, Melita Sauka

Sports Medicine State Agency, Latvia

Caroline van Rossum

National Institute for Public Health and the Environment, The Netherlands

Lene Frost-Andersen

Universitetet I Oslo, Norway

Maria Daniel Vaz de Almeida

Faculdade de Ciencias da Nutricao e Alimenacao da Universidade do

Porto, Portugal

Ewa Halicka

Faculty of Human Nutrition and Consumer Sciences, Warsaw University of

Life Sciences, Poland

Mariana Vlad

Wulf Becker

Institute of Public Health Cluj-Napoca, Romania

National Food Administration, Sweden

Marjan Simčič

Biotechnical Faculty, University of Ljubljana, Slovenia

Barrie Margetts

Institute of Human Nutrition, School of Medicine, University of

Southampton, United Kingdom

Eliza Markidou

Department of Medical and Public Health Services, Ministry of Health,

Cyprus

Liis Kambek

National Institute for Health Development, Estonia 


\section{Collaborating Organizations}

Institute of Sport Science and Sport (ISS) of the Friedrich Alexander University Erlangen-Nuremberg (FAU), Germany.

Unit for Health Services Research and International Health, IRCCS Burlo Garofolo, Trieste, Italy.

World Health Organization Regional Office for Europe (WHO), Copenhagen, Denmark.

Nutrition and Consumer Protection Division, Food and Agriculture Organization of the United Nations (FAO), Rome, Italy. 



\section{Executive Summary}

The aim of the European Nutrition and Health Report 2009 is to provide a comprehensive view of the health and nutrition status in the European Union (EU). It is not intended to generate new data, but rather to collect available and authorized data, published or unpublished, on the nutrition and health situation in the EU countries. Since the release of its predecessor five years ago, two new member states, Bulgaria and Romania, have joined the European Union the latter of which participated in the current report. Moreover, with the exception of Malta and Slovakia, the countries that joined the EU in 2005 also contributed as did Ireland and The Netherlands, not represented in the previous report. With 24 EU member states as well as Norway taking part, the amount of data contained in the second European Nutrition and Health Report 2009 has increased considerably. These countries were grouped into four geographical regions: (1) Denmark, Estonia, Finland, Latvia, Lithuania, Norway, Sweden constitute the North, (2) Belgium-Luxembourg, France, Ireland, The Netherlands, and the UK the West, (3) Central and Eastern Europe is represented by Austria, the Czech Republic, Germany, Hungary, Poland, Romania and Slovenia, and (4) the South is represented by Cyprus, Greece, Italy, Portugal and Spain. Like the previous project, the current project was again funded by the European Commission and coordinated by the Institute of Nutritional Sciences of the University of Vienna, Austria, directed by Professor Ibrahim Elmadfa. The coordinating center compiled the final report of the national data collected by the partners.

- To provide an overview of available data on food and nutrient intake as well as

- identify major national and regional health and nutrition issues were again the major aims of the report.

Specific additional objectives of the ENHR 2009 were

- to describe trends in food supply in the European Union focusing also on the different regions (chapter 4),

- to compare average daily individual food availability at the household level (chapter 5),

- to evaluate individual food consumption in adults (chapter 6) and energy and nutrient intake in all age groups (chapter 7),

- to describe data on diet-related health indicators and status (chapter 8), 
- to analyze food and nutrition policies in countries of the European Union (chapter 9).

The national reports constituting chapter 11 provide more detailed information on the nutrition and health situation in the different participating countries, especially on issues not addressed in the main report.

Besides providing an overview of the nutrition and health situation in the European Union, this report also shows that, despite a considerable improvement of the quality of the methods of data collection and assessment, there is still a need for harmonization of databases and survey methods.

\section{Trends in the Food Supply in Europe}

The Food Balance Sheets (FBS) of the Food and Agriculture Organization of the United Nations (FAO) are agricultural statistics that have so far been published yearly since 1961 up to 2003. To estimate food supply, they take into account data on total production, import and stocks of food as well as its utilization for non-food purposes in a given country. The consistency of methods and the coverage of a large number of countries worldwide make FBS a valuable source of information about the pattern of a country's food supply during a specified reference period. Especially, they allow international comparisons and the detection of international and national trends. In turn, regional differences within one country or between groups of its population cannot be discerned. Data are obtained on a per capita basis without any differentiation between genders and age groups. Additionally, losses due to waste and spoilage can only roughly be accounted for so that supply data are higher than the actual intake. Nevertheless, FBS are a unique source for showing international trends in food supply.

\section{Supply in Europe Over the Last Four Decades}

Cereals and Potatoes. Both, the mean supply of cereals and that of potatoes have been decreasing over the last 40 years. On a regional basis, the supply of cereals increased in the North region, while in the other regions a slight decrease could be observed. The supply of potatoes decreased in the West and Central and East regions and remained relatively constant in the South. In the North, the gap between minimum and maximum supply has been widening.

Fruits, Vegetables and Pulses. In all participating countries on the whole, the mean supplies of fruits and vegetables increased from 1961 to 2003, as well as in every region of the EU. Only in the West, has the supply of vegetables shown a slight decrease. In turn, the amount of pulses available for the food sector showed an undesirable decreasing tendency over the past four decades. However, this was mainly due to strong decreases in the South and the Central and East region, while the North and West region showed slight increases. 
Milk and Milk Products. From 1961 to 2003, the average supply of milk showed an increase that was seen in every region except the North where it remained relatively constant. Nonetheless, in this latter, it was at the highest level compared to all other regions.

Animal Products - Excluding Milk. The average supply of meat, most notably of pork and poultry, eggs as well as fish and seafood increased over the past 40 years. A general increase in the supply of animal products can be observed in all regions, but it was higher in the central and eastern as well as western regions.

Fats, Oils and Sugar. The mean supply of oil crops, vegetable oils as well as the supply of sugar and sweeteners in the participating countries increased since 1961, whereas the supply of animal fats remained relatively constant. The average supply of oil crops increased in every region, with a particularly strong increase of the maximum values in the South region. Both, the supply of animal fats and vegetable oils increased over the past 40 years except in the North region where the supply of animal fats markedly decreased. The supply of sugar and sweeteners also increased. In the North, the gap between minimum and maximum supply widened.

Alcoholic Beverages. While the average supply of beer in the participating countries doubled over the past 40 years, the mean wine supply decreased slightly. The supply of alcoholic beverages in general increased in every region.

Concerning trends in the proportion of macronutrients over the past four decades, no change was observed for protein, while the proportion of fat increased and that of carbohydrates decreased.

In most of the participating countries, the contribution of animal and vegetable food groups in total energy supply changed only slightly from 1961 to 2003, although there was a strong increase during this period in the southern European countries Cyprus, Greece, Italy, Portugal and Spain as well as in Romania. Considering the different regions, the proportion of animal products in total energy supply remained relatively constant in the West region, decreased slightly in the North region, increased in the Central and East region and increased markedly in the South region over the past 40 years.

An increase in most of the food groups can be observed except for pulses, potatoes, wine, cereals and mutton and goat meat. The supply of animal fats remained relatively constant.

\section{Food Availability at Household Level in Europe}

The ability to monitor and compare the dietary habits of different populations is important in the formulation of dietary guidelines and in planning and implementing national policies. In the realm of actions in the field of public health, emphasis should be put on the importance of standardized and comparable dietary data and the promotion of nutrition surveillance systems. 
Household budget surveys (HBSs) are systematically conducted by National Statistical Offices in representative samples and aim at collecting, among others, data on food availability at household level. The use of the national HBSs for nutrition monitoring purposes has been evaluated through the EU-supported Data Food Networking (DAFNE) initiative, which built up a regularly updated food-based databank allowing the: (a) identification of dietary patterns prevailing in Europe and of their sociodemographic determinants [Trichopoulou et al., 2002; Naska et al., 2006], (b) follow-up of time trends in food habits [Trichopoulou and Naska, 2003], and (c) evaluation of nutrition action plans and strategies implemented at national or international level [Trichopoulou, 2001] (http://www.nut.uoa.gr/dafnesoftweb/). The DAFNE network currently interrelates 26 European countries (Albania, Armenia, Austria, Belgium, Croatia, Cyprus, Finland, France, Germany, Greece, Hungary, Ireland, Italy, Latvia, Luxembourg, Malta, Montenegro, Norway, Poland, Portugal, Serbia, Spain, Slovak Republic, Slovenia, Sweden and the UK).

The DAFNE data, comparable between countries, documents regional and social disparities in food habits in Europe. Given the potential of the DAFNE data, a system facilitating the regular update of the database, as well as the expansion of the network to embrace all European countries could provide a ready source of data for monitoring public health nutrition at a reasonable cost.

Despite high variability between single countries, certain regional characteristics in food availability can be discerned. Vegetable and fruit availability was higher in the South, although this was more marked for fruits. Vegetable availability was very high in Cyprus and Greece, but less so in Portugal and Spain. A high availability of fruit was not always associated with a high vegetable availability and vice versa. Cereals and potatoes were on average more available in households of Central and Eastern Europe. Milk and milk products showed the highest availability in the North although for cheese, it was very comparable across regions despite a great variance between single countries. Average availability of meat was highest in the Central and Eastern region and lowest in the North, although interregional differences were not that large. The Central and East region also showed the highest amounts for processed meat, the South the lowest. In this latter, availability of fish and seafood was highest despite a high intraregional variation. It even exceeded the levels of processed meat in all and those of poultry in most countries. In the North, availability of fish and seafood was also above the average of all regions, while it was lowest in the Central and Eastern region. Availability of added lipids was also higher in the South and mostly accounted for by vegetable oils. The West had the lowest availability of this food group especially the vegetable variants (oil and fat). However, apart from the South variability within regions was particularly high for this food group.

Educational level of the household head generally has a strong influence on household food purchases. The present data revealed a lower overall availability of food associated with higher educational level. However, fish and seafood availability was higher in higher educated household heads and there was a similar trend for fruit and vegetables as well. 


\section{Food Consumption in Adults on the Basis of Dietary Surveys in European Countries}

\section{On the Basis of the EFSA Concise Database}

To date, numerous countries have collected data on individual food consumption from dietary surveys. Up to now, there is no fully harmonized European database on individual food consumption. Hence, the European Food Safety Authority (EFSA) has compiled the available data on food consumption in adults from representative dietary surveys in 19 countries of the EU to make data as comparable as possible across the EU. Data are available for adults from 16 of the participating countries of the ENHR 2009 [EFSA, 2008a; EFSA, 2008b].

It has to be noted that differences between countries can be caused by different survey methodologies or different durations of surveys. Another limitation is the diverging assignment of foods to the food groups due to the use of differing nutritional evaluation software. For instance, ingredients- vs. recipe-based processing yields inconsistent results. Main results of the 16 participating countries were:

- The highest consumption of cereals was reported in the Central and East region (292 g/capita/day). The North region (223 g/day) was far below all the regions' average.

- The Central and East region had the highest consumption of added sugar and sugar products ( $46 \mathrm{~g} / \mathrm{capita} /$ day). In the South region, represented by Italy, less than half of this quantity was reported ( $20 \mathrm{~g} /$ day).

- As for sugar and sugar products, the highest intake of fats was reported in the Central and East region ( $39 \mathrm{~g} /$ capita/day). The lowest values were reported in the West region ( $28 \mathrm{~g} /$ day). The other three regions were positioned around $35 \mathrm{~g} /$ day.

- The consumption of starchy roots and potatoes was highest in the Central and East region (165 g/capita/day), followed by the North (126 g/day) and West regions (97 g/day). The value for the South region was exceptionally low (48 g/ day).

- Consumption of vegetables was very high in the South (249 g/capita/day) and Central and East regions (248 g/day). The North region (140 g/day) was far below average.

- The highest intake of fruits was reported in the Central and East (209 g/capita/ day) and South regions (203 g/day). Fruit consumption was considerably lower in the North (129 g/day) and West regions (113 g/day). On average, only four countries (Poland, Germany, Italy, and Austria) have met the recommendation of consuming at least $400 \mathrm{~g}$ of fruits and vegetables per day.

- The consumption of fruit and vegetable juices was reported highest in the Central and East region (667 g/capita/day). The lowest values emerged in the North region (331 g/day).

- The Central and East (633 g/capita/day) and North regions (625 g/day) had the highest values in the category coffee, tea and cocoa. In contrast, only $124 \mathrm{~g} /$ day was accounted for in the South region. 
- The highest consumption of alcoholic beverages was reported in the West region (235 g/capita/day), whereas the lowest values appeared in the South region (126 g/day). The North and Central and East regions showed equal results (181 g/day).

- The consumption of meat and meat products was higher in the Central and East region (194 g/capita/day) than in the West (174 g/day), South (137 g/day), and North regions (134 g/day).

- The highest consumption of fish and seafood was found in the South region (43 g/capita/day). As the Central and East region (18 g/day) is predominantly represented by landlocked countries, the low intake emerged as expected.

- Consumption of eggs was found to be highest in the Central and East region (25 g/capita/day), followed by the South (18 g/day), West (18 g/day), and North regions (17 g/day).

- The highest consumption of milk and dairy-based products was reported in the North region (416 g/capita/day). The lowest intake was found in the South region (212 g/day).

- The differences concerning tap water consumption were relatively small between the West (231 g/capita/day) and South regions ( $206 \mathrm{~g} /$ day). Tap water intake was highest in the North region (630 g/day) and lowest in the Central and East region (136 g/day).

\section{Energy and Nutrient Intake in Europe}

Assessments of individual energy and nutrient intake allow a better characterization of nutrition in a given country than the agricultural statistics previously presented. Such data are regularly collected in the majority of the European nations. Since the last European Nutrition and Health Report, the European Food Safety Authority (EFSA) has taken over the task of gathering the available data aiming for a harmonization. While methodological differences in the nutritional assessment of the participating countries still led to a certain incoherence of the data presented, comparability has improved as especially the age groups were uniform with a slightly higher variability only for children.

Information on individual intake of energy and major nutrients was available for sixteen of the participating countries and an overview is given in the following chapter.

\section{Children}

The energy intake in children was between 6.2 and $11.7 \mathrm{MJ}$ /day in boys and between 5.5 and 10.6 MJ/day in girls and increased with age. The gap between genders became also larger with age. The protein intake contributed between 11.1 and $17.6 \%$ to total energy intake $(\% \mathrm{E})$ and was therefore above the recommendation of $10-15 \% \mathrm{E}$ [WHO, 2003]. Only children from France, Greece (4-6 years), Portugal (7-9 years) and Spain (10-14 years) did not meet the recommendations for carbohydrate intake of 50-75\% $\mathrm{E}$ [WHO, 2007]. In all countries and age groups, the fat intake was above the recommended 
maximum of 30\%E [WHO, 2003]. Apart from Italian boys aged 7-9 years, the mean intake of saturated fatty acids exceeded the recommended maximum of $10 \% \mathrm{E}$ [WHO, 2003] and polyunsaturated fatty acids were generally low. The mean daily intake of retinol equivalents was between 0.4 and $2.4 \mathrm{mg}$. Compared to the $\mathrm{D}-\mathrm{A}-\mathrm{CH}$ reference values, younger children are more likely to reach the recommendations than children from the older age groups. The vitamin D intake in European children was generally low. The recommended intake values for $\alpha$-tocopherol equivalents were between 5.3 and 14.5 $\mathrm{mg} /$ day in boys and 5.1 and $18.1 \mathrm{mg} /$ day in girls. It was particularly low in Denmark, Finland, Ireland and Sweden. In the age group of 10-14 years, only 3 countries reached the reference values (Germany, Poland, and The Netherlands). Vitamin $B_{6}$ and vitamin $\mathrm{B}_{12}$ intake were above the recommendations of the D-A-CH in all countries. The intake of folate equivalents was between 109 and $428 \mu \mathrm{g} /$ day. Only 10- to 14-year-old children from Portugal met the reference intake values [D-A-CH, 2000]. The sodium intake, in form of sodium chloride, was above the recommendations in all countries [WHO, 2003]. The recommendations for calcium intake were better met by younger children. It was especially low in Austrian and Polish children. Intake of iron was sufficient for boys and girls aged up to 10 years but very much below the D-A-CH recommendations in girls from 10 to 14 years apart from Portuguese girls aged 13. For zinc, the recommendations [D-A-CH, 2000] were generally better met in younger age groups and in boys.

\section{Adolescents}

The intake of energy was between 9.5 and $14.5 \mathrm{MJ} /$ day in male adolescents and between 6.8 and 9.7 MJ/day in female adolescents and therefore in general below the reference intake values [D-A-CH, 2000]. The share of protein in total energy intake (12.4-17.8\%E in male and $12.0-18.0 \% \mathrm{E}$ in female adolescents) was within or slightly above the recommended range whereas for carbohydrates, it was in general lower than the recommendation $(39.7-57.0 \% \mathrm{E}$ in male and $38.6-57.0 \% \mathrm{E}$ in female adolescents) [WHO, 2003 and 2007]. Fat contributed $28.0-40.4 \%$ and $25.5-41.9 \%$ of total energy in male and female adolescents, respectively. The fatty acid pattern was unfavorable; the intake of saturated fatty acids was above the recommendation in every country and in both genders, whereas the intake of polyunsaturated fatty acids was below the recommendation [WHO, 2003 and 2009b] in most of the participating countries. In most participating countries, the intake levels of retinol equivalents, vitamin $\mathrm{D}$, a-tocopherol equivalents and folate equivalents were below the recommendations [D-A-CH, 2000]. The intake of calcium, magnesium as well as the intake of iron and iodine in female adolescents was in general lower whereas the intake of sodium was higher than the recommendations in both genders [D-A-CH, 2000; Eurodiet, 2000].

\section{Adults}

Daily energy intake was between 8.5 and $13.9 \mathrm{MJ}$ in men and between 6.3 and 11.4 $\mathrm{MJ}$ in women. In most of the participating countries, it was below the reference values [D-A-CH, 2000]. Protein intake amounted to $13.5-18.5 \% \mathrm{E}$ in men and $13.1-19.3 \% \mathrm{E}$ 
in women and, thus, was within or slightly above the recommended range [WHO, 2003]. Carbohydrates contributed 36.8 and $51.0 \%$ of total energy intake in men and between 37.7 and $51.8 \% \mathrm{E}$ in women. In most of the participating countries, fat intake was above the recommended range $(28.4-45.0 \% \mathrm{E}$ in men and $29.9-47.2 \% \mathrm{E}$ in women) and in general, the fatty acid pattern did not meet the recommendations [WHO, 2003 and 2009] either. Intake of most vitamins was within the recommendations except for vitamin $D, \alpha$-tocopherol equivalents and folate equivalents for which the reference intake levels [D-A-CH, 2000] were not met. In most of the participating countries, intake of calcium, magnesium and iron (women only) was also below the respective recommendations [D-A-CH, 2000].

\section{Elderly}

Daily energy intake was between 7.1 and $13.0 \mathrm{MJ}$ in elderly men and between 5.8 and $10.9 \mathrm{MJ}$ in women. Protein intake was in the range of $13.6-19.0 \% \mathrm{E}$ in men and 13.2$20.0 \% \mathrm{E}$ in women. The recommended intake level for total carbohydrates of $50-75 \% \mathrm{E}$ [WHO, 2007] was only met by elderly from Norway and Portugal as well as by female elderly from Finland and Ireland. Fat contributed between 26.7 and $43.9 \% \mathrm{E}$ in male and between 28.0 and $45.3 \% \mathrm{E}$ in female elderly and was therefore in general above the recommendation [WHO, 2003]. As was the case with adults, the intake was below the respective recommendations for vitamin $\mathrm{D}, \boldsymbol{\alpha}$-tocopherol equivalents and folate equivalents as well as calcium, magnesium and iron (women only) [D-A-CH, 2000; Eurodiet, 2000].

\section{Indicators of Health Status in Europe}

Overweight and obesity are a major health threat in the European Union. It already affects children, in whom prevalence reaches up to $36 \%$ in 10- to 14-year-old Spanish girls and 7- to 9-year-old Italian boys. With increasing age, a gender difference became apparent inasmuch as adolescent girls showed a lower prevalence than their male counterparts. This discrepancy was maintained through adulthood in most countries. $21-37 \%$ of women aged $19-64$ years were overweight, $7-36 \%$ obese. For men, the respective ranges were 35-54 and 6-29\%. The highest prevalence of both, overweight and obesity was found in Greek women and in Cypriot men. Taken together, overweight and obesity affected as much as $73 \%$ of women and $83 \%$ of men in these two countries. In all participating countries, mean body mass index (BMI) exceeded the range of $21-23 \mathrm{~kg} / \mathrm{m}^{2}$ recommended as optimal by the WHO [WHO, 2000; WHO, 2003] with highest values found in Greek women and Lithuanian men. BMI of elderly was higher than in younger adults. However, according to the higher cut-off points for overweight recommended for this population, mean BMI was within the reference range of $24-29 \mathrm{~kg} / \mathrm{m}^{2}$ except in Greek women and Austrian men. These two countries also showed the highest prevalence of overweight and obesity for the respective gender. No marked regional differences were observed in any age group. 
Data on blood lipids were available for adults and elderly from 8 and 6 countries, respectively. In most countries and collectives, total as well as LDL cholesterol concentrations exceeded the reference levels of $<5$ and $<3 \mathrm{mmol} / \mathrm{l}$, respectively. Total cholesterol was within the reference in Greek, Spanish and British adults of both genders (the latter aged 19-34 years), as well as in Romanian men $\geq 19$ years and Finnish men $\geq 65$ years. For LDL cholesterol levels, this was only the case in Lithuanian women aged 19-64 years. HDL cholesterol levels were above the reference value of $>1$ and $1.2 \mathrm{mmol} / \mathrm{l}$ for men and women, respectively. Triglyceride levels exceeded the reference value in 19- to 64-year-old Portuguese and Lithuanian men and Romanians of both genders. The atherogenic quotient (given as total cholesterol/HDL-cholesterol) was within the reference range [Graham et al., 2007].

Data on mortality were obtained from the European Health for All database of the WHO [WHO, 2009a] and was available from ten European countries for 2007. In women, total mortality rate was highest in Latvia (777 deaths/100,000 women alive), followed by Romania and Lithuania (764 and 731 deaths/100,000 women alive, respectively) and lowest in Finland and Austria (445 and 448 deaths/100,000 women alive). In men, the highest total mortality rate was observed in Lithuania with 1,621 deaths/100,000 men alive, followed by Latvia and Romania (1,565 and 1,242 deaths/100,000 men alive), the lowest in The Netherlands and the United Kingdom (708 and 712 deaths/100,000 men alive). Mortality rate were always higher in men than in women.

Most death cases were attributable to cardiovascular diseases (CVD) and malignant neoplasms $(\mathrm{MN})$. Latvia, Lithuania and Romania showed the highest mortality rates from CVD, The Netherlands the lowest. For MN, it was highest in Lithuania and Latvia for men and in the Czech Republic and the UK for women. Greece and Finland showed the lowest MN mortality rates in women, Austria and Finland in men. Mortality from both causes was higher in men than in women.

Data on incidence of malignant neoplasms (MN) in 24 European countries was obtained from the GLOBOCAN database 2002 [Ferlay et al., 2004] from the International Agency for Research on Cancer in Lyon (IARC). Denmark followed by Germany and Sweden had the highest incidence of $\mathrm{MN}$ from all sites except skin in women (496, 467 and 464 new cases/100,000 inhabitants, respectively), Italy followed by Belgium and Hungary in men (586, 585 and 560 new cases/100,000 inhabitants, respectively). Romania had the lowest incidences for both genders (243 and 297 new cases/100,000 inhabitants, respectively). MN incidence was higher in men than women in all countries but Denmark.

In men, cancers of the lung and prostate were most common, breast cancer in women. MN affected men to a higher degree than women, especially in the case of lung cancer. Incidence of $\mathrm{MN}$ of the colon and rectum and the stomach was more comparable in both genders.

Data on the prevalence of diabetes mellitus (DM) of type I and II in 25 European countries in 2007 was obtained from the International Diabetes Federation (IDF) 
[IDF, 2006]. It was highest in Cyprus, affecting $8.9 \%$ of the total population. In turn, the UK and Norway had the lowest prevalence with 2.9 and $3.5 \%$ of the total population, respectively. The rest of the countries could be separated in two blocs, one regrouping countries with a DM prevalence between 5.1 and $5.9 \%$ of the total population, the other countries showing a higher prevalence between 7.6 and $7.9 \%$.

Data on smoking behavior of young, middle-aged and elderly adults of both genders were available from 15-17 countries across Europe. Generally, more smokers were found among men than women. Highest numbers of smokers were observed in young adult men with the maximum value seen in Greece (57.8\%). With age, the proportion of smokers decreased while that of ex-smokers increased in most countries. However, in Denmark adherence to smoking was constant across all age groups.

Alcohol consumption was below the respective recommended maximum intake levels of 10 and $20 \mathrm{~g} /$ day for women and men in most of the 14 European countries for which data were available. Elderly Danish women and Portuguese men showed the highest consumption with 14.0 and $31.8 \mathrm{~g} /$ day, respectively. High proportions of alcohol abstainers among adults and elderly were found in Romania and Lithuania.

In 2004, the highest percentages for respondents never engaging in physical activity were found in Portugal (66\%), Hungary (60\%), and Italy (58\%). The lowest rates were identified in Finland (4\%), Sweden (7\%), and Denmark (17\%). These results confirm the prevalent North-South gradient in regard to leisure-time physical activity in European nations. Age and educational attainment of respondents were also related to the engagement in exercise and sport.

The rate of initiation of breastfeeding was almost 100\% in Norway, Lithuania, Sweden, and Denmark. Ireland was the only country with less than half (46\%) of mothers beginning to breastfeed their newborn in the most recent survey.

The North region reached the highest rates in the categories initiation of breastfeeding and breastfeeding at the ages of 6 and 12 months. The Central and East region had the highest values for exclusive breastfeeding at the age of 6 months and the second highest in all other categories. Overall, the West region had the lowest rates and the South region the second lowest. Mother's milk alone provides all the nutrients an infant needs during the first 6 months of life. Hence, it is the ideal nourishment for this age group and no additional liquid or solid food is needed.

\section{Food and Nutrition Policies in Countries of the European Union}

The inclusion of a chapter addressing Food and Nutrition Policy (FNP) across Europe in the edition of ENHR 2009 highlights the importance of this topic and its potential impact on the nutritional and health status of European citizens. The development of policies addressing food and nutrition in Europe has, in general, shifted from a focus on providing the general population with secure and sufficient amounts of food to interventions related to food safety and the prevention of nutrition-related health problems. 
The objective of this present analysis was to summarize the actions of FNPs currently carried out in countries participating in the ENHR 2009, with special attention to initiatives addressing food fortification and food-based dietary guidelines (FBDG).

A questionnaire incorporating expert feedback was sent to the participating countries. Only the information provided by the partners who responded was included in this report.

Twenty-one of 25 countries provided data about FNP in their countries. All of them had a specific policy document on food and nutrition or various nutritionrelated programs (except Ireland). Intersectorial collaboration was the foundation of developing the FNP in most of the countries, with the lead institution commonly being a Government Ministry. A coordination mechanism existed in all countries except Hungary, Poland and Sweden. FBDG comprised a policy implementation tool existing in all participating countries except for Ireland and Slovenia. Most countries reported a periodical revision of their FBDG and for certain countries, FBDGs were the result of intersectorial collaborations between several institutions. The pyramid was the graphic most countries applied to represent their FBDG. Other countries included specific written guidelines, tables, recommendations or other graphical representations. Mandatory food fortification with iodine in salt was reported in Austria, the Czech Republic, Denmark, Finland, Lithuania, Poland, Romania and Slovenia. In Finland and Sweden fortification of margarine with vitamin D and A, and vitamin D in milk products was mandatory. In Hungary and Austria the fortification (vitamins and minerals) of certain baby products, infant formulas, formulas for special medical treatment and special formulas for obesity treatment had mandatory fortification requirements. The fortification of certain foods was recommended in Hungary, The Netherlands, and Spain.

\section{Conclusions}

An optimal FNP should comprehend issues related to nutrition and health, food safety and food production. All participating countries had strategies addressing the main areas of interest related to health and nutrition. However, in some cases these did not constitute a 'real' policy as actions were not issued by a single body and were distributed throughout a variety of distinct documents. The extension and coordination between relevant sectors involved in FNP development, coordination and implementation need further attention. There is also a need for coordinated management of nutritional data for their application in planning and evaluation, such as in the establishment of appropriate indicators to gauge the degree of policy implementation and effectiveness, or in the identification of areas where an intervention is required. 


\section{Outlook}

The main objective of the ENHR 2009 was to describe and comment on the nutrition and health situation in the European Union. Trends in food supply and food availability were evaluated in countries and geographic regions of the EU, using available data on country level. Based on individual surveys, special emphasis was put on food consumption in adults and energy and nutrient intake of all age groups. Another specific objective of the ENHR 2009 was a comprehensive analysis of food and nutrition policies in countries of the EU.

The most prominent trends, prevalent inadequate health indicators and dietary habits are:

- An increase of the proportion of animal products in total energy supply in Southern and Central-Eastern European countries, a slight decrease in the North and no change in the West.

- A decrease of the average carbohydrate supply of all countries (-5\%), an increase in that of fat to the same extent.

- An excessive average supply and availability of meat and meat products.

- Food availability varied between countries, but certain regional characteristics are discernible:

- higher availability of fruits and vegetables in the South, however the availability of fruit is not always related to that of vegetables,

- higher availability of cereals and potatoes in the Central-Eastern region,

- strikingly high milk availability in the North,

- highest consumption of added sugar (46 g/capita/day) and added lipids (39 g/ capita/day) in Central-Eastern European countries,

- higher consumption of alcoholic beverages in the West region, whereas the South indicated the lowest.

- Energy intake of all age groups was below the reference intake values in most countries. Intake of protein was satisfying, but too low for carbohydrates and excessive for total fat.

- Intake of vitamin $\mathrm{D}$ and folate was generally low in nearly all age groups.

- Intakes of calcium, magnesium, iron in women, and, in some age groups, iodine were generally low. Sodium intake in the form of sodium chloride was above the recommended level in all age groups and countries. 
- An alarmingly high prevalence of overweight and obesity in all countries with no marked regional differences observed in any age group.

- A varying total mortality rate among countries, always higher for men than women. Most deaths were attributed to cardiovascular diseases and malignant neoplasms. Prevalence of diabetes mellitus also varied, ranging from $2.9 \%$ in the UK to $8.9 \%$ in Cyprus.

- Generally, more smokers were found among young adult men than women. With age, the proportion of smokers decreased and that of ex-smokers rose in most countries.

- Alcohol consumption was below the respective recommended maximum intake levels for women and men in most assessed countries.

- With regard to leisure time physical activity, a clear South-North upward gradient was observed which was also associated with age and educational attainment of individuals.

- The North region revealed the highest rate of breastfeeding (initiation and at 6 and 12 months of age), while the West had the lowest.

The food and nutrition policies are not consistent. All participating countries had strategies addressing the main areas of health and nutrition, but, in some cases, these did not constitute a real policy. The extension of and coordination between sectors involved in the development and implementation of food and nutrition policies needs further attention.

Despite a considerable improvement of the quality of data collection and assessment methods, there is still a need for better harmonization of databases and survey methods. The procedures used in this project proved suitable to collect, evaluate, and document information on major indicators of diet and nutrition-related health aspects only. In order to be able to accurately and comprehensively describe the nutritional status of particular population groups, laboratory assessment of the nutritional status is needed. 


\section{Project Background and Methodological Aspects}

The general aim of the European Nutrition and Health Report 2009 was to provide a comprehensive up-to-date documentation on the nutrition and health situation in the Europe Union.

The specific objectives were

- To describe trends in food supply

- To compare average daily individual food availability

- To evaluate food consumption in adults based on representative nutrition surveys

- To evaluate energy and nutrient intake in all age groups based on national/ representative nutrition surveys

- To inform about diet-related health indicators and status

- To analyze food and nutrition policies in European countries.

The methods implied collecting and critically reviewing available data on the most common indicators used for the assessment of the nutrition and health situation of 25 European countries. The main task of the participating partners was the collection of all relevant national data and in consequence the provision of clarifications in describing and analyzing the available data in a comparable manner.

The implementation strategy was a simple, feasible four-step process (fig. 3.1):

Step 1: Selection and definition of nutrition and health indicators to be included in the report

To decide on the nutrition and health indicators to be considered following steps were taken:

- Based on the experience gained from the European Nutrition and Health Report 2004 [Elmadfa et al., 2005] a template with questions concerning the fields of information, survey, methods of analysis, references and cut-off points and others was prepared by the coordinating center and sent out to the participating countries prior to the project's kick-off meeting in October 2007.

- During the kick-off meeting a representative of each country presented available data from the respective country. 


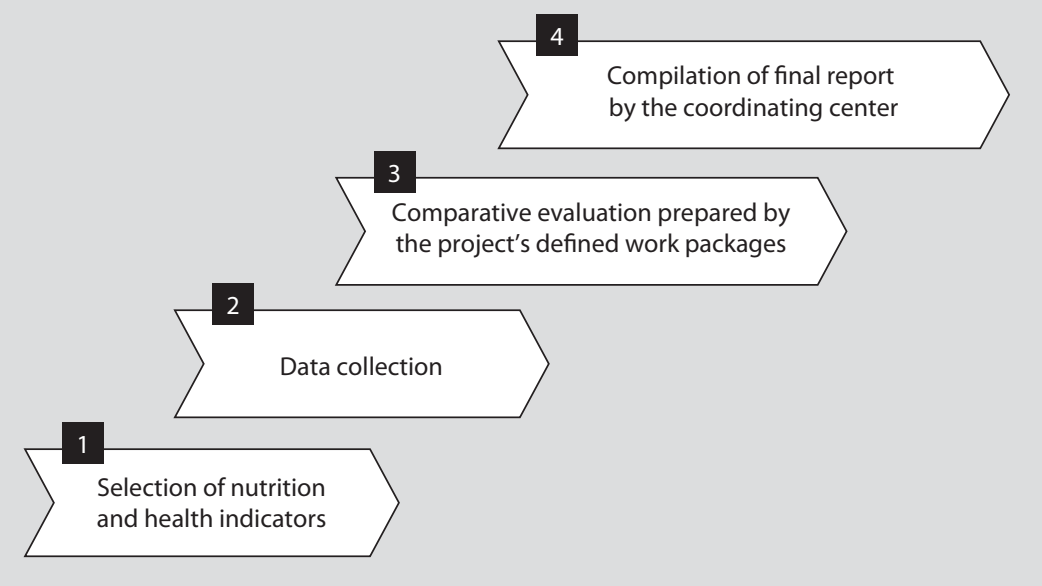

Fig. 3.1. Implementation strategy of the project Report on Nutrition and Health Status.

- After the kick-off meeting the template was revised. A more specific and accurate questionnaire with questions on available data for specified age groups was developed and sent again to the partners. All 25 countries answered the questionnaire, on that background it was possible to decide which health indicators to include in the European Nutrition and Health Report 2009.

- Recommendations and the experience of former European projects such as EFCOSUM [EFCOSUM, 2002], ECHI [ECHI, 2005] and the European Nutrition and Health Report 2004 [Elmadfa et al., 2005] were taken into account.

Step 2: Collection of existing published and authorized unpublished data in each participating country

To facilitate and harmonize data collection on nutrition and health indicators templates were developed and sent to the participating organizations.

In addition, data from already existing databases such as the WHO Health for All database [WHO, 2009a], the EFSA concise food consumption database [EFSA, 2008a] and the Food Balance sheets of the FAO [FAO, 2009] were collected.

Data on food and nutrition policy were collected by a separate questionnaire developed by the project's specific Work Package, Data on food availability at the household level were derived from the DAFNE databank (the task of another project's work package).

Step 3: Comparative evaluation of available data

Data were described and analyzed by the work packages with assistance of project partners. During the final plenary meeting of the project in June 2009 the results were discussed with the consortium.

Step 4: Compilation of the final report by the coordinating center

The main report consists of the chapters 'Trends of Average Food Supply in the European Union', 'Food Availability at the Household Level in the European Union', 


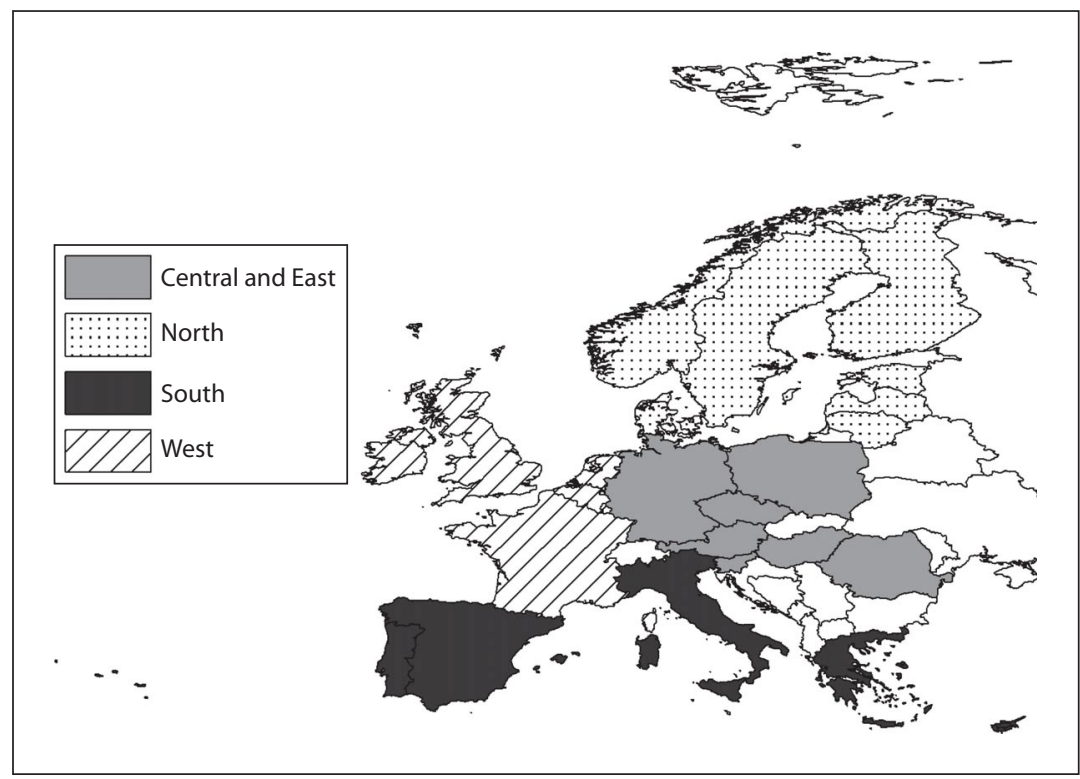

Fig. 3.2. Regions and countries of the European Nutrition and Health Report 2009.

'Food Consumption in Adults on the Basis of Dietary Surveys in European Countries', 'Energy and Nutrient Intake in the European Union', 'Health and Lifestyle Indicators in the European Union, 'Food and Nutrition Policies in European Countries' and a 'General Discussion'. Additional information on the nutrition and health situation in the participating countries is given in the chapter national reports (pp. 250-405).

In order to identify present region-specific data on diet, nutrition, physical activity, and diet-related health indicators of the European Union population following regions were defined and data analyzed accordingly (fig. 3.2):

- North: Denmark, Estonia, Finland, Latvia, Lithuania, Norway, Sweden

- South: Cyprus, Greece, Italy, Portugal, Spain

- Central and East: Austria, Czech Republic, Germany, Hungary, Poland, Romania, Slovenia

- West: UK, Belgium, France, Ireland, The Netherlands, Luxembourg, United Kingdom During the project it was not possible to collect raw data. Not all countries could provide data on nutrition and health indicators according to predefined age groups and to calculate prevalence of overweight and obesity according to the suggested methods. Nevertheless, also those data were included to get the most comprehensive picture. However, data have to be interpreted cautiously taking into consideration that not exactly the same methods were used to obtain the data.

In order to properly interpret data derived from different methods in chapters 7 and 8.1 , no means were calculated but minimum and maximum values were given as a range. 
4

\section{Trends of Average Food Supply in the European Union}

\section{Calculated Trends of Average Food Supply in the European Union}

\section{On the Basis of the FAO Food Balance Sheets}

The Food Balance Sheets (FBS) of the Food and Agriculture Organization of the United Nations (FAO), as part of the FAO's database FAOSTAT, are agricultural statistics which provide detailed information about the pattern of a country's food supply during a specified reference period. Since 1961, FBS have been compiled every year up to 2003. FBS are made up of sources of supply, such as the total quantity of foodstuffs produced in a country and the total quantity imported and adjusted to any change in stocks that may have occurred since the beginning of the reference period as well as its utilization for non-food purposes, such as feed, seed and manufacture.

Because of the equality of the methods used to obtain the data, FBS permit a comparison of international and national data and are the main source used in the assessment and appraisal of the world food situation. Moreover, the comparison in terms of commodities can be helpful for the formulation of the Common Agriculture Policy (CAP) in Europe.

FBS have some limitations. In essence, these data include food consumption at home, food consumption outside of home and wastes along the whole food chain from the production to the end-consumer, even though an attempt to estimate the overall amount of wastes is also made. Compared to methods of collecting data at household or individual level, FBS estimate food consumption only from a food supply perspective and do not differentiate between diets consumed by different population groups such as children, the elderly, or those from disadvantaged backgrounds. Furthermore, they do not give any indication of the differences between ecological zones or geographical areas within a country nor seasonal variations.

Nevertheless, FBS are a unique source for showing international trends in food supply.

Following the European Nutrition and Health Report 2004, this chapter provides an overview of the average food supply of the 25 participating countries based on 
the FBS; data from Belgium and Luxembourg are combined. In the subsequent sections, the highest and lowest supply levels for different food groups (table 4.1) as well as the calculated arithmetic average of the participating countries are presented for each year since 1961. Additionally the year 2003 will be emphasized because the European Nutrition and Health Report 2004 described the supply from 1961 to 2001. It should be noted that data for Belgium-Luxembourg, the Czech Republic, Estonia, Latvia, Lithuania, and Slovenia are only available since 1993 and data for Norway are missing.

In order to compare different regions, the participating countries were grouped as follows:

North: Sweden, Norway, Finland, Estonia, Latvia, Lithuania, Denmark

South: Portugal, Spain, Italy, Cyprus, Greece

West: UK, Belgium-Luxembourg, The Netherlands, France, Ireland

Central and East: Poland, Czech Republic, Slovenia, Romania, Hungary, Austria, Germany

For the comparison of the regions, data were weighted using the number of inhabitants from 2003 [Eurostat, 2009].

Fruits - Excluding Wine

The increasing tendency of the fruit supply described in the report of 2004 also continued on in the years 2002 and 2003 (fig. 4.1). In 2003 the highest supply was observed in The Netherlands (182 kg/capita/year) followed by Greece and Denmark with an average supply of $147 \mathrm{~kg} /$ capita/year. The countries with the lowest supply were Poland (48 kg/capita/year), Latvia (54 kg/capita/year) and Romania (65 kg/capita/year). Comparing the different regions, the supply in the South and West regions was higher than in the North and Central and East regions.

\section{Vegetables}

As shown in figure 4.2, the mean supply of vegetables increased over the past four decades. A South-North gradient can be observed. The supply in Greece (276 kg/capita/year) was in 2003 almost four times higher than in Finland (71 kg/capita/year).

\section{Potatoes}

The average supply of potatoes showed a decreasing tendency over the last 40 years (fig. 4.3). In 2003, the lowest supply was observed in Cyprus (38 kg/capita/year) and Italy $(41 \mathrm{~kg} /$ capita/year), whereas the supply was highest in Poland $(130 \mathrm{~kg} / \mathrm{capita} /$ year) and Latvia (140 kg/capita/year). The supply of potatoes was lowest in the South region and highest in the Central and East and West regions. 
Table 4.1. List of commodities classified by major food groups in FAOSTAT

\begin{tabular}{|c|c|}
\hline Fruits - excluding wine & $\begin{array}{l}\text { plantains, bananas, oranges, lemons and limes, grapefruit and pomelos, tangerines, mandarins, } \\
\text { clementines, satsumas, other citrus fruits, melons, watermelons, apples, apricots, avocados, } \\
\text { cherries, figs, grapes, mangoes, papaya, peaches, pears, persimmons, pineapples, plums, } \\
\text { quinces, blueberries, cranberries, gooseberries, raspberries, strawberries, kiwi, other fruits fresh, } \\
\text { dates, figs dried, prunes currants, raisins, other dried fruits }\end{array}$ \\
\hline Vegetables & $\begin{array}{l}\text { beets, carrots, turnips, rutabagas or swedes, onions green, onions dry, artichokes, tomatoes, } \\
\text { asparagus, cabbage, cauliflower, celery, kale, lettuce, spinach, beans green, broad beans green, } \\
\text { chili peppers, garlic, cucumbers, mushrooms, eggplant, peas green, pumpkins, squash, gourds, } \\
\text { okra, radishes, other }\end{array}$ \\
\hline Cereals & wheat, rye, barley, oats, maize, rice, mixed grains, buckwheat, sorghum, millet, quinoa, other \\
\hline Sugar and sweeteners & $\begin{array}{l}\text { sugar cane, sugar beet, sugar refined, sugar non-centrifugal, molasses, honey, other sugars and } \\
\text { syrups }\end{array}$ \\
\hline Pulses & $\begin{array}{l}\text { beans dry, broad beans dry, peas dry, chick peas, cow peas, pigeon peas, lentils, vetches, lupins, } \\
\text { other }\end{array}$ \\
\hline Oil crops & $\begin{array}{l}\text { soybeans, coconuts (incl. copra), oil palm fruit, groundnuts, olives, rape and mustard seed, } \\
\text { sunflower seed, cottonseed, linseed, hempseed, sesame seed, other }\end{array}$ \\
\hline Vegetable oils & $\begin{array}{l}\text { rape and mustard seed oil, sunflower seed oil, cottonseed oil, linseed oil, hempseed oil, sesame } \\
\text { seed oil, copra and coconut oil, palm kernel oil, palm oil, soybean oil, olive oil, maize oil }\end{array}$ \\
\hline Animal fats & butter, ghee, fish liver oil, whale oil, other \\
\hline Meat & $\begin{array}{l}\text { beef and veal, buffalo, pork, mutton and lamb, goat, horse, chicken, goose, duck, turkey, rabbit, } \\
\text { game, offal }\end{array}$ \\
\hline Fish and seafood & $\begin{array}{l}\text { freshwater fish, demersal fish, pelagic fish, crustaceans, molluscs, aquatic mammals meat, } \\
\text { aquatic plants }\end{array}$ \\
\hline $\begin{array}{l}\text { Milk and milk products - } \\
\text { excluding butter }\end{array}$ & $\begin{array}{l}\text { cow, goat, sheep, buffalo, skim, evaporated unsweetened whole, condensed sweetened whole, } \\
\text { evaporated unsweetened skim, condensed sweetened skim, dried whole, dried skim, cream }\end{array}$ \\
\hline Eggs & chicken, other \\
\hline Alcoholic b & , beer, fermented \\
\hline
\end{tabular}

Source: FAO, 2001

\section{Cereals}

Figure 4.4 shows the mean supply of cereals over the last 40 years, which was decreasing although there was a slight increase as from 1992. In 2003 the highest supply of cereals was observed in Romania (205 kg/capita/year) followed by Italy (163 kg/capita/year) and Lithuania (155 kg/capita/year). The lowest supply levels were reported in Cyprus (98 kg/capita/year), Spain (99 kg/capita/year) and The Netherlands (103 $\mathrm{kg} /$ capita/year). Concerning the different regions, no big difference can be observed. 


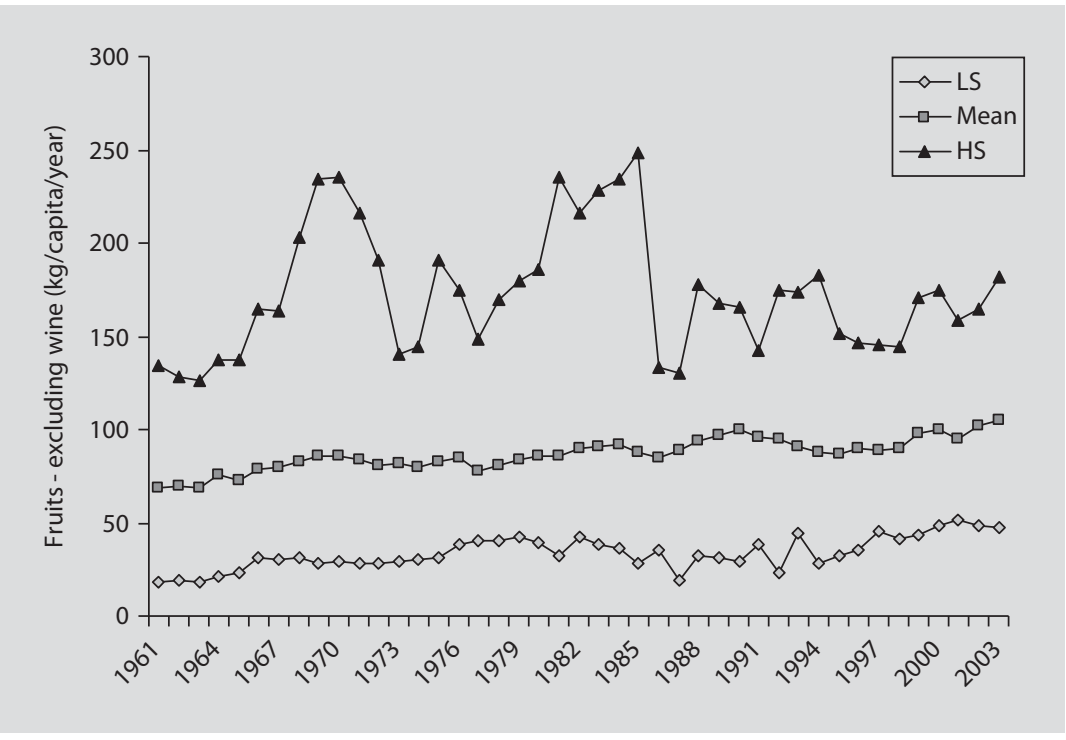

Fig. 4.1. Average supply of fruits excluding wine in the participating countries. $\mathrm{HS}=$ Highest supply; LS = lowest supply. 2003: HS = NL, LS = PL. Source of raw data: FAO, 2009.

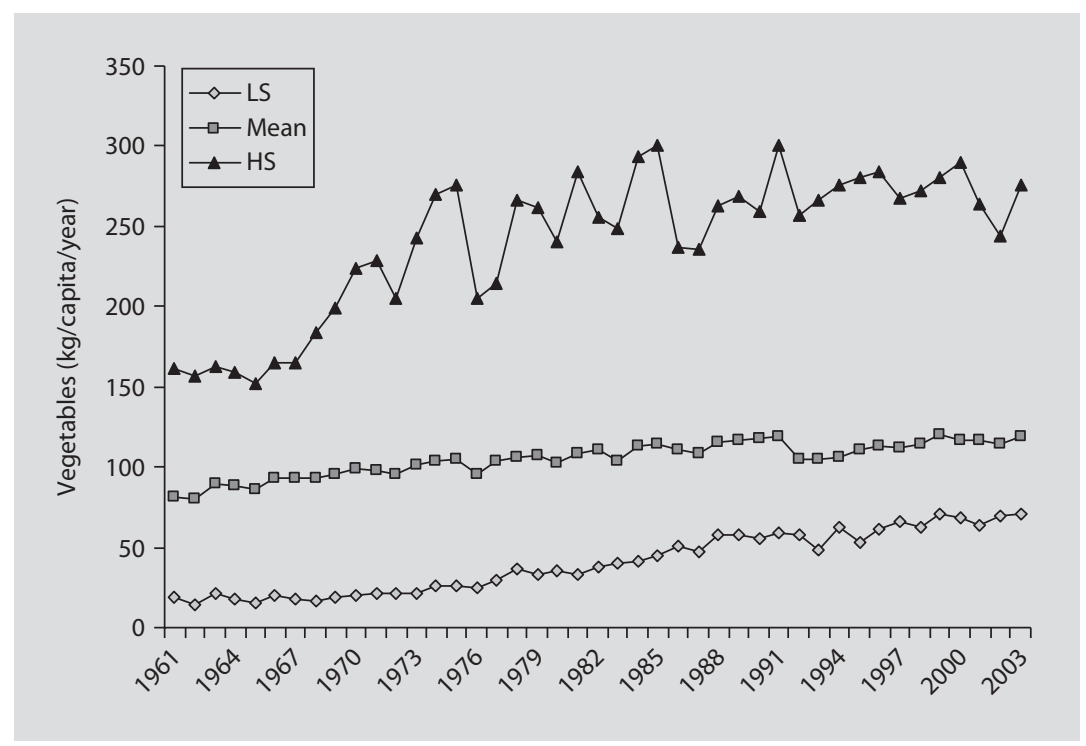

Fig. 4.2. Average supply of vegetables in the participating countries. $H S=$ Highest supply; $L S=$ lowest supply. 2003: HS = GR, LS = FI. Source of raw data: FAO, 2009. 


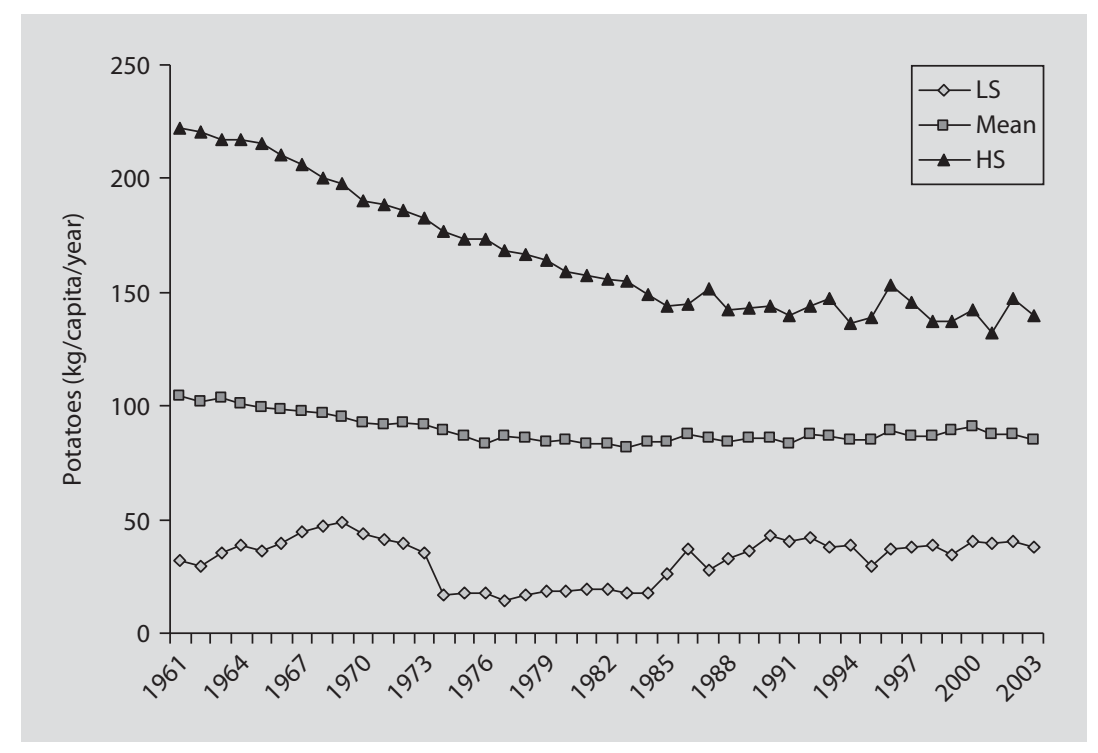

Fig. 4.3. Average supply of potatoes in the participating countries. $H S=$ Highest supply; $L S=$ lowest supply. 2003: HS = LV, LS = CY. Source of raw data: FAO, 2009.

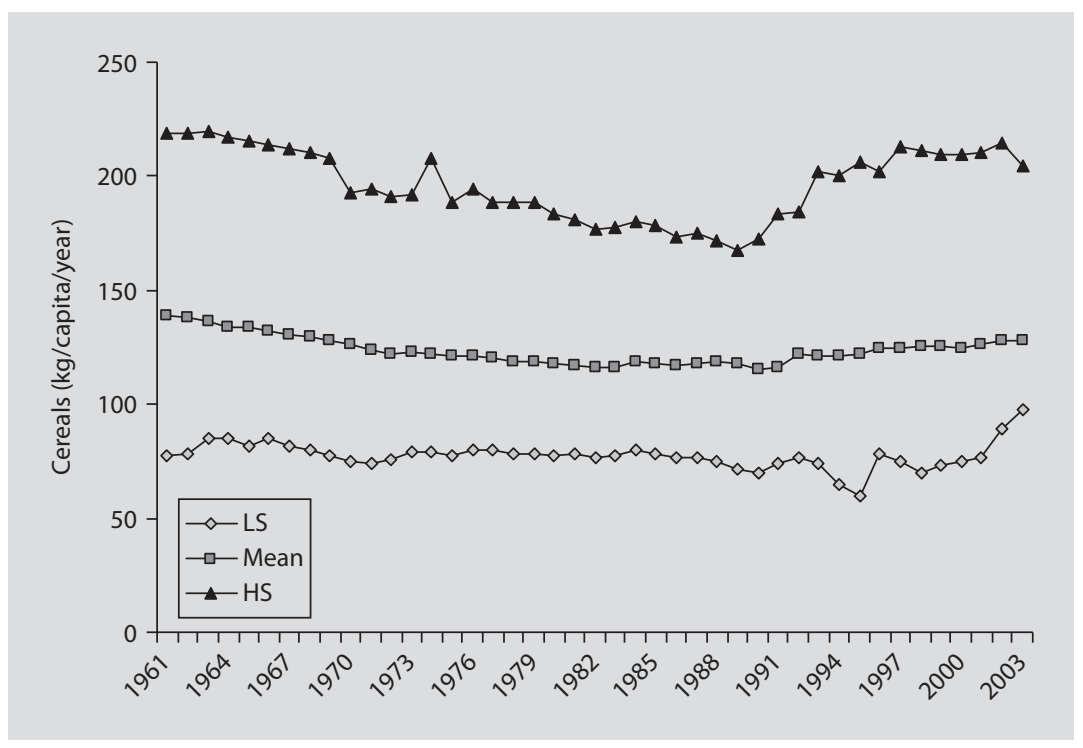

Fig. 4.4. Average supply of cereals in the participating countries. HS = Highest supply; LS = lowest supply. 2003: HS = RO, LS = CY. Source of raw data: FAO, 2009. 


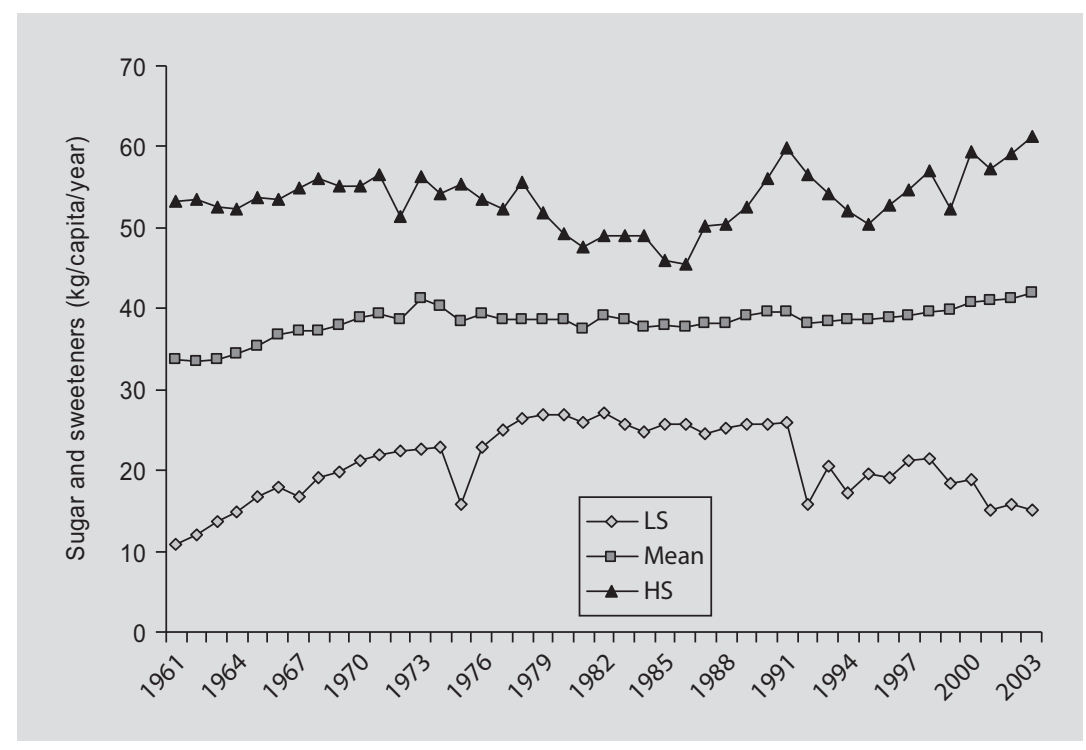

Fig. 4.5. Average supply of sugar and sweeteners in the participating countries. $\mathrm{HS}=$ Highest supply; $\mathrm{LS}=$ lowest supply. 2003: HS = EE, LS = SI. Source of raw data: FAO, 2009.

\section{Sugar and Sweeteners}

As shown in figure 4.5, the mean supply of sugar and sweeteners has increased over the last four decades. In 2003 the supply was highest in Estonia and Denmark with about $60 \mathrm{~kg} /$ capita/year. Low supply levels were found in Slovenia (15 kg/capita/year) and Romania (27 kg/capita/year). The supply was lowest in the South region.

\section{Pulses}

The amount of pulses available in the participating countries showed an undesirable decreasing tendency (fig. 4.6). As was the case with the vegetable supply, a SouthNorth gradient can be observed. The highest supply in 2003 was found in Spain with almost $6 \mathrm{~kg} / \mathrm{capita} /$ year and the lowest supply in Latvia. The supply was highest in the South region followed by the West region. The North and Central and East regions showed the lowest supply.

\section{Oil Crops}

The mean supply of oil crops in the participating countries has increased slightly since 1961 (fig. 4.7). In 2003 the supply was highest in the southern countries such as Spain (7 kg/capita/year), Cyprus (9 kg/capita/year) and Greece (15 kg/capita/year). The lowest supply levels were observed in the Baltic countries and Slovenia (about $1 \mathrm{~kg} / \mathrm{capita} / \mathrm{year}$ ). The supply of oil crops was almost twice as high in the South region as in the West and Central and East regions, and almost three times higher than in the northern countries. 


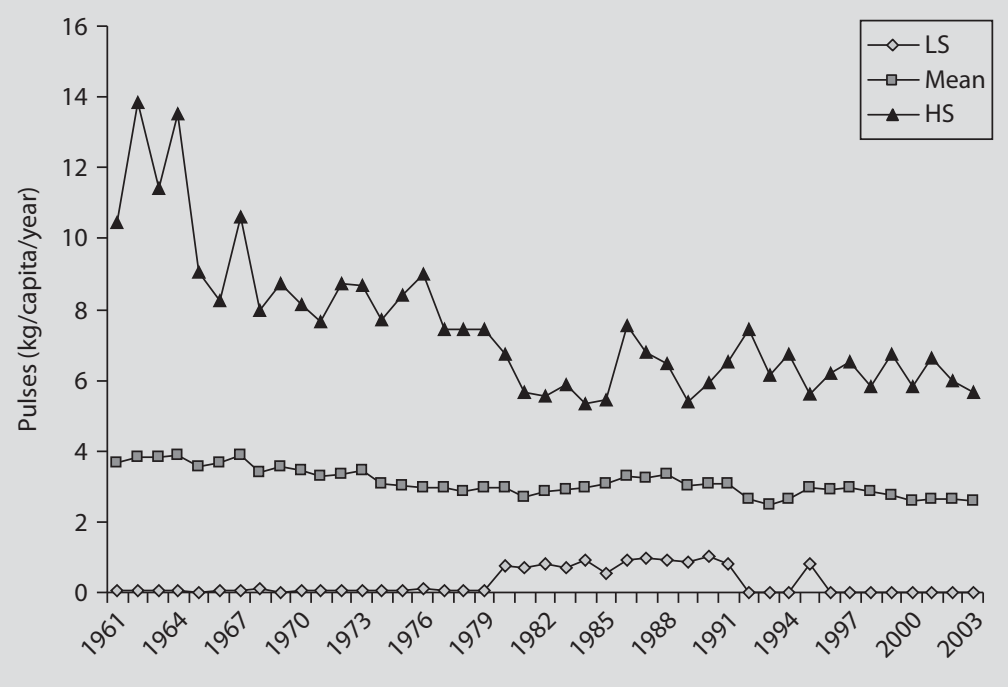

Fig. 4.6. Average supply of pulses in the participating countries. $\mathrm{HS}=$ Highest supply; $\mathrm{LS}=$ lowest supply. 2003: HS = ES, LS = LV. Source of raw data: FAO, 2009.

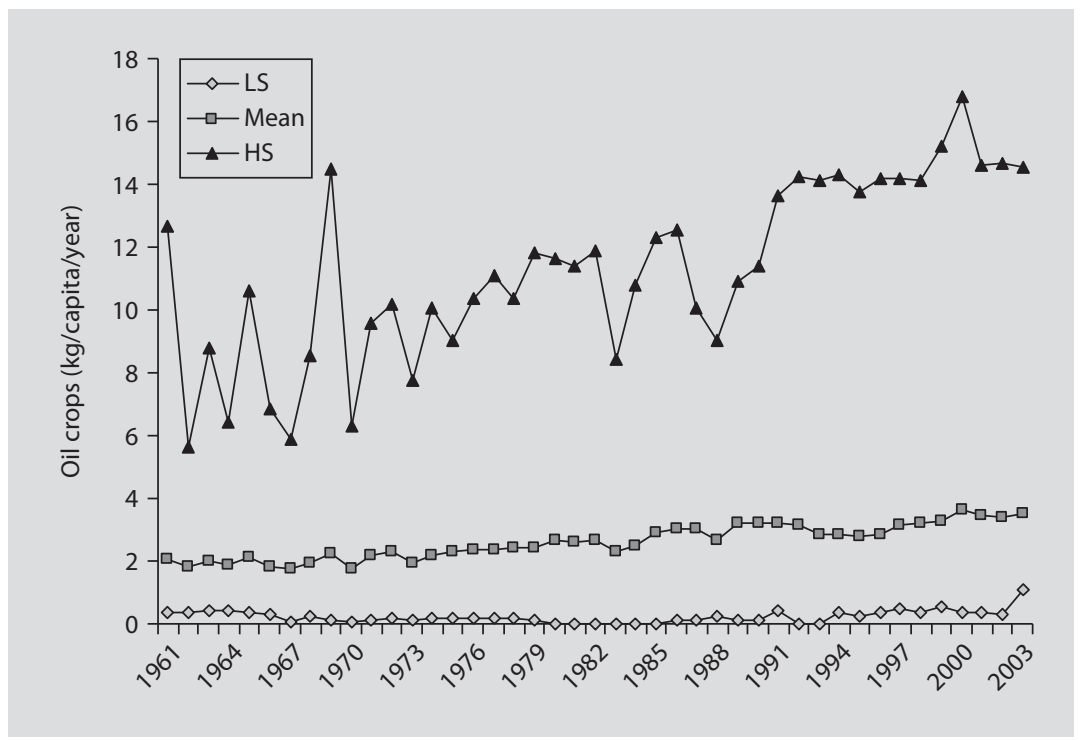

Fig. 4.7. Average supply of oil crops in the participating countries. $\mathrm{HS}=$ Highest supply; $L S=$ lowest supply. 2003: $\mathrm{HS}=\mathrm{GR}, \mathrm{LS}=\mathrm{EE}$. Source of raw data: $\mathrm{FAO}, 2009$. 


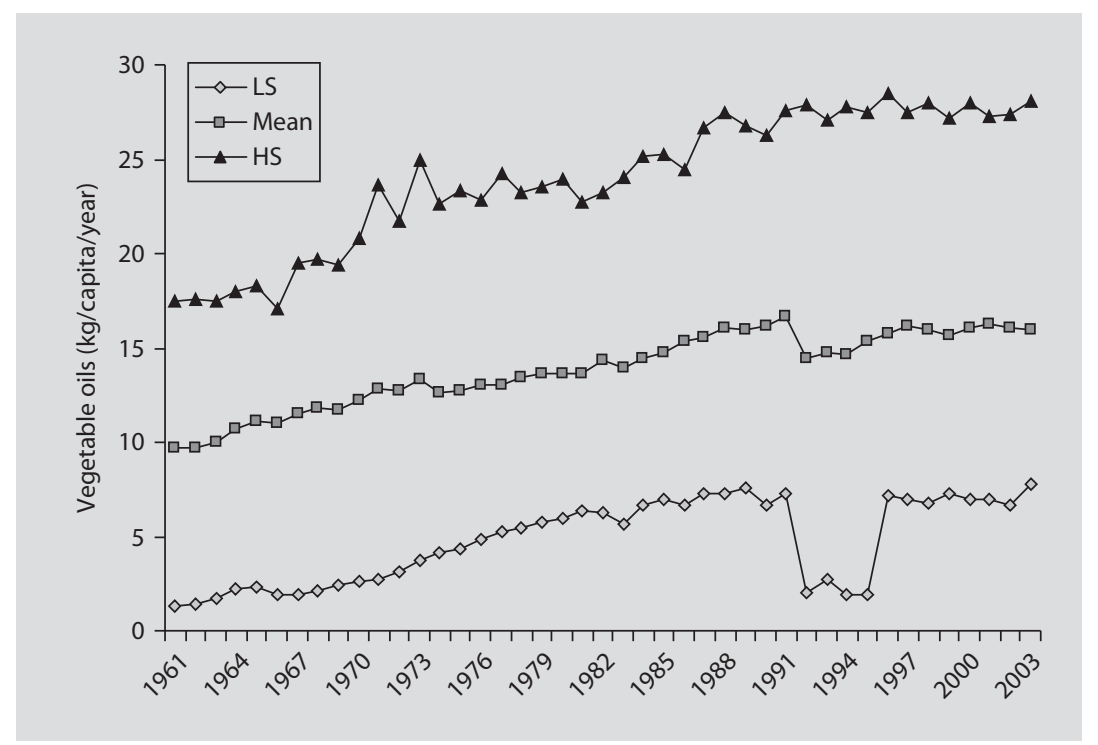

Fig. 4.8. Average supply of vegetable oils in the participating countries. $H S=$ Highest supply; $L S=$ lowest supply. 2003: HS = ES, LS = DK. Source of raw data: FAO, 2009.

\section{Vegetable Oils}

The average supply of vegetable oils in the participating countries increased from 1961 to 2003 (fig. 4.8). As was the case with the supply of oil crops, the highest supply levels in 2003 were found in the southern countries $(25-28 \mathrm{~kg} / \mathrm{capita} /$ year). The lowest supply levels were reported in Denmark, Estonia, and Slovenia ( $<10 \mathrm{~kg} /$ capita/ year). A South-North gradient can also be observed.

\section{Olive Oil}

Although there was an increase of the supply of vegetable oils, the mean supply of olive oil barely changed in the period of 1961-2003 (fig. 4.9). The highest supply was observed in Greece (16 kg/capita/year) followed by Italy (13 kg/capita/year) and Spain (12 kg/capita/year). In general, the southern countries showed a higher supply than the countries in North, West, East, and Central Europe. Romania, Lithuania, and Poland showed the lowest supply.

\section{Animal Fats}

In the past four decades, the supply of animal fats remained relatively constant (fig. 4.10). The highest and lowest supply levels were contrary to the supply of vegetable oils. The southern countries showed the lowest supply. The highest supply in 2003 was reported in Hungary (28 kg/capita/year) and the lowest in Cyprus (3 kg/capita/ year). 


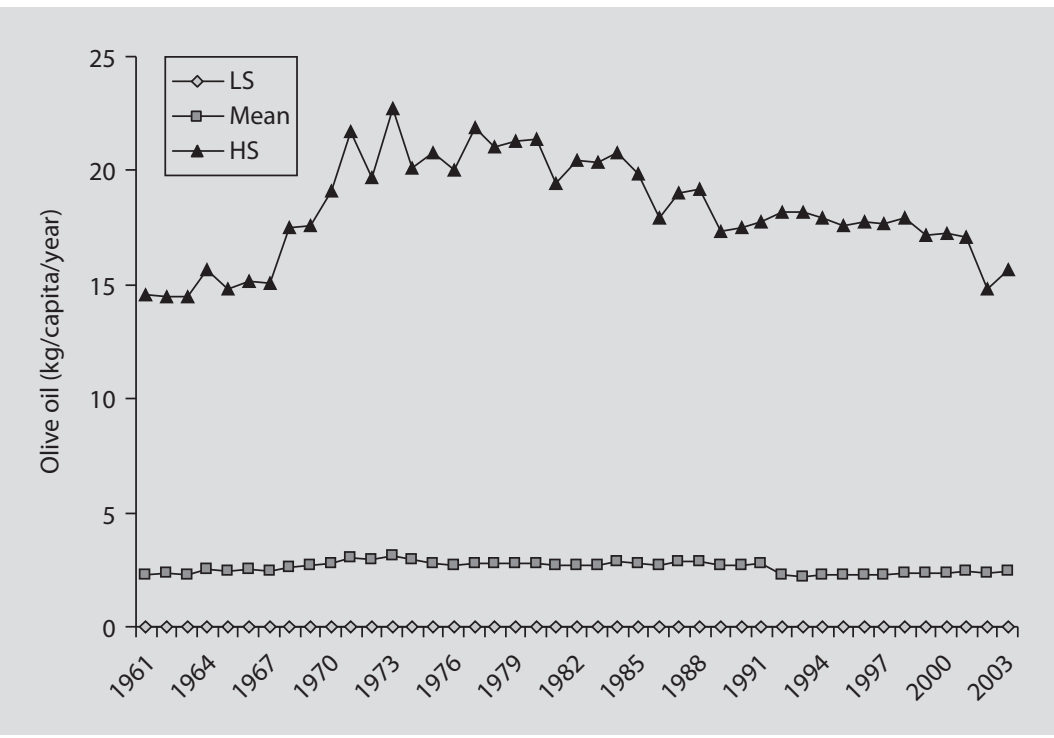

Fig. 4.9. Average supply of olive oil in the participating countries. $H S=$ Highest supply; $L S=$ lowest supply. 2003: HS = GR, LS = RO. Source of raw data: FAO, 2009.

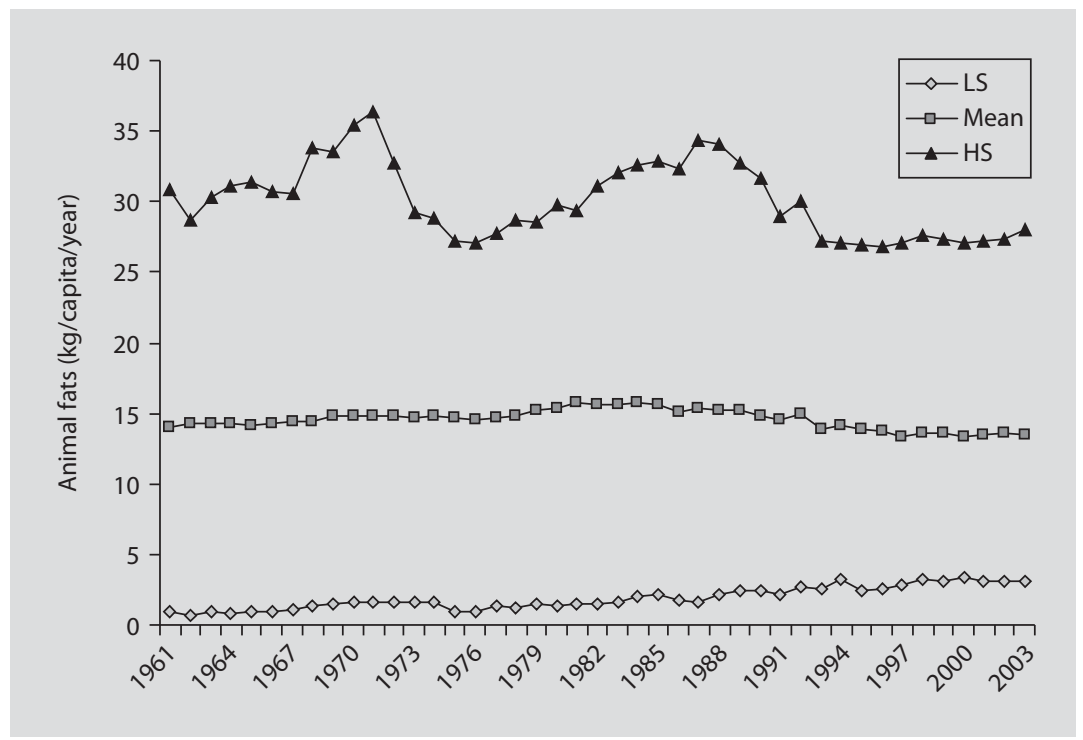

Fig. 4.10. Average supply of animal fats in the participating countries. $H S=$ Highest supply; $L S=$ lowest supply. 2003: HS = HU, LS = CY. Source of raw data: FAO, 2009. 


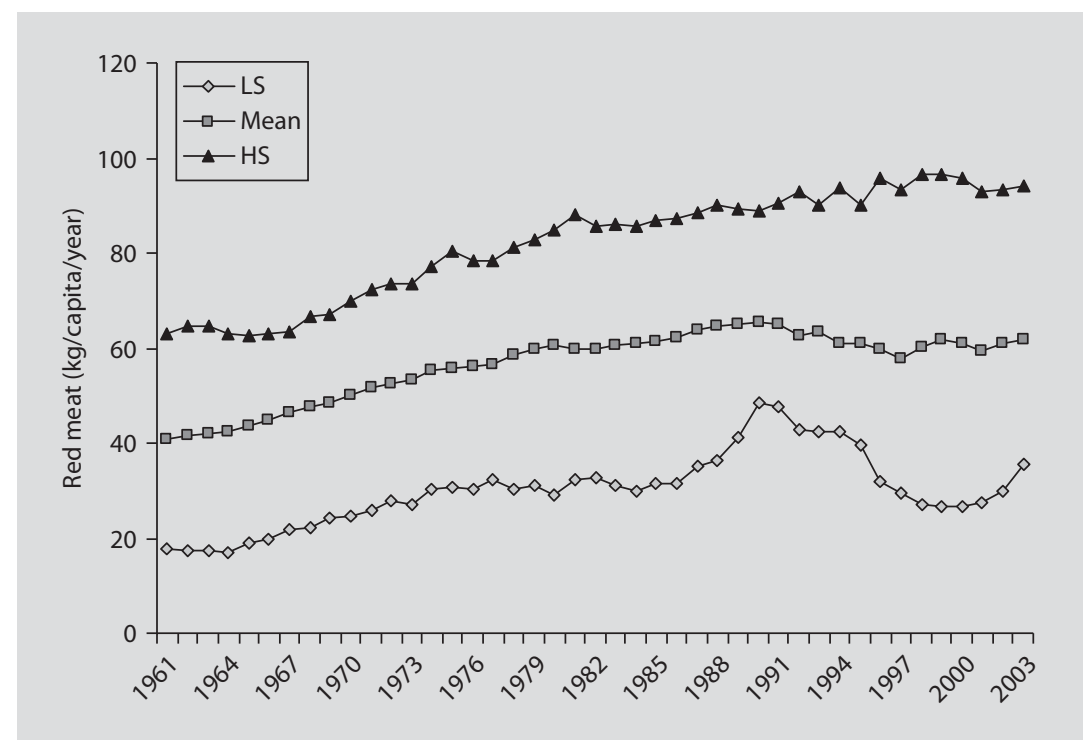

Fig. 4.11. Average supply of red meat in the participating countries. $\mathrm{HS}=$ Highest supply; $L S=$ lowest supply. 2003: HS = AT, LS = LV. Source of raw data: FAO, 2009.

\section{Red Meat}

The supply of red meat has shown an undesirable increase during the past 40 years (fig. 4.11). In 2003 the supply was highest in Austria with $94 \mathrm{~kg} / \mathrm{capita} /$ year and lowest in Latvia with $35 \mathrm{~kg} /$ capita/year. Between the regions, no big difference can be observed.

\section{Bovine Meat}

Figure 4.12 shows the mean supply of bovine meat in the participating countries from 1961 to 2003. It scarcely changed during the past four decades. In 2003, the highest supply was observed in Denmark (29 kg/capita/year) followed by France ( $26 \mathrm{~kg} / \mathrm{capita} /$ year) and Italy (25 kg/capita/year). Hungary showed the lowest supply with $6 \mathrm{~kg} / \mathrm{capita} /$ year. In 2003, the supply of bovine meat was highest in the West region followed by the North and South regions. The lowest supply was found in the East and Central European countries.

\section{Pork}

The supply of pork showed an increase in the past 40 years. The supply nearly doubled from 1961 to 2003 (fig. 4.13). The lowest supply in 2003 was observed in Latvia with $25 \mathrm{~kg} / \mathrm{capita} /$ year and the highest supply in Austria with $74 \mathrm{~kg} / \mathrm{capita} /$ year. Regarding the different regions, no big difference can be observed, although the supply was a little bit lower in the North and West regions than in the South and Central and East regions. 


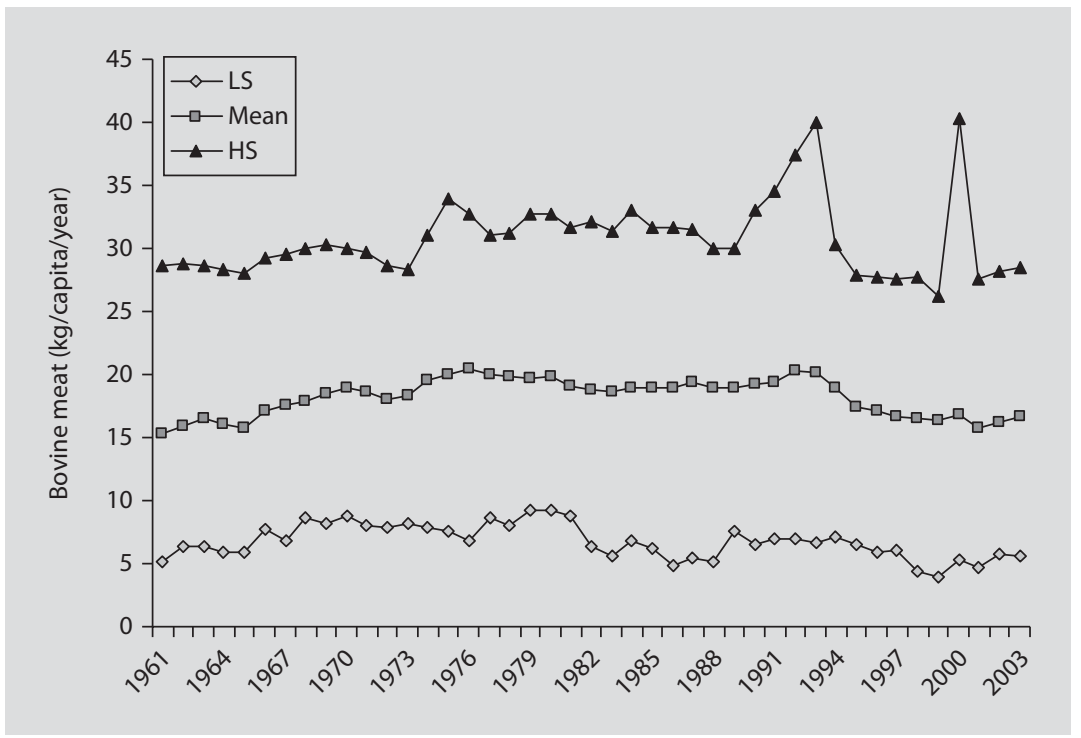

Fig. 4.12. Average supply of bovine meat in the participating countries. $H S=$ Highest supply; $L S=$ lowest supply. 2003: HS = DK, LS = HU. Source of raw data: FAO, 2009.

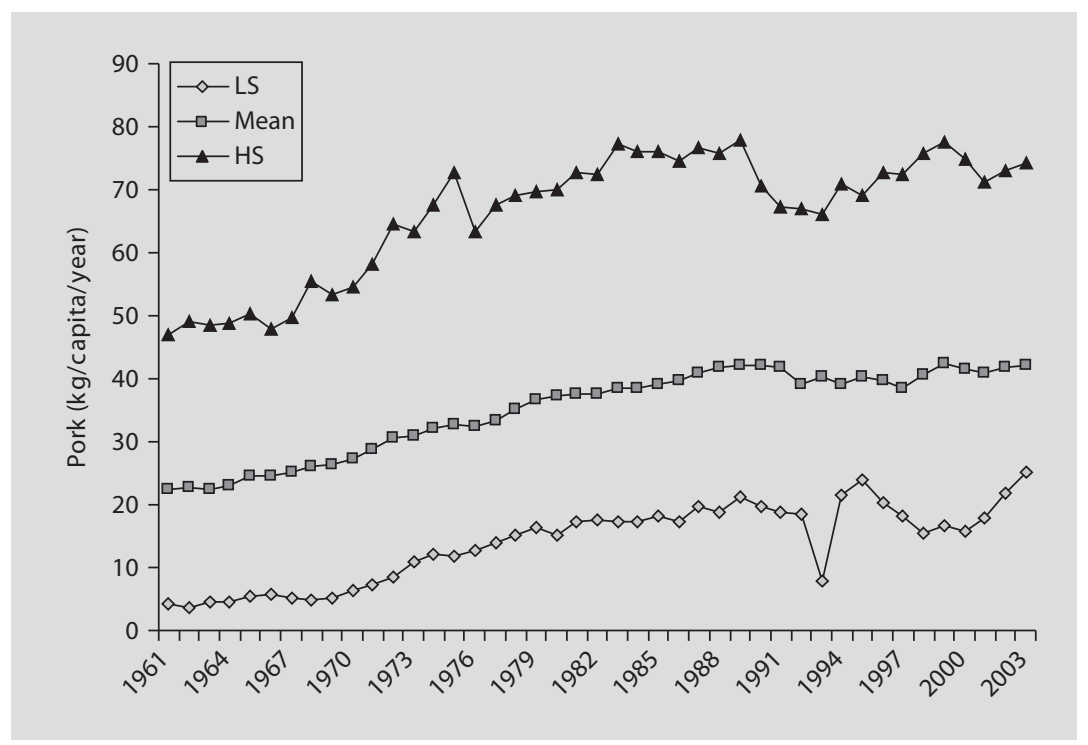

Fig. 4.13. Average supply of pork in the participating countries. HS = Highest supply; LS = lowest supply. 2003: HS = AT, LS = LV. Source of raw data: FAO, 2009. 


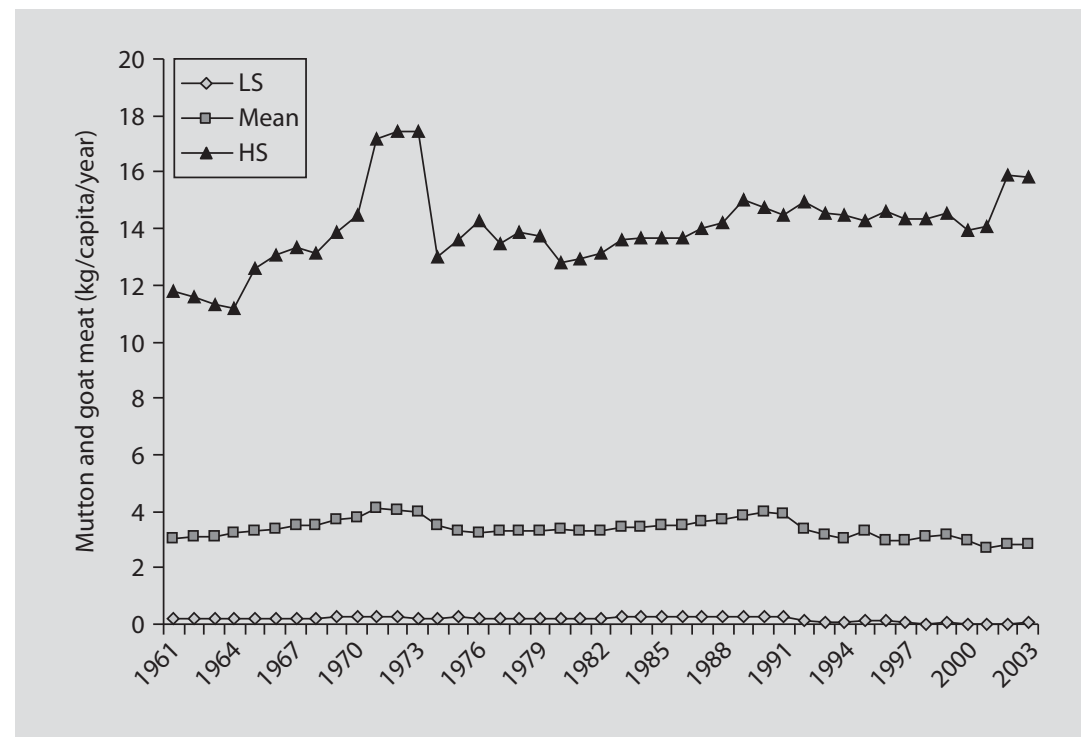

Fig. 4.14. Average supply of mutton and goat meat in the participating countries. $\mathrm{HS}=$ Highest supply; LS = lowest supply. 2003: HS = CY, LS = PL. Source of raw data: FAO, 2009.

\section{Mutton and Goat Meat}

Figure 4.14 shows the average supply of mutton and goat meat in the participating countries during the past four decades. The supply remained relatively constant. In 2003, the supply was at its highest in Cyprus ( $16 \mathrm{~kg} / \mathrm{capita} /$ year) followed by Greece (12 kg/capita/year) and Spain (6 kg/capita/year). The lowest supply was observed in Poland. In the South and West regions the supply was four times higher as in the North and Central and East regions.

\section{Poultry}

In 2003, the mean supply of poultry was almost five times higher than in 1961 (fig. 4.15). Cyprus showed in 2003 the highest supply with $37 \mathrm{~kg} / \mathrm{capita} /$ year and The Netherlands the lowest supply with $9 \mathrm{~kg} / \mathrm{capita} /$ year. Concerning the different regions, no big difference can be observed.

\section{Fish and Seafood}

As shown in figure 4.16, the average supply of fish and seafood in the participating countries increased from 1961 to 2003. The highest supply levels were observed in the northern and southern countries such as Lithuania and Portugal (60 kg/capita/year). Romania showed the lowest supply with $3 \mathrm{~kg} / \mathrm{capita} /$ year. Hence the supply is higher in the North and South regions than in the West region. The Central and East region showed the lowest supply. 


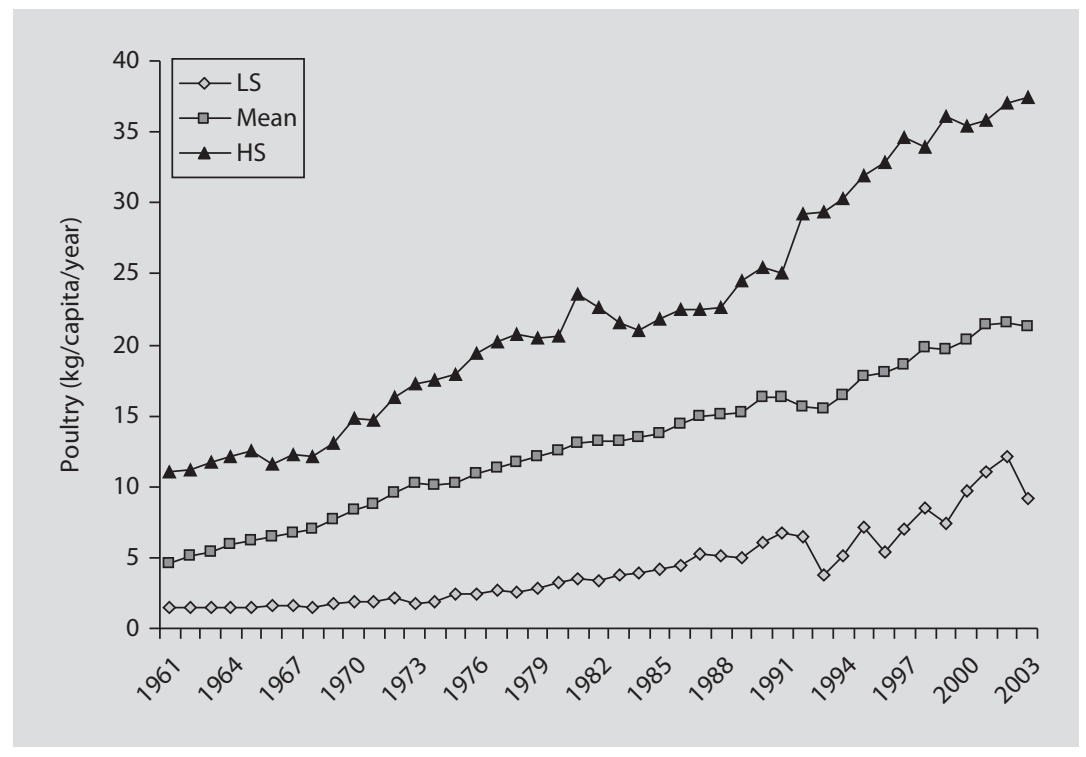

Fig. 4.15. Average supply of poultry in the participating countries. $H S=$ Highest supply; $L S=$ lowest supply. 2003: HS = CY, LS = NL. Source of raw data: FAO, 2009.

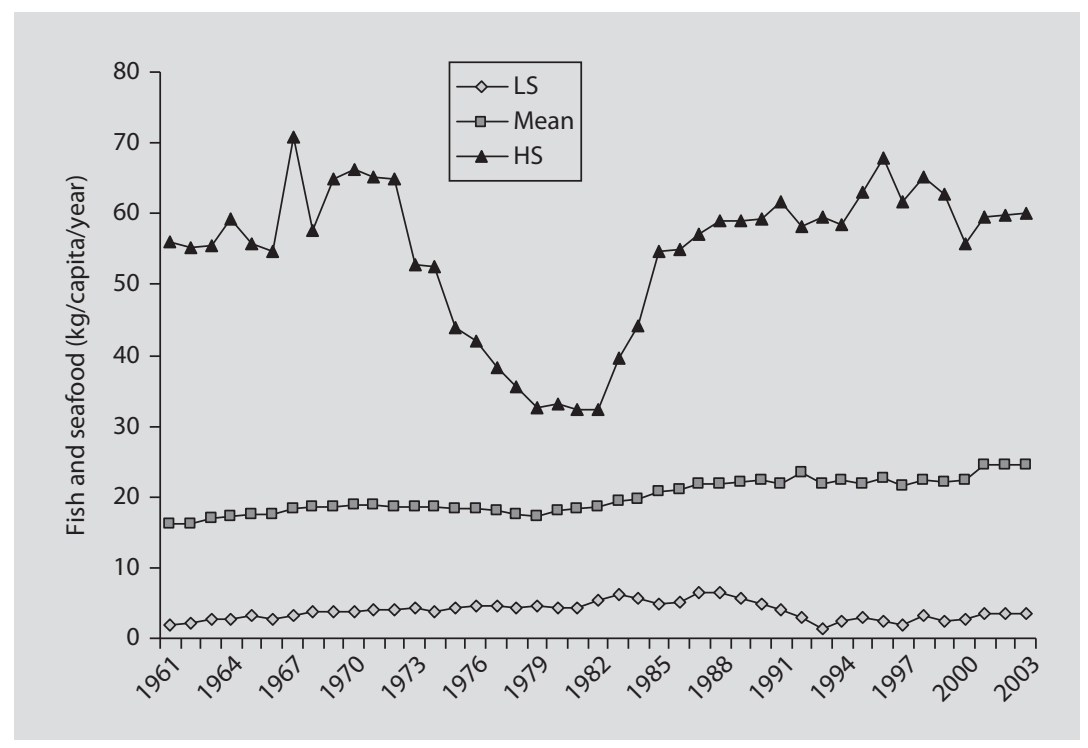

Fig. 4.16. Average supply of fish and seafood in the participating countries. HS = Highest supply; $L S$ = lowest supply. 2003: HS = LT, LS = RO. Source of raw data: FAO, 2009. 


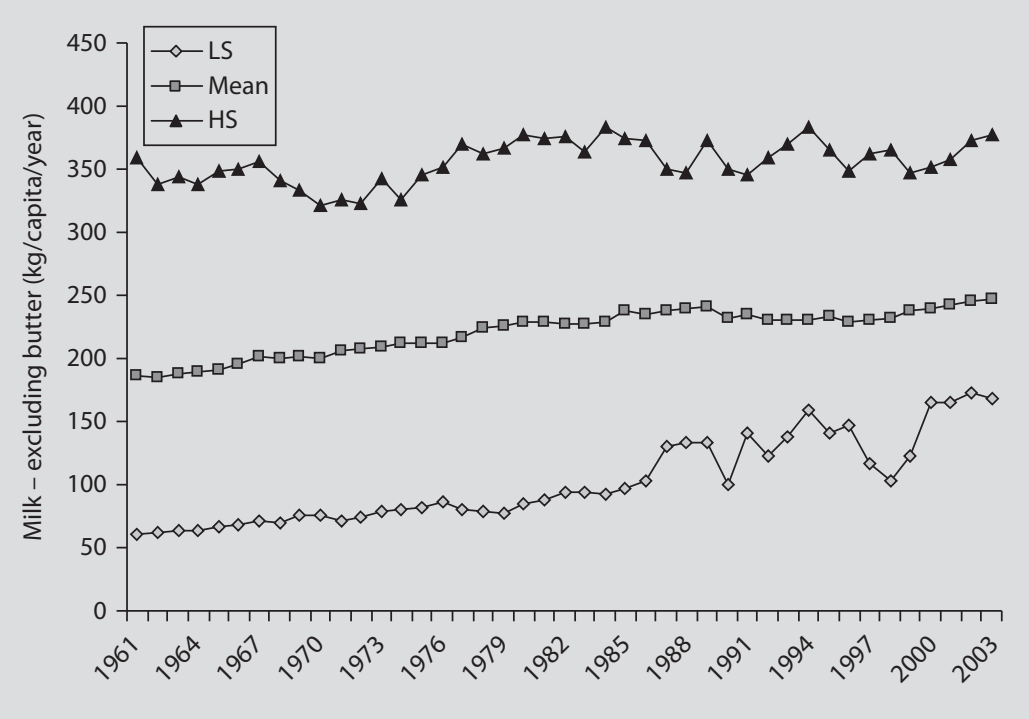

Fig. 4.17. Average supply of milk and milk products - excluding butter in the participating countries. HS = Highest supply; LS = lowest supply. 2003: HS = SE, LS = HU. Source of raw data: FAO, 2009.

\section{Milk and Milk Products - Excluding Butter}

Figure 4.17 shows the amount of milk available in the participating countries in the past 40 years. From 1961 to 2003, an increase was observed. In 2003, the Nordic countries Sweden and Finland showed the highest supply $(378 \mathrm{~kg} / \mathrm{capita} /$ year respectively $356 \mathrm{~kg} / \mathrm{capita} /$ year). The lowest supply was recorded in Hungary with $169 \mathrm{~kg} / \mathrm{capita} /$ year. Regarding the different regions, it can be observed that the supply was higher in the North and West regions than in the South and Central and East regions.

\section{Eggs}

The mean supply of eggs increased slightly over the past four decades (fig. 4.18). In 2003, Denmark and Hungary showed the highest supply (17 kg/capita/year). The lowest supply was observed in Ireland and Slovenia $(7 \mathrm{~kg} / \mathrm{capita} /$ year) followed by Finland with $8 \mathrm{~kg} / \mathrm{capita} / \mathrm{year}$. Concerning the different regions, no big difference was observed.

\section{Beer}

The average supply of beer doubled in the past 40 years in the participating countries (fig. 4.19). In 2003, Ireland showed the highest supply with $190 \mathrm{~kg} / \mathrm{capita} / \mathrm{year}$. The supply was lowest in the southern countries such as Italy (30 kg/capita/year), France (30 kg/capita/year) and Greece (33 kg/capita/year). The highest supply was recorded in the Central and East region followed by the West and North regions. The lowest supply was reported in the South region. 


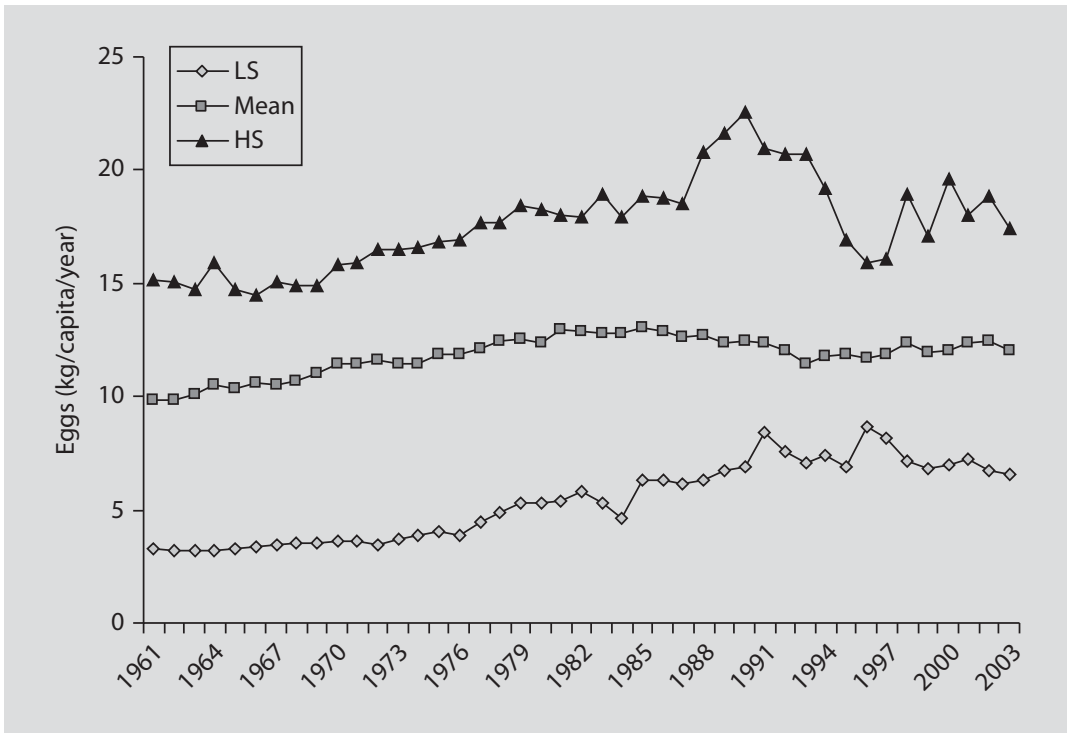

Fig. 4.18. Average supply of eggs in the participating countries. $H S=$ Highest supply; $L S=$ lowest supply. 2003: HS = DK, LS = IE. Source of raw data: FAO, 2009.

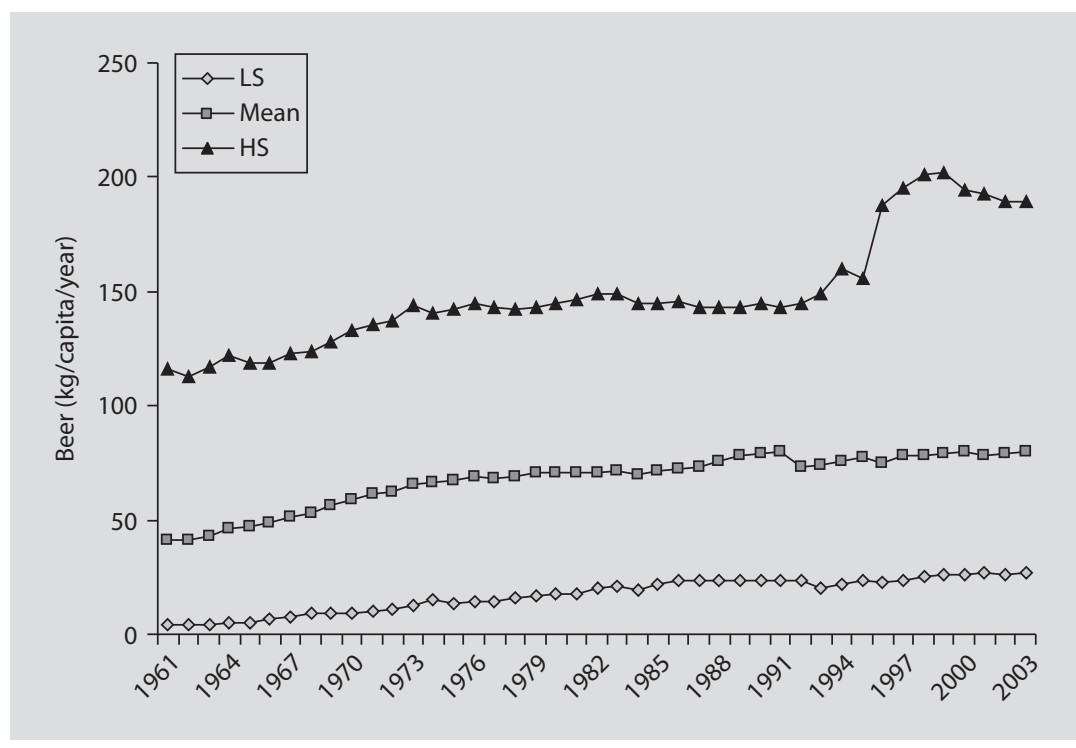

Fig. 4.19. Average supply of beer in the participating countries. HS = Highest supply; $L S=$ lowest supply. 2003: HS = IE, LS = IT. Source of raw data: FAO, 2009. 
Wine

The mean wine supply slightly decreased in the participating countries from 1961 to 2003 (fig. 4.20). In contrast to the beer supply, where the southern countries showed the lowest supply, they showed highest supply in the case of the wine supply (France: $54 \mathrm{~kg} /$ capita/year, Portugal: $53 \mathrm{~kg} /$ capita/year, Italy: $51 \mathrm{~kg} /$ capita/year). The lowest supply was reported in Poland with $2 \mathrm{~kg} /$ capita/year. Regarding the different regions, a South-North gradient can be observed.

\section{Comparison of the Proportion of Macronutrients in Total Energy Supply}

The proportion of macronutrients was calculated using the Atwater conversion factors $(1 \mathrm{~g}$ protein $=4 \mathrm{kcal}, 1 \mathrm{~g}$ carbohydrates $=4 \mathrm{kcal}, 1 \mathrm{~g}$ fat $=9 \mathrm{kcal})$. Carbohydrates (E\%) were calculated by difference. Whereas the FBS provide only data for protein and fat, the difference includes carbohydrates as well as alcohol. Because no generally accepted factor for the alcohol content of alcoholic beverages exists, the calculations were done exclusive of alcohol.

Figure 4.21 shows the comparison of the mean development of the supply of the proportion of macronutrients from 1961 and 2003. Whereas there was no change in the proportion of protein, the proportion of fat increased and the proportion of carbohydrates decreased.

Comparison of the Proportion of Animal and Vegetable Products in Total Energy Supply The proportion of animal and vegetable products in total energy supply slightly changed from 1961 to 2003 in most of the participating countries. Noteworthy is that the proportion of animal products in total energy supply strongly increased over the past four decades in the southern European countries Cyprus, Greece, Italy, Portugal and Spain as well as in Romania, whereas the proportion of vegetable products in total energy supply decreased (cf. table 4.2).

Concerning the different regions, the proportion of animal products in total energy supply remained relatively constant in the West region, decreased slightly in the North region, increased in the Central and East region and increased markedly in the South region over the past 40 years (cf. table 4.3 ).

\section{Comparison of the Supply of Different Food Groups (1961 and 2003)}

Figure 4.22 shows the differences in mean supply of selected food groups in the participating countries. The basis for this graph are the differences of the average supply of the year 2003 (A) from the average supply of the year 1961 (B): Formula: $((\mathrm{A}-\mathrm{B}) / \mathrm{A}) 100=\%$ difference $)$. Concerning most of the food groups, an increase can be observed except for pulses, potatoes, wine, cereals and mutton and goat meat. The supply of animal fats remained relatively constant.

The following figures (fig. 4.23-4.36) show the region-specific comparison indicating the change in the supply of different food groups from 1961 to 2003; for this purpose the minimum and maximum from every region are presented. 


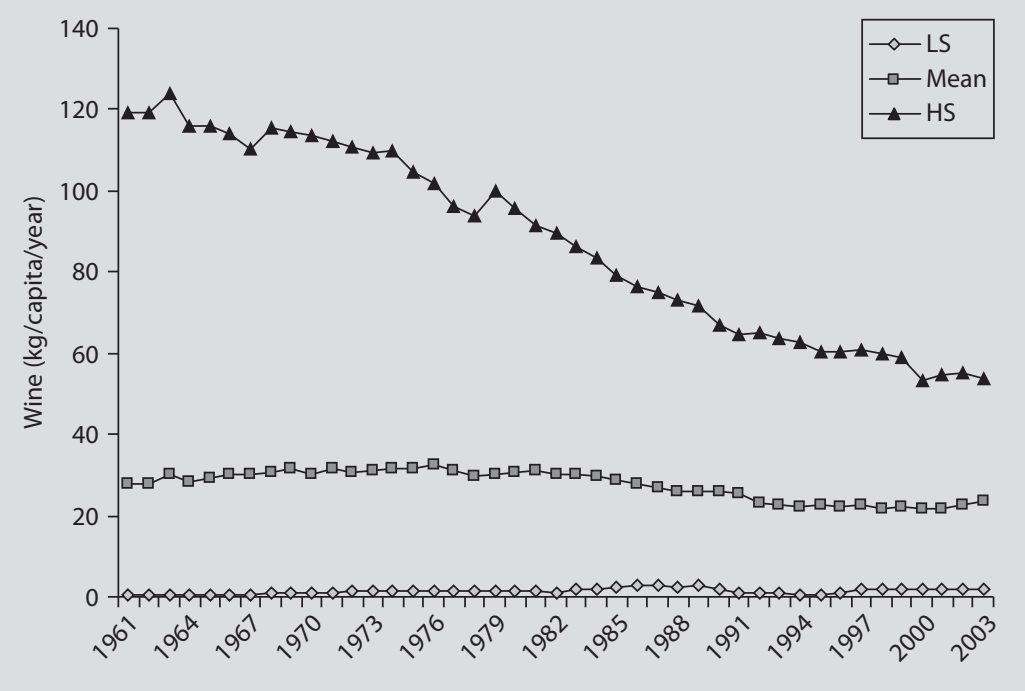

Fig. 4.20. Average supply of wine in the participating countries. $\mathrm{HS}=$ Highest supply; $\mathrm{LS}=$ lowest supply. 2003: HS = FR, LS = PL. Source of raw data: FAO, 2009.

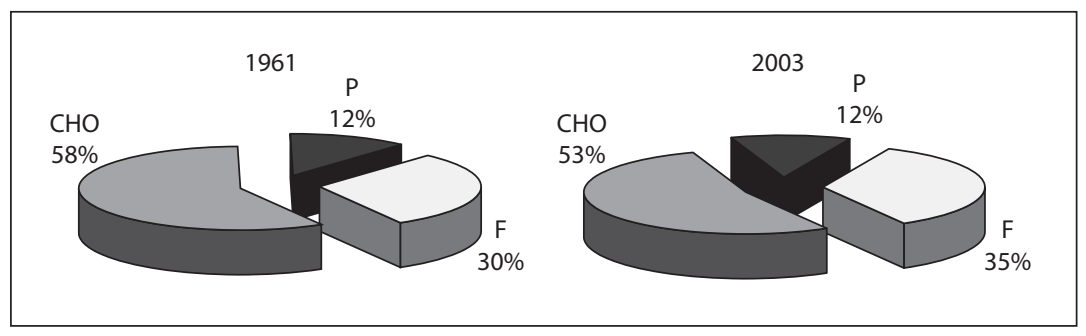

Fig. 4.21. Comparison of the proportion of macronutrients in the participating countries. $P=$ Protein; $\mathrm{CHO}=$ carbohydrates incl. alcohol; $\mathrm{F}=$ fat. Source of raw data: FAO, 2009.

\section{Cereals and Potatoes}

Figures 4.23 and 4.24 show the change in the supply of cereals and potatoes in the four regions. The supply of cereals increased in the North region during the last 40 years; in the other regions a slight decrease can be observed. The supply of potatoes decreased in the West and Central and East regions and stayed relatively constant in the South region. In the North region the gap between minimum and maximum has been widening.

\section{Fruit, Vegetables and Pulses}

The supply of fruit increased from 1961 to 2003 in every region (fig. 4.25). The supply of vegetables increased in the North, South as well as the Central and East regions. In the West region a slight decrease can be observed (fig. 4.26). Concerning the supply of pulses, a decrease can be observed in the South and Central and East regions and an increase in the North and West regions (fig. 4.27). 
Table 4.2. Comparison of the proportion of animal and vegetable products in total energy supply in the participating countries

\begin{tabular}{|c|c|c|c|c|}
\hline & \multicolumn{2}{|c|}{ Animal products, \%E } & \multicolumn{2}{|c|}{ Vegetable products, $\% \mathrm{E}$} \\
\hline & 1961 & 2003 & 1961 & 2003 \\
\hline Austria & 32 & 33 & 68 & 67 \\
\hline Belgium-Luxembourg & 33 & 31 & 67 & 69 \\
\hline Cyprus & 13 & 30 & 87 & 70 \\
\hline Czech Republic & n.a. & 25 & n.a. & 75 \\
\hline Denmark & 36 & 36 & 64 & 64 \\
\hline Estonia & n.a. & 26 & n.a. & 74 \\
\hline Finland & 39 & 37 & 61 & 63 \\
\hline France & 32 & 37 & 68 & 63 \\
\hline Germany & 33 & 31 & 67 & 69 \\
\hline Greece & 13 & 23 & 87 & 77 \\
\hline Hungary & 31 & 32 & 69 & 68 \\
\hline Ireland & 36 & 32 & 64 & 68 \\
\hline Italy & 16 & 26 & 84 & 74 \\
\hline Latvia & n.a. & 28 & n.a. & 72 \\
\hline Lithuania & n.a. & 27 & n.a. & 73 \\
\hline Poland & 28 & 26 & 72 & 74 \\
\hline Portugal & 14 & 29 & 86 & 71 \\
\hline Romania & 15 & 23 & 85 & 77 \\
\hline Slovenia & n.a. & 31 & n.a. & 69 \\
\hline Spain & 13 & 28 & 87 & 72 \\
\hline Sweden & 37 & 36 & 63 & 64 \\
\hline The Netherlands & 30 & 30 & 70 & 70 \\
\hline United Kingdom & 39 & 31 & 61 & 69 \\
\hline
\end{tabular}

Source of raw data: FAO, 2009

Table 4.3. Comparison of the proportion of animal and vegetable products in total energy supply (min-max) in the four different regions

\begin{tabular}{|c|c|c|c|c|}
\hline & \multicolumn{2}{|c|}{ Animal products, \%E } & \multicolumn{2}{|c|}{ Vegetable products, \%E } \\
\hline & 1961 & 2003 & 1961 & 2003 \\
\hline North & $36-39$ & $26-37$ & $61-64$ & $63-74$ \\
\hline South & $13-16$ & $23-30$ & $84-87$ & $70-77$ \\
\hline West & $30-39$ & $30-37$ & $61-70$ & $63-70$ \\
\hline Central and East & $15-33$ & $25-33$ & $67-85$ & $67-77$ \\
\hline
\end{tabular}

Source of raw data: FAO, 2009 


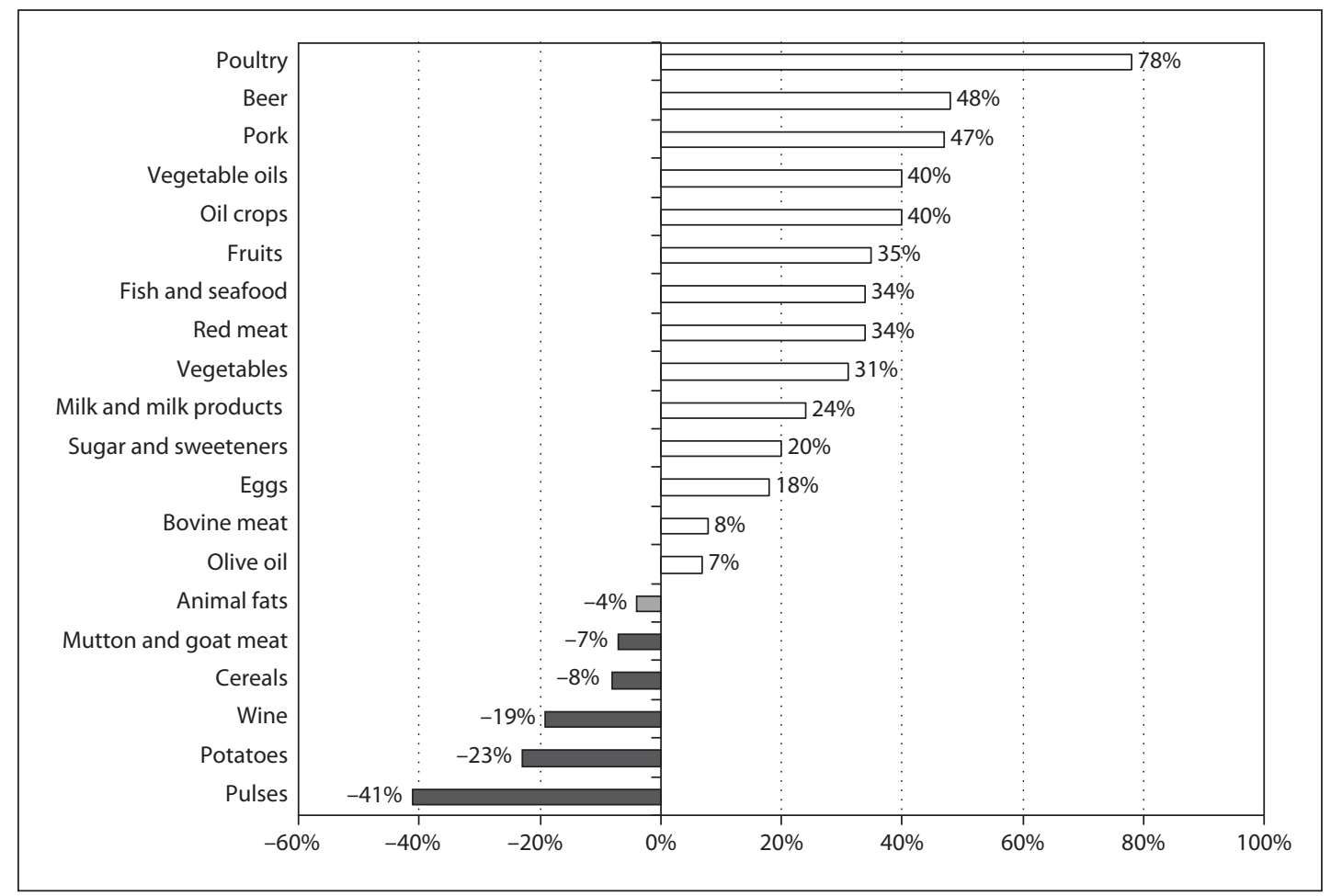

Fig. 4.22. Differences in mean supply of selected food groups in the participating countries (1961 and 2003). Source of raw data: FAO, 2009.

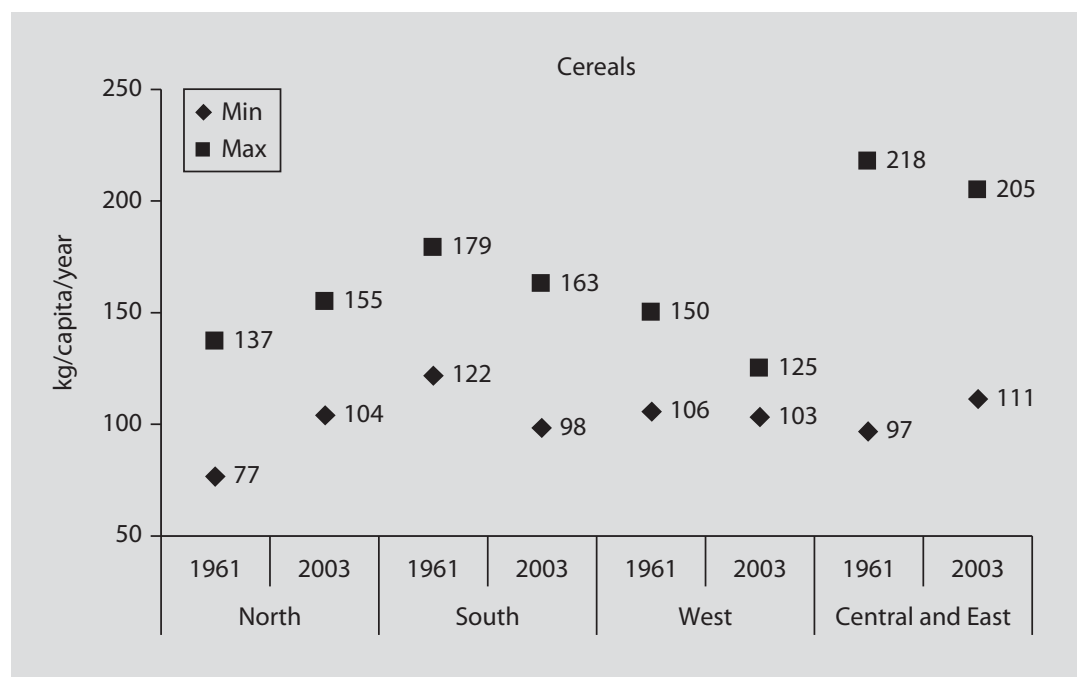

Fig. 4.23. Supply (min-max) of cereals in the four regions (1961 and 2003). Source of raw data: FAO, 2009. 


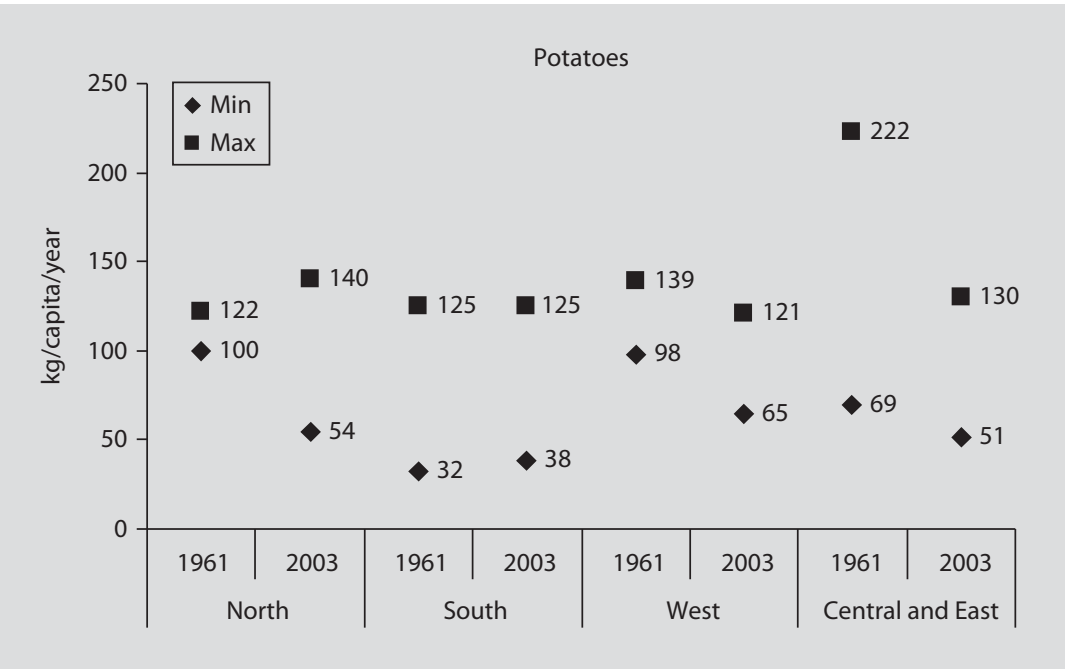

Fig. 4.24. Supply (min-max) of potatoes in the four regions (1961 and 2003). Source of raw data: FAO, 2009.

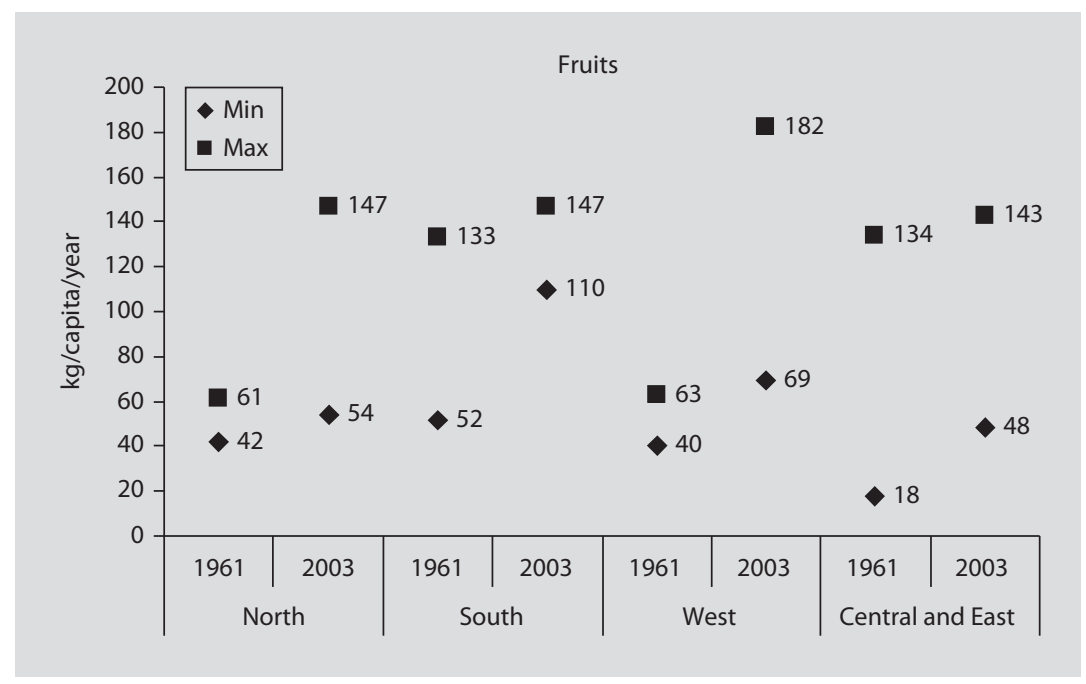

Fig. 4.25. Supply (min-max) of fruits in the four regions (1961 and 2003). Source of raw data: FAO, 2009. 


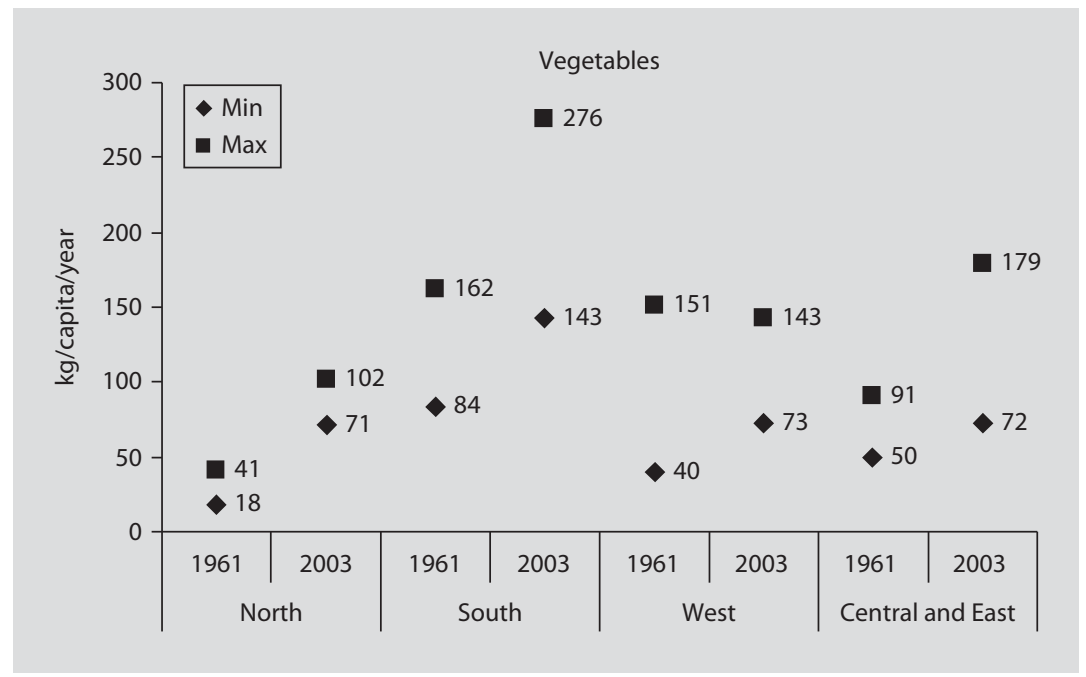

Fig. 4.26. Supply (min-max) of vegetables in the four regions (1961 and 2003). Source of raw data: FAO, 2009.

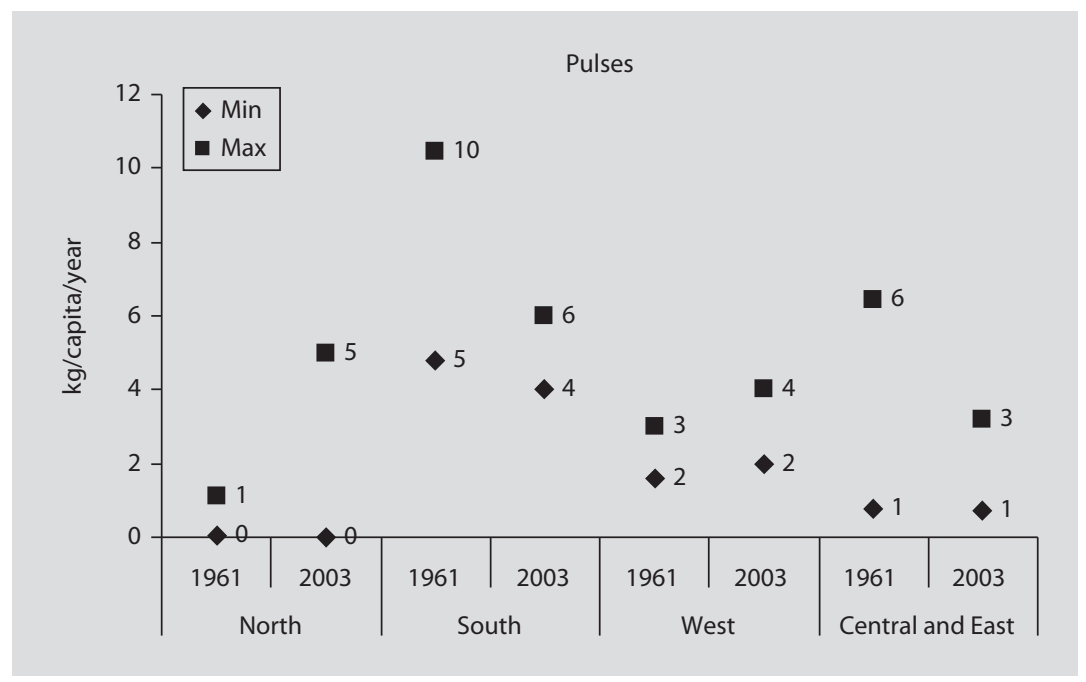

Fig. 4.27. Supply (min-max) of pulses in the four regions (1961 and 2003). Source of raw data: FAO, 2009. 


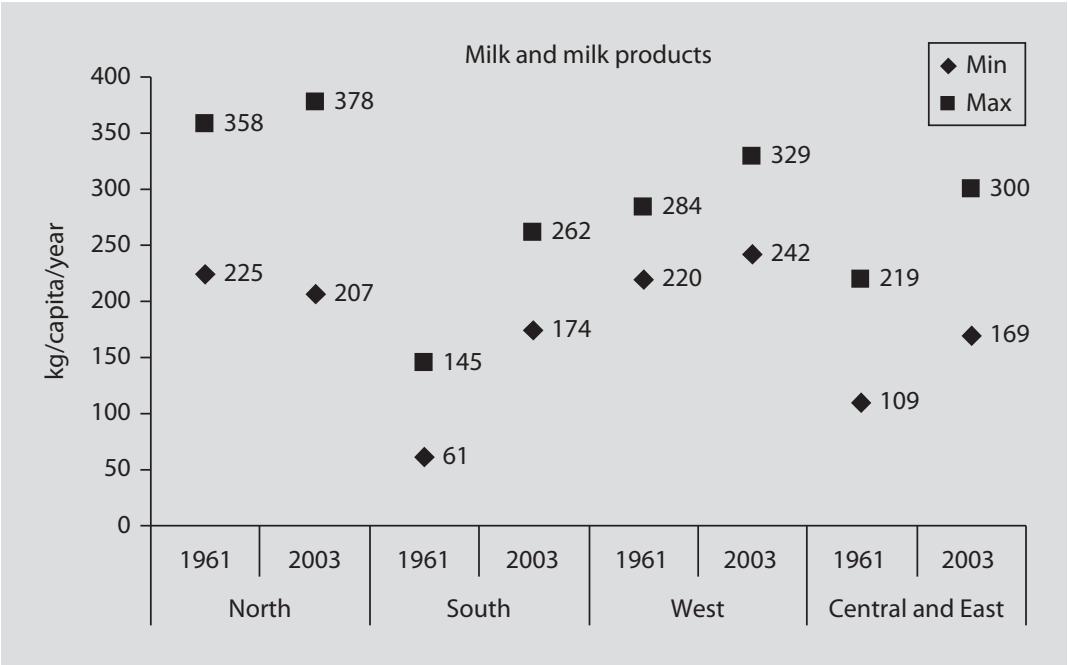

Fig. 4.28. Supply (min-max) of milk and milk products in the four regions (1961 and 2003). Source of raw data: FAO, 2009.

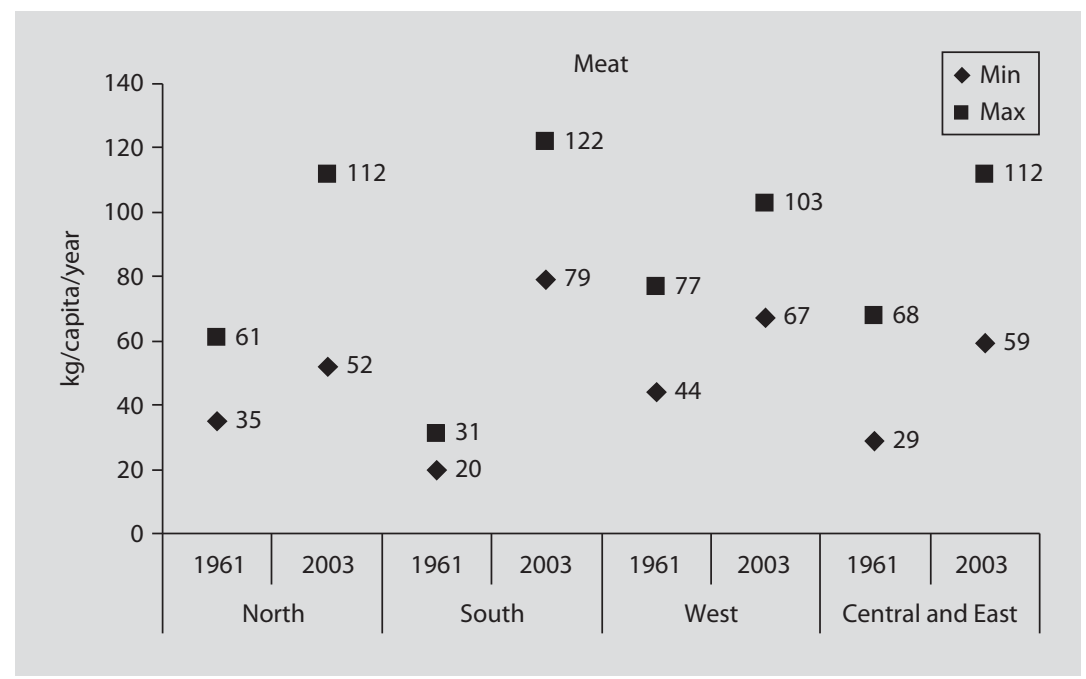

Fig. 4.29. Supply (min-max) of meat in the four regions (1961 and 2003). Source of raw data: FAO, 2009. 


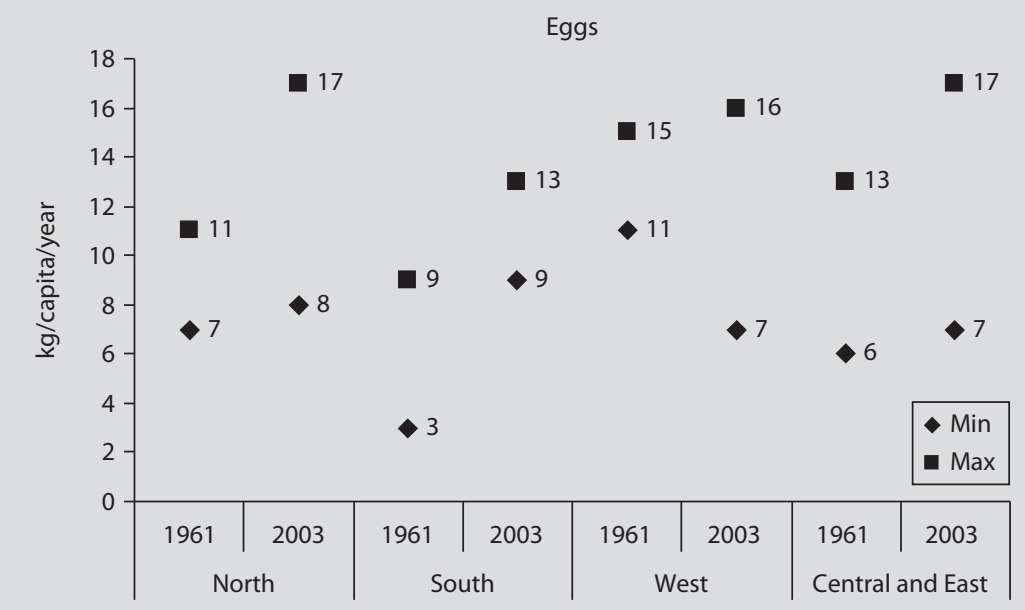

Fig. 4.30. Supply (min-max) of eggs in the four regions (1961 and 2003). Source of raw data: FAO, 2009.

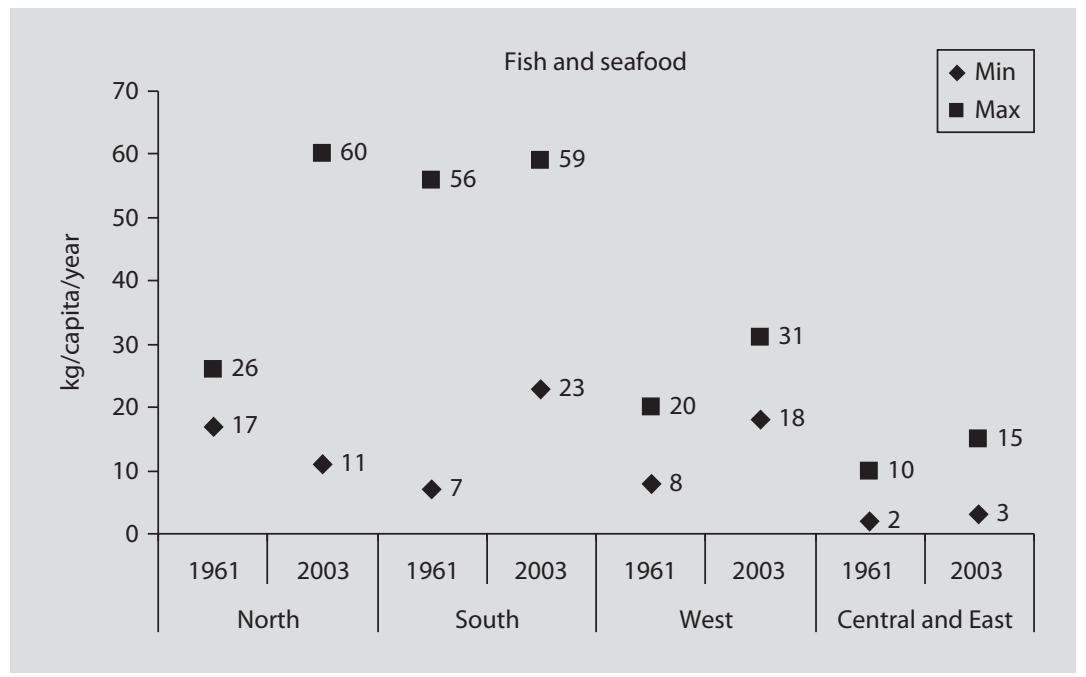

Fig. 4.31. Supply (min-max) of fish and seafood in the four regions (1961 and 2003). Source of raw data: FAO, 2009. 


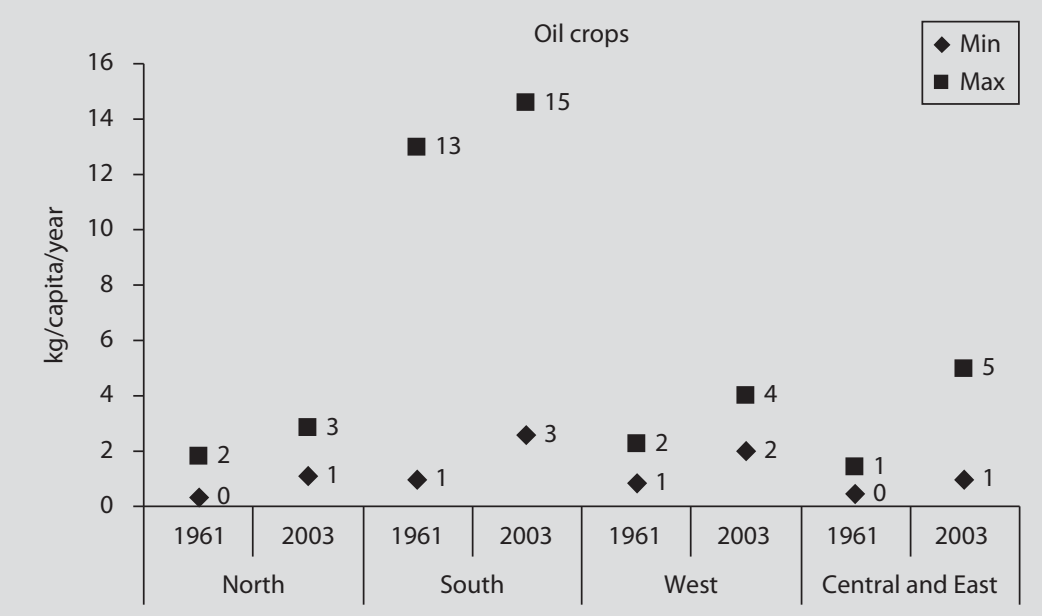

Fig. 4.32. Supply (min-max) of oil crops in the four regions (1961 and 2003). Source of raw data: FAO, 2009.

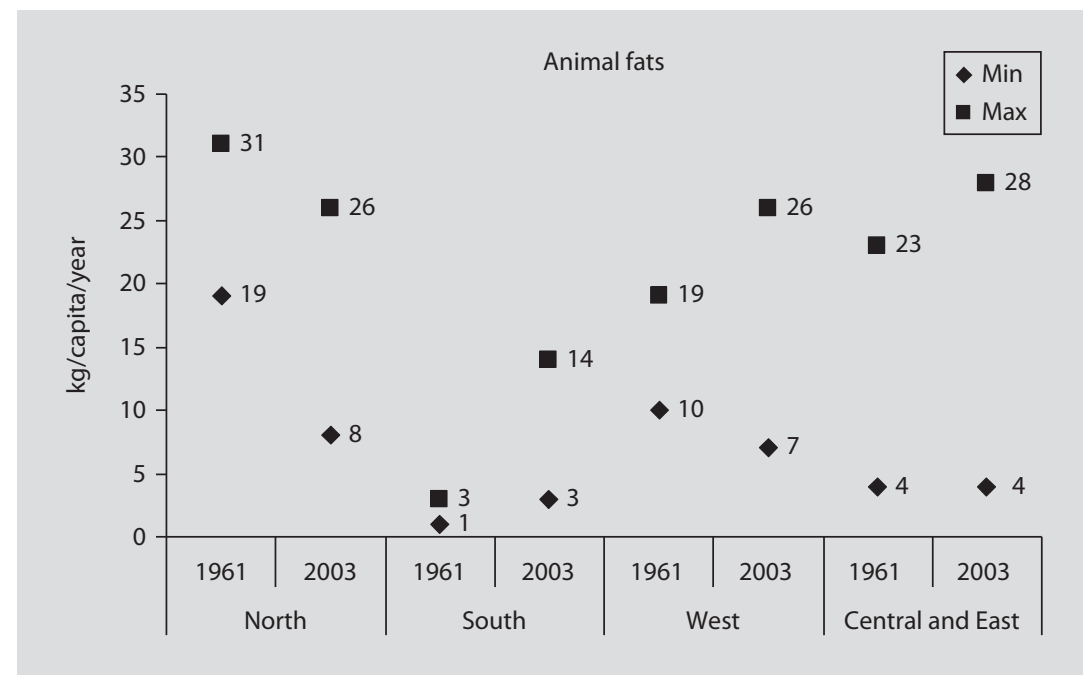

Fig. 4.33. Supply (min-max) of animal fats in the four regions (1961 and 2003). Source of raw data: FAO, 2009. 


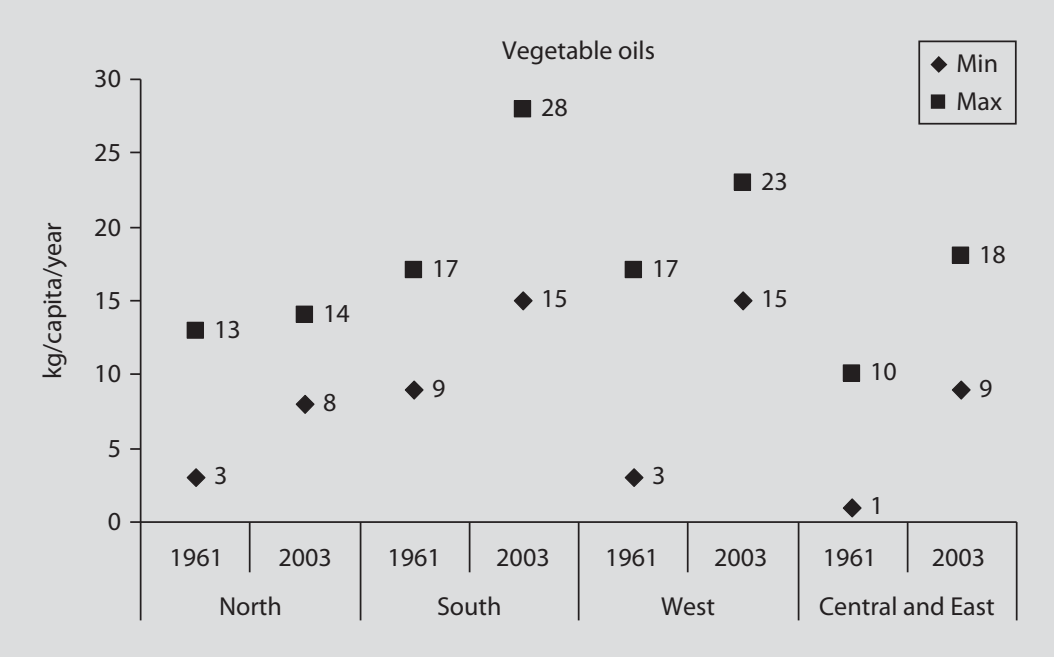

Fig. 4.34. Supply (min-max) of vegetable oils in the four regions (1961 and 2003). Source of raw data: FAO, 2009.

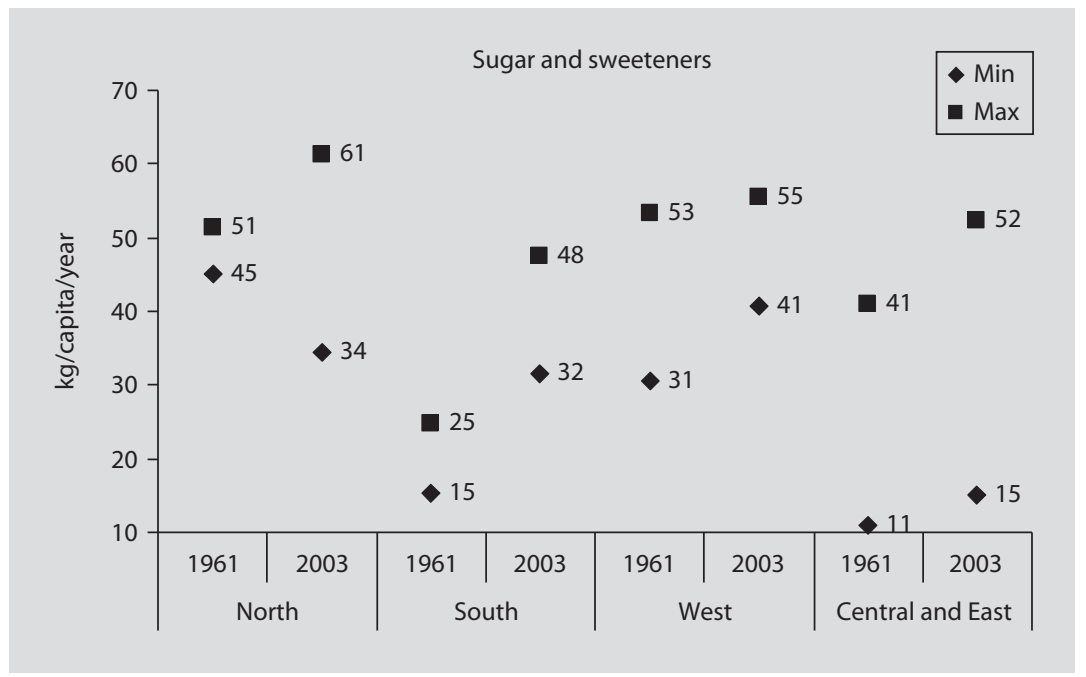

Fig. 4.35. Supply (min-max) of sugar and sweeteners in the four regions (1961 and 2003). Source of raw data: FAO, 2009. 


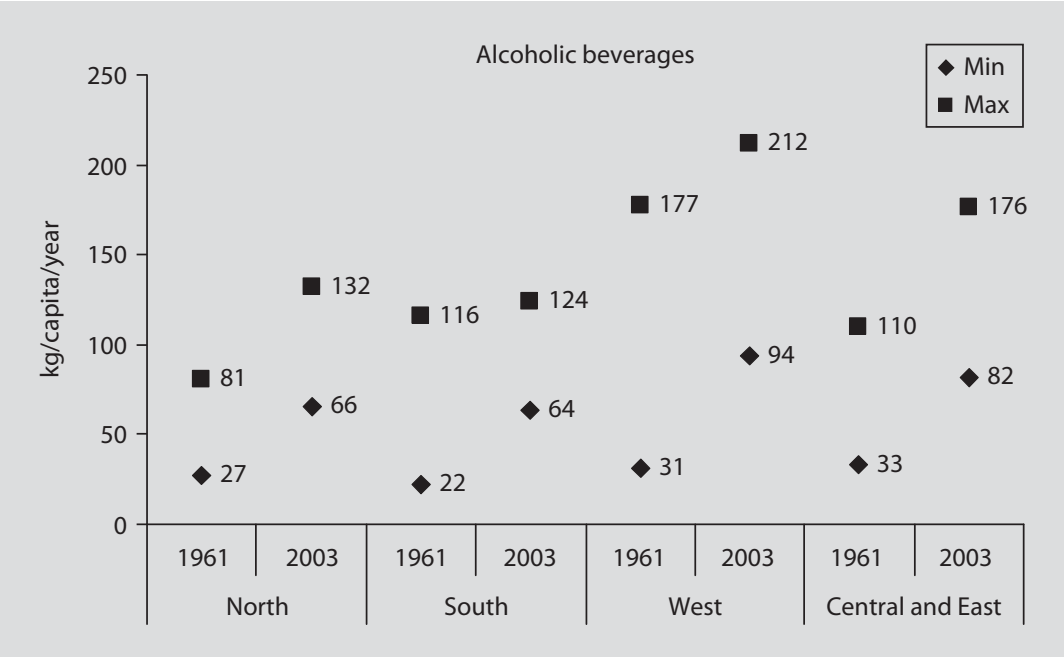

Fig. 4.36. Supply (min-max) of alcoholic beverages in the four regions (1961 and 2003). Source of raw data: $\mathrm{FAO}, 2009$.

\section{Milk and Milk Products}

The supply of milk increased in every region except for the North region where it stayed relatively constant (fig. 4.28).

\section{Animal Products - Excluding Milk}

Figures 4.29-4.31 show the change in the supply of meat, eggs as well as fish and seafood in the four regions. In general an increase in the supply of animal products can be observed.

\section{Fats, Oils and Sugar}

The supply of oil crops increased in every region, whereas there was a strong increase of the maximum values in the South region (fig. 4.32). Both the supply of animal fats and the supply of vegetable oils increased over the past 40 years except for the North region where the supply of animal fats decreased (fig. 4.33,4.34). The supply of sugar and sweeteners increased too. In the North region the gap between minimum and maximum has been widening (fig. 4.35).

\section{Alcoholic Beverages}

As shown in figure 4.36, the supply of alcoholic beverages increased over the past 40 years in every region. 


\section{Food Availability at the Household Level in the European Union'}

\section{The DAFNE Network (Only for the Countries Participating in the ENHR 2009 and Presented in This Report)}

Austria

Institute of Nutritional Sciences,

University of Vienna

I. Elmadfa, H. Friesling,

A. Suchomel

Belgium

University of Gent, Faculty of

Agricultural and Applied Biological

Sciences, Department of Food

Technology and Nutrition

A.M. Remaut - de Winter, AP.

Cueto Eulert

National Institute of Statistics,

Brussels

Buermans, L. Merckx

Cyprus

Department of Medical and Public

Health Services, Ministry of Health

Agrotou A., Markidou E.

Statistical Service of the

Republic of Cyprus

Onisiforou K.
Finland

Statistics Finland, Helsinki, Finland

MA Berg, A. Pajunen

National Public Health Institute,

Helsinki, Finland

T. Hirvonen

France

Unite Observatoire des Consommations

Alimentaires, AFSSA

JL. Volatier, J. Maffre

Germany

Department of Marketing and

Consumer Research TUM Business

School, Technische Universität

München, Munich, Germany

G. Karg, K Gedrich, K. Wagner

Greece (coordinating center)

Department of Hygiene Epidemiology

and Medical Statistics,

School of Medicine, University of Athens

A. Trichopoulou, A. Naska,

E. Oikonomou, K. Tsiotas, V. Bountziouka.

National Statistical Office, Athens

Douros, I. Tsaousi

${ }^{1}$ Naska $A^{\mathrm{a}}$, Oikonomou $\mathrm{E}^{\mathrm{a}}$, Tsiotas $\mathrm{K}^{\mathrm{a}}$, Bountziouka $\mathrm{V}^{\mathrm{a}, \mathrm{b}}$, Trichopoulou $\mathrm{A}^{\mathrm{a}, \mathrm{b}}$ on behalf of the DAFNE network

${ }^{a}$ Dept. of Hygiene, Epidemiology and Medical Statistics, School of Medicine, National and Kapodistrian University of Athens, ${ }^{b}$ Hellenic Health Foundation, Athens, Greece 
Hungary

National Institute of Food Hygiene and

Nutrition, Budapest

Zajkas, M. Korom

Hungarian Central Statistical Office,

Section of Living Standard Statistics

Surveys

E. Menesi

Ireland

National Nutrition Surveillance Centre, University College, Galway, Ireland

C. Kelleher, S. Friel

Central Statistics Office, Cork

K. McCormack

Italy

National Research Institute for

Food and Nutrition

A. Turrini, S. Martines

Italian Statistical Office (ISTAT)

S. Barcherini

Latvia

Food and Veterinary Service

Food Center

D. Šantare, N. Petruhina

Central Statistical Bureau of Latvia

L. Sparite

Luxembourg

Interdisciplinary Nutrition Policy Group

A. Schmitt

Central Statistics Office

J. Langers, M. Zanardelli

Norway

Institute of Nutrition Research,

University of Oslo

K. Trygg, K. Lund-Iversen

Statistics Norway, Oslo

E. Mork
Poland

National Food and Nutrition Institute

W. Sekula, M Ołtarzewski

Portugal

University of Porto

MD Vaz de Almeida, S. Rodrigues

\section{Slovenia}

Institute of Public Health of the

Republic of Slovenia

M. Gabrijelčič Blenkuš, M. Gregorič,

M. Adamič

Statistical Office of the Republic of

Slovenia

M. Remec

Spain

Departamento de Nutricion y

Bromatologia, Facultat de Farmacia,

Universidad Complutense de Madrid

O. Moreiras, C. Cuadrado

Instituto Nacional de Estadistica,

Madrid

M.L. Boned, P. Seoane Spiegelberg

Sweden

Unit for Preventive Nutrition,

Department of Biosciences and

Nutrition, Karolinska Institute

M. Sjöstrom, E. Poortvliet

\section{United Kingdom}

King's College London, Department of

Nutrition and Dietetics, London

Nelson, S. Burr

Ministry of Agriculture, Fisheries and

Food, National Food Survey Branch,

London

D. Rimmer 


\section{Introduction}

Data for nutrition surveillance can be derived from: Food Balance Sheets (FBS), Household Budget Surveys (HBS) and specifically designed Individual Nutrition Surveys (INS). The Food and Agriculture Organization assembled FBS have traditionally been used by countries with no routine information on food consumption or those interested in undertaking worldwide comparisons of overtime trends in the supply of food commodities at the population level [FAO, 2007a]. Household budget surveys are systematically conducted by National Statistical Offices in country representative samples and aim at collecting, among others, data on food availability at household level. Like FBS, the HBS allow inter-country comparisons on a regular basis but, moving from total population to household level, they further allow the description of the current and developing structure of dietary patterns at national or regional level and of population sub-groups defined on the basis of their sociodemographic characteristics [Trichopoulou, 1992]. Individual-based surveys, when participants' intakes are recorded as adequately as possible, constitute the optimal method for assessing dietary patterns and quantifying determinants and consequences of food choices. Being expensive and labor intensive, however, representative individual-based surveys are regularly undertaken in some countries. Furthermore, differences in study design and data analysis reduce the comparability of results at an international level.

The use of the national HBSs for nutrition monitoring purposes has been evaluated through the EU-supported Data Food Networking (DAFNE) initiative, which built up a regularly updated food-based databank allowing the: (a) identification of dietary patterns prevailing in Europe and of their sociodemographic determinants; [Trichopoulou et al., 2002; Naska et al., 2006], (b) follow up of time trends in food habits; [Trichopoulou and Naska, 2003], and (c) evaluation of nutrition action plans and strategies implemented at national or international level [Trichopoulou, 2001]. The DAFNE network is coordinated by the Department of Hygiene and Epidemiology of the University of Athens Medical School and currently interrelates 26 European countries (Albania, Armenia, Austria, Belgium, Croatia, Cyprus, Finland, France, Germany, Greece, Hungary, Ireland, Italy, Latvia, Luxembourg, Malta, Montenegro, Norway, Poland, Portugal, Serbia, Spain, Slovak Republic, Slovenia, Sweden and the $\mathrm{UK})$.

\section{The DAFNE Methodology}

\section{Data Collection}

Through the standardized and nationally representative HBSs, members of the participating households are asked to record in open questionnaires all foods and beverages available during a reference period, including purchases, contributions from 
own production and food items offered to members as gifts. Data on food quantities consumed in restaurants, canteens and similar establishments are, however, not consistently available. Data collection is accomplished within one year, with due attention to capture seasonal variation in food intake. Trained interviewers visit the households regularly to assure complete data recording and to collect further information on socio- demographic and lifestyle characteristics of the household members through standardised interviewer-administered questionnaires [European Communities, 2003].

\section{Integration of the National HBS Data in the DAFNE Databank}

The collected national datasets are centrally analyzed according to the methodology developed and validated in the DAFNE project (fig. 5.1) [Trichopoulou and Naska, 2001]. Briefly, data are read, cleaned and managed to a format suitable for a common between countries analysis. To improve comparability, food and socio demographic data are post-harmonized. In particular, the national HBS food data are classified under 56 detailed common subgroups, which can be further aggregated to 15 main food groupings [Health and Consumer Protection Directorate General, 2005]. With respect to sociodemographic characteristics, focus is, up to now, put on the locality of the dwelling (classified as rural, semi-urban and urban), the educational level of the household head (elementary, secondary and higher), the occupation of the household head (manual, non-manual, unemployed and retired), and on the household's composition, defined on the basis of the number and the age of the members.

Food quantities available for consumption to each household member are calculated by dividing the household availability by the product of the referent time period and the mean household size, under the assumption of equal distribution of food during the survey period and without making allowances for inedible parts, preparation losses, spoilage on the plate or food offered to domestic pets. Weighting factors are incorporated, whenever necessary, to accommodate national sampling schemes.

\section{Conversion of Expenses to Food Quantities}

Since the primary aim of the HBSs is the derivation of national price indices, attention is given to ensure the complete recording of expenses, while data on food quantities (in terms of $\mathrm{kg}$ or liters) could be missing or incomplete.

The method for converting food expenditure to quantity was applied in three cases (Cyprus, Ireland and Sweden) and included the application of retail prices per unit weight, sought from various sources including national and governmental statistics, food organizations, retailers and consumer surveys. Since collection of the prices was made using various sources from differing years, adjustment was made to equivalent prices for the year of HBS data using national Consumer Price Indices. Simple models were devised and validated into which the expenditure data and retail prices were fitted and resulted in the estimated quantity of food item available in each household [Friel et al., 2001]. 


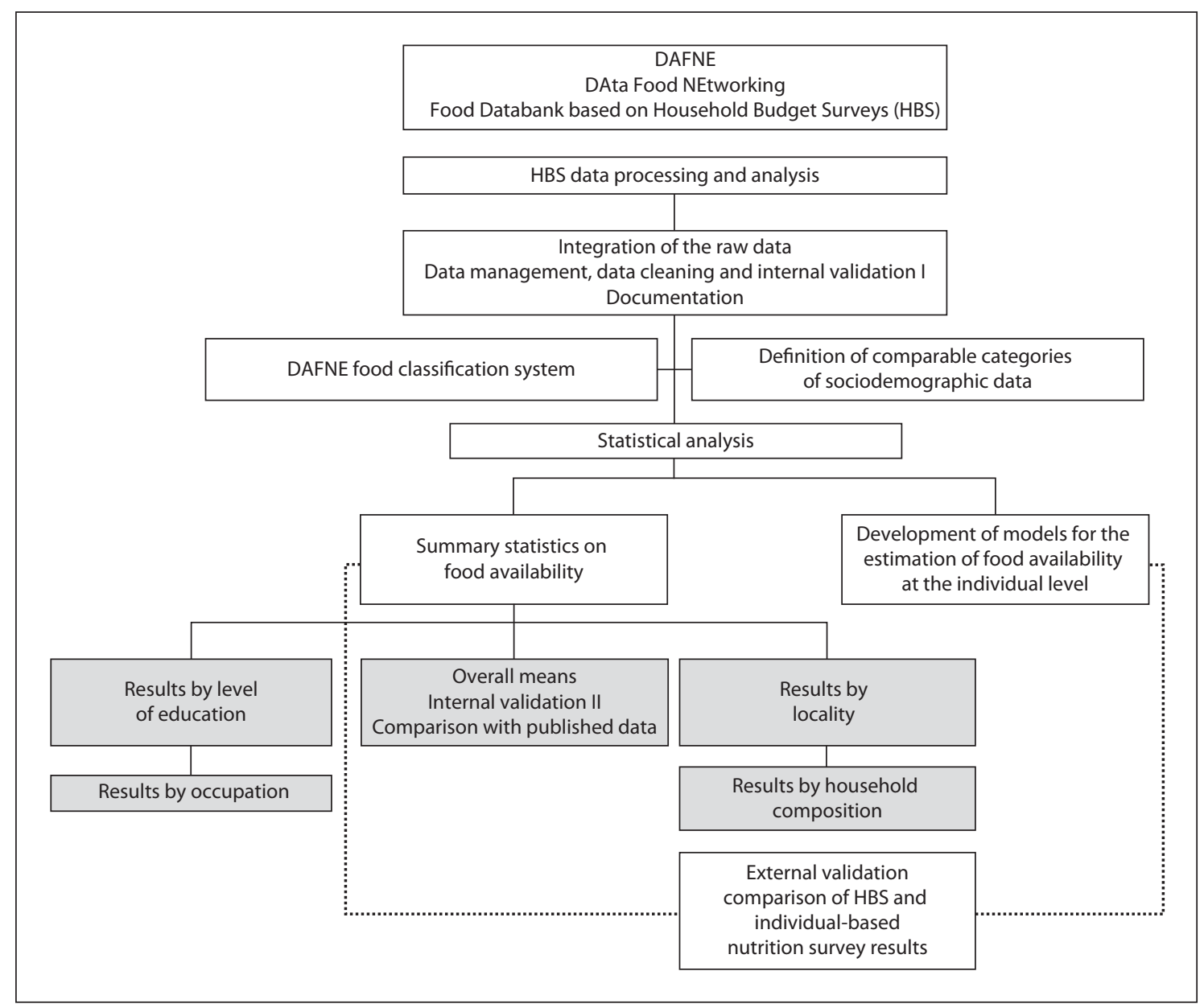

Fig. 5.1. The Household Budget Survey data processing and analysis in the context of the DAFNE initiative.

\section{The DafneSoft Application Tool}

Results on the mean daily per person food availability, based on the DAFNE data, are freely accessible through DafneSoft (http://www.nut.uoa.gr/dafnesoftweb/), an application tool allowing the presentation and exporting of DAFNE data in various formats (tables, bars, pie charts, map presentations) at various levels of detail.

\section{Results Retrieved from the DAFNE Databank - Food Availability in Europe}

Tables 5.1-5.4 present the mean daily individual availability of DAFNE food groups, by European region and country. In tables 5.5-5.7, data on the daily food availability according to the educational level of the household head are also presented. 
Table 5.1. Mean availability of food and beverages in South European countries (unit/person/day)

\begin{tabular}{|c|c|c|c|c|c|}
\hline \multirow[t]{2}{*}{ Survey year } & Cyprus & Greece & Italy & Spain & Portugal \\
\hline & 2003 & 2004-2005 & 1996 & 1998-1999 & 2000 \\
\hline Eggs (pieces) & 0.17 & 0.22 & 0.34 & 0.32 & 0.18 \\
\hline Potatoes, $\mathrm{g}$ & 94 & 124 & 76 & 86 & 180 \\
\hline Pulses, $\mathrm{g}$ & 23 & 14 & 4.1 & 10 & 9.2 \\
\hline Nuts, g & 4.6 & 4.4 & 0.9 & 0.9 & 3.0 \\
\hline Cereals, g & 302 & 246 & 334 & 170 & 239 \\
\hline Milk and milk products, $\mathrm{g}$ & 337 & 296 & 271 & 341 & 275 \\
\hline Cheese, $g$ & 44 & 55 & 49 & 15 & 19 \\
\hline Meat and meat products, $\mathrm{g}$ & 178 & 159 & 136 & 139 & 160 \\
\hline Red meat, $g$ & 98 & 101 & 71 & 55 & 90 \\
\hline Poultry, g & 62 & 42 & 38 & 38 & 44 \\
\hline Processed meat, g & 17 & 13 & 24 & 35 & 17 \\
\hline Vegetables, g & 284 & 283 & 184 & 121 & 137 \\
\hline Fresh vegetables, g & 280 & 256 & 128 & 103 & 126 \\
\hline Processed vegetables, g & 4.0 & 26 & 56 & 18 & 11 \\
\hline Fish and seafood, $\mathrm{g}$ & 18 & 46 & 38 & 61 & 83 \\
\hline Fruits, $\mathrm{g}$ & 252 & 264 & 233 & 195 & 198 \\
\hline Fresh fruit, $g$ & 248 & 263 & 232 & 190 & 196 \\
\hline Processed fruit, $\mathrm{g}$ & 3.5 & 0.82 & 1.4 & 4.8 & 2.3 \\
\hline Fruit and vegetable juices, $\mathrm{ml}$ & 53 & 37 & 9.6 & 30 & 11 \\
\hline Lipids, added, g & 47 & 77 & 63 & 45 & 51 \\
\hline Animal fat, $g$ & 4.0 & 0.88 & 5.6 & 1.0 & 2.7 \\
\hline Vegetable fat, $\mathrm{g}$ & 6.2 & 6.1 & 1.5 & 1.6 & 5.1 \\
\hline Vegetable oils, g & 37 & 69 & 56 & 42 & 42 \\
\hline Beverages, alcoholic, ml & 32 & 60 & 149 & 74 & 125 \\
\hline Beverages, non-alcoholic, ml & 384 & 244 & 824 & 377 & 171 \\
\hline Soft drinks, $\mathrm{ml}$ & 101 & 66 & 42 & 85 & 63 \\
\hline Sugar and sugar products, g & 67 & 34 & 49 & 26 & 30 \\
\hline
\end{tabular}

Source: The DAFNE databank (www.nut.uoa.gr).

Figures 5.2 and 5.3 present the mean daily availability of selected food groups by European region. Values refer to arithmetic means in each region and were calculated by summing the observations (i.e. mean daily availability in each country) and dividing by the number of observations (i.e. number of countries in each region).

The constellation of countries under study possess several interesting characteristics. Cyprus and Greece for example, are Mediterranean countries, which have for centuries been in the cross-road of several historical cultures. Hungary, Latvia and Slovenia were undergoing, to varying extent, through a transition phase during the 
Table 5.2. Mean availability of food and beverages in Central and Eastern European countries (unit/person/day)

\begin{tabular}{|c|c|c|c|c|c|}
\hline \multirow[t]{2}{*}{ Survey year } & Austria & Germany & Hungary & Poland & Slovenia \\
\hline & 1999-2000 & 1998 & 2005 & 1988 & 2002 \\
\hline Eggs (pieces) & 0.50 & 0.36 & 0.48 & 0.61 & 0.38 \\
\hline Potatoes, g & 100 & 115 & 103 & 301 & 110 \\
\hline Pulses, $g$ & n.a. & 0.6 & 3.1 & 3.2 & 5.8 \\
\hline Nuts, $g$ & n.a. & 7.5 & 2.9 & n.a. & 5.5 \\
\hline Cereals, $g$ & 302 & 218 & 253 & 345 & 315 \\
\hline Milk and milk products, $g$ & 284 & 311 & 220 & 381 & 355 \\
\hline Cheese, $g$ & 28 & 55 & 15 & 39 & 36 \\
\hline Meat and meat products, $g$ & 182 & 132 & 160 & 170 & 173 \\
\hline Red meat, g & 65 & 48 & 51 & 62 & 81 \\
\hline Poultry, g & 23 & 16 & 52 & 33 & 31 \\
\hline Processed meat, g & 92 & 59 & 47 & 70 & 54 \\
\hline Vegetables, g & 142 & 180 & 155 & 202 & 173 \\
\hline Fresh vegetables, g & 114 & 142 & 137 & 177 & 148 \\
\hline Processed vegetables, g & 28 & 38 & 18 & 25 & 25 \\
\hline Fish and seafood, $\mathrm{g}$ & 9.3 & 16 & 4.9 & 15 & 13 \\
\hline Fruits, $g$ & 192 & 182 & 119 & 100 & 219 \\
\hline Fresh fruit, $g$ & 186 & 169 & 118 & 96 & 217 \\
\hline Processed fruit, $\mathrm{g}$ & 6.2 & 13 & 1.0 & 4.6 & 2.6 \\
\hline Fruit and vegetable juices, ml & 90 & 123 & 40 & n.a. & 108 \\
\hline Lipids, added, g & 42 & 37 & 50 & 59 & 54 \\
\hline Animal fat, $g$ & 12 & 15 & 13 & 41 & 7.03 \\
\hline Vegetable fat, $\mathrm{g}$ & 11 & 15 & 9.7 & 12 & 6.7 \\
\hline Vegetable oils, g & 20 & 6.4 & 27 & 5.4 & 41 \\
\hline Beverages, alcoholic, ml & 171 & 200 & 58 & n.a. & 141 \\
\hline Beverages, non-alcoholic, ml & 652 & 915 & 577 & 300 & 549 \\
\hline Soft drinks, ml & 116 & 108 & 87 & 34 & 90 \\
\hline Sugar and sugar products, g & 74 & 59 & 51 & 106 & 74 \\
\hline
\end{tabular}

n.a. $=$ Not available

Source: The DAFNE databank (www.nut.uoa.gr).

data collection period. Finland, Norway and Sweden are countries with robust economies and long-lasting national nutrition policies. Furthermore, one should not ignore the diet-related health mandates to which European populations are globally exposed, as well as the increasing prevalence of eating out, which is not considered in these data and has been reported to varyingly concern European populations [Orfanos et al., 2007].

With respect to plant foods, daily availability is higher in the South. Adequate quantities of fruits and vegetables were reported of being available in the Southern 


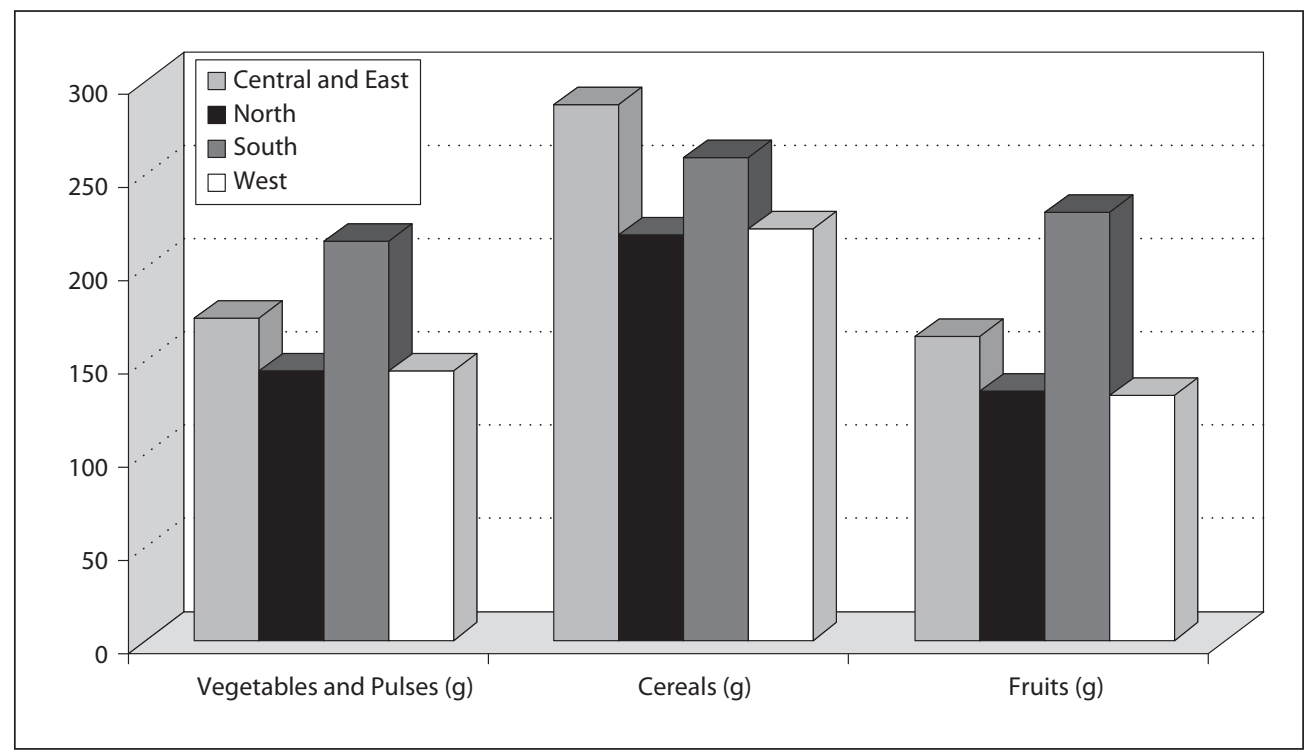

Fig. 5.2. Mean availability (g/person/day) of foods of plant origin by European region. The DAFNE databank.

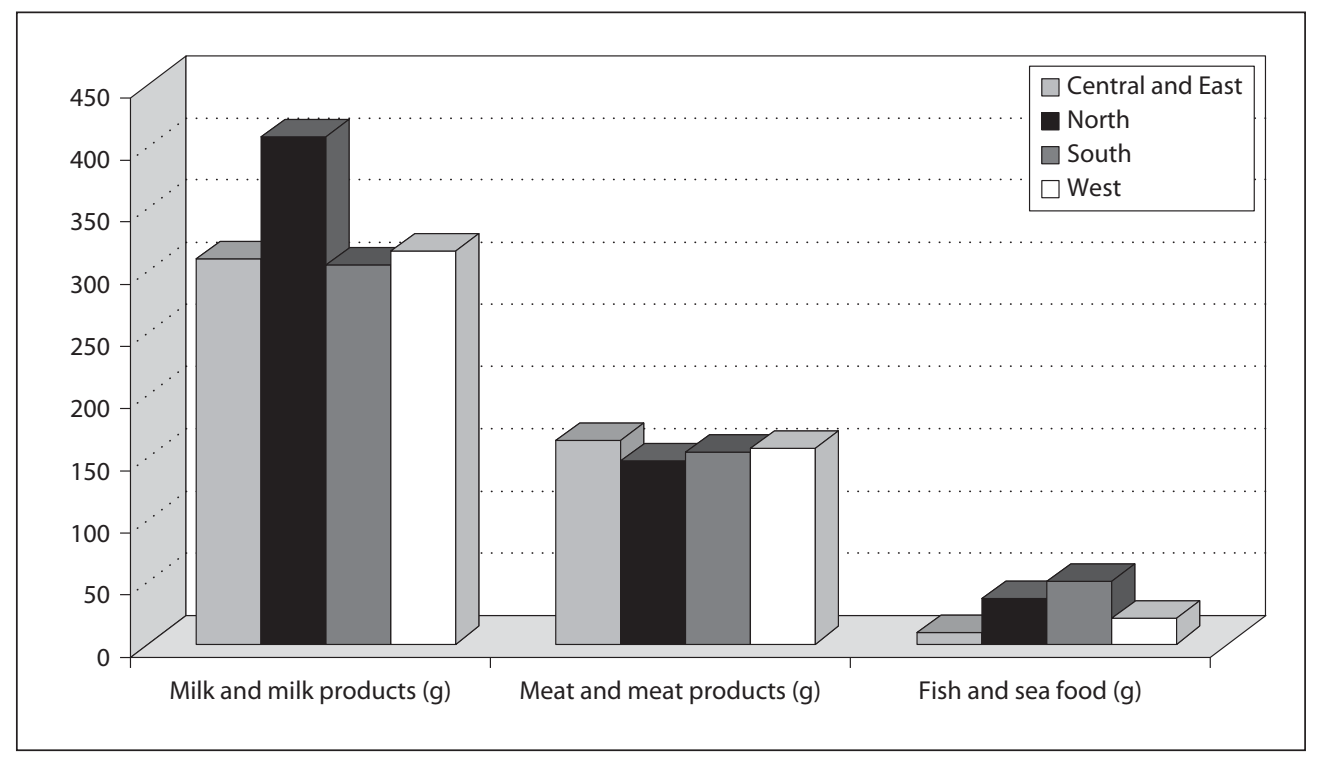

Fig. 5.3. Mean availability ( $g /$ person/day) of foods of animal origin by European region. The DAFNE databank. 
Table 5.3. Mean availability of food and beverages in West European countries (unit/person/day)

\begin{tabular}{llllll}
\hline Survey year & Belgium & France & Ireland & Luxembourg & United Kingdom \\
\cline { 2 - 6 } & 1999 & 1991 & 1999 & 1993 & 1999 \\
\hline Eggs (pieces) & 0.21 & 0.38 & 0.23 & 0.33 & 0.24 \\
Potatoes, g & 97 & 95 & 186 & 163 & 128 \\
Pulses, g & n.a. & 4.9 & 1.1 & 2.3 & 0.99 \\
Nuts, g & 2.6 & 1.7 & 1.2 & 3.1 & 1.7 \\
Cereals, g & 243 & 160 & 290 & 205 & 202 \\
Milk and milk products, g & 256 & 290 & 481 & 235 & 320 \\
Cheese, g & 55 & 48 & 15 & 55 & 20 \\
Meat and meat products, g & 144 & 163 & 166 & 185 & 130 \\
Red meat, g & 54 & 77 & 44 & 83 & 34 \\
Poultry, g & 25 & 36 & 58 & 30 & 33 \\
Processed meat, g & 35 & 33 & 48 & 51 & 25 \\
Vegetables, g & 168 & 183 & 144 & 164 & 150 \\
Fresh vegetables, g & 127 & 157 & 110 & 129 & 103 \\
Processed vegetables, g & 40 & 26 & 34 & 35 & 48 \\
Fish and seafood, g & 24 & 21 & 14 & 28 & 20 \\
Fruits, g & 123 & 157 & 101 & 170 & 106 \\
Fresh fruit, g & 117 & 153 & 94 & 165 & 99 \\
Processed fruit, g & 6.0 & 4.4 & 7.6 & 5.2 & 7.6 \\
Fruit and vegetable juices, ml & 54 & 33 & 57 & 80 & 41 \\
Lipids, added, g & 29 & 41 & 22 & 57 & 26 \\
Animal fat, g & 7.6 & 16 & 8.9 & 22 & 9.7 \\
Vegetable fat, g & 13 & 5.9 & 3.9 & 18 & 11 \\
Vegetable oils, g & 8.4 & 19 & 8.7 & 16 & 202 \\
Beverages, alcoholic, $\mathrm{ml}$ & 135 & 114 & 40 & 175 & 27 \\
Beverages, non-alcoholic, $\mathrm{ml}$ & 500 & 373 & 563 & 1,062 & 181 \\
Soft drinks, ml & 191 & 47 & 122 & 181 & \\
Sugar and sugar products, g & 55 & 44 & 61 & 46 & \\
& & & & & \\
\hline
\end{tabular}

n.a. $=$ Not available

Source: The DAFNE databank (www.nut.uoa.gr).

households and are generally purchased fresh. In Italy, Spain and Portugal and in several other European countries the daily fruit availability is higher than that of vegetables, pointing possibly to a preference for their consumption. In Central and Eastern Europe, part of the deficit appears to be compensated by increased consumption of fruit and vegetable juices. Higher daily availability of cereals and products was reported in Italy, Cyprus and Latvia; although the type of cereals consumed is different. In Italy, pasta and bakery products (incl. pizza) account for $50 \%$, whereas in 
Table 5.4. Mean availability of food and beverages in North European countries (unit/person/day)

\begin{tabular}{|c|c|c|c|c|}
\hline \multirow[t]{2}{*}{ Survey year } & Finland & Latvia & Norway & Sweden \\
\hline & 1998 & 2004 & $\begin{array}{l}1996 / 1997 / \\
1998\end{array}$ & 1996 \\
\hline Eggs (pieces) & 0.31 & 0.55 & 0.34 & 0.40 \\
\hline Potatoes, $\mathrm{g}$ & 111 & 274 & 114 & 85 \\
\hline Pulses, $\mathrm{g}$ & 0.80 & 4.4 & 0.82 & 2.0 \\
\hline Nuts, $g$ & 1.6 & 2.1 & 3.8 & 1.9 \\
\hline Cereals, $\mathrm{g}$ & 205 & 262 & 201 & 200 \\
\hline Milk and milk products, $\mathrm{g}$ & 507 & 292 & 387 & 445 \\
\hline Cheese, $\mathrm{g}$ & 48 & 35 & 39 & 25 \\
\hline Meat and meat products, $\mathrm{g}$ & 149 & 185 & 126 & 128 \\
\hline Red meat, $\mathrm{g}$ & 52 & 67 & 53 & 50 \\
\hline Poultry, g & 12 & 25 & 14 & 18 \\
\hline Processed meat, g & 67 & 85 & 41 & 42 \\
\hline Vegetables, g & 122 & 216 & 109 & 128 \\
\hline Fresh vegetables, $\mathrm{g}$ & 102 & 168 & 84 & 107 \\
\hline Processed vegetables, g & 20 & 48 & 24 & 21 \\
\hline Fish and seafood, $\mathrm{g}$ & 30 & 38 & 50 & 30 \\
\hline Fruits, $g$ & 157 & 120 & 135 & 122 \\
\hline Fresh fruit, $\mathrm{g}$ & 147 & 118 & 129 & 114 \\
\hline Processed fruit, $\mathrm{g}$ & 11 & 2.1 & 6.1 & 8.3 \\
\hline Fruit and vegetable juices, $\mathrm{ml}$ & 82 & 25 & 48 & n.a. \\
\hline Lipids, added, g & 31 & 43 & 32 & 39 \\
\hline Animal fat, $g$ & 11 & 8.6 & 3.6 & 4.2 \\
\hline Vegetable fat, $\mathrm{g}$ & 16 & 9.9 & 26 & 31 \\
\hline Vegetable oils, g & 3.5 & 24 & 1.7 & 3.6 \\
\hline Beverages, alcoholic, ml & 102 & 51 & 73 & n.a. \\
\hline Beverages, non-alcoholic, ml & 531 & 394 & 720 & 492 \\
\hline Soft drinks, ml & 81 & 28 & 177 & 115 \\
\hline Sugar and sugar products, $\mathrm{g}$ & 54 & 72 & 79 & 56 \\
\hline
\end{tabular}

n.a. $=$ Not available

Source: The DAFNE databank (www.nut.uoa.gr).

Cyprus and Latvia bread and rolls correspond to $60 \%$ of the daily cereal availability. Potatoes are mostly a characteristic of Northern/Western diet, as pulses are a characteristic of South food choices.

Regarding added fats and oils, studies have traditionally documented vegetable oils as the lipid of preference in the South and vegetable fat in Central/Northern Europe. The DAFNE results support these findings. Nonetheless, recent data collected in 
Table 5.5. Mean availability of foods and beverages ( $\mathrm{g} \cdot \mathrm{ml} / \mathrm{person} /$ day) by country (survey year) and educational attainment in Central and Eastern European countries

\begin{tabular}{|c|c|c|c|c|c|c|c|c|c|c|c|c|}
\hline & \multicolumn{3}{|c|}{ Austria (1999-2000) } & \multicolumn{3}{|c|}{ Germany (1998) } & \multicolumn{3}{|c|}{ Hungary (2005) } & \multicolumn{3}{|c|}{ Slovenia (2002) } \\
\hline & II/EI & $\mathrm{Sec}$ & $\mathrm{C} / \mathrm{U}$ & II/EI & $\mathrm{Sec}$ & $\mathrm{C} / \mathrm{U}$ & II/EI & $\mathrm{Sec}$ & $\mathrm{C} / \mathrm{U}$ & II/EI & $\mathrm{Sec}$ & $\mathrm{C} / \mathrm{U}$ \\
\hline Cereals and cereal products, $\mathrm{g}$ & 339 & 302 & 305 & n.a. & 216 & 224 & 318 & 244 & 195 & 441 & 289 & 266 \\
\hline Meat and meat products, $\mathrm{g}$ & 197 & 185 & 140 & & 137 & 114 & 183 & 157 & 141 & 247 & 159 & 140 \\
\hline Red meat, $\mathrm{g}$ & 80 & 67 & 38 & & 51 & 40 & 59 & 51 & 38 & 136 & 70 & 60 \\
\hline Poultry, $\mathrm{g}$ & 28 & 23 & 18 & & 16 & 15 & 66 & 48 & 46 & 37 & 31 & 27 \\
\hline Processed meat, $\mathrm{g}$ & 87 & 93 & 83 & & 61 & 52 & 48 & 47 & 47 & 66 & 52 & 49 \\
\hline Fish and seafood, $\mathrm{g}$ & 7.1 & 9.0 & 12 & & 16 & 18 & 3.6 & 5.0 & 6.6 & 10 & 12 & 16 \\
\hline Milk and milk products, $\mathrm{g}$ & 343 & 285 & 267 & & 307 & 322 & 219 & 213 & 246 & 447 & 322 & 358 \\
\hline Cheese, $\mathrm{g}$ & 26 & 28 & 30 & & 53 & 62 & 9.3 & 14 & 25 & 38 & 32 & 46 \\
\hline Vegetables, $\mathrm{g}$ & 193 & 140 & 159 & & 176 & 192 & 178 & 148 & 150 & 229 & 161 & 154 \\
\hline Fresh vegetables, g & 162 & 112 & 123 & & 138 & 151 & 161 & 130 & 128 & 205 & 135 & 130 \\
\hline Proc. vegetables, g & 31 & 27 & 36 & & 37 & 41 & 17 & 18 & 21 & 23 & 26 & 24 \\
\hline Fruit, $\mathrm{g}$ & 260 & 191 & 191 & & 177 & 197 & 114 & 116 & 139 & 241 & 208 & 230 \\
\hline Fresh fruit, $\mathrm{g}$ & 255 & 185 & 182 & & 165 & 183 & 113 & 115 & 137 & 239 & 205 & 226 \\
\hline Processed fruit, $\mathrm{g}$ & 4.9 & 5.9 & 9.9 & & 13 & 14 & 0.57 & 0.93 & 1.6 & 2.3 & 2.4 & 3.4 \\
\hline Fruit and vegetable juices, ml & 94 & 87 & 117 & & 120 & 134 & 22 & 38 & 71 & 94 & 99 & 146 \\
\hline Potatoes, $\mathrm{g}$ & 151 & 103 & 67 & & 117 & 103 & 127 & 100 & 83 & 156 & 102 & 84 \\
\hline Pulses, g & n.a. & n.a. & n.a. & & 0.59 & 0.79 & 4.3 & 2.9 & 2.1 & 8.6 & 5.6 & 3.9 \\
\hline Lipids added, g & 67 & 43 & 30 & & 37 & 34 & 59 & 48 & 41 & 88 & 48 & 39 \\
\hline Animal lipids, g & 14 & 12 & 11 & & 15 & 15 & 20 & 12 & 7.1 & 12 & 6.0 & 4.6 \\
\hline Vegetable fats, $\mathrm{g}$ & 12 & 11 & 6.2 & & 16 & 13 & 10 & 9.4 & 9.8 & 8.9 & 6.1 & 6.1 \\
\hline Vegetable oils, $\mathrm{g}$ & 40 & 20 & 13 & & 6.5 & 5.9 & 29 & 27 & 24 & 67 & 36 & 28 \\
\hline Beverages, alcoholic, ml & 172 & 174 & 142 & & 199 & 212 & 56 & 56 & 70 & 175 & 139 & 114 \\
\hline Beverages, non-alcoholic, ml & 684 & 660 & 551 & & 925 & 887 & 565 & 562 & 648 & 644 & 517 & 545 \\
\hline Soft drinks, ml & 121 & 119 & 86 & & 117 & 81 & 104 & 87 & 65 & 83 & 93 & 88 \\
\hline Sugar and sugar products, $\mathrm{g}$ & 97 & 75 & 57 & & 59 & 56 & 61 & 50 & 41 & 105 & 69 & 57 \\
\hline $\begin{array}{l}\text { n.a. = Not available. } \\
\text { II/EL = Illiterate/Elementary e } \\
\text { Source: } \text { The DAFNE databank }\end{array}$ & N.n & & 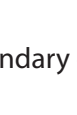 & & & & & & & & & \\
\hline
\end{tabular}

newly emerging markets, such as Slovenia, Latvia and Hungary, indicate higher household availability of oils than fats (animal and vegetable).

In relation to foods of animal origin, the highest daily availability of milk and products, but not cheese, was recorded in Finland, Sweden, Norway and Ireland. Fish and seafood are items whose consumption is shaped through various factors, among which availability/proximity to the sea and cost are dominant. According to the DAFNE data, higher daily fish availability was recorded in Portugal, Spain and Norway. With respect to meat, all countries recorded substantial household acquisitions. The type of 
Table 5.6. Mean availability of foods and beverages ( $\mathrm{g} \cdot \mathrm{ml} /$ person/day) by country (survey year) and educational attainment in South EC

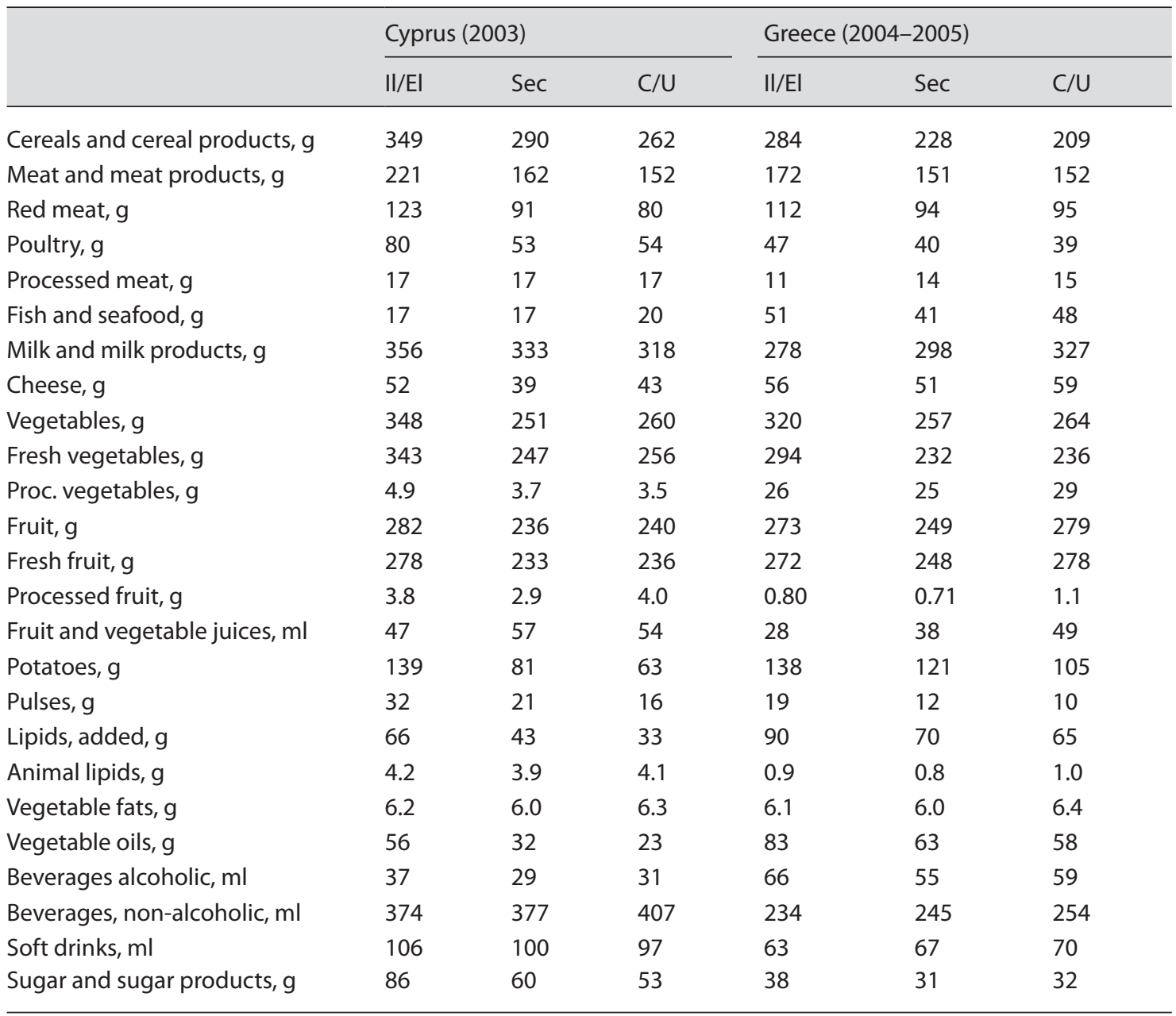

II/EL = Illiterate/Elementary education; $\mathrm{Sec}=$ Secondary education; $\mathrm{C} / \mathrm{U}=$ College/University .

Source: The DAFNE databank (www.nut.uoa.gr).

meat is, however, region-dependent. Red meat is more common in the South (Greece, Cyprus and Portugal) and processed meat is preferred in Central/Eastern European countries, Latvia and Finland. In most cases, the daily availability of poultry is less than half of that of red meat, indicating possible room for recommending the substitution of red with white meat. Exceptions are Hungary and the UK where poultry availability equals that of red meat.

Although out of home consumption of beverages is more common than the inhouse one, the DAFNE data point towards a prevalent acquisition of non-alcoholic 


\begin{tabular}{|c|c|c|c|c|c|c|c|c|}
\hline \multicolumn{3}{|c|}{ Italy (1996) } & \multicolumn{3}{|c|}{ Portugal (2000) } & \multicolumn{3}{|c|}{ Spain (1998-1999) } \\
\hline II/EI & $\mathrm{Sec}$ & $\mathrm{C} / \mathrm{U}$ & II/EI & $\mathrm{Sec}$ & $\mathrm{C} / \mathrm{U}$ & II/EI & $\mathrm{Sec}$ & $\mathrm{C} / \mathrm{U}$ \\
\hline 367 & 320 & 289 & 251 & 201 & 182 & 187 & 157 & 136 \\
\hline 149 & 130 & 118 & 159 & 169 & 155 & 151 & 131 & 113 \\
\hline 77 & 68 & 61 & 89 & 97 & 83 & 60 & 51 & 46 \\
\hline 43 & 35 & 33 & 45 & 41 & 37 & 43 & 34 & 28 \\
\hline 25 & 24 & 20 & 17 & 20 & 17 & 37 & 35 & 30 \\
\hline 38 & 37 & 38 & 81 & 91 & 79 & 63 & 58 & 65 \\
\hline 275 & 269 & 266 & 266 & 313 & 310 & 345 & 331 & 353 \\
\hline 51 & 48 & 51 & 16 & 33 & 26 & 14 & 16 & 17 \\
\hline 191 & 179 & 193 & 137 & 138 & 135 & 125 & 111 & 128 \\
\hline 135 & 123 & 138 & 127 & 126 & 120 & 109 & 92 & 104 \\
\hline 56 & 56 & 55 & 10 & 13 & 15 & 16 & 19 & 24 \\
\hline 242 & 227 & 231 & 193 & 207 & 238 & 206 & 176 & 206 \\
\hline 241 & 226 & 230 & 191 & 205 & 235 & 201 & 171 & 201 \\
\hline 1.2 & 1.5 & 1.3 & 2.2 & 2.6 & 2.9 & 4.6 & 4.9 & 5.2 \\
\hline 6.6 & 11 & 12 & 9.0 & 16 & 17 & 27 & 34 & 32 \\
\hline 85 & 71 & 69 & 188 & 158 & 132 & 102 & 72 & 58 \\
\hline 4.4 & 4.0 & 3.7 & 11 & 5.1 & 3.6 & 12 & 9.0 & 7.1 \\
\hline 71 & 59 & 51 & 53 & 45 & 37 & 50 & 40 & 37 \\
\hline 6.3 & 5.2 & 5.0 & 2.2 & 4.0 & 5.0 & 1.1 & 0.8 & 1.1 \\
\hline 1.6 & 1.5 & 1.2 & 5.1 & 5.7 & 4.4 & 1.5 & 1.7 & 1.9 \\
\hline 64 & 52 & 45 & 46 & 35 & 28 & 48 & 37 & 34 \\
\hline 175 & 136 & 123 & 135 & 90 & 91 & 70 & 77 & 76 \\
\hline 852 & 807 & 811 & 151 & 236 & 243 & 354 & 396 & 422 \\
\hline 39 & 45 & 39 & 60 & 77 & 67 & 83 & 92 & 75 \\
\hline 55 & 46 & 38 & 32 & 23 & 24 & 27 & 25 & 27 \\
\hline
\end{tabular}

beverages (including sugary soft drinks) for household use. With respect to the daily availability of sugar and sugar products in the household, the higher values were recorded in Norway, Austria, Slovenia and Latvia.

Time comparisons, using data presented in the European Nutrition and Health Report 2004 [Elmadfa et al., 2005], were feasible for four countries: Greece (19981999 vs. 2004-2005), Hungary (1991 vs. 2005), Portugal (1995 vs. 2000) and Spain (1990-1991 vs. 1998-1999). In the three Mediterranean countries, household food availability was generally lower in recent years. Exceptions hold for meat, milk and 
Table 5.7. Mean availability of foods and beverages ( $\mathrm{g} \cdot \mathrm{ml} /$ person/day) by country (survey year) and educational attainment in North and Western European countries

\begin{tabular}{|c|c|c|c|c|c|c|}
\hline & \multicolumn{3}{|c|}{ Belgium (1999) } & \multicolumn{3}{|c|}{ Finland (1998) } \\
\hline & II/El. & $\mathrm{Sec}$ & $\mathrm{C} / \mathrm{U}$ & II/El. & $\mathrm{Sec}$ & $\mathrm{C} / \mathrm{U}$ \\
\hline Cereals and cereal products, $\mathrm{g}$ & 272 & 239 & 236 & 223 & 190 & 202 \\
\hline Meat and meat products, $\mathrm{g}$ & 163 & 146 & 135 & 168 & 145 & 133 \\
\hline Red meat, $\mathrm{g}$ & 71 & 55 & 47 & 62 & 49 & 45 \\
\hline Poultry, g & 27 & 24 & 26 & 11 & 12 & 14 \\
\hline Processed meat, $\mathrm{g}$ & 38 & 36 & 32 & 78 & 65 & 57 \\
\hline Fish and seafood, $\mathrm{g}$ & 24 & 23 & 26 & 36 & 26 & 28 \\
\hline Milk and milk products, $\mathrm{g}$ & 232 & 235 & 284 & 564 & 501 & 449 \\
\hline Cheese, $g$ & 48 & 50 & 62 & 44 & 47 & 53 \\
\hline Vegetables, g & 162 & 163 & 175 & 126 & 110 & 134 \\
\hline Fresh vegetables, $\mathrm{g}$ & 122 & 123 & 135 & 106 & 91 & 111 \\
\hline Processed vegetables, $\mathrm{g}$ & 40 & 40 & 40 & 19 & 19 & 23 \\
\hline Fruit, $\mathrm{g}$ & 115 & 115 & 134 & 161 & 145 & 169 \\
\hline Fresh fruit, $\mathrm{g}$ & 110 & 109 & 127 & 151 & 135 & 156 \\
\hline Processed fruit, $\mathrm{g}$ & 5.2 & 5.6 & 6.7 & 10 & 9.4 & 13 \\
\hline Fruit and vegetable juices, $\mathrm{ml}$ & 37 & 46 & 68 & 71 & 82 & 94 \\
\hline Potatoes, $\mathrm{g}$ & 132 & 103 & 81 & 143 & 105 & 81 \\
\hline Pulses, g & n.a. & n.a. & n.a. & 0.87 & 0.68 & 0.86 \\
\hline Lipids, added, g & 36 & 29 & 26 & 39 & 29 & 25 \\
\hline Animal lipids, $\mathrm{g}$ & 11 & 7.1 & 7.0 & 16 & 9.9 & 7.4 \\
\hline Vegetable fats, $\mathrm{g}$ & 17 & 14 & 10 & 19 & 15 & 14 \\
\hline Vegetable oils, g & 8.9 & 8.4 & 8.4 & 3.6 & 3.5 & 3.5 \\
\hline Beverages alcoholic, ml & 127 & 119 & 155 & 86 & 107 & 115 \\
\hline Beverages, non-alcoholic, ml & 463 & 489 & 524 & 588 & 476 & 533 \\
\hline Soft drinks, ml & 163 & 209 & 184 & 74 & 84 & 85 \\
\hline Sugar and sugar products, $\mathrm{g}$ & 56 & 55 & 57 & 65 & 48 & 47 \\
\hline
\end{tabular}

n.a. = Not available; II/EI = Illiterate/Elementary education; $\mathrm{Sec}=$ Secondary education; $\mathrm{C} / \mathrm{U}=\mathrm{College} /$ University Source: The DAFNE databank (www.nut.uoa.gr).

products, vegetables, fruits and beverages (alcoholic and non-alcoholic). In particular, the daily availability of meat and meat products remained stable in Portugal and increased in Greece by $10 \mathrm{~g} /$ person/day, reflecting an increase in processed meat consumption. Vegetable availability increased only in Greece (from 271 to $283 \mathrm{~g} /$ person/day), whereas fruit availability decreased in Greece by $42 \mathrm{~g} /$ person/day and increased in Portugal by $23 \mathrm{~g} /$ person/day. The daily availability of fruit and vegetable juices increased in all countries, triggered probably by the availability of new products in the market. Regarding other beverages, the household daily availability of 


\begin{tabular}{|c|c|c|c|c|c|c|c|c|}
\hline \multicolumn{3}{|c|}{ Ireland (1999) } & \multicolumn{3}{|c|}{ Latvia (2004) } & \multicolumn{3}{|c|}{ Norway (1996/97/98) } \\
\hline II/El. & $\mathrm{Sec}$ & $\mathrm{C} / \mathrm{U}$ & II/EI. & $\mathrm{Sec}$ & $\mathrm{C} / \mathrm{U}$ & II/EI. & $\mathrm{Sec}$ & $\mathrm{C} / \mathrm{U}$ \\
\hline 320 & 275 & 285 & 333 & 258 & 220 & 214 & 198 & 199 \\
\hline 185 & 163 & 151 & 170 & 193 & 174 & 138 & 129 & 114 \\
\hline 49 & 43 & 39 & 57 & 72 & 60 & 61 & 55 & 45 \\
\hline 57 & 58 & 62 & 20 & 26 & 25 & 9.4 & 14 & 15 \\
\hline 61 & 46 & 37 & 82 & 86 & 82 & 46 & 42 & 36 \\
\hline 13 & 13 & 16 & 45 & 37 & 35 & 58 & 50 & 47 \\
\hline 537 & 469 & 443 & 351 & 281 & 280 & 429 & 377 & 387 \\
\hline 13 & 14 & 19 & 35 & 33 & 43 & 39 & 38 & 40 \\
\hline 169 & 137 & 135 & 228 & 214 & 213 & 107 & 104 & 118 \\
\hline 130 & 103 & 105 & 178 & 166 & 165 & 85 & 80 & 91 \\
\hline 39 & 34 & 30 & 49 & 48 & 48 & 22 & 23 & 27 \\
\hline 92 & 96 & 127 & 74 & 115 & 171 & 131 & 129 & 150 \\
\hline 85 & 89 & 118 & 73 & 114 & 167 & 126 & 123 & 143 \\
\hline 7.7 & 6.7 & 9.8 & 1.1 & 1.9 & 3.7 & 5.2 & 5.7 & 7.1 \\
\hline 39 & 57 & 84 & 8.4 & 24 & 44 & 32 & 45 & 63 \\
\hline 238 & 177 & 145 & 359 & 270 & 213 & 178 & 111 & 90 \\
\hline 1.4 & 0.97 & 0.98 & 7.0 & 3.9 & 3.6 & 1.3 & 0.78 & 0.55 \\
\hline 26 & 20 & 20 & 57 & 42 & 33 & 40 & 32 & 27 \\
\hline 13 & 7.7 & 6.7 & 12 & 8.0 & 7.6 & 4.2 & 3.2 & 3.8 \\
\hline 4.5 & 3.7 & 3.7 & 12 & 12 & 6.7 & 35 & 27 & 21 \\
\hline 8.3 & 8.8 & 9.9 & 33 & 24 & 19 & 0.9 & 1.5 & 2.3 \\
\hline 29 & 36 & 61 & 44 & 49 & 60 & 45 & 71 & 92 \\
\hline 686 & 519 & 516 & 366 & 386 & 438 & 896 & 725 & 637 \\
\hline 130 & 123 & 111 & 20 & 26 & 38 & 154 & 193 & 163 \\
\hline 73 & 58 & 52 & 87 & 69 & 65 & 90 & 79 & 76 \\
\hline
\end{tabular}

alcoholic drinks increased in Greece and, interestingly, in Portugal the availability of non-alcoholic beverages in general increased from $129 \mathrm{ml} /$ person/day in 1995 to $171 \mathrm{ml} /$ person/day in 2000 and of soft drinks in particular from $38 \mathrm{ml} /$ person/day in 1995 to $63 \mathrm{ml} /$ person/day in 2000. Data available for Hungary allow comparisons between food availability in the early 1990s and mid-2000s. In most cases, the daily per person availability decreased. Notable increases were, however, noted in the case of vegetables oils (from $15 \mathrm{~g} /$ person/day in 1991 to $27 \mathrm{~g} /$ person/day in 2005) and soft drinks (from $41 \mathrm{ml} /$ person/day in 1991 to $87 \mathrm{ml} /$ person/day in 2005). 
Socio-economic differences in eating practices are often studied in terms of the level of education achieved. Education has been reported to be the strongest and most consistent indicator in assessing socio-economic differences, as it expresses not only the individual's years of schooling, but it might also reflect occupation, income and, even more importantly when it comes to healthy dietary practice, the way an individual perceives current nutritional information [Johansson et al., 1999].

Data presented in tables 5.5-5.7 show that households with heads of higher education generally report lower food acquisitions. Nevertheless, when compared to their lower education counterparts, households of college/university education report higher daily availability of: (a) processed meat in Cyprus and Greece and poultry in Norway, Finland and Latvia; (b) fish and seafood in Central/Eastern Europe and milk, milk products and cheese in particular; (c) fruits but not vegetables in Greece and Portugal; (d) fruits and vegetables in North, West and Central Europe. In Latvia in particular, households of higher education report twice as much daily fruit availability (171 g/person/day), when compared to those of lower education (74 g/person/day). Juices and beverages (alcoholic and non-alcoholic) are purchased more by households of higher education in all countries under study.

\section{Discussion}

From the country members of the DAFNE network, this report presents data for those which are also participants in the ENHR 2009 project. The comparable betweencountries DAFNE data document regional and social disparities in food habits in Europe.

The HBS are not primarily designed to collect nutritional information and the food data bear limitations, which need to be considered when interpreting findings. For example, there are different ways to estimate the per person food availability and methods range from a simple division by the number of household members (as applied in the present analysis) to the application of sophisticated statistical modeling, which have been developed and tested in the DAFNE project [Vasdekis, 2001]. Their application, however, falls beyond the scope of the present report which aims to compare average food choices among different European households. In most cases, no records are collected on the type and quantity of food items and beverages consumed outside the home and not originating from household supplies; food losses and waste, foods given to pets, and meals offered to guests are not consistently collected. Despite their limitations, however, the HBSs provide a resource for the conduct of a wide range of nutritional analyses.

In parallel to enriching the databank, the DAFNE network is also working towards advancing the HBS dietary data. In this context:

(a) The EU supported FAIR-97-3096 project was carried out with the aim to compare individualized HBS data to those collected through INS in four countries, participants 
of the ENHR 2009 project (Belgium, Greece, Norway and the UK) [Trichopoulou and Naska, 2001].

(b) Greek and German DAFNE teams comparatively assessed cost-efficient methods for estimating energy and nutrient availability using HBS data [Naska et al., 2007].

(c) Food supply (FBS) and food availability (HBS) data from 18 DAFNE countries were compared to estimate the extent to which they correlate and were further examined for their ability to predict overall, coronary and cancer mortality through ecological analyses [Naska et al., 2008].

In addition, the FP6-supported HECTOR project (Eating Out: Habits, Determinants, and Recommendations for Consumers and the European Catering Sector) is currently in progress (http://www.nut.uoa.gr/hector/). The project is led by the DAFNE Coordinating Center and, among its objectives, is the exploitation of HBS data on out-of-home food expenditures in order to conceptually assess and monitor the within-home and out-of-home food choices in Europe.

In conclusion, given the potential of the DAFNE data, a system facilitating the regular update of the database, as well as the expansion of the network to embrace all European countries could provide a ready source of data for monitoring public health nutrition in Europe at reasonable cost.

\section{Acknowledgment}

The DAFNE initiative has been supported by the European Commission, in the context of the 'Cooperation in Science and Technology with Central and Eastern European Countries', the 'Agriculture and Agro-Industry, including Fisheries - AIR', the 'Agriculture and Fisheries - FAIR', the 'COST Action 99 - Food Consumption and Composition Data' programs and the Health Monitoring Program of DG-SANCO.

Thanks are due to the Statistical Offices and data providers of all countries presented in this report for supplying the national household budget survey data and supporting documentation, and for their unreserved collaboration.

Statistics Finland was the source of the Finnish HBS. The British HBS data is Crown copyright. It has been made available by the Office for National Statistics (ONS) through the Data Archive, based in the University of Essex. Neither the ONS nor Data Archive bear any responsibility for the analysis or interpretation of the data reported here. 


\title{
Food Consumption in Adults on the Basis of Dietary Surveys in European Countries
}

\author{
Food Consumption in Adults on the Basis of the EFSA Concise Food Consumption \\ Database (EFSA)
}

To date, numerous countries have collected data on individual food consumption from dietary surveys. The assessment of food consumption on the basis of representative dietary surveys as it was done by the EFSA Concise Food Consumption Database has several advantages compared to the use of the FAO Food Balance Sheets (FBS). Food supply as calculated from FBS overestimates food intake. Moreover, FBS do not consider the variability of consumption between individuals (cf. chapter 4).

Up to now, there is no fully harmonized European database on individual food consumption. Hence, the European Food Safety Authority (EFSA) has compiled the available data in order to make it as comparable as possible across Europe. For this purpose, expert members of the participating countries were asked to re-codify their latest and most appropriate dietary survey according to harmonized guidelines. The new EFSA Concise Database is intended for conservative estimates of exposure. Data are available for total population as well as for consumers only. Data for total population are of interest for this report. Consumption data is expressed as daily consumption per capita in grams. At this point, data are available for adults aged 16-64 years only. It is a future goal to create a database that is more comprehensive and provides data for specific population groups (e.g. children, elderly).

To overcome the problem of inhomogeneous categorization, EFSA has created a system using 15 broad food categories. Examples of foods enable a definite assignment to a particular food group. Foods that unmistakably belong to a category were not mentioned in the list of examples (table 6.1).

The consistency of the consumption data was verified by requesting daily energy intake for each individual. Additionally, no exclusion of under-reporters was applied, as the methodologies for this purpose are not yet harmonized across the participating countries [EFSA, 2008a]. However, the methods used, as well as age groups and duration of surveillance, are still inhomogeneous in this compilation (table 6.2). Several countries 
Table 6.1. 15 broad food categories as used in the Concise Database (Foods that unmistakably belong to a particular category are not mentioned here)

\begin{tabular}{|c|c|c|c|}
\hline \multicolumn{3}{|c|}{ Food category } & \multirow{2}{*}{$\begin{array}{l}\text { Examples of items } \\
\text { muesli bars, biscuits, fried rice, buckwheat, quinoa, cereal-based snacks, } \\
\text { popcorn, couscous, paella, pizza, sandwiches, lasagna, quiches, salt cake, } \\
\text { pancakes, spring rolls } \\
\text { Remark: preferably expressed as raw cereals }\end{array}$} \\
\hline 1 & $\begin{array}{l}\text { Cereals and cereal } \\
\text { products }\end{array}$ & solid & \\
\hline 2 & $\begin{array}{l}\text { Sugar and sugar products } \\
\text { including chocolate }\end{array}$ & solid & $\begin{array}{l}\text { jam, marmalade, honey, toppings, chewing gum, toffees, fudges, chocolate, } \\
\text { candies, chocolate confectionery, liquorice, marzipan, sugar-free confectionary, } \\
\text { cocoa powder, meringue, nutritive sweeteners (e.g. fructose) } \\
\text { List of items excluded: intense sweeteners }\end{array}$ \\
\hline 3 & $\begin{array}{l}\text { Fats (vegetable and } \\
\text { animal) }\end{array}$ & solid & $\begin{array}{l}\text { mayonnaise, dressings, sauces (béchamel, hollandaise), low-fat dressings or } \\
\text { mayonnaise, goose fat, coconut extract } \\
\text { List of items excluded: vegetable sauces }\end{array}$ \\
\hline 4 & $\begin{array}{l}\text { Vegetables, nuts, } \\
\text { pulses including carrots, } \\
\text { tomatoes and leafy } \\
\text { vegetables }\end{array}$ & $\begin{array}{l}\text { solid/ } \\
\text { liquid }\end{array}$ & $\begin{array}{l}\text { cooked and raw products, vegetable sauces (tomato sauce, ketchup), vegetable } \\
\text { condiments, vegetable soup, snack nuts, olives, capers, chestnuts, rhubarb, } \\
\text { soy-based products (excl. milk), coconuts, peanut butter, avocado, quorn, tofu } \\
\text { List of items excluded: starchy roots } \\
\text { Remark: preferably expressed as raw vegetable; dehydrated soups expressed } \\
\text { as such }\end{array}$ \\
\hline 5 & Starchy roots or potatoes & solid & $\begin{array}{l}\text { tapioca, cassava, sweet potatoes, starch/potato-based crisps } \\
\text { List of items excluded: carrots }\end{array}$ \\
\hline 6 & Fruits & solid & $\begin{array}{l}\text { dried fruits, canned fruit, fruit mousse, compote } \\
\text { List of items excluded: fruit juice }\end{array}$ \\
\hline 7 & $\begin{array}{l}\text { Fruit and vegetable juices, } \\
\text { soft drinks and bottled } \\
\text { water }\end{array}$ & liquid & \\
\hline 8 & $\begin{array}{l}\text { Coffee, tea, cocoa } \\
\text { (expressed as liquid) }\end{array}$ & liquid & $\begin{array}{l}\text { ice coffee, herbal tea (infusion), water-based cocoa beverage } \\
\text { List of items excluded: ice tea, milk-based chocolate }\end{array}$ \\
\hline 9 & Alcoholic beverages & liquid & \\
\hline 10 & $\begin{array}{l}\text { Meat and meat products, } \\
\text { offal }\end{array}$ & solid & \\
\hline 11 & Fish and seafood & solid & fish eggs \\
\hline 12 & Eggs & solid & $\begin{array}{l}\text { omelette, fried eggs } \\
\text { List of items excluded: fish eggs }\end{array}$ \\
\hline 13 & $\begin{array}{l}\text { Milk- and diary-based } \\
\text { products }\end{array}$ & $\begin{array}{l}\text { solid/ } \\
\text { liquid }\end{array}$ & cold milk chocolate, soy milk \\
\hline 14 & $\begin{array}{l}\text { Miscellaneous/food for } \\
\text { special dietary uses }\end{array}$ & solid & $\begin{array}{l}\text { salt, baker's yeast, vinegar, mustard, condiment sauce (e.g. sweet-sour sauce, } \\
\text { soy sauce), herbs and spices, pepper, gelatine, intense sweeteners, fat-free } \\
\text { sauces, glutamate-based products (e.g. vetsin, marmite)/meal substitutes, food } \\
\text { for special purposes, food supplements (e.g. fish oil, vitamins, brewers'yeast) } \\
\text { List of items excluded: nutritional sweeteners (e.g. fructose) }\end{array}$ \\
\hline 15 & Tap water & liquid & \\
\hline
\end{tabular}

Source: EFSA, 2008b. 
Table 6.2. Age group, method and year(s) of survey - overview of 16 countries

\begin{tabular}{llll}
\hline Country & Age group, years & Method & Year(s) of survey \\
\hline Austria & $19-64$ & 24-hour recall & 2005-2006 \\
\hline Belgium & $16-64$ & 24-hour recall & 2004 \\
\hline Czech Republic & $16-64$ & 24-hour recall & 2003-2004 \\
\hline Denmark & $4-65$ & 7-day precoded food & 2000-2002 \\
\hline Estonia & $16-64$ & diary with open fields & 1997 \\
\hline Finland & $25-64$ & 24-hour recall & 2002 \\
\hline France & $>14$ & 48-hour recall & 1999 \\
\hline Germany & $>17$ & 7-day dietary record & 1998 \\
\hline Hungary & $>17$ & 28-day dietary history & 2003-2004 \\
\hline Ireland & $18-64$ & 3-day dietary record & $1997-1999$ \\
\hline Italy & $16-64$ & 7-day dietary record & $1994-1996$ \\
\hline Netherlands & all ages & 7-day dietary record & $1997-1998$ \\
\hline Norway & $16-79$ & 2-day dietary record & $1993-1997$ \\
\hline Poland & 1-96 & $\begin{array}{l}\text { quantitative food frequency } \\
\text { questionnaire }\end{array}$ & 2000 \\
\hline Sweden & $>16$ & 24-hour recall & 2000-2001 \\
\hline UK & $19-64$ & 7-day dietary record & 7-day dietary record \\
\hline
\end{tabular}

Source: EFSA, 2008b.

provided data for participants $<16$ and/or $>64$ years. If that was the case, only participants aged 16-64 years were included. It has to be noted that differences between countries can be caused by different survey methodologies or different durations of survey.

In 7 of 15 food categories, highest consumption was reported in Poland. To some extent, this might be caused by inhomogeneous methodology across the compared studies. The high level of consumption in Poland might be linked to two important issues. The first one is the time of data collection - the Polish survey was conducted in the peak season of fruit, vegetable, and potato consumption. The second issue relates to the demographical structure in Poland, that implies a high level of food self-supply, since $40 \%$ of the Polish population lives in rural areas. Another limitation is the diverging assignment of foods to the food groups due to the use of differing nutritional evaluation software. For instance, ingredients- vs. recipe- 
Table 6.3. Average consumption and countries with highest and lowest consumption of 15 broad food groups (g/capita/day)

\begin{tabular}{|c|c|c|c|c|}
\hline & $\begin{array}{l}\text { Countries } \\
n\end{array}$ & Mean & Min (country) & Max (country) \\
\hline Cereals and cereal products & 16 & 277 & 153 (Finland) & 330 (Austria) \\
\hline $\begin{array}{l}\text { Sugar and sugar products including } \\
\text { chocolate }\end{array}$ & 16 & 36 & 20 (Italy) & 57 (Poland) \\
\hline Fats (vegetable and animal) & 16 & 34 & 12 (Austria) & 60 (Poland) \\
\hline $\begin{array}{l}\text { Vegetables, nuts, pulses including carrots, } \\
\text { tomatoes and leafy vegetables }\end{array}$ & 16 & 220 & 118 (Sweden) & 295 (Poland) \\
\hline Starchy roots or potatoes & 16 & 119 & 48 (Italy) & 304 (Poland) \\
\hline Fruits & 16 & 166 & 95 (UK) & 282 (Poland) \\
\hline $\begin{array}{l}\text { Fruit and vegetable juices, soft drinks } \\
\text { and bottle water }\end{array}$ & 16 & 495 & 179 (Ireland) & 947 (Germany) \\
\hline Coffee, tea, cocoa (expressed as liquid) & 16 & 521 & 124 (Italy) & 887 (Netherlands) \\
\hline Alcoholic beverages & 16 & 195 & 68 (Poland) & 413 (Czech Republic) \\
\hline Meat and meat products, offal & 16 & 174 & 109 (Norway) & 259 (Poland) \\
\hline Fish and seafood & 16 & 28 & 9 (Hungary) & 63 (Norway) \\
\hline Eggs & 16 & 20 & 5 (Austria) & 31 (Poland) \\
\hline Milk- and dairy-based products & 16 & 266 & 171 (Austria) & 522 (Norway) \\
\hline Miscellaneous/food for special dietary uses & 16 & 15 & 2 (Belgium) & 36 (Germany) \\
\hline Tap water & 13 & 225 & 71 (Germany) & 887 (Finland) \\
\hline
\end{tabular}

Means were calculated as arithmetic averages weighted for total country populations of adults aged 15-64 years at the year of survey.

Source: Eurostat, 2009.

$\mathrm{n}$ (subjects) $=33,463$ (total number of subjects that participated in the surveys of the 16 countries).

Source of raw data: EFSA, 2008b.

based processing yields inconsistent results. Further information on the strengths and limitations of the EFSA Concise Database can be found on the website: http:// www.efsa.europa.eu/EFSA/ScientificPanels/DATEX/efsa_locale-1178620753812_ ConciseEuropeanConsumptionDatabase.htm.

With the exception of tap water (no data from Estonia, Hungary, and Poland), data are available from 16 of the participating countries of the ENHR 2009 for the 15 broad food categories. Countries with the highest and lowest intake as well as mean values are compiled in table 6.3. Means were calculated as arithmetic averages and 
weighted for the total country populations of adults aged 15-64 years at the year of survey [Eurostat, 2009].

The amounts of consumption of the 15 broad food categories in the four predefined ENHR 2009 regions are described below (means of the countries belonging to a region weighted on the basis of total country populations at the year of survey). Denmark, Estonia, Finland, Norway, and Sweden represent the North region; Austria, the Czech Republic, Germany, Hungary, and Poland represent the Central and East region; Belgium, France, Ireland, The Netherlands, and the UK represent the West region; Italy represents the South region.

Cereals and Cereal Products. The highest consumption of this food group was reported in the Central and East region (292 g/capita/day). The North region (223 g/ day) was far below the average of the regions. Especially the countries Austria (330 g/day), France (318 g/day), and Poland (312 g/day), reported high amounts of cereal intake. The lowest values were accounted for Finland (153 g/day), Estonia (186 g/ day), and Norway (182 g/day).

Sugar and Sugar Products, Including Chocolate. The Central and East region had the highest intake of sugar and sugar products ( $46 \mathrm{~g} / \mathrm{capita} /$ day). In the South region, represented only by Italy, less than half of this quantity was reported (20 g/day). Poland (57 g/day), Norway (47 g/day), and Germany (45 g/day) were the countries with the highest values. In addition to Italy ( $20 \mathrm{~g} /$ day), Estonia (21 g/day) and Austria ( $23 \mathrm{~g} /$ day) had satisfyingly low results in this category.

Fats (Vegetable and Animal). As for sugar and sugar products, the highest intake of fats was reported in the Central and East region (39 g/capita/day). The lowest values were reported in the West region ( $28 \mathrm{~g} /$ day). The other three regions were positioned around 35 g/day. Poland (60 g/day), Hungary (54 g/day), and The Netherlands (48 g/ day) were the countries with the highest values. The lowest consumption was reported in Austria (12 g/day), the UK (20 g/day), and Sweden (24 g/day).

Vegetables, Nuts, Pulses Including Carrots, Tomatoes and Leafy Vegetables. Consumption of foods belonging to this category was very high in the South ( $249 \mathrm{~g} /$ capita/day) and Central and East regions (248 g/day). The North region (140 g/day) was far below the average. The highest values appeared in Poland (295 g), Germany (252 g/day), and Italy (249 g/day). Lowest values were reported in Sweden (118 g/day), the Czech Republic (131 g/day), and Finland (135 g/day).

Starchy Roots or Potatoes. The consumption of starchy roots and potatoes was highest in the Central and East region (165 g/capita/day), followed by the North (126 $\mathrm{g} /$ day) and West regions (97 $\mathrm{g} /$ day). The value for the South region was exceptionally low (48 g). Besides an extraordinarily high result for Poland (304 g/day), high values were reported in Ireland ( $229 \mathrm{~g} /$ day) and Estonia (201 g/day). In addition to Italy (48 g/day), Austria ( $59 \mathrm{~g} /$ day) and France (67 g/day) had the lowest values.

Fruits. It is recommended to consume at least five servings of a variety of fruits and colorful vegetables each day [WCRF/AIRC, 2007]. The sum of fruits and vegetables should exceed $400 \mathrm{~g} /$ day [Kushi et al., 2006]. 
The highest intake of fruits was reported in the Central and East (209 g/capita/ day) and South regions (203 g/day). Fruit consumption was considerably lower in the North (129 g/day) and West regions (113 g/day). Leading countries concerning the consumption of fruits were Poland (282 g/day), Italy (203 g), and Austria (202 g/day). The lowest values were assessed for the UK (95 g/day), Ireland (106 g/day), and The Netherlands (107 g/day). On average, only four countries (Poland, Germany, Italy, and Austria) have met the recommendation of consuming at least $400 \mathrm{~g}$ of fruits and vegetables per day.

Fruit and Vegetable Juices, Soft Drinks and Bottled Water. The consumption of products belonging to this category was highest in the Central and East region $(667 \mathrm{~g} /$ capita/day). The lowest values emerged in the North region (331 g/day). Remarkably high values were accounted for Germany ( $947 \mathrm{~g} /$ day) and Belgium (945 g/day), followed by the Czech Republic ( $618 \mathrm{~g} /$ day). The lowest intake was found to be in Ireland (179 g/day), Finland (213 g/day), and Poland (237 g/day).

Coffee, Tea, Cocoa (Expressed as Liquid). The Central and East (633 g/capita/day) and North regions ( $625 \mathrm{~g} /$ day) had the highest values in this category. In contrast, only $124 \mathrm{~g} /$ day were accounted in the South region. The highest consumption was reported in The Netherlands ( $887 \mathrm{~g} /$ day), Denmark ( $836 \mathrm{~g} /$ day), and the UK (725 g/ day). In addition to Italy ( $124 \mathrm{~g} /$ day), Hungary ( $176 \mathrm{~g} /$ day) and France ( $282 \mathrm{~g} /$ day) had the lowest values in this category.

Alcoholic Beverages. The highest consumption of alcoholic beverages was reported in the West region (235 g/capita/day), whereas the lowest values appeared in the South region (126 g/day). The North and Central and East regions showed an equal result (181 g). The Czech Republic (413 g/day), Ireland ( $335 \mathrm{~g} /$ day), and the UK (313 $\mathrm{g} /$ day) were the countries with the highest consumption of alcoholic beverages. The lowest values were reported in Poland (68 g/day), Hungary ( $76 \mathrm{~g} /$ day), and Austria (118 g/day).

Meat and Meat Products, Offal. The consumption of meat and meat products was higher in the Central and East region (194 g/capita/day) than in the West (174 g/day), South (137 g/day), and North regions (134 g/day). The highest values were reported in Poland (259 g/day), France ( $202 \mathrm{~g} /$ day), and the Czech Republic (187 g/day). The lowest intake appeared in Norway (109 g/day), Finland (120 g/day), and Belgium (123 g/day).

Fish and Seafood. The highest consumption of fish and seafood was found in the South region ( $43 \mathrm{~g} / \mathrm{capita} /$ day). As the Central and East region $(18 \mathrm{~g} /$ day) is predominantly represented by landlocked countries, the low intake emerged as expected. An outstandingly high consumption was reported in Norway (63 g/day), followed by Italy ( $43 \mathrm{~g} /$ day) and France ( $37 \mathrm{~g} /$ day). On the other hand, intake of fish and seafood was exceptionally low in Hungary ( 9 g/day) and still lower in The Netherlands (13 g/ day) and Austria (16 g/day).

Eggs. Consumption of eggs was found to be highest in the Central and East region ( $25 \mathrm{~g} /$ capita/day), followed by the South (18 g/day), West (18 g/day), and North 


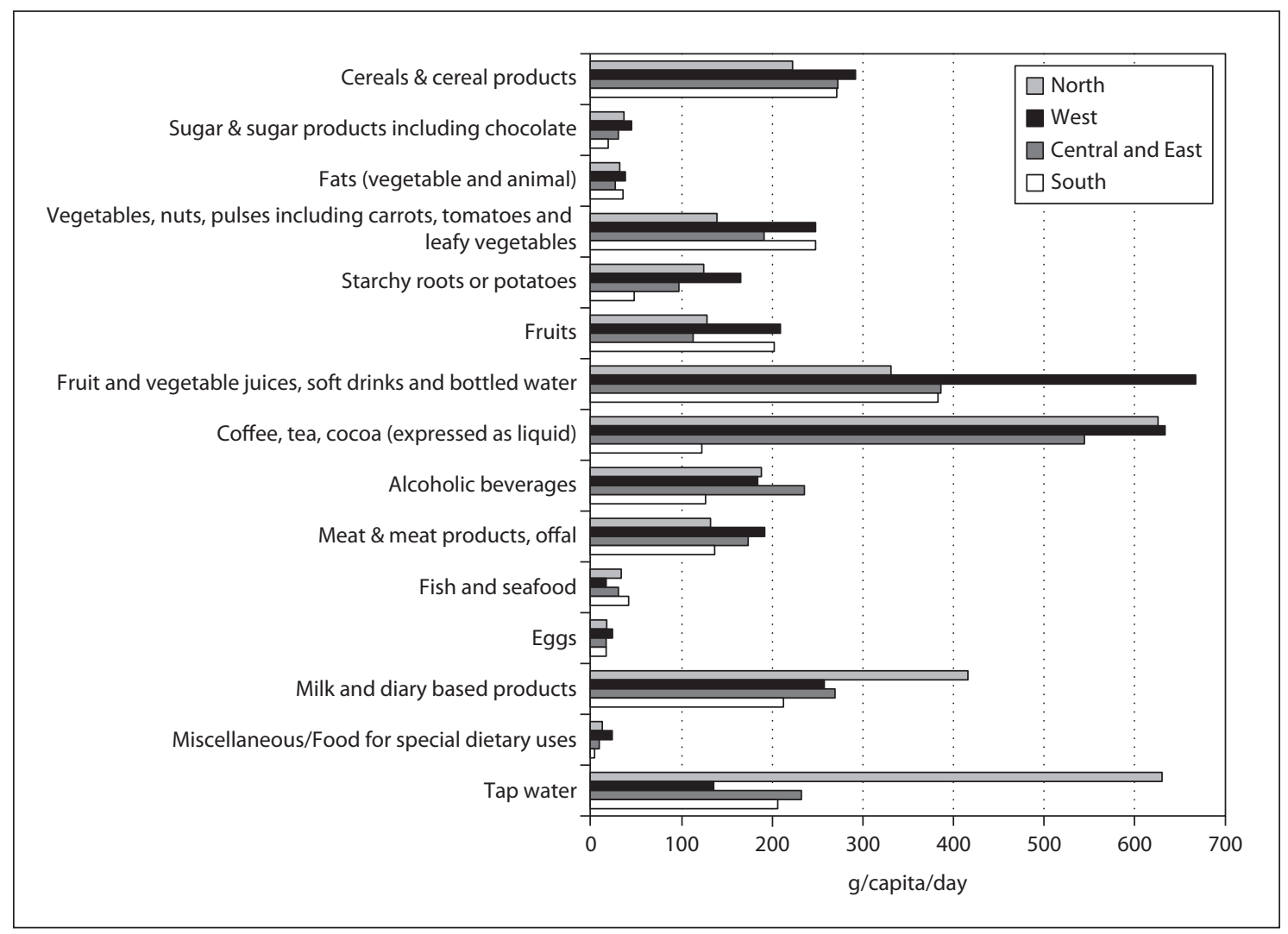

Fig. 6.1. 15 broad food categories - comparison of the four regions of ENHR 2009. Means were calculated as arithmetic averages weighted for total country populations of adults aged 15-64 years in the year of survey. Source: Eurostat, 2009.

regions (17 g/day). The countries Poland ( $31 \mathrm{~g} /$ day), Hungary (27 g/day), and Estonia ( $27 \mathrm{~g} /$ day) had the highest intake of eggs. The lowest values appeared in Austria (5 g/ day), followed by Belgium (10 g/day), and Sweden (14 g/day).

Milk- and Dairy-Based Products. The highest consumption of milk- and dairybased products was reported in the North region ( $416 \mathrm{~g} /$ capita/day). The lowest intake was found in the South region (212 g/day). Norway (522 g/day), Finland (437 g/day), and The Netherlands (388 g/day) were the countries with the highest values in this category. Intake was low in Austria (171 g/day), Poland (181 g/day), and the Czech Republic (186 g/day). Concerning the high intake of milk- and dairy-based products in the northern parts of Europe, it has to be considered that lactose intolerance is less prevalent in this area [EUFIC, 2006].

Miscellaneous/Food for Special Dietary Uses. In this category, highest amounts were accounted for the Central and East region (25 g/capita/day). The lowest values were reported in the South region ( $5 \mathrm{~g} /$ day). The countries with the highest consumption 
were Germany ( $36 \mathrm{~g} /$ day), Finland ( $24 \mathrm{~g} /$ day), and the UK (12 g/day). The lowest results appeared in Belgium ( $2 \mathrm{~g} /$ day), France ( $2 \mathrm{~g} /$ day), and Estonia ( $\mathrm{g}$ /day).

Tap Water. It is recommended to drink at least eight cups of liquid (equal to $1,000-1,500 \mathrm{~g}$ ) per day. More can be beneficial. A sufficient fluid intake requires 21 of water per day [WCRF/AIRC, 2007], or $30 \mathrm{ml} / \mathrm{MJ}$ of consumed food energy [Eurodiet, 2000]. The results shown here refer to the consumption of tap water only.

The differences concerning tap water consumption were relatively small between the West $(231 \mathrm{~g} /$ capita/day) and South regions (206 g/day). Tap water intake was highest in the North region $(630 \mathrm{~g} /$ day) and lowest in the Central and East region (136 g/day). The highest consumption was reported in Finland (887 g/day), Denmark (840 g/day), and Austria (594 g/day). For Germany (71 g/day) and Belgium (100 g/ day) the lowest results emerged.

Figure 6.1 shows an overview of the consumption of the 15 broad food categories in the four regions of ENHR 2009. Means were calculated as arithmetic averages weighted on the basis of the total age-specific population of the countries. 


\subsection{Methodology}

The following chapter will describe the energy and nutrient intake on the individual level by sex and age group, using data from dietary surveys undertaken by the participating countries. Although the comparability of data is limited because of the use of different methods such as 24-hour recalls or Food Frequency Questionnaires, different years and periods of data collection, and different age classifications, they still can give an overview on the nutritional situation in countries of the European Union. Nevertheless, the data have to be interpreted cautiously. At the beginning of every part of this chapter an overview of the surveys which were included is given. If one of the participating countries provided more than one study for the different age groups, the study with the largest sample size followed by the most recent year of the survey were taken into account.

In Europe many different reference values exist [see SCF, 2003]. For example, the 'Dietary Reference Values for Food Energy and Nutrients for the United Kingdom' of the Department of Health [1991] and the 'Dietary reference intakes: energy, proteins, fats, and digestible carbohydrates' of the Health Council of The Netherlands [2001]. In addition to those national reference values, further nutrient based guidelines for groups of countries were developed. Those are the D-A-CH Reference values for the German-speaking countries and central Europe [D-A-CH, 2000] which are applied also in Hungary, Slovenia and the Czech Republic, and the Nordic Nutrition Recommendations [NNR, 2004] for the Nordic Countries respectively. Regarding the coverage of countries the most complete reference values for the participating countries are those published by the World Health Organization/ Food and Agriculture Organization of the United Nations [WHO, 2003], Eurodiet [Eurodiet, 2000] and the Scientific Committee on Food [SCF, 1993]. In this report data will be discussed using the recommendations published by the WHO and Eurodiet. When no reference value was given by those two publications, the intake 
was compared to the $\mathrm{D}-\mathrm{A}-\mathrm{CH}$ reference values. In some cases the $\mathrm{D}-\mathrm{A}-\mathrm{CH}$ and the NNR deviate a lot. For those nutrients intake data were discussed considering both reference values.

Table 7.1 gives an overview on the different nutrient based guidelines which are used in Europe. To give a complete picture the SCF recommendations [SCF, 1993] are given in table 7.1. However, as those recommendations are outdated they will not be discussed.

Updated internationally harmonized recommendations based on up-to-date scientific data are needed. Therefore, the European Commission asked the European Food Safety Authority (EFSA) to advise on values for micronutrient recommendations, after first advising on existing values for energy, macronutrients and dietary fibers in 2005.

The discussion will focus on the comparison between the four different regions North, South, Central and East, and West.

\subsection{Energy and Nutrient Intake in European Children}

\section{Background}

Data on energy and nutrient intake of children were available from 16 countries. It was not possible for all 16 countries to provide data for the same age groups, a situation which makes comparison for this population group especially difficult. Nevertheless, these data give a valuable overview on nutrient and energy intake of European children.

Dietary assessment in the age group 4-6 years was done in most participating countries using dietary records over 2-7 days or recalls (one or repeated 24-hour recalls, 48-hour recall) or as in one case a combination of a 3-day weighed record and one 24-hour recall. In the reported surveys use of supplements was considered only by five countries (table 7.2). Dietary intake data therefore has to be interpreted cautiously as large differences in intake levels in certain nutrients might be caused by this methodological aspect. For this age group data from 7,467 children were available.

Also for the age group 7-9 years assessment methods used were weighed records over 2-7 days ( 8 countries) and recalls, one or repeated 24-hour recalls (5 countries). The use of supplements was considered only by two countries. Data from 9,815 children of 13 countries were available (table 7.3).

In nine countries energy and nutrient intakes in the 10- to 14-year-old children were calculated from weighed records over 2-7 days, in other four countries recalls, one or repeated 24-hour, and from one country only FFQ was used. The use of supplements was considered only by three countries. From this age group data from 8,995 children are available (table 7.4). 
Table 7.1. Comparison of different reference values using the example of adults

\begin{tabular}{|c|c|c|c|c|c|}
\hline & \multicolumn{2}{|c|}{ WHO 2003, 2007, 2009b } & \multicolumn{2}{|l|}{ Eurodiet 2000} & \multirow{2}{*}{$\frac{\text { SCF } 1993^{1}}{M}$} \\
\hline & $M$ & $F$ & $\mathrm{M}$ & $\mathrm{F}$ & \\
\hline \multicolumn{6}{|c|}{ Energy and macronutrients } \\
\hline \multicolumn{6}{|l|}{ Energy } \\
\hline Protein & $10-15 \% \mathrm{E}$ & $10-15 \% E$ & & & 56 g/day \\
\hline Carbohydrates & $50-75 \% \mathrm{E}$ & $50-75 \% \mathrm{E}$ & $>50 \% \mathrm{E}$ & $>50 \% \mathrm{E}$ & \\
\hline Sucrose & $<10 \% \mathrm{E}$ & $<10 \% \mathrm{E}$ & & & \\
\hline Dietary fiber & $>25$ g/day & $>25$ g/day & >25 g/day & $>25$ g/day & \\
\hline Fat & $15-30 \% \mathrm{E}$ & $15-30 \% \mathrm{E}$ & $<30 \% \mathrm{E}$ & $<30 \% \mathrm{E}$ & \\
\hline SFA & $<10 \% \mathrm{E}$ & $<10 \% \mathrm{E}$ & $<10 \% \mathrm{E}$ & $<10 \% \mathrm{E}$ & \\
\hline \multicolumn{6}{|l|}{ MUFA } \\
\hline PUFA & $6-11 \% \mathrm{E}$ & $6-11 \% \mathrm{E}$ & $\begin{array}{l}4-8 \% \text { E n-6 + } 2 \text { g } \\
\text { linolenic }+200 \\
\text { mg very long } \\
\text { chain }\end{array}$ & $\begin{array}{l}4-8 \% \text { E n- } 6+ \\
2 \mathrm{~g} \text { linolenic + } \\
200 \text { mg very } \\
\text { long chain }\end{array}$ & $\begin{array}{l}6 \mathrm{~g} \mathrm{n}-6+1.5 \mathrm{gn}-3 / \\
\text { day }\end{array}$ \\
\hline Cholesterol & <300 mg/day & $<300$ mg/day & & & \\
\hline \multicolumn{6}{|l|}{ Vitamins } \\
\hline Vitamin A & & & & & $\begin{array}{l}500 \mu \mathrm{g} \text { retinol } \\
\text { equivalents/day }\end{array}$ \\
\hline Thiamine & & & & & $1.1 \mathrm{mg} / \mathrm{day}$ \\
\hline Riboflavin & & & & & 1.6 mg/day \\
\hline Niacin & & & & & $\begin{array}{l}18 \text { mg niacin } \\
\text { equivalents/day }\end{array}$ \\
\hline Vitamin $\mathrm{B}_{6}$ & & & & & $1.5 \mathrm{mg} / \mathrm{day}$ \\
\hline Folate & & & $\begin{array}{l}>400 \mu \mathrm{g} / \mathrm{day} \\
\text { (from food) }\end{array}$ & $\begin{array}{l}>400 \mu \mathrm{g} / \text { day } \\
\text { (from food) }\end{array}$ & $\begin{array}{l}200 \mu \mathrm{g} \text { mixed } \\
\text { dietary folates/ } \\
\text { day }\end{array}$ \\
\hline Cobalamin & & & & & $1.4 \mu \mathrm{g} /$ day \\
\hline Ascorbic acid & & & & & 45 mg/day \\
\hline Vitamin D & & & & & 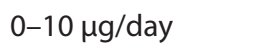 \\
\hline
\end{tabular}

Vitamin E 


\begin{tabular}{|c|c|c|c|c|}
\hline \multirow{2}{*}{$\begin{array}{l}\text { SCF } 1993^{1} \\
F\end{array}$} & \multicolumn{2}{|c|}{$\mathrm{DACH} 2000^{2}$} & \multicolumn{2}{|c|}{ NNR $2004^{4}$} \\
\hline & $M$ & $\mathrm{~F}$ & $M$ & $F$ \\
\hline
\end{tabular}

\begin{tabular}{|c|c|c|c|c|}
\hline & 12.5/12.0/10.5 MJ/day 3 & 10.0/9.5/8.5 MJ/day 3 & 12.3/11.8 MJ/day 5 & 9.4/9.2 MJ/day ${ }^{5}$ \\
\hline \multirow[t]{5}{*}{47 g/day } & 59/59/58 g/day & 48/47/46 g/day & $10-20 \% \mathrm{E}$ & $10-20 \% \mathrm{E}$ \\
\hline & $>50 \% \mathrm{E}$ & $>50 \% \mathrm{E}$ & $50-60 \% \mathrm{E}$ & $50-60 \% \mathrm{E}$ \\
\hline & & & $<10 \% \mathrm{E}$ & $<10 \% \mathrm{E}$ \\
\hline & $>30$ g/day & >30 g/day & 25-35 g/day & 25-35 g/day \\
\hline & $30 \% \mathrm{E}$ & $30 \% \mathrm{E}$ & $25-35 \% \mathrm{E}$ & $25-35 \% \mathrm{E}$ \\
\hline
\end{tabular}

\begin{tabular}{|c|c|c|c|}
\hline & & $10-15 \% \mathrm{E}$ & $10-15 \% \mathrm{E}$ \\
\hline $4.5 \mathrm{~g} \mathrm{n}-6+1 \mathrm{~g} \mathrm{n}-3 /$ day $2.5 \mathrm{n}-6+0.5 \mathrm{n}-3 \% \mathrm{E}$ & $2.5 n-6+0.5 n-3 \% E$ & $5-10 \% \mathrm{E}$ & $5-10 \% E$ \\
\hline
\end{tabular}

\begin{tabular}{|c|c|c|c|c|}
\hline $\begin{array}{l}400 \mu \mathrm{g} \text { retinol } \\
\text { equivalents/day }\end{array}$ & $\begin{array}{l}1 \text { mg retinol } \\
\text { equivalents/day }\end{array}$ & $\begin{array}{l}0.8 \text { mg retinol } \\
\text { equivalents/day }\end{array}$ & $\begin{array}{l}900 \mu \mathrm{g} \text { retinol } \\
\text { equivalents/day }\end{array}$ & $\begin{array}{l}700 \mu \mathrm{g} \text { retinol } \\
\text { equivalents/day }\end{array}$ \\
\hline 0.9 mg/day & 1.3/1.2/1.1 mg/day & $1 \mathrm{mg} / \mathrm{day}$ & 1.4 mg/day & 1.1 mg/day \\
\hline 1.3 mg/day & 1.5/1.4/1.3 mg/day & 1.2 mg/day & 1.7 mg/day & 1.3 mg/day \\
\hline $\begin{array}{l}14 \text { mg niacin } \\
\text { equivalents/day }\end{array}$ & $\begin{array}{l}\text { 17/16/15 mg niacin } \\
\text { equivalents/day }\end{array}$ & $\begin{array}{l}13 \text { mg niacin } \\
\text { equivalents/day }\end{array}$ & $\begin{array}{l}19 \text { mg niacin } \\
\text { equivalents/day }\end{array}$ & $\begin{array}{l}15 \text { mg niacin } \\
\text { equivalents/day }\end{array}$ \\
\hline $1.1 \mathrm{mg} /$ day & 1.5 mg/day & 1.2 mg/day & 1.6 mg/day & $1.2 \mathrm{mg} /$ day \\
\hline $\begin{array}{l}200 \mu \mathrm{g} \text { mixed } \\
\text { dietary folates/ } \\
\text { day }\end{array}$ & $\begin{array}{l}400 \mu \mathrm{g} \text { folate } \\
\text { equivalents/day }\end{array}$ & $\begin{array}{l}400 \mu \mathrm{g} \text { folate } \\
\text { equivalents/day }\end{array}$ & 300 mg/day & $300 \mu \mathrm{g} /$ day \\
\hline $1.4 \mu \mathrm{g} / \mathrm{day}$ & $3 \mu \mathrm{g} /$ day & $3 \mu \mathrm{g} /$ day & $2 \mu \mathrm{g} /$ day & $2 \mu \mathrm{g} /$ day \\
\hline 45 mg/day & 100 mg/day & 100 mg/day & 75 mg/day & 75 mg/day \\
\hline \multirow[t]{2}{*}{$010 \mu \mathrm{g} / \mathrm{day}$} & $5 \mu \mathrm{g} /$ day & $5 \mu \mathrm{g} /$ day & $7.5 \mu \mathrm{g} /$ day & $7.5 \mu \mathrm{g} /$ day \\
\hline & $\begin{array}{l}\text { 15/14/13 mg } \\
\text { a-tocopherol } \\
\text { equivalents/day }\end{array}$ & $\begin{array}{l}12 \text { mg a-tocopherol } \\
\text { equivalents/day }\end{array}$ & $\begin{array}{l}10 \mathrm{mg} \text { a-tocopherol } \\
\text { equivalents/day }\end{array}$ & $\begin{array}{l}8 \mathrm{mg} \\
\text { a-tocopherol } \\
\text { equivalents/day }\end{array}$ \\
\hline
\end{tabular}


Table 7.1. Continued

\begin{tabular}{|c|c|c|c|c|c|}
\hline & \multicolumn{2}{|c|}{ WHO 2003, 2007, 2009b } & \multicolumn{2}{|l|}{ Eurodiet 2000} & \multirow{2}{*}{ SCF $1993^{1}$} \\
\hline & $M$ & $\mathrm{~F}$ & $M$ & $\mathrm{~F}$ & \\
\hline \multicolumn{6}{|l|}{ Minerals } \\
\hline Calcium & & & $>800$ mg/day & $>800$ mg/day & 700 mg/day \\
\hline Magnesium & & & & & $150-500$ mg/day \\
\hline Phosphorus & & & & & 550 mg/day \\
\hline Sodium & $<2 \mathrm{~g} /$ day & $<2$ g/day & $<6$ g salt/day & $<6$ g salt/day & $\begin{array}{l}575-3,500 \mathrm{mg} / \\
\text { day }\end{array}$ \\
\hline Potassium & & & & & 3,100 mg/day \\
\hline Iron & & & & 15 mg/day & \\
\hline Zinc & & & & & 9.5 mg/day \\
\hline Copper & & & & & $1.1 \mathrm{mg} / \mathrm{day}$ \\
\hline Selenium & & & & & $55 \mu \mathrm{g} /$ day \\
\hline lodine & $150 \mu \mathrm{g} /$ day & $150 \mu \mathrm{g} /$ day & $150 \mu \mathrm{g} /$ day & 150 mg/day & 130 mg/day \\
\hline Manganese & & & & & $1-10 \mathrm{mg} / \mathrm{day}$ \\
\hline \multicolumn{6}{|c|}{$\begin{array}{l}{ }^{1} \text { Population Reference Intake; }{ }^{2} \text { reference values refer to age groups } 19-24 \text { years, } 25-50 \text { years and } 51-64 \\
\text { years; }{ }^{3} 19-24 \text { years: PAL } 1.75,25-50 \text { years: PAL } 1.7,51-64 \text { years: PAL } 1.6 ;{ }^{4} \text { reference values refer to age groups } \\
18-30 \text { years and } 31-60 \text { years, recommended intake; }{ }^{5} \text { PAL } 1.6 ;{ }^{6} \text { postmenopause. }\end{array}$} \\
\hline
\end{tabular}

\section{Energy and Macronutrients}

Comparison of Countries

Tables 7.5-7.7 show that in most countries the energy intake increased with increasing age. Exceptions are Portuguese boys and Austrian, Danish, Portuguese and Swedish girls. With increasing age the difference between the energy intake of girls and boys increased as well. The energy intake in male children was 6.2-11.7 MJ/day, in female children 5.5-10.6 MJ/day.

Protein intake in percent of total energy intake was between 11.1 and $17.6 \% \mathrm{E}$. Lowest share of protein intake was found in 7- to 9-year-old children from Poland. Highest intake was found in 10- to 14-year-old girls from Spain. Protein intake (\%E) was above the recommendation of $10-15 \% \mathrm{E}$ in seven countries, namely Finland (only boys), France, Greece, Italy, Portugal, Spain, and Sweden [WHO, 2003].

As already documented in the European Nutrition and Health Report 2004 in Spain and Greece the mean carbohydrate intake of children was particularly low 


\begin{tabular}{|c|c|c|c|c|}
\hline \multirow{2}{*}{$\frac{\text { SCF } 1993^{1}}{F}$} & \multicolumn{2}{|l|}{ DACH $2000^{2}$} & \multicolumn{2}{|l|}{ NNR $2004^{4}$} \\
\hline & M & $\mathrm{F}$ & M & $\mathrm{F}$ \\
\hline 700 mg/day & 1,000 mg/day & 1,000 mg/day & 800 mg/day & $800 \mathrm{mg} /$ day \\
\hline $150-500$ mg/day & $400 / 350 / 350 \mathrm{mg} /$ day & $310 / 300 / 300 \mathrm{mg} /$ day & 350 mg/day & $280 \mathrm{mg} /$ day \\
\hline $550 \mathrm{mg} /$ day & 700 mg/day & 700 mg/day & $600 \mathrm{mg} /$ day & $600 \mathrm{mg} / \mathrm{day}$ \\
\hline $575-3,500 \mathrm{mg} /$ day & 550 mg/day & 550 mg/day & $1-2$ g/day & $1-2 \mathrm{~g} /$ day \\
\hline \multirow[t]{2}{*}{3,100 mg/day } & 830 mg/day & 830 mg/day & $3.5 \mathrm{~g} / \mathrm{day}$ & $3.1 \mathrm{~g} /$ day \\
\hline & 10 mg/day & 15/15/10 mg/day & 9 mg/day & $15 / 9 \mathrm{mg} /$ day \\
\hline \multirow[t]{5}{*}{7 mg/day } & 10 mg/day & 7 mg/day & 9 mg/day & 7 mg/day \\
\hline & $1.0-1.5 \mathrm{mg} /$ day & $1.0-1.5 \mathrm{mg} /$ day & 0.9 mg/day & $0.9 \mathrm{mg} / \mathrm{day}$ \\
\hline & 30-70 $\mu \mathrm{g} /$ day & 30-70 $\mu \mathrm{g} /$ day & $50 \mu \mathrm{g} /$ day & $40 \mu \mathrm{g} /$ day \\
\hline & 200/200/180 ug/day & 200/200/180 ug/day & $150 \mu \mathrm{g} / \mathrm{day}$ & $150 \mu \mathrm{g} /$ day \\
\hline & 2-5 mg/day & 2-5 mg/day & - & - \\
\hline
\end{tabular}

(41-45\%E). Only children from France, Greece (4-6 years), Portugal (7-9 years) and Spain (10-14 years) did not meet the recommendations for carbohydrate intake of $50-75 \% \mathrm{E}$ [WHO, 2007]. With a mean share of sucrose of $15.5 \% \mathrm{E}$ the recommendation of less than $10 \% \mathrm{E}$ was exceeded considerably. The lowest intake was observed for 10- to 14-year-old boys from Sweden (11.7\%E). This value was around twice as high in Portuguese children (21.8-25.4\%E) [WHO, 2003].

The intake of dietary fibers is highly associated with the mean energy intake. So it is lower for younger children as their energy intake is in general lower than in older age groups. The mean intake of dietary fiber which was less than $10 \mathrm{~g} /$ day was particularly low in Irish (all age groups) and Finnish children (aged 4). Only children from Germany (girls aged 12, boys aged 12-14) and Portugal (aged 13) reached the recommendation of at least $25 \mathrm{~g} /$ day [Eurodiet, 2000].

In all countries and age groups, the fat intake expressed in percent of total energy intake was above the recommended maximum of $30 \% \mathrm{E}$ [WHO, 2003]. It was particularly high (above 35\%E) in France (aged 7-9 and 10-14), Greece, Portugal (aged 7-9), 
Table 7.2. Methods and period of dietary assessment in children aged 4-6 years of the participating countries (data available from 7,467 children aged 4-6 years)

\begin{tabular}{|c|c|c|c|c|c|c|}
\hline Country & $\begin{array}{l}\text { Age } \\
\text { group } \\
\text { years }\end{array}$ & Method & $\begin{array}{l}\text { Year of } \\
\text { survey }\end{array}$ & $\mathrm{n}$ & $\begin{array}{l}\text { Survey } \\
\text { included } \\
\text { supplements }\end{array}$ & Reference \\
\hline $\begin{array}{l}\text { Czech } \\
\text { Republic }\end{array}$ & $4-6$ & 48-hour recall & n.a. & 1,087 & no & $\begin{array}{l}\text { Tláskal, Hrstková, } \\
\text { unpubl. data' }\end{array}$ \\
\hline Denmark & $4-6$ & 7-day dietary record & 2000-2002 & 233 & no & $\begin{array}{l}\text { Fagt et al., 2008; } \\
\text { Lyhne et al., } 2005\end{array}$ \\
\hline Finland & $\begin{array}{l}4 \\
6\end{array}$ & $\begin{array}{l}\text { 3-day dietary record } \\
\text { 3-day dietary record }\end{array}$ & $\begin{array}{l}2008 \\
2008\end{array}$ & $\begin{array}{l}554 \\
713\end{array}$ & $\begin{array}{l}\text { yes } \\
\text { yes }\end{array}$ & Kyttälä et al., 2008 \\
\hline France & $4-6$ & $3 \times 24$-hour recall & $2006 / 2007$ & 326 & no & $\begin{array}{l}\text { Castetbon et al., } \\
2009\end{array}$ \\
\hline Germany & 6 & $\begin{array}{l}\text { 3-day dietary record } \\
\text { (random) }\end{array}$ & 2007 & 208 & no & $\begin{array}{l}\text { Mensink et al., } \\
\text { 2007b }\end{array}$ \\
\hline Greece & $1-5$ & $\begin{array}{l}3 \text { days overall, } \\
\text { combination of } \\
\text { weighed food } \\
\text { records and 24-hour } \\
\text { recall or food } \\
\text { diaries (3-day) }\end{array}$ & $2003 / 2004$ & 2,374 & no & $\begin{array}{l}\text { Manios et al., } \\
2006\end{array}$ \\
\hline Ireland & $5-6$ & $\begin{array}{l}\text { 7-day weighed } \\
\text { dietary records } \\
\text { 7-day weighed } \\
\text { dietary records }\end{array}$ & $2003 / 2004$ & 148 & yes & $\begin{array}{l}\text { National Children's } \\
\text { Food Survey, } 2005\end{array}$ \\
\hline Italy & $4-6$ & 7-day dietary record & n.a. & 38 & no & D'Amicis, 2000 \\
\hline Norway & 4 & $\begin{array}{l}\text { 4-day dietary record } \\
\text { (precoded) }\end{array}$ & 2000 & 391 & yes & $\begin{array}{l}\varnothing \text { verby et al., 2003 } \\
\text { (UNGKOST 2000) }\end{array}$ \\
\hline Poland & $4-6$ & 24-hour recall & 2000 & 166 & no & $\begin{array}{l}\text { Szponar et al., 2000, } \\
\text { unpubl. }\end{array}$ \\
\hline Sweden & $4-6$ & 4-day dietary record (open) & 2006 & 590 & no & Becker et al., 2000 \\
\hline $\begin{array}{l}\text { The } \\
\text { Netherlands }\end{array}$ & $4-6$ & 2-day dietary record & $2005 / 2006$ & 639 & no & Ocké et al., 2008 \\
\hline
\end{tabular}


Table 7.3. Methods and period of dietary assessment in children aged 7-9 years of the participating countries (data available from 9,158 children aged 7-9 years)

\begin{tabular}{|c|c|c|c|c|c|c|}
\hline Country & $\begin{array}{l}\text { Age } \\
\text { group } \\
\text { years }\end{array}$ & Method & $\begin{array}{l}\text { Year of } \\
\text { survey }\end{array}$ & $\mathrm{n}$ & $\begin{array}{l}\text { Survey } \\
\text { included } \\
\text { supplements }\end{array}$ & Reference \\
\hline Austria & $7-9$ & $\begin{array}{l}\text { 3-day dietary } \\
\text { record }\end{array}$ & 2007 & 280 & no & $\begin{array}{l}\text { Elmadfa } \\
\text { et al., } 2008\end{array}$ \\
\hline $\begin{array}{l}\text { Czech } \\
\text { Republic }\end{array}$ & $7-9$ & 48-hour recall & n.a. & 1,705 & no & $\begin{array}{l}\text { Tláskal, } \\
\text { Hrstková, } \\
\text { unpubl data }\end{array}$ \\
\hline Denmark & $7-9$ & $\begin{array}{l}\text { 7-day dietary } \\
\text { record }\end{array}$ & $2000-2002$ & 257 & no & $\begin{array}{l}\text { Fagt et al., } \\
\text { 2008; Lyhne } \\
\text { et al., } 2005\end{array}$ \\
\hline France & $7-9$ & $3 \times 24$-hour recall & $2006 / 2007$ & 304 & no & $\begin{array}{l}\text { Castetbon } \\
\text { et al., } 2009\end{array}$ \\
\hline Germany & $7-9$ & $\begin{array}{l}\text { 3-day dietary } \\
\text { record (random) }\end{array}$ & 2007 & 629 & no & $\begin{array}{l}\text { Mensink } \\
\text { et al., 2007b }\end{array}$ \\
\hline Greece & $6-15$ & $\begin{array}{l}3 \times 24 \text {-hour } \\
\text { recall }\end{array}$ & 2005 & 524 & n.a. & $\begin{array}{l}\text { Papandreou } \\
\text { et al., 2006a }\end{array}$ \\
\hline Ireland & $7-9$ & $\begin{array}{l}\text { 7-day weighed } \\
\text { dietary records }\end{array}$ & $2003-2004$ & 224 & yes & $\begin{array}{l}\text { National } \\
\text { Children's } \\
\text { Food Survey, } \\
2005\end{array}$ \\
\hline Italy & $7-9$ & $\begin{array}{l}\text { 7-day dietary } \\
\text { record }\end{array}$ & & 50 & no & D'Amicis, 2000 \\
\hline Norway & 9 & $\begin{array}{l}\text { 4-day dietary } \\
\text { record (precoded) }\end{array}$ & 2000 & 810 & yes & $\begin{array}{l}\varnothing \text { verby } \\
\text { et al., } 2003 \\
\text { (UNGKOST } \\
2000)\end{array}$ \\
\hline Poland & $7-9$ & 24-hour recall & 2000 & 204 & no & $\begin{array}{l}\text { Szponar } \\
\text { et al., 2000, } \\
\text { unpubl. }\end{array}$ \\
\hline Portugal & $7-9$ & 24-hour recall & & 3,044 & no & $\begin{array}{l}\text { Moreira } \\
\text { et al., } 2005\end{array}$ \\
\hline Sweden & $7-9$ & $\begin{array}{l}\text { 4-day dietary } \\
\text { record (open) }\end{array}$ & 2006 & 889 & no & $\begin{array}{l}\text { Enghardt } \\
\text { et al., } 2006\end{array}$ \\
\hline $\begin{array}{l}\text { The } \\
\text { Netherlands }\end{array}$ & $7-9$ & $\begin{array}{l}\text { 2-day dietary } \\
\text { record }\end{array}$ & $1997 / 1998$ & 238 & no & $\begin{array}{l}\text { Ocké } \\
\text { et al., } 2008\end{array}$ \\
\hline
\end{tabular}

n.a. $=$ Not available

${ }^{1}$ Data provided by the Czech Nutrition Society. 
Table 7.4. Methods and period of dietary assessment in children aged 10-14 years of the participating countries (data available from 8,985 children aged $10-14$ years)

\begin{tabular}{|c|c|c|c|c|c|c|}
\hline Country & $\begin{array}{l}\text { Age } \\
\text { group } \\
\text { years }\end{array}$ & Method & $\begin{array}{l}\text { Year of } \\
\text { survey }\end{array}$ & $\mathrm{n}$ & $\begin{array}{l}\text { Survey } \\
\text { included } \\
\text { supplements }\end{array}$ & Reference \\
\hline Austria & $10-14$ & 3-day dietary record & 2007 & 487 & no & Elmadfa et al., 2008 \\
\hline Denmark & $10-14$ & 7-day dietary record & $2000-2002$ & 333 & no & $\begin{array}{l}\text { Fagt et al., 2008; } \\
\text { Lyhne et al., } 2005\end{array}$ \\
\hline France & $10-14$ & $3 \times 24$-hour recall & $2006 / 2007$ & 550 & no & Castetbon et al., 2009 \\
\hline Germany & $\begin{array}{l}10-11 \\
12 \\
13-14\end{array}$ & $\begin{array}{l}\text { 3-day dietary } \\
\text { record (random) } \\
\text { personal interview } \\
\text { personal interview }\end{array}$ & $\begin{array}{l}2007 \\
2007 \\
2007\end{array}$ & $\begin{array}{l}397 \\
217 \\
444\end{array}$ & $\begin{array}{l}\text { no } \\
\text { no } \\
\text { no }\end{array}$ & Mensink et al., 2007b \\
\hline Greece & $\begin{array}{l}12 \text { years } \\
\text { at baseline }\end{array}$ & 7-day dietary record & 1999-2001 & 210 & n.a. & Papandreou et al., 2006a \\
\hline Hungary & $11-14$ & $3 \times 24$-hour recall & $2005 / 2006$ & 235 & no & Bíró et al., 2007 \\
\hline Ireland & $10-12$ & $\begin{array}{l}\text { 7-day weighed } \\
\text { dietary records }\end{array}$ & $2003 / 2004$ & 222 & yes & $\begin{array}{l}\text { The National Teen's } \\
\text { Food Survey, } 2008\end{array}$ \\
\hline Italy & $10-14$ & 7-day dietary record & n.a. & 99 & no & D'Amicis, 2000 \\
\hline Norway & 13 & $\begin{array}{l}\text { 4-day dietary } \\
\text { record (precoded) }\end{array}$ & 2000 & 1,005 & yes & $\begin{array}{l}\text { Øverby et al., } 2003 \text { (UNGKOST } \\
\text { 2000) }\end{array}$ \\
\hline Poland & $10-14$ & 24-hour recall & 2000 & 404 & no & $\begin{array}{l}\text { Szponar et al., 2000, } \\
\text { unpubl. data }\end{array}$ \\
\hline Portugal & 13 & FFQ & n.a. & 2,040 & no & $\begin{array}{l}\text { Ramos E, data collected under } \\
\text { the EPITeen Project from the } \\
\text { Epidemiology Department - } \\
\text { Medical School Oporto } \\
\text { University (personal data } \\
\text { never published) }\end{array}$ \\
\hline Spain & $10-14$ & $2 \times 24$-hour recall & $2002 / 2003$ & 119 & no & $\begin{array}{l}\text { Serra Majem and Ribas 2007; } \\
\text { Serra-Majem L et al., 2007; } \\
\text { Serra Majem et al., } 2006\end{array}$ \\
\hline Sweden & $10-14$ & $\begin{array}{l}\text { 4-day dietary } \\
\text { record (open) }\end{array}$ & 2006 & 1,016 & no & Enghardt et al., 2006 \\
\hline $\begin{array}{l}\text { The } \\
\text { Netherlands }\end{array}$ & $10-14$ & 2-day dietary record & 1997/1998 & 410 & no & Ocké et al., 2008 \\
\hline $\begin{array}{l}\text { United } \\
\text { Kingdom }\end{array}$ & $2-15$ & n.a. & $2003-2005$ & 797 & no & $\begin{array}{l}\text { Low Income Diet and } \\
\text { Health Survey, 2003-2005' }\end{array}$ \\
\hline
\end{tabular}

${ }^{1}$ Data provided by the University of Southampton; n.a. = not available. 
Spain and the United Kingdom. For the prevention of non-communicable diseases not only is the amount of fat of importance but also the quality of fat. Apart from Italian boys aged 7-9 the mean intake of saturated fatty acids exceeded the recommended maximum of $10 \% \mathrm{E}$ and polyunsaturated fatty acids were generally below the intake level recommended for the heart health promotion (6-11\%E) [WHO 2003, 2009b].

Cholesterol intake was above the recommended maximum of $300 \mathrm{mg} /$ day in Germany (girls aged 12, boys aged 12-14), Italy, Poland (girls aged 10-14, boys aged 7-14), Portugal (aged 13) and Spain. A relatively low cholesterol intake was observed in Finland, Norway, Sweden and The Netherlands [WHO, 2003].

\section{Comparison of Regions}

To describe regional differences in dietary intake every participating country was assigned to one of four regions. In order not to compare intake levels of younger children to older children the tables are split into three age groups. However, the tendencies of differences between regions are similar throughout the age groups. Tables 7.8-7.10 show minimum and maximum values for energy, protein, carbohydrates/sugar and dietary fiber in the European regions. Following results have to be interpreted carefully because of different survey methods and age groups. However, despite these limitations trends in different nutrient intake patterns in the regions can be recognized.

Whereas for 7- to 9-year-old children, the South region showed a higher energy intake as well as a higher protein and fat intake (\%) and a lower carbohydrate intake (\%) compared to the other regions. All other regions show otherwise comparable intake levels. The intake of macronutrients in 4- to 14-year-old children can be considered less favorable in the South region than in the other three regions.

For dietary fiber, intake levels were far below the recommended level of $25 \mathrm{~g} /$ day in every region [Eurodiet, 2000].

Regarding fat quality, the South region tended to show in all age intervals the lowest share of saturated fatty acids $(9.4-14.5 \% \mathrm{E})$. Lowest intake of polyunsaturated fatty acids was observed in the North region (3.6\%E). A considerably high cholesterol intake (308-375 mg/day) was observed in the South region (cf. tables 7.11-7.13).

\section{Vitamins}

\section{Comparison of Countries}

The mean daily intake of retinol equivalents was between 0.4 and $2.4 \mathrm{mg}$. Compared to the D-A-CH reference values, younger children are more likely to reach the recommendations than children from the older age groups [D-A-CH, 2000]. In the age group of 10- to 14-year-olds, only 4 countries reached the recommended intake levels (Germany, Norway, Poland, Portugal). The lowest intake levels were observed in children from Ireland (all age groups: $0.3-0.4 \mathrm{mg} /$ day). The intake of $\beta$-carotene was about $1.1-5.2 \mathrm{mg} /$ day. 
Table 7.5. Intake of energy and macronutrients (mean \pm SD) in children aged 4-6 years of European countries (male and female); total $n=5,894$

\begin{tabular}{|c|c|c|c|c|c|c|}
\hline & \multirow{2}{*}{$\begin{array}{l}\text { Age } \\
\text { years }\end{array}$} & \multirow[t]{2}{*}{$\mathrm{n}$} & \multirow{2}{*}{$\begin{array}{l}\text { Energy } \\
\text { MJ }\end{array}$} & \multirow[t]{2}{*}{ Protein $\% \mathrm{E}^{1}$} & \multicolumn{2}{|c|}{ Carbohydrates \% $\mathrm{E}^{1}$} \\
\hline & & & & & total & sucrose \\
\hline \multicolumn{7}{|l|}{ Male } \\
\hline Czech Republic & $4-6$ & 641 & $6.5 \pm 1.3$ & $14.0 \pm 2.2$ & $56.0 \pm 5.8$ & n.a. \\
\hline Denmark & $4-6$ & 117 & $7.8 \pm 1.7$ & $14.0 \pm 1.9$ & $50.0 \pm 4.2$ & $12.0 \pm 4.4$ \\
\hline Finland & $\begin{array}{l}4 \\
6\end{array}$ & $\begin{array}{l}307 \\
364\end{array}$ & $\begin{array}{l}5.8 \pm 1.1 \\
6.7 \pm 1.2\end{array}$ & $\begin{array}{l}15.0 \\
16.0\end{array}$ & $\begin{array}{l}53.0 \\
53.0\end{array}$ & $\begin{array}{l}13.7 \\
13.0\end{array}$ \\
\hline France & $4-6$ & 164 & $6.3 \pm 0.1$ & $15.5 \pm 0.2$ & $49.8 \pm 0.8$ & n.a. \\
\hline Germany & $<7$ & 102 & $7.2 \pm 1.4$ & $13.3 \pm 1.9$ & $53.3 \pm 6.2$ & n.a. \\
\hline Greece & $4-5$ & 356 & $6.2 \pm 0.1$ & $16.4 \pm 2.5$ & $44.8 \pm 6.5$ & n.a. \\
\hline Ireland & $5-6$ & 72 & $6.3 \pm 1.2$ & $13.6 \pm 1.8$ & $52.1 \pm 4.6$ & n.a. \\
\hline Italy & $4-6$ & 21 & $7.4 \pm 1.6$ & $15.8 \pm 2.5$ & $50.0 \pm 5.5$ & n.a. \\
\hline Norway & 4 & 206 & $6.3 \pm 1.5$ & $14.0 \pm 2.0$ & $53.0 \pm 5.0$ & $15.0 \pm 5.0$ \\
\hline Poland & $4-6$ & 82 & $7.9 \pm 2.4$ & $11.1 \pm 2.3$ & $57.0 \pm 7.9$ & $18.5 \pm 5.8$ \\
\hline Sweden & $4-6$ & 302 & $6.5 \pm 1.3$ & $14.4 \pm 2.2$ & $54.2 \pm 4.8$ & $13.8 \pm 4.6$ \\
\hline The Netherlands & $4-6$ & 327 & $6.8 \pm 0.9$ & $13.0 \pm 2.0$ & $56.0 \pm 5.0$ & n.a. \\
\hline All countries (min.-max.) & & & $6.2-7.9$ & $11.1-16.4$ & $44.8-57.0$ & $12.0-18.5$ \\
\hline \multicolumn{7}{|l|}{ Female } \\
\hline Czech Republic & $4-6$ & 446 & $6.5 \pm 1.3$ & $14.0 \pm 2.2$ & $56.0 \pm 5.8$ & n.a. \\
\hline Denmark & $4-6$ & 116 & $7.3 \pm 1.6$ & $13.0 \pm 1.8$ & $50.0 \pm 3.8$ & $12.0 \pm 4.1$ \\
\hline Finland & $\begin{array}{l}4 \\
6\end{array}$ & $\begin{array}{l}307 \\
349\end{array}$ & $\begin{array}{l}5.5 \pm 1.0 \\
6.0 \pm 1.1 \\
\end{array}$ & $\begin{array}{l}15.0 \\
15.0 \\
\end{array}$ & $\begin{array}{l}53.0 \\
53.0 \\
\end{array}$ & $\begin{array}{l}13.6 \\
13.8 \\
\end{array}$ \\
\hline France & $4-6$ & 162 & $6.3 \pm 0.1$ & $15.0 \pm 0.2$ & $48.6 \pm 0.5$ & n.a. \\
\hline Germany & $<7$ & 102 & $6.3 \pm 1.3$ & $13.6 \pm 2.0$ & $53.3 \pm 5.2$ & n.a. \\
\hline Greece & $4-5$ & 389 & $5.9 \pm 0.1$ & $16.3 \pm 2.3$ & $45.2 \pm 6.4$ & n.a. \\
\hline Ireland & $5-6$ & 76 & $5.9 \pm 9.3$ & $13.6 \pm 1.9$ & $51.7 \pm 4.0$ & n.a. \\
\hline Italy & $4-6$ & 17 & $7.4 \pm 1.4$ & $15.6 \pm 1.8$ & $50.3 \pm 4.9$ & n.a. \\
\hline Norway & 4 & 185 & $6.1 \pm 1.2$ & $14.0 \pm 2.0$ & $54.0 \pm 6.0$ & $16.0 \pm 6.0$ \\
\hline Poland & $4-6$ & 84 & $7.1 \pm 2.4$ & $12.0 \pm 2.8$ & $55.6 \pm 7.5$ & $17.9 \pm 6.0$ \\
\hline Sweden & $4-6$ & 288 & $6.1 \pm 1.2$ & $14.4 \pm 2.1$ & $59.4 \pm 5.1$ & $13.7 \pm 4.5$ \\
\hline The Netherlands & $4-6$ & 312 & $6.3 \pm 1.0$ & $13.0 \pm 2.0$ & $57.0 \pm 4.0$ & n.a. \\
\hline All countries (min.-max.) & & & $5.5-7.4$ & $12.0-16.3$ & $45.2-59.4$ & $12.0-17.9$ \\
\hline Reference values & & & $\begin{array}{l}6.4 \text { (male)/ } \\
5.8(\text { female })^{2}\end{array}$ & $10-15 \% \mathrm{E}^{3}$ & $50-75 \% \mathrm{E}^{4}$ & $<10 \% \mathrm{E}^{3}$ \\
\hline
\end{tabular}

n.a. $=$ Not available.

${ }^{1}$ Difference is not necessarily alcohol; ${ }^{2} \mathrm{D}-\mathrm{A}-\mathrm{CH}, 2000 ;{ }^{3} \mathrm{WHO}, 2003 ;{ }^{4} \mathrm{WHO}, 2007 ;{ }^{5} \mathrm{WHO}, 2009 \mathrm{~b} ;{ }^{6}$ Eurodiet, 2000. 


\begin{tabular}{|c|c|c|c|c|c|}
\hline $\begin{array}{l}\text { Dietary fiber } \\
g\end{array}$ & Fat $\% \mathrm{E}^{1}$ & SFA $\% E$ & MUFA \%E & PUFA \%E & $\begin{array}{l}\text { Cholesterol } \\
\mathrm{mg}\end{array}$ \\
\hline $15.3 \pm 4.7$ & $30.0 \pm 5.3$ & n.a. & n.a. & n.a. & $240.7 \pm 111.3$ \\
\hline $17.0 \pm 5.0$ & $34.0 \pm 4.0$ & $15.0 \pm 2.2$ & $12.0 \pm 1.6$ & $5.0 \pm 0.8$ & n.a. \\
\hline $9.6 \pm 3.2$ & 31.0 & 13.7 & 10.6 & 4.0 & $153.0 \pm 53.0$ \\
\hline $11.4 \pm 3.7$ & 31.0 & 13.5 & 10.8 & 4.2 & $180.0 \pm 60.0$ \\
\hline $11.8 \pm 0.5$ & $34.7 \pm 0.84$ & 15.1 & n.a. & n.a. & n.a. \\
\hline $15.7 \pm 4.1$ & $32.6 \pm 6.1$ & $15.3 \pm 2.9$ & $12.5 \pm 2.2$ & $4.8 \pm 1.0$ & $246.8 \pm 103.2$ \\
\hline n.a. & $40.2 \pm 5.0$ & $14.5 \pm 2.9$ & n.a. & n.a. & n.a. \\
\hline $7.3 \pm 2.7$ & $33.8 \pm 4.4$ & $14.5 \pm 2.8$ & $11.3 \pm 1.9$ & $4.5 \pm 1.2$ & $141.4 \pm 55.0$ \\
\hline $14.9 \pm 4.7$ & $34.3 \pm 5.5$ & $10.9 \pm 2.5$ & $10.6 \pm 3.4$ & $4.1 \pm 1.5$ & $327.6 \pm 105.4$ \\
\hline $12.0 \pm 5.0$ & $33.0 \pm 5.0$ & $14.0 \pm 2.0$ & $10.0 \pm 2.0$ & $6.0 \pm 2.0$ & $185.0 \pm 82.0$ \\
\hline $16.8 \pm 5.8$ & $31.9 \pm 7.8$ & $11.6 \pm 3.9$ & $13.7 \pm 4.1$ & $4.5 \pm 2.2$ & $240.0 \pm 145.0$ \\
\hline $12.0 \pm 3.0$ & $31.3 \pm 4.2$ & $14.3 \pm 2.4$ & $11.1 \pm 1.7$ & $3.7 \pm 1.0$ & $196.0 \pm 73.0$ \\
\hline $15.0 \pm 3.0$ & $31.0 \pm 4.0$ & $12.0 \pm 2.0$ & $10.0 \pm 1.0$ & $6.0 \pm 1.0$ & $126.0 \pm 42.0$ \\
\hline $7.3-16.8$ & $30.0-40.2$ & $10.9-15.3$ & $10.0-13.7$ & $3.7-6.0$ & $122.0-327.6$ \\
\hline $15.3 \pm 4.7$ & $30.0 \pm 5.3$ & n.a. & n.a. & n.a. & $240.7 \pm 111.3$ \\
\hline $16.0 \pm 4.0$ & $35.0 \pm 4.0$ & $15.0 \pm 2.4$ & $12.0 \pm 1.5$ & $5.0 \pm 0.8$ & n.a. \\
\hline $9.4 \pm 3.0$ & 31.0 & 13.7 & 10.6 & 4.0 & $139.0 \pm 51.0$ \\
\hline $10.3 \pm 3.3$ & 31.0 & 13.8 & 10.7 & 4.1 & $156.0 \pm 56.0$ \\
\hline $11.5 \pm 0.3$ & $36.3 \pm 0.5$ & 15.8 & n.a. & n.a. & n.a. \\
\hline $15.8 \pm 4.7$ & $32.2 \pm 4.6$ & $15.2 \pm 2.0$ & $12.1 \pm 1.6$ & $4.9 \pm 1.1$ & $201.4 \pm 89.3$ \\
\hline n.a. & $40.5 \pm 5.0$ & $14.4 \pm 2.9$ & n.a. & n.a. & n.a. \\
\hline $6.9 \pm 2.8$ & $34.3 \pm 3.5$ & $15.0 \pm 2.6$ & $11.2 \pm 1.5$ & $4.6 \pm 1.3$ & $145.0 \pm 63.8$ \\
\hline $15.8 \pm 3.9$ & $33.8 \pm 3.5$ & $10.8 \pm 2.0$ & $9.7 \pm 2.3$ & $4.0 \pm 1.2$ & $327.1 \pm 107.0$ \\
\hline $12.0 \pm 6.0$ & $32.0 \pm 5.0$ & $14.0 \pm 3.0$ & $10.0 \pm 2.0$ & $6.0 \pm 2.0$ & $174.0 \pm 65.0$ \\
\hline $14.6 \pm 5.9$ & $32.5 \pm 7.0$ & $12.0 \pm 3.3$ & $13.6 \pm 3.9$ & $4.7 \pm 2.2$ & $245.0 \pm 176.0$ \\
\hline $11.0 \pm 3.0$ & $32.1 \pm 4.6$ & $14.6 \pm 2.5$ & $11.5 \pm 1.9$ & $3.7 \pm 0.9$ & $187.0 \pm 68.0$ \\
\hline $18.0 \pm 3.0$ & $37.0 \pm 4.0$ & $12.0 \pm 2.0$ & $10.0 \pm 1.0$ & $5.0 \pm 1.0$ & $166.0 \pm 116.0$ \\
\hline $6.9-18.0$ & $30.0-40.5$ & $10.8-15.8$ & $9.7-13.6$ & $3.7-6.0$ & $113.0-327.1$ \\
\hline$>25$ g.day $^{-1,6}$ & $15-30 \% E^{3}$ & $<10 \% \mathrm{E}^{3}$ & & $6-11 \% \mathrm{E}^{5}$ & $<300 \mathrm{mg} \cdot \mathrm{d}^{-1,3}$ \\
\hline
\end{tabular}


Table 7.6. Intake of energy and macronutrients (mean \pm SD) in children aged 7-9 years of European countries (male and female): total $n=8,634$

\begin{tabular}{|c|c|c|c|}
\hline \multirow{2}{*}{$\begin{array}{ll}\text { Age } & n \\
\text { years } & \end{array}$} & \multirow{2}{*}{$\begin{array}{l}\text { Energy } \\
\text { MJ }\end{array}$} & \multirow[t]{2}{*}{ Protein $\% \mathrm{E}^{1}$} & Carbohydrates, \% $\mathrm{E}^{1}$ \\
\hline & & & total \\
\hline
\end{tabular}

\section{Male}

\begin{tabular}{|c|c|c|c|c|c|c|}
\hline Austria & $7-9$ & 146 & $6.9 \pm 1.9$ & $14.4 \pm 2.7$ & $51.4 \pm 6.8$ & $16.6 \pm 7.1$ \\
\hline Czech Republic & $7-9$ & 940 & $7.6 \pm 2.8$ & $14.5 \pm 2.4$ & $53.4 \pm 6.7$ & n.a. \\
\hline Denmark & $7-9$ & 139 & $9.3 \pm 1.9$ & $14.0 \pm 1.8$ & $51.0 \pm 3.7$ & $14.0 \pm 5.1$ \\
\hline France & $7-9$ & 160 & $7.6 \pm 0.2$ & $14.7 \pm 0.2$ & $49.3 \pm 0.5$ & n.a. \\
\hline Germany & $7-9$ & 321 & $7.8 \pm 1.6$ & $13.5 \pm 2.1$ & $53.2 \pm 6.1$ & n.a. \\
\hline Ireland & $7-9$ & 110 & $7.4 \pm 1.6$ & $13.6 \pm 2.3$ & n.a. & n.a. \\
\hline Italy & $7-9$ & 29 & $9.1 \pm 1.2$ & $15.5 \pm 1.6$ & n.a. & n.a. \\
\hline Norway & 9 & 402 & $8.6 \pm 2.0$ & $15.0 \pm 2.0$ & $54.0 \pm 6.0$ & $16.0 \pm 6.0$ \\
\hline Poland & $7-9$ & 101 & $9.1 \pm 2.9$ & $11.7 \pm 2.8$ & $56.3 \pm 7.9$ & $17.3 \pm 5.6$ \\
\hline Portugal & $7-9$ & 1,541 & $11.3 \pm 21.4$ & $16.6 \pm 3.8$ & $48.6 \pm 7.8$ & $22.5 \pm 7.1$ \\
\hline Sweden & $7-9$ & 444 & $7.2 \pm 1.5$ & $15.4 \pm 2.2$ & $53.3 \pm 4.9$ & $12.6 \pm 4.2$ \\
\hline The Netherlands & $7-9$ & 104 & $8.4 \pm 1.8$ & $13.5 \pm 2.7$ & $55.3 \pm 7.0$ & n.a. \\
\hline All countries (min.-max.) & & & $7.2-11.3$ & $11.7-16.6$ & $49.3-56.3$ & $12.6-17.3$ \\
\hline \multicolumn{7}{|l|}{ Female } \\
\hline Austria & $7-9$ & 134 & $6.3 \pm 1.6$ & $13.5 \pm 2.7$ & $52.2 \pm 7.0$ & $18.0 \pm 6.9$ \\
\hline Czech Republic & $7-9$ & 765 & $7.6 \pm 2.8$ & $14.5 \pm 2.4$ & $53.4 \pm 6.7$ & n.a. \\
\hline Denmark & $7-9$ & 118 & $8.1 \pm 1.7$ & $14.0 \pm 2.0$ & $52.0 \pm 4.6$ & $14.0 \pm 4.8$ \\
\hline France & $7-9$ & 144 & $6.9 \pm 0.2$ & $15.0 \pm 0.3$ & $48.5 \pm 0.7$ & n.a. \\
\hline Germany & $7-9$ & 308 & $7.0 \pm 1.4$ & $13.6 \pm 2.7$ & $54.2 \pm 6.7$ & n.a. \\
\hline Ireland & $7-9$ & 114 & $6.8 \pm 1.2$ & $13.6 \pm 2.1$ & n.a. & n.a. \\
\hline Italy & $7-9$ & 21 & $7.9 \pm 1.1$ & $15.9 \pm 2.1$ & n.a. & n.a. \\
\hline Norway & 9 & 408 & $7.7 \pm 2.0$ & $14.0 \pm 3.0$ & $55.0 \pm 6.0$ & $18.0 \pm 6.0$ \\
\hline Poland & $7-9$ & 103 & $8.0 \pm 2.5$ & $11.3 \pm 2.5$ & $55.5 \pm 7.7$ & $16.4 \pm 6.2$ \\
\hline Portugal & $7-9$ & 1,503 & $10.6 \pm 11.8$ & $16.6 \pm 3.7$ & $48.3 \pm 7.9$ & $21.8 \pm 7.1$ \\
\hline Sweden & $7-9$ & 445 & $7.2 \pm 1.5$ & $15.4 \pm 2.2$ & $53.3 \pm 4.9$ & $12.6 \pm 4.2$ \\
\hline The Netherlands & $7-9$ & 134 & $7.6 \pm 1.6$ & $13.5 \pm 2.6$ & $52.0 \pm 7.0$ & n.a. \\
\hline All countries (min.-max.) & & & $6.3-10.6$ & $11.3-16.6$ & $48.3-55.5$ & $12.6-21.8$ \\
\hline Reference values & & & $\begin{array}{l}7.9 \text { (male)/ } \\
7.1 \text { (female) }{ }^{2}\end{array}$ & $10-15 \% \mathrm{E}^{3}$ & $50-75 \% \mathrm{E}^{4}$ & $<10 \% \mathrm{E}^{3}$ \\
\hline
\end{tabular}

n.a. $=$ Not available.

${ }^{1}$ Difference is not necessarily alcohol; ${ }^{2}$ D-A-CH, 2000; ${ }^{3}$ WHO, 2003; ${ }^{4}$ WHO, 2007; ${ }^{5}$ WHO, 2009b; ${ }^{6}$ Eurodiet, 2000. 


\begin{tabular}{|c|c|c|c|c|c|}
\hline $\begin{array}{l}\text { Dietary fiber } \\
g\end{array}$ & Fat $\% \mathrm{E}^{1}$ & SFA \%E & MUFA \%E & PUFA \%E & $\begin{array}{l}\text { Cholesterol } \\
\mathrm{mg}\end{array}$ \\
\hline $15.0 \pm 5.9$ & $34.1 \pm 5.3$ & $14.4 \pm 2.7$ & $11.7 \pm 2.4$ & $6.1 \pm 2.0$ & $259.0 \pm 111.1$ \\
\hline n.a. & $32.2 \pm 5.6$ & n.a. & n.a. & n.a. & n.a. \\
\hline $18.0 \pm 6.7$ & $33.0 \pm 3.6$ & $15.0 \pm 2.0$ & $11.0 \pm 1.4$ & $5.0 \pm 0.8$ & n.a. \\
\hline $13.5 \pm 0.4$ & $36.0 \pm 0.4$ & 15.1 & n.a. & n.a. & n.a. \\
\hline $17.5 \pm 5.3$ & $32.4 \pm 5.5$ & $15.1 \pm 2.5$ & $12.5 \pm 2.0$ & $4.8 \pm 1.0$ & $246.0 \pm 98.9$ \\
\hline $9.2 \pm 3.4$ & $33.3 \pm 4.7$ & $14.0 \pm 2.5$ & $11.0 \pm 2.2$ & $4.7 \pm 1.3$ & $176.2 \pm 82.9$ \\
\hline $18.5 \pm 5.1$ & $34.8 \pm 5.9$ & $9.4 \pm 2.2$ & $10.9 \pm 3.1$ & $4.6 \pm 3.1$ & $375.2 \pm 193.3$ \\
\hline $16.0 \pm 7.0$ & $32.0 \pm 5.0$ & $14.0 \pm 3.0$ & $10.0 \pm 2.0$ & $6.0 \pm 2.0$ & $225.0 \pm 103.0$ \\
\hline $19.6 \pm 6.8$ & $32.0 \pm 7.5$ & $12.0 \pm 3.8$ & $13.3 \pm 3.6$ & $4.6 \pm 1.9$ & $315.0 \pm 220.0$ \\
\hline $20.2 \pm 8.1$ & $35.9 \pm 6.6$ & $13.0 \pm 3.3$ & $15.0 \pm 3.4$ & $5.0 \pm 1.7$ & n.a. \\
\hline $13.0 \pm 4.0$ & $31.3 \pm 4.4$ & $14.1 \pm 2.3$ & $11.3 \pm 1.9$ & $3.6 \pm 0.9$ & $216.0 \pm 78.0$ \\
\hline $17.0 \pm 6.0$ & $34.0 \pm 6.0$ & $13.0 \pm 3.0$ & $12.0 \pm 3.0$ & $6.0 \pm 2.0$ & $155.0 \pm 63.0$ \\
\hline $9.2-20.2$ & $31.3-36.0$ & $9.4-15.1$ & $10.0-15.0$ & $3.6-6.1$ & $155.0-375.2$ \\
\hline $14.3 \pm 4.4$ & $34.2 \pm 5.7$ & $14.3 \pm 3.0$ & $11.5 \pm 2.3$ & $6.5 \pm 2.3$ & $261.7 \pm 303.8$ \\
\hline n.a. & $32.2 \pm 5.6$ & n.a. & n.a. & n.a. & n.a. \\
\hline $17.0 \pm 4.7$ & $32.0 \pm 4.4$ & $14.0 \pm 2.3$ & $11.0 \pm 1.8$ & $5.0 \pm 0.7$ & n.a. \\
\hline $12.2 \pm 0.4$ & $36.5 \pm 0.6$ & 15.5 & n.a. & n.a. & n.a. \\
\hline $16.8 \pm 5.4$ & $31.3 \pm 5.8$ & $14.9 \pm 2.6$ & $11.8 \pm 2.2$ & $4.5 \pm 1.1$ & $168.0 \pm 54.0$ \\
\hline $7.6 \pm 3.0$ & $34.4 \pm 4.1$ & $14.6 \pm 2.6$ & $11.1 \pm 1.8$ & $4.9 \pm 1.4$ & $157.2 \pm 65.2$ \\
\hline $15.2 \pm 5.0$ & $34.9 \pm 4.7$ & $10.9 \pm 2.4$ & $9.9 \pm 1.8$ & $4.3 \pm 1.8$ & $308.6 \pm 119.2$ \\
\hline $14.0 \pm 6.0$ & $31.0 \pm 5.0$ & $14.0 \pm 3.0$ & $10.0 \pm 2.0$ & $6.0 \pm 2.0$ & $200.0 \pm 105.0$ \\
\hline $17.4 \pm 6.7$ & $33.2 \pm 7.6$ & $11.8 \pm 3.6$ & $14.2 \pm 3.8$ & $5.1 \pm 2.6$ & $281.0 \pm 194.0$ \\
\hline $19.4 \pm 8.2$ & $36.2 \pm 6.7$ & $13.1 \pm 3.3$ & $15.1 \pm 3.5$ & $5.1 \pm 1.6$ & n.a. \\
\hline $13.0 \pm 4.0$ & $31.3 \pm 4.4$ & $14.1 \pm 2.3$ & $11.3 \pm 1.9$ & $3.6 \pm 0.9$ & $216.0 \pm 78.0$ \\
\hline $15.0 \pm 5.0$ & $34.0 \pm 7.0$ & $14.0 \pm 3.0$ & $12.0 \pm 3.0$ & $7.0 \pm 2.0$ & $147.0 \pm 72.0$ \\
\hline $7.6-19.4$ & $31.0-36.5$ & $11.8-15.5$ & $9.9-15.1$ & $3.6-7.0$ & $147.0-308.6$ \\
\hline$>25 \mathrm{~g} \cdot \mathrm{day}^{-1,6}$ & $15-30 \% \mathrm{E}^{3}$ & $<10 \% \mathrm{E}^{3}$ & & $6-11 \% \mathrm{E}^{5}$ & $<300 \mathrm{mg} \cdot \mathrm{d}^{-1,3}$ \\
\hline
\end{tabular}


Table 7.7. Intake of energy and macronutrients (mean \pm SD) in children aged 10-14 years of European countries (male and female); total $\mathrm{n}=8,405$

\begin{tabular}{|c|c|c|c|c|}
\hline \multirow{2}{*}{$\begin{array}{l}\text { Age } \\
\text { years }\end{array}$} & \multirow[t]{2}{*}{$n$} & \multirow{2}{*}{$\begin{array}{l}\text { Energy } \\
\text { MJ }\end{array}$} & \multirow[t]{2}{*}{ Protein $\% \mathrm{E}^{1}$} & Carbohydrates, $\% \mathrm{E}^{1}$ \\
\hline & & & & total \\
\hline
\end{tabular}

\section{Male}

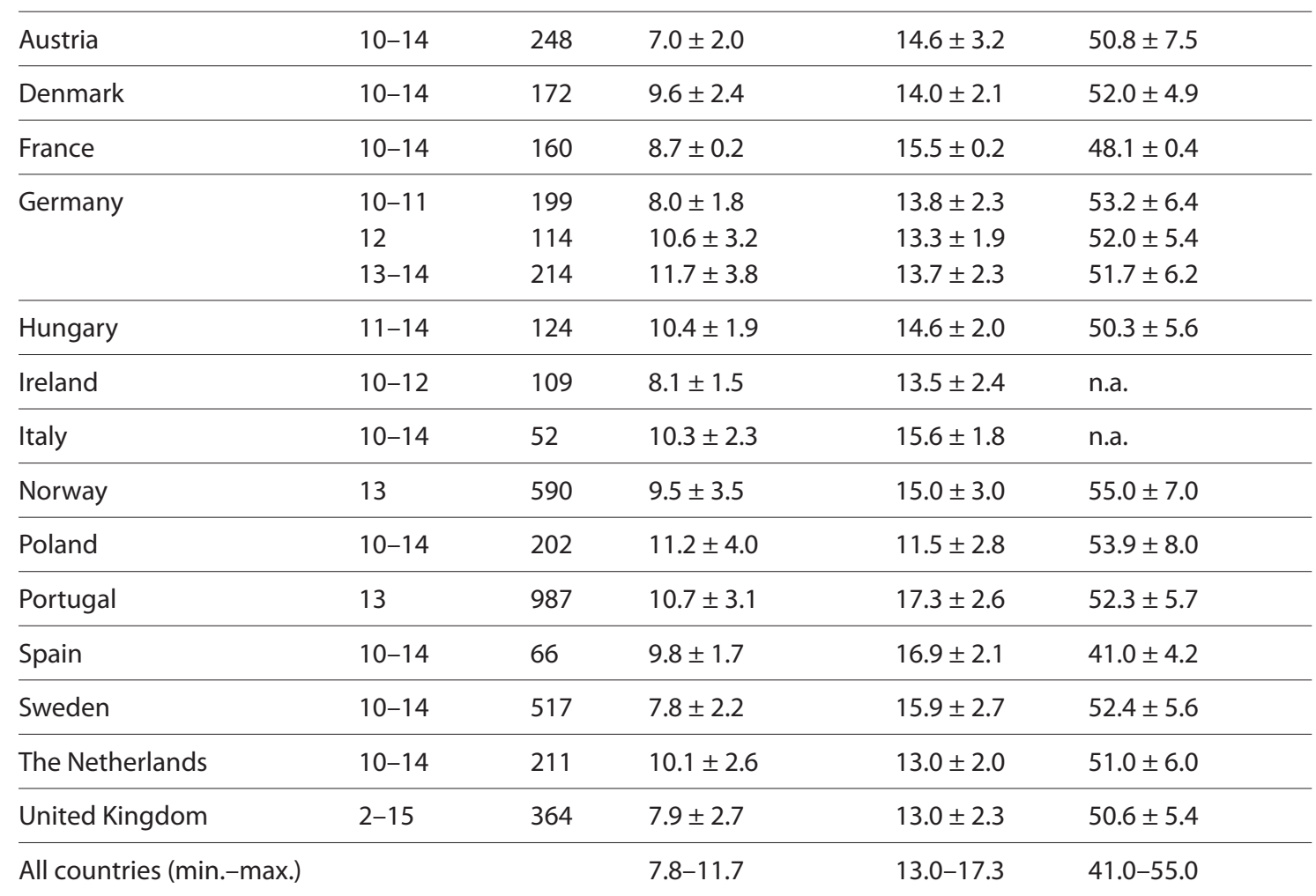

\section{Female}

\begin{tabular}{|c|c|c|c|c|c|}
\hline Austria & $10-14$ & 239 & $6.1 \pm 1.7$ & $14.1 \pm 3.0$ & $52.1 \pm 8.0$ \\
\hline Denmark & $10-14$ & 161 & $8.1 \pm 2.1$ & $14.0 \pm 2.2$ & $52.0 \pm 5.0$ \\
\hline France & $10-14$ & 144 & $7.5 \pm 0.1$ & $15.6 \pm 0.2$ & $48.0 \pm 0.4$ \\
\hline \multirow[t]{3}{*}{ Germany } & $10-11$ & 198 & $7.6 \pm 1.6$ & $13.7 \pm 2.4$ & $53.1 \pm 7.2$ \\
\hline & 12 & 103 & $9.3 \pm 3.2$ & $13.1 \pm 1.9$ & $52.8 \pm 6.6$ \\
\hline & $13-14$ & 230 & $9.5 \pm 2.7$ & $13.1 \pm 2.2$ & $52.7 \pm 6.1$ \\
\hline Hungary & $11-14$ & 111 & $9.2 \pm 1.5$ & $13.9 \pm 1.9$ & $51.7 \pm 5.4$ \\
\hline Ireland & $10-12$ & 113 & $7.0 \pm 1.4$ & $13.6 \pm 2.2$ & n.a. \\
\hline Italy & $10-14$ & 47 & $8.6 \pm 1.5$ & $15.7 \pm 2.0$ & n.a. \\
\hline Norway & 13 & 515 & $8.1 \pm 2.6$ & $14.0 \pm 3.0$ & $55.0 \pm 6.0$ \\
\hline Poland & $10-14$ & 202 & $9.4 \pm 3.4$ & $11.7 \pm 2.7$ & $54.0 \pm 7.7$ \\
\hline
\end{tabular}




\begin{tabular}{|c|c|c|c|c|c|c|}
\hline & Dietary fiber & Fat $\% \mathrm{E}^{1}$ & SFA \%E & MUFA \%E & PUFA \%E & Cholesterol \\
\hline sucrose & & & & & & \\
\hline
\end{tabular}

\begin{tabular}{|c|c|c|c|c|c|c|}
\hline $17.6 \pm 8.2$ & $15.1 \pm 6.1$ & $34.5 \pm 6.2$ & $14.5 \pm 3.3$ & $12.0 \pm 2.5$ & $6.1 \pm 1.9$ & $271.1 \pm 146.4$ \\
\hline $14.0 \pm 5.9$ & $18.0 \pm 6.3$ & $32.0 \pm 4.4$ & $14.0 \pm 2.3$ & $11.0 \pm 1.8$ & $5.0 \pm 0.8$ & n.a. \\
\hline n.a. & $15.2 \pm 0.4$ & $36.3 \pm 0.4$ & 15.1 & n.a. & n.a. & n.a. \\
\hline n.a. & $17.9 \pm 6.0$ & $32.1 \pm 5.8$ & $15.1 \pm 2.6$ & $12.4 \pm 2.2$ & $4.6 \pm 1.0$ & $261.2 \pm 118.1$ \\
\hline n.a. & $25.3 \pm 9.4$ & $33.7 \pm 5.2$ & $15.6 \pm 2.3$ & $12.7 \pm 1.9$ & $5.3 \pm 1.0$ & $343.0 \pm 142.2$ \\
\hline n.a. & $27.7 \pm 12.2$ & $33.5 \pm 5.5$ & $15.5 \pm 2.4$ & $12.7 \pm 2.0$ & $5.2 \pm 1.2$ & $382.4 \pm 165.4$ \\
\hline $11.9 \pm 5.6$ & $20.8 \pm 5.9$ & $35.0 \pm 4.9$ & $10.9 \pm 2.1$ & $10.8 \pm 2.1$ & $8.2 \pm 2.1$ & $347.3 \pm 115.9$ \\
\hline n.a. & $9.4 \pm 3.8$ & $33.3 \pm 4.2$ & $13.9 \pm 2.6$ & $10.9 \pm 1.8$ & $4.6 \pm 1.3$ & $178.6 \pm 68.0$ \\
\hline n.a. & $21.6 \pm 7.6$ & $35.8 \pm 5.3$ & $10.8 \pm 2.4$ & $11.9 \pm 2.9$ & $5.1 \pm 1.5$ & $370.1 \pm 100.6$ \\
\hline $18.0 \pm 8.0$ & $16.0 \pm 8.0$ & $31.0 \pm 6.0$ & $13.0 \pm 3.0$ & $10.0 \pm 2.0$ & $5.0 \pm 2.0$ & $241.0 \pm 134.0$ \\
\hline $15.2 \pm 5.8$ & $24.6 \pm 10.0$ & $34.6 \pm 7.7$ & $12.1 \pm 3.8$ & $14.7 \pm 3.9$ & $5.5 \pm 2.9$ & $408.0 \pm 323.0$ \\
\hline $24.2 \pm 6.0^{3}$ & $25.4 \pm 10.3$ & $32.0 \pm 4.5$ & $10.9 \pm 1.8$ & $13.1 \pm 2.5$ & $5.3 \pm 1.1$ & $377.7 \pm 147.7$ \\
\hline $16.1 \pm 3.3$ & $18.5 \pm 1.6$ & $40.8 \pm 4.1$ & $14.3 \pm 1.8$ & $16.7 \pm 2.5$ & $5.7 \pm 0.9$ & $362.1 \pm 34.4$ \\
\hline $11.7 \pm 5.0$ & $13.0 \pm 4.0$ & $31.7 \pm 4.6$ & $14.1 \pm 2.4$ & $11.6 \pm 2.1$ & $3.7 \pm 1.0$ & $233.0 \pm 92.0$ \\
\hline n.a. & $20.0 \pm 7.0$ & $35.0 \pm 6.0$ & $14.0 \pm 3.0$ & $13.0 \pm 3.0$ & $7.0 \pm 2.0$ & $189.0 \pm 92.0$ \\
\hline $16.9 \pm 6.5$ & $10.9 \pm 4.3$ & $36.3 \pm 5.0$ & $14.5 \pm 2.9$ & n.a. & n.a. & $203.5 \pm 112.3$ \\
\hline $11.7-24.2$ & $9.4-27.7$ & $31.0-40.8$ & $10.8-15.6$ & $10.0-16.7$ & $3.7-8.2$ & $178.6-408.0$ \\
\hline
\end{tabular}

\begin{tabular}{lllllll}
\hline $16.8 \pm 7.3$ & $13.7 \pm 4.3$ & $33.7 \pm 7.0$ & $14.1 \pm 3.6$ & $11.5 \pm 2.8$ & $6.2 \pm 2.3$ & $228.3 \pm 160.4$ \\
\hline $14.0 \pm 5.2$ & $15.0 \pm 4.8$ & $32.0 \pm 4.7$ & $14.0 \pm 2.4$ & $11.0 \pm 1.8$ & $5.0 \pm 0.8$ & n.a. \\
\hline n.a. & $13.8 \pm 0.3$ & $36.4 \pm 0.5$ & 14.9 & n.a. & n.a. & n.a. \\
\hline n.a. & $17.7 \pm 5.5$ & $32.3 \pm 6.3$ & $14.8 \pm 2.7$ & $12.6 \pm 2.5$ & $4.8 \pm 1.2$ & $253.1 \pm 110.1$ \\
n.a. & $25.0 \pm 10.9$ & $33.1 \pm 6.2$ & $15.4 \pm 2.9$ & $12.3 \pm 2.1$ & $5.3 \pm 1.1$ & $301.5 \pm 144.5$ \\
n.a. & $24.4 \pm 8.8$ & $32.9 \pm 5.8$ & $15.4 \pm 2.7$ & $12.3 \pm 2.1$ & $5.1 \pm 1.0$ & $297.7 \pm 117.3$ \\
\hline $12.5 \pm 5.9$ & $20.1 \pm 6.7$ & $34.3 \pm 5.2$ & $10.6 \pm 2.2$ & $10.2 \pm 2.1$ & $8.6 \pm 2.0$ & $291.8 \pm 96.0$ \\
\hline n.a. & $7.7 \pm 3.4$ & $34.5 \pm 4.3$ & $14.0 \pm 2.3$ & $11.4 \pm 1.7$ & $5.1 \pm 1.4$ & $158.1 \pm 65.5$ \\
\hline n.a. & $16.8 \pm 3.9$ & $33.4 \pm 4.5$ & $10.1 \pm 2.3$ & $11.0 \pm 2.3$ & $4.4 \pm 1.9$ & $319.9 \pm 111.0$ \\
\hline $19.0 \pm 7.0$ & $14.0 \pm 7.0$ & $31.0 \pm 5.0$ & $14.0 \pm 3.0$ & $10.0 \pm 2.0$ & $5.0 \pm 2.0$ & $198.0 \pm 106.0$ \\
\hline $15.5 \pm 6.0$ & $20.9 \pm 8.7$ & $34.3 \pm 7.5$ & $12.0 \pm 3.7$ & $14.6 \pm 4.1$ & $5.5 \pm 2.8$ & $321.0 \pm 235.0$ \\
\hline
\end{tabular}


Table 7.7. Continued

\begin{tabular}{|c|c|c|c|c|c|}
\hline & \multirow{2}{*}{$\begin{array}{l}\text { Age } \\
\text { years }\end{array}$} & \multirow[t]{2}{*}{$\mathrm{n}$} & \multirow{2}{*}{$\begin{array}{l}\text { Energy } \\
\text { MJ }\end{array}$} & \multirow[t]{2}{*}{ Protein $\% \mathrm{E}^{1}$} & \multirow{2}{*}{$\frac{\text { Carbohydrates, } \% \mathrm{E}^{1}}{\text { total }}$} \\
\hline & & & & & \\
\hline Portugal & 13 & 1053 & $10.3 \pm 3.2$ & $17.1 \pm 2.9$ & $52.6 \pm 6.2$ \\
\hline Spain & $10-14$ & 53 & $8.4 \pm 0.9$ & $17.6 \pm 1.9$ & $41.6 \pm 3.3$ \\
\hline Sweden & $10-14$ & 499 & $6.9 \pm 1.9$ & $15.4 \pm 2.7$ & $53.2 \pm 5.5$ \\
\hline The Netherlands & $10-14$ & 199 & $8.7 \pm 1.8$ & $13.0 \pm 2.0$ & $52.0 \pm 6.0$ \\
\hline United Kingdom & $2-15$ & 433 & $7.0 \pm 2.1$ & $13.3 \pm 2.4$ & $50.6 \pm 5.1$ \\
\hline All countries (min.-max.) & & & $6.1-10.3$ & $13.0-17.6$ & $41.6-55.0$ \\
\hline Reference values & & & $\begin{array}{l}\text { 9.4/11.2 (male)/ } \\
\text { 8.5/9.4 (female) }{ }^{2}\end{array}$ & $10-15 \% E^{3}$ & $50-75 \% \mathrm{E}^{4}$ \\
\hline
\end{tabular}

${ }^{1}$ Difference is not necessarily alcohol; ${ }^{2} \mathrm{D}-\mathrm{A}-\mathrm{CH}, 2000$, reference values refer to age group $10-12$ years and $13-14$ years; ${ }^{3} \mathrm{WHO}, 2003 ;{ }^{4} \mathrm{WHO}, 2007 ;{ }^{5} \mathrm{WHO}, 2009 \mathrm{~b} ;{ }^{6}$ Eurodiet, 2000; n.a. = not available.

Table 7.8. Intake of energy and macronutrients (min.-max.) in children aged 4-6 years in the four regions

\begin{tabular}{|c|c|c|c|c|c|}
\hline Region/sex & $\begin{array}{l}\text { Energy } \\
\text { MJ }\end{array}$ & $\begin{array}{l}\text { Protein } \\
\% \mathrm{E}\end{array}$ & $\begin{array}{l}\text { Carbohydrates, \%E } \\
\text { (of that sucrose) }\end{array}$ & $\begin{array}{l}\text { Dietary fiber } \\
g\end{array}$ & $\begin{array}{l}\text { Fat } \\
\% \mathrm{E}\end{array}$ \\
\hline \multicolumn{6}{|l|}{ North ${ }^{1}$} \\
\hline Male & $5.8-7.8$ & $14.0-16.0$ & $50.0-54.2(12.0-15.0)$ & $9.6-17.0$ & $31.0-34.0$ \\
\hline Female & $5.5-7.3$ & $13.0-15.0$ & $50.0-59.4(12.0-16.0)$ & $9.4-16.0$ & $31.0-35.0$ \\
\hline \multicolumn{6}{|l|}{ South ${ }^{2}$} \\
\hline Male & $6.2-7.4$ & $15.8-16.4$ & $44.8-50.0$ (n.a.) & $14.9^{5}$ & $34.3-40.2$ \\
\hline Female & $5.9-7.4$ & $15.6-16.3$ & $45.2-50.3$ (n.a.) & $15.8^{5}$ & $33.8-40.5$ \\
\hline \multicolumn{6}{|c|}{ Central and East ${ }^{3}$} \\
\hline Male & $6.5-7.9$ & $11.1-14.0$ & $56.0-57.0\left(18.5^{6}\right)$ & $15.3-16.8$ & $30.0-31.9$ \\
\hline Female & $6.5-7.1$ & $12.0-14.0$ & $55.6-56.0\left(17.9^{6}\right)$ & $14.6-15.3$ & $30.0-32.5$ \\
\hline \multicolumn{6}{|l|}{ West } \\
\hline Male & $6.3-6.8$ & $13.0-15.5$ & 49.8-56.0 (n.a.) & $7.3-15.0$ & $31.0-34.7$ \\
\hline Female & $5.9-6.3$ & $13.0-15.0$ & 48.6-57.0 (n.a.) & $6.9-18.0$ & $31.0-37.0$ \\
\hline
\end{tabular}

${ }^{1}$ North: SE, NO, FI, DK; ${ }^{2}$ South: IT, GR; ${ }^{3}$ Central and East: CZ, PL; ${ }^{4}$ West: NL, FR, IR; 5 only IT; ${ }^{6}$ only PL; n.a. = not available. 


\begin{tabular}{lllllll}
\hline & $\begin{array}{l}\text { Dietary fiber } \\
\mathrm{g}\end{array}$ & Fat \% $\mathrm{E}^{1}$ & SFA \%E & MUFA \%E & PUFA \%E & $\begin{array}{l}\text { Cholesterol } \\
\mathrm{mg}\end{array}$ \\
\hline $25.4 \pm 7.0$ & $25.2 \pm 10.6$ & $32.0 \pm 4.7$ & $10.9 \pm 1.9$ & $13.0 \pm 2.6$ & $5.3 \pm 1.2$ & $359.6 \pm 146.3$ \\
\hline $16.0 \pm 2.5$ & $17.5 \pm 1.1$ & $39.7 \pm 2.9$ & $13.6 \pm 1.2$ & $16.6 \pm 1.2$ & $5.4 \pm 0.4$ & $312.6 \pm 49.9$ \\
\hline $12.9 \pm 5.0$ & $12.0 \pm 4.0$ & $31.3 \pm 4.6$ & $14.0 \pm 2.3$ & $11.4 \pm 2.1$ & $3.7 \pm 1.0$ & $204.0 \pm 87.0$ \\
\hline n.a. & $17.0 \pm 6.0$ & $35.0 \pm 6.0$ & $14.0 \pm 3.0$ & $12.0 \pm 3.0$ & $7.0 \pm 2.0$ & $174.0 \pm 84.0$ \\
\hline $16.7 \pm 6.0$ & $10.0 \pm 3.4$ & $36.0 \pm 4.8$ & $14.4 \pm 3.0$ & n.a. & n.a. & $179.0 \pm 83.1$ \\
\hline $12.5-25.4$ & $7.7-25.2$ & $31.0-39.7$ & $10.1-15.4$ & $10.0-16.6$ & $3.7-8.6$ & $158.1-359.6$ \\
\hline$<10 \% \mathrm{E}^{3}$ & $>25 \mathrm{~g} \cdot \mathrm{d}^{-1,6}$ & $15-30 \% \mathrm{E}^{3}$ & $<10 \% \mathrm{E}^{3}$ & & $6-11 \% \mathrm{E}^{5}$ & $<300 \mathrm{mg} \cdot \mathrm{d}^{-1,3}$ \\
& & & & & & \\
\hline
\end{tabular}

Table 7.9. Intake of energy and macronutrients (min.-max.) in children aged 7-9 years in the four regions

\begin{tabular}{|c|c|c|c|c|c|}
\hline Region/sex & $\begin{array}{l}\text { Energy } \\
\text { MJ }\end{array}$ & $\begin{array}{l}\text { Protein } \\
\% \mathrm{E}\end{array}$ & $\begin{array}{l}\text { Carbohydrates, \%E } \\
\text { (of that sucrose) }\end{array}$ & $\begin{array}{l}\text { Dietary fiber } \\
\text { g }\end{array}$ & $\begin{array}{l}\text { Fat } \\
\% \mathrm{E}\end{array}$ \\
\hline \multicolumn{6}{|l|}{ North $^{1}$} \\
\hline Male & $7.2-9.3$ & $14.0-15.4$ & $51.0-54.0(12.6-16.0)$ & $13.0-18.0$ & $31.3-33.0$ \\
\hline Female & $7.2-8.1$ & $14.0-15.4$ & $52.0-55.0(12.6-18.0)$ & $13.0-17.0$ & $31.0-32.0$ \\
\hline \multicolumn{6}{|l|}{ South ${ }^{2}$} \\
\hline Male & $9.1-11.3$ & $15.5-16.6$ & $48.6(22.5)^{5}$ & $18.5-20.2$ & $34.8-35.9$ \\
\hline Female & $7.9-10.6$ & $15.9-16.6$ & $48.3(21.8)^{5}$ & $15.2-19.4$ & $34.9-36.2$ \\
\hline \multicolumn{6}{|c|}{ Central and East ${ }^{3}$} \\
\hline Male & $7.6-9.1$ & $11.7-14.5$ & $51.4-56.3\left(16.6-17.3^{6}\right)$ & $15.0-19.6^{7}$ & $32.0-34.1$ \\
\hline Female & $6.3-8.0$ & $11.3-14.5$ & $52.2-55.5\left(16.4-18.0^{6}\right)$ & $14.3-17.4^{7}$ & $31.3-34.2$ \\
\hline \multicolumn{6}{|l|}{ West $^{4}$} \\
\hline Male & $7.4-8.4$ & $13.5-14.7$ & $49.3-53.0^{8}$ (n.a.) & $9.2-17.0$ & $33.3-34.0$ \\
\hline Female & $6.8-7.6$ & $13.5-15.0$ & $48.5-52.0^{8}$ (n.a.) & $7.6-15.0$ & $34.0-36.5$ \\
\hline
\end{tabular}

${ }^{1}$ North: SE, NO, DK; ${ }^{2}$ South: $\mathrm{PT}, \mathrm{IT} ;{ }^{3}$ Central and East: $\mathrm{AT}, \mathrm{CZ}, \mathrm{DE}, \mathrm{PL} ;{ }^{4}$ West: $\mathrm{NL}, \mathrm{FR}, \mathrm{IR} ;{ }^{5}$ only $\mathrm{PT} ;{ }^{6}$ only AT and $\mathrm{PL} ;{ }^{7}$ only AT, DE and PL; ${ }^{8}$ only NL and FR; n.a. = not available. 
Table 7.10. Intake of energy and macronutrients (min.-max.) in children aged 10-14 years in the four regions

\begin{tabular}{|c|c|c|c|c|c|}
\hline Region/sex & $\begin{array}{l}\text { Energy } \\
\text { MJ }\end{array}$ & $\begin{array}{l}\text { Protein } \\
\% \mathrm{E}\end{array}$ & $\begin{array}{l}\text { Carbohydrates, \%E } \\
\text { (of that sucrose) }\end{array}$ & $\begin{array}{l}\text { Dietary fiber } \\
\mathrm{g}\end{array}$ & $\begin{array}{l}\text { Fat } \\
\% E\end{array}$ \\
\hline \multicolumn{6}{|l|}{ North $^{1}$} \\
\hline Male & $7.8-9.6$ & $14.0-15.9$ & $52.0-55.0(11.7-18.0)$ & $13.0-18.0$ & $31.0-32.0$ \\
\hline Female & $6.9-8.1$ & $14.0-15.4$ & $52.0-55.0(12.9-19.0)$ & $12.0-15.0$ & $31.0-32.0$ \\
\hline \multicolumn{6}{|l|}{ South ${ }^{2}$} \\
\hline Male & $9.8-10.3$ & $15.6-16.9$ & $41.0(16.1)^{5}$ & $18.5-21.6$ & $35.8-40.8$ \\
\hline Female & $8.4-8.6$ & $15.7-17.6$ & $41.6(16.0)^{5}$ & $16.8-17.5$ & $33.4-39.7$ \\
\hline \multicolumn{6}{|c|}{ Central and East ${ }^{3}$} \\
\hline Male & $7.0-11.7$ & $11.5-14.6$ & $50.8-53.9(15.2-17.6)^{6}$ & $15.1-27.7$ & $32.1-34.6$ \\
\hline Female & $6.1-9.5$ & $11.7-14.1$ & $51.7-54.0(12.5-16.8)^{6}$ & $13.7-25.0$ & $32.3-34.3$ \\
\hline \multicolumn{6}{|l|}{ West ${ }^{4}$} \\
\hline Male & $7.9-10.1$ & $13.0-15.5$ & $48.1-51.0^{7}\left(16.9^{8}\right)$ & $9.4-20.0$ & $33.3-36.3$ \\
\hline Female & $7.0-8.7$ & $13.0-15.6$ & $48.0-52.0^{7}\left(16.7^{8}\right)$ & $7.7-17.0$ & $34.5-36.4$ \\
\hline
\end{tabular}

The vitamin D intake in European children was generally low. Only Finland, Norway and Sweden reached the recommended intake of $5 \mu \mathrm{g} / \mathrm{day}$ [D-A-CH, 2000]. It is remarkable that it was not reached in the 10- to 14 -year-old children by any country. The requirement for preformed vitamin $\mathrm{D}$ intake depends on the amount of endogenously produced vitamin $\mathrm{D}$. Regarding the relatively low sun exposure during winter months and the possibly low sun exposure through a lifestyle that is dominated by indoor activities, these low amounts of dietary vitamin $\mathrm{D}$ intake may be insufficient.

The recommended intake values for $\alpha$-tocopherol equivalents were between 5.3 and $14.5 \mathrm{mg} /$ day in boys and 5.1 and $18.1 \mathrm{mg} /$ day in girls [D-A-CH, 2000]. It was particularly low in Denmark, Finland, Ireland and Sweden. In the age group of 10-14 years, only 3 countries reached the reference values (Germany, Poland, The Netherlands).

The intake of thiamine, riboflavin and niacin equivalents can be regarded as sufficient in almost every participating country. Only children from Austria (7- to 14-yearold girls and 10- to 14-year-old boys) did not meet the recommendation for thiamine and riboflavin [D-A-CH, 2000]. Concerning the intake of niacin equivalents, from 
Table 7.11. Intake of fat, fatty acids and cholesterol (min.-max.) in children aged 4-6 years in the four regions

\begin{tabular}{|c|c|c|c|c|c|}
\hline Region/sex & $\begin{array}{l}\text { Fat } \\
\% \mathrm{E}\end{array}$ & $\begin{array}{l}\text { SFA } \\
\% \mathrm{E}\end{array}$ & $\begin{array}{l}\text { MUFA } \\
\% \mathrm{E}\end{array}$ & $\begin{array}{l}\text { PUFA } \\
\% E\end{array}$ & $\begin{array}{l}\text { Cholesterol } \\
\mathrm{mg}\end{array}$ \\
\hline \multicolumn{6}{|l|}{ North ${ }^{1}$} \\
\hline Male & $31.0-34.0$ & $13.5-15.0$ & $10.0-12.0$ & $3.7-6.0$ & $153.0-186.0^{5}$ \\
\hline Female & $31.0-35.0$ & $13.7-15.0$ & $10.0-12.0$ & $3.7-6.0$ & $139.0-187.0^{5}$ \\
\hline \multicolumn{6}{|l|}{ South ${ }^{2}$} \\
\hline Male & $34.3-40.2$ & $10.9-14.5$ & $10.6^{6}$ & $4.1^{6}$ & $327.6^{6}$ \\
\hline Female & $33.8-40.5$ & $10.8-14.4$ & $9.7^{6}$ & $4.0^{6}$ & $327.1^{6}$ \\
\hline \multicolumn{6}{|c|}{ Central and East ${ }^{3}$} \\
\hline Male & $30.0-31.9$ & $11.6-15.3^{7}$ & $12.5-13.7^{7}$ & $4.5-4.8^{7}$ & $240.0-246.8$ \\
\hline Female & $30.0-32.5$ & $12.0-15.2^{7}$ & $12.1-13.6^{7}$ & $4.7-4.9^{7}$ & $201.4-245.0$ \\
\hline \multicolumn{6}{|l|}{ West ${ }^{4}$} \\
\hline Male & $31.0-34.7$ & $12.0-15.1$ & $10.0-11.3^{8}$ & $4.5-6.0^{8}$ & $122.0-141.4^{8}$ \\
\hline Female & $31.0-36.3$ & $12.0-15.8$ & $10.0-11.2^{8}$ & $4.6-5.0^{8}$ & $113.0-166.0^{8}$ \\
\hline
\end{tabular}

the United Kingdom only values for niacin were available and therefore cannot be compared to the recommended intake values.

Vitamin $\mathrm{B}_{6}$ and vitamin $\mathrm{B}_{12}$ intake were above the $\mathrm{D}-\mathrm{A}-\mathrm{CH} 2000$ recommendations in all countries. The intake of folate equivalents was between 109 and $428 \mu \mathrm{g} / \mathrm{day}$. Only 10- to 14-year-old children from Portugal met the reference intake [D-A-CH, 2000]. In the female group, the intake was below the recommendation in Sweden, Austria, Hungary and The Netherlands.

The intake of ascorbic acid ranged from 57 to $222 \mathrm{mg}$ /day. It was remarkably high in the Czech Republic in all age groups and in German children from 12 to 13 years of age, and especially low in The Netherlands (cf. tables 7.14-7.16).

\section{Comparison of Regions}

In general, the vitamin intake levels did not show big differences across the regions. The vitamin $\mathrm{D}$ intake revealed remarkably high maximum intakes in children aged $4-14$ in the North region (5.1-6.8 $\mu \mathrm{g} /$ day) (cf. table 7.17-7.19). 
Table 7.12. Intake of fat, fatty acids and cholesterol (min.-max.) in children aged 7-9 years in the four regions

\begin{tabular}{|c|c|c|c|c|c|}
\hline Region/sex & $\begin{array}{l}\text { Fat } \\
\% \mathrm{E}\end{array}$ & $\begin{array}{l}\text { SFA } \\
\% \mathrm{E}\end{array}$ & $\begin{array}{l}\text { MUFA } \\
\% E\end{array}$ & $\begin{array}{l}\text { PUFA } \\
\% E\end{array}$ & $\begin{array}{l}\text { Cholesterol } \\
\mathrm{mg}\end{array}$ \\
\hline \multicolumn{6}{|l|}{ North $^{1}$} \\
\hline Male & $31.3-33.0$ & $14.0-15.0$ & $10.0-11.3$ & $3.6-6.0$ & $216.0-225.0^{5}$ \\
\hline Female & $31.0-32.0$ & $14.0-14.1$ & $10.0-11.3$ & $3.6-6.0$ & $200.0-216.0^{5}$ \\
\hline \multicolumn{6}{|l|}{ South ${ }^{2}$} \\
\hline Male & $34.8-35.9$ & $9.4-13.0$ & $10.9-15.0$ & $4.6-5.0$ & $375.2^{6}$ \\
\hline Female & $34.9-36.2$ & $10.9-13.1$ & $9.9-15.1$ & $4.3-5.1$ & $308.6^{6}$ \\
\hline \multicolumn{6}{|c|}{ Central and East ${ }^{3}$} \\
\hline Male & $32.0-34.1$ & $12.0-15.1$ & $11.7-13.3$ & $4.6-6.1$ & $240.0-259.0$ \\
\hline Female & $31.3-34.2$ & $11.8-14.9$ & $11.5-14.2$ & $4.5-6.5$ & $168.0-281.0$ \\
\hline \multicolumn{6}{|l|}{ West ${ }^{4}$} \\
\hline Male & $33.3-34.0$ & $13.0-15.1$ & $11.0-12.0^{7}$ & $4.7-6.0^{7}$ & $141.4-155.0^{7}$ \\
\hline Female & $34.0-36.5$ & $14.0-15.5$ & $11.1-12.0^{7}$ & $4.9-7.0^{7}$ & $147.0-157.2^{7}$ \\
\hline
\end{tabular}

\section{Minerals}

Comparison of Countries

The sodium intake was in all countries above the recommendations. However, the salt intake should be lowered in most countries in order to prevent chronic diseases such as cancer and cardiovascular disease.

The reference values for potassium were met by all countries and all age groups. The recommendations for calcium intake were met better by younger children. It was especially low in Austrian and Polish children [D-A-CH, 2000].

The mean phosphorus intake can be considered as sufficient in most countries. Children from the older age groups are more likely to have an intake below the reference values than the younger children. The intake levels were especially low in Austrian 10- to 14-year-old children.

Intake of iron was sufficient for boys and girls aged up to 10 years but very much below the D-A-CH 2000 recommendations for girls from 10 to 14 years apart from Portuguese girls aged 13 [D-A-CH, 2000]. 
Table 7.13. Intake of fat, fatty acids and cholesterol (min.-max.) in children aged 10-14 years in the four regions

\begin{tabular}{|c|c|c|c|c|c|}
\hline Region/sex & $\begin{array}{l}\text { Fat } \\
\% \mathrm{E}\end{array}$ & $\begin{array}{l}\text { SFA } \\
\% \mathrm{E}\end{array}$ & $\begin{array}{l}\text { MUFA } \\
\% \mathrm{E}\end{array}$ & $\begin{array}{l}\text { PUFA } \\
\% \mathrm{E}\end{array}$ & $\begin{array}{l}\text { Cholesterol } \\
\mathrm{mg}\end{array}$ \\
\hline \multicolumn{6}{|l|}{ North $^{1}$} \\
\hline Male & $31.0-32.0$ & $13.0-14.1$ & $10.0-11.6$ & $3.7-5.0$ & $233.0-241.0^{5}$ \\
\hline Female & $31.0-32.0$ & 14.0 & $10.0-11.4$ & $3.7-5.0$ & $198.0-204.0^{5}$ \\
\hline \multicolumn{6}{|l|}{ South $^{2}$} \\
\hline Male & $35.8-40.8$ & $10.8-14.3$ & $11.9-16.7$ & $5.1-5.7$ & $362.1-370.1$ \\
\hline Female & $33.4-39.7$ & $10.1-13.6$ & $11.0-16.6$ & $4.4-5.4$ & $312.6-319.9$ \\
\hline \multicolumn{6}{|c|}{ Central and East ${ }^{3}$} \\
\hline Male & $32.1-34.6$ & $12.1-15.6$ & $12.0-14.7$ & $4.6-8.2$ & $261.2-408.0$ \\
\hline Female & $32.3-34.3$ & $10.6-15.4$ & $10.2-14.6$ & $4.8-8.6$ & $228.3-321.0$ \\
\hline \multicolumn{6}{|l|}{ West ${ }^{4}$} \\
\hline Male & $33.3-36.3$ & $13.9-15.1$ & $10.9-13.0^{6}$ & $4.6-7.0^{6}$ & $178.6-203.5^{7}$ \\
\hline Female & $34.5-36.4$ & $14.0-14.9$ & $11.4-12.0^{6}$ & $5.1-7.0^{6}$ & $158.1-179.0^{7}$ \\
\hline
\end{tabular}

For zinc generally the recommendations were met better in younger age groups and in boys. Girls from older age groups from Austria, Denmark, Hungary, Ireland, Poland, Spain, Sweden and The Netherlands and boys from Austria and Ireland did not reach the levels of recommendation [D-A-CH, 2000].

The mean dietary intake of iodine was insufficient for children from the Czech Republic, Germany and Poland.

Dietary selenium intake was sufficient for almost all children, where again children from older age groups had a poorer intake than younger children.

The intake of copper and manganese can be regarded as sufficient in all countries except for boys from the United Kingdom. Girls from older age groups from Sweden, Spain, Italy, the United Kingdom, The Netherlands, Ireland, Austria and Poland did not reach the levels of recommendation either (cf. tables 7.20-7.22).

Comparison of Regions

Tables 7.23-7.25 show intake data for selected minerals in the different regions. As was the case with vitamins in general, no big differences could be observed between the regions except for the iodine intake which tended to be higher in the northern 
Table 7.14. Vitamin intake (mean \pm SD) in children aged $\mathbf{4 - 6}$ years of European countries (male and female); total $n=4,857$

\begin{tabular}{lllllll}
\hline & $\begin{array}{l}\text { Age } \\
\text { years }\end{array}$ & $\mathrm{n}$ & $\begin{array}{l}\text { Vitamin } \mathrm{A}^{1} \\
\mathrm{mg}\end{array}$ & $\begin{array}{l}\beta \text {-Carotene } \\
\mathrm{mg}\end{array}$ & $\begin{array}{l}\text { Vitamin D } \\
\mu \mathrm{g}\end{array}$ & $\begin{array}{l}\text { Vitamin } \mathrm{E}^{2} \\
\mathrm{mg}\end{array}$ \\
\hline Male & & & & & & \\
\hline Czech Republic & $4-6$ & 641 & $0.6 \pm 1.6$ & $2.8 \pm 2.6$ & $2.3 \pm 3.0$ & $9.1 \pm 6.4$ \\
\hline Denmark & $4-6$ & 117 & $1.0 \pm 0.5$ & $3.8 \pm 2.7$ & $2.3 \pm 1.7$ & $6.4 \pm 2.0$ \\
\hline Finland & 4 & 307 & $0.6 \pm 0.7$ & $1.7 \pm 1.4$ & $5.9 \pm 3.6$ & $5.3 \pm 2.5$ \\
& 6 & 364 & $0.7 \pm 0.7$ & $1.8 \pm 1.5$ & $6.3 \pm 3.5$ & $6.1 \pm 2.5$ \\
\hline Germany & $<7$ & 102 & $0.8 \pm 0.5$ & $2.4 \pm 2.1$ & $1.8 \pm 1.9$ & $9.8 \pm 4.7$ \\
\hline Ireland & $5-6$ & 72 & $0.4 \pm 0.4$ & $2.0 \pm 1.6$ & $2.4 \pm 2.7$ & $5.8 \pm 4.0$ \\
\hline Italy & $4-6$ & 21 & $0.8 \pm 0.3$ & $2.0 \pm 1.3$ & $2.3 \pm 1.3$ & $6.5 \pm 2.8$ \\
\hline Norway & 4 & 206 & $1.1 \pm 5.6$ & n.a. & $6.8 \pm 5.3$ & $9.0 \pm 5.4^{7}$ \\
\hline Poland & $4-6$ & 82 & $0.9 \pm 1.2$ & $2.4 \pm 3.4$ & $2.0 \pm 1.7$ & $8.8 \pm 5.2$ \\
\hline Sweden & $4-6$ & 302 & $0.8 \pm 0.4$ & $1.2 \pm 1.1$ & $6.7 \pm 4.4$ & $6.0 \pm 2.2$ \\
\hline The Netherlands ${ }^{6}$ & $4-6$ & 327 & $0.5 \pm 0.3$ & n.a. & $2.2 \pm 0.8$ & $8.8 \pm 2.8$ \\
\hline All countries (min.-max.) & & & $0.4-1.1$ & $1.2-3.8$ & $1.8-5.8$ & $5.3-9.8$ \\
\hline Female & & & & & & \\
\hline
\end{tabular}

\section{Female}

\begin{tabular}{|c|c|c|c|c|c|c|}
\hline Czech Republic & $4-6$ & 446 & $0.6 \pm 1.6$ & $2.8 \pm 2.6$ & $2.3 \pm 3.0$ & $9.1 \pm 6.4$ \\
\hline Denmark & $4-6$ & 117 & $0.9 \pm 0.5$ & $3.4 \pm 2.9$ & $2.0 \pm 0.9$ & $6.2 \pm 1.9$ \\
\hline \multirow[t]{2}{*}{ Finland } & 4 & 307 & $0.5 \pm 0.5$ & $1.6 \pm 1.3$ & $6.1 \pm 4.1$ & $5.1 \pm 2.8$ \\
\hline & 6 & 349 & $0.6 \pm 0.6$ & $1.7 \pm 1.4$ & $5.5 \pm 3.2$ & $5.5 \pm 2.3$ \\
\hline Germany & $<7$ & 102 & $0.7 \pm 0.4$ & $2.4 \pm 1.6$ & $1.5 \pm 1.4$ & $8.5 \pm 3.0$ \\
\hline Ireland & $5-6$ & 72 & $0.4 \pm 0.7$ & $1.4 \pm 1.2$ & $1.9 \pm 2.1$ & $5.4 \pm 3.5$ \\
\hline Italy & $4-6$ & 21 & $0.9 \pm 0.7$ & $2.0 \pm 1.3$ & $2.2 \pm 1.2$ & $6.7 \pm 2.2$ \\
\hline Norway & 4 & 185 & $1.2 \pm 0.4$ & n.a. & $7.1 \pm 5.6$ & $9.8 \pm 7.3^{7}$ \\
\hline Poland & $4-6$ & 82 & $1.0 \pm 0.6$ & $2.7 \pm 3.2$ & $1.9 \pm 1.5$ & $8.0 \pm 0.3$ \\
\hline Sweden & $4-6$ & 302 & $0.7 \pm 0.4$ & $1.1 \pm 0.9$ & $6.5 \pm 4.6$ & $5.9 \pm 2.3$ \\
\hline The Netherlands ${ }^{6}$ & $4-6$ & 312 & $0.5 \pm 0.2$ & n.a. & $1.9 \pm 0.6$ & $7.5 \pm 2.3$ \\
\hline All countries (min.-max.) & & & $0.4-1.2$ & $1.1-3.4$ & $1.5-6.5$ & $5.1-9.8$ \\
\hline Reference values $^{5}$ & & & 0.7 & n.a. & 5 & 8 \\
\hline
\end{tabular}

${ }^{1}$ Retinol equivalent $\left(=1 \mathrm{mg}\right.$ retinol $=6 \mathrm{mg}$ all-trans- $\beta$-carotene $=12 \mathrm{mg}$ other carotenoids); ${ }^{2} \mathrm{RRR}-\alpha-$ tocopherol equivalent $(=\mathrm{mg}$ a-tocopherol $+\mathrm{mg} \beta$-tocopherol $\times 0.5+\mathrm{mg} \gamma$-tocopherol $\times 0.25+\mathrm{mg}$ a-tocotrienol $\times 0.33) ;{ }^{3}$ niacin equivalent ( $=1 \mathrm{mg}$ niacin $=60 \mathrm{mg}$ tryptophan); ${ }^{4}$ folate equivalent $(1 \mu \mathrm{g}$ food folate $=0.5 \mu \mathrm{g}$ folic acid (PGA) $=0.6 \mu \mathrm{g}$ folic acid taken with meals); ${ }^{5} \mathrm{D}-\mathrm{A}-\mathrm{CH}, 2000 ;{ }^{6}$ using different conversion factors for vitamin $\mathrm{A}$, vitamin $\mathrm{E}$ and folate; ${ }^{7}$ alpha-tocopherol only; n.a. $=$ not available. 


\begin{tabular}{|c|c|c|c|c|c|c|}
\hline $\begin{array}{l}\text { Thiamine } \\
\text { mg }\end{array}$ & $\begin{array}{l}\text { Riboflavin } \\
\text { mg }\end{array}$ & $\begin{array}{l}\mathrm{Niacin}^{3} \\
\mathrm{mg}\end{array}$ & $\begin{array}{l}\text { Vitamin } \mathrm{B}_{6} \\
\mathrm{mg}\end{array}$ & $\begin{array}{l}\text { Folate }^{4} \\
\mu \mathrm{g}\end{array}$ & $\begin{array}{l}\text { Cobalamin } \\
\mu \mathrm{g}\end{array}$ & $\begin{array}{l}\text { Ascorbic acid } \\
\mathrm{mg}\end{array}$ \\
\hline $1.3 \pm 0.6$ & $1.6 \pm 0.9$ & $22.8 \pm 10.2$ & $1.7 \pm 0.9$ & $190 \pm 110$ & $5.0 \pm 3.9$ & $157 \pm 95$ \\
\hline $1.1 \pm 0.3$ & $1.6 \pm 0.4$ & $18.9 \pm 4.5$ & $1.1 \pm 0.3$ & $256 \pm 72$ & $5.0 \pm 1.7$ & $86 \pm 40$ \\
\hline $1.0 \pm 0.6$ & $1.8 \pm 0.7$ & $19.5 \pm 6.1$ & $1.5 \pm 0.6$ & $135 \pm 52$ & $4.6 \pm 2.6$ & $60 \pm 41$ \\
\hline $1.1 \pm 0.7$ & $2.1 \pm 0.8$ & $22.2 \pm 6.5$ & $1.6 \pm 0.6$ & $166 \pm 61$ & $5.3 \pm 2.8$ & $72 \pm 49$ \\
\hline $1.2 \pm 0.5$ & $1.6 \pm 0.5$ & $20.4 \pm 5.2$ & $1.8 \pm 0.9$ & $213 \pm 91$ & $4.4 \pm 1.9$ & $91 \pm 45$ \\
\hline $1.4 \pm 0.5$ & $1.7 \pm 0.7$ & $15.7 \pm 6.5$ & $1.8 \pm 0.7$ & $225 \pm 116$ & $4.0 \pm 1.9$ & $74 \pm 47$ \\
\hline $0.9 \pm 0.2$ & $1.3 \pm 0.3$ & $24.9 \pm 5.5$ & $1.6 \pm 0.4$ & $198 \pm 66$ & n.a. & $76 \pm 36$ \\
\hline $1.2 \pm 0.5$ & $1.6 \pm 0.8$ & n.a. & n.a. & n.a. & n.a. & $81 \pm 44$ \\
\hline $0.8 \pm 0.3$ & $1.3 \pm 0.5$ & n.a. & $1.3 \pm 0.5$ & $169 \pm 51$ & $2.7 \pm 2.3$ & $66 \pm 46$ \\
\hline $1.1 \pm 0.4$ & $1.6 \pm 0.5$ & $20.9 \pm 5.3$ & $1.5 \pm 0.5$ & $174 \pm 67$ & $4.2 \pm 2.0$ & $90 \pm 71$ \\
\hline $0.9 \pm 0.2$ & $1.4 \pm 0.4$ & n.a. & $1.4 \pm 0.4$ & $120 \pm 29$ & $2.9 \pm 1.2$ & $72 \pm 26$ \\
\hline $0.8-1.4$ & $1.3-2.1$ & $15.7-24.9$ & $1.3-1.8$ & $120-256$ & $2.7-5.3$ & 60-157 \\
\hline $1.3 \pm 0.6$ & $1.6 \pm 0.9$ & $22.8 \pm 10.2$ & $1.7 \pm 0.9$ & $190 \pm 110$ & $5.0 \pm 3.9$ & $157 \pm 95$ \\
\hline $1.0 \pm 0.3$ & $1.4 \pm 0.5$ & $17.0 \pm 4.4$ & $1.0 \pm 0.3$ & $235 \pm 70$ & $4.4 \pm 1.9$ & $83 \pm 37$ \\
\hline $1.0 \pm 0.5$ & $1.7 \pm 0.6$ & $18.4 \pm 5.9$ & $1.5 \pm 0.7$ & $132 \pm 49$ & $4.2 \pm 2.1$ & $61 \pm 41$ \\
\hline $1.0 \pm 0.6$ & $1.8 \pm 0.7$ & $19.5 \pm 5.9$ & $1.5 \pm 0.6$ & $144 \pm 51$ & $4.5 \pm 2.4$ & $61 \pm 36$ \\
\hline $1.0 \pm 0.3$ & $1.3 \pm 0.4$ & $17.9 \pm 4.7$ & $1.9 \pm 0.4$ & $175 \pm 65$ & $3.0 \pm 1.1$ & $91 \pm 51$ \\
\hline $1.2 \pm 0.5$ & $1.6 \pm 0.6$ & $14.4 \pm 6.5$ & $1.7 \pm 0.7$ & $196 \pm 88$ & $4.3 \pm 3.3$ & $77 \pm 47$ \\
\hline $0.9 \pm 0.2$ & $1.3 \pm 0.3$ & $24.6 \pm 4.5$ & $1.6 \pm 0.4$ & $199 \pm 52$ & n.a. & $82 \pm 38$ \\
\hline $1.2 \pm 0.5$ & $1.6 \pm 0.7$ & n.a. & n.a. & n.a. & n.a. & $88 \pm 41$ \\
\hline $0.8 \pm 0.3$ & $1.3 \pm 0.6$ & n.a. & $1.2 \pm 0.5$ & $164 \pm 57$ & $2.9 \pm 3.4$ & $61 \pm 50$ \\
\hline $1.1 \pm 1.5$ & $1.5 \pm 0.9$ & $19.5 \pm 4.8$ & $1.5 \pm 1.6$ & $162 \pm 56$ & $4.7 \pm 14.8$ & $88 \pm 74$ \\
\hline $0.8 \pm 0.2$ & $1.2 \pm 0.3$ & n.a. & $1.2 \pm 0.3$ & $109 \pm 29$ & $2.6 \pm 0.8$ & $65 \pm 26$ \\
\hline $0.8-1.3$ & $1.2-1.8$ & $14.4-24.6$ & $1.0-1.9$ & 109-199 & $2.6-5.0$ & $61-157$ \\
\hline 0.8 & 0.9 & 10 & 0.5 & 300 & 1.5 & 70 \\
\hline
\end{tabular}


Table 7.15. Vitamin intake (mean \pm SD) in children aged 7-9 years of European countries (male and female); total $n=5,438$

$\begin{array}{llllll}\text { Age } & n & \text { Vitamin } A^{1} & \beta \text {-Carotene } & \text { Vitamin D } & \text { Vitamin } E^{2} \\ \text { years } & & \mathrm{mg} & \mathrm{mg} & \mu \mathrm{g} & \mathrm{mg}\end{array}$

\section{Male}

\begin{tabular}{lllllll}
\hline Austria & $7-9$ & 146 & $0.8 \pm 0.6$ & $2.2 \pm 2.9$ & $1.5 \pm 0.8$ & $10.7 \pm 4.4$ \\
\hline Czech Republic & $7-9$ & 940 & $0.8 \pm 1.8$ & $2.5 \pm 1.7$ & $2.7 \pm 12.7$ & $9.4 \pm 5.3$ \\
\hline Denmark & $7-9$ & 139 & $1.0 \pm 0.6$ & $4.1 \pm 3.6$ & $2.5 \pm 1.4$ & $7.2 \pm 2.4$ \\
\hline Germany & $7-9$ & 321 & $1.0 \pm 0.6$ & $3.0 \pm 2.8$ & $1.8 \pm 2.0$ & $10.6 \pm 4.6$ \\
\hline Ireland & $7-9$ & 110 & $0.4 \pm 0.5$ & $2.3 \pm 2.2$ & $2.2 \pm 2.0$ & $7.4 \pm 5.8$ \\
\hline Italy & $7-9$ & 29 & $0.9 \pm 0.7$ & $2.1 \pm 1.6$ & $2.8 \pm 1.3$ & $8.7 \pm 3.3$ \\
\hline Norway & 9 & 402 & $1.3 \pm 0.7$ & n.a. & $6.4 \pm 5.6$ & $10.4 \pm 6.0^{6}$ \\
\hline Poland & $7-9$ & 101 & $1.4 \pm 2.4$ & $2.6 \pm 3.5$ & $2.8 \pm 2.8$ & $10.1 \pm 5.5$ \\
\hline Sweden & $7-9$ & 444 & $0.9 \pm 0.6$ & $1.6 \pm 1.4$ & $5.1 \pm 2.8$ & $6.3 \pm 1.8$ \\
\hline The Netherlands & $7-9$ & 104 & $0.7 \pm 0.5$ & n.a. & $2.9 \pm 1.6$ & $11.2 \pm 5.4$ \\
\hline All countries (min.-max.) & & & $0.4-1.3$ & $1.6-4.1$ & $1.5-6.4$ & $6.3-11.2$ \\
\hline
\end{tabular}

\section{Female}

\begin{tabular}{lllllll}
\hline Austria & $7-9$ & 146 & $0.7 \pm 0.4$ & $1.9 \pm 1.5$ & $1.5 \pm 1.0$ & $10.7 \pm 4.1$ \\
\hline Czech Republic & $7-9$ & 765 & $0.8 \pm 1.8$ & $2.5 \pm 1.7$ & $2.7 \pm 12.7$ & $9.4 \pm 5.3$ \\
\hline Denmark & $7-9$ & 139 & $0.9 \pm 0.5$ & $4.0 \pm 3.3$ & $2.2 \pm 1.1$ & $6.4 \pm 2.0$ \\
\hline Germany & $7-9$ & 321 & $0.9 \pm 0.5$ & $2.8 \pm 2.3$ & $1.7 \pm 1.8$ & $9.4 \pm 4.0$ \\
\hline Ireland & $7-9$ & 110 & $0.4 \pm 0.3$ & $2.0 \pm 1.5$ & $2.4 \pm 2.4$ & $6.2 \pm 3.7$ \\
\hline Italy & $7-9$ & 29 & $0.8 \pm 0.8$ & $2.2 \pm 1.4$ & $2.1 \pm 0.8$ & $9.8 \pm 8.8$ \\
\hline Norway & 9 & 408 & $1.1 \pm 0.6$ & n.a. & $5.1 \pm 4.2$ & $8.5 \pm 4.6^{6}$ \\
\hline Poland & $7-9$ & 101 & $1.0 \pm 0.7$ & $2.7 \pm 3.6$ & $2.3 \pm 1.5$ & $9.9 \pm 5.6$ \\
\hline Sweden & $7-9$ & 444 & $0.7 \pm 0.4$ & $1.6 \pm 1.2$ & $4.8 \pm 2.8$ & $5.9 \pm 2.1$ \\
\hline The Netherlands & $7-9$ & 134 & $0.7 \pm 0.6$ & n.a. & $2.8 \pm 1.3$ & $10.4 \pm 4.8$ \\
\hline All countries (min.-max.) & & & $0.4-1.1$ & $1.6-4.0$ & $1.5-5.1$ & $5.9-13.3$ \\
\hline Reference values 5 & & & 0.8 & n.a. & 5 & 10 (male)/ \\
\hline
\end{tabular}

${ }^{1}$ Retinol equivalent $\left(=1 \mathrm{mg}\right.$ retinol $=6 \mathrm{mg}$ all-trans- $\beta$-carotene $=12 \mathrm{mg}$ other carotenoids) ${ }^{2}$ RRR- $\mathrm{a}$-tocopherol equivalent $(=\mathrm{mg}$ a-tocopherol $+\mathrm{mg} \beta$-tocopherol $\times 0.5+\mathrm{mg}$--tocopherol $\times 0.25+\mathrm{mg}$ a-tocotrienol $\times 0.33)$; ${ }^{3}$ niacin equivalent $(=1 \mathrm{mg}$ niacin $=60 \mathrm{mg}$ tryptophan $) ;{ }^{4}$ folate equivalent $(1 \mu \mathrm{g}$ food folate $=0.5 \mu \mathrm{g}$ folic acid $(\mathrm{PGA})=0.6 \mu \mathrm{g}$ folic acid taken with meals); ${ }^{5} \mathrm{D}-\mathrm{A}-\mathrm{CH}, 2000 ;{ }^{6}$ alpha-tocopherol only; n.a. $=$ not available. 


\begin{tabular}{|c|c|c|c|c|c|c|}
\hline $\begin{array}{l}\text { Thiamine } \\
\mathrm{mg}\end{array}$ & $\begin{array}{l}\text { Riboflavin } \\
\text { mg }\end{array}$ & $\begin{array}{l}\text { Niacin }^{3} \\
\mathrm{mg}\end{array}$ & $\begin{array}{l}\text { Vitamin } B_{6} \\
\text { mg }\end{array}$ & $\begin{array}{l}\text { Folate }^{4} \\
\mu \mathrm{g}\end{array}$ & $\begin{array}{l}\text { Cobalamin } \\
\mu \mathrm{g}\end{array}$ & $\begin{array}{l}\text { Ascorbic acid } \\
\mathrm{mg}\end{array}$ \\
\hline $0.9 \pm 0.4$ & $1.2 \pm 0.5$ & $19.1 \pm 6.1$ & $1.2 \pm 0.5$ & $154 \pm 64$ & $3.6 \pm 1.4$ & $125 \pm 92$ \\
\hline $1.4 \pm 0.9$ & $1.5 \pm 0.7$ & $24.9 \pm 23.8$ & $1.8 \pm 1.3$ & $200 \pm 109$ & $5.3 \pm 5.3$ & $172 \pm 113$ \\
\hline $1.3 \pm 0.3$ & $1.8 \pm 0.6$ & $22.6 \pm 6.1$ & $1.3 \pm 0.4$ & $290 \pm 101$ & $5.4 \pm 2.2$ & $101 \pm 61$ \\
\hline $1.4 \pm 0.6$ & $1.6 \pm 0.7$ & $23.4 \pm 6.8$ & $1.8 \pm 0.8$ & $229 \pm 108$ & $4.2 \pm 1.7$ & $110 \pm 60$ \\
\hline $1.6 \pm 0.5$ & $2.0 \pm 0.7$ & $18.7 \pm 6.1$ & $2.2 \pm 0.7$ & $256 \pm 99$ & $4.8 \pm 2.5$ & $98 \pm 87$ \\
\hline $1.1 \pm 0.2$ & $1.5 \pm 0.3$ & $29.9 \pm 6.5$ & $1.8 \pm 0.5$ & $242 \pm 58$ & n.a. & $102 \pm 61$ \\
\hline $1.5 \pm 0.7$ & $1.9 \pm 0.9$ & n.a. & n.a. & n.a. & n.a. & $102 \pm 56$ \\
\hline $1.0 \pm 0.5$ & $1.6 \pm 0.8$ & n.a. & $1.5 \pm 0.7$ & $214 \pm 102$ & $4.2 \pm 6.4$ & $77 \pm 71$ \\
\hline $1.4 \pm 0.5$ & $1.9 \pm 0.6$ & $27.5 \pm 6.3$ & $2.5 \pm 14.2$ & $204 \pm 66$ & $5.5 \pm 5.2$ & $88 \pm 68$ \\
\hline $0.9 \pm 0.3$ & $1.4 \pm 0.4$ & n.a. & $1.3 \pm 0.5$ & $144 \pm 51$ & n.a. & $63 \pm 48$ \\
\hline $0.9-1.6$ & $1.2-2.0$ & $18.7-29.9$ & $1.2-2.5$ & $144-290$ & $3.6-5.5$ & $63-172$ \\
\hline $0.8 \pm 0.3$ & $1.1 \pm 0.4$ & $16.7 \pm 5.1$ & $1.1 \pm 0.4$ & $145 \pm 55$ & $3.0 \pm 1.4$ & $110 \pm 73$ \\
\hline $1.4 \pm 0.9$ & $1.5 \pm 0.7$ & $24.9 \pm 23.8$ & $1.8 \pm 1.3$ & $200 \pm 109$ & $5.3 \pm 5.3$ & $172 \pm 113$ \\
\hline $1.1 \pm 0.3$ & $1.6 \pm 0.5$ & $20.0 \pm 4.7$ & $1.2 \pm 0.3$ & $264 \pm 80$ & $4.8 \pm 1.8$ & $96 \pm 49$ \\
\hline $1.2 \pm 0.5$ & $1.4 \pm 0.6$ & $20.5 \pm 6.8$ & $1.6 \pm 0.7$ & $212 \pm 88$ & $3.4 \pm 1.5$ & $108 \pm 69$ \\
\hline $1.4 \pm 0.5$ & $1.7 \pm 0.7$ & $16.2 \pm 5.9$ & $1.9 \pm 0.6$ & $226 \pm 90$ & $4.2 \pm 1.9$ & $100 \pm 61$ \\
\hline $0.9 \pm 0.3$ & $1.2 \pm 0.3$ & $25.9 \pm 6.7$ & $1.7 \pm 0.5$ & $211 \pm 63$ & n.a. & $87 \pm 45$ \\
\hline $1.2 \pm 0.5$ & $1.6 \pm 0.7$ & n.a. & n.a. & n.a. & n.a. & $95 \pm 55$ \\
\hline $0.9 \pm 0.4$ & $1.3 \pm 0.5$ & n.a. & $1.3 \pm 0.6$ & $190 \pm 75$ & $2.8 \pm 2.4$ & $71 \pm 55$ \\
\hline $1.3 \pm 0.4$ & $1.6 \pm 0.5$ & $24.6 \pm 6.0$ & $1.6 \pm 0.5$ & $187 \pm 64$ & $4.7 \pm 5.1$ & $86 \pm 83$ \\
\hline $0.9 \pm 0.5$ & $1.4 \pm 0.4$ & n.a. & $1.2 \pm 0.4$ & $133 \pm 55$ & n.a. & $57 \pm 33$ \\
\hline $0.8-1.4$ & $1.1-1.7$ & $16.2-26.3$ & $1.1-1.9$ & $133-264$ & $2.2-5.3$ & $57-172$ \\
\hline 1 & 1.1 & 12 & 0.7 & 300 & 1.8 & 80 \\
\hline
\end{tabular}


Table 7.16. Vitamin intake (mean \pm SD) in children aged 10-14 years of European countries (male and female); total $n=8,026$

\begin{tabular}{|c|c|c|c|c|c|c|}
\hline & $\begin{array}{l}\text { Age } \\
\text { years }\end{array}$ & $n$ & $\begin{array}{l}\text { Vitamin } A^{1} \\
\text { g }\end{array}$ & $\begin{array}{l}\beta \text {-Carotene } \\
\mathrm{mg}\end{array}$ & $\begin{array}{l}\text { Vitamin D } \\
\mu \mathrm{g}\end{array}$ & $\begin{array}{l}\text { Vitamin } \mathrm{E}^{2} \\
\mathrm{mg}\end{array}$ \\
\hline \multicolumn{7}{|l|}{ Male } \\
\hline Austria & $10-14$ & 248 & $0.8 \pm 0.6$ & $1.7 \pm 1.7$ & $1.5 \pm 1.4$ & $10.9 \pm 5.3$ \\
\hline Denmark & $10-14$ & 172 & $0.9 \pm 0.4$ & $3.1 \pm 2.7$ & $2.6 \pm 1.6$ & $7.0 \pm 2.7$ \\
\hline Germany & $\begin{array}{l}10-11 \\
12 \\
13-14\end{array}$ & $\begin{array}{l}199 \\
114 \\
214\end{array}$ & $\begin{array}{l}1.0 \pm 0.5 \\
1.4 \pm 0.7 \\
1.5 \pm 0.9\end{array}$ & $\begin{array}{l}2.9 \pm 2.3 \\
4.4 \pm 3.4 \\
4.8 \pm 3.9\end{array}$ & $\begin{array}{l}2.3 \pm 2.5 \\
2.4 \pm 1.8 \\
2.3 \pm 1.5\end{array}$ & $\begin{array}{l}10.7 \pm 5.2 \\
17.1 \pm 9.6 \\
18.2 \pm 11.4\end{array}$ \\
\hline Ireland & $10-12$ & 109 & $0.4 \pm 0.3$ & $2.7 \pm 2.4$ & $2.2 \pm 2.0$ & $6.9 \pm 3.4$ \\
\hline Italy & $10-14$ & 52 & $0.9 \pm 0.5$ & $2.5 \pm 1.4$ & $3.0 \pm 1.8$ & $11.1 \pm 6.1$ \\
\hline Hungary & $11-14$ & 124 & $0.6 \pm 0.4$ & n.a. & $2.5 \pm 1.1$ & $14.1 \pm 5.0$ \\
\hline Norway & 13 & 490 & $1.2 \pm 2.4$ & n.a. & $4.4 \pm 6.6$ & $9.8 \pm 6.6^{6}$ \\
\hline Poland & $10-14$ & 202 & $1.3 \pm 1.8$ & $2.8 \pm 3.2$ & $3.9 \pm 4.5$ & $14.5 \pm 9.1$ \\
\hline Portugal & 13 & 987 & $2.4 \pm 1.6$ & $1.1 \pm 1.0$ & $4.8 \pm 2.5$ & $9.4 \pm 3.9$ \\
\hline Spain & $10-14$ & 66 & $0.6 \pm 0.1$ & $1.2 \pm 0.3$ & $1.8 \pm 0.5$ & $8.5 \pm 1.5$ \\
\hline Sweden & $10-14$ & 517 & $0.7 \pm 0.4$ & $1.1 \pm 1.0$ & $4.8 \pm 2.8$ & $5.9 \pm 2.1$ \\
\hline The Netherlands & $10-14$ & 211 & $0.7 \pm 0.5$ & n.a. & $3.8 \pm 1.9$ & $13.7 \pm 6.0$ \\
\hline United Kingdom & $2-15$ & 364 & $0.6 \pm 0.8$ & $1.5 \pm 2.0$ & $2.1 \pm 1.3$ & $9.0 \pm 4.4$ \\
\hline $\begin{array}{l}\text { All countries } \\
\text { (min.-max.) }\end{array}$ & & & $0.4-2.4$ & $1.1-4.8$ & $1.5-4.8$ & $5.9-14.5$ \\
\hline Reference values ${ }^{5}$ & & & $0.9 / 1.1$ & n.a. & 5 & $13 / 14$ \\
\hline \multicolumn{7}{|l|}{ Female } \\
\hline Austria & $10-14$ & 248 & $0.6 \pm 0.5$ & $1.4 \pm 2.1$ & $1.2 \pm 0.8$ & $10.0 \pm 4.3$ \\
\hline Denmark & $10-14$ & 172 & $0.7 \pm 0.3$ & $2.8 \pm 2.3$ & $2.2 \pm 1.7$ & $6.1 \pm 2.4$ \\
\hline Germany & $\begin{array}{l}10-11 \\
12 \\
13-14 \\
\end{array}$ & $\begin{array}{l}199 \\
114 \\
214\end{array}$ & $\begin{array}{l}0.9 \pm 0.6 \\
1.5 \pm 0.8 \\
1.5 \pm 0.7\end{array}$ & $\begin{array}{l}2.8 \pm 2.5 \\
5.2 \pm 4.0 \\
4.9 \pm 3.1\end{array}$ & $\begin{array}{l}1.9 \pm 2.1 \\
2.0 \pm 1.5 \\
2.1 \pm 1.8\end{array}$ & $\begin{array}{l}10.8 \pm 5.2 \\
18.1 \pm 14.9 \\
16.1 \pm 9.4\end{array}$ \\
\hline Hungary & $11-14$ & 111 & $0.6 \pm 0.4$ & n.a. & $2.2 \pm 1.0$ & $13.3 \pm 3.6$ \\
\hline Ireland & $10-12$ & 109 & $0.3 \pm 0.3$ & $2.1 \pm 2.1$ & $2.4 \pm 2.3$ & $6.6 \pm 4.3$ \\
\hline Italy & $10-14$ & 52 & $0.8 \pm 0.4$ & $2.0 \pm 1.3$ & $2.9 \pm 2.2$ & $9.2 \pm 2.8$ \\
\hline Norway & 13 & 515 & $1.0 \pm 1.2$ & n.a. & $4.0 \pm 4.9$ & $8.8 \pm 8.6^{6}$ \\
\hline Poland & $10-14$ & 202 & $1.1 \pm 1.2$ & $3.0 \pm 4.9$ & $2.9 \pm 2.4$ & $12.3 \pm 8.1$ \\
\hline Portugal & 13 & 987 & $2.3 \pm 1.4$ & $1.1 \pm 0.9$ & $4.5 \pm 2.5$ & $9.4 \pm 4.0$ \\
\hline
\end{tabular}




\begin{tabular}{|c|c|c|c|c|c|c|}
\hline $\begin{array}{l}\text { Thiamine } \\
\text { mg }\end{array}$ & $\begin{array}{l}\text { Riboflavin } \\
\text { mg }\end{array}$ & $\begin{array}{l}\text { Niacin }^{3} \\
\mathrm{mg}\end{array}$ & $\begin{array}{l}\text { Vitamin } B_{6} \\
\text { mg }\end{array}$ & $\begin{array}{l}\text { Folate }^{4} \\
\mu \mathrm{g}\end{array}$ & $\begin{array}{l}\text { Cobalamin } \\
\mu \mathrm{g}\end{array}$ & $\begin{array}{l}\text { Ascorbic acid } \\
\mathrm{mg}\end{array}$ \\
\hline $0.9 \pm 0.4$ & $1.2 \pm 0.5$ & $19.7 \pm 7.6$ & $1.2 \pm 0.5$ & $149 \pm 59$ & $3.6 \pm 1.9$ & $113 \pm 96$ \\
\hline $1.3 \pm 0.3$ & $1.8 \pm 0.6$ & $24.3 \pm 6.6$ & $1.4 \pm 0.4$ & $298 \pm 100$ & $5.2 \pm 1.9$ & $103 \pm 63$ \\
\hline $1.4 \pm 0.6$ & $1.7 \pm 0.7$ & $23.8 \pm 7.7$ & $1.8 \pm 0.9$ & $233 \pm 116$ & $4.4 \pm 1.9$ & $119 \pm 108$ \\
\hline $2.0 \pm 1.1$ & $2.3 \pm 1.3$ & $34.3 \pm 16.2$ & $2.5 \pm 1.6$ & $328 \pm 183$ & $5.5 \pm 2.6$ & $172 \pm 102$ \\
\hline $2.3 \pm 1.4$ & $2.6 \pm 1.6$ & $40.4 \pm 20.1$ & $3.1 \pm 2.1$ & $380 \pm 259$ & $6.4 \pm 3.0$ & $197 \pm 135$ \\
\hline $1.8 \pm 0.7$ & $2.1 \pm 0.9$ & $20.7 \pm 7.5$ & $2.4 \pm 0.9$ & $271 \pm 109$ & $4.9 \pm 2.2$ & $94 \pm 72$ \\
\hline $1.2 \pm 0.4$ & $1.6 \pm 0.5$ & $34.5 \pm 9.6$ & $2.2 \pm 0.7$ & $290 \pm 106$ & n.a. & $101 \pm 106$ \\
\hline $1.0 \pm 0.3$ & $1.4 \pm 0.4$ & $30.8 \pm 7.8$ & $1.8 \pm 0.5$ & $150.6 \pm 58.1$ & $3.2 \pm 2.5$ & $99.3 \pm 78.9$ \\
\hline $1.4 \pm 0.7$ & $1.7 \pm 0.9$ & n.a. & n.a. & n.a. & n.a. & $106 \pm 74$ \\
\hline $1.3 \pm 0.5$ & $1.7 \pm 0.7$ & n.a. & $1.8 \pm 0.8$ & $250 \pm 96$ & $3.8 \pm 4.5$ & $81 \pm 79$ \\
\hline $2.2 \pm 0.7$ & $2.9 \pm 1.0$ & $28.3 \pm 9.5$ & $2.8 \pm 1.0$ & $428 \pm 183$ & $11.8 \pm 7.8$ & $161 \pm 94$ \\
\hline $1.6 \pm 0.3$ & $1.9 \pm 0.4$ & $21.1 \pm 4.6$ & $2.0 \pm 0.4$ & $236 \pm 42$ & $4.7 \pm 0.9$ & $73 \pm 34$ \\
\hline $1.4 \pm 1.2$ & $1.8 \pm 1.3$ & $27.7 \pm 7.4$ & $1.8 \pm 1.2$ & $191 \pm 69$ & $4.9 \pm 3.4$ & $82 \pm 120$ \\
\hline $1.2 \pm 0.6$ & $1.6 \pm 0.6$ & n.a. & $1.5 \pm 0.5$ & $170 \pm 56$ & n.a. & $76 \pm 46$ \\
\hline $1.6 \pm 0.7$ & $1.7 \pm 0.8$ & $8.7 \pm 5.1^{7}$ & $2.0 \pm 0.8$ & $202.3 \pm 81$ & $4.4 \pm 2.2$ & $75 \pm 52$ \\
\hline $0.9-2.3$ & $1.2-2.9$ & $8.7-40.4$ & $1.2-2.8$ & $149-428$ & $3.2-11.8$ & 73-197 \\
\hline $1.2 / 1.4$ & $1.4 / 1.6$ & $15 / 18$ & $1.0 / 1.4$ & 400 & $2.0 / 3.0$ & $90 / 100$ \\
\hline $0.8 \pm 0.3$ & $1.1 \pm 0.5$ & $16.9 \pm 6.2$ & $1.1 \pm 0.5$ & $138 \pm 53$ & $2.9 \pm 1.4$ & $113 \pm 67$ \\
\hline $1.0 \pm 0.3$ & $1.4 \pm 0.5$ & $19.4 \pm 5.6$ & $1.1 \pm 0.3$ & $238 \pm 70$ & $4.0 \pm 1.8$ & $94 \pm 47$ \\
\hline $1.3 \pm 0.5$ & $1.5 \pm 0.6$ & $21.6 \pm 5.7$ & $1.6 \pm 0.6$ & $219 \pm 86$ & $3.8 \pm 1.6$ & $110 \pm 61$ \\
\hline $1.9 \pm 1.3$ & $2.3 \pm 1.6$ & $32.5 \pm 18.4$ & $2.5 \pm 1.9$ & $360 \pm 234$ & $4.9 \pm 2.8$ & $222 \pm 243$ \\
\hline $1.8 \pm 1.1$ & $2.1 \pm 1.3$ & $31.1 \pm 13.8$ & $2.4 \pm 1.4$ & $326 \pm 164$ & $4.8 \pm 2.3$ & $201 \pm 12$ \\
\hline $0.9 \pm 0.3$ & $1.2 \pm 0.4$ & $26.3 \pm 5.9$ & $1.6 \pm 0.5$ & $140 \pm 65$ & $2.2 \pm 1.1$ & $94.3 \pm 70.0$ \\
\hline $1.7 \pm 3.4$ & $1.9 \pm 3.4$ & $17.1 \pm 6.9$ & $2.3 \pm 3.5$ & $217 \pm 99$ & $4.1 \pm 2.6$ & $96 \pm 77$ \\
\hline $1.0 \pm 0.2$ & $1.3 \pm 0.3$ & $28.8 \pm 6.0$ & $1.8 \pm 0.3$ & $234 \pm 55$ & n.a. & $85 \pm 36$ \\
\hline $1.2 \pm 0.9$ & $1.5 \pm 1.1$ & n.a. & n.a. & n.a. & n.a. & $103 \pm 81$ \\
\hline $1.1 \pm 0.4$ & $1.4 \pm 0.6$ & n.a. & $1.6 \pm 0.7$ & $224 \pm 93$ & $2.9 \pm 3.5$ & $88 \pm 87$ \\
\hline $2.1 \pm 0.7$ & $2.8 \pm 1.0$ & $26.6 \pm 8.9$ & $2.7 \pm 0.9$ & $412 \pm 177$ & $11.1 \pm 6.8$ & $170 \pm 96$ \\
\hline
\end{tabular}


Table 7.16. Continued

\begin{tabular}{lllllll}
\hline & $\begin{array}{l}\text { Age } \\
\text { years }\end{array}$ & $\mathrm{n}$ & $\begin{array}{l}\text { Vitamin } \mathrm{A}^{1} \\
\mathrm{~g}\end{array}$ & $\begin{array}{l}\beta \text {-Carotene } \\
\mathrm{mg}\end{array}$ & $\begin{array}{l}\text { Vitamin } \mathrm{D} \\
\mu \mathrm{g}\end{array}$ & $\begin{array}{l}\text { Vitamin } \mathrm{E}^{2} \\
\mathrm{mg}\end{array}$ \\
\hline Spain & $10-14$ & 53 & $0.5 \pm 0.1$ & $1.1 \pm 0.4$ & $1.6 \pm 0.5$ & $7.6 \pm 1.4$ \\
\hline Sweden & $10-14$ & 517 & $0.6 \pm 0.4$ & $1.1 \pm 1.1$ & $4.4 \pm 2.6$ & $5.6 \pm 2.1$ \\
\hline The Netherlands & $10-14$ & 199 & $0.7 \pm 0.6$ & n.a. & $3.2 \pm 1.4$ & $12.0 \pm 5.2$ \\
\hline United Kingdom & $2-15$ & 364 & $0.6 \pm 0.6$ & $1.5 \pm 1.3$ & $1.9 \pm 1.1$ & $8.0 \pm 3.7$ \\
\hline $\begin{array}{l}\text { All countries } \\
\text { (min.-max.) }\end{array}$ & & & $0.3-2.3$ & $1.1-5.2$ & $1.2-4.5$ & $5.6-18.1$ \\
\hline $\begin{array}{l}\text { Reference values } \\
5\end{array}$ & & & $0.9 / 1.0$ & n.a. & 5 & $11 / 12$ \\
\hline
\end{tabular}

${ }^{1}$ Retinol equivalent $\left(=1 \mathrm{mg}\right.$ retinol $=6 \mathrm{mg}$ all-trans- $\beta$-carotene $=12 \mathrm{mg}$ other carotenoids); ${ }^{2}$ RRR- $\mathrm{a}$ tocopherol equivalent $(=\mathrm{mg}$ a-tocopherol $+\mathrm{mg} \beta$-tocopherol $\times 0.5+\mathrm{mg} \gamma$-tocopherol $\times 0.25+\mathrm{mg}$ a-tocotrienol $\times 0.33) ;{ }^{3}$ niacin equivalent $\left(=1 \mathrm{mg}\right.$ niacin $=60 \mathrm{mg}$ tryptophan); ${ }^{4}$ folate equivalent $(1 \mu \mathrm{g}$ food folate $=0.5 \mu \mathrm{g}$ folic acid (PGA) $=0.6 \mu \mathrm{g}$ folic acid taken with meals); ${ }^{5} \mathrm{D}-\mathrm{A}-\mathrm{CH}, 2000$, reference values refer to age group $10-12$ years and $13-14$ years; ${ }^{6}$ alpha-tocopherol only; ${ }^{7}$ niacin; n.a. $=$ not available.

Table 7.17. Intake of selected vitamins (min.-max.) in children aged 4-6 years in the four regions

\begin{tabular}{lllll}
\hline Region/sex & $\begin{array}{l}\text { Vitamin } \mathrm{B}_{6} \\
\mathrm{mg}\end{array}$ & $\begin{array}{l}\text { Folate } \\
\mu \mathrm{g}^{5}\end{array}$ & $\begin{array}{l}\text { Cobalamin } \\
\mathrm{gg}\end{array}$ & $\begin{array}{l}\text { Vitamin D } \\
\mu \mathrm{g}\end{array}$ \\
\hline North $^{1}$ & & & \\
\hline Male & $1.1-1.6^{6}$ & $135-256^{6}$ & $4.2-5.3$ & $2.3-6.8$ \\
\hline Female & $1.0-1.5^{6}$ & $132-235^{6}$ & $4.2-4.7$ & $2.0-6.5$ \\
\hline South & & & \\
\hline Male & 1.6 & 198 & n.a. & 2.3 \\
\hline Female & 1.6 & 199 & n.a. & 2.2 \\
\hline Central and East $^{3}$ & & & & \\
\hline Male & $1.5-1.8$ & $190-214$ & $4.2-5.0$ & $1.8-2.3$ \\
\hline Female & $1.2-1.9$ & $164-190$ & $2.9-5.0$ & $1.5-2.3$ \\
\hline West ${ }^{4}$ & & & & \\
\hline Male & $1.3-1.8$ & $120-225$ & $2.9-4.0$ & $2.2-2.4$ \\
\hline Female & $1.2-1.7$ & $109-196$ & $2.6-4.3$ & 1.9 \\
\hline
\end{tabular}

${ }^{1}$ North: SE, NO, FI, DK; ${ }^{2}$ South: IT; ${ }^{3}$ Central and East: CZ, DE, PL; ${ }^{4}$ West: NL, IR; ${ }^{5}$ folate equivalent $(1 \mu \mathrm{g}$ food folate $=0.5 \mu \mathrm{g}$ folic acid $(P G A)=0.6 \mu \mathrm{g}$ folic acid taken with meals); ${ }^{6}$ only $\mathrm{SE}, \mathrm{FI}$ and DK; n.a. $=$ not available. 


\begin{tabular}{|c|c|c|c|c|c|c|}
\hline $\begin{array}{l}\text { Thiamine } \\
\mathrm{mg}\end{array}$ & $\begin{array}{l}\text { Riboflavin } \\
\mathrm{mg}\end{array}$ & $\begin{array}{l}\mathrm{Niacin}^{3} \\
\mathrm{mg}\end{array}$ & $\begin{array}{l}\text { Vitamin } \mathrm{B}_{6} \\
\mathrm{mg}\end{array}$ & $\begin{array}{l}\text { Folate }^{4} \\
\mu \mathrm{g}\end{array}$ & $\begin{array}{l}\text { Cobalamin } \\
\mu \mathrm{g}\end{array}$ & $\begin{array}{l}\text { Ascorbic acid } \\
\mathrm{mg}\end{array}$ \\
\hline $1.3 \pm 0.2$ & $1.6 \pm 0.2$ & $18.8 \pm 3.7$ & $1.9 \pm 0.4$ & $207 \pm 39$ & $3.9 \pm 0.6$ & $77 \pm 37$ \\
\hline $1.2 \pm 0.4$ & $1.4 \pm 0.6$ & $23.7 \pm 6.7$ & $1.5 \pm 0.6$ & $174 \pm 61$ & $4.0 \pm 1.8$ & $78 \pm 79$ \\
\hline $1.0 \pm 0.4$ & $1.4 \pm 0.5$ & n.a. & $1.3 \pm 0.4$ & $156 \pm 57$ & n.a. & $77 \pm 60$ \\
\hline $1.4 \pm 0.5$ & $1.4 \pm 0.6$ & $6.9 \pm 3.7^{7}$ & $1.9 \pm 0.7$ & $186 \pm 69$ & $3.9 \pm 1.1$ & $81 \pm 56$ \\
\hline $0.8-1.9$ & $1.1-2.8$ & $6.9-32.5$ & $1.1-2.7$ & $140-360$ & $2.2-11.1$ & $77-222$ \\
\hline $1.0 / 1.1$ & $1.2 / 1.3$ & $13 / 15$ & $1.0 / 1.4$ & 400 & $2.0 / 3.0$ & $90 / 100$ \\
\hline
\end{tabular}

Table 7.18. Intake of selected vitamins (min.-max.) in children aged 7-9 years in the four regions

\begin{tabular}{lllll}
\hline Region/sex & $\begin{array}{l}\text { Vitamin } \mathrm{B}_{6} \\
\mathrm{mg}\end{array}$ & $\begin{array}{l}\text { Folate } \\
\mu \mathrm{g}^{5}\end{array}$ & $\begin{array}{l}\text { Cobalamin } \\
\mu \mathrm{g}\end{array}$ & $\begin{array}{l}\text { Vitamin D } \\
\mu \mathrm{g}\end{array}$ \\
\hline North $^{1}$ & & & & \\
\hline Male & $1.3-2.5^{6}$ & $204-290^{6}$ & $5.4-5.5^{6}$ & $2.5-6.4$ \\
\hline Female & $1.2-1.6^{6}$ & $187-264^{6}$ & $4.7-4.8^{6}$ & $2.2-5.1$ \\
\hline South & & & & \\
\hline Male & 1.8 & 242 & n.a. & 2.8 \\
\hline Female & 1.7 & 211 & n.a. & 2.1 \\
\hline Central and East $^{3}$ & & & & \\
\hline Male & $1.2-1.8$ & $154-229$ & $3.6-5.3$ & $1.5-2.8$ \\
\hline Female & $1.1-1.8$ & $145-212$ & $2.8-5.3$ & $1.5-2.7$ \\
\hline West & & & & \\
\hline Male & $1.3-2.2$ & $144-256$ & $4.8^{7}$ & $2.2-2.9$ \\
\hline Female & $1.2-1.9$ & $133-226$ & $4.2^{7}$ & $2.4-2.8$ \\
\hline
\end{tabular}

${ }^{1}$ North: SE, NO, DK; ${ }^{2}$ South: $\mathrm{IT}^{3}{ }^{3}$ Central and East: AT, CZ, DE, PL; ${ }^{4}$ West: NL, IR; ${ }^{5}$ folate equivalent $(1 \mu \mathrm{g}$ food folate $=0.5 \mu \mathrm{g}$ folic acid $(\mathrm{PGA})=0.6 \mu \mathrm{g}$ folic acid taken with meals); ${ }^{6}$ only SE and DK; ${ }^{7}$ only IR; n.a. $=$ not available. 
Table 7.19. Intake of selected vitamins (min.-max.) in children aged 10-14 years in the four regions

\begin{tabular}{|c|c|c|c|c|}
\hline Region/sex & $\begin{array}{l}\text { Vitamin } B_{6} \\
\mathrm{mg}\end{array}$ & $\begin{array}{l}\text { Folate } \\
\mu \mathrm{g}^{5}\end{array}$ & $\begin{array}{l}\text { Cobalamin } \\
\mu \mathrm{g}\end{array}$ & $\begin{array}{l}\text { Vitamin D } \\
\mu \mathrm{g}\end{array}$ \\
\hline \multicolumn{5}{|l|}{ North $^{1}$} \\
\hline Male & $1.4-1.8^{6}$ & $191-298^{6}$ & $4.9-5.2^{6}$ & $2.6-4.8$ \\
\hline Female & $1.1-1.5^{6}$ & $174-238^{6}$ & $4.0^{6}$ & $2.2-4.4$ \\
\hline \multicolumn{5}{|l|}{ South ${ }^{2}$} \\
\hline Male & $2.0-2.8$ & $236-428$ & $4.7-11.8^{7}$ & $1.7-4.8$ \\
\hline Female & $1.8-2.7$ & $207-412$ & $3.9-11.1^{7}$ & $1.6-4.5$ \\
\hline \multicolumn{5}{|c|}{ Central and East ${ }^{3}$} \\
\hline Male & $1.2-3.1$ & 149-380 & $3.2-6.4$ & $1.5-3.9$ \\
\hline Female & $1.1-2.5$ & $138-360$ & $2.2-4.9$ & $1.2-2.9$ \\
\hline \multicolumn{5}{|l|}{ West ${ }^{4}$} \\
\hline Male & $1.5-2.4$ & $170-271$ & $4.4-4.9^{8}$ & $2.1-3.8$ \\
\hline Female & $1.3-2.3$ & $156-217$ & $3.9-4.1^{8}$ & $1.9-3.2$ \\
\hline \multicolumn{5}{|c|}{$\begin{array}{l}{ }^{1} \text { North: SE, NO, DK; }{ }^{2} \text { South: IT, PT, ES; }{ }^{3} \text { Central and East: AT, DE, PL; }{ }^{4} \text { West: UK, NL, IR, } \\
\text { HU; }{ }^{5} \text { Folate equivalent }(1 \mu \mathrm{g} \text { food folate }=0.5 \mu \mathrm{g} \text { folic acid }(\mathrm{PGA})=0.6 \mu \mathrm{g} \text { folic acid } \\
\text { taken with meals); }{ }^{6} \text { only SE and } \mathrm{DK} ;{ }^{7} \text { only PT and } \mathrm{ES} ;{ }^{8} \text { only UK and IR; n.a. = not } \\
\text { available. }\end{array}$} \\
\hline
\end{tabular}

European countries. No data on iodine intake were available for the South. Intake levels in 10- to 14-year-old children in the Central and East region showed an inhomogeneous picture. For example, the minimum as well as the maximum intake of calcium (593-1,381 mg/day) and phosphorus (807-1,615 mg/day) can be observed in this region. The intake levels of selenium showed a wide rage but kept within the recommendation except for central and east Europe where the maximum exceeded the recommended range in 4- to 9-year-old children [D-A-CH, 2000].

\subsection{Energy and Nutrient Intake in European Adolescents}

\section{Background}

Although about half of the participating countries had data on energy and nutrient intake of European adolescents, this population group was the group with the fewest 
data available. 13 of the 25 participating countries had data on energy, macronutrient and mineral intake and 12 had data on vitamin intake. The methods which were used to assess the energy and nutrient intake, the periods of data collection and sample sizes are shown in table 7.26.

\section{Energy and Macronutrients}

\section{Comparison of Countries}

The energy intake was between 9.5 and $14.5 \mathrm{MJ} /$ day in male adolescents and between 6.8 and 9.7 MJ/day in female adolescents. Despite the high prevalence of overweight and obesity (cf. chapter 8 ) the intake of energy was in general below the reference intake values of 13.0 (males) and $10.5 \mathrm{MJ} /$ day (females) [D-A-CH, 2000]. It must be noted, that these reference values refer to a physical activity level (PAL) of 1.75, which was on average probably not achieved.

The share of protein in total energy intake was between 12.4 and $17.8 \% \mathrm{E}$ in male and between 12.0 and $18.0 \% \mathrm{E}$ in female adolescents and was therefore within or slightly above the recommended range of the WHO [WHO, 2003] in the participating countries.

The intake of carbohydrates $(\% \mathrm{E})$ was in general within the recommendation of the WHO (WHO, 2007). Male adolescents from Denmark, Norway, Poland, Slovenia, and The Netherlands as well as female adolescents from Belgium, Denmark, Germany, Norway, Poland, Slovenia, The Netherlands and the United Kingdom met the recommendation. The intake of sucrose was above the recommendation of less than $10 \% \mathrm{E}$ [WHO, 2003] in every country. The intake of dietary fibers was between 13.7 and $33.0 \mathrm{~g} /$ day in male and between 10.2 and $27.0 \mathrm{~g} /$ day in female adolescents. In general, the intake was lower than the Eurodiet recommendation [Eurodiet, 2000] of ingesting more than $25 \mathrm{~g}$ dietary fibers per day. Only male German, Norwegian and Polish adolescents as well as male and female Slovenian adolescents met the recommendation.

The intake of fat was between 28.0 and $40.4 \% \mathrm{E}$ in male and between 25.5 and $41.9 \% \mathrm{E}$ in female adolescents. The intake of female adolescents was in general lower than that of male adolescents. The intake of saturated fatty acids was in every country and in both sexes above the recommendation [WHO, 2003], whereas the intake of polyunsaturated fatty acids was in every country except for male adolescents from Belgium, The Netherlands and Poland and female adolescents from Belgium, The Netherlands, Slovenia and Spain below the recommendation [WHO, 2009b]. The cholesterol intake was between 219 and $566 \mathrm{mg} /$ day in male adolescents and between 183 and $340 \mathrm{mg} /$ day in female adolescents (cf. table 7.27).

\section{Comparison of Regions}

Table 7.28 shows the intake of energy and macronutrients (min.-max.) in the four regions. In general the intake of energy was higher in the Central and East region (CE) than in the other regions. The share of protein and fat in total energy intake 
Table 7.20. Mineral intake (mean \pm SD) in children aged 4-6 years of European countries (male and female)

$\begin{array}{llllll}\begin{array}{l}\text { Age } \\ \text { years }\end{array} & \mathrm{n} & \text { Sodium } & \text { Potassium } & \text { Calcium } & \text { Phosphorus } \\ \mathrm{g} & \mathrm{g} & \mathrm{mg} & \mathrm{mg}\end{array}$

\section{Male}

\begin{tabular}{lllllll}
\hline Czech Republic & $4-6$ & 641 & $2.5 \pm 0.9$ & $2.7 \pm 1.0$ & $809 \pm 240$ & $1,183 \pm 285$ \\
\hline Denmark & $4-6$ & 117 & $3.0 \pm 0.8$ & $2.5 \pm 0.6$ & $1008 \pm 273$ & $1,260 \pm 289$ \\
\hline Finland & 4 & 307 & $2.0 \pm 0.5$ & $2.4 \pm 0.6$ & $983 \pm 306$ & $1,116 \pm 274$ \\
\hline France & 6 & 364 & $2.3 \pm 0.5$ & $2.8 \pm 0.6$ & $1,103 \pm 302$ & $1,284 \pm 271$ \\
\hline Germany & $4-6$ & 164 & $2.1 \pm 0.1$ & n.a. & $813 \pm 21$ & n.a. \\
\hline Greece & $<7$ & 102 & $1.8 \pm 0.6$ & $2.3 \pm 0.6$ & $900 \pm 200$ & $1,000 \pm 200$ \\
\hline Ireland & $4-5$ & 745 & n.a. & n.a. & 1,024 & n.a. \\
\hline Italy & $5-6$ & 72 & $1.9 \pm 0.4$ & $2.0 \pm 0.4$ & $837 \pm 257$ & $944 \pm 236$ \\
\hline Norway & $4-6$ & 21 & $3.4 \pm 1.5$ & $2.4 \pm 0.8$ & $793 \pm 212$ & $1,085 \pm 280$ \\
\hline Poland & 4 & 206 & n.a. & n.a. & $687 \pm 217$ & n.a. \\
\hline Sweden & $4-6$ & 82 & $2.5 \pm 0.9$ & $2.5 \pm 0.7$ & $604 \pm 301$ & $882 \pm 273$ \\
\hline The Netherlands & $4-6$ & 302 & $2.1 \pm 0.5$ & $2.4 \pm 0.6$ & $889 \pm 288$ & $1,042 \pm 262$ \\
\hline All countries (min.-max.) & $4-6$ & 327 & n.a. & $2.3 \pm 0.4$ & $856 \pm 270$ & $1,067 \pm 239$ \\
\hline Female & & & $1.8-3.4$ & $2.0-2.8$ & $604-1,103$ & $882-1,284$ \\
\hline
\end{tabular}

\section{Female}

\begin{tabular}{lllllll}
\hline Czech Republic & $4-6$ & 446 & $2.5 \pm 0.9$ & $2.7 \pm 1.0$ & $809 \pm 240$ & $1,183 \pm 285$ \\
\hline Denmark & $4-6$ & 117 & $2.7 \pm 0.7$ & $2.3 \pm 0.6$ & $886 \pm 301$ & $1,115 \pm 307$ \\
\hline Finland & 4 & 307 & $1.8 \pm 0.5$ & $2.3 \pm 0.5$ & $930 \pm 255$ & $1,052 \pm 237$ \\
\hline France & 6 & 349 & $2.0 \pm 0.6$ & $2.5 \pm 0.6$ & $991 \pm 286$ & $1,137 \pm 272$ \\
\hline Germany & $4-6$ & 162 & $2.1 \pm 0.1$ & n.a. & $783 \pm 20$ & n.a. \\
\hline Greece & $<7$ & 102 & $1.7 \pm 0.5$ & $2.0 \pm 0.5$ & $800 \pm 200$ & $900 \pm 200$ \\
\hline Ireland & $4-5$ & 745 & n.a. & n.a. & 1024 & n.a. \\
\hline Italy & $5-6$ & 72 & $1.8 \pm 1.5$ & $1.9 \pm 0.5$ & $794 \pm 214$ & $904 \pm 206$ \\
\hline Norway & $4-6$ & 21 & $3.4 \pm 1.1$ & $2.3 \pm 0.4$ & $701 \pm 180$ & $1,063 \pm 211$ \\
\hline Poland & 4 & 185 & n.a. & n.a. & $673 \pm 212$ & n.a. \\
\hline Sweden & $4-6$ & 82 & $2.4 \pm 0.9$ & $2.4 \pm 0.9$ & $606 \pm 309$ & $877 \pm 324$ \\
\hline The Netherlands & $4-6$ & 302 & $2.0 \pm 0.5$ & $2.2 \pm 0.6$ & $819 \pm 257$ & $966 \pm 228$ \\
\hline All countries (min.-max.) & $4-6$ & 312 & n.a. & $2.0 \pm 0.4$ & $750 \pm 209$ & $950 \pm 195$ \\
\hline Reference values ${ }^{3}$ & & & $1.7-3.4$ & $1.9-2.7$ & $606-1024$ & $900-1,183$ \\
\hline
\end{tabular}

${ }^{1}$ Sum of males and females; ${ }^{2}$ iodised salt was not taken into account in each country; ${ }^{3} \mathrm{D}-\mathrm{A}-\mathrm{CH}, 2000 ;{ }^{4} \mathrm{WHO}$, 2003; n.a. = not available. 


\begin{tabular}{|c|c|c|c|c|c|c|}
\hline $\begin{array}{l}\text { Magnesium } \\
\text { mg }\end{array}$ & $\begin{array}{l}\text { Iron } \\
\text { mg }\end{array}$ & $\begin{array}{l}\text { Zinc } \\
\text { mg }\end{array}$ & $\begin{array}{l}\text { lodine } \\
\mu g^{2}\end{array}$ & $\begin{array}{l}\text { Copper } \\
\text { mg }\end{array}$ & $\begin{array}{l}\text { Manganese } \\
\mathrm{mg}\end{array}$ & $\begin{array}{l}\text { Selenium } \\
\mu \mathrm{g}\end{array}$ \\
\hline $267 \pm 70$ & $10.6 \pm 3.6$ & $7.2 \pm 1.8$ & $69 \pm 31$ & $1.3 \pm 3.3$ & n.a. & $61 \pm 41$ \\
\hline $265 \pm 63$ & $7.8 \pm 1.9$ & $8.8 \pm 2.0$ & $173 \pm 48$ & n.a. & n.a. & $31 \pm 8$ \\
\hline $211 \pm 52$ & $8.0 \pm 3.6$ & $8.0 \pm 2.7$ & $196 \pm 57$ & n.a. & n.a. & $39 \pm 12$ \\
\hline $246 \pm 67$ & $8.6 \pm 2.8$ & $9.1 \pm 2.8$ & $223 \pm 53$ & n.a. & n.a. & $45 \pm 13$ \\
\hline n.a. & n.a. & n.a. & n.a. & n.a. & n.a. & n.a. \\
\hline $265 \pm 67$ & $10.0 \pm 2.9$ & $7.7 \pm 1.6$ & $77 \pm 28$ & n.a. & n.a. & n.a. \\
\hline 188.0 & 10.0 & 8.4 & n.a. & 0.7 & n.a. & n.a. \\
\hline $171 \pm 41$ & $8.7 \pm 3.6$ & $6.0 \pm 2.5$ & $142 \pm 75$ & $0.7 \pm 0.4$ & $1.4 \pm 0.5$ & $23 \pm 7$ \\
\hline $171 \pm 33$ & $8.8 \pm 2.4$ & $9.2 \pm 2.6$ & n.a. & $1.0 \pm 0.4$ & n.a. & $29 \pm 10$ \\
\hline n.a. & $7.8 \pm 3.8$ & n.a. & n.a. & n.a. & n.a. & n.a. \\
\hline $216 \pm 66$ & $8.0 \pm 3.3$ & $6.8 \pm 2.0$ & $87 \pm 37$ & $0.9 \pm 0.3$ & $3.3 \pm 1.3$ & $27 \pm 9$ \\
\hline $216 \pm 51$ & $8.3 \pm 3.4$ & $7.8 \pm 2.3$ & n.a. & n.a. & n.a. & $26 \pm 9$ \\
\hline $219 \pm 45$ & $7.3 \pm 1.7$ & $6.0 \pm 1.4$ & n.a. & $0.8 \pm 0.2$ & n.a. & $27 \pm 6$ \\
\hline $171-267$ & $7.3-10.6$ & $6.0-9.2$ & $69-223$ & $0.7-2.2$ & $1.4-3.3$ & $23-61$ \\
\hline $267 \pm 70$ & $10.6 \pm 3.6$ & $7.2 \pm 1.8$ & $69 \pm 31$ & $1.3 \pm 3.3$ & n.a. & $61 \pm 41$ \\
\hline $235 \pm 55$ & $6.9 \pm 1.7$ & $7.7 \pm 1.9$ & $151 \pm 49$ & n.a. & n.a. & $28 \pm 8$ \\
\hline $199 \pm 41$ & $7.0 \pm 2.7$ & $7.2 \pm 2.0$ & $183 \pm 47$ & n.a. & n.a. & $35 \pm 10$ \\
\hline $216 \pm 50$ & $7.7 \pm 3.3$ & $8.2 \pm 3.1$ & $197 \pm 55$ & n.a. & n.a. & $39 \pm 11$ \\
\hline n.a. & n.a. & n.a. & n.a. & n.a. & n.a. & n.a. \\
\hline $246 \pm 60$ & $8.8 \pm 2.2$ & $7.3 \pm 1.9$ & $70 \pm 23$ & n.a. & n.a. & n.a. \\
\hline 188 & 10.0 & 8.4 & n.a. & 0.7 & n.a. & n.a. \\
\hline $166 \pm 38$ & $7.8 \pm 2.9$ & $5.6 \pm 1.5$ & $137 \pm 65$ & $0.7 \pm 0.6$ & $1.3 \pm 0.5$ & $23 \pm 8$ \\
\hline $164 \pm 40$ & $10.1 \pm 3.6$ & $8.9 \pm 2.1$ & n.a. & $1.0 \pm 0.3$ & n.a. & $28 \pm 7$ \\
\hline n.a. & $7.4 \pm 3.9$ & n.a. & n.a. & n.a. & n.a. & n.a. \\
\hline $205 \pm 73$ & $7.4 \pm 3.6$ & $6.4 \pm 2.3$ & $82 \pm 32$ & $0.9 \pm 0.4$ & $3.0 \pm 1.2$ & $29 \pm 27$ \\
\hline $199 \pm 45$ & $7.7 \pm 2.8$ & $7.1 \pm 1.9$ & n.a. & n.a. & n.a. & $24 \pm 9$ \\
\hline $191 \pm 39$ & $6.8 \pm 1.9$ & $5.3 \pm 1.0$ & n.a. & $0.7 \pm 0.1$ & n.a. & $24 \pm 5$ \\
\hline $166-267$ & $6.8-10.6$ & $5.3-8.9$ & 70-198 & $0.7-2.0$ & $1.3-3.0$ & 24-61 \\
\hline 120 & 8 & 5 & 120 & $0.5-1.0$ & $1.5-2.0$ & $15-45$ \\
\hline
\end{tabular}


Table 7.21. Mineral intake (mean \pm SD) in children aged 7-9 years of European countries (male and female)

\begin{tabular}{|c|c|c|c|c|c|c|}
\hline & $\begin{array}{l}\text { Age } \\
\text { years }\end{array}$ & $\mathrm{n}$ & $\begin{array}{l}\text { Sodium } \\
\mathrm{g}\end{array}$ & $\begin{array}{l}\text { Potassium } \\
\mathrm{g}\end{array}$ & $\begin{array}{l}\text { Calcium } \\
\mathrm{mg}\end{array}$ & $\begin{array}{l}\text { Phosphorus } \\
\mathrm{mg}\end{array}$ \\
\hline \multicolumn{7}{|l|}{ Male } \\
\hline Austria & $7-9$ & 146 & $2.8 \pm 1.0$ & $1.9 \pm 0.7$ & $732 \pm 294$ & $960 \pm 306$ \\
\hline Czech Republic $^{1}$ & $7-9$ & 940 & $3.0 \pm 2.4$ & $2.9 \pm 1.2$ & $882 \pm 280$ & $1,295 \pm 440$ \\
\hline Denmark & $7-9$ & 139 & $3.5 \pm 1.0$ & $3.0 \pm 0.9$ & $1,207 \pm 388$ & $1,455 \pm 403$ \\
\hline France & $7-9$ & 160 & $2.6 \pm 0.1$ & n.a. & $855 \pm 22$ & n.a. \\
\hline Germany & $7-9$ & 321 & $2.2 \pm 0.6$ & $2.4 \pm 0.6$ & $900 \pm 300$ & $1,100 \pm 300$ \\
\hline Ireland & $7-9$ & 110 & $2.3 \pm 0.8$ & $2.4 \pm 0.6$ & $943 \pm 325$ & $1,098 \pm 309$ \\
\hline Italy & $7-9$ & 29 & $4.2 \pm 1.4$ & $2.7 \pm 0.7$ & $904 \pm 232$ & $1,253 \pm 189$ \\
\hline Norway & 9 & 402 & n.a. & n.a. & $917 \pm 338$ & n.a. \\
\hline Poland & $7-9$ & 101 & $3.0 \pm 1.1$ & $2.7 \pm 0.9$ & $628 \pm 356$ & $1031 \pm 370$ \\
\hline Portugal & $7-9$ & 1,541 & n.a. & n.a. & $1,174 \pm 430$ & n.a. \\
\hline Sweden & $7-9$ & 444 & $2.9 \pm 0.7$ & $2.9 \pm 0.7$ & $1,024 \pm 352$ & $1,300 \pm 329$ \\
\hline The Netherlands & $7-9$ & 104 & n.a. & $2.8 \pm 0.8$ & $914 \pm 324$ & $1,273 \pm 303$ \\
\hline All countries (min.-max.) & & & $2.2-4.2$ & $1.9-3.0$ & $732-1,207$ & $960-1,455$ \\
\hline \multicolumn{7}{|l|}{ Female } \\
\hline Austria & $7-9$ & 146 & $2.5 \pm 0.8$ & $1.8 \pm 0.6$ & $631 \pm 249$ & $851 \pm 262$ \\
\hline Czech Republic $^{1}$ & $7-9$ & 765 & $3.0 \pm 2.4$ & $2.9 \pm 1.2$ & $882 \pm 280$ & $1,295 \pm 440$ \\
\hline Denmark & $7-9$ & 139 & $3.0 \pm 0.8$ & $2.7 \pm 0.7$ & $1,094 \pm 366$ & $1,288 \pm 334$ \\
\hline France & $7-9$ & 144 & $2.4 \pm 0.1$ & n.a. & $799 \pm 23$ & n.a. \\
\hline Germany & $7-9$ & 321 & $1.9 \pm 0.6$ & $2.2 \pm 0.7$ & $800 \pm 300$ & $1,000 \pm 300$ \\
\hline Ireland & $7-9$ & 110 & $2.1 \pm 0.6$ & $2.1 \pm 0.5$ & $857 \pm 283$ & $991 \pm 254$ \\
\hline Italy & $7-9$ & 29 & $3.7 \pm 0.9$ & $2.4 \pm 0.5$ & $715 \pm 170$ & $1,091 \pm 244$ \\
\hline Norway & 9 & 408 & n.a. & n.a. & $756 \pm 273$ & n.a. \\
\hline Poland & $7-9$ & 101 & $2.8 \pm 1.0$ & $2.5 \pm 0.9$ & $548 \pm 283$ & $892 \pm 295$ \\
\hline Portugal & $7-9$ & 1,053 & n.a. & n.a. & $1126 \pm 419$ & n.a. \\
\hline Sweden & $7-9$ & 444 & $2.6 \pm 0.6$ & $2.6 \pm 0.7$ & $894 \pm 306$ & $1,155 \pm 288$ \\
\hline The Netherlands & $7-9$ & 134 & n.a. & $2.6 \pm 0.6$ & $901 \pm 296$ & $1,185 \pm 283$ \\
\hline All countries (min.-max.) & & & $1.9-3.7$ & $1.8-2.9$ & $631-1126$ & $851-1,295$ \\
\hline Reference values ${ }^{3}$ & & & $0.46 /<2^{4}$ & 1.6 & 900 & 800 \\
\hline
\end{tabular}

${ }^{1}$ Male and female; ${ }^{2}$ iodised salt was not taken into account in each country; ${ }^{3} \mathrm{D}-\mathrm{A}-\mathrm{CH}, 2000 ;{ }^{4} \mathrm{WHO}, 2003$; n.a. = not available. 


\begin{tabular}{|c|c|c|c|c|c|c|}
\hline $\begin{array}{l}\text { Magnesium } \\
\text { mg }\end{array}$ & $\begin{array}{l}\text { Iron } \\
\text { mg }\end{array}$ & $\begin{array}{l}\text { Zinc } \\
\text { mg }\end{array}$ & $\begin{array}{l}\text { lodine } \\
\mu g^{2}\end{array}$ & $\begin{array}{l}\text { Copper } \\
\mathrm{mg}\end{array}$ & $\begin{array}{l}\text { Manganese } \\
\mathrm{mg}\end{array}$ & $\begin{array}{l}\text { Selenium } \\
\mu \mathrm{g}\end{array}$ \\
\hline $230 \pm 82$ & $9.2 \pm 3.4$ & $8.0 \pm 2.5$ & $143 \pm 54$ & $1.5 \pm 0.5$ & $3.0 \pm 1.3$ & n.a. \\
\hline $301 \pm 103$ & $11.8 \pm 5.1$ & $8.0 \pm 3.4$ & $73 \pm 82$ & $1.7 \pm 5.7$ & n.a. & n.a. \\
\hline $303 \pm 89$ & $8.9 \pm 2.2$ & $10.3 \pm 2.6$ & $203 \pm 60$ & n.a. & n.a. & $36 \pm 11$ \\
\hline n.a. & n.a. & n.a. & n.a. & n.a. & n.a. & n.a. \\
\hline $298 \pm 77$ & $11.4 \pm 3.1$ & $8.7 \pm 2.2$ & $84 \pm 29$ & n.a. & n.a. & n.a. \\
\hline $211 \pm 56$ & $10.3 \pm 3.5$ & $7.0 \pm 2.3$ & $165 \pm 78$ & $0.9 \pm 0.4$ & $1.7 \pm 0.8$ & $27 \pm 10$ \\
\hline $204 \pm 37$ & $11.1 \pm 2.8$ & $10.9 \pm 2.1$ & n.a. & $1.2 \pm 0.3$ & n.a. & $41 \pm 14$ \\
\hline n.a. & $10.3 \pm 4.8$ & n.a. & n.a. & n.a. & n.a. & n.a. \\
\hline $242 \pm 79$ & $9.9 \pm 4.5$ & $8.2 \pm 2.9$ & $95 \pm 40$ & $1.0 \pm 0.4$ & $3.7 \pm 1.4$ & $34 \pm 18$ \\
\hline n.a. & n.a. & n.a. & n.a. & n.a. & n.a. & n.a. \\
\hline $262 \pm 62$ & $9.5 \pm 3.3$ & $10.4 \pm 2.8$ & n.a. & n.a. & n.a. & $34 \pm 11$ \\
\hline $247 \pm 64$ & $8.4 \pm 2.2$ & $7.5 \pm 2.0$ & n.a. & $0.9 \pm 0.2$ & n.a. & $34 \pm 13$ \\
\hline 204-303 & $8.4-11.8$ & $7.0-10.9$ & $73-203$ & $0.9-2.8$ & $1.7-3.7$ & $27-41$ \\
\hline $209 \pm 61$ & $8.2 \pm 2.4$ & $7.1 \pm 2.2$ & $128 \pm 45$ & $1.4 \pm 0.4$ & $2.9 \pm 1.2$ & n.a. \\
\hline $301 \pm 103$ & $11.8 \pm 5.1$ & $8.0 \pm 3.4$ & $73 \pm 82$ & $1.7 \pm 5.7$ & n.a. & $58 \pm 35$ \\
\hline $266 \pm 64$ & $7.7 \pm 1.7$ & $8.9 \pm 2.1$ & $179 \pm 52$ & n.a. & n.a. & $32 \pm 9$ \\
\hline n.a. & n.a. & n.a. & n.a. & n.a. & n.a. & n.a. \\
\hline $273 \pm 73$ & $10.2 \pm 2.8$ & $7.8 \pm 1.9$ & $78 \pm 31$ & n.a. & n.a. & n.a. \\
\hline $187 \pm 42$ & $8.9 \pm 2.5$ & $6.4 \pm 1.9$ & $137 \pm 60$ & $0.8 \pm 0.3$ & $1.6 \pm 0.6$ & $26 \pm 9$ \\
\hline $166 \pm 37$ & $9.8 \pm 3.0$ & $9.4 \pm 3.1$ & n.a. & $1.1 \pm 0.3$ & n.a. & $33 \pm 12$ \\
\hline n.a. & $9.2 \pm 4.4$ & n.a. & n.a. & n.a. & n.a. & n.a. \\
\hline $214 \pm 73$ & $8.5 \pm 3.3$ & $7.1 \pm 2.4$ & $90 \pm 40$ & $0.9 \pm 0.3$ & $3.4 \pm 1.3$ & $31 \pm 25$ \\
\hline n.a. & n.a. & n.a. & n.a. & n.a. & n.a. & n.a. \\
\hline $236 \pm 54$ & $8.3 \pm 2.7$ & $9.3 \pm 2.5$ & n.a. & n.a. & n.a. & $30 \pm 10$ \\
\hline $220 \pm 54$ & $7.8 \pm 2.4$ & $7.0 \pm 2.0$ & n.a. & $0.8 \pm 0.2$ & n.a. & $30 \pm 10$ \\
\hline $166-301$ & $7.7-11.8$ & $6.4-9.4$ & 73-179 & $0.8-2.6$ & $1.6-3.4$ & $26-58$ \\
\hline 170 & 10 & 7.0 & 140 & $1.0-1.5$ & $2.0-3.0$ & $20-50$ \\
\hline
\end{tabular}


Table 7.22. Mineral intake (mean \pm SD) in children aged 10 -14 years of European countries (male and female)

\begin{tabular}{|c|c|c|c|c|c|c|}
\hline & Age, years & $\mathrm{n}$ & Sodium, $\mathrm{g}$ & Potassium, $\mathrm{g}$ & Calcium, mg & Phosphorus, mg \\
\hline \multicolumn{7}{|l|}{ Male } \\
\hline Austria & $10-14$ & 248 & $2.7 \pm 1.0$ & $1.9 \pm 0.7$ & $701 \pm 278$ & $964 \pm 297$ \\
\hline Denmark & $10-14$ & 172 & $3.7 \pm 1.1$ & $3.0 \pm 0.8$ & $1,193 \pm 392$ & $1,472 \pm 413$ \\
\hline France & $10-14$ & 160 & $3.0 \pm 0.1$ & n.a. & $961 \pm 25$ & n.a. \\
\hline \multirow[t]{3}{*}{ Germany } & $10-11$ & 199 & $2.3 \pm 0.8$ & $2.3 \pm 0.6$ & $900 \pm 300$ & $1,100 \pm 300$ \\
\hline & 12 & 114 & $3.1 \pm 1.1$ & $3.4 \pm 1.2$ & $1,280 \pm 451$ & $1,458 \pm 466$ \\
\hline & $13-14$ & 214 & $3.5 \pm 1.4$ & $3.6 \pm 1.3$ & $1,381 \pm 554$ & $1,615 \pm 578$ \\
\hline Ireland & $10-12$ & 109 & $2.4 \pm 0.6$ & $2.5 \pm 0.6$ & $990 \pm 343$ & $1,180 \pm 312$ \\
\hline Italy & $10-14$ & 52 & $5.0 \pm 1.6$ & $3.2 \pm 0.9$ & $879 \pm 312$ & $1,395 \pm 391$ \\
\hline Norway & 13 & 490 & n.a. & n.a. & $936 \pm 498$ & n.a. \\
\hline Poland & $10-14$ & 202 & $4.0 \pm 2.6$ & $3.3 \pm 1.3$ & $686 \pm 397$ & $1,227 \pm 463$ \\
\hline Portugal & 13 & 987 & $2.5 \pm 0.9$ & $4.0 \pm 1.3$ & $1,167 \pm 452$ & $1,704 \pm 518$ \\
\hline Spain & $10-14$ & 66 & $3.3 \pm 0.6$ & $2.9 \pm 0.5$ & $959 \pm 276$ & $1,458 \pm 316$ \\
\hline Sweden & $10-14$ & 517 & $2.9 \pm 0.8$ & $2.8 \pm 0.8$ & $949 \pm 401$ & $1,259 \pm 381$ \\
\hline The Netherlands & $10-14$ & 211 & n.a. & $3.3 \pm 1.0$ & $1,023 \pm 405$ & $1,493 \pm 417$ \\
\hline United Kingdom & $2-15$ & 364 & $2.5 \pm 1.0$ & $2.4 \pm 0.8$ & $852 \pm 348$ & $1,118 \pm 374$ \\
\hline All countries (min.-max.) & & & $2.3-5.0$ & $1.9-4.0$ & $701-1,381$ & $964-1,704$ \\
\hline Reference values ${ }^{2}$ & & & $0.51 / 0.55 /<2^{3}$ & $1.7 / 1.9$ & $1,100 / 1,200$ & 1,250 \\
\hline \multicolumn{7}{|l|}{ Female } \\
\hline Austria & $10-14$ & 248 & $2.4 \pm 0.8$ & $1.7 \pm 0.5$ & $600 \pm 241$ & $807 \pm 256$ \\
\hline Denmark & $10-14$ & 172 & $3.1 \pm 1.0$ & $2.6 \pm 0.7$ & $1,003 \pm 357$ & $1,184 \pm 363$ \\
\hline France & $10-14$ & 144 & $2.7 \pm 0.1$ & n.a. & $821 \pm 24$ & n.a. \\
\hline \multirow[t]{3}{*}{ Germany } & $10-11$ & 199 & $2.2 \pm 0.7$ & $2.3 \pm 0.7$ & $900 \pm 300$ & $1,100 \pm 300$ \\
\hline & 12 & 114 & $2.7 \pm 0.9$ & $3.2 \pm 1.3$ & $1,172 \pm 451$ & $1,291 \pm 466$ \\
\hline & $13-14$ & 214 & $2.8 \pm 0.8$ & $3.2 \pm 1.0$ & $1,238 \pm 413$ & $1,324 \pm 429$ \\
\hline Hungary & $11-14$ & 111 & $4.8 \pm 1.9$ & $2.5 \pm 0.7$ & $696 \pm 238$ & $988 \pm 221$ \\
\hline Ireland & $10-12$ & 109 & $2.2 \pm 0.8$ & $2.2 \pm 0.5$ & $803 \pm 301$ & $977 \pm 275$ \\
\hline Italy & $10-14$ & 52 & $4.3 \pm 1.4$ & $2.5 \pm 0.3$ & $769 \pm 186$ & $1169 \pm 213$ \\
\hline Norway & 13 & 515 & n.a. & n.a. & $785 \pm 359$ & n.a. \\
\hline Poland & $10-14$ & 202 & $3.3 \pm 1.2$ & $2.9 \pm 1.2$ & $593 \pm 359$ & $1,038 \pm 396$ \\
\hline Portugal & 13 & 987 & $2.4 \pm 0.9$ & $4.0 \pm 1.3$ & $1,130 \pm 465$ & $1,636 \pm 528$ \\
\hline Spain & $10-14$ & 53 & $2.8 \pm 0.7$ & $2.6 \pm 0.4$ & $849 \pm 159$ & $1,297 \pm 155$ \\
\hline Sweden & $10-14$ & 517 & $2.5 \pm 0.7$ & $2.4 \pm 0.7$ & $805 \pm 307$ & $1,079 \pm 319$ \\
\hline The Netherlands & $10-14$ & 199 & n.a. & $2.8 \pm 0.8$ & $905 \pm 331$ & $1,298 \pm 319$ \\
\hline United Kingdom & $2-15$ & 364 & $2.3 \pm 0.8$ & $2.2 \pm 0.7$ & $743 \pm 299$ & $983 \pm 303$ \\
\hline All countries (min.-max.) & & & $2.2-4.8$ & $1.7-4.0$ & $600-1,238$ & $807-1,636$ \\
\hline Reference values ${ }^{2}$ & & & $0.51 / 0.55 /<2^{3}$ & $1.7 / 1.9$ & $1,100 / 1,200$ & 1,250 \\
\hline
\end{tabular}

${ }^{1}$ lodised salt was not taken into account in each country; ${ }^{2} \mathrm{D}-\mathrm{A}-\mathrm{CH}, 2000$, reference values refer to age group 10-12 years and 13-14 years; ${ }^{3}$ WHO, 2003; n.a. = not available. 


\begin{tabular}{|c|c|c|c|c|c|c|}
\hline Magnesium, mg & Iron, mg & Zinc, mg & lodine, $\mu g^{1}$ & Copper, mg & Manganese, mg & Selenium, $\mu \mathrm{g}$ \\
\hline $235 \pm 79$ & $9.2 \pm 3.2$ & $8.3 \pm 2.6$ & $134 \pm 50$ & $1.5 \pm 0.5$ & $3.2 \pm 1.5$ & n.a. \\
\hline $306 \pm 87$ & $9.4 \pm 2.4$ & $10.8 \pm 2.8$ & $209 \pm 62$ & n.a. & n.a. & $37 \pm 12$ \\
\hline n.a. & n.a. & n.a. & n.a. & n.a. & n.a. & n.a. \\
\hline $296 \pm 80$ & $11.7 \pm 3.6$ & $9.0 \pm 2.3$ & $89 \pm 35$ & n.a. & n.a. & n.a. \\
\hline $443 \pm 139$ & $15.5 \pm 5.6$ & $12.3 \pm 4.1$ & $102 \pm 36$ & n.a. & n.a. & n.a. \\
\hline $503 \pm 204$ & $17.9 \pm 7.0$ & $13.8 \pm 4.9$ & $106 \pm 36$ & n.a. & n.a. & n.a. \\
\hline $224 \pm 61$ & $11.5 \pm 4.4$ & $7.8 \pm 2.8$ & $168 \pm 84$ & $0.9 \pm 0.4$ & $1.8 \pm 0.8$ & $29 \pm 11$ \\
\hline $229 \pm 68$ & $13.3 \pm 5.7$ & $12.4 \pm 3.4$ & n.a. & $1.5 \pm 0.5$ & n.a. & $42 \pm 18$ \\
\hline n.a. & $11.5 \pm 7.6$ & n.a. & n.a. & n.a. & n.a. & n.a. \\
\hline $291 \pm 111$ & $11.9 \pm 7.0$ & $10.1 \pm 3.7$ & $125 \pm 64$ & $1.3 \pm 0.6$ & $4.6 \pm 1.7$ & $42 \pm 21$ \\
\hline $375 \pm 118$ & $19.4 \pm 6.9$ & $14.6 \pm 4.5$ & $134 \pm 74$ & $1.9 \pm 0.8$ & $3.8 \pm 1.5$ & $110 \pm 39$ \\
\hline $290 \pm 46$ & $12.9 \pm 1.9$ & $10.2 \pm 1.8$ & n.a. & n.a. & n.a. & n.a. \\
\hline $254 \pm 74$ & $9.2 \pm 3.1$ & $10.5 \pm 3.2$ & n.a. & n.a. & n.a. & $33 \pm 12$ \\
\hline $288 \pm 85$ & $10.4 \pm 3.0$ & $9.3 \pm 2.9$ & n.a. & $1.1 \pm 0.3$ & n.a. & $40 \pm 14$ \\
\hline $200 \pm 67$ & $9.6 \pm 4.0$ & $7.0 \pm 2.6$ & $154 \pm 76$ & $0.8 \pm 0.3$ & $2.0 \pm 0.8$ & n.a. \\
\hline $200-503$ & $9.2-19.4$ & $7.0-14.6$ & $102-209$ & $0.8-2.9$ & $1.8-4.6$ & $29-110$ \\
\hline $230 / 310$ & 12 & $9.0 / 9.5$ & $180 / 200$ & $1.0-1.5$ & $2.0-5.0$ & $25-60$ \\
\hline $209 \pm 66$ & $8.1 \pm 2.4$ & $7.1 \pm 2.1$ & $123 \pm 48$ & $1.4 \pm 0.5$ & $2.8 \pm 1.1$ & n.a. \\
\hline $256 \pm 73$ & $7.7 \pm 2.1$ & $8.6 \pm 2.5$ & $171 \pm 57$ & n.a. & n.a. & $30 \pm 10$ \\
\hline n.a. & n.a. & n.a. & n.a. & n.a. & n.a. & n.a. \\
\hline $296 \pm 87$ & $10.8 \pm 2.7$ & $8.4 \pm 2.2$ & $85 \pm 36$ & n.a. & n.a. & n.a. \\
\hline $412 \pm 147$ & $14.8 \pm 6.1$ & $10.9 \pm 3.7$ & $100 \pm 48$ & n.a. & n.a. & n.a. \\
\hline $429 \pm 121$ & $14.8 \pm 4.7$ & $11.3 \pm 3.7$ & $94 \pm 30$ & n.a. & n.a. & n.a. \\
\hline $324 \pm 89$ & $10.0 \pm 2.9$ & $7.2 \pm 1.9$ & n.a. & $0.9 \pm 0.3$ & n.a. & n.a. \\
\hline $189 \pm 46$ & $8.9 \pm 3.3$ & $6.5 \pm 1.8$ & $129 \pm 61$ & $0.8 \pm 0.3$ & $1.7 \pm 0.6$ & $27 \pm 10$ \\
\hline $197 \pm 44$ & $10.5 \pm 2.1$ & $10.7 \pm 2.2$ & n.a. & $1.2 \pm 0.4$ & n.a. & $36 \pm 11$ \\
\hline n.a. & $10.3 \pm 9.7$ & n.a. & n.a. & n.a. & n.a. & n.a. \\
\hline $246 \pm 90$ & $10.0 \pm 5.1$ & $8.7 \pm 3.2$ & $96 \pm 44$ & $1.1 \pm 0.4$ & $4.1 \pm 1.6$ & $34 \pm 21$ \\
\hline $366 \pm 122$ & $18.3 \pm 6.6$ & $13.9 \pm 4.5$ & $127 \pm 78$ & $1.9 \pm 0.7$ & $3.7 \pm 1.6$ & $104 \pm 39$ \\
\hline $268 \pm 36$ & $11.3 \pm 1.3$ & $8.7 \pm 0.9$ & n.a. & n.a. & n.a. & n.a. \\
\hline $224 \pm 63$ & $7.9 \pm 2.8$ & $8.8 \pm 2.7$ & n.a. & n.a. & n.a. & $28 \pm 10$ \\
\hline $244 \pm 64$ & $9.1 \pm 2.4$ & $7.7 \pm 2.1$ & n.a. & $0.9 \pm 0.3$ & n.a. & $34 \pm 11$ \\
\hline $181 \pm 57$ & $8.3 \pm 3.0$ & $6.1 \pm 2.1$ & $137 \pm 72$ & $0.7 \pm 0.3$ & $1.8 \pm 0.7$ & n.a. \\
\hline $181-429$ & $7.7-14.8$ & $6.1-13.9$ & $85-171$ & $0.7-2.8$ & $1.7-4.1$ & $28-104$ \\
\hline $250 / 310$ & 15 & 7 & $180 / 200$ & $1.0-1.5$ & $2.0-5.0$ & $25-60$ \\
\hline
\end{tabular}


Table 7.23. Intake of selected minerals (min.-max.) in children aged 4-6 years in the four regions

\begin{tabular}{|c|c|c|c|c|c|c|}
\hline Region/sex & Calcium, mg & Phosphorus, mg & Iron, mg & Zinc, $\mu \mathrm{g}$ & lodine, $\mu \mathrm{g}$ & Selenium, $\mu \mathrm{g}$ \\
\hline \multicolumn{7}{|l|}{ North ${ }^{1}$} \\
\hline Male & $687-1,103$ & $1,042-1,284^{5}$ & $7.8-8.6$ & $9.8-9.1^{5}$ & $173-223^{6}$ & $26-45^{5}$ \\
\hline Female & $673-991$ & $966-1,137^{5}$ & $6.9-7.7$ & $7.1-8.2^{5}$ & $151-197^{6}$ & $24-39^{5}$ \\
\hline \multicolumn{7}{|l|}{ South ${ }^{2}$} \\
\hline Male & $793-1,024$ & $1,085^{7}$ & $8.8-10.0$ & $8.4-9.2$ & n.a. & $29^{7}$ \\
\hline Female & $701-1,024$ & $1,063^{7}$ & $10.0-10.1$ & $8.4-8.9$ & n.a. & $28^{7}$ \\
\hline \multicolumn{7}{|c|}{ Central and East ${ }^{3}$} \\
\hline Male & $604-900$ & $882-1,183$ & $8.0-10.6$ & $6.8-7.7$ & $69-87$ & $27-61^{8}$ \\
\hline Female & $606-809$ & $877-1,183$ & $7.4-10.6$ & $6.4-7.3$ & $69-82$ & $29-61^{8}$ \\
\hline \multicolumn{7}{|l|}{ West } \\
\hline Male & $813-856$ & $944-1,067^{9}$ & $7.3-8.7^{9}$ & $6.0^{9}$ & $142^{10}$ & $23-27^{9}$ \\
\hline Female & $750-794$ & $904-950^{9}$ & $6.8-7.8^{9}$ & $5.3-5.6^{9}$ & $137^{10}$ & $23-24^{9}$ \\
\hline
\end{tabular}

${ }^{1}$ North: SE, NO, FI, DK; ${ }^{2}$ South: IT, GR; ${ }^{3}$ Central and East: CZ, DE, PL; ${ }^{4}$ West: NL, FR, IR; 5 only SE, FI and $\mathrm{DK} ;{ }^{6}$ only $\mathrm{FI}$ and $\mathrm{DK} ;{ }^{7}$ only IT; ${ }^{8}$ only $\mathrm{CZ}$ and $\mathrm{PL} ;{ }^{9}$ only $\mathrm{NL}$ and IR; ${ }^{10}$ only IR; n.a. = not available.

Table 7.24. Intake of selected minerals (min.-max.) in children aged 7-9 years in the four regions

\begin{tabular}{|c|c|c|c|c|c|c|}
\hline Region/sex & Calcium, mg & Phosphorus, mg & Iron, mg & Zinc, $\mu \mathrm{g}$ & lodine, $\mu \mathrm{g}$ & Selenium, $\mu \mathrm{g}$ \\
\hline \multicolumn{7}{|l|}{ North ${ }^{1}$} \\
\hline Male & $936-1,207$ & $1,300-1,455^{5}$ & $8.9-10.3$ & $10.3-10.4^{5}$ & $203^{6}$ & $34-36^{5}$ \\
\hline Female & $756-1,094$ & $1,155-1,288^{5}$ & $7.7-9.2$ & $8.9-9.3^{5}$ & $179^{6}$ & $30-32^{5}$ \\
\hline \multicolumn{7}{|l|}{ South ${ }^{2}$} \\
\hline Male & $904-1,174$ & $1,253^{7}$ & $11.1^{7}$ & $10.9^{7}$ & n.a. & $41^{7}$ \\
\hline Female & $715-1,126$ & $1,091^{7}$ & $9.8^{7}$ & $9.4^{7}$ & n.a. & $33^{7}$ \\
\hline \multicolumn{7}{|c|}{ Central and East ${ }^{3}$} \\
\hline Male & $628-900$ & $960-1,295$ & $9.2-11.8$ & $8.0-8.7$ & $73-143$ & $34-58^{8}$ \\
\hline Female & $548-882$ & $851-1,295$ & $8.2-11.8$ & $7.1-8.0$ & $73-128$ & $31-58^{8}$ \\
\hline \multicolumn{7}{|l|}{ West $^{4}$} \\
\hline Male & $855-943$ & $1,098-1,273^{9}$ & $8.4-10.3^{9}$ & $7.0-7.5^{9}$ & $165^{10}$ & $27-34^{9}$ \\
\hline Female & $857-901$ & $991-1,185^{9}$ & $7.8-8.9^{9}$ & $6.4-7.0^{9}$ & $137^{10}$ & $26-30^{9}$ \\
\hline
\end{tabular}

${ }^{1}$ North: SE, NO, DK; ${ }^{2}$ South: IT, PT; ${ }^{3}$ Central and East: AT, CZ, DE, PL; ${ }^{4}$ West: NL, FR, IR; ${ }^{5}$ only SE and DK; ${ }^{6}$ only $\mathrm{FI} ;{ }^{7}$ only IT; ${ }^{8}$ only $\mathrm{CZ}$ and $\mathrm{PL} ;{ }^{9}$ only NL and IR; ${ }^{10}$ only IR; n.a. = not available. 
Table 7.25. Intake of selected minerals (min.-max.) in children aged 10-14 years in the four regions

\begin{tabular}{|c|c|c|c|c|c|c|}
\hline Region/sex & Calcium, mg & Phosphorus, mg & Iron, mg & Zinc, $\mu \mathrm{g}$ & lodine, $\mu \mathrm{g}$ & Selenium, $\mu \mathrm{g}$ \\
\hline \multicolumn{7}{|l|}{ North ${ }^{1}$} \\
\hline Male & $936-1,193$ & $1,259-1,472^{5}$ & $9.2-11.5$ & $10.5-10.8^{5}$ & $209^{6}$ & $33-36^{5}$ \\
\hline Female & $785-1,003$ & $1,079-1,184^{5}$ & $7.7-10.3$ & $8.6-8.8^{5}$ & $171^{6}$ & $28-30^{5}$ \\
\hline \multicolumn{7}{|l|}{ South ${ }^{2}$} \\
\hline Male & 879-959 & $1,395-1,458$ & $12.8-13.3$ & $10.1-12.4$ & n.a. & $42^{7}$ \\
\hline Female & $769-849$ & 1,169-1,297 & $10.5-11.3$ & $8.7-10.7$ & n.a. & $36^{7}$ \\
\hline \multicolumn{7}{|c|}{ Central and East ${ }^{3}$} \\
\hline Male & $686-1,381$ & $964-1,615$ & $9.2-17.9$ & $8.3-13.8$ & 89-134 & $42^{8}$ \\
\hline Female & $593-1,238$ & $807-1,324$ & $8.1-14.8$ & $7.1-11.3$ & $85-123^{12}$ & $34^{8}$ \\
\hline \multicolumn{7}{|l|}{ West ${ }^{4}$} \\
\hline Male & $852-1,023$ & $1,118-1,493^{9}$ & $9.6-11.5^{9}$ & $7.0-9.3^{9}$ & $154-168^{10}$ & $29-40^{11}$ \\
\hline Female & $743-905$ & $977-1,298^{9}$ & $8.3-9.1^{9}$ & $6.1-7.7^{9}$ & $129-137^{10}$ & $27-34^{11}$ \\
\hline
\end{tabular}

${ }^{1}$ North: SE, NO, DK; ${ }^{2}$ South: IT, ES; ${ }^{3}$ Central and East: AT, DE, PL (female: HU); ${ }^{4}$ West: UK, NL, FR, IR; ${ }^{5}$ only SE and $\mathrm{DK} ;{ }^{6}$ only $\mathrm{DK} ;{ }^{7}$ only IT; ${ }^{8}$ only $\mathrm{PL} ;{ }^{9}$ only UK, NL and IR; ${ }^{10}$ only UK and IR; ${ }^{11}$ only $\mathrm{NL}$ and IR; ${ }^{12}$ only AT, DE and PL; n.a. = not available.

was higher in the South region and the share of carbohydrates in total energy intake was lower than in the other regions. The intake of sucrose was in general high (10$17 \% \mathrm{E})$, but the highest values were reported in the West region. The lowest intake values of dietary fibers were observed in the West region (10-23 g/day) and highest in the Central and East region, but the intake was as mentioned above lower than the Eurodiet recommendation [Eurodiet, 2000].

The fatty acid pattern was in general unfavorable. The share of saturated fatty acids (SFA) in total energy intake was in every region higher than the recommendation [WHO, 2003] and the share of polyunsaturated fatty acids (PUFA) in total energy intake was lower than the recommended intake range [WHO, 2009b]. Only in the West region the intake was close to the recommendation. The intake of cholesterol was desirable lower in countries of the West region than in the other regions (cf. table 7.29).

\section{Vitamins}

\section{Comparison of Countries}

The intake of retinol equivalents was between 0.3 and $1.8 \mathrm{mg} / \mathrm{day}$ in male and between 0.3 and $1.6 \mathrm{mg} /$ day in female adolescents. Only adolescents from Germany, 
Table 7.26. Methods and period of dietary assessment in adolescents of the participating countries (data available from 12,716 adolescents)

\begin{tabular}{|c|c|c|c|c|c|c|}
\hline Country & $\begin{array}{l}\text { Age group } \\
\text { years }\end{array}$ & Method & $\begin{array}{l}\text { Year of } \\
\text { survey }\end{array}$ & $\mathrm{n}$ & $\begin{array}{l}\text { Intake from } \\
\text { dietary } \\
\text { supplements } \\
\text { is included }\end{array}$ & Reference \\
\hline Austria & 14-19 & 24-hour recall & $2003 / 2004$ & 2,949 & no & $\begin{array}{l}\text { Elmadfa et al., } \\
2008\end{array}$ \\
\hline Belgium & $15-18$ & 24-hour recall & 2004 & 3,249 & no & $\begin{array}{l}\text { Devriese et al., } \\
2006\end{array}$ \\
\hline Denmark & $15-18$ & 7-day dietary record & 2000-2002 & 199 & no & Lyhne et al., 2005 \\
\hline France & $15-17$ & $3 \times 24$-hour recall & $2006 / 2007$ & 403 & no & $\begin{array}{l}\text { Castetbon } \\
\text { et al., } 2009\end{array}$ \\
\hline Germany & $15-18$ & $\begin{array}{l}\text { dietary history } \\
\text { interview }\end{array}$ & 2005-2007 & 1,203 & no & $\begin{array}{l}\text { Max Rubner- } \\
\text { Institut (eds), } \\
\text { 2008a, 2008b }\end{array}$ \\
\hline Ireland & $13-17$ & $\begin{array}{l}\text { 7-day semi-weighed } \\
\text { dietary record }\end{array}$ & 2005-2006 & 441 & yes & $\begin{array}{l}\text { Morgan et al., } \\
2007\end{array}$ \\
\hline Italy & $15-18$ & 7-day dietary record & n.a. & 99 & no & D'Amicis, 2000 \\
\hline Norway & $15-18$ & FFQ & 2000 & 134 & yes & $\begin{array}{l}\text { Johansson and } \\
\text { Solvoll, } 1997\end{array}$ \\
\hline Poland & $15-18$ & 24-hour recall & 2000 & 349 & no & $\begin{array}{l}\text { Szponar et al., } \\
2000 \text { (unpubl. } \\
\text { data) }\end{array}$ \\
\hline Slovenia & $14-17$ & FFQ & n.a. & 2,813 & no & $\begin{array}{l}\text { Fidler Mis et al. } \\
\text { (unpubl. data' }{ }^{1} \text { ) }\end{array}$ \\
\hline Spain & $15-18$ & $2 \times 24$-hour recall & 2002-2003 & 118 & no & $\begin{array}{l}\text { Serra Majem and } \\
\text { Ribas 2007; } \\
\text { Serra-Majem L } \\
\text { et al., 2007; } \\
\text { Serra Majem } \\
\text { et al., } 2006\end{array}$ \\
\hline The Netherlands & $15-18$ & 2-day dietary record & 1997/1998 & 361 & no & $\begin{array}{l}\text { Hulshof and van } \\
\text { Staveren, } 1991\end{array}$ \\
\hline United Kingdom & $16-24$ & n.a. & 2003-2005 & 398 & yes & $\begin{array}{l}\text { Low Income Diet } \\
\text { and } \\
\text { Health Survey, } \\
2003-2005^{2}\end{array}$ \\
\hline
\end{tabular}

n.a. $=$ Not available. ${ }^{1}$ Data provided by the Biotechnical faculty, University of Ljubljana; ${ }^{2}$ data provided by the University of Southampton. 
Norway, Poland and Slovenia met the recommendation [D-A-CH, 2000]. The intake of $\beta$-carotene was between 1.2 and $4.7 \mathrm{mg} /$ day in male and between 1.0 and $4.7 \mathrm{mg} /$ day in female adolescents. In general, the intake was higher in the male group.

The intake of vitamin $\mathrm{D}$ was below the recommendation [D-A-CH, 2000] in every participating country except for Norway, both male and female, and Poland, only males. Under the climatic conditions in the Nordic countries there are indications that exposure to sunlight is insufficient for enough vitamin $\mathrm{D}$ to be formed in the skin and for vitamin $\mathrm{D}$ status to be maintained during the winter months. Therefore, the recommendation of the Nordic countries [NNR, 2004] is higher (7.5 $\mu \mathrm{g} /$ day) than the recommendation for Central Europe [D-A-CH, 2000].

The $\alpha$-tocopherol equivalents intake levels were between 6.8 and $20.8 \mathrm{mg} /$ day in male and between 6.0 and $15.5 \mathrm{mg} /$ day in female adolescents. German, Polish and Slovenian adolescents as well as male adolescents from The Netherlands met the recommendation [D-A-CH, 2000].

The recommendations for the intake of thiamine, riboflavin and niacin equivalents were met by almost every participating country as compared with the D-A-CH reference values [D-A-CH, 2000]. From the United Kingdom only values for preformed niacin were available and therefore cannot be compared to the recommended intake value. The recommendations for vitamin $\mathrm{B}_{6}$ and cobalamin were met by almost every participating country, too. Only Danish male adolescents were slightly below the recommendation for vitamin $\mathrm{B}_{6}$ [D-A-CH, 2000].

The intake of folate equivalents was between 190 and $365 \mu \mathrm{g} /$ day in male adolescents and between 154 and $298 \mu \mathrm{g} /$ day in female adolescents. Compared with the D-A-CH reference values, every participating country was below the recommendation [D-A-CH, 2000]. In the Nordic countries an intake of $300 \mu \mathrm{g} / \mathrm{day}$ (400 $\mu \mathrm{g} /$ day for women of fertile age) [NNR, 2004] is recommended. This recommendation was only met by male adolescents from Denmark, Germany, Italy and Poland.

The D-A-CH reference values recommend an intake of ascorbic acid of $100 \mathrm{mg} / \mathrm{day}$ [D-A-CH, 2000]. Adolescents from Austria, Belgium, Ireland, Spain, The Netherlands and the United Kingdom as well as female adolescents from Poland were below this recommendation. Compared with the NNR [NNR, 2004] every country was close to the recommended intake of $75 \mu \mathrm{g} /$ day (cf. table 7.30).

\section{Comparison of Regions}

The intake of vitamin $\mathrm{B}_{6}$ was higher in the Central and East region than in the other regions. The lowest intake was reported in the North region, but also the highest intake of folate equivalents and vitamin D. Concerning cobalamin, no big difference between the regions could be observed (cf. table 7.31). 
Table 7.27. Intake of energy and macronutrients (mean \pm SD) in adolescents of European countries (male and female)

\begin{tabular}{|c|c|c|c|c|}
\hline \multirow{2}{*}{$\begin{array}{l}\text { Age } \\
\text { years }\end{array}$} & \multirow[t]{2}{*}{$\mathrm{n}$} & \multirow{2}{*}{$\begin{array}{l}\text { Energy } \\
\text { MJ }\end{array}$} & \multirow{2}{*}{$\begin{array}{l}\text { Protein } \\
\% E^{1}\end{array}$} & Carbohydrates, \%E \\
\hline & & & & total \\
\hline
\end{tabular}

\section{Male}

\begin{tabular}{llllll}
\hline Austria & $14->19$ & 1,527 & $11.5 \pm 3.0$ & $16.1 \pm 4.0$ & $46.1 \pm 9.9$ \\
\hline Belgium & $15-18$ & n.a. & $11.1 \pm 2.6$ & $13.5 \pm 2.0$ & $48.5 \pm 4.4$ \\
\hline Denmark & $15-18$ & 78 & $10.3 \pm 2.7$ & $14.0 \pm 2.7$ & $50.0 \pm 6.1$ \\
\hline France & $15-18$ & 181 & $10.2 \pm 0.4$ & $15.7 \pm 0.3$ & $48.7 \pm 0.6$ \\
\hline Germany & $15-18$ & 598 & $12.2 \pm 3.8$ & $13.4 \pm 2.2$ & $49.6 \pm 6.5$ \\
\hline Ireland & $13-17$ & 224 & $9.5 \pm 2.4$ & $15.2 \pm 2.5$ & n.a. \\
\hline Italy & $15-18$ & 52 & $11.7 \pm 2.5$ & $16.1 \pm 2.0$ & n.a. \\
\hline Norway & $15-18$ & 72 & $13.9 \pm 6.1$ & $15.0 \pm 3.0$ & $53.0 \pm 7.0$ \\
\hline Poland & $15-18$ & 174 & $14.5 \pm 4.7$ & $12.4 \pm 3.0$ & $50.7 \pm 7.4$ \\
\hline Slovenia & $15-18$ & 1,010 & $12.7 \pm 6.9$ & $15.0 \pm 3.0$ & $57.0 \pm 9.0$ \\
\hline Spain & $15-18$ & 61 & $10.7 \pm 2.0$ & $17.8 \pm 2.6$ & $39.7 \pm 4.7$ \\
\hline The Netherlands & $15-18$ & 180 & $11.4 \pm 3.1$ & $13.0 \pm 3.0$ & $50.0 \pm 7.0$ \\
\hline United Kingdom & $16-24$ & 150 & $10.6 \pm 3.4$ & $13.9 \pm 2.9$ & $49.3 \pm 6.8$ \\
\hline All countries (min.-max.) & & $9.5-14.5$ & $12.4-17.8$ & $39.7-57.0$ \\
\hline Fomalo & 150 & & & 5.0 \\
\hline
\end{tabular}

\section{Female}

\begin{tabular}{llllll}
\hline Austria & $14->19$ & 1,422 & $8.5 \pm 2.2$ & $14.7 \pm 4.1$ & $47.3 \pm 10.3$ \\
\hline Belgium & $15-18$ & n.a. & $7.7 \pm 1.6$ & $13.6 \pm 2.1$ & $50.3 \pm 5.5$ \\
\hline Denmark & $15-18$ & 121 & $8.2 \pm 2.0$ & $14.0 \pm 2.6$ & $52.0 \pm 5.2$ \\
\hline France & $15-18$ & 222 & $6.8 \pm 0.2$ & $15.6 \pm 0.2$ & $48.8 \pm 0.7$ \\
\hline Germany & $15-18$ & 605 & $8.8 \pm 2.9$ & $12.9 \pm 2.0$ & $52.7 \pm 6.4$ \\
\hline Ireland & $13-17$ & 217 & $7.1 \pm 1.9$ & $14.3 \pm 2.6$ & n.a. \\
\hline Italy & $15-18$ & 47 & $8.7 \pm 1.5$ & $16.0 \pm 3.6$ & n.a. \\
\hline Norway & $15-18$ & 62 & $9.3 \pm 3.5$ & $15.0 \pm 3.0$ & $55.0 \pm 5.0$ \\
\hline Poland & $15-18$ & 175 & $9.5 \pm 3.5$ & $12.0 \pm 2.9$ & $54.2 \pm 8.5$ \\
\hline Slovenia & $15-18$ & 1,214 & $9.7 \pm 5.1$ & $14.0 \pm 3.0$ & $57.0 \pm 8.0$ \\
\hline Spain & $15-18$ & 57 & $7.9 \pm 1.1$ & $18.0 \pm 2.5$ & $38.6 \pm 3.7$ \\
\hline The Netherlands & $15-18$ & 181 & $8.9 \pm 2.2$ & $14.0 \pm 3.0$ & $50.0 \pm 7.0$ \\
\hline
\end{tabular}




\begin{tabular}{lllllll}
\hline & Dietary fiber & Fat & SFA & MUFA & PUFA & Cholesterol \\
\cline { 1 - 2 } & $\mathrm{g}$ & $\% \mathrm{E}^{1}$ & $\% \mathrm{E}$ & $\% \mathrm{E}$ & $\% \mathrm{E}$ & $\mathrm{mg}$ \\
\hline
\end{tabular}

\begin{tabular}{lllllll}
\hline $16.1 \pm 8.9$ & $15.6 \pm 7.1$ & $36.5 \pm 8.9$ & $18.3 \pm 6.2$ & $11.1 \pm 3.4$ & $4.7 \pm 2.6$ & $375.0 \pm 204.0$ \\
\hline n.a. & n.a. & $36.0 \pm 3.5$ & $14.1 \pm 2.4$ & $12.8 \pm 1.4$ & $6.5 \pm 1.5$ & n.a. \\
\hline $13.0 \pm 7.2$ & $18.0 \pm 6.4$ & $32.0 \pm 4.7$ & $14.0 \pm 2.6$ & $11.0 \pm 1.7$ & $5.0 \pm 0.8$ & n.a. \\
\hline n.a. & $16.9 \pm 0.8$ & $34.2 \pm 0.5$ & 14.0 & n.a. & n.a. & n.a. \\
\hline n.a. & $26.1 \pm 10.7$ & $34.6 \pm 5.8$ & n.a. & n.a. & n.a. & $400.5 \pm 162.4$ \\
\hline n.a. & $17.6 \pm 7.7$ & $35.5 \pm 5.2$ & $14.5 \pm 2.8$ & $12.6 \pm 2.3$ & $5.6 \pm 1.6$ & n.a. \\
\hline n.a. & $23.9 \pm 9.1$ & $37.0 \pm 7.1$ & $11.2 \pm 2.4$ & $12.9 \pm 4.1$ & $5.3 \pm 2.2$ & $437.6 \pm 129.6$ \\
\hline $15.0 \pm 8.0$ & $27.0 \pm 13.0$ & $31.0 \pm 6.0$ & $13.0 \pm 3.0$ & $11.0 \pm 2.0$ & $5.0 \pm 2.0$ & $358.0 \pm 174.0$ \\
\hline $12.5 \pm 5.0$ & $32.6 \pm 12.5$ & $36.8 \pm 6.9$ & $12.1 \pm 3.8$ & $16.0 \pm 3.8$ & $6.3 \pm 2.9$ & $566.0 \pm 366.0$ \\
\hline $11.0 \pm 4.0$ & $33.0 \pm 21.0$ & $28.0 \pm 7.0$ & $13.0 \pm 3.0$ & $10.3 \pm 3.0$ & $5.0 \pm 2.0$ & $300.0 \pm 188.0$ \\
\hline $14.8 \pm 3.4$ & $18.9 \pm 1.8$ & $40.4 \pm 3.8$ & $13.8 \pm 1.8$ & $16.7 \pm 2.3$ & $5.7 \pm 0.9$ & $382.4 \pm 47.8$ \\
\hline n.a. & $23.0 \pm 10.0$ & $35.0 \pm 6.0$ & $14.0 \pm 3.0$ & $13.0 \pm 3.0$ & $7.0 \pm 3.0$ & $219.0 \pm 98.0$ \\
\hline $17.5 \pm 8.5$ & $13.7 \pm 8.5$ & $36.8 \pm 5.9$ & $13.6 \pm 3.0$ & n.a. & n.a. & $292.7 \pm 154.9$ \\
\hline $11.0-17.5$ & $13.7-33.0$ & $28.0-40.4$ & $11.2-18.3$ & $10.3-16.7$ & $4.7-7.0$ & $219.0-566.0$ \\
\hline
\end{tabular}

\begin{tabular}{lllllll}
\hline $15.7 \pm 8.7$ & $13.8 \pm 6.0$ & $37.4 \pm 9.6$ & $19.1 \pm 6.7$ & $11.1 \pm 3.5$ & $4.8 \pm 2.9$ & $267.0 \pm 141.0$ \\
\hline n.a. & n.a. & $25.5 \pm 5.0$ & $14.4 \pm 2.4$ & $12.8 \pm 2.1$ & $6.6 \pm 2.1$ & n.a. \\
\hline $14.0 \pm 6.9$ & $16.0 \pm 5.5$ & $30.0 \pm 4.6$ & $13.0 \pm 2.5$ & $10.0 \pm 1.7$ & $4.0 \pm 0.8$ & n.a. \\
\hline n.a. & $12.7 \pm 0.3$ & $35.3 \pm 0.6$ & 14.2 & n.a. & n.a. & n.a. \\
\hline n.a. & $23.1 \pm 8.3$ & $33.0 \pm 5.9$ & n.a. & n.a. & n.a. & $269.0 \pm 115.8$ \\
\hline n.a. & $13.3 \pm 4.3$ & $35.9 \pm 4.8$ & $14.2 \pm 2.5$ & $12.9 \pm 2.2$ & $5.9 \pm 1.6$ & n.a. \\
\hline n.a. & $17.6 \pm 4.7$ & $33.7 \pm 4.7$ & $10.2 \pm 2.3$ & $10.9 \pm 2.4$ & $5.2 \pm 1.7$ & $340.3 \pm 102.0$ \\
\hline $12.0 \pm 6.0$ & $22.0 \pm 8.0$ & $28.0 \pm 5.0$ & $12.0 \pm 2.0$ & $10.0 \pm 2.0$ & $5.0 \pm 2.0$ & $236.0 \pm 117.0$ \\
\hline $14.1 \pm 5.7$ & $23.0 \pm 8.9$ & $33.8 \pm 8.1$ & $11.7 \pm 4.2$ & $14.4 \pm 4.5$ & $5.4 \pm 2.6$ & $326.0 \pm 255.0$ \\
\hline $13.0 \pm 4.0$ & $27.0 \pm 18.0$ & $29.0 \pm 7.0$ & $13.0 \pm 3.0$ & $10.0 \pm 3.0$ & $6.0 \pm 2.0$ & $218.0 \pm 139.0$ \\
\hline $15.4 \pm 2.9$ & $16.2 \pm 2.0$ & $41.9 \pm 3.7$ & $13.7 \pm 1.3$ & $17.7 \pm 1.9$ & $6.0 \pm 1.0$ & $313.9 \pm 45.5$ \\
\hline n.a. & $19.0 \pm 7.0$ & $36.0 \pm 6.0$ & $14.0 \pm 3.0$ & $13.0 \pm 3.0$ & $7.0 \pm 2.0$ & $183.0 \pm 86.0$ \\
\hline
\end{tabular}


Table 7.27. Continued

\begin{tabular}{llllll} 
& $\begin{array}{l}\text { Age } \\
\text { years }\end{array}$ & $\mathrm{n}$ & $\begin{array}{l}\text { Energy } \\
\mathrm{MJ}\end{array}$ & $\begin{array}{l}\text { Protein } \\
\% \mathrm{E}^{1}\end{array}$ & $\begin{array}{l}\text { Carbohydrates, \% } \mathrm{E}^{1} \\
\text { total }\end{array}$ \\
\hline United Kingdom & $16-24$ & 248 & $7.0 \pm 2.2$ & $14.5 \pm 3.7$ & $50.5 \pm 6.8$ \\
\hline All countries (min.-max.) & & $6.8-9.7$ & $12.0-18.0$ & $38.6-57.0$ \\
\hline Reference values & & $\begin{array}{l}13.0 \text { (male)/ } \\
10.5 \text { (female) }\end{array}$ & $10-15 \% \mathrm{E}^{3}$ & $50-75 \% \mathrm{E}^{4}$ \\
\hline
\end{tabular}

${ }^{1}$ Difference is not necessarily alcohol; ${ }^{2} \mathrm{D}-\mathrm{A}-\mathrm{CH}, 2000$, reference values refer to age group $15-18$ years (PAL 1.75); ${ }^{3} \mathrm{WHO}, 2003 ;{ }^{4} \mathrm{WHO}, 2007 ;{ }^{5} \mathrm{WHO}, 2009 \mathrm{~b} ;{ }^{6}$ Eurodiet, 2000; n.a. = not available.

Table 7.28. Intake of energy and macronutrients (min.-max.) in adolescents in the four regions

\begin{tabular}{|c|c|c|c|c|c|}
\hline Region/sex & $\begin{array}{l}\text { Energy } \\
\text { MJ }\end{array}$ & $\begin{array}{l}\text { Protein } \\
\% \mathrm{E}\end{array}$ & $\begin{array}{l}\text { Carbohydrates, \%E } \\
\text { (of that sucrose) }\end{array}$ & $\begin{array}{l}\text { Dietary fiber } \\
\mathrm{g}\end{array}$ & $\begin{array}{l}\text { Fat } \\
\% \mathrm{E}\end{array}$ \\
\hline \multicolumn{6}{|l|}{ North ${ }^{1}$} \\
\hline Male & $10.3-13.9$ & $14.0-15.0$ & $50.0-53.0(13.0-15.0)$ & $18.0-27.0$ & $31.0-32.0$ \\
\hline Female & $8.2-9.3$ & $14.0-15.0$ & $52.0-55.0(12.0-14.0)$ & $16.0-22.0$ & $28.0-30.0$ \\
\hline \multicolumn{6}{|l|}{ South $^{2}$} \\
\hline Male & $10.7-11.7$ & $16.1-17.8$ & $39.7^{5}(14.8)^{5}$ & $18.9-23.9$ & $37.0-40.4$ \\
\hline Female & $7.9-8.7$ & $16.0-18.0$ & $38.6^{5}(15.4)^{5}$ & $16.2-17.6$ & $33.7-41.9$ \\
\hline \multicolumn{6}{|c|}{ Central and East ${ }^{3}$} \\
\hline Male & $11.5-14.5$ & $12.4-16.1$ & $46.1-57.0(11.0-16.1)$ & $15.6-33.0$ & $28.0-36.8$ \\
\hline Female & $8.5-9.7$ & $12.0-14.7$ & $47.3-57.0(13.0-15.7)$ & $13.8-27.0$ & $29.0-37.4$ \\
\hline \multicolumn{6}{|l|}{ West ${ }^{4}$} \\
\hline Male & $9.5-11.4$ & $13.0-15.7$ & $48.5-50.0^{6}(17.5)^{7}$ & $13.7-23.0$ & $34.2-36.8$ \\
\hline Female & $6.8-8.9$ & $13.6-15.6$ & $48.8-50.5^{6}(16.5)^{7}$ & $10.2-19.0$ & $35.0-36.0$ \\
\hline
\end{tabular}




\begin{tabular}{lllllll}
\hline & $\begin{array}{l}\text { Dietary fiber } \\
\mathrm{g}\end{array}$ & $\begin{array}{l}\text { Fat } \\
\% \mathrm{E}^{1}\end{array}$ & $\begin{array}{l}\text { SFA } \\
\% \mathrm{E}\end{array}$ & $\begin{array}{l}\text { MUFA } \\
\% \mathrm{E}\end{array}$ & $\begin{array}{l}\text { PUFA } \\
\% \mathrm{E}\end{array}$ & $\begin{array}{l}\text { Cholesterol } \\
\mathrm{mg}\end{array}$ \\
\hline $16.5 \pm 8.9$ & $10.2 \pm 4.2$ & $35.0 \pm 6.7$ & $12.9 \pm 3.4$ & n.a. & n.a. & $187.0 \pm 93.9$ \\
\hline $12.0-16.5$ & $10.2-27.0$ & $25.5-41.9$ & $10.2-19.1$ & $10.0-17.7$ & $4.0-7.0$ & $183.0-340.3$ \\
\hline$<10 \% \mathrm{E}^{3}$ & $>25{\mathrm{~g} \cdot \mathrm{d}^{-1,6}}^{2}$ & $15-30 \% \mathrm{E}^{3}$ & $<10 \% \mathrm{E}^{3}$ & & $6-11 \% \mathrm{E}^{4}$ & $<300{\mathrm{mg} \cdot \mathrm{d}^{-1,3}}$
\end{tabular}

Table 7.29. Intake of fat, fatty acids and cholesterol (min.-max.) in adolescents in the four regions

\begin{tabular}{|c|c|c|c|c|c|}
\hline Region/sex & $\begin{array}{l}\text { Fat } \\
\% \mathrm{E}\end{array}$ & $\begin{array}{l}\text { SFA } \\
\% \mathrm{E}\end{array}$ & $\begin{array}{l}\text { MUFA } \\
\% \mathrm{E}\end{array}$ & $\begin{array}{l}\text { PUFA } \\
\% \mathrm{E}\end{array}$ & $\begin{array}{l}\text { Cholesterol } \\
\mathrm{mg}\end{array}$ \\
\hline \multicolumn{6}{|l|}{ North $^{1}$} \\
\hline Male & $31.0-32.0$ & $13.0-14.0$ & 11.0 & 5.0 & $358^{5}$ \\
\hline Female & $28.0-30.0$ & $12.0-13.0$ & 10.0 & $4.0-5.0$ & $236^{5}$ \\
\hline \multicolumn{6}{|l|}{ South ${ }^{2}$} \\
\hline Male & $37.0-40.4$ & $11.2-13.8$ & $12.9-16.7$ & $5.3-5.7$ & $383-438$ \\
\hline Female & $33.7-41.9$ & $10.2-13.7$ & $10.9-17.7$ & $5.2-6.0$ & $314-340$ \\
\hline \multicolumn{6}{|c|}{ Central and East ${ }^{3}$} \\
\hline Male & $28.0-36.8$ & $12.1-18.3^{6}$ & $10.3-16.0^{6}$ & $4.7-6.3^{6}$ & $300-566$ \\
\hline Female & $29.0-37.4$ & $11.7-19.1^{6}$ & $10.0-14.0^{6}$ & $4.8-6.0^{6}$ & $218-326$ \\
\hline \multicolumn{6}{|l|}{ West ${ }^{4}$} \\
\hline Male & $34.2-36.8$ & $13.6-14.5$ & $12.6-13.0^{7}$ & $5.6-7.0^{7}$ & $219-293^{8}$ \\
\hline Female & $35.0-36.0$ & $12.9-14.4$ & $12.8-13.0^{7}$ & $5.9-7.0^{7}$ & $183-187^{8}$ \\
\hline
\end{tabular}


Table 7.30. Vitamin intake (mean \pm SD) in adolescents of European countries (male and female)

\begin{tabular}{|c|c|c|c|c|c|c|}
\hline & $\begin{array}{l}\text { Age } \\
\text { years }\end{array}$ & $\mathrm{n}$ & $\begin{array}{l}\text { Vitamin } A^{1} \\
\text { mg }\end{array}$ & $\begin{array}{l}\beta \text {-Carotene } \\
\mathrm{mg}\end{array}$ & $\begin{array}{l}\text { Vitamin D } \\
\mu \mathrm{g}\end{array}$ & $\begin{array}{l}\text { Vitamin } \mathrm{E}^{2} \\
\mathrm{mg}\end{array}$ \\
\hline \multicolumn{7}{|l|}{ Male } \\
\hline Austria & $>13$ & 1,527 & $1.0 \pm 1.3$ & $1.5 \pm 1.7$ & $2.0 \pm 1.8$ & $11.8 \pm 8.4$ \\
\hline Belgium & $15-18$ & n.a. & n.a. & n.a. & n.a. & n.a. \\
\hline Denmark & $15-18$ & 78 & $0.9 \pm 0.5$ & $2.6 \pm 2.2$ & $2.6 \pm 1.6$ & $6.8 \pm 2.5$ \\
\hline Germany & $15-18$ & 598 & $1.8 \pm 1.1$ & $4.7 \pm 3.4$ & $2.8 \pm 1.9$ & $18.7 \pm 11.1$ \\
\hline Ireland & $13-17$ & 224 & $0.4 \pm 0.3$ & $2.9 \pm 2.8$ & $3.0 \pm 2.6$ & $9.3 \pm 6.4$ \\
\hline Italy & $15-18$ & 52 & $1.0 \pm 0.4$ & $2.6 \pm 1.5$ & $3.3 \pm 1.9$ & $12.1 \pm 3.8$ \\
\hline Norway & $15-18$ & 72 & $1.8 \pm 1.2^{5}$ & n.a. & $7.5 \pm 6.3$ & n.a. \\
\hline Poland & $15-18$ & 174 & $2.3 \pm 4.3$ & $4.2 \pm 4.2$ & $5.5 \pm 4.2$ & $20.8 \pm 12.2$ \\
\hline Slovenia & $15-18$ & 1,010 & $1.4 \pm 1.1$ & $3.1 \pm 3.2$ & $4.0 \pm 6.0$ & $15.0 \pm 11.0$ \\
\hline Spain & $15-18$ & 61 & $0.6 \pm 0.1$ & $1.2 \pm 0.3$ & $1.8 \pm 0.5$ & $9.0 \pm 1.9$ \\
\hline The Netherlands & $15-18$ & 180 & $0.9 \pm 0.9$ & n.a. & $4.4 \pm 2.2$ & $16.3 \pm 8.4$ \\
\hline United Kingdom & $16-24$ & 150 & $0.6 \pm 0.4$ & $1.4 \pm 1.3$ & $3.0 \pm 1.9$ & $11.9 \pm 5.5$ \\
\hline All countries (min.-max.) & & & $0.4-1.8$ & $1.2-4.7$ & $1.8-7.5$ & $6.8-20.8$ \\
\hline Reference values $^{6}$ & & & 1.1 & n.a. & 5 & 15 \\
\hline \multicolumn{7}{|l|}{ Female } \\
\hline Austria & $>13$ & 1,422 & $0.8 \pm 1.0$ & $1.5 \pm 1.9$ & $1.7 \pm 1.6$ & $9.6 \pm 6.5$ \\
\hline Belgium & $15-18$ & n.a. & n.a. & n.a. & n.a. & n.a. \\
\hline Denmark & $15-18$ & 121 & $0.7 \pm 0.3$ & $3.0 \pm 2.7$ & $2.1 \pm 1.7$ & $6.0 \pm 1.9$ \\
\hline Germany & $15-18$ & 605 & $1.6 \pm 0.8$ & $4.7 \pm 2.8$ & $2.0 \pm 1.3$ & $15.5 \pm 8.5$ \\
\hline Ireland & $13-17$ & 217 & $0.3 \pm 0.3$ & $2.3 \pm 1.9$ & $2.3 \pm 2.2$ & $8.0 \pm 9.7$ \\
\hline Italy & $15-18$ & 47 & $0.8 \pm 0.4$ & $2.2 \pm 1.0$ & $3.0 \pm 2.0$ & $11.0 \pm 3.8$ \\
\hline Norway & $15-18$ & 62 & $1.6 \pm 1.0^{5}$ & n.a. & $7.1 \pm 6.6$ & n.a. \\
\hline Poland & $15-18$ & 175 & $1.6 \pm 2.6$ & $4.2 \pm 6.8$ & $3.0 \pm 3.1$ & $12.9 \pm 7.3$ \\
\hline Slovenia & $15-18$ & 1,214 & $1.1 \pm 0.9$ & $2.7 \pm 2.1$ & $3.0 \pm 3.0$ & $14.0 \pm 10.0$ \\
\hline Spain & $15-18$ & 57 & $0.6 \pm 0.1$ & $1.0 \pm 0.3$ & $1.5 \pm 0.5$ & $8.3 \pm 2.0$ \\
\hline The Netherlands & $15-18$ & 181 & $0.8 \pm 0.7$ & n.a. & $3.3 \pm 1.8$ & $11.7 \pm 5.2$ \\
\hline United Kingdom & $16-24$ & 248 & $0.6 \pm 0.4$ & $1.7 \pm 1.7$ & $2.0 \pm 1.2$ & $8.0 \pm 3.7$ \\
\hline All countries (min.-max.) & & & $0.3-1.6$ & $1.0-4.7$ & $1.5-7.1$ & $6.0-15.5$ \\
\hline Reference values ${ }^{6}$ & & & 0.9 & n.a. & 5 & 12 \\
\hline
\end{tabular}

${ }^{1}$ Retinol equivalent $\left(=1 \mathrm{mg}\right.$ retinol $=6 \mathrm{mg}$ all-trans- $\beta$-carotene $=12 \mathrm{mg}$ other carotenoids) ${ }^{2}$ RRR- $\alpha$-Tocopherol equivalent $(=\mathrm{mg}$ a-tocopherol $+\mathrm{mg} \beta$-tocopherol $\times 0.5+\mathrm{mg}$-tocopherol $\times 0.25+\mathrm{mg}$ a-tocotrienol $\times 0.33$ ); ${ }^{3}$ niacin equivalent $(=1 \mathrm{mg}$ niacin $=60 \mathrm{mg}$ tryptophan $) ;{ }^{4}$ folate equivalent $(1 \mu \mathrm{g}$ food folate $=0.5 \mu \mathrm{g}$ folic acid $(P G A)=0.6 \mu$ g folic acid taken with meals); ${ }^{5}$ values refer to a-tocopherol, only; ${ }^{6} \mathrm{D}-\mathrm{A}-\mathrm{CH}, 2000$, reference values refer to age groups $15-18$ years; ${ }^{7}$ niacin; n.a. $=$ not available. 


\begin{tabular}{|c|c|c|c|c|c|c|}
\hline $\begin{array}{l}\text { Thiamine } \\
\mathrm{mg}\end{array}$ & $\begin{array}{l}\text { Riboflavin } \\
\mathrm{mg}\end{array}$ & $\begin{array}{l}\mathrm{Niacin}^{3} \\
\mathrm{mg}\end{array}$ & $\begin{array}{l}\text { Vitamin } B_{6} \\
\mathrm{mg}\end{array}$ & $\begin{array}{l}\text { Folate }^{4} \\
\mu \mathrm{g}\end{array}$ & $\begin{array}{l}\text { Cobalamin } \\
\mu \mathrm{g}\end{array}$ & $\begin{array}{l}\text { Ascorbic acid } \\
\mathrm{mg}\end{array}$ \\
\hline $1.4 \pm 0.7$ & $1.5 \pm 0.8$ & $38.0 \pm 17.0$ & $2.4 \pm 2.5$ & $195 \pm 88$ & $5.3 \pm 3.1$ & $91 \pm 96$ \\
\hline n.a. & n.a. & n.a. & n.a. & n.a. & n.a. & $90 \pm 42$ \\
\hline $1.4 \pm 0.4$ & $1.8 \pm 0.7$ & $28.0 \pm 7.9$ & $1.5 \pm 0.4$ & $306 \pm 96$ & $5.1 \pm 2.2$ & $100 \pm 60$ \\
\hline $2.3 \pm 1.4$ & $2.6 \pm 1.6$ & $41.4 \pm 19.8$ & $3.1 \pm 1.9$ & $360 \pm 214$ & $6.4 \pm 2.8$ & $167 \pm 108$ \\
\hline $2.3 \pm 2.4$ & $2.6 \pm 2.5$ & $43.3 \pm 14.6$ & $3.1 \pm 2.5$ & $320 \pm 157$ & $6.0 \pm 3.2$ & $98 \pm 87$ \\
\hline $1.4 \pm 0.3$ & $1.7 \pm 0.4$ & $40.0 \pm 8.7$ & $2.3 \pm 0.6$ & $336 \pm 104$ & n.a. & $129 \pm 74$ \\
\hline $2.1 \pm 0.9$ & $2.7 \pm 1.2$ & $23.0 \pm 12.0$ & n.a. & n.a. & n.a. & $146 \pm 105$ \\
\hline $1.8 \pm 0.7$ & $2.3 \pm 1.4$ & n.a. & $2.5 \pm 1.0$ & $365 \pm 192$ & $6.2 \pm 11.3$ & $120 \pm 80$ \\
\hline $1.5 \pm 0.9$ & $2.0 \pm 1.2$ & $37.0 \pm 22.0$ & $2.3 \pm 1.4$ & $288 \pm 176$ & $7.5 \pm 5.9$ & $201 \pm 164$ \\
\hline $1.7 \pm 0.3$ & $1.9 \pm 0.4$ & $23.6 \pm 3.9$ & $2.2 \pm 0.4$ & $237 \pm 38$ & $4.9 \pm 1.3$ & $73 \pm 28$ \\
\hline $1.3 \pm 0.6$ & $1.6 \pm 0.7$ & n.a. & $1.8 \pm 0.7$ & $190 \pm 70$ & n.a. & $71 \pm 49$ \\
\hline $1.8 \pm 0.8$ & $1.9 \pm 1.2$ & $12.2 \pm 8.8^{7}$ & $2.7 \pm 1.4$ & $281 \pm 140$ & $5.6 \pm 3.2$ & $80 \pm 56$ \\
\hline $1.3-2.3$ & $1.5-2.6$ & $12.2-43.3$ & $1.5-3.1$ & $190-365$ & $4.9-7.5$ & $71-201$ \\
\hline 1.3 & 1.5 & 17 & 1.6 & 400 & 3 & 100 \\
\hline $1.0 \pm 0.5$ & $1.1 \pm 0.6$ & $26.0 \pm 12.0$ & $1.7 \pm 1.7$ & $154 \pm 69$ & $3.5 \pm 2.2$ & $90 \pm 93$ \\
\hline n.a. & n.a. & n.a. & n.a. & n.a. & n.a. & $88 \pm 38$ \\
\hline $1.0 \pm 0.3$ & $1.4 \pm 0.5$ & $20.0 \pm 5.8$ & $1.2 \pm 0.4$ & $262 \pm 87$ & $3.8 \pm 2.0$ & $103 \pm 63$ \\
\hline $1.7 \pm 1.0$ & $2.0 \pm 1.2$ & $29.0 \pm 14.2$ & $2.4 \pm 1.5$ & $298 \pm 159$ & $4.0 \pm 1.8$ & $164 \pm 84$ \\
\hline $1.9 \pm 3.2$ & $2.0 \pm 3.1$ & $30.5 \pm 10.8$ & $2.5 \pm 3.1$ & $230 \pm 129$ & $4.2 \pm 3.1$ & $92 \pm 100$ \\
\hline $1.1 \pm 0.3$ & $1.4 \pm 0.3$ & $29.6 \pm 8.0$ & $1.9 \pm 0.6$ & $254 \pm 70$ & n.a. & $107 \pm 56$ \\
\hline $1.9 \pm 1.0$ & $2.3 \pm 1.2$ & $21.0 \pm 13.0$ & n.a. & n.a. & n.a. & $149 \pm 85$ \\
\hline $1.1 \pm 0.5$ & $1.5 \pm 0.8$ & n.a. & $1.7 \pm 0.7$ & $244 \pm 106$ & $3.7 \pm 6.1$ & $94 \pm 73$ \\
\hline $1.1 \pm 0.6$ & $1.5 \pm 0.9$ & $27.0 \pm 14.0$ & $1.8 \pm 1.0$ & $242 \pm 141$ & $5.2 \pm 4.0$ & $205 \pm 178$ \\
\hline $1.3 \pm 0.2$ & $1.5 \pm 0.2$ & $18.0 \pm 3.1$ & $1.7 \pm 0.3$ & $196 \pm 31$ & $4.0 \pm 0.6$ & $75 \pm 28$ \\
\hline $1.1 \pm 0.7$ & $1.4 \pm 0.6$ & n.a. & $1.4 \pm 0.5$ & $166 \pm 58$ & n.a. & $81 \pm 49$ \\
\hline $1.3 \pm 0.5$ & $1.2 \pm 0.6$ & $7.3 \pm 5.2^{7}$ & $1.9 \pm 0.9$ & $196 \pm 99$ & $3.6 \pm 2.0$ & $67 \pm 55$ \\
\hline $1.0-1.9$ & $1.2-2.3$ & $7.3-30.5$ & $1.2-2.5$ & $154-298$ & $3.5-5.2$ & $67-205$ \\
\hline 1.0 & 1.1 & 13 & 1.2 & 400 & 3 & 100 \\
\hline
\end{tabular}


Table 7.31. Intake of selected vitamins (min.-max.) in adolescents in the four regions

\begin{tabular}{|c|c|c|c|c|}
\hline Region/sex & $\begin{array}{l}\text { Vitamin } \mathrm{B}_{6} \\
\mathrm{mg}\end{array}$ & $\begin{array}{l}\text { Folate } \\
\mu g^{5}\end{array}$ & $\begin{array}{l}\text { Cobalamin } \\
\mu \mathrm{g}\end{array}$ & $\begin{array}{l}\text { Vitamin D } \\
\mu g\end{array}$ \\
\hline \multicolumn{5}{|l|}{ North ${ }^{1}$} \\
\hline Male & $1.5^{6}$ & $306^{6}$ & $5.1^{6}$ & $2.6-7.5$ \\
\hline Female & $1.2^{6}$ & $262^{6}$ & $3.8^{6}$ & $2.1-7.1$ \\
\hline \multicolumn{5}{|l|}{ South ${ }^{2}$} \\
\hline Male & $2.2-2.3$ & $237-336$ & $4.9^{7}$ & $1.8-3.3$ \\
\hline Female & $1.7-1.9$ & $196-254$ & $4.0^{7}$ & $1.5-3.0$ \\
\hline \multicolumn{5}{|c|}{ Central and East ${ }^{3}$} \\
\hline Male & $2.3-3.1$ & $195-365$ & $5.3-7.5$ & $2.0-5.5$ \\
\hline Female & $1.7-2.4$ & $154-298$ & $3.5-5.2$ & $1.7-3.0$ \\
\hline \multicolumn{5}{|l|}{ West ${ }^{4}$} \\
\hline Male & $1.8-3.1$ & $190-320$ & $4.2-6.0^{8}$ & $3.0-4.4$ \\
\hline Female & $1.4-2.5$ & $166-230$ & $3.6-4.2^{8}$ & $2.0-3.3$ \\
\hline \multicolumn{5}{|c|}{$\begin{array}{l}{ }^{1} \text { North: NO, DK; }{ }^{2} \text { South: ES, IT; }{ }^{3} \text { Central and East: } \mathrm{AT}, \mathrm{DE}, \mathrm{PL}, \mathrm{SL} ;{ }^{4} \text { West: UK, NL, IR; }{ }^{5} \text { folate equivalent } \\
\left(1 \mu \mathrm{g} \text { food folate }=0.5 \mu \mathrm{g} \text { folic acid }(\mathrm{PGA})=0.6 \mu \mathrm{g} \text { folic acid taken with meals); }{ }^{6} \text { only } \mathrm{DK} ;{ }^{7} \text { only } \mathrm{ES}\right. \\
{ }^{8} \text { only UK and IR. }\end{array}$} \\
\hline
\end{tabular}

\section{Minerals}

Comparison of Countries

The intake of sodium was between 2.9 and $6.5 \mathrm{~g} /$ day in male adolescents and between 2.2 and $4.5 \mathrm{~g} /$ day in female adolescents. In order to prevent chronic diseases it is recommended by the WHO [WHO, 2003] not to ingest more than $2 \mathrm{~g}$ sodium per day. Every country was above this recommendation.

With the exception of female adolescents from Slovenia and Austria the potassium intake was within the recommendation [D-A-CH, 2000].

The intake of calcium was between 806 and 1,447 mg/day in male adolescents and between 645 and 1,040 mg/day in female adolescents. Only male adolescents from Denmark, Germany, Norway and Slovenia were within the recommended calcium intake levels [D-A-CH, 2000]. Compared with the recommendation for the Nordic countries [NNR, 2004], all male adolescents met the recommendation of $800 \mathrm{mg} /$ day. In the female group this recommendation was not met by adolescents from Austria, Belgium, France, Ireland, Poland and the United Kingdom. 
The intake of phosphorus was between 1,413 and 1,705 $\mathrm{mg} /$ day in male adolescents and therefore within the recommendation [D-A-CH, 2000]. In the female group the intake was between 962 and 1,356 mg/day, but only female adolescents from Slovenia, Spain and The Netherlands met the recommendation [D-A-CH, 2000].

Apart from Germany, Norway (only male) and Slovenia (both male and female) the intake of magnesium was below the recommendation [D-A-CH, 2000].

The intake of iron was between 10.2 and $19 \mathrm{mg} /$ day in male adolescents and between 7.8 and $14.0 \mathrm{mg} /$ day in female adolescents. With the exception of Denmark and The Netherlands every country met the recommended $12 \mathrm{mg} /$ day [D-A-CH, 2000] in the male group. The recommendation for women is higher (15 mg/day) because of iron losses during menstruation. The intake in the female group was in most countries below the recommendation [D-A-CH, 2000].

The recommended intake for zinc $(10 \mathrm{mg} /$ day for male and $7 \mathrm{mg} /$ day for female adolescents [D-A-CH, 2000]) was met by all participating countries except for the United Kingdom.

The intake of iodine was between 93 and $221 \mu \mathrm{g} /$ day in male adolescents and between 115 and $182 \mu \mathrm{g} /$ day in female adolescents. With the exception of Ireland all participating countries met the recommendation of $150 \mu \mathrm{g}$ iodine per day in the male group [Eurodiet, 2000]. In the female group, the intake was in general lower. Only female adolescents from Denmark and Slovenia were within the recommendation.

The recommended intake for copper is between 1.0 and $1.5 \mathrm{mg} /$ day [D-A-CH, 2000]. In the male group every country except for The Netherlands and the United Kingdom was above this recommended level. In the female group every country met the recommendation except for Ireland and the United Kingdom where the intake was below and Slovenia where the intake was above the recommended level.

The intake of manganese was between 2.5 and $6.1 \mathrm{mg} /$ day in male adolescents and between 1.9 and $4.4 \mathrm{mg} /$ day in female adolescents. Female Irish as well as male Polish and Slovenian adolescents did not meet the recommendation [D-A-CH, 2000].

Data concerning the selenium intake were available only from Denmark, Ireland, Italy, Poland and The Netherlands. The intake was in every country within the recommendation [D-A-CH, 2000] (cf. table 7.32).

\section{Comparison of Regions}

Table 7.33 shows the intake of selected minerals (min.-max.) in the four regions. The intake of calcium was lower in female than in male adolescents in all regions. The intake was higher in the North region than in the other regions. Concerning the intake of phosphorus no big difference between the regions could be observed. The intake values of iron ranged in average from $8 \mathrm{mg} /$ day in female adolescents of the North region to $19 \mathrm{mg} /$ day in male adolescents from the Central and East region. In general, the intake of zinc was lower in the West region than in the other regions. Data for iodine were only available in three out of the four regions. In comparison 
Table 7.32. Mineral intake (mean \pm SD) in adolescents of European countries (male and female)

\begin{tabular}{|c|c|c|c|c|c|c|}
\hline & $\begin{array}{l}\text { Age } \\
\text { years }\end{array}$ & $\mathrm{n}$ & $\begin{array}{l}\text { Sodium } \\
\mathrm{g}\end{array}$ & $\begin{array}{l}\text { Potassium } \\
\mathrm{g}\end{array}$ & $\begin{array}{l}\text { Calcium } \\
\mathrm{mg}\end{array}$ & $\begin{array}{l}\text { Phosphorus } \\
\mathrm{mg}\end{array}$ \\
\hline \multicolumn{7}{|l|}{ Male } \\
\hline Austria & $>13$ & 1,527 & n.a. & $2.4 \pm 0.9$ & $863 \pm 478$ & n.a. \\
\hline Belgium & $15-18$ & n.a. & n.a. & n.a. & $869 \pm 358$ & n.a. \\
\hline Denmark & $15-18$ & 78 & $4.0 \pm 1.1$ & $3.3 \pm 1.0$ & $1,209 \pm 487$ & $1,525 \pm 472$ \\
\hline France & $15-18$ & 181 & $3.5 \pm 0.2$ & n.a. & $974 \pm 46$ & n.a. \\
\hline Germany & $15-18$ & 598 & $3.6 \pm 1.2$ & $3.7 \pm 1.2$ & $1,283 \pm 446$ & n.a. \\
\hline Ireland & $13-17$ & 224 & $2.9 \pm 0.8$ & $3.0 \pm 0.9$ & $1,070 \pm 409$ & $1,413 \pm 430$ \\
\hline Italy & $15-18$ & 52 & $6.5 \pm 2.2$ & $3.4 \pm 1.0$ & $1,001 \pm 332$ & $1,605 \pm 372$ \\
\hline Norway & $15-18$ & 72 & n.a. & n.a. & $1,447 \pm 688$ & n.a. \\
\hline Poland & $15-18$ & 174 & $5.7 \pm 2.0$ & $4.4 \pm 1.6$ & $806 \pm 519$ & $1,646 \pm 597$ \\
\hline Slovenia & $15-18$ & 1,010 & $4.5 \pm 2.8$ & $4.0 \pm 2.3$ & $1,238 \pm 783$ & $1,705 \pm 1000$ \\
\hline Spain & $15-18$ & 61 & $3.5 \pm 0.8$ & $3.0 \pm 0.5$ & $941 \pm 252$ & $1,596 \pm 323$ \\
\hline The Netherlands & $15-18$ & 180 & n.a. & $3.6 \pm 1.2$ & $1,086 \pm 473$ & $1,697 \pm 523$ \\
\hline United Kingdom & $16-24$ & 150 & $3.5 \pm 1.4$ & $3.0 \pm 1.1$ & $1,032 \pm 535$ & $1,468 \pm 541$ \\
\hline All countries (min.-max.) & & & $2.9-6.5$ & $2.4-4.4$ & $806-1,447$ & $1,413-1,705$ \\
\hline Reference values ${ }^{2}$ & & & $0.55 /<2^{4}$ & 2 & 1,200 & 1,250 \\
\hline \multicolumn{7}{|l|}{ Female } \\
\hline Austria & $>13$ & 1,422 & n.a. & $1.9 \pm 0.8$ & $710 \pm 395$ & n.a. \\
\hline Belgium & $15-18$ & n.a. & n.a. & n.a. & $700 \pm 216$ & n.a. \\
\hline Denmark & $15-18$ & 121 & $3.2 \pm 0.9$ & $2.6 \pm 0.8$ & $1,040 \pm 382$ & $1,194 \pm 362$ \\
\hline France & $15-18$ & 222 & $2.4 \pm 0.1$ & n.a. & $732 \pm 25$ & n.a. \\
\hline Germany & $15-18$ & 605 & $2.5 \pm 0.9$ & $3.0 \pm 1.0$ & $1,022 \pm 369$ & n.a. \\
\hline Ireland & $13-17$ & 217 & $2.2 \pm 0.7$ & $2.3 \pm 0.7$ & $738 \pm 328$ & $1,000 \pm 331$ \\
\hline Italy & $15-18$ & 47 & $4.5 \pm 1.3$ & $2.7 \pm 0.6$ & $803 \pm 181$ & $1,208 \pm 272$ \\
\hline Norway & $15-18$ & 62 & n.a. & n.a. & $1,025 \pm 406$ & n.a. \\
\hline Poland & $15-18$ & 175 & $3.6 \pm 1.4$ & $1.2 \pm 3.3$ & $678 \pm 435$ & $1,124 \pm 455$ \\
\hline Slovenia & $15-18$ & 1,214 & $3.6 \pm 2.3$ & $3.3 \pm 1.9$ & $1,018 \pm 595$ & $1,265 \pm 702$ \\
\hline Spain & $15-18$ & 57 & $2.6 \pm 0.8$ & $2.5 \pm 0.4$ & $810 \pm 206$ & $1,272 \pm 206$ \\
\hline The Netherlands & $15-18$ & 181 & n.a. & $3.0 \pm 0.8$ & $911 \pm 396$ & $1,356 \pm 379$ \\
\hline United Kingdom & $16-24$ & 248 & $2.3 \pm 0.8$ & $2.2 \pm 0.7$ & $645 \pm 282$ & $962 \pm 317$ \\
\hline All countries (min.-max.) & & & $2.2-4.5$ & $1.2-3.3$ & $645-1,040$ & $962-1,356$ \\
\hline Reference values $^{2}$ & & & $0.55 /<2^{4}$ & 2 & 1,200 & 1,250 \\
\hline
\end{tabular}

${ }^{1}$ lodised salt was not taken into account in each country; ${ }^{2} \mathrm{D}-\mathrm{A}-\mathrm{CH}, 2000$, reference values refer to age groups 15-18 years; ${ }^{3}$ Eurodiet, 2000; ${ }^{4}$ WHO, 2003; n.a. $=$ not available. 


\begin{tabular}{|c|c|c|c|c|c|c|}
\hline $\begin{array}{l}\text { Magnesium } \\
\mathrm{mg}\end{array}$ & $\begin{array}{l}\text { Iron } \\
\text { mg }\end{array}$ & $\begin{array}{l}\text { Zinc } \\
\mathrm{mg}\end{array}$ & $\begin{array}{l}\text { lodine } \\
\mu g^{1}\end{array}$ & $\begin{array}{l}\text { Copper } \\
\mathrm{mg}\end{array}$ & $\begin{array}{l}\text { Manganese } \\
\mathrm{mg}\end{array}$ & $\begin{array}{l}\text { Selenium } \\
\mu \mathrm{g}\end{array}$ \\
\hline $302 \pm 110$ & $12.6 \pm 4.5$ & $12.2 \pm 4.4$ & $172 \pm 72$ & n.a. & n.a. & n.a. \\
\hline n.a. & $12.5 \pm 2.9$ & n.a. & n.a. & n.a. & n.a. & n.a. \\
\hline $338 \pm 101$ & $10.2 \pm 2.8$ & $11.7 \pm 3.3$ & $221 \pm 74$ & n.a. & n.a. & $39 \pm 11$ \\
\hline n.a. & n.a. & n.a. & n.a. & n.a. & n.a. & n.a. \\
\hline $440 \pm 132$ & $15.8 \pm 5.1$ & $12.9 \pm 4.4$ & $93 \pm 33$ & n.a. & n.a. & n.a. \\
\hline $271 \pm 92$ & $14.1 \pm 11.5$ & $10.2 \pm 3.7$ & $185 \pm 117$ & $2.3 \pm 17.2$ & $2.5 \pm 1.5$ & $49 \pm 102$ \\
\hline $260 \pm 60$ & $14.7 \pm 3.6$ & $14.5 \pm 3.6$ & n.a. & $1.7 \pm 0.5$ & n.a. & $50 \pm 15$ \\
\hline $415 \pm 167$ & $15.1 \pm 7.9$ & n.a. & n.a. & n.a. & n.a. & n.a. \\
\hline $388 \pm 141$ & $16.7 \pm 7.8$ & $14.5 \pm 5.0$ & $161 \pm 71$ & $1.7 \pm 0.7$ & $6.1 \pm 2.2$ & $59 \pm 27$ \\
\hline $467 \pm 282$ & $19.0 \pm 11.0$ & $15.2 \pm 8.5$ & $196 \pm 114$ & $2.5 \pm 1.4$ & $5.6 \pm 3.9$ & n.a. \\
\hline $309 \pm 47$ & $13.4 \pm 1.6$ & $10.8 \pm 1.7$ & n.a. & n.a. & n.a. & n.a. \\
\hline $328 \pm 114$ & $11.3 \pm 3.8$ & $10.4 \pm 3.4$ & n.a. & $1.2 \pm 0.4$ & n.a. & $44 \pm 17$ \\
\hline $270 \pm 94$ & $12.1 \pm 5.1$ & $9.3 \pm 3.3$ & $196 \pm 109$ & $1.2 \pm 0.4$ & $2.7 \pm 1.2$ & n.a. \\
\hline $260-467$ & $10.2-19.0$ & $9.3-15.2$ & $93-221$ & $1.2-3.4$ & $2.5-6.1$ & $39-59$ \\
\hline 400 & 12 & 10.0 & $150^{3}$ & $1.0-2.5$ & $2-5$ & $30-70$ \\
\hline $244 \pm 86$ & $9.6 \pm 3.4$ & $9.1 \pm 3.4$ & $140 \pm 61$ & n.a. & n.a. & n.a. \\
\hline n.a. & $9.6 \pm 2.3$ & n.a. & n.a. & n.a. & n.a. & n.a. \\
\hline $264 \pm 78$ & $7.8 \pm 2.3$ & $8.9 \pm 2.6$ & $175 \pm 56$ & n.a. & n.a. & $31 \pm 12$ \\
\hline n.a. & n.a. & n.a. & n.a. & n.a. & n.a. & n.a. \\
\hline $347 \pm 103$ & $12.0 \pm 3.9$ & $9.3 \pm 3.1$ & $78 \pm 29$ & n.a. & n.a. & n.a. \\
\hline $196 \pm 62$ & $10.7 \pm 11.6$ & $7.2 \pm 3.1$ & $115 \pm 75$ & $0.8 \pm 0.4$ & $1.9 \pm 0.8$ & $30 \pm 13$ \\
\hline $198 \pm 36$ & $10.8 \pm 2.2$ & $11.0 \pm 2.7$ & n.a. & $1.2 \pm 0.3$ & n.a. & $38 \pm 15$ \\
\hline $316 \pm 112$ & $12.7 \pm 6.5$ & n.a. & n.a. & n.a. & n.a. & n.a. \\
\hline $273 \pm 101$ & $10.7 \pm 5.2$ & $9.4 \pm 3.6$ & $115 \pm 55$ & $1.2 \pm 0.5$ & $4.4 \pm 1.7$ & $37 \pm 20$ \\
\hline $369 \pm 204$ & $14.0 \pm 8.0$ & $10.8 \pm 5.7$ & $182 \pm 102$ & $2.1 \pm 1.1$ & $4.2 \pm 2.8$ & n.a. \\
\hline $264 \pm 45$ & $10.9 \pm 1.6$ & $8.5 \pm 0.9$ & n.a. & n.a. & n.a. & n.a. \\
\hline $260 \pm 75$ & $9.7 \pm 3.1$ & $8.4 \pm 2.6$ & n.a. & $1.0 \pm 0.3$ & n.a. & $36 \pm 12$ \\
\hline $186 \pm 61$ & $8.3 \pm 3.3$ & $6.4 \pm 2.4$ & $126 \pm 74$ & $0.8 \pm 0.3$ & $2.0 \pm 0.9$ & n.a. \\
\hline $186-369$ & $7.8-14.0$ & $6.4-11.0$ & 115-182 & $0.8-2.1$ & $1.9-4.4$ & $30-38$ \\
\hline 350 & 15 & 7 & $150^{3}$ & $1.0-1.5$ & $2-5$ & $30-70$ \\
\hline
\end{tabular}


to the other regions a lower intake was observed in the Central and East region. Selenium intake values were only reported from one to two countries within the regions.

\subsection{Energy and Nutrient Intake in European Adults}

\section{Background}

Twenty-one of the participating countries of the European Nutrition and Health report 2009 could provide data concerning the energy and nutrient intake of adults. Due to the fact that different dietary assessment methods were used, attention should be paid when comparing the data (cf. table 7.34).

\section{Energy and Macronutrients}

\section{Comparison of Countries}

The daily energy intake was between 8.5 and 13.9 MJ in males and between 6.3 and 11.4 MJ in females. The reference intake values [D-A-CH, 2000] range from 10.5 to $12.5 \mathrm{MJ} /$ day in males and from 8.5 to $10.0 \mathrm{MJ} /$ day in females. The intake was in most of the participating countries below these reference values. This may partly be due to under-reporting of food intake. Regarding the high prevalence of overweight and obesity in adults, it can be assumed that most adults did not meet the recommended physical activity level with according low energy expenditure.

The share of protein in total energy intake was between 13.5 and $18.5 \% \mathrm{E}$ in males and between 13.1 and $19.3 \% \mathrm{E}$ in females and was therefore within or slightly above the recommended range of the WHO [WHO, 2003] in the participating countries.

The share of carbohydrates in total energy intake was between 36.8 and $51.0 \% \mathrm{E}$ in males and between 37.7 and 51.8\% E in females. Only males from Norway and females from the Czech Republic, Finland, Norway, Poland and Portugal were within the recommended range of the WHO of $50-75 \% \mathrm{E} /$ day [WHO, 2007] whereas the share of sucrose in total energy intake was above the recommendation in Lithuania, Spain and the United Kingdom, in both groups males and females, as well as in Polish men and Austrian and Finnish women [WHO, 2003]. The dietary fiber intake was in most of the participating countries lower than the recommendation of Eurodiet [Eurodiet, 2000]. Only German, Norwegian and Polish males met the recommendation.

The share of fat in total energy intake was between 28.4 and $45.0 \% \mathrm{E}$ in males and between 29.9 and $47.2 \% \mathrm{E}$ in females. Thus, the majority of the participating countries were above the recommended range of the WHO [WHO, 2003]. In general, the fatty acid pattern did not meet the recommendations of the WHO 2009. The share of saturated fatty acids (SFA) in total energy intake was in general above the recommendation $(<10 \% \mathrm{E})$ and the share of polyunsaturated fatty acids (PUFA) in total energy intake was below the recommended intake range $(6-11 \% \mathrm{E})$ in most of the 
Table 7.33. Intake of selected minerals (min.-max.) in adolescents in the four regions

\begin{tabular}{|c|c|c|c|c|c|c|}
\hline Region/sex & $\begin{array}{l}\text { Calcium } \\
\mathrm{mg}\end{array}$ & $\begin{array}{l}\text { Phosphorus } \\
\mathrm{mg}\end{array}$ & $\begin{array}{l}\text { Iron } \\
\text { mg }\end{array}$ & $\begin{array}{l}\text { Zinc } \\
\mu \mathrm{g}\end{array}$ & $\begin{array}{l}\text { lodine } \\
\mu \mathrm{g}\end{array}$ & $\begin{array}{l}\text { Selenium } \\
\mu \mathrm{g}\end{array}$ \\
\hline \multicolumn{7}{|l|}{ North $^{1}$} \\
\hline Male & $1,209-1,447$ & $1,525^{5}$ & $10.2-15.1$ & $11.7^{5}$ & $221^{5}$ & $39^{5}$ \\
\hline Female & $1,025-1,040$ & $1,194^{5}$ & $7.8-12.7$ & $8.9^{5}$ & $175^{5}$ & $31^{5}$ \\
\hline \multicolumn{7}{|l|}{ South $^{2}$} \\
\hline Male & $941-1,001$ & $1,596-1,605$ & $13.4-14.7$ & $10.8-14.5$ & n.a. & $50^{6}$ \\
\hline Female & $803-810$ & $1,208-1,272$ & $10.8-10.9$ & $8.5-11.0$ & n.a. & $38^{6}$ \\
\hline \multicolumn{7}{|c|}{ Central and East ${ }^{3}$} \\
\hline Male & $806-1,283$ & $1,646-1,705^{7}$ & $12.6-19.0$ & $12.2-15.2$ & $93-196$ & $59^{8}$ \\
\hline Female & $678-1,021$ & $1,124-1,265^{7}$ & $9.6-14.0$ & $9.1-10.8$ & $78-182$ & $37^{8}$ \\
\hline \multicolumn{7}{|l|}{ West $^{4}$} \\
\hline Male & $869-1,086$ & $1,413-1,697^{9}$ & $11.3-14.1^{10}$ & $9.3-10.4^{9}$ & $185-196^{11}$ & $44-49^{12}$ \\
\hline Female & $645-911$ & $962-1,356^{9}$ & $8.3-10.7^{10}$ & $6.4-8.4^{9}$ & $115-126^{11}$ & $30-36^{12}$ \\
\hline
\end{tabular}

participating countries [WHO, 2003, 2009b]. The intake of cholesterol was between 211 and $800 \mathrm{mg} /$ day in males and between 176 and $680 \mathrm{mg} /$ day in females. The intake was higher in males than in females. In the male group only Finnish, Greek and Dutch males as well as males aged 35-54 years from the United Kingdom were within the recommendation [WHO, 2003]. In the female group, females from Hungary, Italy, Lithuania, Poland, Portugal as well as Romania exceeded it (cf. table 7.35).

\section{Comparison of Regions}

Table 7.36 shows the intake of energy and macronutrients (min.-max.) in the four regions. Whereas there is no big difference between the regions concerning the energy intake and share of protein in total energy intake as well as the relatively low intake of dietary fibers, the share of carbohydrates in total energy intake is lower and the share of sucrose in total energy intake is higher in the South region than in the other regions. The highest as well as the lowest intake values of fat in total energy intake were observed in the South region $(28-47 \% \mathrm{E})$. 
Table 7.34. Methods and period of dietary assessment in adults of the participating countries (data available from 80,103 adults)

\begin{tabular}{|c|c|c|c|c|c|c|}
\hline Country & $\begin{array}{l}\text { Age group } \\
\text { years }\end{array}$ & Method & $\begin{array}{l}\text { Year of } \\
\text { survey }\end{array}$ & $\mathrm{n}$ & $\begin{array}{l}\text { Intake from } \\
\text { dietary } \\
\text { supplements } \\
\text { is included }\end{array}$ & Reference \\
\hline Austria & $19-64$ & 24-hour recall & 2007 & 2,123 & no & Elmadfa et al., 2008 \\
\hline Belgium & $19-64$ & 24-hour recall & 2004 & 3,249 & no & Devriese et al., 2006 \\
\hline Czech Republic & $19-64$ & n.a. & n.a. & 2,140 & n.a. & Cífková et al., 2004 \\
\hline Denmark & $19-64$ & 7-day dietary record & 2000-2002 & 2,769 & n.a. & Lyhne et al., 2005 \\
\hline Estonia & $19-64$ & 24-hour recall & 1997 & 2,012 & n.a. & $\begin{array}{l}1997 \text { National Dietary } \\
\text { Survey }\end{array}$ \\
\hline Finland & $19-64$ & 48-hour recall & 2007 & 1,576 & no & $\begin{array}{l}\text { The National Findiet } \\
2007 \text { Survey }\end{array}$ \\
\hline France & $19-64$ & $3 \times 24$-hour recall & $2006 / 2007$ & 2,351 & no & Castetbon et al., 2009 \\
\hline Germany & $19-64$ & $\begin{array}{l}\text { dietary history } \\
\text { interview }\end{array}$ & 2005-2007 & 10928 & no & $\begin{array}{l}\text { Max Rubner-Institut } \\
\text { (eds), 2008a, 2008b }\end{array}$ \\
\hline Greece & $\begin{array}{l}19-64 \\
22 \pm 2\end{array}$ & $\begin{array}{l}\text { FFQ } \\
\text { 24-hour recall }\end{array}$ & $\begin{array}{l}1994-1999 \\
1989-2001\end{array}$ & $\begin{array}{l}20,399 \\
951\end{array}$ & $\begin{array}{l}\text { no } \\
\text { yes }\end{array}$ & $\begin{array}{l}\text { EPIC study - Greek } \\
\text { cohort }^{1}\end{array}$ \\
\hline Hungary & $\geq 18$ & 3-day dietary record & $2003 / 2004$ & 1,179 & no & $\begin{array}{l}\text { Rodler et al., 2007; } \\
\text { Zajkás et al., 2007; } \\
\text { Bíró et al., } 2007\end{array}$ \\
\hline Ireland & $\begin{array}{l}18-64 \\
19-64\end{array}$ & $\begin{array}{l}\text { FFQ } \\
\text { 7-day dietary record } \\
\text { (estimated) }\end{array}$ & $\begin{array}{l}2007 \\
1997-1999\end{array}$ & $\begin{array}{l}7,813 \\
1,379\end{array}$ & $\begin{array}{l}\text { yes } \\
\text { yes }\end{array}$ & $\begin{array}{l}\text { SLÁN, } 2007 \\
\text { SLÁN, 1997/1998 }\end{array}$ \\
\hline Italy & $19-64$ & 7-day dietary record & n.a. & 1,461 & no & D’Amicis, 2000 \\
\hline Latvia & $19-64$ & 24-hour recall & 1997 & 2,308 & no & Pomerlau et al., 1999 \\
\hline Lithuania & $19-64$ & 24-hour recall & 2007 & 1,936 & no & unpubl. data ${ }^{2}$ \\
\hline Norway & $19-64$ & FFQ & 1997 & 2,196 & yes & Norkost, 1997 \\
\hline Poland & $19-64$ & 24-hour recall & 2000 & 2,440 & no & $\begin{array}{l}\text { Szponar et al., } 2000 \\
\text { (unpubl.) }\end{array}$ \\
\hline Portugal & $\geq 18$ & FFQ & n.a. & 2,389 & no & $\begin{array}{l}\text { EPIPorto. study held by } \\
\text { the Department of } \\
\text { Hygiene and } \\
\text { Epidemiology, } \\
\text { University of Porto } \\
\text { Medical School }\end{array}$ \\
\hline Romania & $19-64$ & personal interview & 2006 & 518 & no & $\begin{array}{l}\text { National synthesis. } \\
2006\end{array}$ \\
\hline
\end{tabular}


Table 7.34. Continued

\begin{tabular}{|c|c|c|c|c|c|c|}
\hline Country & $\begin{array}{l}\text { Age group } \\
\text { years }\end{array}$ & Method & $\begin{array}{l}\text { Year of } \\
\text { survey }\end{array}$ & $\mathrm{n}$ & $\begin{array}{l}\text { Intake from } \\
\text { dietary } \\
\text { supplements } \\
\text { is included }\end{array}$ & Reference \\
\hline Spain & $19-64$ & $2 \times 24$-hour recall & $2002-2003$ & 1,581 & no & $\begin{array}{l}\text { Serra Majem and } \\
\text { Ribas. 2007; Serra- } \\
\text { Majem et al, 2007; } \\
\text { Serra-Majem et al., } 2006\end{array}$ \\
\hline Sweden & $19-64$ & 7-day dietary record & 1997/1998 & 1,092 & no & Becker et al., 2002 \\
\hline The Netherlands & $19-64$ & 2-day dietary record & 1997/1998 & 3,948 & no & $\begin{array}{l}\text { Hulshof and van } \\
\text { Steveren, } 1991\end{array}$ \\
\hline United Kingdom & $\begin{array}{l}25-34 \\
35-44 \\
45-54 \\
55-64\end{array}$ & $\begin{array}{l}\text { n.a. } \\
\text { n.a. } \\
\text { n.a. } \\
\text { n.a. }\end{array}$ & $\begin{array}{l}2003-2005 \\
2003-2005 \\
2003-2005 \\
2003-2005\end{array}$ & $\begin{array}{l}414 \\
501 \\
388 \\
425\end{array}$ & $\begin{array}{l}\text { n.a. } \\
\text { n.a. } \\
\text { n.a. } \\
\text { n.a. }\end{array}$ & $\begin{array}{l}\text { Low income diet } \\
\text { and health survey, } \\
2003-2005^{3}\end{array}$ \\
\hline
\end{tabular}

1 unpulished data estimated for the purposes of the ENHR 2009 project; ${ }^{2}$ data provided by the Institute of Biochemical Research of Kaunas University of Medicine; ${ }^{3}$ data provided by the University of Southampton; n.a. = not available.

Concerning fatty acids the highest intake values of SFA were found in the Central and East region. The share of PUFA in total energy intake was relatively similar in the four regions. The intake of cholesterol was higher in the Central and East region than in the other regions (cf. table 7.37).

\section{Vitamins}

\section{Comparison of Countries}

The intake of retinol equivalents was between 0.5 and $2.2 \mathrm{mg} /$ day in males and between 0.5 and $2.0 \mathrm{mg} /$ day in females. Only in Greece, Hungary, Spain and the United Kingdom (25-54 years) the intake was below the recommendation [D-A-CH, 2000] in both males and females, as well as in Finland, Ireland and Latvia, but only in females. The daily intake of $\beta$-carotene was between 1.4 and $5.3 \mathrm{mg} /$ day in males and between 1.4 and $5.6 \mathrm{mg} /$ day in females.

With a vitamin D intake of $1.6-10.9$ and $1.2-10.1 \mu \mathrm{g} /$ day most of the countries were below the recommended intake of $5 \mu \mathrm{g} /$ day [D-A-CH, 2000]. Only Finnish, Lithuanian, Norwegian and Polish males as well as Finnish and Norwegian females met the recommendation. The recommended intake of the Nordic countries of $7.5 \mu \mathrm{g} /$ day $[\mathrm{NNR}, 2004]$ was only met by Norwegian adults. 
Table 7.35. Intake of energy and macronutrients (mean \pm SD) in adults of European countries (male and female)

\begin{tabular}{|c|c|c|c|c|}
\hline \multirow{2}{*}{$\begin{array}{l}\text { Age } \\
\text { years }\end{array}$} & \multirow[t]{2}{*}{$n$} & \multirow{2}{*}{$\begin{array}{l}\text { Energy } \\
\text { MJ }\end{array}$} & \multirow{2}{*}{$\begin{array}{l}\text { Protein } \\
\% E^{1}\end{array}$} & Carbohydrates, $\% \mathrm{E}^{1}$ \\
\hline & & & & total \\
\hline
\end{tabular}

Male

\begin{tabular}{|c|c|c|c|c|c|}
\hline Austria & $19-64$ & 778 & $9.0 \pm 3.1$ & $16.8 \pm 4.9$ & $42.5 \pm 10.5$ \\
\hline Belgium & $19-64$ & n.a. & $12.2 \pm 3.4$ & $15.1 \pm 2.8$ & $42.4 \pm 6.8$ \\
\hline Czech Republic & 19-64 & 1,064 & $12.4 \pm 3.7$ & $14.1 \pm 4.0$ & $49.5 \pm 10.5$ \\
\hline Denmark & $19-64$ & 1,283 & $10.6 \pm 2.9$ & $14.1 \pm 2.2$ & $44.1 \pm 6.4$ \\
\hline Estonia & $19-64$ & 899 & $9.6 \pm 4.8$ & $14.7 \pm 4.7$ & $42.8 \pm 13.9$ \\
\hline Finland & $19-64$ & 730 & $9.2 \pm 3.0$ & $16.8 \pm 3.7$ & $47.1 \pm 8.8$ \\
\hline France & 19-64 & 852 & $10.0 \pm 0.1$ & $16.3 \pm 0.1$ & $43.4 \pm 0.3$ \\
\hline Germany & 19-64 & 4,912 & $11.0 \pm 4.3$ & $14.6 \pm 3.2$ & $45.3 \pm 8.4$ \\
\hline Greece & 19-64 & 8,365 & $10.4 \pm 3.0$ & $14.1 \pm 1.7$ & $37.9 \pm 5.9$ \\
\hline Hungary & $\geq 18$ & 473 & $11.7 \pm 2.4$ & $14.7 \pm 2.0$ & $45.0 \pm 6.6$ \\
\hline Ireland & 19-64 & 662 & $11.0 \pm 3.1$ & $15.5 \pm 2.7$ & $43.5 \pm 6.4$ \\
\hline Italy & $19-64$ & 660 & $10.3 \pm 1.9$ & $16.2 \pm 2.6$ & n.a. \\
\hline Latvia & 19-64 & 1,065 & $10.8 \pm 5.0$ & $13.7 \pm 4.2$ & $42.4 \pm 11.8$ \\
\hline Lithuania & $19-64$ & 849 & $10.3 \pm 4.3$ & $16.5 \pm 5.2$ & $38.9 \pm 9.3$ \\
\hline Norway & $19-64$ & 1,050 & $11.1 \pm 3.9$ & $16.0 \pm 2.0$ & $51.0 \pm 6.0$ \\
\hline Poland & $19-64$ & 1,106 & $13.4 \pm 4.5$ & $13.5 \pm 3.1$ & $48.0 \pm 8.4$ \\
\hline Portugal & $19-64$ & 917 & $9.9 \pm 2.3$ & $17.6 \pm 2.4$ & $47.0 \pm 6.8$ \\
\hline Romania & $19-64$ & 177 & $13.9 \pm 5.2$ & $17.8 \pm 3.8$ & $43.3 \pm 9.4$ \\
\hline Spain & $19-64$ & 706 & $9.1 \pm 1.7$ & $18.5 \pm 2.4$ & $36.9 \pm 5.8$ \\
\hline Sweden & $19-64$ & 517 & $9.9 \pm 2.7$ & $15.7 \pm 2.2$ & $46.2 \pm 5.7$ \\
\hline The Netherlands & $19-64$ & 1,836 & $11.1 \pm 2.9$ & $14.7 \pm 3.1$ & $44.2 \pm 7.5$ \\
\hline \multirow[t]{4}{*}{ United Kingdom } & $25-34$ & 119 & $10.1 \pm 3.4$ & $15.5 \pm 3.2$ & $48.0 \pm 7.2$ \\
\hline & $35-44$ & 152 & $9.0 \pm 3.3$ & $15.5 \pm 3.3$ & $48.4 \pm 7.9$ \\
\hline & $45-54$ & 143 & $8.7 \pm 3.2$ & $16.5 \pm 4.0$ & $47.0 \pm 8.0$ \\
\hline & $55-64$ & 189 & $8.5 \pm 2.6$ & $17.1 \pm 3.7$ & $46.9 \pm 7.4$ \\
\hline All countries (min.-max.) & & & $8.5-13.9$ & $13.5-18.5$ & $36.8-51.0$ \\
\hline Reference values & & & $12.5 / 12.0 / 10.5^{2}$ & $10-15 \% \mathrm{E}^{3}$ & $50-75 \% \mathrm{E}^{4}$ \\
\hline
\end{tabular}

Female

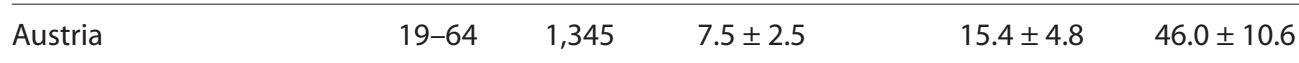




\begin{tabular}{|c|c|c|c|c|c|c|}
\hline & Dietary fiber & Fat $\% \mathrm{E}^{1}$ & SFA & MUFA & PUFA & Cholesterol \\
\hline sucrose & g & & $\% \mathrm{E}$ & $\% \mathrm{E}$ & $\% \mathrm{E}$ & $\mathrm{mg}$ \\
\hline
\end{tabular}

\begin{tabular}{|c|c|c|c|c|c|c|}
\hline $8.7 \pm 6.4$ & $19.5 \pm 9.9$ & $37.4 \pm 8.9$ & $14.3 \pm 4.3$ & $13.0 \pm 3.8$ & $7.9 \pm 3.7$ & $352.5 \pm 227.0$ \\
\hline n.a. & n.a. & $36.5 \pm 5.6$ & $14.6 \pm 3.0$ & $13.3 \pm 2.2$ & $6.7 \pm 2.1$ & n.a. \\
\hline n.a. & $18.7 \pm 37.5$ & $31.3 \pm 7.8$ & n.a. & n.a. & n.a. & $383.0 \pm 204.6$ \\
\hline $8.9 \pm 5.5$ & $20.9 \pm 7.9$ & $33.1 \pm 5.6$ & $14.2 \pm 2.8$ & $11.7 \pm 2.2$ & $4.8 \pm 0.9$ & n.a. \\
\hline n.a. & $20.7 \pm 12.2$ & $36.5 \pm 12.6$ & $14.1 \pm 5.8$ & $13.9 \pm 5.9$ & $5.6 \pm 3.0$ & $347.7 \pm 275.3$ \\
\hline $9.7 \pm 5.9$ & $24.0 \pm 11.0$ & $33.1 \pm 7.9$ & $12.9 \pm 4.1$ & $12.0 \pm 3.9$ & $5.9 \pm 2.2$ & $256.0 \pm 152.0$ \\
\hline n.a. & $18.7 \pm 0.4$ & $35.5 \pm 0.3$ & 14.4 & n.a. & n.a. & n.a. \\
\hline n.a. & $26.7 \pm 12.6$ & $35.9 \pm 7.7$ & n.a. & n.a. & n.a. & $395.8 \pm 209.0$ \\
\hline n.a. & n.a. & $45.0 \pm 5.5$ & $12.7 \pm 2.5$ & $21.9 \pm 4.0$ & $6.4 \pm 2.6$ & $282.9 \pm 116.2$ \\
\hline $7.9 \pm 5.2$ & $24.2 \pm 6.6$ & $38.2 \pm 5.9$ & $11.7 \pm 2.4$ & $12.5 \pm 2.9$ & $8.8 \pm 2.4$ & $463.0 \pm 177.2$ \\
\hline n.a. & $23.2 \pm 8.5$ & $34.8 \pm 5.7$ & n.a. & n.a. & n.a. & n.a. \\
\hline n.a. & $21.8 \pm 6.5$ & $35.0 \pm 6.0$ & $10.6 \pm 2.4$ & $12.8 \pm 3.6$ & $4.8 \pm 2.2$ & $378.4 \pm 133.3$ \\
\hline n.a. & n.a. & $42.7 \pm 11.7$ & n.a. & n.a. & n.a. & n.a. \\
\hline $10.8 \pm 5.6$ & $20.9 \pm 12.4$ & $44.9 \pm 9.1$ & $13.5 \pm 7.0$ & $16.9 \pm 8.0$ & $8.9 \pm 5.4$ & $477.9 \pm 264.7$ \\
\hline $9.0 \pm 6.0$ & $25.0 \pm 10.0$ & $31.0 \pm 6.0$ & $12.0 \pm 3.0$ & $11.0 \pm 2.0$ & $6.0 \pm 2.0$ & $344.0 \pm 143.0$ \\
\hline $11.0 \pm 5.2$ & $29.7 \pm 11.4$ & $36.7 \pm 7.9$ & $12.3 \pm 3.9$ & $16.2 \pm 4.3$ & $5.7 \pm 2.4$ & $553.2 \pm 382.4$ \\
\hline n.a. & $23.5 \pm 9.0$ & $28.4 \pm 4.7$ & $8.8 \pm 2.0$ & $12.3 \pm 2.3$ & $4.8 \pm 0.9$ & $324.4 \pm 106.1$ \\
\hline n.a. & n.a. & $38.9 \pm 9.0$ & $26.3 \pm 5.0$ & n.a. & n.a. & $800.0 \pm 430.0$ \\
\hline $15.1 \pm 4.2$ & $19.3 \pm 3.4$ & $39.9 \pm 3.8$ & $12.3 \pm 1.7$ & $17.6 \pm 2.3$ & $5.9 \pm 1.0$ & $375.4 \pm 53.7$ \\
\hline $8.6 \pm 4.3$ & $18.0 \pm 6.4$ & $34.4 \pm 5.2$ & $14.6 \pm 2.7$ & $12.7 \pm 2.1$ & $4.7 \pm 1.4$ & $345.4 \pm 117.1$ \\
\hline n.a. & $24.4 \pm 9.0$ & $36.5 \pm 6.4$ & $14.3 \pm 3.2$ & $12.8 \pm 2.8$ & $7.0 \pm 2.3$ & $250.0 \pm 125.0$ \\
\hline $15.1 \pm 8.1$ & $14.0 \pm 5.7$ & $36.5 \pm 6.5$ & $13.8 \pm 3.5$ & n.a. & n.a. & $317.3 \pm 172.4$ \\
\hline $15.9 \pm 11.3$ & $11.7 \pm 5.3$ & $36.1 \pm 7.1$ & $13.6 \pm 3.6$ & n.a. & n.a. & $268.8 \pm 148.5$ \\
\hline $14.8 \pm 9.2$ & $12.3 \pm 6.3$ & $36.4 \pm 7.4$ & $13.8 \pm 3.7$ & n.a. & n.a. & $210.9 \pm 107.7$ \\
\hline $13.4 \pm 8.4$ & $13.0 \pm 5.7$ & $36.0 \pm 7.5$ & $13.7 \pm 3.8$ & n.a. & n.a. & $319.1 \pm 161.7$ \\
\hline $7.9-15.9$ & $11.7-29.7$ & $28.4-45.0$ & $8.8-26.3$ & $11.0-21.9$ & $4.7-8.9$ & $210.9-800.0$ \\
\hline$<10 \% \mathrm{E}^{3}$ & $>25 \mathrm{~g} \cdot \mathrm{d}^{-1,6}$ & $15-30 \% \mathrm{E}^{3}$ & $<10 \% \mathrm{E}^{3}$ & & $6-11 \% \mathrm{E}^{5}$ & $<300 \mathrm{mg} \cdot \mathrm{d}^{-1,3}$ \\
\hline $10.9 \pm 6.5$ & $20.1 \pm 9.3$ & $37.3 \pm 8.8$ & $14.9 \pm 4.6$ & $12.3 \pm 3.6$ & $8.1 \pm 4.0$ & $282.8 \pm 175.8$ \\
\hline
\end{tabular}


Table 7.35. Continued

\begin{tabular}{|c|c|c|c|c|c|}
\hline & \multirow{2}{*}{$\begin{array}{l}\text { Age } \\
\text { years }\end{array}$} & \multirow[t]{2}{*}{$n$} & \multirow{2}{*}{$\begin{array}{l}\text { Energy } \\
\text { MJ }\end{array}$} & \multirow{2}{*}{$\begin{array}{l}\text { Protein } \\
\% \mathrm{E}^{1}\end{array}$} & \multirow{2}{*}{$\frac{\text { Carbohydrates, } \% \mathrm{E}^{1}}{\text { total }}$} \\
\hline & & & & & \\
\hline Belgium & n.a. & n.a. & $8.1 \pm 2.2$ & $16.0 \pm 3.1$ & $45.2 \pm 6.8$ \\
\hline Czech Republic & $19-64$ & 1,094 & $9.7 \pm 3.0$ & $14.7 \pm 7.7$ & $53.9 \pm 12.0$ \\
\hline Denmark & $19-64$ & 1,486 & $8.2 \pm 2.2$ & $14.3 \pm 2.3$ & $47.5 \pm 6.1$ \\
\hline Estonia & $19-64$ & 1,113 & $6.9 \pm 3.2$ & $15.0 \pm 4.4$ & $47.2 \pm 12.5$ \\
\hline Finland & $19-64$ & 846 & $6.8 \pm 2.0$ & $17.2 \pm 4.1$ & $50.2 \pm 8.3$ \\
\hline France & $19-64$ & 1,499 & $7.2 \pm 0.1$ & $17.0 \pm 0.1$ & $44.4 \pm 0.2$ \\
\hline Germany & $19-64$ & 6,016 & $8.1 \pm 2.5$ & $14.4 \pm 2.6$ & $48.7 \pm 7.4$ \\
\hline Greece & $19-64$ & 12,034 & $8.3 \pm 2.4$ & $14.4 \pm 1.7$ & $39.5 \pm 5.4$ \\
\hline Hungary & $\geq 18$ & 706 & $9.2 \pm 1.8$ & $14.6 \pm 1.9$ & $48.0 \pm 5.8$ \\
\hline Ireland & $19-64$ & 717 & $7.6 \pm 2.0$ & $15.6 \pm 2.9$ & $45.1 \pm 6.1$ \\
\hline Italy & $19-64$ & 801 & $8.4 \pm 1.6$ & $16.3 \pm 2.5$ & n.a. \\
\hline Latvia & $19-64$ & 1,238 & $7.5 \pm 3.4$ & $13.7 \pm 4.8$ & $44.6 \pm 11.9$ \\
\hline Lithuania & $19-64$ & 1,087 & $7.4 \pm 3.0$ & $16.7 \pm 6.2$ & $42.9 \pm 10.3$ \\
\hline Norway & $19-64$ & 1,146 & $8.1 \pm 2.7$ & $16.0 \pm 3.0$ & $51.0 \pm 6.0$ \\
\hline Poland & $19-64$ & 1,334 & $8.4 \pm 3.0$ & $13.1 \pm 3.5$ & $51.8 \pm 9.1$ \\
\hline Portugal & $19-64$ & 1,472 & $8.7 \pm 2.1$ & $19.0 \pm 2.4$ & $50.1 \pm 5.9$ \\
\hline Romania & $19-64$ & 341 & $11.4 \pm 4.9$ & $17.1 \pm 3.6$ & $43.6 \pm 9.7$ \\
\hline Spain & $19-64$ & 875 & $7.1 \pm 1.5$ & $19.3 \pm 2.7$ & $37.7 \pm 5.3$ \\
\hline Sweden & $19-64$ & 575 & $7.8 \pm 1.8$ & $16.0 \pm 2.3$ & $47.4 \pm 5.3$ \\
\hline The Netherlands & $19-64$ & 2,112 & $8.4 \pm 2.3$ & $15.6 \pm 3.8$ & $44.7 \pm 7.9$ \\
\hline \multirow[t]{4}{*}{ United Kingdom } & $25-34$ & 295 & $7.0 \pm 2.1$ & $15.1 \pm 3.3$ & $48.3 \pm 7.3$ \\
\hline & $35-44$ & 349 & $6.6 \pm 2.1$ & $16.0 \pm 3.5$ & $48.2 \pm 7.2$ \\
\hline & $45-54$ & 245 & $6.4 \pm 2.2$ & $16.6 \pm 4.1$ & $47.7 \pm 7.3$ \\
\hline & $55-64$ & 236 & $6.3 \pm 2.0$ & $17.0 \pm 3.6$ & $47.7 \pm 7.0$ \\
\hline All countries (min.-max.) & & & $6.3-11.4$ & $13.1-19.3$ & $37.7-51.8$ \\
\hline Reference values & & & $10.0 / 9.5 / 8.5^{2}$ & $10-15 \% \mathrm{E}^{3}$ & $50-75 \% \mathrm{E}^{4}$ \\
\hline
\end{tabular}

${ }^{1}$ Difference is not necessarily alcohol; ${ }^{2} \mathrm{D}-\mathrm{A}-\mathrm{CH}, 2000$, reference values refer to age groups $19-24$ years (PAL 1.75), 25-50 years (PAL 1.70) and 51-64 years (PAL 1.60); ${ }^{3} \mathrm{WHO}, 2003 ;{ }^{4} \mathrm{WHO}, 2007 ;{ }^{5} \mathrm{WHO}, 2009 \mathrm{~b} ;{ }^{6}$ Eurodiet, 2000; n.a. = not available. 


\begin{tabular}{|c|c|c|c|c|c|c|}
\hline sucrose & $\begin{array}{l}\text { Dietary fiber } \\
\mathrm{g}\end{array}$ & Fat $\% E^{1}$ & $\begin{array}{l}\text { SFA } \\
\% E\end{array}$ & $\begin{array}{l}\text { MUFA } \\
\% E\end{array}$ & $\begin{array}{l}\text { PUFA } \\
\% E\end{array}$ & $\begin{array}{l}\text { Cholesterol } \\
\mathrm{mg}\end{array}$ \\
\hline n.a. & n.a. & $35.1 \pm 6.4$ & $14.6 \pm 3.2$ & $12.8 \pm 2.2$ & $6.7 \pm 2.7$ & n.a. \\
\hline n.a. & $20.4 \pm 63.6$ & $31.2 \pm 8.4$ & n.a. & n.a. & n.a. & $277.0 \pm 157.4$ \\
\hline $9.8 \pm 5.7$ & $18.8 \pm 6.6$ & $31.6 \pm 5.3$ & $13.6 \pm 2.8$ & $10.9 \pm 2.1$ & $4.7 \pm 0.9$ & n.a. \\
\hline n.a. & $16.2 \pm 8.8$ & $36.3 \pm 11.2$ & $14.4 \pm 5.3$ & $13.5 \pm 5.5$ & $5.5 \pm 3.0$ & $251.9 \pm 196.5$ \\
\hline $10.5 \pm 5.1$ & $21.0 \pm 9.0$ & $31.2 \pm 7.4$ & $12.0 \pm 3.9$ & $10.9 \pm 3.2$ & $5.7 \pm 2.2$ & $176.0 \pm 93.0$ \\
\hline n.a. & $15.7 \pm 0.2$ & $36.7 \pm 0.2$ & 14.7 & n.a. & n.a. & n.a. \\
\hline n.a. & $24.7 \pm 9.6$ & $34.8 \pm 6.7$ & n.a. & n.a. & n.a. & $278.8 \pm 2.1$ \\
\hline n.a. & n.a. & $47.2 \pm 4.7$ & $13.2 \pm 2.6$ & $22.9 \pm 4.1$ & $6.9 \pm 2.9$ & $227.6 \pm 92.3$ \\
\hline $8.6 \pm 4.8$ & $21.7 \pm 5.6$ & $36.8 \pm 5.4$ & $11.0 \pm 2.3$ & $11.4 \pm 2.5$ & $9.2 \pm 2.3$ & $327.2 \pm 125.4$ \\
\hline n.a. & $17.4 \pm 5.9$ & $35.6 \pm 5.8$ & n.a. & n.a. & n.a. & n.a. \\
\hline n.a. & $18.9 \pm 6.1$ & $33.0 \pm 5.8$ & $10.1 \pm 2.5$ & $11.6 \pm 3.2$ & $4.5 \pm 1.7$ & $310.8 \pm 101.6$ \\
\hline n.a. & n.a. & $40.1 \pm 11.3$ & n.a. & n.a. & n.a. & n.a. \\
\hline $14.0 \pm 6.6$ & $15.6 \pm 9.9$ & $41.9 \pm 9.5$ & $12.9 \pm 7.0$ & $15.7 \pm 7.6$ & $8.7 \pm 5.4$ & $318.8 \pm 209.1$ \\
\hline $9.0 \pm 6.0$ & $21.0 \pm 8.0$ & $31.0 \pm 6.0$ & $12.0 \pm 3.0$ & $11.0 \pm 2.0$ & $5.0 \pm 2.0$ & $260.0 \pm 101.0$ \\
\hline $13.7 \pm 6.6$ & $19.7 \pm 7.9$ & $34.7 \pm 8.3$ & $11.7 \pm 4.1$ & $15.0 \pm 4.5$ & $5.6 \pm 2.6$ & $322.2 \pm 244.5$ \\
\hline n.a. & $23.7 \pm 9.4$ & $29.9 \pm 4.5$ & $9.4 \pm 2.0$ & $13.0 \pm 2.3$ & $4.9 \pm 0.9$ & $302.3 \pm 102.5$ \\
\hline n.a. & n.a. & $39.7 \pm 7.7$ & $24.8 \pm 3.8$ & n.a. & n.a. & $680.0 \pm 340.0$ \\
\hline $17.3 \pm 3.7$ & $16.9 \pm 3.4$ & $40.2 \pm 4.3$ & $12.4 \pm 1.9$ & $17.9 \pm 2.4$ & $5.7 \pm 1.0$ & $285.6 \pm 39.7$ \\
\hline $9.0 \pm 3.7$ & $16.1 \pm 4.8$ & $33.8 \pm 4.8$ & $14.3 \pm 2.3$ & $12.3 \pm 2.0$ & $4.7 \pm 1.4$ & $289.7 \pm 95.3$ \\
\hline n.a. & $20.1 \pm 7.1$ & $36.9 \pm 6.9$ & $14.6 \pm 3.4$ & $13.1 \pm 3.0$ & $6.7 \pm 2.4$ & $201.0 \pm 99.0$ \\
\hline $15.0 \pm 8.3$ & $10.0 \pm 3.6$ & $36.6 \pm 6.5$ & $13.9 \pm 3.7$ & n.a. & n.a. & $218.3 \pm 118.4$ \\
\hline $13.5 \pm 8.4$ & $10.0 \pm 4.2$ & $35.7 \pm 6.9$ & $13.5 \pm 3.2$ & n.a. & n.a. & $205.0 \pm 104.0$ \\
\hline $12.9 \pm 9.0$ & $10.3 \pm 4.4$ & $35.7 \pm 6.9$ & $13.7 \pm 3.6$ & n.a. & n.a. & $210.9 \pm 107.7$ \\
\hline $11.1 \pm 7.4$ & $11.1 \pm 4.5$ & $35.3 \pm 7.3$ & $13.8 \pm 4.3$ & n.a. & n.a. & $226.5 \pm 122.5$ \\
\hline $8.6-17.3$ & $10.0-24.7$ & $29.9-47.2$ & $9.4-24.8$ & $10.9-22.9$ & $4.5-9.2$ & $176.0-680.0$ \\
\hline$<10 \% \mathrm{E}^{3}$ & $>25 \mathrm{~g} \cdot \mathrm{d}^{-1,6}$ & $15-30 \% \mathrm{E}^{3}$ & $<10 \% \mathrm{E}^{3}$ & & $6-11 \% \mathrm{E}^{5}$ & $<300 \mathrm{mg} \cdot \mathrm{d}^{-1,3}$ \\
\hline
\end{tabular}


Table 7.36. Intake of energy and macronutrients (min.-max.) in adults in the four regions

\begin{tabular}{|c|c|c|c|c|c|}
\hline Region/sex & $\begin{array}{l}\text { Energy } \\
\text { MJ }\end{array}$ & $\begin{array}{l}\text { Protein } \\
\% \mathrm{E}\end{array}$ & $\begin{array}{l}\text { Carbohydrates, \%E } \\
\text { (of that sucrose) }\end{array}$ & $\begin{array}{l}\text { Dietary fiber } \\
\mathrm{g}\end{array}$ & $\begin{array}{l}\text { Fat } \\
\% \mathrm{E}\end{array}$ \\
\hline \multicolumn{6}{|l|}{ North ${ }^{1}$} \\
\hline Male & $9.2-11.1$ & $13.7-16.8$ & $42.4-51.0\left(8.6-10.8^{5}\right)$ & $18.0-25.0^{6}$ & $31.0-44.9$ \\
\hline Female & $6.8-8.2$ & $13.7-17.2$ & $42.9-51.0\left(9.0-14.0^{5}\right)$ & $15.6-21.0^{6}$ & $31.0-41.9$ \\
\hline \multicolumn{6}{|l|}{ South ${ }^{2}$} \\
\hline Male & $9.1-10.4$ & $14.1-18.5$ & $36.8-47.0^{7}\left(15.1^{8}\right)$ & $19.3-23.5^{9}$ & $28.4-45.0$ \\
\hline Female & $7.1-8.7$ & $14.4-19.3$ & $37.7-50.1^{7}\left(17.3^{8}\right)$ & $16.9-23.7^{9}$ & $29.9-47.2$ \\
\hline \multicolumn{6}{|c|}{ Central and East ${ }^{3}$} \\
\hline Male & $9.0-13.9$ & $13.5-17.8$ & $42.5-49.5\left(7.9-11.0^{10}\right)$ & $18.7-29.7^{11}$ & $31.3-38.9$ \\
\hline Female & $7.5-11.4$ & $13.1-17.1$ & $43.6-53.9\left(10.9-13.7^{10}\right)$ & $19.7-24.7^{11}$ & $31.2-39.7$ \\
\hline \multicolumn{6}{|l|}{ West $^{4}$} \\
\hline Male & $9.1-12.2$ & $14.7-16.3$ & $42.4-47.6^{12}\left(14.8^{13}\right)$ & $12.8-24.4^{14}$ & $34.8-36.5$ \\
\hline Female & $6.6-8.4$ & $15.6-17.0$ & $44.4-48.0^{12}\left(13.1^{13}\right)$ & $10.4-20.1^{14}$ & $35.1-36.9$ \\
\hline \multicolumn{6}{|c|}{ 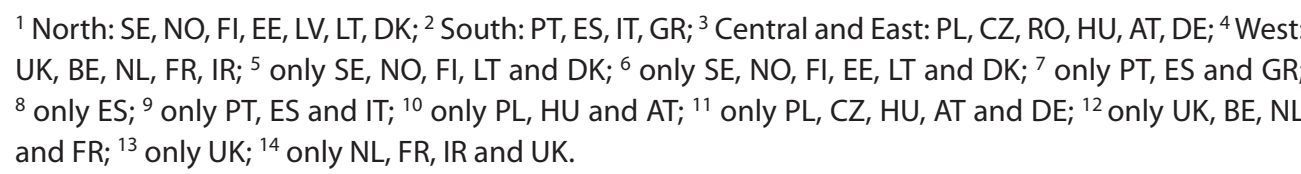 } \\
\hline
\end{tabular}

The intake of $\alpha$-tocopherol equivalents was between 3.3 and $17.4 \mathrm{mg} /$ day in males and 4.2 and $16.1 \mathrm{mg} /$ day in females. The recommendations range from 13 to $15 \mathrm{mg} /$ day for males and $12 \mathrm{mg} /$ day for females [D-A-CH, 2000]. Only Austrian, German, Hungarian, Lithuanian, Polish and Dutch males as well as females from Austria, Germany, Hungary and Lithuania met the recommendations.

The intake of thiamine was between 1.1 and $2.3 \mathrm{mg} /$ day in males and between 0.9 and $2.1 \mathrm{mg} /$ day in females, that of riboflavin between 1.4 and $2.4 \mathrm{mg} /$ day and 1.2 and $2.8 \mathrm{mg} /$ day and that of vitamin $\mathrm{B}_{6}$ between 1.6 and $3.5 \mathrm{mg} /$ day and 1.3 and $2.1 \mathrm{mg} /$ day. Therefore, the intake levels were within the recommendations except for Hungarian females in whom the intake of thiamine was slightly below the recommendation [D-A-CH, 2000].

The intake of folate equivalents was between 203 and $494 \mu \mathrm{g} /$ day in males and between 131 and $392 \mu \mathrm{g} / \mathrm{day}$ in females, therefore the $\mathrm{D}-\mathrm{A}-\mathrm{CH}$ reference value of $400 \mu \mathrm{g} /$ day $[\mathrm{D}-\mathrm{A}-\mathrm{CH}, 2000$ ] was not met by all countries. Compared with the recommendation for the Nordic countries $-300 \mu \mathrm{g} /$ day ( 400 for women of fertile 
Table 7.37. Intake of fat, fatty acids and cholesterol (min.-max.) in adults in the four regions

\begin{tabular}{|c|c|c|c|c|c|}
\hline Region/sex & $\begin{array}{l}\text { Fat } \\
\% \mathrm{E}\end{array}$ & $\begin{array}{l}\text { SFA } \\
\% \mathrm{E}\end{array}$ & $\begin{array}{l}\text { MUFA } \\
\% E\end{array}$ & $\begin{array}{l}\text { PUFA } \\
\% E\end{array}$ & $\begin{array}{l}\text { Cholesterol } \\
\mathrm{mg}\end{array}$ \\
\hline \multicolumn{6}{|l|}{ North ${ }^{1}$} \\
\hline Male & $31.0-44.9$ & $12.0-14.6^{5}$ & $11.0-16.9^{5}$ & $4.7-8.9^{5}$ & $256.0-477.9^{6}$ \\
\hline Female & $31.0-41.9$ & $12.0-14.4^{5}$ & $10.9-15.7^{5}$ & $4.7-8.7^{5}$ & $176.0-318.8^{6}$ \\
\hline \multicolumn{6}{|l|}{ South ${ }^{2}$} \\
\hline Male & $28.4-45$ & $8.8-12.7$ & $12.3-21.9$ & $4.8-6.4$ & $282.9-378.4$ \\
\hline Female & 29.9-47.2 & $9.4-13.2$ & $13.0-22.9$ & $4.5-6.9$ & $227.6-310.8$ \\
\hline \multicolumn{6}{|c|}{ Central and East ${ }^{3}$} \\
\hline Male & $31.3-38.9$ & $11.7-26.3^{7}$ & $12.5-16.2^{8}$ & $5.7-8.8$ & $352.5-800.0$ \\
\hline Female & $31.2-39.7$ & $11.7-24.8^{7}$ & $14.0-15.0^{8}$ & $5.6-9.2$ & $277.0-680.0$ \\
\hline \multicolumn{6}{|l|}{ West $^{4}$} \\
\hline Male & $34.8-36.5$ & $13.7-14.6$ & $12.8-13.3^{9}$ & $6.7-7.0^{9}$ & $250.0-279.0^{10}$ \\
\hline Female & $35.1-36.9$ & $13.7-14.7$ & $12.8-13.1^{9}$ & $6.7^{9}$ & $201.0-215.2^{10}$ \\
\hline $\begin{array}{l}{ }^{1} \text { North: SE, } \\
\text { UK, BE, NL, } \\
{ }^{8} \text { only PL, HU }\end{array}$ & $\begin{array}{l}E \text { E, LV, LT, D } \\
\text { ly SE, NO, } \\
\text { T; }{ }^{9} \text { only BE }\end{array}$ & $\begin{array}{l}\text { uth: PT, ES, I } \\
\text { LT and DK; } \\
\mathrm{VL}^{10} \text { only U }\end{array}$ & $\begin{array}{l}{ }^{3} \text { Central ar } \\
\text { SE, NO, FI, } \\
\text { NL. }\end{array}$ & $\begin{array}{l}\text { : PL, CZ, R } \\
\mathrm{d} \mathrm{LT;}{ }^{7} \text { onl }\end{array}$ & $\begin{array}{l}\text {, AT, DE; }{ }^{4} \text { West: } \\
\text { RO, HU and AT; }\end{array}$ \\
\hline
\end{tabular}

age) - Austrian, Finnish, Greek, Hungarian, Spanish, Swedish and Dutch males as well as males aged 35-64 years from the United Kingdom did not meet the recommendation. In the female group, the recommendation of $300 \mu \mathrm{g} / \mathrm{day}$ was only met by Estonian and Portuguese females [NNR, 2004].

The recommendation for Central Europe for ascorbic acid is $100 \mathrm{mg} / \mathrm{day}$ [D-A-CH, 2000]. It was only met by Austrian, Czech, Danish, German, Greek, Irish, Italian, Norwegian and Portuguese adults in both men and women, as well as by Finnish and Spanish females. The recommended intake of the Nordic countries of $75 \mathrm{mg} /$ day was met by almost all participating countries except for Latvia, Lithuania and the United Kingdom (55-64 years) [NNR, 2004] (cf. table 7.38).

\section{Comparison of Regions}

The intake of selected vitamins in the four regions is shown in table 7.39. In general, no big differences between the regions could be observed. However the lowest intake levels of cobalamin and the highest intake levels of vitamin D were reported in the North region. 
Table 7.38. Vitamin intake (mean \pm SD) in adults of European countries (male and female)

\begin{tabular}{|c|c|c|c|c|c|c|}
\hline & Age years & $\mathrm{n}$ & $\begin{array}{l}\text { Vitamin } A^{1} \\
\mathrm{mg}\end{array}$ & $\begin{array}{l}\beta \text {-Carotene } \\
\mathrm{mg}\end{array}$ & $\begin{array}{l}\text { Vitamin D } \\
\mu \mathrm{g}\end{array}$ & $\begin{array}{l}\text { Vitamin } E^{2} \\
m g\end{array}$ \\
\hline \multicolumn{7}{|l|}{ Male } \\
\hline Austria & $19-64$ & 778 & $1.0 \pm 1.2$ & $2.8 \pm 3.1$ & $1.8 \pm 3.8$ & $17.4 \pm 10.3$ \\
\hline Belgium & $19-64$ & n.a. & n.a. & n.a. & n.a. & n.a. \\
\hline Czech Republic & $19-64$ & 1,064 & n.a. & n.a. & n.a. & $3.3 \pm 2.7$ \\
\hline Denmark & $19-64$ & 1,283 & $1.2 \pm 0.8$ & $3.5 \pm 3.5$ & $3.6 \pm 2.8$ & $7.6 \pm 3.1$ \\
\hline Estonia & $19-64$ & 899 & $1.9 \pm 6.0$ & n.a. & $3.6 \pm 6.2$ & $9.2 \pm 9.2$ \\
\hline Finland & $19-64$ & 730 & $1.0 \pm 1.4$ & n.a. & $7.1 \pm 5.7$ & $10.3 \pm 4.8$ \\
\hline Germany & 19-64 & 4,912 & $2.1 \pm 1.4$ & $5.3 \pm 4.2$ & $3.8 \pm 3.6$ & $16.2 \pm 11.3$ \\
\hline Greece & $22 \pm 2$ & 500 & $0.9 \pm 1.3$ & n.a. & n.a. & $8.9 \pm 15.2$ \\
\hline Hungary & $\geq 18$ & 473 & $0.6 \pm 0.8$ & $2.2 \pm 1.9$ & $2.2 \pm 1.2$ & $16.0 \pm 5.8$ \\
\hline Ireland & $19-64$ & 662 & $1.0 \pm 0.9$ & $2.5 \pm 2.1$ & $3.7 \pm 3.4$ & $11.2 \pm 37.2$ \\
\hline Italy & $19-64$ & 660 & $1.3 \pm 1.4$ & $3.1 \pm 1.9$ & $3.5 \pm 2.3$ & $11.6 \pm 5.0$ \\
\hline Latvia & $19-64$ & 1,065 & $1.0 \pm 2.0$ & n.a. & n.a. & n.a. \\
\hline Lithuania & $19-64$ & 849 & $1.3 \pm 2.9$ & $3.5 \pm 7.0$ & $6.3 \pm 9.7$ & $16.8 \pm 11.1$ \\
\hline Norway & $19-64$ & 1,050 & $2.0 \pm 1.3^{5}$ & n.a. & $10.9 \pm 10.7$ & n.a. \\
\hline Poland & $19-64$ & 1,106 & $1.8 \pm 3.6$ & $3.6 \pm 3.4$ & $5.9 \pm 6.1$ & $16.7 \pm 9.6$ \\
\hline Portugal & $19-64$ & 917 & $1.5 \pm 0.8$ & n.a. & $3.6 \pm 1.7$ & $8.4 \pm 2.7$ \\
\hline Romania & $19-64$ & 177 & $2.2 \pm 1.1$ & n.a. & n.a. & n.a. \\
\hline Spain & $19-64$ & 706 & $0.6 \pm 0.1$ & $1.4 \pm 0.5$ & $1.6 \pm 0.8$ & $9.5 \pm 2.0$ \\
\hline Sweden & $19-64$ & 517 & $1.3 \pm 0.9^{5}$ & $1.7 \pm 3.3$ & $6.1 \pm 2.7$ & $7.9 \pm 2.7$ \\
\hline The Netherlands & $19-64$ & 1,836 & $1.1 \pm 1.2$ & n.a. & $4.6 \pm 2.5$ & $14.8 \pm 7.0$ \\
\hline \multirow[t]{4}{*}{ United Kingdom } & $25-34$ & 119 & $0.9 \pm 1.4$ & $2.5 \pm 2.5$ & $2.9 \pm 1.7$ & $10.5 \pm 5.5$ \\
\hline & $35-44$ & 152 & $0.8 \pm 1.5$ & $1.7 \pm 1.7$ & $2.8 \pm 1.9$ & $9.1 \pm 4.5$ \\
\hline & $45-54$ & 143 & $0.8 \pm 0.7$ & $1.9 \pm 2.1$ & $3.2 \pm 2.0$ & $10.0 \pm 6.8$ \\
\hline & $55-64$ & 189 & $1.3 \pm 2.4$ & $2.3 \pm 2.5$ & $3.7 \pm 3.0$ & $9.7 \pm 5.3$ \\
\hline All countries (min.-max.) & & & $0.5-2.2$ & $1.4-5.3$ & $1.6-10.9$ & $3.3-17.4$ \\
\hline Reference values ${ }^{6}$ & & & 1.0 & n.a. & 5.0 & $15 / 14 / 13$ \\
\hline \multicolumn{7}{|l|}{ Female } \\
\hline Austria & $19-64$ & 1,345 & $1.2 \pm 1.8$ & $3.5 \pm 4.0$ & $1.8 \pm 2.8$ & $16.1 \pm 9.1$ \\
\hline Belgium & n.a. & n.a. & n.a. & n.a. & n.a. & n.a. \\
\hline Czech Republic & $19-64$ & 1,094 & n.a. & n.a. & n.a. & $4.2 \pm 4.1$ \\
\hline
\end{tabular}




\begin{tabular}{|c|c|c|c|c|c|c|}
\hline $\begin{array}{l}\text { Thiamine } \\
\mathrm{g}\end{array}$ & $\begin{array}{l}\text { Riboflavin } \\
\mathrm{mg}\end{array}$ & $\begin{array}{l}\mathrm{Niacin}^{3} \\
\mathrm{mg}\end{array}$ & $\begin{array}{l}\text { Vitamin } B_{6} \\
\text { mg }\end{array}$ & $\begin{array}{l}\text { Folate }^{4} \\
\mu \mathrm{g}\end{array}$ & $\begin{array}{l}\text { Cobalamin } \\
\mu \mathrm{g}\end{array}$ & $\begin{array}{l}\text { Ascorbic acid } \\
\mathrm{mg}\end{array}$ \\
\hline $1.4 \pm 0.7$ & $1.4 \pm 0.6$ & $16.8 \pm 8.4$ & $1.8 \pm 1.0$ & $203 \pm 107$ & $5.4 \pm 4.3$ & $130 \pm 120$ \\
\hline n.a. & n.a. & n.a. & n.a. & n.a. & n.a. & $88 \pm 36$ \\
\hline n.a. & n.a. & n.a. & n.a. & n.a. & n.a. & $111 \pm 119$ \\
\hline $1.4 \pm 0.5$ & $1.8 \pm 0.7$ & $33.5 \pm 9.4$ & $1.6 \pm 0.5$ & $323 \pm 120$ & $5.8 \pm 3.3$ & $102 \pm 56$ \\
\hline n.a. & n.a. & $32.0 \pm 32.0$ & $2.0 \pm 2.0$ & $494 \pm 494$ & n.a. & $82 \pm 96$ \\
\hline $1.5 \pm 0.6$ & $2.1 \pm 0.9$ & $35.0 \pm 12.0$ & $2.1 \pm 0.9$ & $270 \pm 120$ & $6.6 \pm 6.5$ & $98 \pm 88$ \\
\hline $1.9 \pm 1.4$ & $1.4 \pm 0.7$ & $41.3 \pm 20.9$ & $2.7 \pm 1.9$ & $321 \pm 202$ & $6.6 \pm 3.7$ & $153 \pm 106$ \\
\hline $2.2 \pm 2.1$ & $2.2 \pm 1.3$ & $21.7 \pm 11.0$ & $1.9 \pm 1.3$ & $283 \pm 190$ & $5.3 \pm 11.4$ & $146 \pm 130$ \\
\hline $1.1 \pm 0.3$ & $1.4 \pm 0.5$ & $38.8 \pm 9.9$ & $1.9 \pm 0.5$ & $152 \pm 53$ & $4.0 \pm 2.9$ & $79 \pm 50$ \\
\hline $2.3 \pm 1.5$ & $2.2 \pm 1.5$ & $28.2 \pm 9.9$ & $3.5 \pm 1.9$ & $332 \pm 128$ & $5.4 \pm 3.7$ & $116 \pm 223$ \\
\hline $1.2 \pm 0.3$ & $1.7 \pm 0.5$ & $36.7 \pm 8.2$ & $2.2 \pm 0.6$ & $315 \pm 91$ & n.a. & $122 \pm 65$ \\
\hline $1.6 \pm 1.0$ & $1.7 \pm 1.1$ & $17.0 \pm 11.0$ & n.a. & n.a. & n.a. & $64 \pm 63$ \\
\hline $1.6 \pm 0.9$ & $1.7 \pm 0.9$ & $19.1 \pm 11.1$ & $1.8 \pm 0.9$ & $344 \pm 358$ & $1.9 \pm 6.8$ & $69 \pm 53$ \\
\hline $2.0 \pm 1.2$ & $2.4 \pm 1.4$ & $24.2 \pm 14.8$ & n.a. & n.a. & n.a. & $140 \pm 95$ \\
\hline $1.9 \pm 0.9$ & $2.1 \pm 1.1$ & n.a. & $2.6 \pm 1.1$ & $329 \pm 146$ & $5.6 \pm 8.7$ & $98 \pm 84$ \\
\hline $1.9 \pm 0.5$ & $2.0 \pm 0.6$ & $23.6 \pm 5.9$ & $2.1 \pm 0.6$ & $302 \pm 130$ & $9.3 \pm 4.1$ & $116 \pm 54$ \\
\hline $2.0 \pm 0.8$ & $3.6 \pm 2.4$ & n.a. & n.a. & n.a. & n.a. & $82 \pm 48$ \\
\hline $1.3 \pm 0.2$ & $1.6 \pm 0.3$ & $21.8 \pm 3.2$ & $2.0 \pm 0.4$ & $237 \pm 45$ & $5.0 \pm 1.0$ & $97 \pm 37$ \\
\hline $1.6 \pm 0.5$ & $1.9 \pm 0.6$ & $38.9 \pm 10.2$ & $2.2 \pm 0.6$ & $232 \pm 73$ & $6.8 \pm 3.8$ & $79 \pm 45$ \\
\hline $1.5 \pm 0.8$ & $1.7 \pm 0.7$ & n.a. & $1.9 \pm 0.7$ & $215 \pm 98$ & n.a. & $79 \pm 49$ \\
\hline $1.7 \pm 0.7$ & $1.9 \pm 0.9$ & $11.4 \pm 8.4^{7}$ & $2.8 \pm 1.3$ & $310 \pm 150$ & $5.7 \pm 3.7$ & $78 \pm 66$ \\
\hline $1.5 \pm 0.7$ & $1.7 \pm 0.8$ & $10.3 \pm 7.9^{7}$ & $2.3 \pm 1.2$ & $268 \pm 137$ & $5.4 \pm 4.7$ & $56 \pm 48$ \\
\hline $1.6 \pm 0.7$ & $1.8 \pm 0.8$ & $9.7 \pm 6.6^{7}$ & $2.4 \pm 1.1$ & $281 \pm 131$ & $5.5 \pm 2.7$ & $59 \pm 50$ \\
\hline $1.7 \pm 0.6$ & $1.9 \pm 0.9$ & $9.2 \pm 6.2^{7}$ & $2.4 \pm 0.9$ & $287 \pm 124$ & $7.7 \pm 8.7$ & $71 \pm 53$ \\
\hline $1.1-2.3$ & $1.4-2.4$ & $9.2-41.3$ & $1.6-3.5$ & $203-494$ & $1.9-9.3$ & 64-153 \\
\hline $1.3 / 1.2 / 1.1$ & $1.5 / 1.4 / 1.3$ & $17 / 16 / 15$ & 1.5 & 400 & 3.0 & 100 \\
\hline $1.1 \pm 0.5$ & $1.3 \pm 0.6$ & $12.7 \pm 6.2$ & $1.5 \pm 0.8$ & $204 \pm 102$ & $3.9 \pm 4.6$ & $133 \pm 105$ \\
\hline n.a. & n.a. & n.a. & n.a. & n.a. & n.a. & $92 \pm 44$ \\
\hline n.a. & n.a. & n.a. & n.a. & n.a. & n.a. & $138 \pm 142$ \\
\hline
\end{tabular}


Table 7.38. Continued

\begin{tabular}{|c|c|c|c|c|c|c|}
\hline & Age years & $\mathrm{n}$ & $\begin{array}{l}\text { Vitamin } A^{1} \\
\text { mg }\end{array}$ & $\begin{array}{l}\beta \text {-Carotene } \\
\mathrm{mg}\end{array}$ & $\begin{array}{l}\text { Vitamin D } \\
\mu \mathrm{g}\end{array}$ & $\begin{array}{l}\text { Vitamin } \mathrm{E}^{2} \\
\mathrm{mg}\end{array}$ \\
\hline Denmark & $19-64$ & 1,486 & $1.0 \pm 0.7$ & $4.6 \pm 4.5$ & $2.8 \pm 2.3$ & $6.6 \pm 2.5$ \\
\hline Estonia & $19-64$ & 1,113 & $1.4 \pm 1.4$ & n.a. & $2.8 \pm 4.6$ & $7.0 \pm 4.7$ \\
\hline Finland & $19-64$ & 846 & $0.7 \pm 0.7$ & n.a. & $5.2 \pm 4.2$ & $8.1 \pm 3.7$ \\
\hline Germany & $19-64$ & 6,016 & $1.8 \pm 1.0$ & $5.6 \pm 4.0$ & $2.0 \pm 1.3$ & $13.4 \pm 6.7$ \\
\hline Greece & $22 \pm 2$ & 451 & $0.9 \pm 1.3$ & n.a. & n.a. & $6.2 \pm 4.6$ \\
\hline Hungary & $\geq 18$ & 706 & $0.5 \pm 0.8$ & $2.3 \pm 2.0$ & $1.9 \pm 1.1$ & $13.9 \pm 5.0$ \\
\hline Ireland & $19-64$ & 717 & $0.5 \pm 0.8$ & $2.3 \pm 1.6$ & $3.7 \pm 8.7$ & $11.0 \pm 27.4$ \\
\hline Italy & $19-64$ & 801 & $1.1 \pm 1.1$ & $3.1 \pm 1.9$ & $2.8 \pm 1.8$ & $10.8 \pm 5.1$ \\
\hline Latvia & $19-64$ & 1,238 & $0.8 \pm 1.6$ & n.a. & n.a. & n.a. \\
\hline Lithuania & $19-64$ & 1,087 & $1.0 \pm 3.1$ & $3.0 \pm 8.1$ & $3.9 \pm 7.0$ & $12.3 \pm 8.1$ \\
\hline Norway & $19-64$ & 1,146 & $1.9 \pm 1.2^{5}$ & n.a. & $10.1 \pm 9.1$ & n.a. \\
\hline Poland & $19-64$ & 1,334 & $1.2 \pm 2.5$ & $2.8 \pm 3.2$ & $3.4 \pm 4.6$ & $11.2 \pm 6.8$ \\
\hline Portugal & $19-64$ & 1,472 & $1.6 \pm 0.8$ & n.a. & $3.5 \pm 1.6$ & $8.2 \pm 2.6$ \\
\hline Romania & $19-64$ & 341 & $2.0 \pm 0.9$ & n.a. & n.a. & n.a. \\
\hline Spain & $19-64$ & 875 & $0.5 \pm 0.1$ & $1.4 \pm 0.6$ & $1.2 \pm 0.6$ & $8.4 \pm 1.8$ \\
\hline Sweden & $19-64$ & 575 & $1.1 \pm 0.6^{5}$ & $1.8 \pm 1.2$ & $4.8 \pm 1.9$ & $6.8 \pm 2.0$ \\
\hline The Netherlands & $19-64$ & 2,112 & $0.8 \pm 0.8$ & n.a. & $3.2 \pm 1.7$ & $11.5 \pm 5.7$ \\
\hline \multirow[t]{4}{*}{ United Kingdom } & $25-34$ & 295 & $0.7 \pm 1.0$ & $2.0 \pm 1.8$ & $2.1 \pm 1.4$ & $7.9 \pm 3.4$ \\
\hline & $35-44$ & 349 & $0.8 \pm 1.3$ & $2.0 \pm 2.7$ & $2.3 \pm 2.1$ & $7.7 \pm 4.0$ \\
\hline & $45-54$ & 245 & $0.7 \pm 0.7$ & $2.0 \pm 2.1$ & $2.5 \pm 2.4$ & $7.7 \pm 4.4$ \\
\hline & $55-64$ & 236 & $1.1 \pm 1.7$ & $2.5 \pm 2.3$ & $2.9 \pm 1.9$ & $7.5 \pm 4.1$ \\
\hline All countries (min.-max.) & & & $0.5-2.0$ & $1.4-5.6$ & $1.2-10.1$ & $4.2-16.1$ \\
\hline Reference values $^{6}$ & & & 0.8 & n.a. & 5.0 & 12 \\
\hline
\end{tabular}

${ }^{1}$ Retinol equivalent $\left(=1 \mathrm{mg}\right.$ retinol $=6 \mathrm{mg}$ all-trans- $\beta$-carotene $=12 \mathrm{mg}$ other carotenoids); ${ }^{2}$ RRR- $\alpha$ tocopherol equivalent $(=\mathrm{mg}$ a-tocopherol $+\mathrm{mg} \beta$-tocopherol $\times 0.5+\mathrm{mg} \gamma$-tocopherol $\times 0.25+\mathrm{mg}$ a-tocotrienol $\times 0.33) ;{ }^{3}$ niacin equivalent $\left(=1 \mathrm{mg}\right.$ niacin $=60 \mathrm{mg}$ tryptophan); ${ }^{4}$ folate equivalent $(1 \mu \mathrm{g}$ food folate $=0.5 \mu \mathrm{g}$ folic acid $(\mathrm{PGA})=0.6 \mu \mathrm{g}$ folic acid taken with meals); ${ }^{5}$ values refer to a-tocopherol, only; ${ }^{6} \mathrm{D}-\mathrm{A}-\mathrm{CH}, 2000$, reference values refer to age groups $19-24$ years, $25-50$ years and $51-64$ years; ${ }^{7}$ Niacin; n.a. $=$ not available. 


\begin{tabular}{|c|c|c|c|c|c|c|}
\hline $\begin{array}{l}\text { Thiamine } \\
\mathrm{g}\end{array}$ & $\begin{array}{l}\text { Riboflavin } \\
\mathrm{mg}\end{array}$ & $\begin{array}{l}\mathrm{Niacin}^{3} \\
\mathrm{mg}\end{array}$ & $\begin{array}{l}\text { Vitamin } \mathrm{B}_{6} \\
\mathrm{mg}\end{array}$ & $\begin{array}{l}\text { Folate }^{4} \\
\mu \mathrm{g}\end{array}$ & $\begin{array}{l}\text { Cobalamin } \\
\mu \mathrm{g}\end{array}$ & $\begin{array}{l}\text { Ascorbic acid } \\
\text { mg }\end{array}$ \\
\hline $1.1 \pm 0.3$ & $1.5 \pm 0.6$ & $25.8 \pm 7.3$ & $1.3 \pm 0.4$ & $296 \pm 111$ & $4.3 \pm 2.6$ & $107 \pm 61$ \\
\hline n.a. & n.a. & $23.4 \pm 11.6$ & $1.5 \pm 0.8$ & $392 \pm 219$ & n.a. & $82 \pm 81$ \\
\hline $1.1 \pm 0.4$ & $1.6 \pm 0.6$ & $26.0 \pm 8.0$ & $1.7 \pm 0.6$ & $226 \pm 88$ & $4.5 \pm 3.4$ & $118 \pm 82$ \\
\hline $1.4 \pm 0.7$ & $1.8 \pm 0.9$ & $29.4 \pm 11.2$ & $2.0 \pm 1.0$ & $277 \pm 124$ & $4.4 \pm 2.1$ & $153 \pm 84$ \\
\hline $1.3 \pm 0.9$ & $1.5 \pm 1.0$ & $14.0 \pm 7.7$ & $1.3 \pm 0.7$ & $221 \pm 184$ & $3.8 \pm 9.7$ & $145 \pm 120$ \\
\hline $0.9 \pm 0.3$ & $1.2 \pm 0.5$ & $30.4 \pm 7.5$ & $1.6 \pm 0.4$ & $131 \pm 47$ & $2.8 \pm 2.4$ & $80 \pm 52$ \\
\hline $2.1 \pm 4.1$ & $2.0 \pm 3.4$ & $20.7 \pm 9.9$ & $3.3 \pm 6.4$ & $260 \pm 144$ & $4.1 \pm 3.6$ & $108 \pm 183$ \\
\hline $1.0 \pm 0.3$ & $1.5 \pm 0.5$ & $30.0 \pm 7.3$ & $1.8 \pm 0.5$ & $283 \pm 100$ & n.a. & $113 \pm 58$ \\
\hline $1.0 \pm 0.6$ & $1.3 \pm 0.9$ & $12.0 \pm 8.0$ & n.a. & n.a. & n.a. & $70 \pm 65$ \\
\hline $1.0 \pm 0.6$ & $1.3 \pm 0.8$ & $13.0 \pm 8.1$ & $1.4 \pm 0.7$ & $238 \pm 231$ & $1.0 \pm 2.7$ & $66 \pm 62$ \\
\hline $1.8 \pm 1.1$ & $2.1 \pm 1.2$ & $21.2 \pm 13.4$ & n.a. & n.a. & n.a. & $149 \pm 88$ \\
\hline $1.1 \pm 0.5$ & $1.4 \pm 0.8$ & n.a. & $1.6 \pm 0.7$ & $221 \pm 105$ & $3.5 \pm 6.3$ & $80 \pm 68$ \\
\hline $1.7 \pm 0.4$ & $2.1 \pm 0.6$ & $21.6 \pm 5.3$ & $2.1 \pm 0.6$ & $304 \pm 144$ & $8.8 \pm 4.0$ & $131 \pm 63$ \\
\hline $1.5 \pm 0.7$ & $2.8 \pm 1.8$ & n.a. & n.a. & n.a. & n.a. & $76 \pm 41$ \\
\hline $1.1 \pm 0.2$ & $1.4 \pm 0.3$ & $17.3 \pm 2.6$ & $1.7 \pm 0.3$ & $220 \pm 48$ & $4.0 \pm 0.8$ & $108 \pm 42$ \\
\hline $1.3 \pm 0.3$ & $1.6 \pm 0.5$ & $30.6 \pm 7.5$ & $1.9 \pm 0.5$ & $215 \pm 65$ & $5.9 \pm 5.4$ & $90 \pm 50$ \\
\hline $1.2 \pm 1.1$ & $1.5 \pm 0.6$ & n.a. & $1.5 \pm 0.5$ & $174 \pm 78$ & n.a. & $84 \pm 54$ \\
\hline $1.2 \pm 0.5$ & $1.3 \pm 0.6$ & $6.9 \pm 6.0^{7}$ & $1.9 \pm 1.3$ & $202 \pm 83$ & $4.3 \pm 2.6$ & $62 \pm 49$ \\
\hline $1.3 \pm 0.6$ & $1.5 \pm 0.8$ & $7.0 \pm 4.9^{7}$ & $1.8 \pm 0.8$ & $204 \pm 91$ & $4.7 \pm 5.3$ & $60 \pm 45$ \\
\hline $1.3 \pm 0.5$ & $1.4 \pm 0.7$ & $6.4 \pm 4.3^{7}$ & $1.7 \pm 0.7$ & $213 \pm 95$ & $4.6 \pm 3.4$ & $63 \pm 50$ \\
\hline $1.4 \pm 0.5$ & $1.5 \pm 0.7$ & $7.2 \pm 4.4^{7}$ & $1.8 \pm 0.6$ & $225 \pm 88$ & $5.3 \pm 5.4$ & $72 \pm 56$ \\
\hline $0.9-2.1$ & $1.2-2.8$ & $6.4-30.6$ & $1.3-2.1$ & $131-392$ & $1.0-8.8$ & $62-153$ \\
\hline 1.0 & 1.2 & 13 & 1.2 & 400 & 3.0 & 100 \\
\hline
\end{tabular}


Table 7.39. Intake of selected vitamins (min.-max.) in adults in the four regions

\begin{tabular}{|c|c|c|c|c|}
\hline Region/sex & $\begin{array}{l}\text { Vitamin } \mathrm{B}_{6} \\
\mathrm{mg}\end{array}$ & $\begin{array}{l}\text { Folate } \\
\mu g^{5}\end{array}$ & $\begin{array}{l}\text { Cobalamin } \\
\mu \mathrm{g}\end{array}$ & $\begin{array}{l}\text { Vitamin D } \\
\mu \mathrm{g}\end{array}$ \\
\hline \multicolumn{5}{|l|}{ North ${ }^{1}$} \\
\hline Male & $1.6-2.2^{6}$ & $232-494^{6}$ & $1.9-6.8^{7}$ & $3.6-10.9$ \\
\hline Female & $1.3-1.9^{6}$ & $215-392^{6}$ & $1.0-5.9^{7}$ & $2.8-10.1$ \\
\hline \multicolumn{5}{|l|}{ South ${ }^{2}$} \\
\hline Male & $1.9-2.2$ & $237-315$ & $5.3-9.3^{8}$ & $1.6-3.6^{9}$ \\
\hline Female & $1.3-2.1$ & $220-304$ & $3.8-8.8^{8}$ & $1.2-3.5^{9}$ \\
\hline \multicolumn{5}{|c|}{ Central and East ${ }^{3}$} \\
\hline Male & $1.8-2.7$ & $203-329$ & $4.0-6.6$ & $1.8-5.9$ \\
\hline Female & $1.5-2.0$ & $131-277$ & $2.8-4.4$ & $1.8-3.4$ \\
\hline \multicolumn{5}{|l|}{ West ${ }^{4}$} \\
\hline Male & $1.9-3.5$ & $215-332$ & $5.4-6.1^{10}$ & $3.2-4.6$ \\
\hline Female & $1.5-3.3$ & $174-260$ & $4.1-4.7^{10}$ & $2.5-3.7$ \\
\hline \multicolumn{5}{|c|}{$\begin{array}{l}{ }^{1} \text { North: } \mathrm{SE}, \mathrm{NO}, \mathrm{FI}, \mathrm{EE}, \mathrm{LT}, \mathrm{DK} ;{ }^{2} \text { South: } \mathrm{PT}, \mathrm{ES}, \mathrm{IT}, \mathrm{GR} ;{ }^{3} \text { Central and East: } \mathrm{PL}, \mathrm{HU}, \mathrm{AT}, \mathrm{DE} ;{ }^{4} \text { West: } \mathrm{UK}, \mathrm{NL}, \mathrm{IR} \text {; } \\
{ }^{5} \text { Folate equivalent }(1 \mu \mathrm{g} \text { food folate }=0.5 \mu \mathrm{g} \text { folic acid }(\mathrm{PGA})=0.6 \mu \mathrm{g} \text { folic acid taken with meals); } \\
{ }^{6} \text { only } \mathrm{SE}, \mathrm{EE}, \mathrm{FI}, \mathrm{LT} \text { and } \mathrm{DK} ;{ }^{7} \text { only } \mathrm{SE}, \mathrm{FI}, \mathrm{LT} \text { and } \mathrm{DK} ;{ }^{8} \text { only PT, ES and } \mathrm{GR} ;{ }^{9} \text { only PT, ES and IT; }{ }^{10} \text { only IR } \\
\text { and } \mathrm{UK} \text {. }\end{array}$} \\
\hline
\end{tabular}

\section{Minerals}

Comparison of Countries

The intake of sodium was between 3.6 and $7.3 \mathrm{~g} /$ day in males and between 1.7 and $5.6 \mathrm{~g} /$ day in females. Therefore, every participating country was above the D-A-CH recommended minimum intake level of $0.55 \mathrm{~g} /$ day [D-A-CH, 2000]. The recommendation of the WHO of $<2$ g sodium per day was only met by Greek females [WHO, 2003].

Compared with the D-A-CH reference values [D-A-CH, 2000], the potassium, manganese and phosphorus intake levels were within the recommendations except for Polish females in whom the intake of manganese was slightly above the recommendation.

The intake of calcium was between 687 and $1,171 \mathrm{mg} /$ day in males and between 508 and 1,047 mg/day in females. Males from Denmark, Finland, Germany, Norway, Sweden and The Netherlands as well as females from Finland and Germany met the 
recommendation for Central Europe of 1,000 mg/day [D-A-CH, 2000]. The recommendation for the Nordic countries is lower ( $800 \mathrm{mg} /$ day [NNR, 2004]). This recommendation was not met by Austrian, Czech, Estonian, Hungarian and Polish men as well as by females from Austria, Belgium, the Czech Republic, Estonia, Greece, Hungary, Ireland, Latvia, Lithuania, Poland, Romania, Spain and the United Kingdom.

Compared with the D-A-CH reference values for magnesium the group of males from almost one-third of the participating countries and the female group from half of the participating countries did not meet the recommendation [D-A-CH, 2000].

The iron intake was between 10.6 and $26.9 \mathrm{mg} /$ day in males and between 8.2 and $22.2 \mathrm{mg} /$ day in females. Males from all participating countries met the recommendation of $10 \mathrm{mg} /$ day, in the female group only Czech, Portuguese and Romanian women met the reference value of $15 \mathrm{mg} /$ day [D-A-CH, 2000].

With the exception of the United Kingdom (35-64 years in males and 25-54 years in females) the zinc intake was above the recommendation [D-A-CH, 2000].

The iodine intake was between 67 and $264 \mathrm{mg} /$ day in males and between 48 and $200 \mathrm{mg} /$ day in females. All countries were within the recommendation, except for German and Lithuanian adults, both males and females, as well as females from Portugal [Eurodiet, 2000].

The copper intake was between 1.1 and $2.3 \mathrm{mg} /$ day in males and between 0.1 and $2.2 \mathrm{mg} /$ day in females. The intake of males in Austria, Finland, Italy, Lithuania and Poland was above the recommended intake range of $1.0-1.5 \mathrm{mg} /$ day. In the female group, in Austria the intake was above and in the United Kingdom below the recommended intake range [D-A-CH, 2000].

The intake of selenium was within the recommendation in all participating countries except for Finnish females in whom the intake was slightly above the recommended intake range of 30-70 $\mu \mathrm{g} / \mathrm{day}$ [D-A-CH, 2000] (cf. table 7.40).

Comparison of Regions

The intake data of selected minerals in the four regions is shown in table 7.41. Concerning the intake values of calcium, phosphorus and iron no big difference between the regions could be observed. The intake of zinc was lower in the West region than in the other regions and that of selenium was lower in the North region.

\subsection{Energy and Nutrient Intake in European Elderly}

\section{Background}

More than $70 \%$ of the participating countries had data on energy and nutrient intake of people older than 64 years. In contrast to the European Nutrition and Health Report 2004 , the age groups in the current report are more homogeneous, only the methods used for the assessment of dietary intake data were different (cf. table 7.42). 
Table 7.40. Mineral intake (mean \pm SD) in adults of European countries (male and female)

\begin{tabular}{|c|c|c|c|c|c|c|}
\hline & $\begin{array}{l}\text { Age } \\
\text { years }\end{array}$ & $n$ & $\begin{array}{l}\text { Sodium } \\
\mathrm{g}\end{array}$ & $\begin{array}{l}\text { Potassium } \\
\mathrm{g}\end{array}$ & $\begin{array}{l}\text { Calcium } \\
\mathrm{mg}\end{array}$ & $\begin{array}{l}\text { Phosphorus } \\
\text { mg }\end{array}$ \\
\hline \multicolumn{7}{|l|}{ Male } \\
\hline Austria & $19-64$ & 778 & $3.9 \pm 1.6$ & $2.7 \pm 1.1$ & $782 \pm 445$ & $1,302 \pm 492$ \\
\hline Belgium & $19-64$ & n.a. & n.a. & n.a. & $847 \pm 326$ & n.a. \\
\hline Czech Republic & $19-64$ & 1,064 & n.a. & n.a. & $687 \pm 512$ & n.a. \\
\hline Denmark & $19-64$ & 1,283 & $4.2 \pm 1.3$ & $3.8 \pm 1.1$ & $1,055 \pm 448$ & $1,519 \pm 479$ \\
\hline Estonia & 19-64 & 899 & $3.6 \pm 2.3$ & $3.8 \pm 1.8$ & $717 \pm 543$ & n.a. \\
\hline Finland & $19-64$ & 730 & $3.3 \pm 1.2$ & $4.2 \pm 1.3$ & $1,202 \pm 592$ & $1,778 \pm 622$ \\
\hline France & $19-64$ & 852 & $3.9 \pm 0.1$ & n.a. & $981 \pm 16$ & n.a. \\
\hline Germany & $19-64$ & 4,912 & $3.5 \pm 1.5$ & $3.9 \pm 1.4$ & $1,171 \pm 556$ & n.a. \\
\hline Greece & $22 \pm 2$ & 500 & $2.6 \pm 1.5$ & $3.1 \pm 1.5$ & $991 \pm 614$ & $1,389 \pm 619$ \\
\hline Hungary & $\geq 18$ & 473 & $7.3 \pm 1.7$ & $3.0 \pm 0.9$ & $717 \pm 319$ & $1,290 \pm 303$ \\
\hline Ireland & $19-64$ & 662 & n.a. & n.a. & $949 \pm 354$ & $1,645 \pm 463$ \\
\hline Italy & $19-64$ & 660 & $5.8 \pm 2.4$ & $3.4 \pm 0.8$ & $947 \pm 309$ & $1,467 \pm 311$ \\
\hline Latvia & $19-64$ & 1,065 & n.a. & $4.3 \pm 2.2$ & $855 \pm 547$ & $1,485 \pm 687$ \\
\hline Lithuania & $19-64$ & 849 & $4.3 \pm 2.3$ & $3.3 \pm 1.4$ & $812 \pm 446$ & $1,403 \pm 603$ \\
\hline Norway & $19-64$ & 1,050 & n.a. & n.a. & $1068 \pm 460$ & n.a. \\
\hline Poland & $19-64$ & 1,106 & $6.0 \pm 2.3$ & $4.4 \pm 1.5$ & $666 \pm 423$ & $1,596 \pm 568$ \\
\hline Portugal & $19-64$ & 917 & $3.8 \pm 1.0$ & $3.8 \pm 0.9$ & $883 \pm 354$ & $1,434 \pm 381$ \\
\hline Romania & $19-64$ & 177 & n.a. & n.a. & $875 \pm 497$ & n.a. \\
\hline Spain & $19-64$ & 706 & $3.0 \pm 0.7$ & $3.0 \pm 0.5$ & $830 \pm 200$ & $1,418 \pm 217$ \\
\hline Sweden & $19-64$ & 517 & $3.6 \pm 0.9$ & $3.5 \pm 0.9$ & $1069 \pm 395$ & $1,577 \pm 427$ \\
\hline The Netherlands & $19-64$ & 1,836 & n.a. & $4.1 \pm 1.1$ & $1082 \pm 464$ & $1,755 \pm 511$ \\
\hline \multirow[t]{4}{*}{ United Kingdom } & $25-34$ & 119 & $3.4 \pm 1.5$ & $3.2 \pm 1.2$ & $934 \pm 445$ & $1,464 \pm 523$ \\
\hline & $35-44$ & 152 & $2.9 \pm 1.2$ & $2.9 \pm 1.0$ & $853 \pm 371$ & $1,264 \pm 449$ \\
\hline & $45-54$ & 143 & $3.1 \pm 1.3$ & $2.9 \pm 1.0$ & $875 \pm 408$ & $1,306 \pm 473$ \\
\hline & $55-64$ & 189 & $2.9 \pm 1.1$ & $3.1 \pm 0.9$ & $899 \pm 340$ & $1,323 \pm 387$ \\
\hline All countries (min.-max.) & & & $2.6-7.3$ & $2.7-4.4$ & $687-1,171$ & $1264-1,778$ \\
\hline Reference values ${ }^{2}$ & & & $0.55 /<2^{4}$ & 2 & 1,000 & 700 \\
\hline \multicolumn{7}{|l|}{ Female } \\
\hline Austria & $19-64$ & 1,345 & $3.1 \pm 1.4$ & $2.6 \pm 1.0$ & $774 \pm 404$ & $1,102 \pm 421$ \\
\hline Belgium & n.a. & n.a. & n.a. & n.a. & $750 \pm 260$ & n.a. \\
\hline
\end{tabular}




\begin{tabular}{|c|c|c|c|c|c|c|}
\hline $\begin{array}{l}\text { Magnesium } \\
\mathrm{mg}\end{array}$ & $\begin{array}{l}\text { Iron } \\
\text { mg }\end{array}$ & $\begin{array}{l}\text { Zinc } \\
\text { mg }\end{array}$ & $\begin{array}{l}\text { lodine } \\
\mu g^{1}\end{array}$ & $\begin{array}{l}\text { Copper } \\
\mathrm{mg}\end{array}$ & $\begin{array}{l}\text { Manganese } \\
\mathrm{mg}\end{array}$ & $\begin{array}{l}\text { Selenium } \\
\mu \mathrm{g}\end{array}$ \\
\hline $338 \pm 132$ & $13.4 \pm 5.1$ & $12.2 \pm 4.8$ & $199 \pm 112$ & $2.3 \pm 1.0$ & $4.7 \pm 2.8$ & n.a. \\
\hline n.a. & $13.3 \pm 3.1$ & n.a. & n.a. & n.a. & n.a. & n.a. \\
\hline n.a. & $19.3 \pm 18.3$ & n.a. & n.a. & n.a. & n.a. & n.a. \\
\hline $394 \pm 115$ & $11.1 \pm 3.3$ & $12.3 \pm 3.6$ & $213 \pm 74$ & n.a. & n.a. & $42 \pm 14$ \\
\hline $348 \pm 179$ & $15.3 \pm 10.0$ & $12.4 \pm 6.8$ & $264 \pm 170$ & n.a. & n.a. & n.a. \\
\hline $428 \pm 134$ & $13.6 \pm 5.7$ & $13.6 \pm 4.8$ & $253 \pm 220$ & $1.6 \pm 0.7$ & n.a. & $73 \pm 27$ \\
\hline n.a. & n.a. & n.a. & n.a. & n.a. & n.a. & n.a. \\
\hline $465 \pm 162$ & $15.5 \pm 5.8$ & $12.6 \pm 4.9$ & $108 \pm 46$ & n.a. & n.a. & n.a. \\
\hline $281 \pm 127$ & $13.7 \pm 6.4$ & n.a. & n.a. & n.a. & n.a. & n.a. \\
\hline $442 \pm 117$ & $12.5 \pm 3.4$ & $10.2 \pm 2.8$ & n.a. & $1.4 \pm 0.9$ & $3.0 \pm 7.9$ & n.a. \\
\hline $354 \pm 116$ & $14.4 \pm 5.5$ & $11.6 \pm 4.4$ & n.a. & $1.5 \pm 0.8$ & n.a. & n.a. \\
\hline $230 \pm 58$ & $14.7 \pm 3.9$ & $12.9 \pm 3.2$ & n.a. & $1.6 \pm 0.7$ & n.a. & $48 \pm 20$ \\
\hline $383 \pm 197$ & $19.0 \pm 10.0$ & n.a. & n.a. & n.a. & n.a. & n.a. \\
\hline $353 \pm 155$ & $14.3 \pm 7.0$ & $13.4 \pm 6.4$ & $67 \pm 67$ & $1.8 \pm 1.1$ & $4.9 \pm 4.3$ & n.a. \\
\hline $392 \pm 121$ & $13.4 \pm 5.1$ & n.a. & n.a. & n.a. & n.a. & n.a. \\
\hline $393 \pm 127$ & $17.1 \pm 10.6$ & $14.6 \pm 5.6$ & $181 \pm 91$ & $1.6 \pm 0.6$ & $6.1 \pm 2.3$ & $64 \pm 33$ \\
\hline $354 \pm 97$ & $16.7 \pm 4.6$ & n.a. & n.a. & n.a. & n.a. & n.a. \\
\hline $372 \pm 157$ & $26.9 \pm 11.0$ & n.a. & n.a. & n.a. & n.a. & n.a. \\
\hline $309 \pm 50$ & $13.0 \pm 1.8$ & $9.4 \pm 1.4$ & n.a. & n.a. & n.a. & n.a. \\
\hline $348 \pm 94$ & $12.4 \pm 3.4$ & $12.7 \pm 3.4$ & n.a. & n.a. & n.a. & $36 \pm 12$ \\
\hline $380 \pm 109$ & $12.9 \pm 4.2$ & $11.3 \pm 3.6$ & n.a. & $1.3 \pm 0.4$ & n.a. & $52 \pm 22$ \\
\hline $289 \pm 109$ & $11.7 \pm 5.0$ & $10.0 \pm 4.2$ & $213 \pm 111$ & $1.2 \pm 0.6$ & $2.9 \pm 1.3$ & n.a. \\
\hline $256 \pm 106$ & $10.9 \pm 5.4$ & $8.6 \pm 3.6$ & $197 \pm 109$ & $1.1 \pm 0.7$ & $2.8 \pm 1.5$ & n.a. \\
\hline $257 \pm 95$ & $10.6 \pm 4.6$ & $9.1 \pm 3.8$ & $203 \pm 101$ & $1.1 \pm 0.5$ & $3.0 \pm 1.8$ & n.a. \\
\hline $259 \pm 90$ & $11.5 \pm 4.2$ & $9.5 \pm 3.0$ & $209 \pm 95$ & $1.3 \pm 1.4$ & $3.1 \pm 1.4$ & n.a. \\
\hline $256-465$ & $10.6-26.9$ & $8.6-14.6$ & $67-264$ & $1.1-2.3$ & $2.8-6.1$ & $36-73$ \\
\hline $400 / 350 / 350$ & 10 & 10 & $150^{3}$ & $1.0-1.5$ & $2-5$ & $30-70$ \\
\hline $306 \pm 113$ & $11.9 \pm 4.4$ & $10.0 \pm 3.7$ & $192 \pm 107$ & $2.2 \pm 0.9$ & $5.0 \pm 3.0$ & n.a. \\
\hline n.a. & $9.9 \pm 2.3$ & n.a. & n.a. & n.a. & n.a. & n.a. \\
\hline
\end{tabular}


Table 7.40. Continued

\begin{tabular}{|c|c|c|c|c|c|c|}
\hline & $\begin{array}{l}\text { Age } \\
\text { years }\end{array}$ & $\mathrm{n}$ & $\begin{array}{l}\text { Sodium } \\
\mathrm{g}\end{array}$ & $\begin{array}{l}\text { Potassium } \\
\mathrm{g}\end{array}$ & $\begin{array}{l}\text { Calcium } \\
\mathrm{mg}\end{array}$ & $\begin{array}{l}\text { Phosphorus } \\
\mathrm{mg}\end{array}$ \\
\hline Czech Republic & $19-64$ & 1,094 & n.a. & n.a. & $679 \pm 456$ & n.a. \\
\hline Denmark & $19-64$ & 1,486 & $3.1 \pm 0.9$ & $3.2 \pm 0.9$ & $990 \pm 389$ & $1,238 \pm 370$ \\
\hline Estonia & 19-64 & 1,113 & $2.5 \pm 1.5$ & $3.1 \pm 1.3$ & $590 \pm 362$ & n.a. \\
\hline Finland & 19-64 & 846 & $2.4 \pm 0.8$ & $3.4 \pm 1.0$ & $1007 \pm 450$ & $1,363 \pm 448$ \\
\hline France & $19-64$ & 1,499 & $2.8 \pm 0.0$ & n.a. & $841 \pm 13$ & n.a. \\
\hline Germany & $19-64$ & 6,016 & $2.5 \pm 0.9$ & $3.3 \pm 1.0$ & $1047 \pm 389$ & n.a. \\
\hline Greece & $22 \pm 2$ & 451 & $1.7 \pm 1.0$ & $2.4 \pm 1.1$ & $744 \pm 438$ & n.a. \\
\hline Hungary & $\geq 18$ & 706 & $5.6 \pm 1.8$ & $2.7 \pm 1.1$ & $656 \pm 276$ & $1,047 \pm 254$ \\
\hline Ireland & 19-64 & 717 & n.a. & n.a. & $742 \pm 299$ & $1,161 \pm 318$ \\
\hline Italy & 19-64 & 801 & $5.0 \pm 2.4$ & $2.9 \pm 0.8$ & $851 \pm 264$ & $1,213 \pm 268$ \\
\hline Latvia & $19-64$ & 1,238 & n.a. & $3.1 \pm 1.6$ & $742 \pm 456$ & $1,081 \pm 493$ \\
\hline Lithuania & 19-64 & 1,087 & $2.8 \pm 15$ & $2.5 \pm 1.0$ & $674 \pm 338$ & $1,034 \pm 456$ \\
\hline Norway & $19-64$ & 1,146 & n.a. & n.a. & $833 \pm 340$ & n.a. \\
\hline Poland & $19-64$ & 1,334 & $3.4 \pm 1.4$ & $3.1 \pm 1.1$ & $508 \pm 317$ & $1,017 \pm 405$ \\
\hline Portugal & $19-64$ & 1,472 & $3.5 \pm 0.9$ & $3.6 \pm 1.0$ & $963 \pm 395$ & $1,422 \pm 415$ \\
\hline Romania & 19-64 & 341 & n.a. & n.a. & $792 \pm 409$ & n.a. \\
\hline Spain & 19-64 & 875 & $2.2 \pm 0.5$ & $2.7 \pm 0.5$ & $778 \pm 170$ & $1,197 \pm 187$ \\
\hline Sweden & 19-64 & 575 & $2.9 \pm 0.8$ & $3.0 \pm 0.8$ & $922 \pm 300$ & $1,285 \pm 322$ \\
\hline The Netherlands & 19-64 & 2,112 & n.a. & $3.4 \pm 0.9$ & $965 \pm 393$ & $1,383 \pm 387$ \\
\hline \multirow[t]{4}{*}{ United Kingdom } & $25-34$ & 295 & $2.2 \pm 0.8$ & $2.3 \pm 0.7$ & $687 \pm 284$ & $1,021 \pm 320$ \\
\hline & $35-44$ & 349 & $2.2 \pm 0.8$ & $2.3 \pm 0.8$ & $717 \pm 348$ & $1,019 \pm 353$ \\
\hline & $45-54$ & 245 & $2.1 \pm 0.8$ & $2.4 \pm 0.8$ & $740 \pm 330$ & $1,026 \pm 356$ \\
\hline & $55-64$ & 236 & $2.1 \pm 0.8$ & $2.4 \pm 0.7$ & $751 \pm 300$ & $1,037 \pm 334$ \\
\hline All countries (min.-max.) & & & $1.7-5.6$ & $2.3-3.6$ & $508-1,047$ & $1,017-1,422$ \\
\hline Reference values ${ }^{2}$ & & & $0.55 /<2^{4}$ & 2 & 1000 & 700 \\
\hline
\end{tabular}

${ }^{1}$ lodised salt was not taken into account in each country; ${ }^{2} \mathrm{D}-\mathrm{A}-\mathrm{CH}, 2000$, reference values refer to age groups 19-24 years, $25-50$ years and $51-64$ years; ${ }^{3}$ Eurodiet, $2000 ;{ }^{4}$ WHO, 2003; n.a. $=$ not available. 


\begin{tabular}{|c|c|c|c|c|c|c|}
\hline $\begin{array}{l}\text { Magnesium } \\
\text { mg }\end{array}$ & $\begin{array}{l}\text { Iron } \\
\text { mg }\end{array}$ & $\begin{array}{l}\text { Zinc } \\
\text { mg }\end{array}$ & $\begin{array}{l}\text { lodine } \\
\mu g^{1}\end{array}$ & $\begin{array}{l}\text { Copper } \\
\text { mg }\end{array}$ & $\begin{array}{l}\text { Manganese } \\
\mathrm{mg}\end{array}$ & $\begin{array}{l}\text { Selenium } \\
\mu \mathrm{g}\end{array}$ \\
\hline n.a. & $16.5 \pm 18.8$ & n.a. & n.a. & n.a. & n.a. & n.a. \\
\hline $325 \pm 88$ & $8.9 \pm 2.5$ & $9.7 \pm 2.6$ & $175 \pm 58$ & n.a. & n.a. & $34 \pm 11$ \\
\hline $261 \pm 109$ & $11.7 \pm 6.8$ & $9.1 \pm 4.5$ & $200 \pm 121$ & n.a. & n.a. & n.a. \\
\hline $340 \pm 102$ & $10.3 \pm 3.4$ & $10.0 \pm 3.3$ & $194 \pm 121$ & $1.3 \pm 0.5$ & n.a. & $54 \pm 19$ \\
\hline n.a. & n.a. & n.a. & n.a. & n.a. & n.a. & n.a. \\
\hline $382 \pm 348$ & $12.6 \pm 3.8$ & $9.7 \pm 3.1$ & $101 \pm 40$ & n.a. & n.a. & n.a. \\
\hline $213 \pm 102$ & $9.7 \pm 5.4$ & n.a. & n.a. & n.a. & n.a. & n.a. \\
\hline $372 \pm 100$ & $9.8 \pm 2.6$ & $7.8 \pm 2.2$ & n.a. & $1.0 \pm 0.5$ & $2.5 \pm 8.0$ & n.a. \\
\hline $255 \pm 83$ & $14.1 \pm 18.7$ & $8.5 \pm 5.0$ & n.a. & $1.2 \pm 0.7$ & n.a. & n.a. \\
\hline $197 \pm 53$ & $11.9 \pm 3.4$ & $10.7 \pm 2.8$ & n.a. & $1.3 \pm 0.5$ & n.a. & $39 \pm 16$ \\
\hline $285 \pm 143$ & $13.0 \pm 7.0$ & n.a. & n.a. & n.a. & n.a. & n.a. \\
\hline $263 \pm 113$ & $9.9 \pm 5.4$ & $9.3 \pm 4.8$ & $48 \pm 48$ & $1.4 \pm 1.1$ & $3.8 \pm 5.5$ & n.a. \\
\hline $303 \pm 90$ & $12.2 \pm 5.6$ & n.a. & n.a. & n.a. & n.a. & n.a. \\
\hline $263 \pm 91$ & $10.7 \pm 6.5$ & $8.9 \pm 3.5$ & $118 \pm 55$ & $1.1 \pm 0.4$ & $4.2 \pm 1.7$ & $38 \pm 21$ \\
\hline $328 \pm 98$ & $15.0 \pm 4.4$ & n.a. & n.a. & n.a. & n.a. & n.a. \\
\hline $306 \pm 129$ & $22.2 \pm 9.2$ & n.a. & n.a. & n.a. & n.a. & n.a. \\
\hline $269 \pm 51$ & $10.5 \pm 1.7$ & $7.8 \pm 1.2$ & n.a. & n.a. & n.a. & n.a. \\
\hline $293 \pm 68$ & $10.3 \pm 3.4$ & $9.9 \pm 2.4$ & n.a. & n.a. & n.a. & $31 \pm 11$ \\
\hline $301 \pm 86$ & $10.6 \pm 3.2$ & $9.0 \pm 2.8$ & n.a. & $1.0 \pm 0.3$ & n.a. & $41 \pm 19$ \\
\hline $193 \pm 60$ & $8.2 \pm 3.2$ & $6.7 \pm 2.2$ & $142 \pm 73$ & $0.9 \pm 0.4$ & $2.2 \pm 0.9$ & n.a. \\
\hline $192 \pm 65$ & $8.5 \pm 3.7$ & $6.8 \pm 2.4$ & $146 \pm 83$ & $0.9 \pm 0.5$ & $2.3 \pm 1.2$ & n.a. \\
\hline $195 \pm 72$ & $8.3 \pm 3.5$ & $6.7 \pm 2.6$ & $161 \pm 88$ & $0.8 \pm 0.5$ & $2.5 \pm 1.2$ & n.a. \\
\hline $199 \pm 64$ & $9.0 \pm 3.2$ & $7.1 \pm 2.6$ & $158 \pm 80$ & $0.1 \pm 1.1$ & $2.6 \pm 1.1$ & n.a. \\
\hline $192-372$ & $8.2-22.2$ & $6.7-10.7$ & $48-200$ & $0.1-2.2$ & $2.2-5.0$ & $31-54$ \\
\hline $310 / 300 / 300$ & $15 / 15 / 10$ & 7 & $150^{3}$ & $1.0-1.5$ & $2-5$ & $30-70$ \\
\hline
\end{tabular}


Table 7.41. Intake of selected minerals (min.-max.) in adults in the four regions

\begin{tabular}{|c|c|c|c|c|c|c|}
\hline Region/sex & $\begin{array}{l}\text { Calcium } \\
\mathrm{mg}\end{array}$ & $\begin{array}{l}\text { Phosphorus } \\
\text { mg }\end{array}$ & $\begin{array}{l}\text { Iron } \\
\text { mg }\end{array}$ & $\begin{array}{l}\text { Zinc } \\
\mu \mathrm{g}\end{array}$ & $\begin{array}{l}\text { lodine } \\
\mu \mathrm{g}\end{array}$ & $\begin{array}{l}\text { Selenium } \\
\mu \mathrm{g}\end{array}$ \\
\hline \multicolumn{7}{|l|}{ North $^{1}$} \\
\hline Male & 717-1,202 & $1,403-1,778^{5}$ & $11.1-19.0$ & $12.3-13.6^{6}$ & $67-264^{7}$ & $42-73^{8}$ \\
\hline Female & $590-1,007$ & $1,034-1363^{5}$ & $8.9-13.0$ & $9.1-10.0^{6}$ & $48-200^{7}$ & $31-54^{8}$ \\
\hline \multicolumn{7}{|l|}{ South ${ }^{2}$} \\
\hline Male & 830-991 & $1,389-1,467$ & $13.0-16.7$ & $9.4-12.9^{9}$ & n.a. & $48^{10}$ \\
\hline Female & $744-963$ & $1,197-1,422^{19}$ & $9.7-15.0$ & $7.8-10.7^{9}$ & n.a. & $39^{10}$ \\
\hline \multicolumn{7}{|c|}{ Central and East ${ }^{3}$} \\
\hline Male & $666-1,171$ & $1,290-1,596^{11}$ & $12.5-26.9$ & $10.2-14.6^{12}$ & $108-199^{13}$ & $64^{14}$ \\
\hline Female & $508-1,047$ & $1,017-1,102^{11}$ & $9.8-22.2$ & $7.8-10.0^{12}$ & $101-192^{13}$ & $38^{14}$ \\
\hline \multicolumn{7}{|l|}{ West ${ }^{4}$} \\
\hline Male & $847-1,082$ & $1,339-1,755^{15}$ & $11.2-14.4^{16}$ & $9.3-11.6^{15}$ & $205^{17}$ & $52^{18}$ \\
\hline Female & $724-965$ & $1,026-1,383^{15}$ & $8.5-14.1^{16}$ & $6.8-9.0^{15}$ & $152^{17}$ & $48^{18}$ \\
\hline \multicolumn{7}{|c|}{$\begin{array}{l}{ }^{1} \text { North: SE, NO, EE, FI, LV, LT, DK; }{ }^{2} \text { South: PT, ES, IT, GR; }{ }^{3} \text { Central and East: AT, CZ, DE, HU, PL, RO; }{ }^{4} \text { West: } \\
\text { BE, NL, FR, IR, UK; }{ }^{5} \text { only SE, FI, LV, LT and DK; }{ }^{6} \text { only SE, EE, FI, LT and DK; }{ }^{7} \text { only EE, FI, LT and DK; }{ }^{8} \text { only } \\
\text { SE, FI and DK; }{ }^{9} \text { only ES and IT; }{ }^{10} \mathrm{IT} ;{ }^{11} \text { only AT, HU and PL; }{ }^{12} \text { only AT, DE, HU and PL; }{ }^{13} \text { only AT, DE and } \\
\mathrm{PL} ;{ }^{14} \text { only PL; }{ }^{15} \text { only NL, IR and UK; }{ }^{16} \text { only BE, NL, IR and UK; }{ }^{17} \text { only UK; }{ }^{18} \text { only NL; }{ }^{19} \text { only PT, ES and } \\
\text { IT. }\end{array}$} \\
\hline
\end{tabular}

Table 7.42. Methods and period of dietary assessment in elderly of the participating countries (data available from 19,328 elderly)

\begin{tabular}{lllllll}
\hline Country & $\begin{array}{l}\text { Age group } \\
\text { years }\end{array}$ & Method & $\begin{array}{l}\text { Year of } \\
\text { survey }\end{array}$ & n & $\begin{array}{l}\text { Intake from } \\
\text { dietary } \\
\text { supplements } \\
\text { is included }\end{array}$ & Reference \\
\hline Austria & $>64$ & $\begin{array}{l}\text { 3-day dietary } \\
\text { record }\end{array}$ & 2007 & 349 & no & Elmadfa et al., 2008 \\
\hline Belgium & $>64$ & 24-hour recall & 2004 & 3,249 & no & Devriese et al., 2006 \\
\hline Denmark & $>64$ & $\begin{array}{l}\text { 7-day dietary } \\
\text { record }\end{array}$ & $2000-2002$ & 329 & no & Lyhne et al., 2005 \\
\hline Finland & $>64$ & $\begin{array}{l}\text { 48-hour recall } \\
\text { France }\end{array}$ & 2007 & 463 & no & The National Findiet \\
\hline $65-74$ & $\begin{array}{l}3 \times 24-\text {-hour } \\
\text { recall }\end{array}$ & $2006 / 2007$ & 349 & no & Castetbon et al., 2009 \\
\hline
\end{tabular}


Table 7.42. Continued

\begin{tabular}{|c|c|c|c|c|c|c|}
\hline Country & $\begin{array}{l}\text { Age group } \\
\text { years }\end{array}$ & Method & $\begin{array}{l}\text { Year of } \\
\text { survey }\end{array}$ & $\mathrm{n}$ & $\begin{array}{l}\text { Intake from } \\
\text { dietary } \\
\text { supplements } \\
\text { is included }\end{array}$ & Reference \\
\hline Germany & $>64$ & $\begin{array}{l}\text { dietary history } \\
\text { interview }\end{array}$ & $2005-2007$ & 3,031 & no & $\begin{array}{l}\text { Max Rubner-Institut } \\
\text { (eds), 2008a, 2008b }\end{array}$ \\
\hline Greece & $>64$ & FFQ & 1994-1999 & 6,108 & no & $\begin{array}{l}\text { EPIC study - } \\
\text { Greek cohort }{ }^{1}\end{array}$ \\
\hline Hungary & $\geq 60$ & $\begin{array}{l}\text { 3-day dietary } \\
\text { record }\end{array}$ & $2003 / 2004$ & 373 & no & unpubl. data ${ }^{2}$ \\
\hline Ireland & $>64$ & FFQ & 2007 & 1,322 & no & $\begin{array}{l}\text { SLÁN, 2007 } \\
\text { SLÁN, 1997/1998 }\end{array}$ \\
\hline Italy & $>64$ & $\begin{array}{l}\text { 7-day dietary } \\
\text { record }\end{array}$ & & 167 & no & D'Amicis, 2000 \\
\hline Norway & $>64$ & FFQ & 1997 & 342 & yes & Norkost 1997 \\
\hline Poland & $>64$ & 24-hour recall & 2000 & 453 & no & $\begin{array}{l}\text { Szponar et al., } 2000 \\
\text { (unpubl.) }\end{array}$ \\
\hline Portugal & $>64$ & FFQ & & 585 & no & $\begin{array}{l}\text { EPIPorto, Study held } \\
\text { by the Department } \\
\text { of Hygiene and } \\
\text { Epidemiology, } \\
\text { University of Porto } \\
\text { Medical School }\end{array}$ \\
\hline Romania & $>64$ & $\begin{array}{l}\text { personal } \\
\text { interview }\end{array}$ & 2006 & 518 & no & $\begin{array}{l}\text { National synthesis, } \\
2006\end{array}$ \\
\hline Spain & $>64$ & $\begin{array}{l}2 \times 24 \text {-hour } \\
\text { recall }\end{array}$ & $2002-2003$ & 342 & no & $\begin{array}{l}\text { Serra Majem and } \\
\text { Ribas, 2007; Serra- } \\
\text { Majem L et al, 2007; } \\
\text { Serra Majem et al., } \\
2006\end{array}$ \\
\hline Sweden & $65-74$ & $\begin{array}{l}\text { 7-day dietary } \\
\text { record }\end{array}$ & 1997/1998 & 122 & no & Becker et al., 2002 \\
\hline The Netherlands & $>64$ & $\begin{array}{l}\text { 2-day dietary } \\
\text { record }\end{array}$ & 1997/1998 & 421 & no & $\begin{array}{l}\text { Hulshof and van } \\
\text { Staveren, } 1991\end{array}$ \\
\hline United Kingdom & $\begin{array}{l}65-74 \\
\geq 75\end{array}$ & $\begin{array}{l}\text { n.a. } \\
\text { n.a. }\end{array}$ & $\begin{array}{l}2003-2005 \\
2003-2005\end{array}$ & $\begin{array}{l}397 \\
408\end{array}$ & $\begin{array}{l}\text { n.a. } \\
\text { n.a. }\end{array}$ & $\begin{array}{l}\text { Low income diet and } \\
\text { health survey, 2003- } \\
2005^{3}\end{array}$ \\
\hline
\end{tabular}

${ }^{1}$ unpubl. data estimated for the purposes of the ENHR 2009 project; ${ }^{2}$ data provided by the National Institute of Food Safety and Nutrition, Hungary; ${ }^{3}$ data provided by the University of Southampton; n.a. $=$ not available. 
Table 7.43. Intake of energy and macronutrients (mean \pm SD) in elderly of European countries (male and female)

\begin{tabular}{lllll}
$\begin{array}{l}\text { Age } \\
\text { years }\end{array}$ & $n$ & Energy $M J$ & $\begin{array}{l}\text { Protein } \\
\% E^{1}\end{array}$ & $\frac{\text { Carbohydrates, } \% E^{1}}{\text { total }}$ \\
\hline
\end{tabular}

\section{Male}

\begin{tabular}{|c|c|c|c|c|c|c|}
\hline Austria & $>64$ & 147 & $7.7 \pm 2.4$ & $14.9 \pm 3.1$ & $41.7 \pm 7.5$ & $10.0 \pm 5.3$ \\
\hline Belgium & $>64$ & n.a. & $8.9 \pm 2.3$ & $15.2 \pm 2.7$ & $40.7 \pm 7.4$ & n.a. \\
\hline Denmark & $>64$ & 165 & $9.7 \pm 2.9$ & $14.0 \pm 2.1$ & $42.0 \pm 5.9$ & $7.0 \pm 4.1$ \\
\hline Finland & $>64$ & 229 & $7.7 \pm 2.3$ & $17.4 \pm 3.8$ & $49.7 \pm 8.9$ & $9.3 \pm 5.6$ \\
\hline France & $>64$ & 130 & $9.0 \pm 0.2$ & $16.5 \pm 0.3$ & $44.0 \pm 0.9$ & n.a. \\
\hline Germany & $>64$ & 1,469 & $9.2 \pm 2.5$ & $14.5 \pm 2.6$ & $44.6 \pm 7.3$ & n.a. \\
\hline Greece & $>64$ & 2,508 & $8.5 \pm 2.5$ & $14.1 \pm 1.7$ & $39.8 \pm 5.9$ & n.a. \\
\hline Hungary & $>59$ & 138 & $10.5 \pm 2.3$ & $14.8 \pm 2.1$ & $45.6 \pm 6.5$ & $6.5 \pm 4.0$ \\
\hline Ireland & $>64$ & 580 & $8.6 \pm 3.3$ & $19.0 \pm 8.0$ & $49.0 \pm 4.2$ & n.a. \\
\hline Italy & $>64$ & 60 & $9.4 \pm 2.2$ & $15.8 \pm 2.4$ & n.a. & n.a. \\
\hline Norway & $>64$ & 176 & $9.0 \pm 3.1$ & $16.0 \pm 2.0$ & $51.0 \pm 6.0$ & $8.0 \pm 5.0$ \\
\hline Poland & $>64$ & 176 & $10.3 \pm 3.6$ & $13.6 \pm 3.3$ & $50.0 \pm 9.5$ & $12.0 \pm 7.1$ \\
\hline Portugal & $>64$ & 246 & $9.3 \pm 2.2$ & $17.5 \pm 2.4$ & n.a. & n.a. \\
\hline Romania & $>64$ & 177 & $13.0 \pm 4.1$ & $17.2 \pm 3.5$ & $41.2 \pm 7.7$ & n.a. \\
\hline Spain & $>64$ & 163 & $7.1 \pm 1.3$ & $18.7 \pm 2.9$ & $39.1 \pm 5.8$ & $16.8 \pm 4.7$ \\
\hline Sweden & $65-74$ & 64 & $9.6 \pm 2.8$ & $15.6 \pm 1.9$ & $47.4 \pm 6.1$ & $7.8 \pm 3.0$ \\
\hline The Netherlands & $>64$ & 185 & $9.4 \pm 2.4$ & $16.0 \pm 3.0$ & $43.0 \pm 7.0$ & n.a. \\
\hline \multirow[t]{2}{*}{ United Kingdom } & $65-74$ & 144 & $7.7 \pm 2.5$ & $16.8 \pm 3.3$ & $46.8 \pm 6.7$ & $12.0 \pm 6.9$ \\
\hline & $>74$ & 124 & $7.4 \pm 2.3$ & $16.5 \pm 3.7$ & $47.5 \pm 7.0$ & $14.1 \pm 6.9$ \\
\hline All countries (min.-max.) & & & $7.1-13.0$ & $13.6-19.0$ & $39.1-51.0$ & $6.5-16.8$ \\
\hline \multicolumn{7}{|l|}{ Female } \\
\hline Austria & $>64$ & 202 & $7.1 \pm 1.7$ & $15.0 \pm 2.5$ & $45.9 \pm 6.1$ & $12.6 \pm 4.7$ \\
\hline Belgium & $>64$ & n.a. & $6.4 \pm 1.5$ & $16.3 \pm 3.3$ & $42.7 \pm 6.4$ & n.a. \\
\hline Denmark & $>64$ & 164 & $7.8 \pm 1.9$ & $14.0 \pm 2.1$ & $46.0 \pm 6.2$ & $9.0 \pm 5.3$ \\
\hline Finland & $>64$ & 234 & $5.9 \pm 1.7$ & $17.6 \pm 3.4$ & $51.2 \pm 8.0$ & $9.3 \pm 5.1$ \\
\hline France & $>64$ & 219 & $6.7 \pm 0.1$ & $17.5 \pm 0.3$ & $45.4 \pm 0.6$ & n.a. \\
\hline Germany & $>64$ & 1,562 & $7.3 \pm 2.1$ & $14.4 \pm 2.7$ & $48.5 \pm 7.2$ & n.a. \\
\hline Greece & $>64$ & 3,600 & $6.8 \pm 2.1$ & $14.4 \pm 1.8$ & $41.8 \pm 5.6$ & n.a. \\
\hline
\end{tabular}




\begin{tabular}{|c|c|c|c|c|c|}
\hline $\begin{array}{l}\text { Dietary fiber } \\
\mathrm{g}\end{array}$ & Fat $\% \mathrm{E}^{1}$ & SFA \%E & MUFA \%E & PUFA \%E & $\begin{array}{l}\text { Cholesterol } \\
\mathrm{mg}\end{array}$ \\
\hline $16.7 \pm 5.9$ & $37.6 \pm 5.8$ & $15.6 \pm 3.1$ & $12.7 \pm 3.4$ & $7.4 \pm 2.2$ & $329.6 \pm 136.0$ \\
\hline n.a. & $37.9 \pm 7.7$ & $15.6 \pm 4.4$ & $13.5 \pm 2.9$ & $6.7 \pm 2.6$ & n.a. \\
\hline $21.0 \pm 8.8$ & $33.0 \pm 5.5$ & $14.0 \pm 3.0$ & $12.0 \pm 2.1$ & $5.0 \pm 1.0$ & n.a. \\
\hline $24.0 \pm 10.0$ & $31.4 \pm 7.9$ & $12.0 \pm 4.5$ & $11.1 \pm 3.5$ & $5.7 \pm 2.0$ & $217.0 \pm 115.0$ \\
\hline $22.3 \pm 0.8$ & $33.7 \pm 0.7$ & 13.6 & n.a. & n.a. & n.a. \\
\hline $27.3 \pm 9.9$ & $36.0 \pm 6.7$ & n.a. & n.a. & n.a. & $329.6 \pm 133.9$ \\
\hline n.a. & $43.9 \pm 5.4$ & $12.1 \pm 2.6$ & $21.0 \pm 4.2$ & $6.7 \pm 3.1$ & $214.8 \pm 96.4$ \\
\hline $24.2 \pm 6.5$ & $37.5 \pm 5.9$ & $11.4 \pm 2.5$ & $12.0 \pm 2.9$ & $8.7 \pm 2.3$ & $418.2 \pm 182.2$ \\
\hline $24.6 \pm 12.1$ & $34.0 \pm 6.5$ & $13.0 \pm 3.9$ & $10.0 \pm 2.3$ & $6.0 \pm 2.1$ & $361.3 \pm 197.8$ \\
\hline $22.1 \pm 7.6$ & $33.2 \pm 6.5$ & $10.0 \pm 3.0$ & $13.1 \pm 4.0$ & $4.8 \pm 2.5$ & $313.1 \pm 97,3$ \\
\hline $24.0 \pm 9.0$ & $31.0 \pm 6.0$ & $12.0 \pm 3.0$ & $11.0 \pm 2.0$ & $5.0 \pm 2.0$ & $316.0 \pm 132.0$ \\
\hline $23.0 \pm 9.2$ & $36.0 \pm 9.2$ & $12.8 \pm 4.2$ & $15.4 \pm 4.9$ & $5.3 \pm 2.6$ & $433.0 \pm 311.0$ \\
\hline $25.9 \pm 10.4$ & $26.7 \pm 4.6$ & $7.8 \pm 1.9$ & $11.9 \pm 2.3$ & $4.6 \pm 0.9$ & $276.0 \pm 95.4$ \\
\hline n.a. & $41.0 \pm 9.0$ & $25.3 \pm 4.3$ & $15.7 \pm 4.7$ & n.a. & $810.0 \pm 350.0$ \\
\hline $19.6 \pm 3.8$ & $35.6 \pm 4.2$ & $10.3 \pm 1.6$ & $16.6 \pm 2.7$ & $5.2 \pm 1.3$ & $265.5 \pm 28.5$ \\
\hline $19.8 \pm 7.2$ & $33.9 \pm 5.9$ & $15.0 \pm 3.2$ & $12.2 \pm 2.2$ & $4.2 \pm 0.8$ & $383.1 \pm 182.6$ \\
\hline $24.0 \pm 10.0$ & $37.0 \pm 7.0$ & $15.0 \pm 4.0$ & $12.0 \pm 3.0$ & $7.0 \pm 2.0$ & $231.0 \pm 107.0$ \\
\hline $12.1 \pm 4.7$ & $36.5 \pm 6.9$ & $14.4 \pm 4.1$ & n.a. & n.a. & $280.7 \pm 162.4$ \\
\hline $11.9 \pm 5.4$ & $36.1 \pm 6.4$ & $14.5 \pm 4.0$ & n.a. & n.a. & $268.8 \pm 148.5$ \\
\hline $11.9-27.3$ & $26.7-43.9$ & $7.8-25.3$ & $11.0-21.0$ & $4.2-8.7$ & $214.8-810.0$ \\
\hline $17.5 \pm 6.2$ & $37.7 \pm 5.2$ & $16.4 \pm 3.2$ & $12.3 \pm 2.0$ & $6.9 \pm 2.1$ & $323.8 \pm 118.8$ \\
\hline n.a. & $37.5 \pm 6.4$ & $16.4 \pm 4.2$ & $13.4 \pm 2.5$ & $5.9 \pm 2.1$ & n.a. \\
\hline $20.0 \pm 6.7$ & $32.0 \pm 5.8$ & $14.0 \pm 3.0$ & $11.0 \pm 2.2$ & $5.0 \pm 1.0$ & n.a. \\
\hline $21.0 \pm 9.0$ & $30.4 \pm 7.3$ & $11.4 \pm 3.9$ & $10.4 \pm 3.2$ & $5.6 \pm 1.9$ & $159.0 \pm 82.0$ \\
\hline $18.5 \pm 0.5$ & $34.5 \pm 0.6$ & 13.3 & n.a. & n.a. & n.a. \\
\hline $24.9 \pm 9.1$ & $35.5 \pm 6.7$ & n.a. & n.a. & n.a. & $252.33 \pm 279.0$ \\
\hline n.a. & $45.3 \pm 5.0$ & $12.3 \pm 2.5$ & $21.8 \pm 4.4$ & $7.0 \pm 3.3$ & $173.4 \pm 76.1$ \\
\hline
\end{tabular}


Table 7.43. Continued

\begin{tabular}{lllllll}
\hline & $\begin{array}{l}\text { Age } \\
\text { years }\end{array}$ & $\mathrm{n}$ & Energy MJ & \multicolumn{2}{l}{$\begin{array}{l}\text { Protein } \\
\% \mathrm{E}^{1}\end{array}$} & \multicolumn{2}{l}{\begin{tabular}{l} 
Carbohydrates, \% $\mathrm{E}^{1}$ \\
\cline { 6 - 8 }
\end{tabular}} & & & & & total & sucrose \\
\hline Hungary & $>59$ & 235 & $8.8 \pm 1.7$ & $14.5 \pm 1.8$ & $48.6 \pm 5.9$ & $8.0 \pm 5.0$ \\
\hline Ireland & $>64$ & 742 & $8.1 \pm 3.1$ & $19.0 \pm 8.1$ & $50.0 \pm 4.1$ & n.a. \\
\hline Italy & $>64$ & 107 & $7.6 \pm 1.6$ & $16.8 \pm 2.5$ & n.a. & n.a. \\
\hline Norway & $>64$ & 166 & $7.0 \pm 2.0$ & $17.0 \pm 3.0$ & $52.0 \pm 6.0$ & $8.0 \pm 4.0$ \\
\hline Poland & $>64$ & 277 & $8.1 \pm 2.7$ & $13.2 \pm 3.5$ & $52.6 \pm 8.6$ & $13.2 \pm 6.3$ \\
\hline Portugal & $>64$ & 339 & $8.0 \pm 1.9$ & $18.7 \pm 2.3$ & n.a. & n.a. \\
\hline Romania & $>64$ & 341 & $10.9 \pm 3.4$ & $16.3 \pm 3.0$ & $43.9 \pm 9.2$ & n.a. \\
\hline Spain & $>64$ & 179 & $5.8 \pm 0.7$ & $20.0 \pm 2.0$ & $39.1 \pm 5.0$ & $18.5 \pm 4.1$ \\
\hline Sweden & $65-74$ & 58 & $7.9 \pm 2.0$ & $16.3 \pm 2.6$ & $48.4 \pm 5.2$ & $8.7 \pm 3.6$ \\
\hline The Netherlands & $>64$ & 236 & $7.5 \pm 1.9$ & $17.0 \pm 4.0$ & $44.0 \pm 7.0$ & n.a. \\
\hline United Kingdom & $65-74$ & 253 & $6.2 \pm 1.8$ & $17.2 \pm 3.8$ & $48.0 \pm 7.0$ & $11.4 \pm 7.0$ \\
\hline All countries (min.-max.) & $>74$ & 284 & $5.9 \pm 1.7$ & $16.6 \pm 3.7$ & $47.6 \pm 6.0$ & $12.4 \pm 6.0$ \\
\hline Reference values & & & $5.8-10.9$ & $13.2-20.0$ & $39.1-52.6$ & $8.0-18.5$ \\
\hline
\end{tabular}

${ }^{1}$ Difference is not necessarily alcohol; ${ }^{2} \mathrm{D}-\mathrm{A}-\mathrm{CH}, 2000$, reference values refer to age groups $\geq 65$ years (PAL 1.60); ${ }^{3}$ WHO, 2003; ${ }^{4}$ WHO, 2007; ${ }^{5}$ WHO, 2009b; ${ }^{6}$ Eurodiet, 2000; n.a. = not available.

\section{Energy and Macronutrients}

\section{Comparison of Countries}

The daily energy intake was between 7.1 and $13.0 \mathrm{MJ}$ in male and between 5.8 and 10.9 MJ in female elderly. As the $\mathrm{D}-\mathrm{A}-\mathrm{CH}$ reference values $(9.5 \mathrm{MJ} /$ day in male and $7.5 \mathrm{MJ} /$ day in female elderly) apply for a physical activity level (PAL) of 1.6 and elderly are a very inhomogeneous group (ranging from go-goes to no-goes), it is very difficult to compare the intake with the reference values [D-A-CH, 2000].

The share of protein in total energy intake per day was in the range of 13.6 and 19.0\%E in male and 13.2 and 20.0\%E in female elderly. Only Austrian, Danish, German, Greek, Hungarian and Polish male as well as Danish, German, Greek, Hungarian and Polish female elderly were within the recommended intake range (10-15\%E) of the WHO [WHO, 2003].

The share of total carbohydrates in total energy intake per day was between 39.1 and 51.0\% $\mathrm{E}$ in male and between 39.1 and $52.6 \% \mathrm{E}$ in female elderly. The recommended 


\begin{tabular}{|c|c|c|c|c|c|}
\hline $\begin{array}{l}\text { Dietary fiber } \\
\mathrm{g}\end{array}$ & Fat $\% \mathrm{E}^{1}$ & SFA \%E & MUFA \%E & PUFA \%E & $\begin{array}{l}\text { Cholesterol } \\
\mathrm{mg}\end{array}$ \\
\hline $21.7 \pm 5.4$ & $36.3 \pm 5.5$ & $10.9 \pm 2.9$ & $11.3 \pm 2.5$ & $9.1 \pm 2.2$ & $297.0 \pm 112.5$ \\
\hline $24.6 \pm 11.3$ & $33.0 \pm 6.7$ & $12.0 \pm 3.8$ & $10.0 \pm 2.4$ & $5.0 \pm 2.5$ & $305.8 \pm 145.9$ \\
\hline $18.8 \pm 5.6$ & $30.5 \pm 5.9$ & $9.4 \pm 2.6$ & $11.8 \pm 3.0$ & $4.4 \pm 1.9$ & $279.3 \pm 121.7$ \\
\hline $21.0 \pm 7.0$ & $30.0 \pm 6.0$ & $12.0 \pm 3.0$ & $11.0 \pm 2.0$ & $5.0 \pm 1.0$ & $260.0 \pm 91.0$ \\
\hline $19.1 \pm 7.0$ & $34.1 \pm 7.9$ & $12.6 \pm 4.0$ & $14.3 \pm 4.4$ & $4.8 \pm 2.3$ & $317.0 \pm 204.0$ \\
\hline $23.7 \pm 10.4$ & $28.0 \pm 4.8$ & $8.4 \pm 2.1$ & $12.3 \pm 2.4$ & $4.8 \pm 1.0$ & $247.2 \pm 85.2$ \\
\hline n.a. & $39.9 \pm 8.1$ & $24.9 \pm 4.7$ & $15.0 \pm 3.4$ & n.a. & $690.0 \pm 280.0$ \\
\hline $17.5 \pm 3.8$ & $37.4 \pm 2.9$ & $10.8 \pm 1.1$ & $17.5 \pm 1.8$ & $5.3 \pm 1.0$ & $209.3 \pm 25.8$ \\
\hline $19.8 \pm 7.6$ & $33.2 \pm 4.1$ & $14.3 \pm 2.2$ & $12.0 \pm 1.5$ & $4.6 \pm 1.4$ & $309.7 \pm 120.0$ \\
\hline $21.0 \pm 6.0$ & $37.0 \pm 7.0$ & $15.0 \pm 4.0$ & $12.0 \pm 3.0$ & $7.0 \pm 3.0$ & $195.0 \pm 86.0$ \\
\hline $11.2 \pm 4.3$ & $34.8 \pm 6.9$ & $13.9 \pm 4.2$ & n.a. & n.a. & $220.1 \pm 102.2$ \\
\hline $10.3 \pm 4.0$ & $35.8 \pm 6.5$ & $15.0 \pm 4.2$ & n.a. & n.a. & $215.9 \pm 101.7$ \\
\hline $10.3-24.9$ & $28.0-45.3$ & $8.4-24.9$ & $10.0-21.8$ & $4.4-9.1$ & $159.0-690.0$ \\
\hline$>25 \mathrm{~g} \cdot \mathrm{d}^{-1,6}$ & $15-30 \% \mathrm{E}^{3}$ & $<10 \% \mathrm{E}^{3}$ & & $6-11 \% \mathrm{E}^{5}$ & $<300 \mathrm{mg} \cdot \mathrm{d}^{-1,3}$ \\
\hline
\end{tabular}

intake level of 50-75\%E of the WHO [WHO, 2007] was only met by elderly from Norway and Portugal and by female elderly from Finland and Ireland. Whereas the proportion of total carbohydrates in total energy intake was lower than the recommendation, the share of sucrose in total energy intake was above the recommended level in both elderly males and females from Poland, Spain and the United Kingdom. In general, the intake of dietary fibers was below the recommended level ( $>25 \mathrm{~g} /$ day) of Eurodiet [Eurodiet, 2000]. Only Portuguese and German male elderly met the recommendation.

The share of fat in total energy intake was above the recommendation of the WHO [WHO, 2003] in almost every country. Only Portuguese elderly, both males and females, and Norwegian female elderly ingested less than $30 \% \mathrm{E}$ fat. The share of saturated fatty acids (SFA) in total energy intake was between 7.8 and $25.3 \% \mathrm{E}$ in male and between 8.4 and $24.9 \% \mathrm{E}$ in female elderly and therefore in most participating countries in average above the recommended level $(<10 \% \mathrm{E})$ with the exception of Portugal, both groups males and females, and Italy, only females. Due to the generally high intake of SFA, the share of polyunsaturated fatty acids (PUFA) in total energy intake 
Table 7.44. Intake of energy and macronutrients (min.-max.) in elderly in the four regions

\begin{tabular}{|c|c|c|c|c|c|}
\hline Region/sex & $\begin{array}{l}\text { Energy } \\
\text { MJ }\end{array}$ & Protein \%E & $\begin{array}{l}\text { Carbohydrates, \%E } \\
\text { (of that sucrose) }\end{array}$ & $\begin{array}{l}\text { Dietary fiber } \\
\mathrm{g}\end{array}$ & $\begin{array}{l}\text { Fat } \\
\% \mathrm{E}\end{array}$ \\
\hline \multicolumn{6}{|l|}{ North $^{1}$} \\
\hline Male & $7.7-9.7$ & $14.0-17.4$ & $42.0-51.0(7.0-9.3)$ & $19.8-24.0$ & $31.0-33.9$ \\
\hline Female & $5.9-7.9$ & $14.0-17.6$ & $46.0-52.0(8.0-9.3)$ & $19.8-21.0$ & $30.0-33.2$ \\
\hline \multicolumn{6}{|l|}{ South $^{2}$} \\
\hline Male & $7.1-9.4$ & $14.1-18.7$ & $39.1-39.8^{5}\left(16.8^{6}\right)$ & $19.6-25.9^{7}$ & $26.7-43.9$ \\
\hline Female & $5.8-8.0$ & $14.4-20.0$ & $39.1-41.8^{5}\left(18.5^{6}\right)$ & $17.5-23.7^{7}$ & $28.0-45.3$ \\
\hline \multicolumn{6}{|c|}{ Central and East ${ }^{3}$} \\
\hline Male & $7.7-13.0$ & $13.6-17.2$ & $41.2-50.0\left(6.5-12.0^{8}\right)$ & $16.7-27.3^{9}$ & $36.0-41.0$ \\
\hline Female & $7.1-10.9$ & $13.2-16.3$ & $43.9-52.6\left(8.0-13.2^{8}\right)$ & $17.5-24.9^{9}$ & $34.1-39.9$ \\
\hline \multicolumn{6}{|l|}{ West $t^{4}$} \\
\hline Male & $7.6-9.4$ & $15.2-19.0$ & $40.7-49.0\left(13.1^{10}\right)$ & $12.0-24.6^{11}$ & $33.7-37.9$ \\
\hline Female & $6.1-8.1$ & $16.3-19.0$ & $42.7-50.0\left(11.9^{10}\right)$ & $10.8-24.6^{11}$ & $33.0-37.5$ \\
\hline
\end{tabular}

was below the recommended range in almost every country. Only elderly males from Austria, Belgium, Greece, Hungary, Ireland and The Netherlands as well as elderly females from Austria, Greece, Hungary and The Netherlands met the recommendation of ingesting between 6 and 11\% $\mathrm{E}$ of PUFA [WHO, 2009b]. The cholesterol intake was between 215 and $810 \mathrm{mg} /$ day in male and between 159 and $690 \mathrm{mg} /$ day in female elderly. Elderly from Finland, Greece, Portugal, Spain, The Netherlands and the United Kingdom, both males and females, as well as elderly females from Germany, Hungary, Italy and Norway were within the recommendation [WHO, 2003] (cf. table 7.43).

\section{Comparison of Regions}

Table 7.44 shows the intake of energy and macronutrients distribution (min-max) in the four regions. Generally, no remarkable differences between the regions could be observed. It is notable that both lowest and highest relative intake values of fat could be found in the South region.

Concerning fatty acids, the highest intake values of SFA were found in the Central and East region. The share of PUFA in total energy intake was also higher in the Central and East region than in the other regions. The intake of cholesterol was higher in the Central and East region than in the other regions (cf. table 7.45). 
Table 7.45. Intake of fat, fatty acids and cholesterol (min.-max.) in elderly in the four regions

\begin{tabular}{|c|c|c|c|c|c|}
\hline Region/sex & $\begin{array}{l}\text { Fat } \\
\% \mathrm{E}\end{array}$ & $\begin{array}{l}\text { SFA } \\
\% E\end{array}$ & $\begin{array}{l}\text { MUFA } \\
\% \mathrm{E}\end{array}$ & $\begin{array}{l}\text { PUFA } \\
\% E\end{array}$ & $\begin{array}{l}\text { Cholesterol } \\
\text { mg }\end{array}$ \\
\hline \multicolumn{6}{|l|}{ North $^{1}$} \\
\hline Male & $31.0-33.9$ & $12.0-15.0$ & $11.0-12.2$ & $4.2-5.7$ & $217.0-383.1^{5}$ \\
\hline Female & $30.0-33.2$ & $11.4-14.3$ & $10.4-12.0$ & $4.6-5.6$ & $159.0-309.7^{5}$ \\
\hline \multicolumn{6}{|l|}{ South ${ }^{2}$} \\
\hline Male & 26.7-43.9 & $7.8-12.1$ & $11.9-21.0$ & $4.6-6.7$ & $214.8-313.1$ \\
\hline Female & $28.0-45.3$ & $8.4-12.3$ & $12.3-21.8$ & $4.4-7.0$ & $173.4-279.3$ \\
\hline \multicolumn{6}{|c|}{ Central and East ${ }^{3}$} \\
\hline Male & $36.0-41.0$ & $11.4-25.3^{6}$ & $12.0-15.7^{6}$ & $5.3-8.7^{7}$ & $329.6-810.0$ \\
\hline Female & $34.1-39.9$ & $10.9-24.9^{6}$ & $11.3-15.0^{6}$ & $4.8-9.1^{7}$ & $252.3-690.0$ \\
\hline \multicolumn{6}{|l|}{ West $^{4}$} \\
\hline Male & $33.7-37.9$ & $13.0-15.6$ & $10.0-13.5^{8}$ & $6.0-7.0^{8}$ & $231.0-361.3^{9}$ \\
\hline Female & $33.0-37.5$ & $12.0-16.4$ & $10.0-13.4^{8}$ & $5.0-7.0^{8}$ & $195.0-305.8^{9}$ \\
\hline
\end{tabular}

\section{Vitamins}

\section{Comparison of Countries}

The intake of retinol equivalents in elderly of the participating countries was on average above the recommendation [D-A-CH, 2000]. Only Spanish and Hungarian elderly, both males and females, as well as Finnish female elderly did not meet the reference value. The daily intake of $\beta$-carotene was between 1.3 and $4.8 \mathrm{mg} /$ day in male and between 1.3 and $5.0 \mathrm{mg} /$ day in female elderly.

The vitamin D intake was below the recommended intake of $10 \mu \mathrm{g} /$ day [Eurodiet, 2000] in almost all countries. Only in Norway was it clearly above this recommendation. The recommended vitamin $\mathrm{D}$ intake for all individuals above the age of 60 years in the Nordic countries [NNR, 2004] is also $10 \mu \mathrm{g} /$ day. In this recommendation the rather short time spent outdoor and the age related diminished vitamin $\mathrm{D}$ synthesis are taken into account.

The daily intake of a-tocopherol equivalents was between 6.3 and $13.7 \mathrm{mg} / \mathrm{day}$ in male and between 6.7 and $13.7 \mathrm{mg}$ /day in female elderly. The recommendation is $12 \mathrm{mg}$ /day for men and $11 \mathrm{mg}$ /day for women D-A-CH, 2000. Only male elderly from Austria, Hungary and The Netherlands as well as female elderly from Austria, Germany, Hungary and The Netherlands met this recommendation. 
Table 7.46. Vitamin intake (mean \pm SD) in elderly of European countries (male and female)

\begin{tabular}{|c|c|c|c|c|c|c|}
\hline & $\begin{array}{l}\text { Age } \\
\text { years }\end{array}$ & $n$ & $\begin{array}{l}\text { Vitamin } A^{1} \\
m g\end{array}$ & $\begin{array}{l}\beta \text {-Carotene } \\
\mathrm{mg}\end{array}$ & $\begin{array}{l}\text { Vitamin D } \\
\mu \mathrm{g}\end{array}$ & $\begin{array}{l}\text { Vitamin } \mathrm{E}^{2} \\
\mathrm{mg}\end{array}$ \\
\hline \multicolumn{7}{|l|}{ Male } \\
\hline Austria & $>64$ & 147 & $1.2 \pm 1.6$ & $2.2 \pm 1.7$ & $2.9 \pm 4.5$ & $13.0 \pm 4.2$ \\
\hline Belgium & $>64$ & n.a. & n.a. & n.a. & n.a. & n.a. \\
\hline Denmark & $>64$ & 165 & $1.2 \pm 0.9$ & $2.9 \pm 2.4$ & $3.9 \pm 3.1$ & $7.1 \pm 3.1$ \\
\hline Finland & $>64$ & 229 & $0.8 \pm 1.1$ & n.a. & $9.0 \pm 8.2$ & $8.7 \pm 3.9$ \\
\hline Germany & $>64$ & 1,469 & $2.1 \pm 1.1$ & $4.8 \pm 2.8$ & $4.4 \pm 4.1$ & $6.3 \pm 6.3$ \\
\hline Hungary & $>59$ & 138 & $0.6 \pm 0.8$ & $2.4 \pm 2.1$ & $2.1 \pm 1.3$ & $15.1 \pm 5.2$ \\
\hline Ireland & $>64$ & 580 & $1.3 \pm 1.2$ & n.a. & $3.5 \pm 2.1$ & $7.6 \pm 4.6$ \\
\hline Italy & $>64$ & 60 & $1.2 \pm 0.9$ & $3.6 \pm 2.2$ & $2.5 \pm 1.7$ & $11.5 \pm 4.4$ \\
\hline Norway & $>64$ & 176 & $2.5 \pm 1.4^{5}$ & n.a. & $15.0 \pm 11.4$ & n.a. \\
\hline Poland & $>64$ & 176 & $1.5 \pm 3.3$ & $2.8 \pm 2.7$ & $4.4 \pm 5.9$ & $11.5 \pm 6.4$ \\
\hline Portugal & $>64$ & 246 & $1.6 \pm 0.8$ & n.a. & $3.4 \pm 1.6$ & $8.3 \pm 2.7$ \\
\hline Romania & $>64$ & 177 & $2.2 \pm 0.9$ & n.a. & n.a. & n.a. \\
\hline Spain & $>64$ & 163 & $0.5 \pm 0.1$ & $1.3 \pm 0.4$ & $0.7 \pm 0.1$ & $8.4 \pm 2.3$ \\
\hline Sweden & $65-74$ & 64 & $1.5 \pm 0.7^{5}$ & $2.0 \pm 1.8$ & $7.1 \pm 2.6$ & $7.6 \pm 2.7$ \\
\hline The Netherlands & $>64$ & 185 & $1.1 \pm 0.8$ & n.a. & $4.8 \pm 2.9$ & $13.7 \pm 6.5$ \\
\hline \multirow[t]{2}{*}{ United Kingdom } & $65-74$ & 144 & $1.0 \pm 1.0$ & $2.5 \pm 2.4$ & $3.1 \pm 2.1$ & $8.8 \pm 5.8$ \\
\hline & $>74$ & 124 & $1.3 \pm 1.9$ & $2.5 \pm 2.4$ & $3.7 \pm 2.4$ & $9.0 \pm 6.0$ \\
\hline All countries (min.- max.) & & & $0.5-2.5$ & $1.3-4.8$ & $0.7-15.0$ & $6.3-13.7$ \\
\hline \multicolumn{7}{|l|}{ Female } \\
\hline Austria & $>64$ & 202 & $1.3 \pm 1.7$ & $2.4 \pm 2.5$ & $2.4 \pm 2.1$ & $12.5 \pm 4.5$ \\
\hline Belgium & $>64$ & n.a. & n.a. & n.a. & n.a. & n.a. \\
\hline Denmark & $>64$ & 164 & $1.0 \pm 0.7$ & $4.1 \pm 3.4$ & $3.1 \pm 2.1$ & $6.7 \pm 2.6$ \\
\hline Finland & $>64$ & 234 & $0.8 \pm 1.2$ & n.a. & $6.5 \pm 4.6$ & $7.3 \pm 3.2$ \\
\hline Germany & $>64$ & 1,562 & $1.7 \pm 0.8$ & $5.0 \pm 3.7$ & $3.4 \pm 2.8$ & $12.6 \pm 6.5$ \\
\hline Hungary & $>59$ & 235 & $0.4 \pm 0.5$ & $2.1 \pm 1.4$ & $1.8 \pm 1.0$ & $13.3 \pm 4.2$ \\
\hline Ireland & $>64$ & 742 & $1.3 \pm 0.9$ & n.a. & $3.2 \pm 1.9$ & $7.8 \pm 4.6$ \\
\hline Italy & $>64$ & 107 & $1.2 \pm 1.4$ & $2.7 \pm 1.8$ & $2.4 \pm 1.8$ & $10.6 \pm 4.4$ \\
\hline Norway & $>64$ & 166 & $2.3 \pm 1.3^{5}$ & n.a. & $12.9 \pm 11.8$ & n.a. \\
\hline Poland & $>64$ & 277 & $1.2 \pm 2.4$ & $2.5 \pm 2.6$ & $2.9 \pm 2.9$ & $9.3 \pm 5.3$ \\
\hline
\end{tabular}




\begin{tabular}{|c|c|c|c|c|c|c|}
\hline $\begin{array}{l}\text { Thiamine } \\
\text { mg }\end{array}$ & $\begin{array}{l}\text { Riboflavin } \\
\mathrm{mg}\end{array}$ & $\begin{array}{l}\text { Niacin }^{3} \\
\mathrm{mg}\end{array}$ & $\begin{array}{l}\text { Vitamin } B_{6} \\
\mathrm{mg}\end{array}$ & $\begin{array}{l}\text { Folate }^{4} \\
\mu \mathrm{g}\end{array}$ & $\begin{array}{l}\text { Cobalamin } \\
\mu \mathrm{g}\end{array}$ & $\begin{array}{l}\text { Ascorbic acid } \\
\mathrm{mg}\end{array}$ \\
\hline $1.0 \pm 0.3$ & $1.2 \pm 0.4$ & $25.0 \pm 8.2$ & $1.2 \pm 0.4$ & $166 \pm 68$ & $4.7 \pm 2.3$ & $102 \pm 59$ \\
\hline n.a. & n.a. & n.a. & n.a. & n.a. & n.a. & $91 \pm 43$ \\
\hline $1.2 \pm 0.5$ & $1.7 \pm 0.6$ & $30.5 \pm 8.4$ & $1.5 \pm 0.5$ & $308 \pm 122$ & $6.0 \pm 3.3$ & $95 \pm 52$ \\
\hline $1.3 \pm 0.5$ & $1.8 \pm 0.8$ & $30.0 \pm 11.0$ & $2.0 \pm 1.2$ & $243 \pm 102$ & $6.5 \pm 6.0$ & $92 \pm 72$ \\
\hline $1.4 \pm 0.5$ & $1.8 \pm 0.7$ & $32.7 \pm 9.6$ & $2.2 \pm 0.7$ & $276 \pm 93$ & $5.9 \pm 2.5$ & $142 \pm 70$ \\
\hline $0.9 \pm 0.3$ & $1.2 \pm 0.4$ & $34.2 \pm 8.6$ & $1.6 \pm 0.1$ & $139 \pm 49$ & $3.6 \pm 2.8$ & $79 \pm 53$ \\
\hline $2.1 \pm 1.0$ & $2.0 \pm 0.9$ & $24.1 \pm 10.7$ & $3.0 \pm 1.1$ & $343 \pm 148$ & n.a. & n.a. \\
\hline $1.1 \pm 0.3$ & $1.6 \pm 0.4$ & $33.2 \pm 8.7$ & $2.1 \pm 0.6$ & $325 \pm 146$ & n.a. & $125 \pm 75$ \\
\hline $1.8 \pm 1.1$ & $2.1 \pm 1.2$ & $21.6 \pm 13.7$ & n.a. & n.a. & n.a. & $139 \pm 81$ \\
\hline $1.3 \pm 0.6$ & $1.8 \pm 1.0$ & n.a. & $1.9 \pm 0.8$ & $255 \pm 132$ & $5.4 \pm 9.1$ & $73 \pm 57$ \\
\hline $1.8 \pm 0.5$ & $1.9 \pm 0.6$ & $22.0 \pm 5.8$ & $2.1 \pm 0.6$ & $312 \pm 156$ & $8.2 \pm 3.8$ & $121 \pm 57$ \\
\hline $1.6 \pm 0.7$ & $3.2 \pm 1.7$ & n.a. & n.a. & n.a. & n.a. & $77 \pm 39$ \\
\hline $1.0 \pm 0.2$ & $1.3 \pm 0.3$ & $16.4 \pm 2.7$ & $1.7 \pm 0.3$ & $236 \pm 47$ & $3.8 \pm 0.6$ & $126 \pm 50$ \\
\hline $1.5 \pm 0.4$ & $1.9 \pm 0.6$ & $37.9 \pm 11.7$ & $2.3 \pm 0.7$ & $240 \pm 75$ & $8.0 \pm 3.9$ & $93 \pm 50$ \\
\hline $1.4 \pm 0.7$ & $1.6 \pm 0.5$ & n.a. & $1.8 \pm 0.5$ & $204 \pm 78$ & n.a. & $88 \pm 56$ \\
\hline $1.5 \pm 0.6$ & $1.7 \pm 0.8$ & $8.7 \pm 6.0^{8}$ & $2.2 \pm 0.9$ & $263 \pm 116$ & $5.8 \pm 4.2$ & $63 \pm 48$ \\
\hline $1.5 \pm 0.6$ & $1.7 \pm 0.7$ & $8.0 \pm 4.9^{8}$ & $2.0 \pm 0.8$ & $248 \pm 106$ & $6.6 \pm 6.2$ & $59 \pm 40$ \\
\hline $0.9-2.1$ & $1.2-3.2$ & $8.0-37.9$ & $1.2-3.0$ & 139-343 & $3.1-8.2$ & 59-142 \\
\hline $0.9 \pm 0.3$ & $1.2 \pm 0.4$ & $22.7 \pm 5.7$ & $1.2 \pm 0.4$ & $166 \pm 62$ & $4.2 \pm 2.4$ & $115 \pm 60$ \\
\hline n.a. & n.a. & n.a. & n.a. & n.a. & n.a. & $87 \pm 44$ \\
\hline $1.0 \pm 0.3$ & $1.5 \pm 0.6$ & $25.6 \pm 7.0$ & $1.3 \pm 0.4$ & $297 \pm 112$ & $4.8 \pm 2.7$ & $115 \pm 67$ \\
\hline $1.0 \pm 0.4$ & $1.5 \pm 0.6$ & $23.0 \pm 7.0$ & $1.5 \pm 0.7$ & $210 \pm 96$ & $5.2 \pm 4.8$ & $97 \pm 68$ \\
\hline $1.2 \pm 0.6$ & $1.6 \pm 0.8$ & $25.9 \pm 0.7$ & $1.8 \pm 0.9$ & $276 \pm 93$ & $4.3 \pm 2.0$ & $148 \pm 81$ \\
\hline $0.9 \pm 0.4$ & $1.2 \pm 0.6$ & $28.6 \pm 7.6$ & $1.6 \pm 0.7$ & $125 \pm 43$ & $2.5 \pm 2.0$ & $72 \pm 40$ \\
\hline $2.0 \pm 0.8$ & $1.8 \pm 0.8$ & $22.2 \pm 10.4$ & $2.9 \pm 1.2$ & $335 \pm 145$ & n.a. & n.a. \\
\hline $0.9 \pm 0.2$ & $1.4 \pm 0.5$ & $28.8 \pm 6.8$ & $1.7 \pm 0.4$ & $279 \pm 174$ & n.a. & $101 \pm 47$ \\
\hline $1.8 \pm 1.4$ & $2.1 \pm 1.5$ & $20.8 \pm 16.1$ & n.a. & n.a. & n.a. & $160 \pm 87$ \\
\hline $1.0 \pm 0.5$ & $1.5 \pm 0.8$ & n.a. & $1.5 \pm 0.6$ & $213 \pm 101$ & $3.4 \pm 5.7$ & $68 \pm 53$ \\
\hline
\end{tabular}


Table 7.46. Continued

\begin{tabular}{lllllll}
\hline & $\begin{array}{l}\text { Age } \\
\text { years }\end{array}$ & $\mathrm{n}$ & $\begin{array}{l}\text { Vitamin } \mathrm{A}^{1} \\
\mathrm{mg}\end{array}$ & $\begin{array}{l}\beta \text {-Carotene } \\
\mathrm{mg}\end{array}$ & $\begin{array}{l}\text { Vitamin D } \\
\mu \mathrm{g}\end{array}$ & $\begin{array}{l}\text { Vitamin }^{2} \\
\mathrm{mg}\end{array}$ \\
\hline Portugal & $>64$ & 339 & $1.6 \pm 0.8$ & $\mathrm{n} . \mathrm{a}$. & $3.3 \pm 1.5$ & $7.7 \pm 2.5$ \\
\hline Romania & $>64$ & 341 & $1.9 \pm 0.7$ & n.a. & n.a. & n.a. \\
\hline Spain & $>64$ & 179 & $0.4 \pm 0.0$ & $1.3 \pm 0.3$ & $0.7 \pm 0.1$ & $7.5 \pm 1.7$ \\
\hline Sweden & $65-74$ & 58 & $1.4 \pm 0.7^{5}$ & $2.3 \pm 1.3$ & $6.1 \pm 2.0$ & $7.1 \pm 2.4$ \\
\hline The Netherlands & $>64$ & 236 & $0.8 \pm 0.5$ & n.a. & $3.6 \pm 2.1$ & $11.6 \pm 6.6$ \\
\hline United Kingdom & $65-74$ & 253 & $1.1 \pm 1.5$ & $2.5 \pm 2.6$ & $2.8 \pm 1.8$ & $7.2 \pm 3.7$ \\
\hline All countries (min.-max.) & & 284 & $1.2 \pm 1.8$ & $2.4 \pm 2.2$ & $2.7 \pm 1.8$ & $7.5 \pm 5.9$ \\
\hline Reference values & & & $0.4-2.3$ & $1.3-5.0$ & $0.7-12.9$ & $6.7-13.7$ \\
\hline
\end{tabular}

${ }^{1}$ Retinol equivalent $\left(=1 \mathrm{mg}\right.$ retinol $=6 \mathrm{mg}$ all-trans- $\beta$-carotene $=12 \mathrm{mg}$ other carotenoids); ${ }^{2}$ RRR- $\alpha$ tocopherol equivalent $(=\mathrm{mg}$ a-tocopherol $+\mathrm{mg} \beta$-tocopherol $\times 0.5+\mathrm{mg} \gamma$-tocopherol $\times 0.25+\mathrm{mg}$ a-tocotrienol $\times 0.33) ;{ }^{3}$ niacin equivalent $\left(=1 \mathrm{mg}\right.$ niacin $=60 \mathrm{mg}$ tryptophan); ${ }^{4}$ folate equivalent $(1 \mu \mathrm{g}$ food folate $=0.5 \mu \mathrm{g}$ folic acid $(\mathrm{PGA})=0.6 \mu \mathrm{g}$ folic acid taken with meals); ${ }^{5}$ values refer to a-tocopherol, only; ${ }^{6} \mathrm{D}-\mathrm{A}-\mathrm{CH}, 2000$, reference values refer to age groups $\geq 65$ years; ${ }^{7}$ Eurodiet, $2000 ;{ }^{8}$ niacin; n.a. $=$ not available.

The daily intake of thiamine was at the recommendation of $1.0 \mathrm{mg} /$ day in almost every participating country, only elderly from Hungary, both males and females, as well as female elderly from Austria and Italy were slightly below the reference value [D-A-CH, 2000].

The intakes of riboflavin, niacin, vitamin $\mathrm{B}_{6}$ and cobalamin were in general above the recommendations in almost every country, except Austria where the vitamin $\mathrm{B}_{6}$ intake in male elderly was below and Hungary where the cobalamin intake in female elderly was slightly below the recommendation. From the United Kingdom only values for niacin were available and therefore cannot be compared to the recommended intake value.

The intake of folate equivalents was between 139 and $343 \mu \mathrm{g} /$ day in male and between 121 and $335 \mu \mathrm{g} /$ day in female elderly. So the intake values were below the recommended $400 \mu \mathrm{g} /$ day [D-A-CH, 2000]. The recommendation for the Nordic countries of $300 \mu \mathrm{g} /$ day [NNR, 2004] was only met by male elderly from Denmark, Ireland, Italy and Portugal as well as by female elderly from Ireland (cf. table 7.46). 


\begin{tabular}{lllllll}
\hline $\begin{array}{l}\text { Thiamine } \\
\mathrm{mg}\end{array}$ & $\begin{array}{l}\text { Riboflavin } \\
\mathrm{mg}\end{array}$ & $\begin{array}{l}\text { Niacin }^{3} \\
\mathrm{mg}\end{array}$ & $\begin{array}{l}\text { Vitamin } \mathrm{B}_{6} \\
\mathrm{mg}\end{array}$ & $\begin{array}{l}\text { Folate } \\
\mu \mathrm{g}\end{array}$ & $\begin{array}{l}\text { Cobalamin } \\
\mu \mathrm{g}\end{array}$ & $\begin{array}{l}\text { Ascorbic acid } \\
\mathrm{mg}\end{array}$ \\
\hline $1.6 \pm 0.4$ & $1.9 \pm 0.6$ & $20.1 \pm 5.1$ & $2.0 \pm 0.6$ & $290 \pm 163$ & $7.5 \pm 4.1$ & $118 \pm 57$ \\
\hline $1.4 \pm 0.5$ & $2.6 \pm 1.3$ & n.a. & n.a. & n.a. & n.a. & $76 \pm 40$ \\
\hline $1.0 \pm 0.2$ & $1.3 \pm 0.2$ & $14.4 \pm 1.2$ & $1.5 \pm 0.2$ & $216 \pm 28$ & $3.5 \pm 0.5$ & $115 \pm 38$ \\
\hline $1.3 \pm 0.4$ & $1.7 \pm 0.5$ & $31.9 \pm 8.3$ & $2.0 \pm 0.6$ & $237 \pm 81$ & $7.4 \pm 4.1$ & $116 \pm 68$ \\
\hline $1.1 \pm 0.6$ & $1.5 \pm 0.5$ & n.a. & $1.4 \pm 0.4$ & $177 \pm 75$ & n.a. & $95 \pm 53$ \\
\hline $1.4 \pm 0.5$ & $1.5 \pm 0.6$ & $6.8 \pm 3.9^{8}$ & $1.8 \pm 0.6$ & $225 \pm 89$ & $5.1 \pm 4.0$ & $71 \pm 51$ \\
$1.3 \pm 0.5$ & $1.6 \pm 0.7$ & $6.7 \pm 3.9^{8}$ & $1.6 \pm 0.6$ & $212 \pm 88$ & $5.3 \pm 5.3$ & $60 \pm 39$ \\
\hline $0.9-1.4$ & $1.2-2.6$ & $6.7-31.9$ & $1.2-2.9$ & $121-335$ & $2.5-7.5$ & $60-160$ \\
\hline 1.0 & 1.2 & 13 & 1.4 (male)/ & 400 & 3.0 & 100 \\
\hline
\end{tabular}

Table 7.47. Intake of selected vitamins (min.-max.) in elderly in the four regions

\begin{tabular}{|c|c|c|c|c|}
\hline Region/sex & Vitamin $\mathrm{B}_{6}, \mathrm{mg}$ & Folate, $\mu \mathrm{g}^{5}$ & Cobalamin, $\mu \mathrm{g}$ & Vitamin $D, \mu \mathrm{g}$ \\
\hline \multicolumn{5}{|l|}{ North ${ }^{1}$} \\
\hline Male & $1.5-2.3^{6}$ & $240-308^{6}$ & $6.0-8.0^{6}$ & $3.9-15.0$ \\
\hline Female & $1.3-2.0^{6}$ & $210-297^{6}$ & $4.8-7.4^{6}$ & $3.1-12.9$ \\
\hline \multicolumn{5}{|l|}{ South ${ }^{2}$} \\
\hline Male & $1.7-2.1$ & $236-325$ & $3.8-8.2^{7}$ & $0.7-3.4$ \\
\hline Female & $1.5-2.0$ & $216-290$ & $3.5-7.5^{7}$ & $0.6-3.3$ \\
\hline \multicolumn{5}{|c|}{ Central and East ${ }^{3}$} \\
\hline Male & $1.2-2.2$ & 139-276 & $3.6-5.9$ & $2.1-4.4$ \\
\hline Female & $1.2-1.8$ & $125-276$ & $2.5-4.3$ & $1.8-3.4$ \\
\hline \multicolumn{5}{|l|}{ West $t^{4}$} \\
\hline Male & $1.8-3.0$ & $204-343$ & $6.2^{8}$ & $3.4-4.8$ \\
\hline Female & $1.4-2.9$ & $177-335$ & $5.2^{8}$ & $2.8-3.6$ \\
\hline
\end{tabular}


Table 7.48. Mineral intake (mean \pm SD) in elderly of European countries (male and female)

\begin{tabular}{|c|c|c|c|c|c|c|}
\hline & $\begin{array}{l}\text { Age } \\
\text { years }\end{array}$ & $n$ & $\begin{array}{l}\text { Sodium } \\
\mathrm{g}\end{array}$ & $\begin{array}{l}\text { Potassium } \\
\mathrm{g}\end{array}$ & $\begin{array}{l}\text { Calcium } \\
\mathrm{mg}\end{array}$ & $\begin{array}{l}\text { Phosphorus } \\
\mathrm{mg}\end{array}$ \\
\hline \multicolumn{7}{|l|}{ Male } \\
\hline Austria & $>64$ & 147 & $3.4 \pm 0.9$ & $2.2 \pm 0.7$ & $649 \pm 295$ & $1,059 \pm 390$ \\
\hline Belgium & $>64$ & n.a. & n.a. & n.a. & $698 \pm 281$ & n.a. \\
\hline Denmark & $>64$ & 165 & $3.6 \pm 1.2$ & $3.6 \pm 1.1$ & $874 \pm 359$ & $1,371 \pm 470$ \\
\hline Finland & $>64$ & 229 & $3.1 \pm 1.3$ & $3.7 \pm 1.1$ & $1,032 \pm 521$ & n.a. \\
\hline France & $>64$ & 130 & $4.1 \pm 0.2$ & n.a. & $893 \pm 28$ & n.a. \\
\hline Germany & $>64$ & 1,469 & $3.1 \pm 0.9$ & $3.5 \pm 0.9$ & $970 \pm 357$ & n.a. \\
\hline Hungary & $>59$ & 138 & $6.7 \pm 1.7$ & $2.8 \pm 0.9$ & $670 \pm 294$ & $1,164 \pm 285$ \\
\hline Ireland & $>64$ & 580 & $3.4 \pm 1.7$ & $3.8 \pm 1.4$ & $892 \pm 403$ & $1,526 \pm 543$ \\
\hline Italy & $>64$ & 60 & $5.9 \pm 2.5$ & $3.3 \pm 0.9$ & $936 \pm 258$ & $1,381 \pm 329$ \\
\hline Norway & $>64$ & 176 & n.a. & n.a. & $861 \pm 349$ & n.a. \\
\hline Poland & $>64$ & 176 & $4.6 \pm 1.8$ & $3.5 \pm 1.3$ & $580 \pm 315$ & $1,257 \pm 458$ \\
\hline Portugal & $>64$ & 246 & $3.7 \pm 1.0$ & $3.8 \pm 1.0$ & $853 \pm 316$ & $1,367 \pm 379$ \\
\hline Romania & $>64$ & 177 & n.a. & n.a. & $931 \pm 365$ & n.a. \\
\hline Spain & $>64$ & 163 & $2.3 \pm 0.5$ & $2.8 \pm 0.4$ & $757 \pm 152$ & $1,165 \pm 187$ \\
\hline Sweden & $65-74$ & 64 & $3.4 \pm 1.0$ & $3.7 \pm 1.1$ & $1,071 \pm 406$ & $1,571 \pm 475$ \\
\hline The Netherlands & $>64$ & 185 & n.a. & $3.8 \pm 1.1$ & $1,024 \pm 414$ & $1,576 \pm 472$ \\
\hline \multirow[t]{2}{*}{ United Kingdom } & $65-74$ & 144 & $2.7 \pm 1.2$ & $2.7 \pm 0.9$ & $843 \pm 351$ & $1,184 \pm 406$ \\
\hline & $>74$ & 124 & $2.6 \pm 1.1$ & $2.5 \pm 0.9$ & $803 \pm 288$ & $1,132 \pm 358$ \\
\hline All countries (min.-max.) & & & $2.3-7.0$ & $2.2-3.8$ & $627-1,071$ & $1,059-1,576$ \\
\hline \multicolumn{7}{|l|}{ Female } \\
\hline Austria & $>64$ & 202 & $3.2 \pm 0.9$ & $2.2 \pm 0.6$ & $693 \pm 276$ & $984 \pm 291$ \\
\hline Belgium & $>64$ & n.a. & n.a. & n.a. & $615 \pm 225$ & n.a. \\
\hline Denmark & $>64$ & 164 & $2.7 \pm 0.8$ & $3.3 \pm 0.9$ & $900 \pm 366$ & $1,190 \pm 383$ \\
\hline Finland & $>64$ & 234 & $2.3 \pm 0.7$ & $3.2 \pm 0.9$ & $900 \pm 426$ & n.a. \\
\hline France & $>64$ & 219 & $2.9 \pm 0.1$ & n.a. & $818 \pm 22$ & n.a. \\
\hline Germany & $>64$ & 1,562 & $2.4 \pm 0.7$ & $3.1 \pm 0.9$ & $918 \pm 342$ & n.a. \\
\hline Hungary & $>59$ & 235 & $5.4 \pm 1.3$ & $2.6 \pm 1.5$ & $626 \pm 264$ & $998 \pm 259$ \\
\hline Ireland & $>64$ & 742 & $3.1 \pm 1.3$ & $3.7 \pm 1.3$ & $823 \pm 370$ & $1,404 \pm 516$ \\
\hline Italy & $>64$ & 107 & $5.2 \pm 3.2$ & $2.9 \pm 0.7$ & $795 \pm 250$ & $1,164 \pm 23$ \\
\hline
\end{tabular}




\begin{tabular}{|c|c|c|c|c|c|c|}
\hline $\begin{array}{l}\text { Magnesium } \\
\text { mg }\end{array}$ & $\begin{array}{l}\text { Iron } \\
\text { mg }\end{array}$ & $\begin{array}{l}\text { Zinc } \\
\text { mg }\end{array}$ & $\begin{array}{l}\text { lodine } \\
\mu g^{1}\end{array}$ & $\begin{array}{l}\text { Copper } \\
\mathrm{mg}\end{array}$ & $\begin{array}{l}\text { Manganese } \\
\mathrm{mg}\end{array}$ & $\begin{array}{l}\text { Selenium } \\
\mu \mathrm{g}\end{array}$ \\
\hline $269 \pm 89$ & $10.9 \pm 3.4$ & $9.4 \pm 3.0$ & $188 \pm 64$ & $1.9 \pm 0.6$ & $4.3 \pm 1.7$ & n.a. \\
\hline n.a. & $11.6 \pm 2.8$ & n.a. & n.a. & n.a. & n.a. & n.a. \\
\hline $364 \pm 118$ & $10.7 \pm 3.7$ & $11.0 \pm 3.6$ & $194 \pm 65$ & n.a. & n.a. & $39 . \pm 13$ \\
\hline $379 \pm 115$ & $12.6 \pm 5.6$ & $12.3 \pm 4.3$ & $226 \pm 94$ & $1.4 \pm 0.5$ & n.a. & $66 \pm 26$ \\
\hline n.a. & n.a. & n.a. & n.a. & n.a. & n.a. & n.a. \\
\hline $403 \pm 109$ & $13.6 \pm 3.9$ & $10.9 \pm 3.2$ & $107 \pm 40$ & n.a. & n.a. & n.a. \\
\hline $403 \pm 107$ & $11.6 \pm 3.5$ & $9.0 \pm 2.6$ & n.a. & $1.2 \pm 1.1$ & $2.1 \pm 0.8$ & n.a. \\
\hline $330 \pm 125$ & $12.6 \pm 6.8$ & $11.9 \pm 5.0$ & $169 \pm 74$ & $1.4 \pm 0.9$ & $4.0 \pm 1.7$ & $62 \pm 27$ \\
\hline $226 \pm 72$ & $14.6 \pm 4.5$ & $12.2 \pm 3.5$ & n.a. & $1.5 \pm 0.5$ & n.a. & $43 \pm 18$ \\
\hline $333 \pm 93$ & $12.3 \pm 5.6$ & n.a. & n.a. & n.a. & n.a. & n.a. \\
\hline $300 \pm 100$ & $13.8 \pm 14.0$ & $11.0 \pm 4.3$ & $152 \pm 73$ & $1.3 \pm 0.5$ & $4.9 \pm 1.7$ & $51 \pm 28$ \\
\hline $354 \pm 108$ & $16.3 \pm 4.8$ & n.a. & n.a. & n.a. & n.a. & n.a. \\
\hline $341 \pm 99$ & $25.2 \pm 7.0$ & n.a. & n.a. & n.a. & n.a. & n.a. \\
\hline $285 \pm 48$ & $11.2 \pm 1.3$ & $7.5 \pm 1.0$ & n.a. & n.a. & n.a. & n.a. \\
\hline $350 \pm 97$ & $12.1 \pm 3.6$ & $12.0 \pm 3.6$ & n.a. & n.a. & n.a. & $40 \pm 14$ \\
\hline $332 \pm 101$ & $11.4 \pm 3.8$ & $10.0 \pm 3.3$ & n.a. & $1.1 \pm 0.4$ & n.a. & $48 \pm 24$ \\
\hline $234 \pm 88$ & $10.0 \pm 3.6$ & $8.5 \pm 3.0$ & $195 \pm 92$ & $1.1 \pm 0.6$ & $3.0 \pm 1.5$ & n.a. \\
\hline $221 \pm 83$ & $10.2 \pm 3.7$ & $8.2 \pm 2.9$ & $184 \pm 79$ & $1.1 \pm 0.8$ & $3.0 \pm 1.4$ & n.a. \\
\hline $221-403$ & $10.0-25.2$ & $7.5-12.3$ & $107-226$ & $1.1-1.9$ & $2.2-4.9$ & $39-62$ \\
\hline $256 \pm 70$ & $10.3 \pm 2.9$ & $8.8 \pm 2.5$ & $190 \pm 60$ & $1.9 \pm 0.5$ & $4.4 \pm 2.0$ & n.a. \\
\hline n.a. & $8.7 \pm 2.6$ & n.a. & n.a. & n.a. & n.a. & n.a. \\
\hline $318 \pm 78$ & $8.5 \pm 2.2$ & $9.1 \pm 2.6$ & $167 \pm 56$ & n.a. & n.a. & $34 \pm 11$ \\
\hline $313 \pm 95$ & $9.9 \pm 3.6$ & $9.4 \pm 2.9$ & $182 \pm 62$ & $1.2 \pm 0.5$ & n.a. & $49 \pm 17$ \\
\hline n.a. & n.a. & n.a. & n.a. & n.a. & n.a. & n.a. \\
\hline $348 \pm 102$ & $11.4 \pm 3.5$ & $8.8 \pm 2.8$ & $97 \pm 38$ & n.a. & n.a. & n.a. \\
\hline $351 \pm 103$ & $9.2 \pm 2.5$ & $7.3 \pm 2.1$ & n.a. & $0.9 \pm 0.5$ & $2.9 \pm 12.6$ & n.a. \\
\hline $313 \pm 123$ & $12.1 \pm 6.3$ & $11.2 \pm 4.9$ & $155 \pm 67$ & $1.3 \pm 0.7$ & $3.9 \pm 1.6$ & $55 \pm 24$ \\
\hline $184 \pm 38$ & $11.5 \pm 3.1$ & $9.8 \pm 2.5$ & n.a. & $1.2 \pm 0.5$ & n.a. & $37 \pm 15$ \\
\hline
\end{tabular}


Table 7.48. Continued

\begin{tabular}{lllllll}
\hline & $\begin{array}{l}\text { Age } \\
\text { years }\end{array}$ & $\mathrm{n}$ & $\begin{array}{l}\text { Sodium } \\
\mathrm{g}\end{array}$ & $\begin{array}{l}\text { Potassium } \\
\mathrm{g}\end{array}$ & $\begin{array}{l}\text { Calcium } \\
\mathrm{mg}\end{array}$ & $\begin{array}{l}\text { Phosphorus } \\
\mathrm{mg}\end{array}$ \\
\hline Norway & $>64$ & 166 & n.a. & n.a. & $776 \pm 266$ & n.a. \\
\hline Poland & $>64$ & 277 & $3.2 \pm 1.2$ & $2.9 \pm 1.0$ & $533 \pm 286$ & $1,002 \pm 363$ \\
\hline Portugal & $>64$ & 339 & $3.3 \pm 0.9$ & $3.4 \pm 1.0$ & $904 \pm 375$ & $1,312 \pm 414$ \\
\hline Romania & $>64$ & 341 & n.a. & n.a. & $846 \pm 334$ & n.a. \\
\hline Spain & $>64$ & 179 & $1.8 \pm 0.4$ & $2.6 \pm 0.4$ & $712 \pm 108$ & $1,045 \pm 112$ \\
\hline Sweden & $65-74$ & 58 & $2.9 \pm 0.7$ & $3.3 \pm 0.9$ & $937 \pm 312$ & $1,357 \pm 394$ \\
\hline The Netherlands & $>64$ & 236 & n.a. & $3.3 \pm 0.8$ & $959 \pm 359$ & $1,338 \pm 340$ \\
\hline United Kingdom & $65-74$ & 253 & $2.1 \pm 0.7$ & $2.4 \pm 0.7$ & $735 \pm 249$ & $1,011 \pm 277$ \\
\hline All countries (min.-max.) & $>74$ & 284 & $2.0 \pm 0.7$ & $2.2 \pm 0.6$ & $714 \pm 256$ & $959 \pm 281$ \\
\hline Reference values & & & $1.8-6.0$ & $2.2-3.7$ & $533-959$ & $959-1,404$ \\
\hline
\end{tabular}

${ }^{1}$ lodised salt was not taken into account in each country; ${ }^{2} \mathrm{D}-\mathrm{A}-\mathrm{CH}, 2000$, reference values refer to age groups 265. years; ${ }^{3}$ Eurodiet, 2000; ${ }^{4}$ WHO, 2003; n.a. $=$ not available.

Comparison of Regions

As shown in table 7.47, the highest intake values of vitamin $\mathrm{B}_{6}$ can be observed in the West region. Concerning the intake of folate equivalents no remarkable differences between the regions were found, but the lowest intake values were reported in the Central and East region. The intake of vitamin D was higher in the North region than in the other regions.

\section{Minerals}

\section{Comparison of Countries}

With a daily intake of sodium between 2.3 and $7.0 \mathrm{~g} /$ day (males) and 1.8 and $6.0 \mathrm{~g} /$ day (females) every country was above the recommended minimum intake level of $0.55 \mathrm{~g} /$ day [D-A-CH, 2000]. The recommendation of the WHO of $<2 \mathrm{~g}$ sodium per day was only met by Spanish females [WHO, 2003].

The intake of potassium was between 2.2 and $3.8 \mathrm{~g} /$ day in male and between 2.2 and $3.7 \mathrm{mg} /$ day in female elderly, that of phosphorus between 1,059 and $1,576 \mathrm{mg} /$ day and 959 and $1,404 \mathrm{mg} /$ day, that of manganese between 2.2 and $4.9 \mathrm{mg} /$ day and 2.4 and $4.4 \mathrm{mg} / \mathrm{day}$ and that of selenium between 39 and $62 \mu \mathrm{g} / \mathrm{day}$ and 34 and $55 \mu \mathrm{g} /$ day. Therefore, the intake levels were within the recommendations [D-A-CH, 2000]. 


\begin{tabular}{|c|c|c|c|c|c|c|}
\hline $\begin{array}{l}\text { Magnesium } \\
\text { mg }\end{array}$ & $\begin{array}{l}\text { Iron } \\
\text { mg }\end{array}$ & $\begin{array}{l}\text { Zinc } \\
\text { mg }\end{array}$ & $\begin{array}{l}\text { lodine } \\
\mu g^{1}\end{array}$ & $\begin{array}{l}\text { Copper } \\
\text { mg }\end{array}$ & $\begin{array}{l}\text { Manganese } \\
\mathrm{mg}\end{array}$ & $\begin{array}{l}\text { Selenium } \\
\mu \mathrm{g}\end{array}$ \\
\hline $277 \pm 69$ & $10.8 \pm 4.6$ & n.a. & n.a. & n.a. & n.a. & n.a. \\
\hline $240 \pm 84$ & $9.8 \pm 6.2$ & $8.4 \pm 3.5$ & $116 \pm 53$ & $1.0 \pm 0.4$ & $4.2 \pm 1.6$ & $36 \pm 19$ \\
\hline $312 \pm 105$ & $14.2 \pm 4.7$ & n.a. & n.a. & n.a. & n.a. & n.a. \\
\hline $291 \pm 85$ & $20.9 \pm 6.5$ & n.a. & n.a. & n.a. & n.a. & n.a. \\
\hline $261 \pm 50$ & $9.6 \pm 1.0$ & $6.8 \pm 0.7$ & n.a. & n.a. & n.a. & n.a. \\
\hline $323 \pm 90$ & $11.8 \pm 4.9$ & $10.5 \pm 2.9$ & n.a. & n.a. & n.a. & $37 \pm 14$ \\
\hline $284 \pm 74$ & $10.1 \pm 2.8$ & $8.9 \pm 2.5$ & n.a. & $1.0 \pm 0.3$ & n.a. & $39 \pm 15$ \\
\hline $197 \pm 58$ & $9.0 \pm 3.4$ & $7.0 \pm 2.4$ & $163 \pm 73$ & $0.9 \pm 0.6$ & $2.7 \pm 1.0$ & n.a. \\
\hline $179 \pm 55$ & $8.8 \pm 3.4$ & $6.7 \pm 2.6$ & $155 \pm 64$ & $0.9 \pm 0.8$ & $2.4 \pm 1.0$ & n.a. \\
\hline $179-348$ & $8.5-20.9$ & $6.7-11.2$ & $97-190$ & $0.9-1.9$ & $2.4-4.4$ & $34-55$ \\
\hline $\begin{array}{l}350 \text { (male)/ } \\
300 \text { (female) }\end{array}$ & 10 & $\begin{array}{l}10 \text { (male)/ } \\
7 \text { (female) }\end{array}$ & $150^{3}$ & $1.0-1.5$ & $2-5$ & $30-70$ \\
\hline
\end{tabular}

In male elderly, the intake of calcium was between 627 and 1,071 $\mathrm{mg} /$ day and in female elderly between 533 and $959 \mathrm{mg} /$ day. With the exception of male elderly from Finland, Sweden and The Netherlands, all countries were below the D-A-CH reference value of $1,000 \mathrm{mg} /$ day [D-A-CH, 2000]. Compared with the recommended intake for the Nordic countries of $800 \mathrm{mg} /$ day [NNR, 2004], elderly from Austria, Belgium, Hungary, Poland and Spain as well as female elderly from the United Kingdom were below this recommendation.

The intake of magnesium was between 221 and $403 \mathrm{mg} /$ day in male and between 179 and $348 \mathrm{mg} /$ day in female elderly. Almost half of the participating countries were below the recommendation of $350 \mathrm{mg} /$ day (male elderly) and $300 \mathrm{mg} /$ day (female elderly).

The daily intake of iron was between 10.0 and $25.2 \mathrm{mg}$ /day in male and between 8.5 and $20.9 \mathrm{mg} /$ day in female elderly. Compared with the $\mathrm{D}-\mathrm{A}-\mathrm{CH}$ reference value of $10 \mathrm{mg} /$ day [D-A-CH, 2000] male elderly met the recommendation whereas it was met in the female group only by Austrian, German, Irish, Italian, Norwegian, Portuguese, Romanian, Swedish and Dutch elderly.

Male elderly ingested between 6.7 and $11.2 \mathrm{mg}$ zinc/day and female elderly between 7.5 and $12.3 \mathrm{mg}$ zinc/day. Male and female elderly from Spain and the United Kingdom as well as male elderly from Austria and Hungary did not meet the recommended value of $10 \mathrm{mg} / \mathrm{day}$ [D-A-CH, 2000]. 
Table 7.49. Intake of selected minerals (min.-max.) in elderly in the four regions

\begin{tabular}{|c|c|c|c|c|c|c|}
\hline Region/sex & $\begin{array}{l}\text { Calcium } \\
\mathrm{mg}\end{array}$ & $\begin{array}{l}\text { Phosphorus } \\
\mathrm{mg}\end{array}$ & $\begin{array}{l}\text { Iron } \\
\text { mg }\end{array}$ & $\begin{array}{l}\text { Zinc } \\
\mu \mathrm{g}\end{array}$ & $\begin{array}{l}\text { lodine } \\
\mu \mathrm{g}\end{array}$ & $\begin{array}{l}\text { Selenium } \\
\mu \mathrm{g}\end{array}$ \\
\hline \multicolumn{7}{|l|}{ North $^{1}$} \\
\hline Male & $861-1,071$ & $1,371-1,571^{5}$ & $10.7-12.6$ & $11.0-12.3^{6}$ & $194-226^{7}$ & $39-66^{6}$ \\
\hline Female & $776-937$ & $1,190-1,357^{5}$ & $8.5-11.8$ & $9.1-10.5^{6}$ & $167-182^{7}$ & $34-49^{6}$ \\
\hline \multicolumn{7}{|l|}{ South ${ }^{2}$} \\
\hline Male & $757-936$ & $1,165-1,381$ & $11.2-16.3$ & $7.5-12.2^{8}$ & n.a. & $43^{9}$ \\
\hline Female & $712-904$ & $1,045-1,312$ & $9.6-14.2$ & $6.8-9.8^{8}$ & n.a. & $37^{9}$ \\
\hline \multicolumn{7}{|c|}{ Central and East ${ }^{3}$} \\
\hline Male & $580-970$ & $1,059-1,257^{10}$ & $10.9-25.2$ & $9.0-11.0^{11}$ & $107-188^{12}$ & $51^{13}$ \\
\hline Female & $533-918$ & $99-1,002^{10}$ & $9.2-20.9$ & $7.3-8.8^{11}$ & $96.9-189.6^{12}$ & $36^{13}$ \\
\hline \multicolumn{7}{|l|}{ West ${ }^{4}$} \\
\hline Male & $698-1,024$ & $1,158-1,576^{14}$ & $10.1-12.6^{15}$ & $8.4-11.9^{14}$ & $169-189^{16}$ & $48-62^{17}$ \\
\hline Female & 615-959 & $985-1,404^{14}$ & $8.7-12.1^{15}$ & $6.9-11.2^{14}$ & $155-159^{16}$ & $39-55^{17}$ \\
\hline \multicolumn{7}{|c|}{ 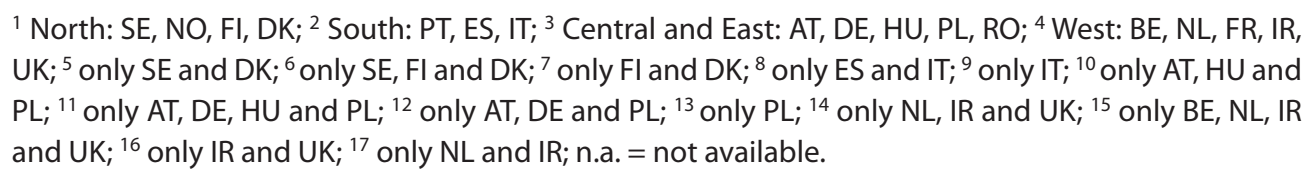 } \\
\hline
\end{tabular}

Every country except for Germany, both males and females, and Poland, only females, was within the recommendation for iodine of Eurodiet of $150 \mu \mathrm{g} / \mathrm{day}$ [Eurodiet, 2000].

The daily intake of copper was between 1.1 and $1.9 \mathrm{mg} /$ day in male and between 0.9 and $1.9 \mathrm{mg} /$ day in female elderly. The intake was slightly below the recommended intake range of $1.0-1.5 \mathrm{mg} /$ day [D-A-CH, 2000] in female elderly from Hungary and the United Kingdom and above the recommended intake range in male and female elderly from Austria (cf. table 7.48).

\section{Comparison of Regions}

Table 7.49 shows the intake of selected minerals in elderly in the four regions. The calcium intake as well as the intake of phosphorus, zinc and iodine was higher in the North region than in the other regions. The highest intake values of iron were observed in the Central and East region. 
8

\section{Health and Lifestyle Indicators in the European Union}

\subsection{Prevalence of Overweight and Obesity}

\section{Background}

Overweight and obesity are known to be major risk factors for nutrition related chronic diseases such as type 2 diabetes, cardiovascular disease, hypertension and stroke. Energy intake that exceeds energy expenditure (a positive energy balance) leads to weight gain and so to overweight and obesity. As already described in the European Nutrition and Health Report 2004, in some countries the average energy intake decreases but prevalence of overweight and obesity is increasing. Thus, a more sedentary lifestyle causing less energy expenditure than the habitual energy intake might be the major factor responsible for increasing prevalence of overweight and obesity.

Overweight and obesity were assessed using the body mass index (BMI, $\mathrm{kg} / \mathrm{m}^{2}$ ). The World Health Organization [WHO, 2000] defines overweight in adults as a BMI $>25 \mathrm{~kg} / \mathrm{m}^{2}$ and obesity as a BMI $>30 \mathrm{~kg} / \mathrm{m}^{2}$.

As already mentioned in the European Nutrition and Health Report 2004, differences in the prevalence of overweight and obesity throughout different countries might be due to different methods of data collection. Therefore, results of calculations will be presented separately based on data derived from self-reported body height and body weight and data measured by trained staff, respectively. It has to be considered that it was not possible for all countries to calculate data for the same age groups. Differences in the prevalence of overweight and obesity could partly be due to this fact. Tables 8.18.3 give information on the included study populations. Another point to be kept in mind is the possibly different age distribution within the age groups that might have an influence on the mean BMI and prevalence of overweight and obesity in the countries.

For those countries, where the age groups did not fit perfectly to the predefined (4-6, $7-9,10-14,15-18,19-64,65+$ years), data were assigned to the most appropriate age groups. Exact data for age groups for the countries can be seen in the tables of the Annex.

\section{Prevalence of Overweight and Obesity in Children and Adolescents}

The calculated BMI changes considerably with age and is also dependent on sex. Because of the different cut-off points needed for the classification of body weight, 
Table 8.1. Study populations for assessment of overweight and obesity in children and adolescents (3-19 years) in the participating countries. Self-reported: $n=23,517$ from 9 countries; measured: $n=119,669$ from 14 countries

\begin{tabular}{lll}
\hline Country & Reference/survey name & Participants, $n$ \\
\cline { 1 - 1 } & female male \\
\hline
\end{tabular}

\section{Self-reported}

\begin{tabular}{|c|c|c|c|c|}
\hline Austria & Austrian Study on Nutritional Status (ASNS) & 177 & 233 & $10-14$ \\
\hline & Austrian Study on Nutritional Status (ASNS) & 1,021 & 1,129 & $15-18$ \\
\hline Belgium & Food Consumption Survey, Belgium, 2004 & 1,623 & 1,626 & $15-18$ \\
\hline \multirow[t]{4}{*}{ Denmark } & $\begin{array}{l}\text { Danish National Survey on Dietary Habits and Physical } \\
\text { Activity }\end{array}$ & 114 & 113 & $4-6$ \\
\hline & $\begin{array}{l}\text { Danish National Survey on Dietary Habits and Physical } \\
\text { Activity }\end{array}$ & 114 & 136 & $7-9$ \\
\hline & $\begin{array}{l}\text { Danish National Survey on Dietary Habits and Physical } \\
\text { Activity }\end{array}$ & 158 & 166 & $10-14$ \\
\hline & $\begin{array}{l}\text { Danish National Survey on Dietary Habits and Physical } \\
\text { Activity }\end{array}$ & 120 & 78 & $15-18$ \\
\hline Estonia & Eesti Kooliõpilaste Tervisekäitumise Uuring, 2005/2006 & 2,134 & 2,070 & $10-14,15-18$ \\
\hline Italy & $\begin{array}{l}\text { Health Behavior in School-Aged Children (HBSC), } \\
\text { international report from the } 2005 / 2006 \text { survey }\end{array}$ & 1,946 & 1,974 & $11-15$ \\
\hline Latvia & $\begin{array}{l}\text { Health Behavior in School-Aged Children (HBSC), } \\
\text { international report from the 2005/2006 survey }\end{array}$ & 1,778 & 1,790 & $11-15$ \\
\hline \multirow[t]{3}{*}{ Lithuania } & \multirow{2}{*}{$\begin{array}{l}\text { Health Behavior in School-Aged Children (HBSC), } \\
\text { international report from the } 2005 / 2006 \text { survey }\end{array}$} & 552 & 532 & 11 \\
\hline & & 626 & 656 & 13 \\
\hline & $\begin{array}{l}\text { Health Behavior in School-Aged Children (HBSC), } \\
\text { international report from the } 2005 / 2006 \text { survey }\end{array}$ & 754 & 716 & 15 \\
\hline Norway & UNGKOST 2000 & n.a. & n.a. & $4-14$ \\
\hline Sweden & $\begin{array}{l}\text { Dietary habits and nutrient intake in Swedish 4-year-old } \\
\text { children and schoolchildren in grade } 2 \text { and 5. Riksmaten } \\
\text { [Enghardt et al., 2006] }\end{array}$ & 611 & 570 & $4-14$ \\
\hline
\end{tabular}

\section{Measured $^{2}$}

\begin{tabular}{lllll}
\hline Austria & Austrian Study on Nutritional Status (ASNS) & 469 & 512 & $7-14$ \\
\hline Belgium & Flemish Growth Charts, Flanders, 2004 & 9,186 & 8,864 & $4-18$ \\
\hline France & French Nutrition and Health Survey (ENNS), 2006-2007 & 2,273 & 1,604 & $4-14$ \\
\hline Germany & $\begin{array}{l}\text { German Health Interview and Examination Survey for } \\
\text { Children and Adolescents, 2003-2006 }\end{array}$ & 7,267 & 7,569 & $3-17$ \\
\hline Greece & Kosti et al. [2008b] & 987 & 1,021 & $12-17$ \\
\hline
\end{tabular}


Table 8.1. Continued

\begin{tabular}{|c|c|c|c|c|}
\hline \multirow[t]{2}{*}{ Country } & \multirow[t]{2}{*}{ Reference/survey name } & \multicolumn{2}{|c|}{ Participants, $n$} & \multirow[t]{2}{*}{ Age, years } \\
\hline & & female & male & \\
\hline \multirow[t]{2}{*}{ Hungary } & $\begin{array}{l}\text { Representative Survey in Metropolitan Primary and } \\
\text { Secondary Schools in 2005-2006 }\end{array}$ & 927 & 1,003 & $7-14$ \\
\hline & $\begin{array}{l}\text { Representative Survey in Metropolitan Primary and } \\
\text { Secondary Schools in 2005-2006 }\end{array}$ & 603 & 670 & $15-19$ \\
\hline Italy & Okkio alla salute & 21,692 & 23,019 & $8-9$ \\
\hline Latvia & $\begin{array}{l}\text { Liekās kermeña masas un aptaukošanās problēma Latvijas } \\
\text { skolēniem (overweight and obesity problem in Latvian } \\
\text { schoolchildren) }\end{array}$ & 6,486 & 6,593 & $7-17$ \\
\hline Poland & $\begin{array}{l}\text { Household Food Consumption and Anthropometric Survey, } \\
2000\end{array}$ & 559 & 541 & $3-18$ \\
\hline \multirow[t]{3}{*}{ Portugal } & $\begin{array}{l}\text { Estado nutricional de crianças e oferta alimentar do pré- } \\
\text { escolar do município de Coimbra, Portugal, } 2001\end{array}$ & 1,190 & 1,210 & $3-6$ \\
\hline & $\begin{array}{l}\text { Prevalence of overweight and obesity in 7- to 9-year-old } \\
\text { Portuguese children: trends in BMI from } 1970 \text { to } 2002\end{array}$ & 2,274 & 2,237 & $7-9$ \\
\hline & $\begin{array}{l}\text { Family and school determinants of overweight in 13-year- } \\
\text { old Portuguese adolescents; Uso de tabaco em } \\
\text { adolescentes portugueses de } 13 \text { anos }\end{array}$ & 1,053 & 987 & 13 \\
\hline Slovenia & Fidler Mis et al.: Dietary intake of Slovenian adolescents & 1,214 & 1,010 & $15-18$ \\
\hline Spain & Catalan Nutrition Survey (ENCAT), 2002-2003 & 103 & 124 & $10-18$ \\
\hline The Netherlands & DNFCS, young children, 2005/06 & 312 & 327 & $4-6$ \\
\hline $\mathrm{UK}^{3}$ & Health Surveys for England, 2006 & 2,806 & 2,977 & $0-15$ \\
\hline \multicolumn{5}{|c|}{$\begin{array}{l}\text { 1 Body weight and body height reported by the participants. } \\
{ }^{2} \text { Body weight and body height measured by researchers. } \\
{ }^{3} \text { UK national BMI classification. }\end{array}$} \\
\hline
\end{tabular}

a general definition of overweight and obesity for children and adolescents cannot be used. The applied methods led to establishing cut-off points to define overweight and obesity in the studied children population in a defined region. Therefore, they result in considerably diverging prevalence of overweight and obesity [Freisling and Elmadfa, 2007]. For international comparability, the cut-off points by Cole et al. [2000] are recommended and therefore applied in all countries except for the United Kingdom (UK National BMI percentiles classification). Fig. 8.1 shows the prevalence of overweight and obesity in children and adolescents from the participating European countries. Data are presented ordered by age groups. 


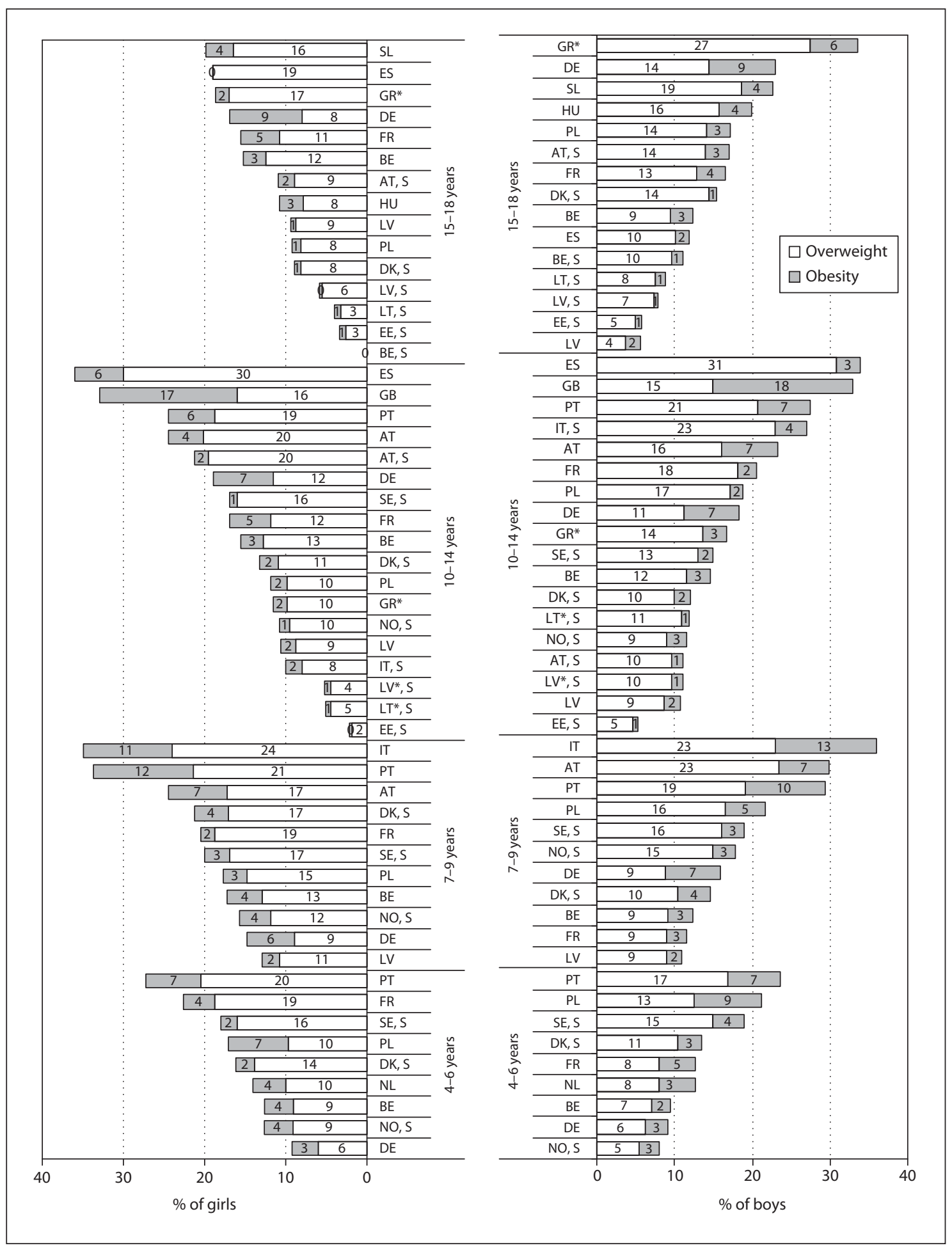

Fig. 8.1. Overweight and obesity in European children and adolescents of different age subgroups $(4-6,7-9,10-14,15-18$ years). S = BMl calculated from self-reported height and weight. Classification according to Cole et al. [2000]. *Estimated from mean values for different age groups. 
Table 8.2. Study populations for assessment of overweight and obesity in adults (19-64 years) in the participating countries. Self-reported: $n=86,936$ from 14 countries; measured: $n=73,926$ from 14 countries

Country Survey name $\quad \frac{\text { Participants, } n}{\text { female male }}$

\section{Self-reported}

\begin{tabular}{|c|c|c|c|c|}
\hline Austria & Austrian Study on Nutritional Status (ASNS) & 1,438 & 834 & $19-64$ \\
\hline Belgium & Food Consumption Survey, Belgium, 2004 & 1,623 & 1,626 & $19-63$ \\
\hline Denmark & $\begin{array}{l}\text { Danish National Survey on Dietary Habits and Physical } \\
\text { Activity }\end{array}$ & 2,124 & 1,936 & $19-64$ \\
\hline Estonia & Estonian Health Interview Survey, 2006 & 3,114 & 2,872 & $19-63$ \\
\hline Germany & National Telephone Health Survey (GSTeL03), 2004 & 4,302 & 4,016 & $18-65$ \\
\hline Hungary & 3rd Hungarian Nutrition Survey, 2003-2004 & 706 & 473 & $18+$ \\
\hline Ireland & Survey of Lifestyle, Attitudes and Nutrition, 2007 & 3,960 & 4,178 & $18-64$ \\
\hline Italy & Health-for-All (ISTAT, updated 2009) & n.a. & n.a. & $18+$ \\
\hline Lithuania & Health behavior among Lithuanian adult population, 2006 & 1,002 & 705 & $20-64$ \\
\hline Norway & Norkost, 1997 & n.a. & n.a & $19-63$ \\
\hline Poland & Low income & 200 & 40 & $19-63$ \\
\hline Portugal & $\begin{array}{l}\text { Data computed from the database of the Inquérito nacional } \\
\text { de saúde, 2005-2006. INE, INSA. }\end{array}$ & 21,299 & 19,894 & $20-64$ \\
\hline Sweden & $\begin{array}{l}\text { National public health survey. Health on equal terms? } 2007 \\
\text { National Institute of Public Health, Sweden }\end{array}$ & 2,329 & 1,824 & $19-64$ \\
\hline The Netherlands & CBS Statline, 2007 & 3,240 & 3,201 & $19-65$ \\
\hline \multicolumn{5}{|l|}{ Measured $^{2}$} \\
\hline Cyprus & CyDA 1st Epidemiological Study & 256 & 187 & $19-64$ \\
\hline Czech Republic & n.a. ${ }^{3}$ & 1,090 & 1,039 & $25-64$ \\
\hline Finland & FINRISK, 2007. Lääkärilehti, 2008 & 3,979 & 3,984 & $19-64$ \\
\hline France & French Nutrition and Health Survey (ENNS), 2006-2007 & 2,273 & 1,604 & $19-64$ \\
\hline Germany & German Nutrition Survey II, 2005-2007 & 5,544 & 4,625 & $19-64$ \\
\hline Greece & EPIC study, 1994-1999 & 12,034 & 8,365 & $19-64$ \\
\hline Ireland & Survey of Lifestyle, Attitudes and Nutrition 2007 & 891 & 949 & $19-64$ \\
\hline Latvia & Nutrition and Lifestyle in the Baltic Republics & 1,230 & 1,062 & $19-64$ \\
\hline Lithuania & National Nutrition Survey & 1,087 & 849 & $19-64$ \\
\hline Poland & $\begin{array}{l}\text { Household Food Consumption and Anthropometric Survey } \\
2000\end{array}$ & 1,469 & 1,346 & $18-64$ \\
\hline
\end{tabular}


Table 8.2. Continued

\begin{tabular}{|c|c|c|c|c|}
\hline \multirow[t]{2}{*}{ Country } & \multirow[t]{2}{*}{ Survey name } & \multicolumn{2}{|c|}{ Participants, n } & \multirow[t]{2}{*}{ Age, years } \\
\hline & & female & male & \\
\hline Portugal & $\begin{array}{l}\text { Overweight and obesity in Portugal: national prevalence in } \\
2003-2005\end{array}$ & 4,320 & 3,796 & $19-64$ \\
\hline Romania & National synthesis, 2006 & 341 & 177 & $19-64$ \\
\hline Spain & Catalan Nutrition Survey (ENCAT), 2002-2003 & 793 & 663 & $19-64$ \\
\hline UK & Health Surveys for England, 2006 & 4,925 & 5,048 & $19-64$ \\
\hline $\begin{array}{l}{ }^{1} \text { Body we } \\
{ }^{2} \text { Body we } \\
{ }^{3} \text { Informat }\end{array}$ & $\begin{array}{l}\text { body height reported by the participants. } \\
\text { body height measured by researchers. } \\
\text { e partner organization of the Czech Republic. }\end{array}$ & & & \\
\hline
\end{tabular}

The prevalence of overweight and obesity was already high in young children. For example in 4- to 6-year-old girls it ranged from 9 to $27 \%$, in 4 - to 6-year-old boys from 8 to $24 \%$. The highest prevalence was found in Spanish 10- to 14-year-old girls and 7 - to 9-year-old Italian boys (36\%). The prevalence of overweight and obesity derived from self-reported data tend to be considerably lower than data derived from measured body height and weight; for example in 10- to 14-year-old girls the highest prevalence derived by self-reported data was 22\% (Austria), whereas it was 36\% (Spain) derived from measured data.

\section{BMI, Overweight and Obesity in Adults}

Table 8.4 shows the mean BMI for European countries. The mean BMI was generally lower in women than in men except for Greece and Latvia. The lowest mean BMI was found for Hungarian 18 - to 34 -year-old women $\left(22.9 \mathrm{~kg} / \mathrm{m}^{2}\right.$, self-reported data), the highest for Greek women $\left(28.6 \mathrm{~kg} / \mathrm{m}^{2}\right.$, measured data).

The WHO suggested a median BMI of $21-23 \mathrm{~kg} / \mathrm{m}^{2}$ as an optimum for a population to reduce the risk of chronic diseases and obesity, where a median BMI of $21 \mathrm{~kg} / \mathrm{m}^{2}$ is considered as being more beneficial for affluent societies with a more sedentary lifestyle and a BMI of $23 \mathrm{~kg} / \mathrm{m}^{2}$ for developing countries [WHO, 2000, 2003]. Eurodiet [2000] recommends a mean BMI of $21-22 \mathrm{~kg} / \mathrm{m}^{2}$. The range was exceeded by every country in both men and women; only Austrian and Polish women (median BMI 22.7 and $22.8 \mathrm{~kg} / \mathrm{m}^{2}$ ) - both derived from selfreported data on body height and body weight - kept in the range of the WHO recommendation.

Table 8.5 shows that the BMIs in the defined European regions did not differ substantially, neither for self-reported nor for measured data. 
Table 8.3. Study populations for assessment of overweight and obesity in elderly (65+ years) in the participating countries. Self-reported: $n=27,874$ from 13 countries; measured: $n=25,805$ from 10 countries

\begin{tabular}{|c|c|c|c|c|}
\hline Country & Survey name & Female & Male & Age, years \\
\hline \multicolumn{5}{|l|}{ Self-reported ${ }^{1}$} \\
\hline Austria & Austrian Study on Nutritional Status - OESES.sen07 & 484 & 204 & $65+$ \\
\hline Belgium & Food Consumption Survey, Belgium, 2004 & 1,623 & 1,626 & $65+$ \\
\hline Denmark & Danish National Survey on Dietary Habits and Physical Activity & 162 & 164 & $65+$ \\
\hline \multirow[t]{3}{*}{ Estonia } & Estonian Health Interview Survey, 2006 & 3,114 & 2,872 & $65+$ \\
\hline & Health Behavior among Estonian Adult Population, 2004 & 1,633 & 1,203 & $65+$ \\
\hline & Health Behavior among Estonian Adult Population, 2006 & 1,603 & 1,030 & $65+$ \\
\hline Germany $^{1}$ & National Telephone Health Survey (GSTeL03), 2004 & 4,302 & 4,016 & $65+$ \\
\hline Ireland & Survey of Lifestyle, Attitudes and Nutrition, 2007 & 786 & 891 & $65+$ \\
\hline Italy & Health-for-All database (ISTAT, updated in 2009) & n.a. & n.a. & $65+$ \\
\hline \multirow[t]{3}{*}{ Lithuania } & $\begin{array}{l}\text { Statistics Lithuania. Health survey results of Lithuanian } \\
\text { population in } 2005\end{array}$ & n.a. & n.a. & $65-74$ \\
\hline & $\begin{array}{l}\text { Statistics Lithuania. Health survey results of Lithuanian } \\
\text { population in } 2005\end{array}$ & n.a. & n.a. & $75-84$ \\
\hline & $\begin{array}{l}\text { Statistics Lithuania. Health survey results of Lithuanian } \\
\text { population in } 2005\end{array}$ & n.a. & n.a. & $85+$ \\
\hline Norway & Norkost 1997 & n.a. & n.a. & $65+$ \\
\hline Poland & Low income & 200 & 40 & $65+$ \\
\hline Sweden & $\begin{array}{l}\text { National public health survey. Health on equal terms? } 2007 \\
\text { National Institute of Public Health, Sweden }\end{array}$ & 700 & 597 & $65+$ \\
\hline \multirow[t]{2}{*}{ The Netherlands } & CBS Statline, 2007 & 105 & 98 & $65+$ \\
\hline & DNFCS-3, 1997/98 & 236 & 185 & $65+$ \\
\hline \multirow[t]{2}{*}{ UK } & Health Surveys for England, 2006 & n.a. & n.a. & $65-74$ \\
\hline & Health Surveys for England, 2006 & n.a. & n.a. & $75+$ \\
\hline \multicolumn{5}{|l|}{ Measured $^{2}$} \\
\hline Austria & Austrian Study on Nutritional Status - OESES.sen07 & 484 & 204 & $65+$ \\
\hline Cyprus & CyDA 1st Epidemiological Study & 256 & 187 & $65+$ \\
\hline Finland & FINRISK, 2007. Lääkärilehti, 2008 & 3,979 & 3,984 & $65+$ \\
\hline France & French Nutrition and Health Survey (ENNS), 2006-2007 & 2,273 & 1,604 & $65-74$ \\
\hline Germany & German Nutrition Survey II, 2005-2007 & 1,435 & 1,377 & $65+$ \\
\hline Greece & EPIC study, 1994-1999 & 3,600 & 2,508 & $65+$ \\
\hline
\end{tabular}


Table 8.3. Continued

\begin{tabular}{lllll}
\hline Country & Survey name & Female & Male & Age, years \\
\hline Ireland & Survey of Lifestyle, Attitudes and Nutrition, 2007 & 220 & 179 & $65+$ \\
\hline Poland & $\begin{array}{l}\text { Household Food Consumption and Anthropometric Survey, } \\
2000\end{array}$ & 1,469 & 1,346 & $65+$ \\
\hline Portugal & $\begin{array}{l}\text { Data collected for PhD diss. thesis in Faculty of Nutrition and } \\
\text { Food Sciences, Oporto University }\end{array}$ & 192 & 194 & $65+$ \\
\hline Spain & Catalan Nutrition Survey (ENCAT), 2002-2003 & 161 & 153 & $65+$ \\
\hline $\begin{array}{l}\text { 'Body weight and body height reported by the participants. } \\
\text { 2Body weight and body height measured by researchers. }\end{array}$ & & & \\
\hline
\end{tabular}

Fig. 8.2 shows a remarkable prevalence of overweight and obesity in adults. As was the BMI, the prevalence of overweight and obesity was generally higher in men than in women. The highest prevalence for overweight and obesity was seen in Greek women (73\%) and Cyprian men (83\%). The lowest prevalence was found for Norwegian and Austrian women (31\%) and for men from Sweden $(38 \%)$.

The difference in prevalence of overweight and obesity between countries that provided measured data and countries that provided self-reported data was considerable. Whereas the maximum prevalence of overweight and obesity in men was $82 \%$ (minimum 51\%) from measured data it was 58\% (minimum 40\%) from self-reported data; in women it was $61 \%$ (minimum $40 \%$ ) for measured data and $49 \%$ (minimum $25 \%$ ) for self-reported data. Thus, interpretation of data must be undertaken carefully. No considerable differences in the prevalence of overweight and obesity between the regions could be observed.

A comparison of prevalence of overweight and obesity between data from the European Nutrition and Health Report 2004 and 2009 was possible for 10 countries. For women and for men, no big change in the prevalence could be observed (table 8.6).

\section{BMI, Overweight and Obesity in Elderly (65+ Years)}

The mean BMI of elderly people as well as the prevalence of overweight and obesity was higher than in the younger adults. Table 8.7 shows the mean BMI of the elderly of European countries. The lowest mean BMI was seen in Danish women $\left(24.7 \mathrm{~kg} / \mathrm{m}^{2}\right)$, the highest in Polish men $\left(28.5 \mathrm{~kg} / \mathrm{m}^{2}\right)$.

During the last years it has been shown that for the elderly population a higher BMI than for younger adults is associated with positive health outcomes. Therefore the National Research Council of the USA suggests a range of $24-29 \mathrm{~kg} / \mathrm{m}^{2}$ for over 
Table 8.4. BMI self-reported and measured (median, mean, SD) in European 19- to 64-year-old men and women

\begin{tabular}{|c|c|c|c|c|c|c|c|}
\hline \multirow[t]{2}{*}{ Country } & \multirow[t]{2}{*}{ Age, years } & \multicolumn{3}{|l|}{ Women } & \multicolumn{3}{|l|}{ Men } \\
\hline & & median & mean & SD & median & mean & SD \\
\hline
\end{tabular}

Self-reported

\begin{tabular}{|c|c|c|c|c|c|c|c|}
\hline Austria & 19-64 & 22.7 & 23.8 & 4.5 & 25.3 & 25.8 & 3.7 \\
\hline Belgium & 19-63 & 23.1 & 24.2 & 4.6 & 25.3 & 25.6 & 4.0 \\
\hline Denmark & 19-64 & 23.2 & 24.1 & 4.3 & 24.9 & 25.5 & 3.5 \\
\hline Estonia & 19-63 & 24.2 & 25.3 & 5.5 & 25.6 & 26.3 & 4.5 \\
\hline \multirow[t]{4}{*}{ Hungary } & $\begin{array}{l}\text { Mean of age } \\
\text { groups }\end{array}$ & n.a. & 25.3 & n.a. & n.a. & 27.6 & n.a. \\
\hline & $18-34$ & n.a. & 22.9 & 4.5 & n.a. & 24.7 & 3.8 \\
\hline & $35-59$ & n.a. & 26.1 & 4.6 & n.a. & 26.8 & 3.9 \\
\hline & $60+$ & n.a. & 27.0 & 4.1 & n.a. & 27.6 & 3.9 \\
\hline \multirow[t]{3}{*}{ Ireland } & $\begin{array}{l}\text { Mean of age } \\
\text { groups }\end{array}$ & n.a. & 25.2 & n.a. & n.a. & 26.5 & n.a. \\
\hline & $18-44$ & 23.1 & 24.2 & 4.5 & 25.0 & 25.5 & 4.2 \\
\hline & $45-64$ & 25.4 & 26.2 & 4.8 & 27.1 & 27.4 & 3.7 \\
\hline Lithuania & $20-64$ & 24.9 & 25.7 & 4.9 & 25.8 & 26.6 & 4.5 \\
\hline Norway & 19-63 & 23.4 & 23.9 & 3.9 & 24.8 & 25.2 & 3.3 \\
\hline Poland & $19-63$ & 22.8 & 23.8 & 4.3 & 24.9 & 25.7 & 2.7 \\
\hline Portugal & $20-64$ & 24.5 & 25.3 & 4.8 & 25.6 & 26.0 & 3.8 \\
\hline Sweden & 19-64 & 23.8 & 24.7 & 4.5 & 25.5 & 26.2 & 3.9 \\
\hline \multirow[t]{2}{*}{ The Netherlands } & 19-64 & 23.9 & 24.8 & 4.5 & 24.7 & 24.9 & 3.4 \\
\hline & & \multicolumn{3}{|c|}{$\min .22 .9, \max .27 .0$} & \multicolumn{3}{|c|}{$\min .24 .9, \max .27 .6$} \\
\hline \multicolumn{8}{|l|}{ Measured } \\
\hline Cyprus & $19-64$ & 26.8 & $26.8^{1}$ & n.a. & 28.6 & $28.6^{1}$ & \\
\hline Czech Republic & $19-64$ & 26.5 & 27.4 & 5.7 & 27.6 & 28.6 & 4.4 \\
\hline Finland & $25-64$ & & 26.9 & 5.4 & & 27.4 & 4.2 \\
\hline France & 19-64 & 23.8 & 25.1 & 0.2 & 25.6 & 26.0 & 0.2 \\
\hline Germany & 19-64 & 24.3 & 25.6 & 5.1 & 26.2 & 26.7 & 4.7 \\
\hline Greece & 19-64 & 28.0 & 28.6 & 5.3 & 27.9 & 28.2 & 3.8 \\
\hline Ireland & 19-64 & n.a. & 25.8 & 4.8 & n.a. & 26.9 & 4.0 \\
\hline Latvia & 19-64 & n.a. & 25.8 & 4.9 & n.a. & 25.5 & 3.7 \\
\hline
\end{tabular}


Table 8.4. Continued

\begin{tabular}{|c|c|c|c|c|c|c|c|}
\hline \multirow[t]{2}{*}{ Country } & \multirow[t]{2}{*}{ Age, years } & \multicolumn{3}{|l|}{ Women } & \multicolumn{3}{|l|}{ Men } \\
\hline & & median & mean & $\mathrm{SD}$ & median & mean & SD \\
\hline Lithuania & $19-64$ & 24.6 & 25.5 & 5.0 & 25.6 & 26.0 & 3.7 \\
\hline Poland & $19-64$ & 24.3 & 25.3 & 5.0 & 25.5 & 26.1 & 4.0 \\
\hline Portugal & $19-64$ & 24.7 & 25.2 & 4.6 & 25.7 & 26.2 & 3.7 \\
\hline Romania & $19-64$ & 24.8 & 25.2 & 4.3 & 26.2 & 26.8 & 5.0 \\
\hline Spain & $19-64$ & 24.0 & 24.8 & 4.5 & 26.0 & 26.2 & 3.8 \\
\hline \multirow[t]{7}{*}{ UK } & $\begin{array}{l}\text { Mean of age } \\
\text { groups }\end{array}$ & n.a. & 26.4 & n.a. & n.a. & 27.1 & n.a. \\
\hline & $16-24$ & n.a. & 24.0 & 6.0 & n.a. & 24.1 & 6.3 \\
\hline & $25-34$ & n.a. & 25.9 & 5.7 & n.a. & 26.7 & 5.4 \\
\hline & $35-44$ & n.a. & 26.8 & 5.5 & n.a. & 27.8 & 5.1 \\
\hline & $45-54$ & n.a. & 27.6 & 5.3 & n.a. & 28.0 & 4.6 \\
\hline & $55-64$ & n.a. & 28.0 & 4.8 & n.a. & 28.6 & 4.5 \\
\hline & & \multicolumn{3}{|c|}{$\min .24 .8, \max .28 .6$} & \multicolumn{3}{|c|}{$\min .25 .5, \max .28 .6$} \\
\hline
\end{tabular}

Table 8.5. Maximum and minimum values of the mean BMI in adults aged 19-64 years the European regions by sex

\begin{tabular}{|c|c|c|c|c|c|}
\hline & & \multicolumn{2}{|c|}{ Self-reported } & \multicolumn{2}{|l|}{ Measured } \\
\hline & & minimum & maximum & minimum & maximum \\
\hline \multirow[t]{2}{*}{ Central and East } & Female & 23.8 & 25.3 & 25.2 & 27.4 \\
\hline & Male & 25.7 & 27.6 & 26.1 & 28.6 \\
\hline \multirow[t]{2}{*}{ North } & Female & 23.9 & 25.7 & 25.5 & 26.9 \\
\hline & Male & 25.1 & 26.6 & 25.5 & 27.4 \\
\hline \multirow[t]{2}{*}{ South } & Female & 23.3 & 25.3 & 24.8 & 28.6 \\
\hline & Male & 25.4 & 26.0 & 26.2 & 28.2 \\
\hline \multirow[t]{2}{*}{ West } & Female & 24.2 & 25.2 & 25.1 & 26.4 \\
\hline & Male & 24.9 & 26.5 & 26.0 & 27.1 \\
\hline
\end{tabular}




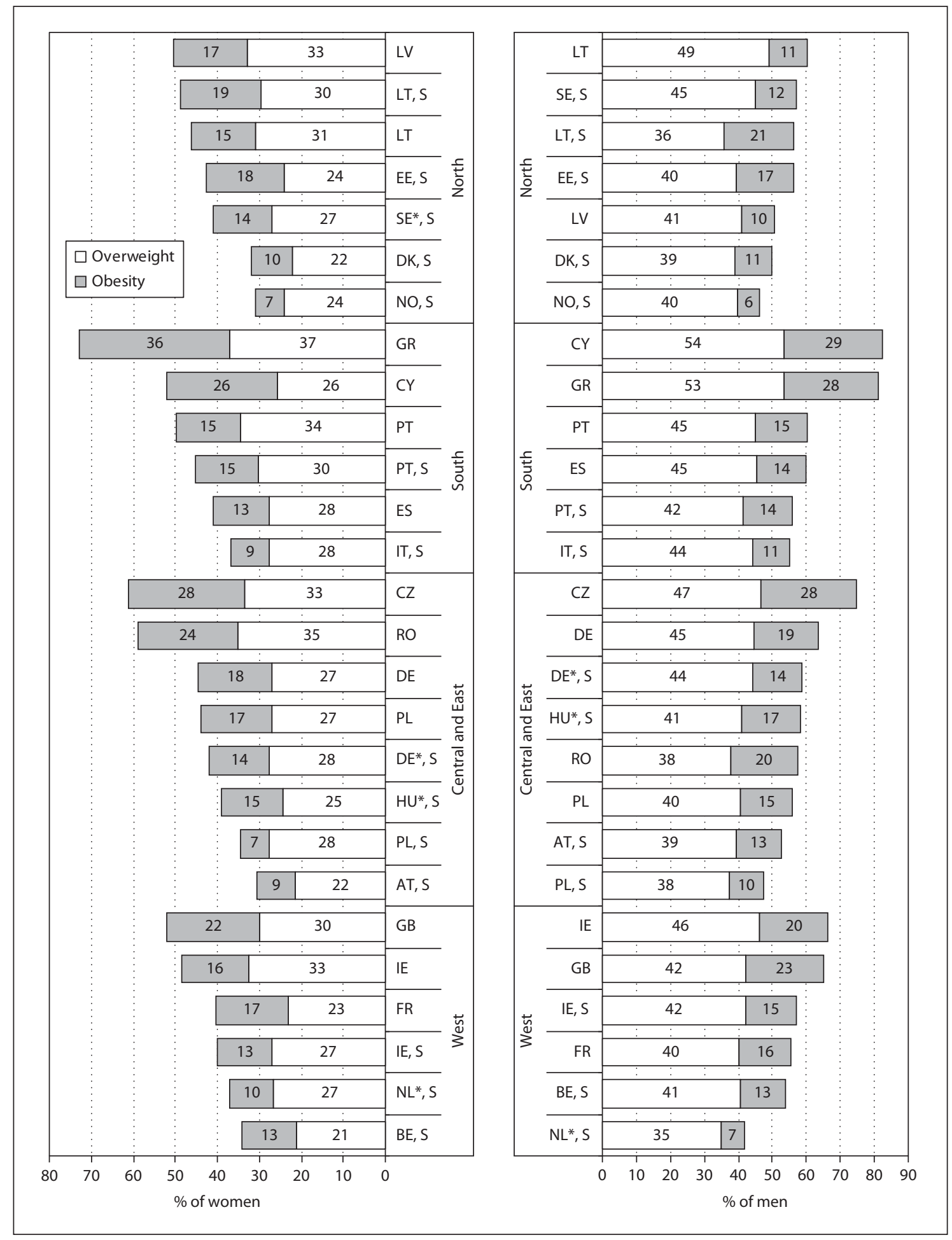

Fig. 8.2. Overweight and obesity in European 19- to 64-year-old adults ordered by region. S = BMI calculated from self-reported height and weight. *Estimated from mean values for different age groups. 
Table 8.6. Comparison of prevalence of overweight and obesity in men and women aged 19-64 years in 2004 and 2009

\begin{tabular}{|c|c|c|c|c|c|}
\hline 2004 & & & 2009 & & \\
\hline BMI & overweight \% & obesity \% & BMI & overweight \% & obesity \% \\
\hline
\end{tabular}

\section{Men}

\begin{tabular}{lllllll}
\hline UK & $\mathrm{m}$ & 41 & 25 & $\mathrm{~s}$ & 46 & 27 \\
\hline Spain & $\mathrm{m}$ & 45 & 13 & $\mathrm{~m}$ & 45 & 14 \\
\hline Portugal & $\mathrm{m}$ & 41 & 13 & $\mathrm{~m}$ & 45 & 15 \\
\hline Italy & $\mathrm{s}$ & 42 & 9 & $\mathrm{~s}$ & 44 & 11 \\
\hline Hungary & $\mathrm{m}$ & 42 & 21 & $\mathrm{~s}$ & 42 & 20 \\
\hline $\begin{array}{l}\text { Germany (mean West/ } \\
\text { East) }\end{array}$ & $\mathrm{m}$ & 47 & 20 & $\mathrm{~m}$ & 45 & 19 \\
\hline Finland & $\mathrm{n} . \mathrm{i}$. & 49 & 19 & $\mathrm{~m}$ & 48 & 22 \\
\hline France & $\mathrm{m}$ & 47 & 10 & $\mathrm{~m}$ & 40 & 16 \\
\hline Belgium & $\mathrm{s}$ & 40 & 10 & $\mathrm{~s}$ & 41 & 13 \\
\hline Austria & $\mathrm{s}$ & 35 & 6 & $\mathrm{~s}$ & 39 & 13
\end{tabular}

Women

\begin{tabular}{lllllll}
\hline UK & $\mathrm{m}$ & 33 & 20 & $\mathrm{~s}$ & 33 & 25 \\
\hline Spain & $\mathrm{m}$ & 32 & 16 & $\mathrm{~m}$ & 28 & 13 \\
\hline Portugal & $\mathrm{m}$ & 31 & 15 & $\mathrm{~m}$ & 34 & 15 \\
\hline Italy & $\mathrm{s}$ & 26 & 9 & $\mathrm{~s}$ & 28 & 10 \\
\hline Hungary & $\mathrm{m}$ & 28 & 21 & $\mathrm{~s}$ & 30 & 17 \\
\hline Germany (mean West/ & $\mathrm{m}$ & 32 & 23 & $\mathrm{~m}$ & 27 & 18 \\
East) & $\mathrm{n} . \mathrm{i}$. & 33 & 18 & $\mathrm{~m}$ & 33 & 23 \\
\hline Finland & $\mathrm{m}$ & 21 & 7 & $\mathrm{~m}$ & 23 & 17 \\
\hline France & $\mathrm{s}$ & 26 & 12 & $\mathrm{~s}$ & 21 & 13 \\
\hline Belgium & $\mathrm{s}$ & 20 & 6 & $\mathrm{~s}$ & 22 & 9
\end{tabular}

$\mathrm{m}=\mathrm{BMI}$ calculated from measured body height and weight; $\mathrm{s}=\mathrm{BMI}$ calculated from self-reported body height and weight; $n$.i. = method used not indicated. 
Table 8.7. BMI self-reported and measured (median, mean, SD) in European men and women from 65+ years

\begin{tabular}{|c|c|c|c|c|c|c|c|}
\hline \multirow[t]{2}{*}{ Country } & \multirow[t]{2}{*}{ Age years } & \multicolumn{3}{|l|}{ Women } & \multicolumn{3}{|l|}{ Men } \\
\hline & & median & mean & SD & median & mean & SD \\
\hline \multicolumn{8}{|l|}{ Self-reported } \\
\hline Austria & $65+$ & 26.0 & 26.7 & 4.9 & 27.6 & 27.8 & 3.6 \\
\hline Belgium & $65+$ & 25.3 & 25.7 & 4.4 & 25.7 & 25.9 & 3.5 \\
\hline Denmark & $65+$ & 24.5 & 24.7 & 4.4 & 25.5 & 26.0 & 3.2 \\
\hline Estonia & $65+$ & 27.0 & 27.6 & 5.0 & 25.8 & 26.3 & 3.9 \\
\hline Ireland & $65+$ & 24.8 & 25.2 & 4.5 & 26.0 & 26.4 & 3.9 \\
\hline Lithuania & $65+$ & n.a. & 26.4 & n.a. & n.a. & 25.9 & n.a. \\
\hline Norway & $65+$ & 25.0 & 25.7 & 4.7 & 24.6 & 25.1 & 2.8 \\
\hline Poland & $65+$ & 25.0 & 25.7 & 3.9 & 27.9 & 28.5 & 4.6 \\
\hline Portugal & $65+$ & 26.1 & 26.6 & 4.9 & 26.3 & 26.0 & 3.9 \\
\hline Sweden & $65+$ & 25.2 & 25.6 & 3.8 & 26.2 & 26.3 & 3.0 \\
\hline The Netherlands & $65+$ & 26.0 & 26.8 & 4.6 & 25.2 & 25.4 & 2.9 \\
\hline \multirow[t]{2}{*}{ UK } & $65+$ & n.a. & 28.1 & n.a. & n.a. & 27.7 & n.a. \\
\hline & & \multicolumn{3}{|c|}{$\min .24 .7, \max .28 .1$} & \multicolumn{3}{|c|}{$\min .25 .1, \max .28 .5$} \\
\hline \multicolumn{8}{|l|}{ Measured } \\
\hline Austria & $65+$ & 28.0 & 28.7 & 4.9 & 29.1 & 29.6 & 3.9 \\
\hline Cyprus & $65+$ & 26.8 & n.a. & n.a. & 28.6 & n.a. & n.a. \\
\hline Finland & $65+$ & n.a. & 28.5 & 5.0 & n.a. & 28.0 & 2.8 \\
\hline France & $65-74$ & 25.4 & 26.4 & 0.4 & 28.0 & 28.0 & 0.4 \\
\hline Germany & $65+$ & 27.8 & 28.3 & 5.0 & 28.1 & 28.4 & 3.7 \\
\hline Greece & $65+$ & 30.3 & 30.6 & 4.9 & 28.1 & 28.2 & 4.0 \\
\hline Ireland & $65+$ & 28.0 & 28.1 & 5.0 & 27.5 & 27.8 & 4.1 \\
\hline Poland & $65+$ & 28.0 & 28.1 & 5.0 & 26.3 & 26.3 & 3.9 \\
\hline Portugal & $65+$ & 27.7 & 27.8 & 4.5 & 27.4 & 27.2 & 3.8 \\
\hline \multirow[t]{2}{*}{ Spain } & $65+$ & 28.6 & 28.4 & 4.3 & 27.6 & 27.6 & 3.6 \\
\hline & & \multicolumn{3}{|c|}{$\min .26 .4, \max .30 .6$} & \multicolumn{3}{|c|}{$\min .26 .3, \max .29 .6$} \\
\hline
\end{tabular}




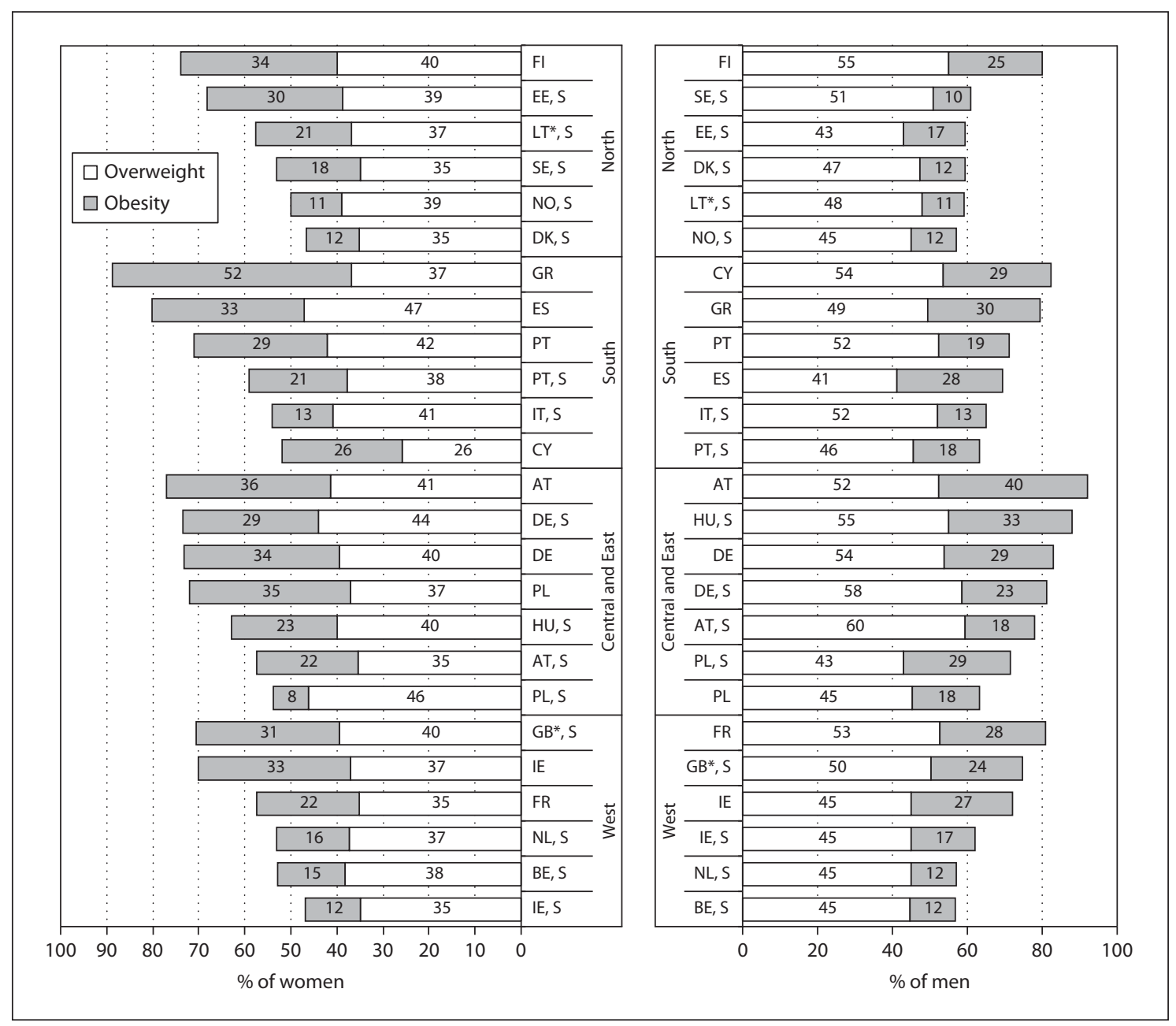

Fig. 8.3. Overweight and obesity in European elderly (65+ years) ordered by region. $S=B M I$ calculated from self-reported height and weight. *Estimated from mean values for different age groups.

65-year-olds as normal body weight [NRC, 1989]. Applying these (individually based) recommendations on the mean of the countries, it can be seen that the mean BMIs of all countries were within this range except for Greek women $\left(30.6 \mathrm{~kg} / \mathrm{m}^{2}\right)$.

The lowest prevalence for overweight and obesity was found in elderly women from Denmark, Italy and Ireland (47\%), for Norwegian and Belgian men (48\%; the highest prevalence was seen in Greek women (89\%) and Austrian men (92\%) (fig. 8.3). No big differences between the mean prevalence of overweight and obesity could be observed between the regions. 


\subsection{Blood Lipids $^{1}$}

In tables 8.8-8.9, the blood lipid status of the different European countries is shown. These data were available from 8 European countries for adults of both sexes (1964 years of age) and from 6 European countries for elderly women and men ( $\geq 65$ years of age). A detailed description of the surveys conducted in the individual countries is given in chapter 7, 'Energy and Nutrient Intake in the European Union', as well as in chapter 11, 'National Reports'. The guidelines of the European Society of Cardiology (ESC) were applied as reference values [Graham et al., 2007].

Except for 19- to 34-year-old women and men from the UK and Greek adult women, the average total cholesterol levels were in all European countries at or above the reference value of $5.0 \mathrm{mmol} / \mathrm{l}$ (table 8.8 ). For elderly women and men ( $\geq 65$ years), the average total cholesterol levels were also equal or above the reference value across Europe (table 8.9).

The average levels of LDL-Cholesterol for both sexes in all European countries were equal or above the reference value of $3.0 \mathrm{mmol} / \mathrm{l}$; the reported values for adult men were slightly higher than for adult women. The average levels of HDL-Cholesterol of all European countries were clearly above the reference value of at least $1.2 \mathrm{mmol} / \mathrm{l}$ for women and $1.0 \mathrm{mmol} / \mathrm{l}$ for men (tables 8.8, 8.9). Except for Romanian adults and adult men from Lithuania and Portugal, levels of triacylglycerides were below the upper level of $1.7 \mathrm{mmol} / \mathrm{l}$ across Europe. The atherogenic quotient (calculated from average values of each country) was higher in men than in women but values for both sexes were within the normal range (3-5 $\mathrm{mmol} / \mathrm{l})$.

\subsection{Mortality/Morbidity}

The data about mortality of the different European countries originate from the European Health-for-All database of the WHO [WHO, 2009a]. Within this database, data about mortality rates were available from 10 European countries from 2007. Data are presented as age standardized death rate per 100,000 alive and represents what the crude rate would have been if the population had the same age distribution as the standard European population (fig. 8.4).

The highest total mortality rate in women was found in Latvia (777 deaths/100,000 women alive), followed by Romania and Lithuania (764 and 731 deaths/100,000 women alive, respectively) (fig. 8.4). The lowest mortality rates in women were observed in Finland and Austria (445 and 448 deaths/100,000 women alive). In men, the highest mortality rate was in Lithuania with 1,621 deaths/100,000 men alive, followed by Latvia and Romania (1,565 and 1,242 deaths/100,000 men alive). The lowest

${ }_{1}$ Chapters 8.2, 8.3, 8.4 and 8.5 by Krems C, Strassburg A: Max Rubner-Institut, Federal Research Institute of Nutrition and Food, Karlsruhe, Germany (carolin.krems@mri.bund.de). 
Table 8.8. Blood lipids in serum/plasma in different European countries (mmol/l), women and men 19-64 years of age

\begin{tabular}{|c|c|c|c|c|c|c|c|}
\hline Country & Survey name & $\begin{array}{l}\text { Survey } \\
\text { year }\end{array}$ & $\begin{array}{l}\text { Total cholesterol } \\
\text { mean } \pm S D\end{array}$ & $\begin{array}{l}\text { LDL chol. } \\
\text { mean } \pm S D\end{array}$ & $\begin{array}{l}\text { HDL chol. } \\
\text { mean } \pm S D\end{array}$ & $\begin{array}{l}\text { Trigly- } \\
\text { cerides } \\
\text { mean } \pm S D\end{array}$ & $\begin{array}{l}\text { Total chol./ } \\
\text { HDL chol. }{ }^{5}\end{array}$ \\
\hline
\end{tabular}

\section{Women}

\begin{tabular}{|c|c|c|c|c|c|c|c|}
\hline Finland $^{1}$ & FINRISK Study & 2007 & $5.2 \pm 1.0$ & & & & \\
\hline Greece & ATTICA Study & 2001-2002 & $4.9 \pm 1.1$ & $3.1 \pm 0.9$ & $1.4 \pm 0.4$ & $1.2 \pm 0.6$ & 3.5 \\
\hline Lithuania & $\begin{array}{l}\text { Countrywide Integrated } \\
\text { Non-Communicable } \\
\text { Diseases Intervention } \\
\text { Survey }\end{array}$ & 2007 & $5.2 \pm 1.1$ & $3.0 \pm 1.0$ & $1.4 \pm 0.4$ & $1.5 \pm 0.7$ & 3.7 \\
\hline Portugal & $\begin{array}{l}\text { Epidemiological study to } \\
\text { characterize the lipid } \\
\text { profile of the Portuguese } \\
\text { population }\end{array}$ & 2001 & 5.5 & 3.6 & 1.5 & 1.2 & 3.7 \\
\hline Spain & Catalan Nutrition Survey & $2002-2003$ & $5.0 \pm 1.3$ & $3.1 \pm 1.1$ & $1.5 \pm 0.4$ & $0.9 \pm 0.5$ & 3.3 \\
\hline \multirow[t]{3}{*}{$\mathrm{UK}^{3}$} & $\begin{array}{l}\text { Low-income diet and } \\
\text { health survey }\end{array}$ & $\begin{array}{l}\text { 2003-2005 } \\
\text { a) }\end{array}$ & $4.9 \pm 1.0$ & & $1.4 \pm 0.3$ & & 3.5 \\
\hline & & b) & $5.4 \pm 1.0$ & & $1.5 \pm 0.3$ & & 3.6 \\
\hline & & c) & $6.2 \pm 1.4$ & & $1.5 \pm 0.4$ & & 4.1 \\
\hline $\begin{array}{l}\text { Reference } \\
\text { value }^{4}\end{array}$ & & & $<5.0$ & $<3.0$ & $>1.2$ & $<1.7$ & \\
\hline
\end{tabular}

\section{Men}

\begin{tabular}{|c|c|c|c|c|c|c|c|}
\hline Finland $^{1}$ & FINRISK Study & 2007 & $5.3 \pm 1.0$ & & & & \\
\hline France & $\begin{array}{l}\text { French Nutrition and } \\
\text { Health Survey }\end{array}$ & 2006-2007 & $5.4 \pm 0.7$ & $3.4 \pm 0.1$ & $1.4 \pm 0.0$ & $1.4 \pm 0.9$ & 3.9 \\
\hline Greece & ATTICA Study & 2001-2002 & $5.0 \pm 1.1$ & $3.2 \pm 1.0$ & $1.2 \pm 0.4$ & $1.6 \pm 0.6$ & 4.2 \\
\hline Lithuania $^{1}$ & $\begin{array}{l}\text { Countrywide Integrated } \\
\text { Non-communicable } \\
\text { Diseases Intervention } \\
\text { Survey }\end{array}$ & 2007 & $5.2 \pm 1.1$ & $3.2 \pm 1.0$ & $1.3 \pm 0.4$ & $1.7 \pm 0.9$ & 4.0 \\
\hline
\end{tabular}


Table 8.8. Continued

\begin{tabular}{|c|c|c|c|c|c|c|c|}
\hline Country & Survey name & $\begin{array}{l}\text { Survey } \\
\text { year }\end{array}$ & $\begin{array}{l}\text { Total cholesterol } \\
\text { mean } \pm \text { SD }\end{array}$ & $\begin{array}{l}\text { LDL chol. } \\
\text { mean } \pm \text { SD }\end{array}$ & $\begin{array}{l}\mathrm{HDL} \text { chol. } \\
\text { mean } \pm \mathrm{SD}\end{array}$ & $\begin{array}{l}\text { Trigly- } \\
\text { cerides } \\
\text { mean } \pm S D\end{array}$ & $\begin{array}{l}\text { Total chol./ } \\
\text { HDL chol. }\end{array}$ \\
\hline Portugal & $\begin{array}{l}\text { Epidemiological study to } \\
\text { characterize the lipid } \\
\text { profile of the Portuguese } \\
\text { population }\end{array}$ & 2001 & 5.5 & 3.6 & 1.2 & 1.9 & 4.6 \\
\hline Romania $^{2}$ & $\begin{array}{l}\text { Nutritional status in adult } \\
\text { population in relation to } \\
\text { the food intake }\end{array}$ & 2002 & $5.0 \pm 1.4$ & & $1.4 \pm 0.3$ & $1.7 \pm 1.2$ & 3.6 \\
\hline Spain & Catalan Nutrition Survey & $2002-2003$ & $5.0 \pm 1.0$ & $3.3 \pm 0.8$ & $1.2 \pm 0.3$ & $1.2 \pm 1.0$ & 4.2 \\
\hline \multirow[t]{3}{*}{$\mathrm{UK}^{3}$} & $\begin{array}{l}\text { Low-income diet and } \\
\text { health survey }\end{array}$ & $\begin{array}{l}\text { 2003-2005 } \\
\text { a) }\end{array}$ & $4.9 \pm 1.0$ & & $1.4 \pm 0.3$ & & 3.5 \\
\hline & & b) & $5.9 \pm 1.5$ & & $1.3 \pm 0.4$ & & 4.5 \\
\hline & & c) & $5.7 \pm 1.2$ & & $1.3 \pm 0.4$ & & 4.4 \\
\hline $\begin{array}{l}\text { Reference } \\
\text { value }^{4}\end{array}$ & & & $<5.0$ & $<3.0$ & $>1.0$ & $<1.7$ & \\
\hline \multicolumn{8}{|c|}{$\begin{array}{l}{ }^{1} \text { Age: } 25-64 \text { years. } \\
{ }^{2} \text { Age: } 55-64 \text { years. } \\
{ }^{3} \text { Age: a) } 19-34 \text { years, b) } 35-49 \text { years, c) } 50-64 \text { years. } \\
{ }^{4} \text { Graham et al. [2007]. } \\
{ }^{5} \text { Calculated from average total cholesterol and } \mathrm{HDL}\end{array}$} \\
\hline
\end{tabular}

mortality rates in men were observed for The Netherlands and the UK (708 and 712 deaths/100,000 men alive). In all European countries, men had a higher mortality rate than women.

Cardiovascular diseases (CVD) were the leading cause of death across the European countries [WHO, 2009a]. The highest mortality rates from CVD were found for women and men from Latvia, Lithuania and Romania, respectively (fig. 8.4). The lowest rates of deaths from CVD were observed for women and men from The Netherlands (134 and 210 deaths/100,000 subjects alive, respectively). In all European countries, men showed higher mortality rates from CVD than women. In particular, theses differences were very large in Latvia, Lithuania and Romania.

The second most frequent cause of death across Europe was malignant neoplasms (MN) [WHO, 2009a]. In women, the highest mortality rate from MN was found in the Czech Republic, followed by UK and The Netherlands with only minor differences (155, 154 and 151 deaths/100,000 women alive, respectively) (fig. 8.4). The 
Table 8.9. Blood lipids in serum/plasma in different European countries $(\mathrm{mmol} / \mathrm{L})$, women and men $65+$ years of age

\begin{tabular}{|c|c|c|c|c|c|c|c|}
\hline Country & Survey name & $\begin{array}{l}\text { Survey } \\
\text { year }\end{array}$ & $\begin{array}{l}\text { Total cholesterol } \\
\text { mean } \pm S D\end{array}$ & $\begin{array}{l}\text { LDL-chol. } \\
\text { mean } \pm S D\end{array}$ & $\begin{array}{l}\mathrm{HDL}-\mathrm{chol} \\
\text { mean } \pm \mathrm{SD}\end{array}$ & $\begin{array}{l}\text { Trigly- } \\
\text { cerides } \\
\text { mean } \pm S D\end{array}$ & $\begin{array}{l}\text { Total chol./ } \\
\text { HDL-chol. }^{2}\end{array}$ \\
\hline \multicolumn{8}{|l|}{ Women } \\
\hline Austria & $\begin{array}{l}\text { Nutritional Status in } \\
\text { Elderly }\end{array}$ & 2007 & $5.7 \pm 0.8$ & $3.6 \pm 0.7$ & $1.6 \pm 0.4$ & $1.2 \pm 0.6$ & 3.6 \\
\hline Finland & FINRISK Study & 2007 & $5.5 \pm 1.0$ & & 1.6 & & \\
\hline France & $\begin{array}{l}\text { French Nutrition and } \\
\text { Health Survey }\end{array}$ & $2006-2007$ & $5.9 \pm 0.1$ & $3.6 \pm 0.1$ & $1.7 \pm 0.0$ & $1.2 \pm 0.1$ & 3.5 \\
\hline Romania & $\begin{array}{l}\text { Nutritional status in } \\
\text { adult population in } \\
\text { relation to the food } \\
\text { intake }\end{array}$ & 2002 & $5.1 \pm 1.2$ & & & $1.6 \pm 1.1$ & \\
\hline Spain & Catalan Nutrition Survey & $2002-2003$ & $5.8 \pm 1.0$ & $3.7 \pm 0.9$ & $1.4 \pm 0.4$ & $1.3 \pm 0.7$ & 4.1 \\
\hline UK & $\begin{array}{l}\text { Low-income diet and } \\
\text { health survey }\end{array}$ & $2003-2005$ & $5.9 \pm 1.4$ & & $1.6 \pm 0.4$ & & 3.7 \\
\hline \multicolumn{8}{|l|}{ Men } \\
\hline Austria & $\begin{array}{l}\text { Nutritional Status in } \\
\text { Elderly }\end{array}$ & 2007 & $5.4 \pm 0.9$ & $3.4 \pm 0.8$ & $1.4 \pm 0.3$ & $1.4 \pm 0.7$ & 3.9 \\
\hline Finland & FINRISK Study & 2007 & $5.0 \pm 1.0$ & & 1.3 & & \\
\hline France & $\begin{array}{l}\text { French Nutrition and } \\
\text { Health Survey }\end{array}$ & $2006-2007$ & $5.2 \pm 0.2$ & $3.2 \pm 0.1$ & $1.3 \pm 0.1$ & $1.5 \pm 0.1$ & 4.0 \\
\hline Romania & $\begin{array}{l}\text { Nutritional status in } \\
\text { adult population in } \\
\text { relation to the food } \\
\text { intake }\end{array}$ & 2002 & $5.0 \pm 1.2$ & & & $1.6 \pm 1.0$ & \\
\hline Spain & $\begin{array}{l}\text { Catalan Nutrition and } \\
\text { Health Survey }\end{array}$ & $2002-2003$ & $5.2 \pm 1.0$ & $3.3 \pm 0.7$ & $1.3 \pm 0.4$ & $1.2 \pm 0.7$ & 3.9 \\
\hline UK & $\begin{array}{l}\text { Low-income diet and } \\
\text { health survey }\end{array}$ & $2003-2005$ & $5.2 \pm 1.2$ & & $1.3 \pm 0.4$ & & 4.0 \\
\hline $\begin{array}{l}\text { Reference } \\
\text { value }^{1}\end{array}$ & & & $<5.0$ & $<3.0$ & $\begin{array}{l}f>1.2 \\
m>1.0\end{array}$ & $<1.7$ & \\
\hline
\end{tabular}


lowest mortality rate in women was observed in Finland and Greece (113 and 114 deaths $/ 100,000$ women alive, respectively). In general, the differences between the mortality rates from $\mathrm{MN}$ in women across the different European countries were rather small. In men, the highest mortality rate from $\mathrm{MN}$ was found in Lithuania followed by Latvia and Slovenia (306, 291 and 288 deaths/100,000 men alive, respectively). Compared to the results of women, the lowest rate of deaths from $\mathrm{MN}$ in men was found in Finland (178 deaths/100,000 men alive). In all European countries, men died more often from $\mathrm{MN}$ than women.

\section{Incidence of Malignant Neoplasms}

To illustrate the annual incidence of MN, the GLOBOCAN database 2002 was used (fig. 8.5-8.7) [Ferlay et al., 2004]. Within this database, data from 24 European countries are included and estimates for the year 2002 are presented. These estimates are based on the most recent data available at the International Agency for Research on Cancer in Lyon (IARC), but more recent data could be available from other sources. However, this database was used because other available databases deliver information from only a few countries.

Except for Denmark, the incidence of MN was higher in men than in women across all European countries (fig. 8.5). The order of the countries regarding the incidence of $\mathrm{MN}$ was different in men and women. In women, the highest incidence of $\mathrm{MN}$ was found in Denmark (496 new cases/100,000 inhabitants) followed by Germany and Sweden (467 and 464 new cases/100,000 inhabitants, respectively), whereas the highest incidence of MN in men was observed in Italy (586 new cases/100,000 inhabitants) followed by Belgium and Hungary (585 and 560 new cases/100,000 inhabitants, respectively). The lowest incidence of $\mathrm{MN}$ was found in Romania for both women and men (243 and 297 new cases/100,000 inhabitants, respectively).

The incidence of $\mathrm{MN}$ of the stomach was higher in men in comparison with women across Europe (fig. 8.6). The highest incidences of $\mathrm{MN}$ of the stomach were found in Portugal and Estonia for both women and men. The lowest incidence of MN of the stomach was observed in Denmark in women and men, respectively 8 and 13 new cases/100,000 inhabitants.

The incidence of $\mathrm{MN}$ of the colon and rectum across Europe are shown in fig. 8.6. In most countries the incidence of new cases was higher in men compared to women. But these differences were smaller compared with the incidence of MN of the stomach. In women, the highest incidence of $\mathrm{MN}$ of the colon and rectum was found in Germany and Norway (77 and 74 new cases/100,000 inhabitants, respectively). In men, the highest incidence of $\mathrm{MN}$ of the colon and rectum was observed for the Czech Republic and Hungary (88 and 85 new cases/100,000 inhabitants). The lowest incidence for both women and men was found in Romania (25 and 32 new cases/100,000 inhabitants, respectively).

Particularly large differences between women and men were found for the incidence of $\mathrm{MN}$ of the lung (fig. 8.7). Men showed up to 9 times higher rates in 

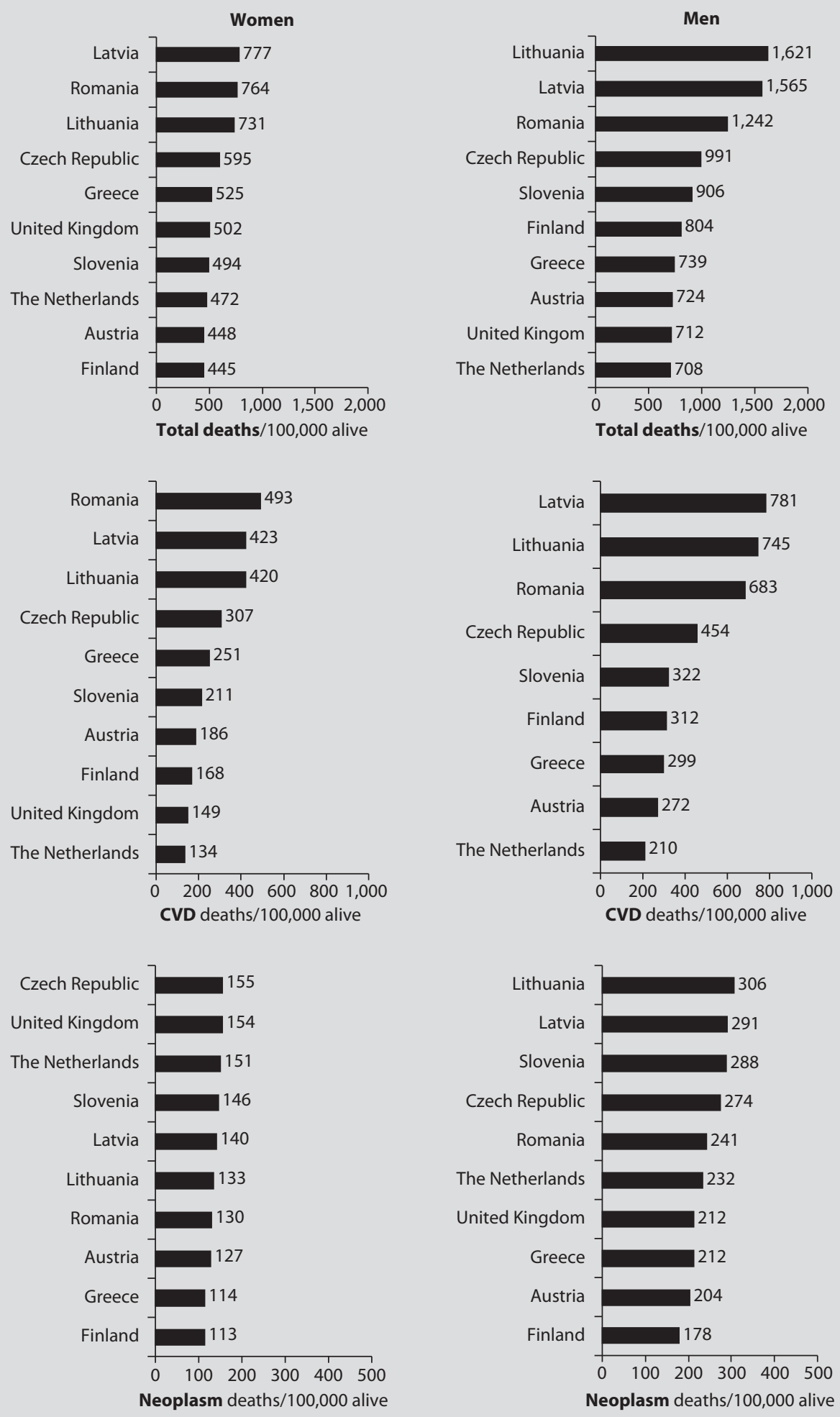

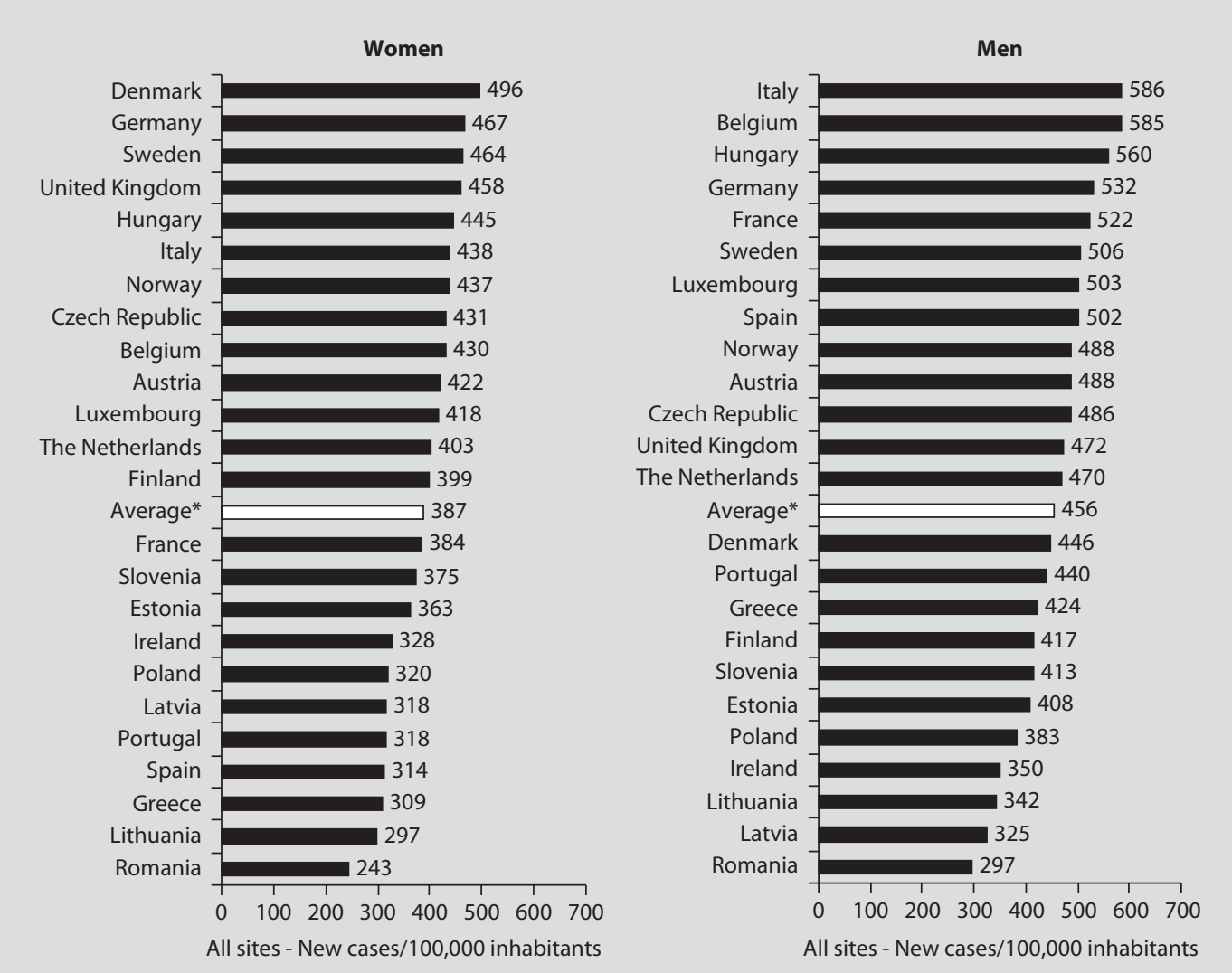

Fig. 8.5. Incidence of $\mathbf{M N}$ (excl. skin) in European countries indicated as new cases/100,000 inhabitants. Source: GLOBOCAN 2002 [Ferlay et al., 2004]. *Average of countries.

comparison with women. This corresponds with the results of smoking behavior (tables 8.5-8.10), showing that men smoked more often than women and that the proportion of ex-smokers was particularly high in older men. The highest incidence of MN of the lung in women was found in Denmark and the UK (56 and 51 new cases/100,000 inhabitants, respectively), whereas the highest incidence in men was observed in Hungary and Belgium (138 and 130 new cases/100,000 inhabitants, respectively). The lowest incidence was found in Spanish women and in Swedish men (10 and 40 new cases/100,000 inhabitants, respectively).

Except for Sweden, Finland, Norway, Austria and Portugal, all European countries showed a higher incidence of $\mathrm{MN}$ of the breast in comparison with $\mathrm{MN}$ of the prostate. The highest incidences of $\mathrm{MN}$ of the breast and prostate were found in

Fig. 8.4. Total mortality rate, mortality from CVD and from neoplasms (deaths/100,000 alive) in 2007 in European countries. Source: European Health-for-All database. Copenhagen, WHO Regional Office for Europe, 2009a. 


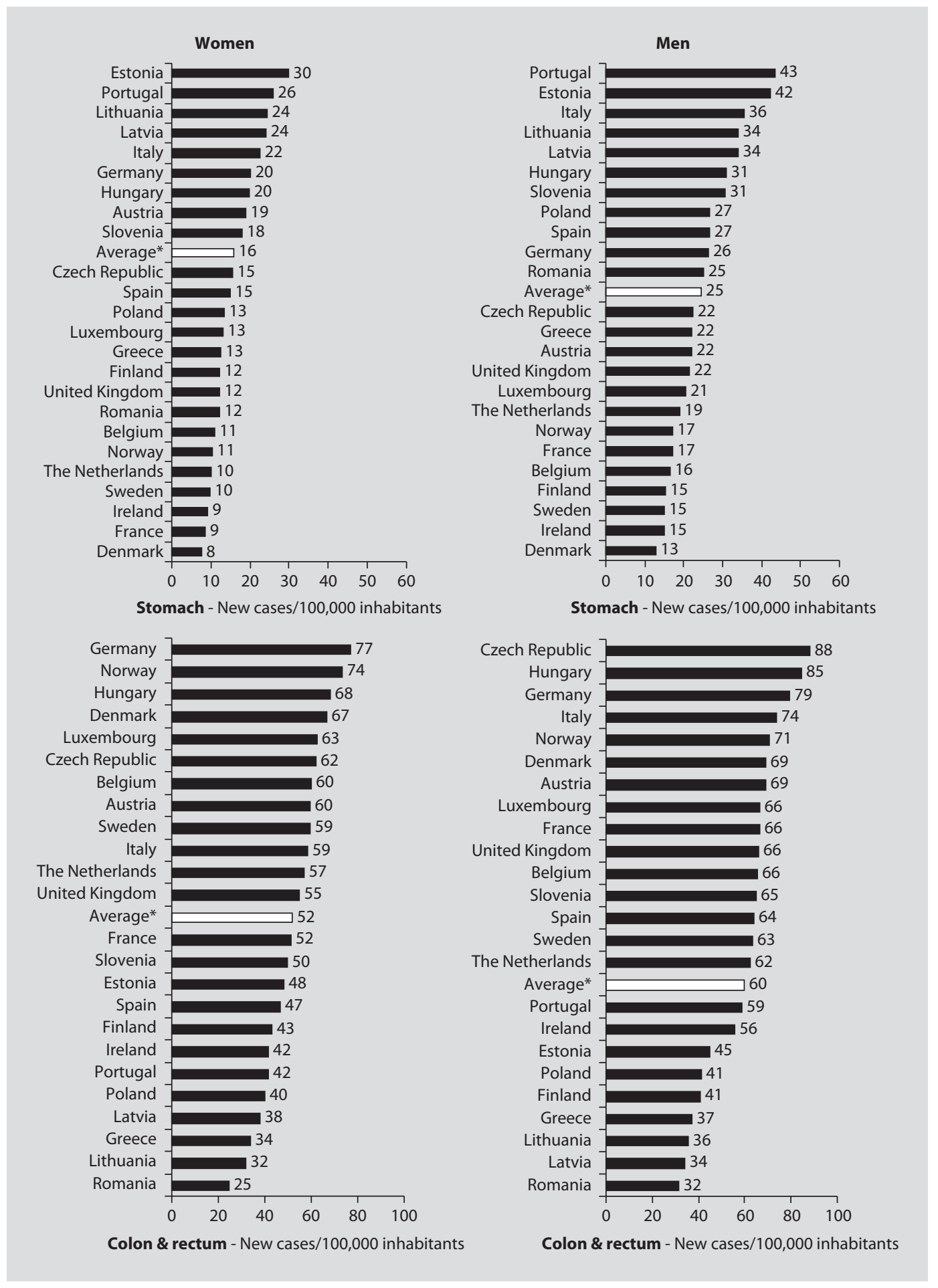

Fig. 8.6. Incidence of $M N$ of the stomach and colon and rectum in European countries indicated as new cases/100,000 inhabitants. Source: GLOBOCAN 2002 [Ferlay et al., 2004]. *Average of countries. 


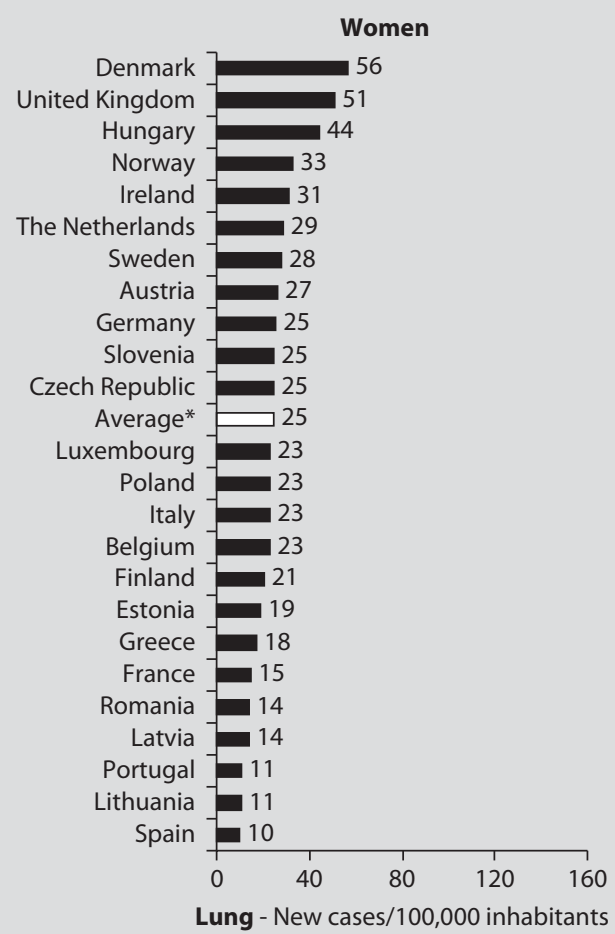

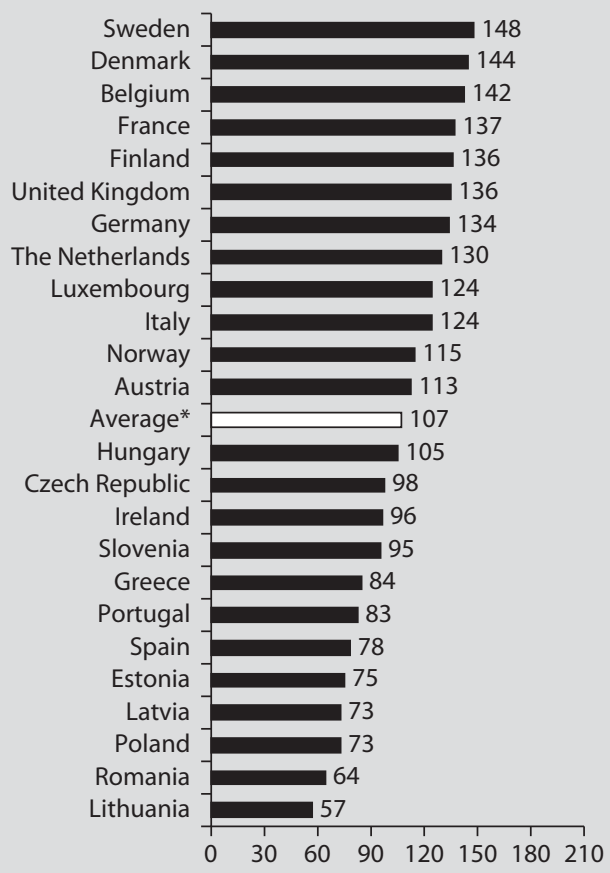

Breast - New cases/100,000 inhabitants
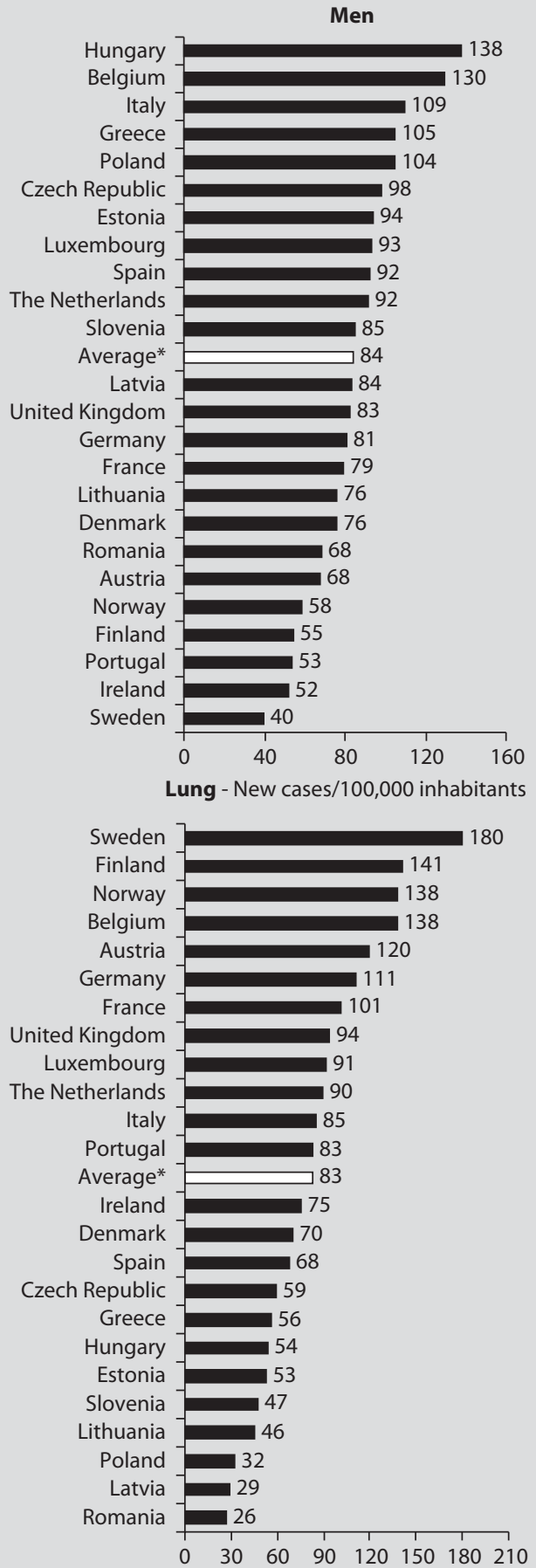

Prostate - New cases/100,000 inhabitants

Fig. 8.7. Incidence of $\mathbf{M N}$ of the lung, prostate and breast in European countries indicated as new cases/100,000 inhabitants. Source: GLOBOCAN 2002 [Ferlay et al., 2004]. *Average of countries. 
Table 8.10. Proportion of smokers and ex-smokers in different European countries, women and men 19-41 years of age

\begin{tabular}{|c|c|c|c|c|c|c|}
\hline \multirow[t]{2}{*}{ Country } & \multirow[t]{2}{*}{ Survey name } & \multirow[t]{2}{*}{ Survey year } & \multicolumn{2}{|l|}{ Women } & \multicolumn{2}{|l|}{ Men } \\
\hline & & & $\begin{array}{l}\text { current } \\
\text { smokers \% }\end{array}$ & $\begin{array}{l}\text { ex- } \\
\text { smokers \% }\end{array}$ & $\begin{array}{l}\text { current } \\
\text { smokers \% }\end{array}$ & $\begin{array}{l}\text { ex- } \\
\text { smokers \% }\end{array}$ \\
\hline Austria & $\begin{array}{l}\text { Austrian Study on Nutritional } \\
\text { Status - ÖSES.pal07 }\end{array}$ & 2007 & 16.4 & 52.4 & 20.8 & 42.2 \\
\hline Belgium & Health Interview Survey & 2004 & 29.5 & 13.8 & 37.7 & 12.4 \\
\hline Czech Republic & & & 28.1 & 13.9 & 39.3 & 13.9 \\
\hline Denmark & $\begin{array}{l}\text { Danish National Survey on } \\
\text { Dietary Habits and Physical } \\
\text { Activity }\end{array}$ & 2000-2002 & 38.1 & 15.0 & 36.3 & 13.6 \\
\hline Estonia & $\begin{array}{l}\text { Estonian Health Interview } \\
\text { Survey }\end{array}$ & 2006 & 23.5 & 9.6 & 46.8 & 15.4 \\
\hline Finland $^{1}$ & FINRISK Study & 2002 & 28.0 & 15.5 & 29.0 & \\
\hline France & $\begin{array}{l}\text { French Nutrition and Health } \\
\text { Survey }\end{array}$ & 2006-2007 & 36.8 & 20.1 & 44.8 & 18.4 \\
\hline Germany & $\begin{array}{l}\text { German National Nutrition } \\
\text { Survey II }\end{array}$ & 2005-2007 & 33.6 & 14.3 & 44.3 & 14.6 \\
\hline Greece & EPIC Study & 1994-1999 & 42.3 & 8.7 & 57.8 & 18.1 \\
\hline Ireland $^{2}$ & $\begin{array}{l}\text { Dietary Habits of the Irish } \\
\text { Population }\end{array}$ & 2007 & 30.5 & & 37.5 & \\
\hline Italy & $\begin{array}{l}\text { Italian National Food } \\
\text { Consumption Survey }\end{array}$ & 2005-2006 & 7.0 & 6.0 & 8.0 & 8.0 \\
\hline Lithuania & $\begin{array}{l}\text { Health Behaviour among } \\
\text { Lithuanian Adult Population }\end{array}$ & 2008 & 17.2 & 8.6 & 43.0 & 12.1 \\
\hline Portugal $^{3}$ & National Health Survey & 2005-2006 & 19.8 & 9.7 & 41.8 & 15.3 \\
\hline Spain & Catalan Nutrition Survey & $2002-2003$ & 41.6 & 17.4 & 47.3 & 12.7 \\
\hline Sweden & $\begin{array}{l}\text { Dietary Habits and Nutrient } \\
\text { Intake in Sweden }\end{array}$ & 1997-1998 & 24.0 & 18.0 & 10.0 & 19.0 \\
\hline The Netherlands ${ }^{4}$ & Statistics Netherlands, Statline & 2007 & 27.9 & & 38.3 & \\
\hline \multirow[t]{3}{*}{$\mathrm{UK}^{5}$} & General Household Survey & 2006 a) & 29.0 & 11.0 & 33.0 & 11.0 \\
\hline & & b) & 26.0 & 17.0 & 33.0 & 16.0 \\
\hline & & c) & 25.0 & 18.0 & 26.0 & 20.0 \\
\hline
\end{tabular}

\footnotetext{
${ }^{1}$ Age: $25-44$ years.
}

${ }^{2}$ Age: $18-44$ years.

${ }^{3}$ Age: $20-44$ years.

${ }^{4}$ Age: $25-44$ years.

${ }^{5}$ Age: a) 20-24 years, b) 25-34 years, c) 35-49 years. 


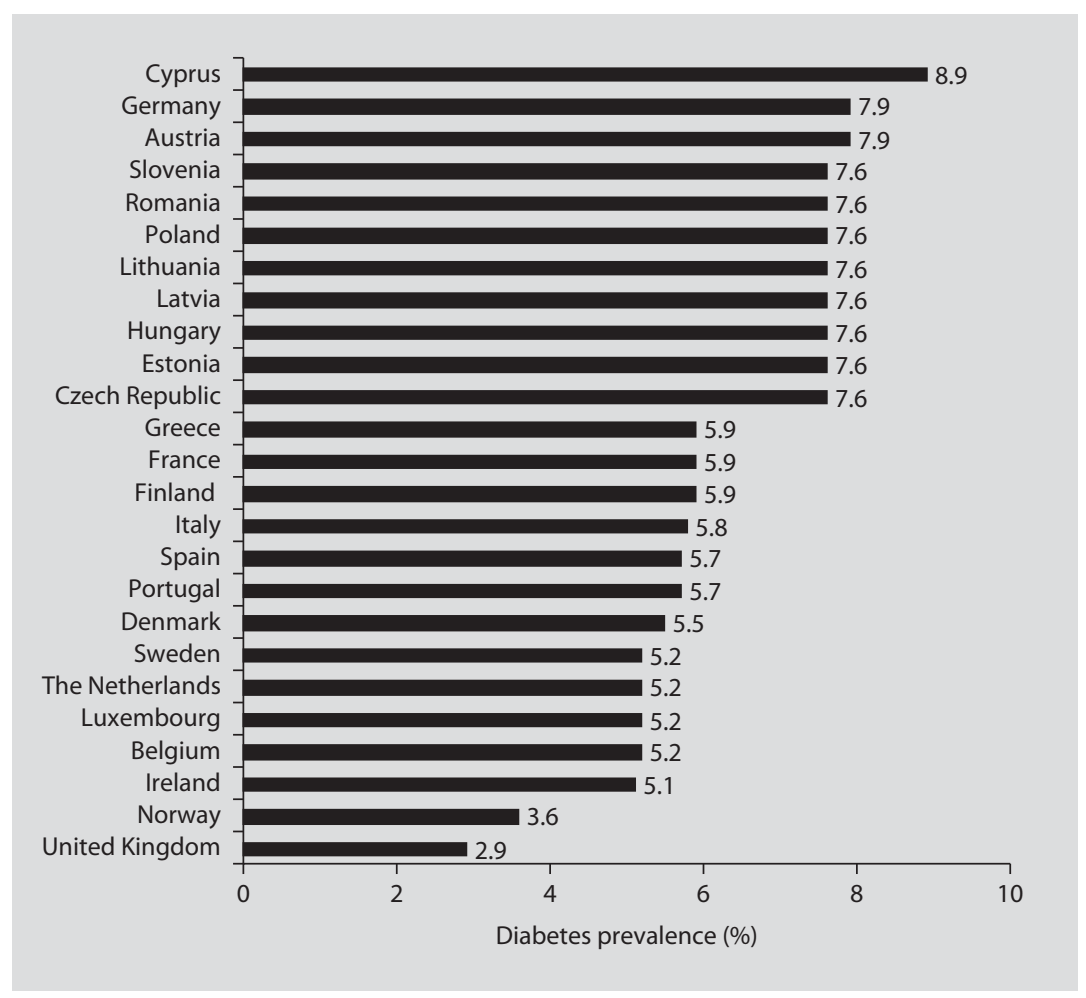

Fig. 8.8. Prevalence of DM type 1 and 2 of European countries (males and females) in 2007. Source: IDF, 2009.

Sweden (148 and 180 new cases/100,000 inhabitants, respectively) (fig. 8.7). The lowest incidence of $\mathrm{MN}$ of the breast was found in Lithuania and Romania (57 and 64 new cases/100,000 inhabitants, respectively) and the lowest incidence of $\mathrm{MN}$ of the prostate was observed in Romania and Latvia (26 and 29 new cases/100,000 inhabitants, respectively).

\section{Prevalence of Diabetes Mellitus}

Data from the International Diabetes Federation (IDF) 2009 are used to present the prevalence of Diabetes mellitus (DM) (types 1 and 2) in European countries (fig. 8.8). Therefore, literature, which was published before March 2006, was collected within the database Medline and the internet. Additionally the members of the IDF were asked for relevant data. If there were no data available for one country, data were extrapolated. In 2007, information about DM was obtainable from 25 European countries but not separately for women and men. The highest prevalence was found in Cyprus with $8.9 \%$ of the total population. The lowest prevalence was observed in the UK with $2.9 \%$ of the total population, followed by Norway with a prevalence of $\mathrm{DM}$ of 3.5\%. The other countries built two sections. The countries which correspond 
to one section showed a prevalence of DM with $5.1-5.9 \%$ of the total population and for the countries of the other section a higher prevalence of DM between 7.6 and $7.9 \%$ with only minor differences was observed.

\subsection{Smoking Behavior}

Data about smoking behavior were available for both women and men from 15 to 17 countries across the European Union. In tables 8.10-8.12, proportions of smokers and ex-smokers in the different countries are shown separately for young adults, adults of middle age and elderly.

In most countries, the proportion of smokers was higher in men compared with women for all age groups. In Sweden, adult women smoked more often than men. This survey was conducted in 1997/1998, whereas the data collection of the other surveys took place between 2002 and 2008. In addition, the proportion of smokers was a little bit higher in young adult women in Denmark in comparison with young adult men as well as in elderly Austrian women in comparison with elderly Austrian men.

The highest proportion of smokers was found among adult men across the European Union, particularly in young adult men in Greece with $57.8 \%$, whereas the highest proportion of ex-smokers was shown in elderly men. Across all countries a reduction of smoking was observed from the younger to the older age groups except for Danish men. Danish men showed nearly the same proportion of smokers in all age groups. Elderly men showed a higher proportion of ex-smokers in comparison with adult men. In elderly women, the proportion of ex-smokers was particularly low. The proportion of smokers was notably low in Italy for all age groups in comparison with the other countries (3.0-9.0\%).

\subsection{Alcohol Consumption}

Data on alcohol consumption (g/day) was available from 14 European countries (table 8.13). The average alcohol consumption of women was $<10 \mathrm{~g} /$ day in all European countries except for adult and elderly Danish women and middleaged women from The Netherlands. In women, the highest alcohol consumption was found for elderly Danish women with $14.0 \mathrm{~g} /$ day. In men, the average alcohol consumption was $<20 \mathrm{~g} /$ day across the European countries except for men from Portugal and Ireland as well as Danish adult and elderly men. Accordingly, the highest alcohol consumption was found for Portuguese men with $31.8 \mathrm{~g} /$ day.

Table 8.13 shows the proportions of alcohol abstainers from 8 to 9 European countries for adults and elderly. The proportion of alcohol abstainers differed widely between the European countries. This can be explained by the fact that the definition

of alcohol abstainers in the studies might have been different. The highest proportion 
Table 8.11. Proportion of smokers and ex-smokers in different European countries, women and men 42-64 years of age

\begin{tabular}{|c|c|c|c|c|c|c|}
\hline \multirow[t]{2}{*}{ Country } & \multirow[t]{2}{*}{ Survey name } & \multirow[t]{2}{*}{ Survey year } & \multicolumn{2}{|l|}{ Women } & \multicolumn{2}{|l|}{ Men } \\
\hline & & & $\begin{array}{l}\text { current } \\
\text { smokers \% }\end{array}$ & $\begin{array}{l}\text { ex- } \\
\text { smokers \% }\end{array}$ & $\begin{array}{l}\text { current } \\
\text { smokers \% }\end{array}$ & $\begin{array}{l}\text { ex- } \\
\text { smokers \% }\end{array}$ \\
\hline Austria & $\begin{array}{l}\text { Austrian Study on Nutritional } \\
\text { Status - ÖSES.pal07 }\end{array}$ & 2007 & 26.7 & 51.5 & 27.2 & 50.3 \\
\hline Belgium & Health Interview Survey & 2004 & 24.8 & 20.6 & 34.6 & 31.5 \\
\hline Czech Republic & & & 24.9 & 14.3 & 35.2 & 32.6 \\
\hline Denmark & $\begin{array}{l}\text { Danish National Survey on } \\
\text { Dietary Habits and Physical } \\
\text { Activity }\end{array}$ & $2000-2002$ & 35.5 & 39.6 & 38.1 & 32.4 \\
\hline Estonia & $\begin{array}{l}\text { Estonian Health Interview } \\
\text { Survey }\end{array}$ & 2006 & 18.1 & 13.0 & 44.0 & 25.1 \\
\hline France & $\begin{array}{l}\text { French Nutrition and Health } \\
\text { Survey }\end{array}$ & 2006-2007 & 23.1 & 19.7 & 29.0 & 43.8 \\
\hline Germany & $\begin{array}{l}\text { German National Nutrition } \\
\text { Survey II }\end{array}$ & 2005-2007 & 27.1 & 22.5 & 35.0 & 30.9 \\
\hline Greece & EPIC Study & 1994-1999 & 15.4 & 5.9 & 42.5 & 33.0 \\
\hline Ireland ${ }^{1}$ & $\begin{array}{l}\text { Dietary Habits of the Irish } \\
\text { Population }\end{array}$ & 2007 & 27.0 & & 23.0 & \\
\hline Italy & $\begin{array}{l}\text { Italian National Food } \\
\text { Consumption Survey }\end{array}$ & 2005-2006 & 7.0 & 12.0 & 9.0 & 24.0 \\
\hline Lithuania & $\begin{array}{l}\text { Health Behaviour among } \\
\text { Lithuanian Adult Population }\end{array}$ & 2008 & 11.5 & 7.9 & 34.1 & 24.9 \\
\hline Portugal $^{1}$ & National Health Survey & 2005-2006 & 9.3 & 7.3 & 20.0 & 12.5 \\
\hline Spain & Catalan Nutrition Survey & $2002-2003$ & 18.5 & 21.3 & 31.6 & 36.1 \\
\hline Sweden & $\begin{array}{l}\text { Dietary Habits and Nutrient } \\
\text { Intake in Sweden }\end{array}$ & 1997-1998 & 27.0 & 32.0 & 17.0 & 35.0 \\
\hline The Netherlands ${ }^{1}$ & Statistics Netherlands, Statline & 2007 & 26.7 & & 32.4 & \\
\hline UK & General Household Survey & 2006 & 22.0 & 25.0 & 23.0 & 31.0 \\
\hline
\end{tabular}

${ }^{1}$ Age: $45-64$ years.

of alcohol abstainers for adults showed Romania and the lowest proportions of alcohol abstainers for adults were found in Estonia and Germany. The highest proportions of alcohol abstainers for elderly women and men were found in Lithuania and Romania. The lowest proportions of alcohol abstainers in elderly women and men 
Table 8.12. Proportion of smokers and ex-smokers in different European countries, women and men $\mathbf{6 5 +}$ years of age

\begin{tabular}{|c|c|c|c|c|c|c|}
\hline \multirow[t]{2}{*}{ Country } & \multirow[t]{2}{*}{ Survey name } & \multirow[t]{2}{*}{ Survey year } & \multicolumn{2}{|l|}{ Women } & \multicolumn{2}{|l|}{ Men } \\
\hline & & & $\begin{array}{l}\text { current } \\
\text { smokers \% }\end{array}$ & $\begin{array}{l}\text { ex- } \\
\text { smokers \% }\end{array}$ & $\begin{array}{l}\text { current } \\
\text { smokers \% }\end{array}$ & $\begin{array}{l}\text { ex- } \\
\text { smokers \% }\end{array}$ \\
\hline Austria & $\begin{array}{l}\text { Austrian Study on Nutritional } \\
\text { Status - ÖSES.sen } 07\end{array}$ & 2007 & 5.1 & 8.9 & 3.5 & 62.3 \\
\hline Belgium & Health Interview Survey & 2004 & 9.2 & 12.0 & 19.6 & 44.7 \\
\hline Denmark & $\begin{array}{l}\text { Danish National Survey on } \\
\text { Dietary Habits and Physical } \\
\text { Activity }\end{array}$ & $2000-2002$ & 31.5 & 32.1 & 36.5 & 47.6 \\
\hline Estonia & $\begin{array}{l}\text { Estonian Health Interview } \\
\text { Survey }\end{array}$ & 2006 & 4.1 & 6.1 & 20.1 & 43.6 \\
\hline France & $\begin{array}{l}\text { French Nutrition and Health } \\
\text { Survey }\end{array}$ & 2006-2007 & 5.6 & 10.0 & 9.8 & 53.2 \\
\hline Germany & $\begin{array}{l}\text { German National Nutrition } \\
\text { Survey II }\end{array}$ & 2005-2007 & 7.7 & 15.6 & 12.6 & 46.0 \\
\hline Greece & EPIC Study & 1994-1999 & 2.2 & 3.0 & 22.6 & 46.4 \\
\hline Ireland & $\begin{array}{l}\text { Dietary Habits of the Irish } \\
\text { Population }\end{array}$ & 2007 & 13.0 & & 17.0 & \\
\hline Italy & $\begin{array}{l}\text { Italian National Food } \\
\text { Consumption Survey }\end{array}$ & 2005-2006 & 3.0 & 9.0 & 5.0 & 41.0 \\
\hline \multirow[t]{2}{*}{ Lithuania } & Health Survey & 2005 a) & 1.3 & 2.9 & 22.7 & 37.1 \\
\hline & & b) & 0.4 & 3.8 & 11.1 & 54.3 \\
\hline Portugal & National Health Survey & 2005-2006 & 0.9 & 1.4 & 10.5 & 43.7 \\
\hline Spain & Catalan Nutrition Survey & 2002-2003 & 4.5 & 4.5 & 17.2 & 56.4 \\
\hline Sweden & $\begin{array}{l}\text { Dietary Habits and Nutrient } \\
\text { Intake in Sweden }\end{array}$ & 1997-1998 & 9.0 & 21.0 & 12.0 & 45.0 \\
\hline The Netherlands & Statistics Netherlands, Statline & 2007 & 14.3 & & 19.9 & \\
\hline UK$^{2}$ & General Household Survey & 2006 & 12.0 & 30.0 & 13.0 & 49.0 \\
\hline
\end{tabular}

${ }^{1}$ Age: a) 65-74 years, b) $\geq 75$ years.

${ }^{2}$ Age: $\geq 60$ years 
Table 8.13. Alcohol consumption in different European countries, women and men

\begin{tabular}{|c|c|c|c|c|c|c|c|c|c|c|c|}
\hline \multirow[t]{3}{*}{ Country } & \multirow[t]{3}{*}{ Survey name } & \multirow[t]{3}{*}{ Survey year } & \multirow{3}{*}{$\begin{array}{l}\text { Age } \\
\text { years }\end{array}$} & \multicolumn{4}{|l|}{ Women } & \multicolumn{4}{|l|}{ Men } \\
\hline & & & & \multirow[t]{2}{*}{$\mathrm{n}$} & \multicolumn{3}{|c|}{$\begin{array}{l}\text { alcohol consumption } \\
\text { g/day }\end{array}$} & \multirow[t]{2}{*}{$\mathrm{n}$} & \multicolumn{3}{|c|}{$\begin{array}{l}\text { alcohol consumption } \\
\text { g/day }\end{array}$} \\
\hline & & & & & mean $\pm S D$ & P50 & P95 & & mean $\pm S D$ & P50 & P95 \\
\hline Austria & $\begin{array}{l}\text { Austrian Study on } \\
\text { Nutritional Status }\end{array}$ & 2007 & $19-64$ & 1,345 & $3.6 \pm 8.7$ & 0.0 & 21.1 & 778 & $10.8 \pm 20.4$ & 0.3 & 59.4 \\
\hline Czech Republic & & & $19-64$ & 1,094 & $3.1 \pm 6.5$ & 0.0 & 12.5 & 1,046 & $18.1 \pm 19.9$ & 12.1 & 55.7 \\
\hline \multirow[t]{3}{*}{ Denmark } & $\begin{array}{l}\text { Danish National } \\
\text { Survey on } \\
\text { Dietary Habits } \\
\text { and Physical } \\
\text { Activity }\end{array}$ & 2000-2002 & $15-18$ & 121 & $5.6 \pm 10.4$ & 0.0 & 30.3 & 78 & $11.3 \pm 19.0$ & 3.2 & 47.7 \\
\hline & & & $19-64$ & 1,486 & $12.9 \pm 13.0$ & 9.5 & 39.1 & 1,283 & $25.5 \pm 24.4$ & 19.7 & 68.1 \\
\hline & & & $65-75$ & 164 & $14.0 \pm 12.8$ & 12.3 & 38.1 & 165 & $28.6 \pm 23.2$ & 23.7 & 70.1 \\
\hline Estonia & $\begin{array}{l}\text { Estonian Health } \\
\text { Interview Survey }\end{array}$ & 2006 & $15-64$ & 3,323 & $2.3 \pm 6.2$ & 1.0 & 8.7 & 3,111 & $12.8 \pm 34.4$ & 5.0 & 13.7 \\
\hline France & $\begin{array}{l}\text { French Nutrition } \\
\text { and Health } \\
\text { Survey }\end{array}$ & 2007-2007 & 19-74 & 2,483 & $4.9 \pm 0.3$ & 1.0 & 21.4 & 1,585 & $16.6 \pm 0-9$ & 10.1 & 54.8 \\
\hline Germany & $\begin{array}{l}\text { German National } \\
\text { Nutrition } \\
\text { Survey II }\end{array}$ & 2005-2007 & $15-80$ & 8,183 & $5.2 \pm 8.2$ & 2.1 & 20.9 & 6,976 & $15.3 \pm 19.7$ & 9.2 & 51.0 \\
\hline \multirow[t]{2}{*}{ Greece } & EPIC Study & 1994-1999 & $16-64$ & 12,043 & $3.5 \pm 6.4$ & 1.2 & 15.7 & 8,365 & $19.6 \pm 25.3$ & 11.5 & 66.5 \\
\hline & & & $\geq 65$ & 3,600 & $2.3 \pm 5.2$ & 0.1 & 10.2 & 2,508 & $13.9 \pm 20.0$ & 7.9 & 49.4 \\
\hline Hungary & Dietary Survey & 2003-2004 & $\geq 19$ & 706 & $6.7 \pm 5.9$ & & & 473 & $15.4 \pm 13.3$ & & \\
\hline Ireland & $\begin{array}{l}\text { North/South } \\
\text { Ireland Food } \\
\text { Consumption } \\
\text { Survey }\end{array}$ & 1997-1999 & $18-64$ & 698 & $9.3 \pm 13.0$ & & & 613 & $22.9 \pm 29.8$ & & \\
\hline Italy & $\begin{array}{l}\text { Italian National } \\
\text { Food } \\
\text { Consumption } \\
\text { Survey }\end{array}$ & 2005-2006 & & 1,822 & $4.5 \pm 8.3$ & 0.0 & 23.8 & 1,501 & $12.4 \pm 16.6$ & 4.3 & 44.3 \\
\hline Latvia & $\begin{array}{l}\text { Nutrition and } \\
\text { Lifestyle in the } \\
\text { Baltic Republics }\end{array}$ & 1997 & $15-64$ & 1,235 & $3.0 \pm 7.0$ & & & 1,065 & $15.0 \pm 24.0$ & & \\
\hline
\end{tabular}


Table 8.13. Continued

\begin{tabular}{|c|c|c|c|c|c|c|c|c|c|c|c|}
\hline \multirow[t]{3}{*}{ Country } & \multirow[t]{3}{*}{ Survey name } & \multirow[t]{3}{*}{ Survey year } & \multirow{3}{*}{$\begin{array}{l}\text { Age } \\
\text { years }\end{array}$} & \multicolumn{4}{|c|}{ Women } & \multicolumn{4}{|l|}{ Men } \\
\hline & & & & \multirow[t]{2}{*}{$n$} & \multicolumn{3}{|c|}{$\begin{array}{l}\text { alcohol consumption } \\
\text { g/day }\end{array}$} & \multirow[t]{2}{*}{$\mathrm{n}$} & \multicolumn{3}{|c|}{$\begin{array}{l}\text { alcohol consumption } \\
\text { g/day }\end{array}$} \\
\hline & & & & & mean $\pm S D$ & P50 & P95 & & mean $\pm S D$ & P50 & P95 \\
\hline Lithuania & $\begin{array}{l}\text { Health Behavior } \\
\text { among Lithuanian } \\
\text { Adult Population }\end{array}$ & 2006 & $20-65$ & 1,016 & $1.7 \pm 3.6$ & 0.4 & 6.9 & 723 & $16.4 \pm 31.6$ & 8.3 & 56.7 \\
\hline Norway & Norkost Study & 1997 & $19-65$ & 1,208 & $3.9 \pm 6.0$ & 1.9 & 14.1 & 1,122 & $8.3 \pm 12.6$ & 4.8 & 27.5 \\
\hline Portugal & EPI Porto & & $\geq 18$ & 1,472 & $6.8 \pm 11.9$ & & 29.0 & 917 & $31.8 \pm 31.6$ & & 93.2 \\
\hline Spain & $\begin{array}{l}\text { Catalan Nutrition } \\
\text { Survey }\end{array}$ & 2002-2003 & $\geq 15$ & 1,111 & $2.6 \pm 5.2$ & 0.5 & 12.4 & 930 & $8.4 \pm 12.2$ & 3.0 & 30.1 \\
\hline Sweden & $\begin{array}{l}\text { Dietary Habits } \\
\text { and Nutrient } \\
\text { Intake in Sweden }\end{array}$ & 1997-1998 & & & $7.6 \pm 9.8$ & 5.0 & 23.2 & & $12.7 \pm 13.4$ & 9.4 & 39.8 \\
\hline \multirow[t]{5}{*}{$\begin{array}{l}\text { The } \\
\text { Netherlands }\end{array}$} & $\begin{array}{l}\text { Dutch National } \\
\text { Food } \\
\text { Consumption } \\
\text { Survey-3 }\end{array}$ & 1997/1998 & $16-18$ & 139 & $3.0 \pm 7.0$ & 0.0 & 17.0 & 142 & $8.0 \pm 19.0$ & 0.0 & 44.0 \\
\hline & & & $19-21$ & 128 & $3.0 \pm 9.0$ & 0.0 & 18.0 & 130 & $14.0 \pm 27.0$ & 0.0 & 66.0 \\
\hline & & & $22-49$ & 1,472 & $8.0 \pm 15.0$ & 0.0 & 38.0 & 1,252 & $18.0 \pm 24.0$ & 9.0 & 66.0 \\
\hline & & & $50-64$ & 512 & $10.0 \pm 15.0$ & 0.0 & 40.0 & 454 & $19.0 \pm 22.0$ & 12.0 & 63.0 \\
\hline & & & $\geq 65$ & 236 & $6.0 \pm 11.0$ & 0.0 & 30.0 & 185 & $17.0 \pm 20.0$ & 12.0 & 51.0 \\
\hline
\end{tabular}

$\mathrm{P} 50=50$ th percentile, $\mathrm{P95}=$ 95 th percentile.

were observed in Germany and France. In addition, elderly men from Estonia showed a low proportion of alcohol abstainers.

\subsection{Overview of European Cross-National Data on Physical Activity²}

Concept of Health-Enhancing Physical Activity - Recommendations and Surveillance Current public health recommendations state that adults should engage in a minimum of 30 minutes of moderate-intensity aerobic (endurance) physical activity on

${ }^{2}$ Rütten A, Abu-Omar K, Gelius P: Institute of Sport Science and Sport, FAU Erlangen-Nuremberg (alfred.ruetten@sport.uni-erlangen.de). 
5 days per week, or in vigorous aerobic physical activity for a minimum of 20 minutes on 3 days per week. Additionally, adults should engage in activities to increase muscular strength on 2 days per week [Haskell et al., 2007].

Since 1997, public health recommendations for physical activity issued by the American College of Sport Medicine (ACSM) have shifted from a specific focus on exercise-related physical activity to the broader concept of physical activity integrated in daily living [Pate et al., 1997]. Within this concept, types of physical activity that have beneficial health effects (health-enhancing physical activity (HEPA)) can take place in the domains of work, transportation/commuting, at home, or during leisuretime. The expansion of the old concept of exercise to the current concept of daily physical activity was warranted by increasing evidence about the health benefits of physical activities of moderate intensity [Blair et al., 1992].

Regarding the assessment of physical activity on the population level, the HEPA concept has stimulated the development of questionnaires that allow for the assessment of physical activity during leisure-time and in the three other domains (work, transport, home). Most prominently, the International Physical Activity Questionnaire (IPAQ) and the Global Physical Activity Questionnaire (GPAQ) have been designed to capture physical activity across all domains. Both questionnaires have been tested for reliability and validity in international studies [IPAQ: Craig et al., 2003; GPAQ: Armstrong and Bull, 2006] and have been shown to have measurement properties comparable to other self-assessment instruments utilized to assess physical activity.

\section{Limitations of Physical Activity Assessment}

In general, one has to acknowledge that self-assessment of physical activity suffers from a number of limitations and that attempts to make assessments across different domains might have contributed to difficulties in generating robust cross-national surveillance data on physical activity. Among these limitations are (1) sensitivity of information on physical activity derived from respondents to wording differences in questions, (2) recall problems of respondents, especially regarding physical activity of moderate intensity, (3) potential over-reporting of the duration of physical activity bouts, (4) limited comparability of physical activity levels across nations and cultures due to differing physical activity concepts, and (5) limited comparability of results across surveys due to methodological peculiarities of studies.

\section{National Surveillance Systems in Europe}

On the national level, surveillance systems tracking the population's physical activity levels as part of behavioral risk factor assessment for chronic diseases have been adopted in a number of nations in the European region. While in some nations these surveillance systems were already established some 20 years ago and collect data annually (e.g. Finland), other nations have only recently established such systems (e.g. Italy), are still in the process of implementing such systems (e.g. Germany), or do not 
feature such systems at all. Some of these systems utilize a framework that was originally developed in the USA as part of the Behavioral Risk Factor Surveillance System. While these systems are generally useful to describe physical activity behavior within nations, data generated by these systems do not permit description of physical activity patterns across nations. This is mainly due to differences in the physical activity concepts operationalized (sport/exercise, HEPA), dimensions of physical activity assessed (e.g. type, duration, frequency, intensity), wording of survey questions, and response categories provided (e.g. Likert scales, duration in hours/minutes). The EUPASS project has demonstrated that such national surveillance instruments for physical activity assessment hardly yield any comparable results and that Spearman's rho correlation coefficient between the different survey instruments is in general low [Rütten et al., 2003]. In order to describe physical activity patterns across nations, it is thus advisable to rely on cross-national datasets instead.

\section{Cross-National Surveillance Systems in Europe}

Starting with the European Food Study [de Almeida et al., 1999; Martinez et al., 2001], the European Physical Activity Surveillance System [Rütten et al., 2003], Eurobarometer 58.2 [Rütten and Abu-Omar, 2004], Eurobarometer 62.0, Eurobarometer 64.3, Eurobarometer 67.3, and the European Health Interview Survey (EHIS), there are now numerous datasets available that allow to describe crossnational physical activity rates of populations in Europe. While each of these datasets yields important information on physical activity levels, it remains difficult to compare results across these surveys. Some studies assessed only leisure-time physical activity (e.g. European Food Study, Eurobarometer 62.0), while other studies utilize the IPAQ (e.g. Eurobarometer 58.2) or the IPAQ in slightly modified versions (Eurobarometer 64.3). For the European region, the IPAQ questionnaire has been the primary means to assess physical activity levels of populations in recent years.

\section{Adults - Selected Findings}

The following section highlights some of the findings regarding physical activity patterns that can be identified in the available cross-national datasets. The European Food Study assessed physical activity with the Paffenbarger questionnaire [Paffenbarger et al., 1978]. This questionnaire provides information on leisure-time physical activity and allows calculating metabolic equivalents (METs) in order to quantify the amount of leisure-time physical activity. Data were gathered in 1997 in the then $15 \mathrm{EU}$ member states by face-to-face interviews. In each nation, about 1,000 interviews were conducted. Fig. 8.9 indicates the percentage of adults reporting to practice any type of leisure-time physical activity. Overall, $73.1 \%$ of adults in the EU Member States reported they were engaging in leisure-time physical activity. The rate declined with age $(82.8 \%$ among respondents aged $15-24,64.5 \%$ among respondents $\geq 65$ years of age) and increased with educational attainment ( $64.1 \%$ of respondents with a degree lesser than high school, $81.4 \%$ of respondents with a high school degree or more). 


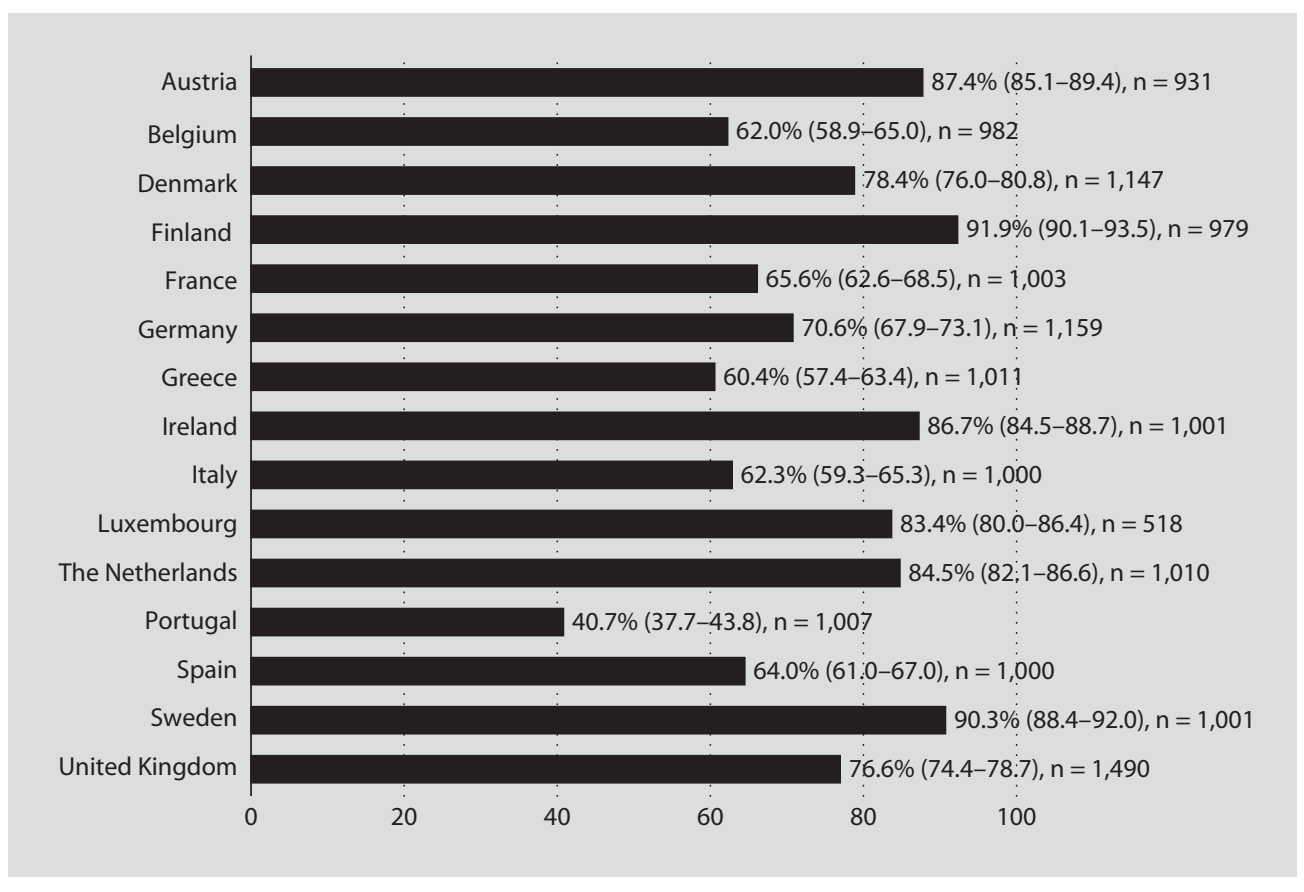

Fig. 8.9. Percentage of adult population practicing any leisure-time physical activity Source: Martinez-Gonzalez et al. [2001].

Across nations, respondents in Northern European countries reported comparably higher rates of engagement in leisure-time physical activity than respondents in Southern European countries. This has been referred to as the North-South gradient of leisure-time physical activity in Europe [Martinez-Gonzalez et al., 2001].

In 2002, D.G. Sanco of the European Commission collected Eurobarometer data on physical activity across the 15 member states. Physical activity was assessed with the short form of the IPAQ (last 7 days' version). The IPAQ allows calculating METs in order to quantify the amount of physical activity. METs represent multiples of the resting metabolic rate. A MET-hour is computed by multiplying the MET score of an activity by the hours performed. With the IPAQ short-form, physical activity is assessed across the different domains. Data were gathered by face-to-face interviews, the sample size was 1,000 respondents in each nation, and the average response rate was 54.6\%. Results were reported in median METs per week (MET-h/week). Men reported higher MET-h/week than women (28.4 for men, 23.1 for women), and MET-h/week declined with age (33.7 for respondents age 15-24, 14.0 for respondents 65 years of age and older). Reported MET-h/week increased with reported gross household income (20.1 for respondents in the lowest national gross household income quartile, 27.8 for respondents in the highest national gross household income 


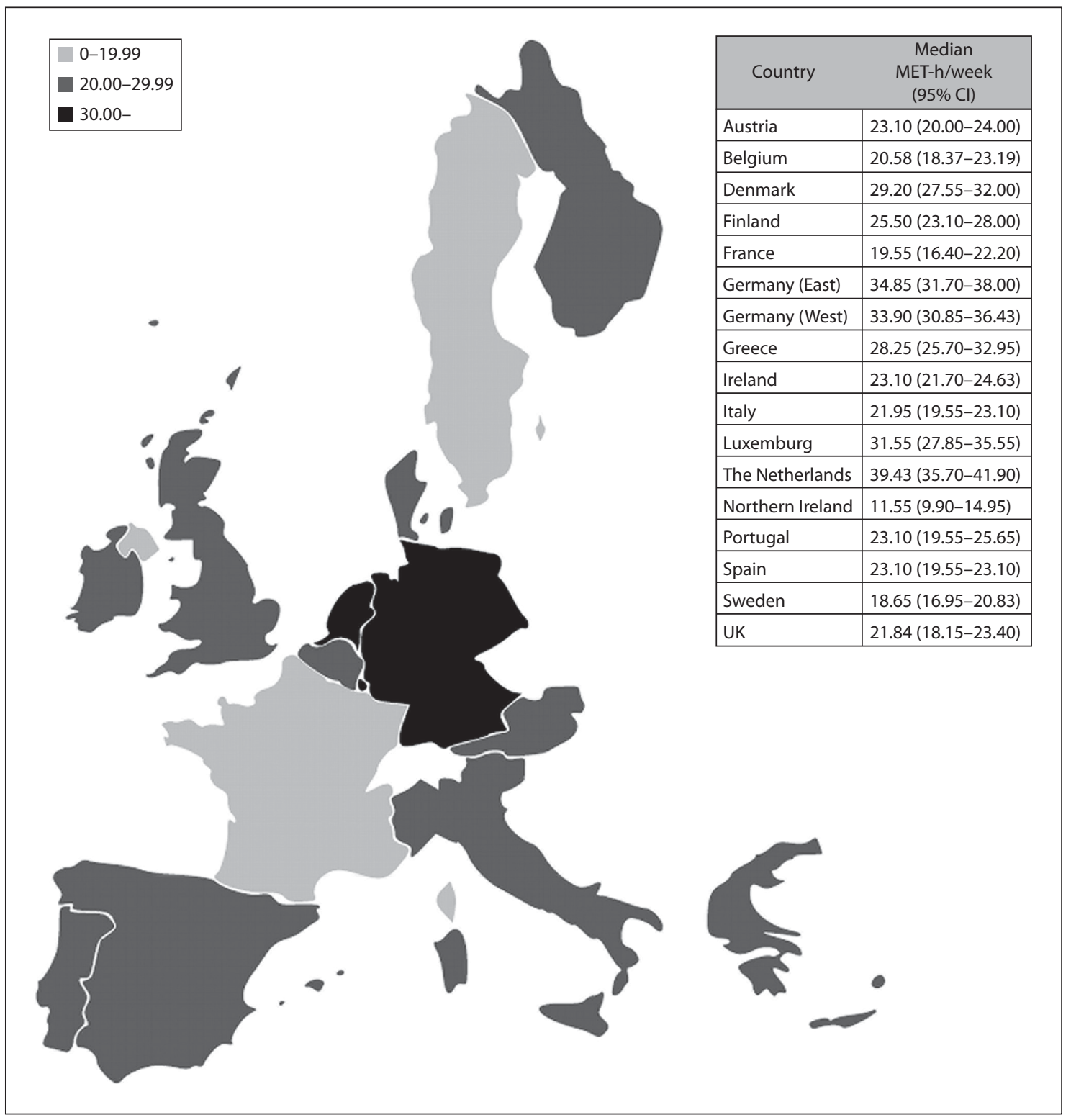

Fig. 8.10. Median MET-h/week for individuals aged 15 years and older in the member states of the European Union. Source: Rütten and Abu-Omar [2004].

quartile). Across nations (fig. 8.10), respondents in The Netherlands, Germany, and Luxembourg reported highest MET-h/week, while respondents in Northern Ireland, Sweden, and France reported lowest MET-h/week. No clear geographical trend emerged across the countries participating in the ENHR 2009 [Rütten and AbuOmar, 2004]. 
In 2004, Eurobarometer 62.0 asked if respondents engaged in exercise or practiced sport. Answer categories were ' 3 times a week or more, ' $1-2$ times a week, ' $1-3$ times a month', 'less often', or 'never'. Fieldwork was carried out in the 27 member states of the European Union, two affiliated nations (Croatia, Turkey), and Northern Cyprus. In each nation, about 1,000 respondents were interviewed face-to-face. Fig. 8.11 depicts results by nation for the percentage of respondents reporting never to engage in exercise or play sport. In Portugal (66\%), Hungary (60\%), and Italy $(58 \%)$, the highest percentages of respondents never engaging in exercise or sport were found. The lowest rates were identified in Finland (4\%), Sweden (7\%), and Denmark (17\%). These results confirm the prevalent North-South gradient with regard to leisure-time physical activity in European nations. Age and educational attainment of respondents were also related to the engagement in exercise and sport. While $60 \%$ of those aged 15-24 reported to engage in exercise or sport at least one time per week, this proportion decreased to 41\% (age 25-39), 34\% (age 40-54), and $28 \%$ (age 55 and older) in the older aged brackets. While only $20 \%$ of respondents who had finished full-time education at the age of 15 years or earlier reported to engage in exercise or sport at least one time per week, this percentage increased to $32 \%$ (for respondents finishing full-time education at age 16-19) and 50\% (for respondents finishing full-time education at the age $\geq 20$ ) for those with higher levels of educational attainment [European Commission, 2004]. The social gradient regarding physical activity behavior has also been confirmed by the EUROTHINE project. On average, the rate of those reporting at least sufficient levels of activity was 24 absolute percentage points higher in the quartile with the highest educational attainment compared to the quartile with the lowest educational attainment [Demarest et al., 2007].

Some other surveys provide additional data on physical activity levels in European nations. Among others, the Special Eurobarometer 64.3 on Health and Food contained modified IPAQ questions on physical activity, and Eurobarometer 67.3 on Health and Long-Term Care in the European Union had some items regarding engagement in leisure-time physical activity. Due to differences in the wording of questions, these data are not fully comparable to the previous surveys. Thus, to this point, no crossnational data that would allow investigating time trends in physical activity behavior exist for the European Union.

\section{Children and Adolescents - Selected Findings}

Beyond the assessment of physical activity levels of adults, there have also been some efforts to generate data for subpopulations such as children, adolescents, and older people. Regarding children and adolescents, the Health Behavior among School-aged Children (HBSC) Survey of the WHO has collected four rounds of data on physical activity in the European region, the USA, and Canada in the years 2005/2006. Questions related to physical activity behavior pertained to the number of days with a minimum of 60 minutes of physical activity per week, the number of days with 


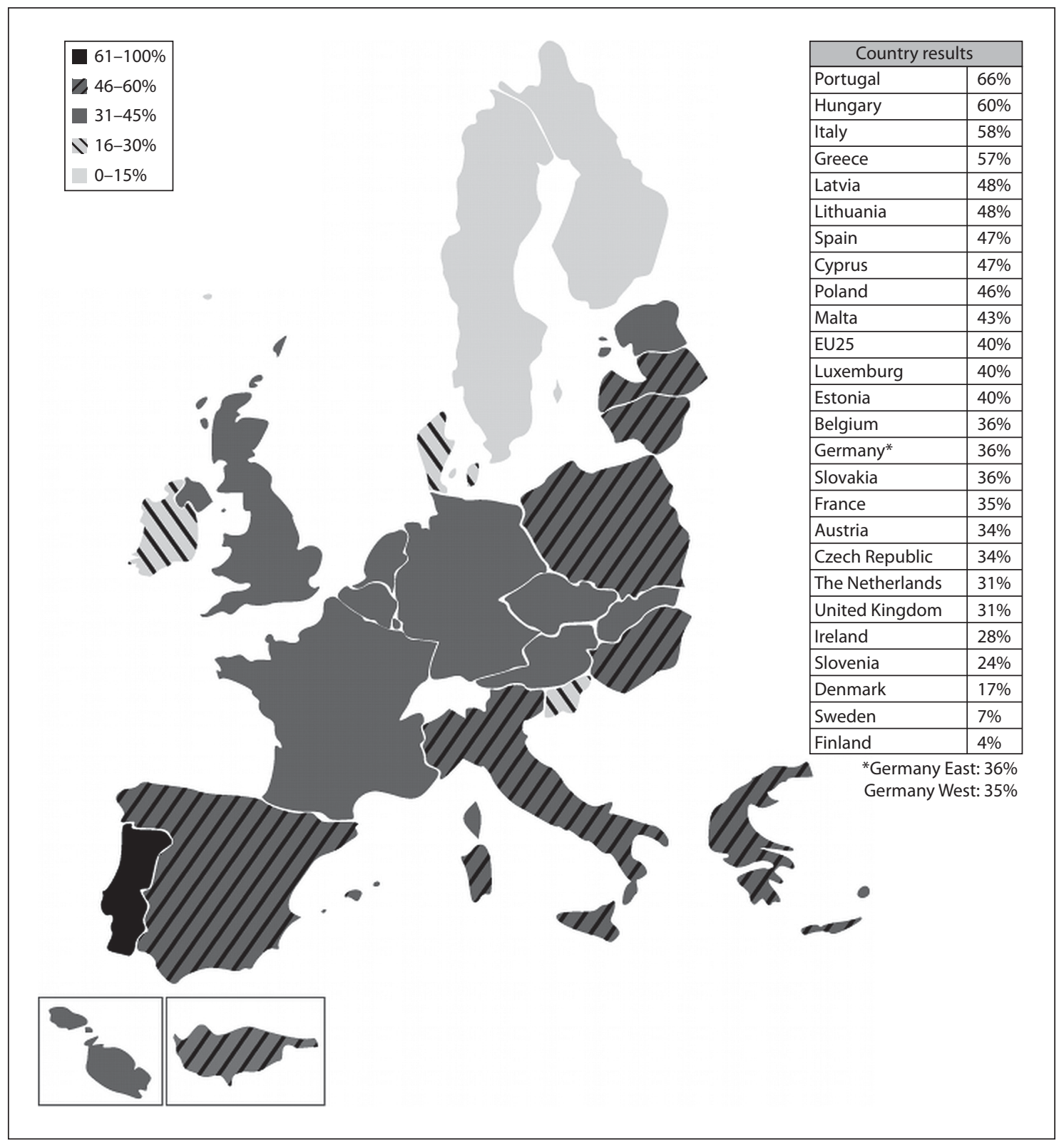

Fig. 8.11. Special Eurobarometer 213, Q48.5 'How often do you exercise or play sport?' Answer: Never. Source: European Commission, 2004.

active commuting to school (walking, biking) per week, and the number of days per week with physical education classes. These questions have been (partly) validated by Prochaska et al. [2001]. Data were collected in schools, using self-assessment questionnaires. In 40 mostly European nations, about 5,000 written questionnaires per 
nation were conducted among 11-, 13-, and 15-year-old children/adolescents. HBSC reported the percentage of children/adolescents who stated to engage in a minimum of 60 minutes of physical activity on each day of the week, which is the amount of physical activity recommended by the ACSM for children and adolescents [US Department of Health and Human Services, 2005].

Results indicate that pronounced differences in levels of daily moderate-to-vigorous physical activity exist. $26 \%$ of 11 -year-olds reported to engage in at least 60 minutes of physical activity on 7 days of the week; this percentage decreased to $20 \%$ among 13 -year-olds and 16\% among 15-year-olds. Across all age groups, a higher percentage of boys than girls reported to engage in 60 minutes of physical activity per day. Among 11-year-olds, the highest percentages were reported in Ireland (38\% for girls, $51 \%$ for boys) and Finland (37\% for girls, $48 \%$ for boys). Lowest percentages were found in Luxembourg (13\% for girls, $18 \%$ for boys) and Italy (13\% for girls, $23 \%$ for boys). Among 13-year-olds, Ireland ranked highest again, while France and Norway ranked lowest. Among 15-year-olds, the Czech Republic and Latvia ranked highest, and France and Portugal ranked lowest among European nations (fig. 8.12, 8.13) [WHO, 2008a].

\section{Elderly - Selected Findings}

For older people, there seem to be only limited cross-national data on physical activity available for Europe. The Survey of the Health, Ageing and Retirement project (SHARE) collected survey and functional test data on physical activity of individuals aged 50 years and older in 11 European nations in the years 2004 and 2006/7. Within the survey, physical activity was assessed with items on how often individuals would perform vigorous or moderate physical activity. Answer categories ranged from 'more than once a week' to 'hardly ever, or never'. Results indicate that the risk of becoming inactive is associated to increasing age and female gender. Older people from Southern European nations were more likely to become inactive compared to those of Northern European nations [Mohd Hairi et al., 2008].

\section{Outlook and Recommendations for the Future}

Recently, there have been efforts to harmonize survey questions and methods of data collection regarding physical activity and other health indicators across the European region. These efforts might, in the future, result in a European Health Monitoring System. The European Community Health Indicators (ECHI) project and the subsequent ECHI monitoring project have proposed both a core list and an extended list of health indicators to be implemented across European nations. With regard to physical activity, ECHI suggests to use, among others, the IPAQ, items on active leisure-time physical activity, and physical activity of an intensity to make a person start sweating. Eurostat has also collected a first round of data related to physical activity as part of the EHIS in 2007. EHIS assessed physical activity utilizing a modified IPAQ version. EHIS plans to collect physical activity 


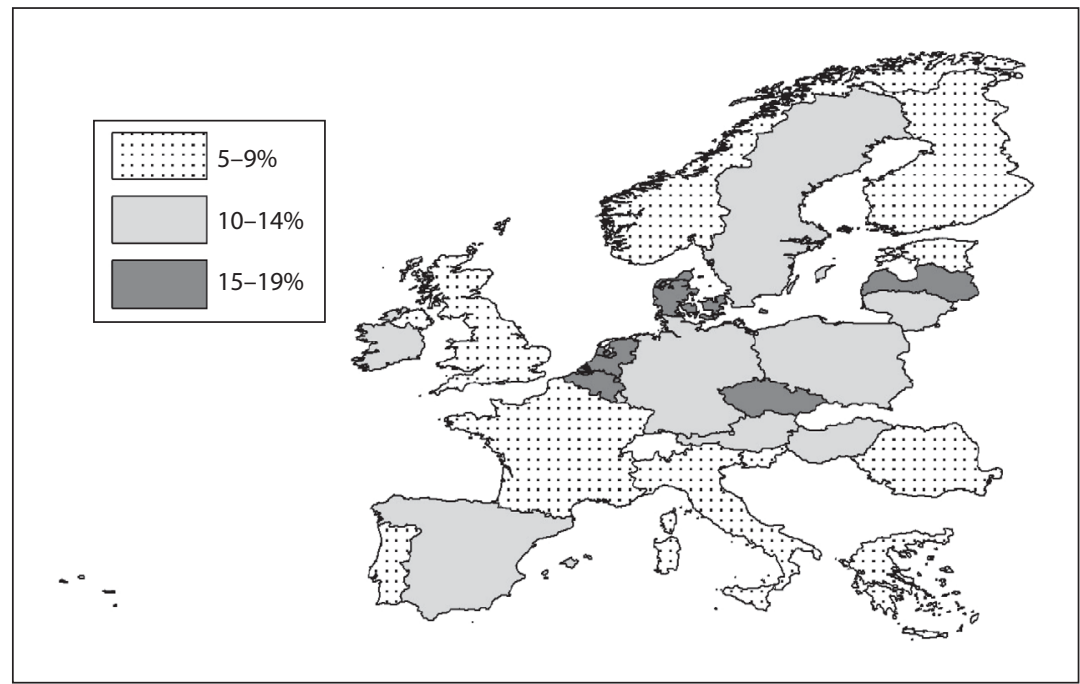

Fig. 8.12. Percentage of $\mathbf{1 5}$-year-old girls who reported at least $1 \mathrm{~h}$ of moderate-to-vigorous activity daily. Source: WHO [2008a].

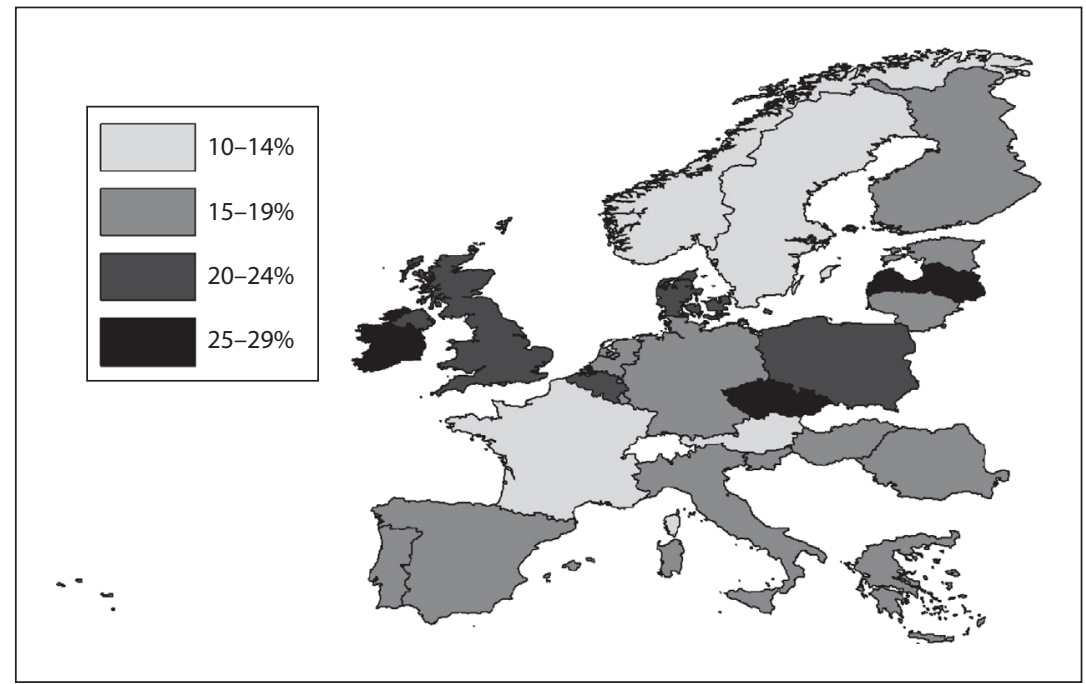

Fig. 8.13. Percentage of $\mathbf{1 5}$-year-old boys who reported at least $1 \mathrm{~h}$ of moderate-to-vigorous activity daily. Source: WHO [2008a]. 
related data biannually in all EU member states. Also, the WHO and European Commission jointly work on a project to monitor progress on improving nutrition and physical activity and to prevent obesity in the EU. This project is currently developing an inventory of existing national and cross-national datasets pertaining to the issue.

Regarding future physical activity assessment in the European Union, the abovementioned projects are of high relevance and might influence the development of policies for the promotion of physical activity. However, in order to do so, several important issues ought to be addressed: (1) Current European cross-national data suffer from a limited comparability. This is partly caused by differences in the wording of questions, even if instruments that were designed to standardize physical activity assessment (such as the IPAQ) are utilized. The Eurobarometer 64.3 and the EHIS survey for instance utilize modified IPAQ versions that yield data difficult to compare with data generated through the original IPAQ. In order to avoid surveys yielding differing or conflicting results regarding physical activity levels of populations, it might thus be important to coordinate the utilization of instruments for physical activity assessment across agencies responsible for conducting cross-national surveys in Europe. (2) Currently, methodologies employed to collect data related to physical activity vary across European surveys. This pertains to the method of data collection (e.g. face-to-face interviews, telephone interviews), response rates achieved, and routines of data cleaning, analysis, and data reporting. These factors might influence results regarding physical activity prevalence across the European region. Thus, it may be useful to agree on standards regarding the collection, analysis, and reporting of physical activity related data across surveys. (3) There is a need for cross-national survey data that enable the identification of time trends in physical activity behavior of populations. Potentially, such data could yield information on the output of policies geared at promoting physical activity on the population level and could assist in formulating and adjusting/improving such policies on national or supranational levels. While the ECHI and EHIS projects are presumably in the process of developing such surveillance systems, these efforts should be coordinated with attempts by WHO and other organizations to develop a European and Global health monitoring system. (4) Beyond these rather methodological issues, current concepts for physical activity assessment should be discussed and potentially be revised. For example, the currently employed concept of HEPA assumes that moderate or vigorous-intensity aerobic physical activity is important to promote and maintain health, independent of the domain it takes place in [Blair et al., 1996]. Following this view, survey instruments for physical activity assessment would not need to address domain-specific physical activity. The IPAQ short version is one example for such an instrument. However, a number of studies suggest that domain-specific differences in the health effects of physical activity might indeed exist, and that physical activity performed during leisure-time might potentially yield greater health benefits compared to physical activity in other domains [Oppert et al., 2006; Abu-Omar and Rütten, 2008]. 
This might imply that instruments such as the GPAQ or the IPAQ long-form that allow for assessing domain-specific levels of physical activity are better suited to monitor physical activity that is related to health outcomes on the population level. (5) Considering the current limitations of providing consistent trend data on physical activity across European nations, it might also be of interest to investigate complementary tools to monitor physical activity levels of populations, and progresses of policy efforts for promoting physical activity. In order to acquire more robust data regarding physical activity levels, some international projects are currently investigating the feasibility of objective assessments of physical activity levels as part of surveillance systems, e.g. via pedometer or accelerometer. With respect to the evaluation of the effectiveness of physical activity promotion efforts, the WHO is in the process of developing tools that allow monitoring the implementation of policies for the promotion of physical activity in different nations [WHO, 2008b]. Also, the forthcoming European Guidelines for Physical Activity Promotion will stress the importance of monitoring such policy efforts. Especially in the light of the current problems in providing consistent surveillance data on physical activity and the high relevance of policy efforts to promote physical activity, tools to monitor the progress in the implementation of such policies might be key to combating sedentary lifestyles successfully.

\subsection{Breastfeeding ${ }^{3,4}$}

Mother milk alone provides all nutrients an infant needs during the first 6 months of life. Hence, it is the ideal nourishment for this age group and no additional liquid or solid food is needed. Mother milk contains antibodies that help babies to combat disease and protect them from diarrhea and acute respiratory infections. Moreover, the immune system and response to vaccination of newborns benefit from breastfeeding [Unicef, 2009].

The WHO recommends exclusive breastfeeding for the first 6 months of life, as a public health recommendation, with introduction of complementary foods and continued breastfeeding thereafter [WHO, 2001]. A prolongation of breastfeeding after 6 months up to 2 years of age and beyond helps to ensure good nutritional status and protects against illnesses. For HIV/AIDS-positive women, breastfeeding carries the risk of mother-to-child transmission and is therefore not recommended, unless replacement feeding is not acceptable, feasible, affordable, sustainable and safe [Unicef, 2009].

3 Source of data: Protection, promotion and support of breastfeeding in Europe: pilot testing the Blueprint for Action. A project funded by the European Commission from 2005-2008.

${ }^{4}$ Putz P (Coordinating Centre), Cattaneo A (Unit for Health Services Research and International Health, IRCCS Burlo Garofolo, Trieste, Italy). 


\section{Data on Breastfeeding in European Countries}

Data has been collected for rates and trends of initiation of breastfeeding, any kind of breastfeeding at the age of 6 months, exclusive breastfeeding at 6 months and breastfeeding at 12 months for two 5-year periods: 1998-2002 and 2003-2007. Except for Cyprus and Greece, all collaborating countries in the ENHR 2009 consortium had data for at least one of the mentioned indicators at the time of data acquisition. It has to be mentioned that the definitions and methods of data collection were not harmonized across countries and thus care is needed when making comparisons between the countries. Figure legends 8.1 and 8.3 illustrate differences in the definitions of indicators. Differences in breastfeeding practices were compared across European regions also. Countries were grouped to four regions as follows. North: DK, EE, FI, LV, LT, NO, SE; West: BE, FR, IE, LU, NL, UK; Central and East: AT, CZ, DE, HU, PL, RO, SI; South: CY, GR, IT, PT, ES.

The rate of initiation of breastfeeding was almost 100\% in Norway, Lithuania, Sweden, and Denmark. Ireland was the only country with less than half (46\%) of mothers beginning to breastfeed their newborn in the most recent survey. From the countries that were able to provide a trend for this indicator, remarkable enhancements were gained in Latvia (+59\%), Ireland (+13\%), and France (+13\%). Marginal declines were observed in Austria (-3\%) and Slovenia (-1\%) (fig. 8.14).

Highest rates for any kind of breastfeeding at the age of 6 months in the most recent studies were reached in the Scandinavian countries (Norway 80\%, Sweden $72 \%$, Finland 60\%). Lowest values appeared in the UK (25\%), Belgium (25\%), and Portugal (29\%). Again, a high increase was reported from Latvia (+26\%). Minor gains were, among others, reported in Finland $(+6 \%)$ and Lithuania $(+5 \%)$. Concerning this indicator, no country that could provide a trend had a decline (fig. 8.15).

From those countries that gathered data on exclusive breastfeeding at the age of 6 months in the recent time span (2003-2007), Latvia (34\%), Lithuania (26\%), and Denmark (25\%) reported the highest rates. Low values appeared in Finland (1\%) and the UK (3\%). Only a few countries had data from both pictured time spans. Whereas the rate increased clearly in Lithuania $(+12 \%)$, Latvia $(+10 \%)$, and The Netherlands $(+6 \%)$, a remarkable decline was reported for Austria (-36\%). This, however, is due to a change of definitions between surveys (fig. 8.16).

The rate of breastfeeding at the age of 12 months was at least twice as high in Norway (36\%) and Finland (36\%) than in any other country that gathered data for this indicator. Lowest values were reported in Belgium (4\%). Each country that could provide a trend for this rate had an increase (Latvia $+9 \%$, Austria $+6 \%$, UK $+5 \%$, Lithuania $+4 \%$ ) (fig. 8.17).

Across the four described indicators, remarkable increases between the two survey periods were reported in Latvia. This is probably due to changes in survey definitions and methods.

After the completion of the dataset from which all the data described above were taken, more recent national data were published. In a national representative 


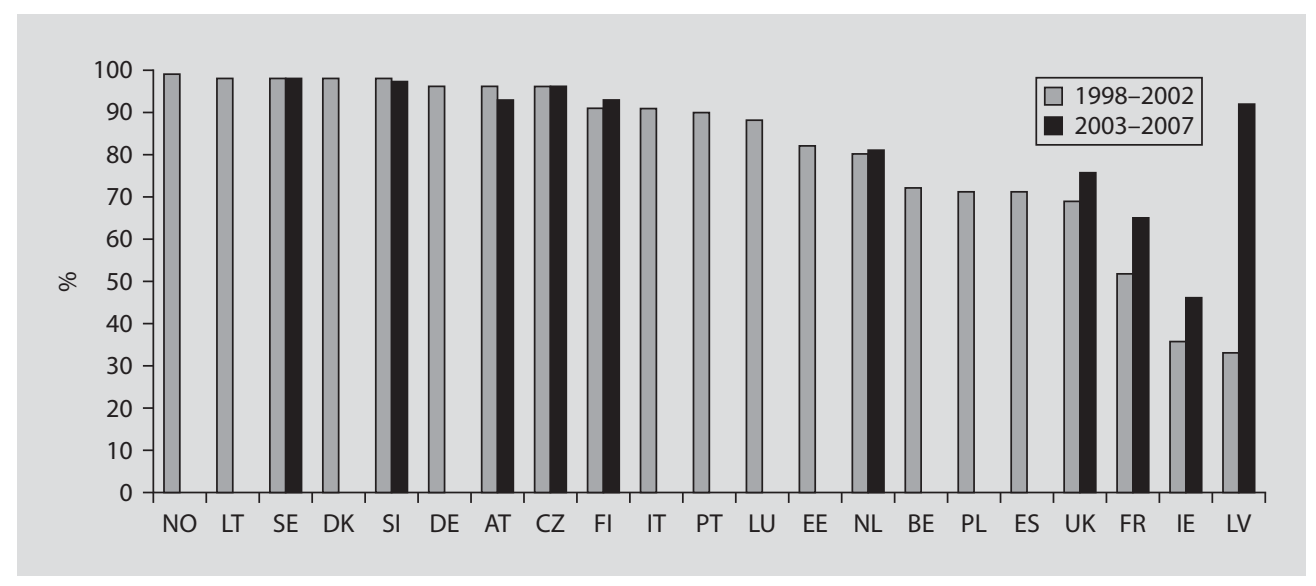

Fig. 8.14. Rates and trends of initiation of breastfeeding.

Data missing from: $\mathrm{CY}, \mathrm{GR}, \mathrm{HU}$, and $\mathrm{RO}$

$\mathrm{SI}, \mathrm{SE}$ and MT: any breastfeeding at discharge

PL and IE: exclusive breastfeeding at discharge

ES, EE and LV: any breastfeeding at six weeks

FI: any breastfeeding at age less than 1 month

NL: initiation of exclusive breastfeeding

All other countries: initiation of any breastfeeding.

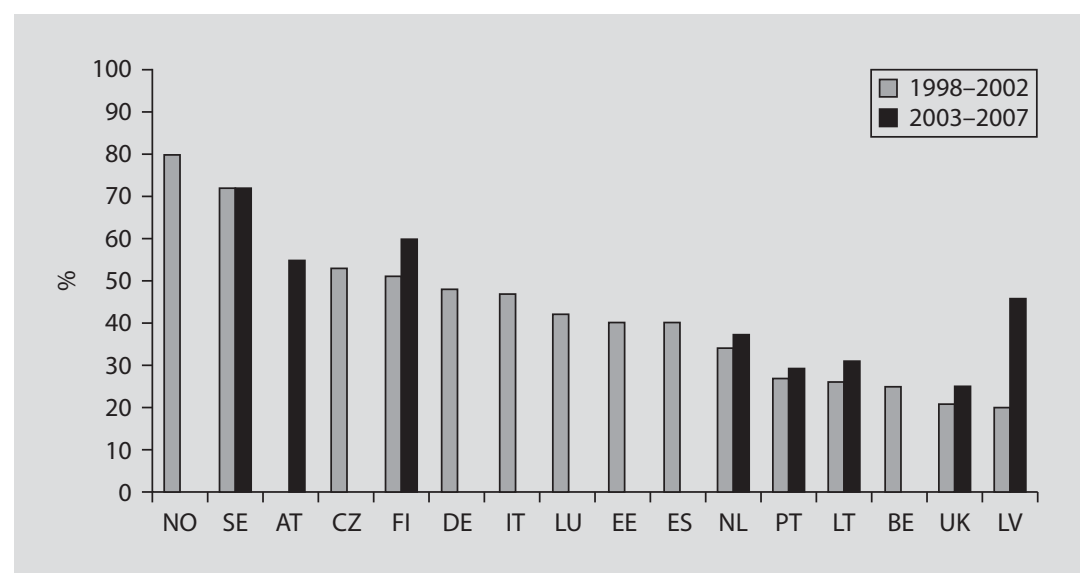

Fig. 8.15. Rates and trends of any breastfeeding at the age of 6 months. Data missing from DK, FR, $\mathrm{GR}, \mathrm{HU}, \mathrm{IE}, \mathrm{PL}, \mathrm{RO}, \mathrm{SI}$. 


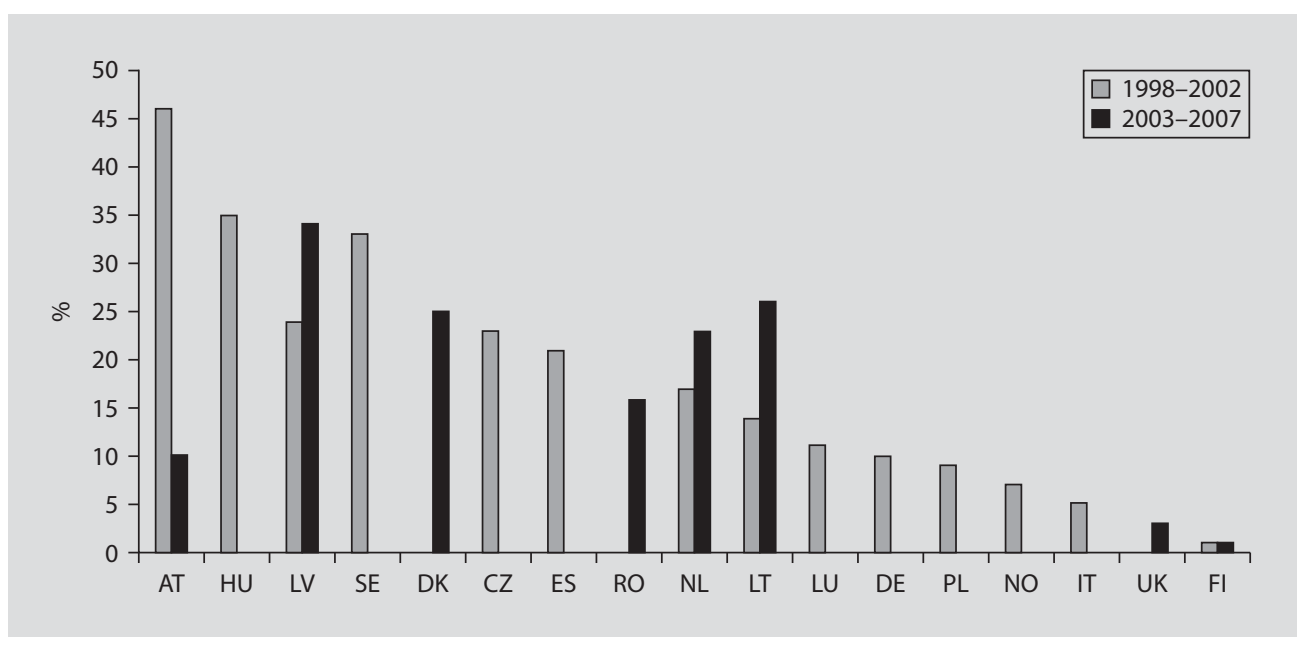

Fig. 8.16. Rates and trends of exclusive breastfeeding at the age of 6 months.

Exclusive breastfeeding: the infant receives only breast milk from his/her mother or a wet nurse, or expressed milk, and no other liquids or solids with the exception of drops or syrups consisting of vitamins, mineral supplements, or medicine (WHO, 2001).

Data missing from: $B E, E E$, FR, GR, IE, PT, SI

DK and SE: full breastfeeding

UK and NL (2007): exclusive breastfeeding at five months.

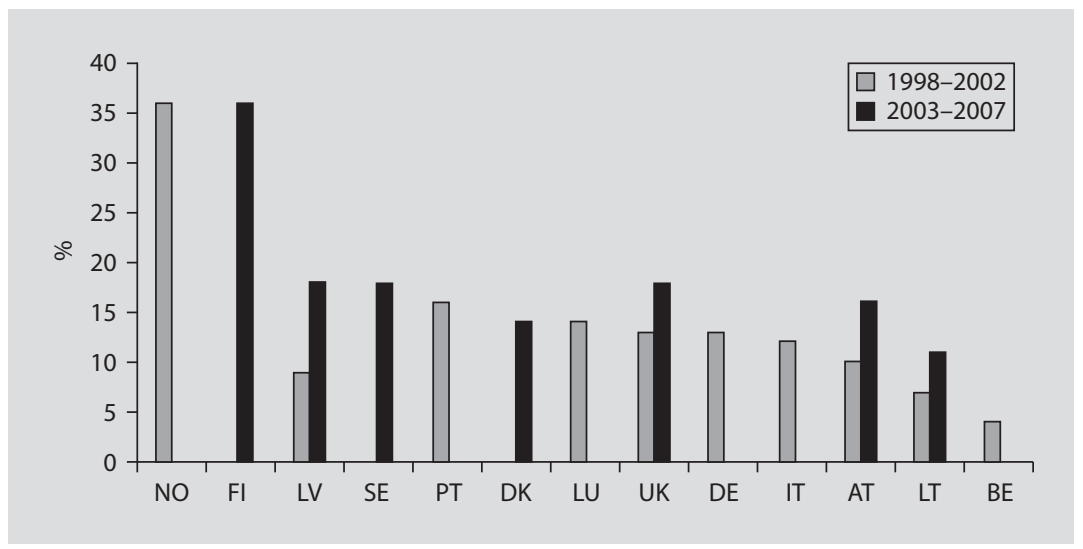

Fig. 8.17. Rates and trends of breastfeeding the age of 12 months.

Data missing from: $\mathrm{CY}, \mathrm{GR}, \mathrm{HU}$, and $\mathrm{RO}$

$\mathrm{SI}, \mathrm{SE}$ and MT: any breastfeeding at discharge

PL and IE: exclusive breastfeeding at discharge

ES, EE and LV: any breastfeeding at six weeks

Fl: any breastfeeding at age less than 1 month

$\mathrm{NL}$ : initiation of exclusive breastfeeding

All other countries: initiation of any breastfeeding. 


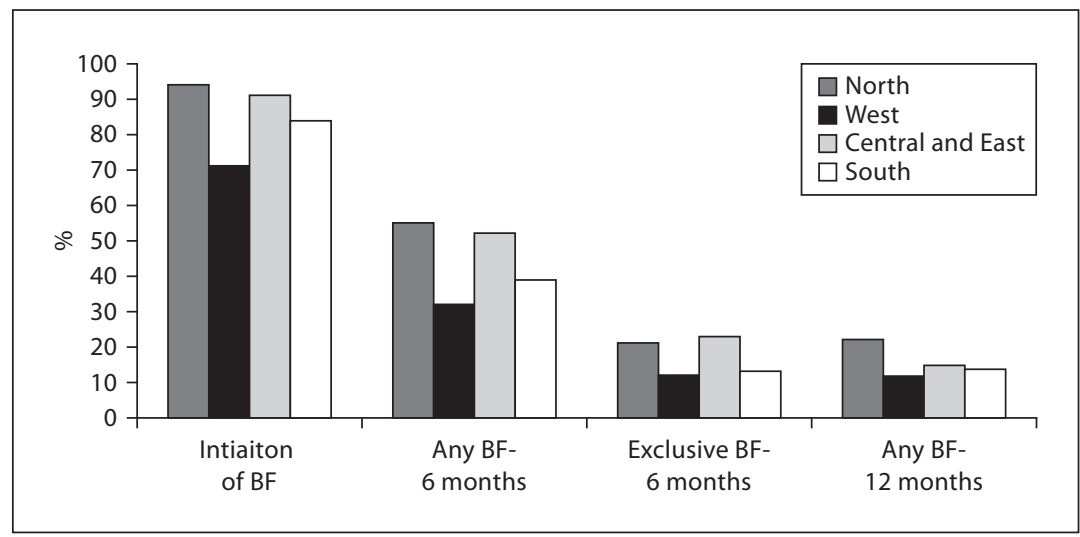

Fig. 8.18. Overview on the established regions of ENHR 2009 for four indicators of breastfeeding (BF). Mean values were calculated from the countries with available data.

Results from the most recent studies were used.

survey that was carried out in Norway in 2006-2007, the rate of initiation was $98 \%$, any breastfeeding at the age of 6 months $80 \%$ and at 12 months $46 \%$, while exclusive breastfeeding at 6 months was $9 \%$. The last two values showed an increase compared to the survey carried out 10 years before [Øverby et al., 2008a, b]. For Italy, the Italian Pediatric Association carried out a national survey in 2008. The initiation rate was $89 \%$, the rate of any breastfeeding at the age of 6 months $52 \%$ and the one at 12 months $24 \%$, while exclusive breastfeeding at 6 months was estimated at 2\% [Navone et al., 2008]. Data for the period 2004-2005 are discussed in the Italian national report (see chapter 11) referring to the results of the 'Survey on Health Conditions' [ISTAT, 2006]. For Hungary, in a recent study carried out by the Hungarian network of midwives, following rates were reported: initiation of breastfeeding 98\%, any kind at the age of 6 months $94 \%$ and at 12 months $36 \%$. The rate of exclusive breastfeeding at the age of 6 months was 37\%. A possible explanation for the high rates reported in Hungary is the length of maternity leave, which is 3 years and thus the longest in the EU [Hungarian National Centre for Healthcare Audit and Inspection, 2008]. In Greece, several studies have been conducted after 2001 and have reported breastfeeding rates [Pechlivani et al., 2005; Theofilogiannakou et al., 2006; Bakoula et al., 2007; Ladomenou, 2007]. In the most recent one study conducted during 2004 in the town of Heraklion, at the island of Crete, breastfeeding rates in a representative sample of 1,049 mothers (representing the $29 \%$ of the total births) for overall breastfeeding were $60.7,35.3,16.7$ and $4.5 \%$ at months 1, 3, 6 and 12 and for exclusive breastfeeding 24.6, 17.2, 10.2 and 3.5\% at the same time points, respectively [Ladomenou, 2007]. Unfortunately these data were gathered with different definitions and methods and it is difficult to compare them with the previous ones. 
Figure 8.18 shows an overview on the established regions of ENHR 2009 for the four indicators as described above. With the exception of exclusive breastfeeding at the age of 6 months, the North region reached the highest values in all categories. The Central and East region had the highest rates for exclusive breastfeeding at the age of 6 months and the second highest rates in each other category. Overall, the West region had the lowest values and the South region the second lowest. 


\section{Food and Nutrition Policies in European Countries ${ }^{1}$}

\section{Introduction}

The development of policies addressing food and nutrition in Europe has, in general, been determined by the course of key historical events necessitating action in one form or another. During the post-war era in the 1950s, food policies addressed the need of providing the population at large with secure and sufficient amounts of food. The re-establishment of a strong agriculture sector to meet this need relied on robust national and regional measures as well as the expansion and consolidation of the food-processing industry. The European Economic Communities in 1962 and in 1970, respectively, established the Common Agriculture Policy (CAP) and the Common Fisheries Policy (CFP), which aimed to tackle food production in Europe and shaped most European countries' food availability and consumption. By the 1980s, in Western Europe these policies were clearly successful and in fact, the problem of surplus food production had to be dealt with. Simultaneously, the sociopolitical changes occurring in Eastern Europe during the 1980s and 1990s brought about policy challenges on food supply and distribution. By the 1990s, higher rates of nutrition-related diseases were detected throughout Europe, encompassing both deficiency and chronic diseases as well as increasing rates of foodborne diseases (intolerances, allergies, poisoning, ...).

Currently, the public health challenges facing Europe include high rates of noncommunicable diseases such as obesity, cardiovascular diseases, cancer and other chronic degenerative pathologies, as a result of a nutrition transition. Certain sectors of the population still remain vulnerable to food insecurity, having inadequate access to foods and thus increased risk for nutrient deficiencies. Moreover, the rise in foodborne disease and the appearance of new pathogens such as bovine spongiform encephalopathy (BSE) have captured the attention of policymakers in recent years.

\footnotetext{
${ }^{1}$ Román Viñas B, Ngo J, Ribas-Barba L, Serra-Majem L. Nutrition Research Foundation, Barcelona, Spain (fin@pcb.ub.es).
} 
Thus, it can be seen that the initial priorities of policies with a focus on addressing the food supply have evolved to take on other issues related to food safety and nutritionrelated health issues. This expansion and proliferation of policy and related actions has, in part, contributed to a certain level of confusion when it comes to defining what a food and nutrition policy (FNP) consists of.

As such, the evolution of these trends led to the development of the 'First Action Plan for Food and Nutrition Policy, WHO European Region, 2000-2005' [WHO, 2000], established in September 2000 by the WHO Regional Committee for Europe, representing the 51 Member States in the European Region. Later, a Second Action Plan 'WHO European Action Plan for Food and Nutrition Policy 2007-2012' [WHO, 2008] was developed to assist with the implementation process of the policies. The Action Plan emphasizes the importance of developing FNPs that protect and promote health and reduce the burden of foodborne disease, while simultaneously contributing to socioeconomic development and a sustainable environment. It focuses on collaboration and interrelatedness of different sectors in the formulation and implementation of such policies.

The objective of this present analysis was to describe the actions of FNPs currently carried out in countries participating in the ENHR 2009, with special attention to initiatives addressing food fortification and food-based dietary guidelines.

\section{Methods}

A draft questionnaire was sent to national and international experts in Europe that addressed FNPs, so as to receive input and advice on the appropriateness of the method. A final questionnaire incorporating the proposed modifications was sent to the participating ENHR 2009 countries. Only the information provided by the partners who responded was included in this report.

\section{Results}

Food and Nutrition Policy in the Participating Countries

Twenty-one partners answered the questionnaire (Austria, the Czech Republic, Denmark, Estonia, Finland, France, Germany, Greece, Hungary, Ireland, Italy, Latvia, Lithuania, Norway, Poland, Portugal, Romania, Slovenia, Spain, Sweden, The Netherlands). All the participating countries that responded to the questionnaire had a specific policy document on food and nutrition except Greece, Poland and Portugal, which reported having various nutrition-related programs. Denmark had a policy document on Health and Nutrition and a policy document on Food. Ireland is currently working on a new policy to be published in 2009. Table 9.1 shows the names of the FNP documents presented by each participating country and the institutions involved 
Table 9.1. Food and nutrition policies, institutions involved in their developement and coordination and examples of implementation actions developed in the participating countries as reported in the questionnaire

\begin{tabular}{|c|c|c|c|}
\hline Country & Name of the food and nutrition policy document & Year of elaboration & Year of implementation \\
\hline Austria & $\begin{array}{l}\text { 1. The Austrian strategy for sustainable development } \\
\text { 2. Health and food safety law } \\
\text { 3. Codex alimentairius austriacus (food fortification) }\end{array}$ & $\begin{array}{l}\text { 1. } 2002 \\
\text { 2. } 2002-2004 \\
\text { 3. since } 1975 \text { part of } \\
\text { the Austrian Food Law }\end{array}$ & \\
\hline
\end{tabular}

Czech - Long-term programme of Czech population health 2002

Republic state improving health for all in 21 st century

\begin{tabular}{|c|c|c|c|}
\hline Denmark & $\begin{array}{l}\text { 1. Healthy throughout life - the target and strategies } \\
\text { for the public health policy of the Government of } \\
\text { Denmark 2002-2010 } \\
\text { 2. National Action plan against obesity } \\
\text { 3. Health for children and youth } \\
\text { 4. Health, food and physical activity - Nordic Plan of } \\
\text { Action on better health and Quality of life through } \\
\text { diet and physical activity } \\
\text { 5. Food Policy in Consumer Perspective (17-11-2006) } \\
\text { (Redegørelse af } 17 / 1106 \text { om fødevarepolitik i et } \\
\text { forbrugerperspektiv) }\end{array}$ & $\begin{array}{l}1.2002 \\
2.2002 \\
3.2003 \\
4.2006 \\
5.2006\end{array}$ & $\begin{array}{l}\text { 1. ongoing } \\
\text { 2. ongoing } \\
\text { 3. ongoing } \\
\text { 4. ongoing } \\
\text { 5. ongoing }\end{array}$ \\
\hline Estonia & $\begin{array}{l}\text { 1. National Health development plan } 2009-2020 \\
\text { 2. National strategy for prevention of cardiovascular } \\
\text { disease } 2005-2020 \\
\text { 3. National cancer strategy } 2007-2015\end{array}$ & $\begin{array}{l}\text { 1. } 2008 \\
\text { 2. } 2005 \\
\text { 3. } 2007\end{array}$ & $\begin{array}{l}\text { 1. } 2009-2020 \\
\text { 2. } 2005-2020 \\
\text { 3. } 2007-2015\end{array}$ \\
\hline
\end{tabular}




\begin{tabular}{|c|c|c|}
\hline $\begin{array}{l}\text { Institutions involved in the } \\
\text { elaboration: leader and others }\end{array}$ & Institutes involved in the coordination & Examples of implementation \\
\hline $\begin{array}{l}\text { - M of Agriculture, M of Health } \\
\text { - UVI, Austrian Nutrition Society } \\
\text { - Chamber of Labour Consumer's } \\
\text { Organization, Food industry }\end{array}$ & $\begin{array}{l}\text { - } M \text { of Health } \\
\text { - } M \text { of Health, } M \text { of Agriculture and } \\
\text { different stakeholders of the private } \\
\text { sector (Austrian Platform of nutrition) }\end{array}$ & $\begin{array}{l}\text { - Foundation of the Austrian } \\
\text { Agency for Health and Food } \\
\text { Safety }\end{array}$ \\
\hline $\begin{array}{l}\text { - Czech Republic Government (M of } \\
\text { Health) } \\
\text { - M of Agriculture, M of Education etc. } \\
\text { - Czech Medical Society, Czech Nutrition } \\
\text { Society, etc. } \\
\text { - Association of consumers } \\
\text { - Hygienic services } \\
\text { - Association of producers } \\
\text { - etc. }\end{array}$ & $-M$ of Health & \\
\hline
\end{tabular}

1. $M$ of Health and Prevention

2. M of Health and Prevention

3. $M$ of Health and Prevention

4. Nordic Council of Ministers

5. M of Food, Agriculture and Fisheries

\section{11 Ministries took part.}

1. $M$ of Health and Prevention

5. M of Food, Agriculture and Fisheries
1. M of Social Affairs

2. M of Social Affairs

3. M of Social Affairs

1. M. for Population and Ethnic Affairs, M. of Defence, $M$. of the Environment, $M$. of the Interior, $M$. of Education and Research, M. of Agriculture, M. of Culture, $M$. of Justice, M. of Economic Affairs and Communications./National Institute for Health Development/Citizens' associations/Local governments 2. Public Health Dept. of the M. of Social Affairs, Health Information and Analysis Dept. of the M. of Social Affairs, M. of Finance, M. of Education and Research,

\section{M of Social Affairs}

2. National Institute for Health Development

3. National Institute for Health

Development
1. Local government level: Develop and implement a concept of support activities to ensure avalilability of nutritious diets to vulnerable groups 2. Collection and systematisation of healthy eating recommendations for target groups with different needs./Information campaigns for promoting the consumption of fruits and vegetables, rye bread and products with a low fat and common salt content targeted at young people and adults 3. Promotion of safe and beneficial food choices 
Table 9.1. Continued

Country Name of the food and nutrition policy document $\quad$ Year of elaboration $\quad$ Year of implementation

Finland - Action programme for implementing national nutrition recommendations

\begin{tabular}{llll}
\hline France & 1. National Nutrition and Health Program & $1.1998-2000$ & 1.2001 \\
& (Programme National Nutrition Santé, PNNS) & 2.2003 & 2.2004
\end{tabular}

2. 2004 Public Health Law (Loi relative à la politique de santé publique de 2004, LSP) 
Institutions involved in the

M. of Culture, $M$. of Agriculture, $M$. of the

Interior, Estonian Health Insurance Fund./

National Institute for Health

Development, Dept. of Food Processing of

the Tallinn University of Technology/

Estonian Employers' Confederation,

Association of Estonian Cities, Association

of Rural Municipalities of Estonia,

Estonian Heart Association, Estonian

Cardiac Society, Estonian Chamber of

County Doctors, Estonian Health

Promotion Society, Estonian Society of

Family Doctors

3. Estonian Health Insurance Fund, Health

Protection Inspectorate/National Institute

for Health Development/Estonian Cancer

Society/Local governments, regional

hospitals

- M of Agriculture and Health

- Social Affairs and Health; Environment;

Employment and the Economy

- Several organizations

- National Public Health Institute, The

Social Insurance Institution of Finland -

representatives from some universities,

hospital and food producer's

organizations

1. Interministerial nutrition policy committee

2. M of Health (LSP)

1. M of Agriculture, $M$ of Education and Research, $M$ of sports, $M$ of Economy, and Governmental Health Agencies /

Consumer organizations / Industry
- National Nutrition Counsel

- Social Affairs and Health; Environment;

Employment and the Economy

- Several organizations

- National Public Health Institute, The Social Insurance Institution of Finland representatives from some universities, hospital and food producer's

organizations
- Diabetes prevention programme

- New guidelines for school lunches
1. Steering Committee / Ministries, governmental health agencies, French national health insurance system, research and educational institutions, food and agricultural industry, consumer organizations, and scientific experts
1. Publication and dissemination of dietary guidelines for general population (distribution of about 6 million FBDG for general population and 700,000 guides for healthcare professionals since 2001) Periodical mass media campaigns to support the PNNS recommendations (At least 5 a day, etc.)

2. Compulsory inclusion of health messages in manufactured/ processed food advertisements (television, radio, cinema, press, and internet). Prohibition of vending machines in schools 
Table 9.1. Continued

\begin{tabular}{llll}
\hline Country & Name of the food and nutrition policy document & Year of elaboration & Year of implementation \\
\hline Germany & $-\begin{array}{l}\text { National Action Plan on the prevention of } \\
\text { unhealthy eating, lack of exercise, overweight and } \\
\text { related diseases titled 'IN FORM' - Germany's } \\
\text { Initiative for a healthy diet and more exercise }\end{array}$ & 2007-2008 & \\
& &
\end{tabular}

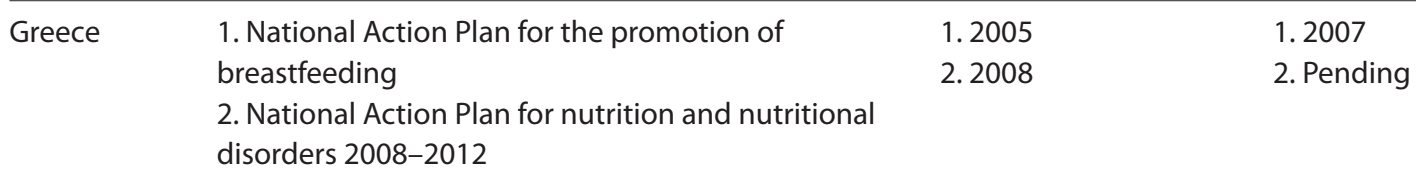




\begin{tabular}{|c|c|c|}
\hline $\begin{array}{l}\text { Institutions involved in the } \\
\text { elaboration: leader and others }\end{array}$ & Institutes involved in the coordination & Examples of implementation \\
\hline $\begin{array}{l}\text { - Federal M for Food, Agriculture and } \\
\text { Consumer Protection. Federal M for } \\
\text { Health } \\
\text { - Federal states, Federal Government/ } \\
\text { states working group, Municipalities } \\
\text { and Civilian population (by Plattform } \\
\text { für Ernährung und Bewegung/platform } \\
\text { for nutrition and physical activity as } \\
\text { well as Bundesvereinigung Prävention } \\
\text { und Gesundheitsförderung/Federal } \\
\text { Association regarding prevention and } \\
\text { health promotion) }\end{array}$ & - An office of both ministries & $\begin{array}{l}\text { - Nationale Verzehrsstudie II } \\
\text { (National Nutrition Survey II) } \\
\text { - Besser essen - mehr bewegen - } \\
\text { Kinderleichtregionen (eat healthy, } \\
\text { exercise more) } \\
\text { - Qualitätsstandards für die } \\
\text { Verpflegung in } \\
\text { Kindertageseinrichtungen } \\
\text { (quality standards for food } \\
\text { provision in daycare centres) } \\
\text {-'10 rules of the German Nutrition } \\
\text { Association' (FBDG) }\end{array}$ \\
\hline $\begin{array}{l}\text { 1. The Hellenic M of Health and Social } \\
\text { Solidarity } \\
\text { 2. The Hellenic M of Health and Social } \\
\text { Solidarity } \\
\text { 1. Greek Midwives Association. / 1st } \\
\text { Department of Paediatrics, University of } \\
\text { Athens Medical School, National School } \\
\text { of Public Health } \\
\text { 2. The Hellenic Food Authority (EFET) of } \\
\text { the M of Rural Development and Food. / } \\
\text { The Greek Diabetic Society, the Greek } \\
\text { Society of Dieticians and Food } \\
\text { Technologists, Hellenic Medical Society } \\
\text { of Obesity, Hellenic Cancer Society, } \\
\text { Hellenic Society of Endocrinology, } \\
\text { Hellenic Society of Health Promotion } \\
\text { and Health Education, Hellenic Society } \\
\text { of Nutrition and Foods, Hellenic Society } \\
\text { of Pediatrics. / EK.POI.ZO (Union of } \\
\text { Consumers for Quality of Life). / NGO } \\
\text { ANASA (Non-profit organization for } \\
\text { Eating Disorders). / WHO Collaborating } \\
\text { Center, Dept. of Hygiene, Epidemiology } \\
\text { and Medical Statistics, School of } \\
\text { Medicine, University of Athens, National } \\
\text { School of Public Health, Federation of } \\
\text { Hellenic Food Industries (SEVT) } \\
\text { Aristidis Daskalopoulos Foundation } \\
\text { Hellenic Health Foundation }\end{array}$ & $\begin{array}{l}\text { 1. The coordination mechanism is } \\
\text { provided by the National Breastfeeding } \\
\text { Committee that has an advisory role and } \\
\text { has been appointed by the M of Health } \\
\text { and Social Solidarity } \\
\text { 2.The National Nutrition Policy } \\
\text { Committee will be responsible for the } \\
\text { coordination of the Action Plan / The M of } \\
\text { Rural Development and Food, } \\
\text { The Hellenic Food Authority (EFET) of the } \\
\text { M of Rural Development and Food, } \\
\text { The M of Development, Department of } \\
\text { Consumer Affairs } \\
\text { The Center of Control and Prevention of } \\
\text { Diseases (KELPNO) } \\
\text { The University of Athens Medical School, } \\
\text { The Federation of Hellenic Food } \\
\text { Industries (SEVT) } \\
\text { A Consumers' Association named EK.POI. } \\
\text { ZO quality of life }\end{array}$ & $\begin{array}{l}\text { 1. In } 2007, \text { The M of Health and } \\
\text { Social Solidarity appointed one } \\
\text { person from the hospital personnel } \\
\text { (a pediatrician or midwife) to be } \\
\text { responsible for all breastfeeding- } \\
\text { related issues in every maternity } \\
\text { and pediatric hospital of the } \\
\text { country. A nationwide study has } \\
\text { been implemented by the Institute } \\
\text { of Child Health with the aim to } \\
\text { measure breastfeeding rates at } 0,3 \\
\text { and } 6 \text { months in a national } \\
\text { representative sample of the Greek } \\
\text { infant population }\end{array}$ \\
\hline
\end{tabular}


Table 9.1. Continued

\begin{tabular}{lll}
\hline Country Name of the food and nutrition policy document $\quad$ Year of elaboration $\quad$ Year of implementation \\
\hline
\end{tabular}

Hungary National Nutrition Policy of Hungary 2004

\begin{tabular}{llll}
\hline Italy National Health Plan 2003-2005 & $1.2000-2002$ & 1.2005 onward \\
2. National Health Plan 2006-2008 & 2.2005 &
\end{tabular}

3. National Health Plan 2009 
Institutions involved in the elaboration: leader and others

- National Institute for Food and Nutrition Science

$-M$ of Health

- Hungarian Society of Nutrition Science

- Complex Committee of Food Science of the Hungarian Academy of Science; Central Research Institute of Food Science

Institutes involved in the coordination

Examples of implementation

- Healthy eating recommendation for catering

- School Buffet Program

- Nationwide survey on school nutritional environment, 2008

- Nationwide survey on pre-school nutritional environment, 2009

- Application of EC regulation about food labelling

- Preparation of nutritional guidelines for adults, 2004 (updated version will be published in 2009)

- Preparation of nutritional guidelines for pregnant women, 2009

- Healthy School Canteen Program, 2005

- Decree on hospital catering, by the Ministry of Health, 2007 and nationwide survey about the implementation in 2008

1. The Health Department of the $M$ of Labour, Health and Welfare is the governmental body in charge of delivering the National Health Plan. Gaining Health is implemented under the coordination by the General Direction of Health Prevention and the General Direction of Communication and Institutional Relationships. Centro Nazionale per la prevenzione e il Controllo delle Malattie (CCM) [National centre for prevention and control of diseases], and within the Superior Institute for Health, the Centro Nazionale di Epidemiologia, Sorveglianza e Promozione della Salute (CNESPS) [National Centre of Epidemiology, Surveillance and Health Promotion]
- The Dietary Guidelines are formulated and disseminated by the INRAN. The Nutrients and Energy Recommendations are formulated by the Italian Human Nutrition Society and INRAN. INRAN has institutionally in charge of nutrition education The Regions have the Department for Prevention

The Local Health Districts have the Hygiene, Food and Nutrition Services Universities and Schools host several education and training courses

- Even though a national coordination committee has not been established, different stakeholders expressed interests and gave their voluntary support to the promotion of health
- Guadagnare salute [Gaining Health] including: OKkio alla salute overweight and (obesity among children) / Frutta Snack

- Progressi delle Aziende Sanitarie per la Salute in Italia (PASSI) [The Italian Behavioural Risk Factor Surveillance System] 
Table 9.1. Continued

Country Name of the food and nutrition policy document $\quad$ Year of elaboration $\quad$ Year of implementation

\begin{tabular}{lll}
\hline Latvia & Healthy Nutrition 2003-2013. Concept of the & Accepted with order of \\
Cabinet of Ministers & Cabinet of Ministers \\
& No.556 on September \\
& 4 th, 2003
\end{tabular}

\begin{tabular}{lll}
\hline Lithuania & - State Food and Nutrition Strategy and Action Plan & 2003 \\
& for 2003-2010
\end{tabular}


Institutions involved in the

Institutes involved in the coordination

Examples of implementation

elaboration: leader and others

All the health policies actions are

implemented at territorial level,

behaviours as part of the Corporate

Social Responsibility.

mainly regions that have specific

For ex. the Italian Association of Food

Departments for the Prevention and

Industry (Federalimentare) prepared a

Promotion of Heath / Conference

dossier to illustrate the enterprises

Government-Regions

contribution, they have also

Inter-ministerial committees (particularly,

M. of Agricultural, Food and Forestry

implemented the Food for Life Italian

Platform.

Policies, and the M. of Education,

NGOs and private food distribution

University and Research) with the

chains are active towards disadvantaged

involvement of all the listed

social groups

stakeholders according to their

expertise and interest

$-M$ of Health

$-M$ of Helath

- Latvian Food Center, Nutrition Council of $\mathrm{MoH}$, Health Promotion Center, $\mathrm{M}$ of Agriculture, $M$ of Education and Science
Latvian Association of Local and Regional Governments (LALRG), Food and

Veterinary Service (FVS)

$M$ of Economics

Nutrition Council of $\mathrm{MoH}$

Professional associations

UNICEF

WHO
The State program for improvement of nutrition of schoolchildren, School Milk programme.

Prohibition of sales of sweets containing sweeteners, colors and drinks containing sweeteners, colors, coffeine in pre-schools and schools.

Recommendations for using of fruits and vegetables (2007) Implementation of Baby-Friendly Hospital Initiative

- Implementation of Baby-Friendly Hospital Initiative

- The State program for improvement of nutrition of preschool and school children and Science, M. of Social Security and

Labour, M. of Environment, State

Food and Veterinary Service,

Department of Physical Education

and Sports.

- Lithuanian Society of Cardiology,

Lithuanian Heart Association,

Lithuanian Society of

Endocrinology

- Lithuanian Consumer Institute

- Club of Obese people in Vilnius
- M of Health, Department of Public Health

- M of Agriculture, M of Social Security and Labour, $\mathrm{M}$ of Education and Science, M of Environment, State Food and Veterinary Service, Department of Physical Education and Sports 
Table 9.1. Continued

\begin{tabular}{llll}
\hline Country & Name of the food and nutrition policy document & Year of elaboration & Year of implementation \\
\hline Norway & & & \\
& 1. The Norwegian Action Plan on Nutrition (2007- & 1.2005 & 1.2007 \\
2011) Recipe for a healthier diet was launched by & 2.2002 & 2.2003 \\
the Government in January 2007. & 3.2004 & 3.2005 \\
& 2. St.meld.nr.16 (2002-2003) 'Resept for et sunnere & & \\
Norge' Folkehelsepolitikken. (White paper No. 16 & \\
(2002-2003) Prescription of a healthier Norway. & \\
Public Health Nutrition). & \\
3. Global strategy on diet, physical activity and & \\
health, WHO 2004
\end{tabular}

\begin{tabular}{llll}
\hline Poland & $\begin{array}{l}\text { 1. National programme for the prevention of } \\
\text { overweight, obesity and non-communicable } \\
\text { diseases through diet, physical activity }\end{array}$ & 1.2006 & 2.2006 \\
2. National Health Programme - operational \\
objective 3: 'Improvement of Diet and Health \\
Quality of Food and Decrease the Prevalence of \\
Obesity' and 4: 'Increase of physical activity of \\
population'
\end{tabular}

\begin{tabular}{llll}
\hline Portugal & 1. M of Health. National Health Plan 2004-2010 & 1.2004 & 1. Since 2004 \\
2. M of Health, General Directorate of Health. & 2.2003 & 2. Since 2003 \\
National Intervention Programme on Health-Related & 3.2006 & 3. Since 2006 \\
Lifestyles Determinants. & 4.2007 & 4. Since 2007 \\
$\begin{array}{l}\text { 3. M of Health, General Directorate of Health. School } \\
\text { Health programme and Healthy food promotion }\end{array}$ & & \\
within schools. & \\
4. M of Health, General Directorate of Health. & \\
Platform against obesity & & \\
\hline
\end{tabular}




\begin{tabular}{|c|c|c|}
\hline $\begin{array}{l}\text { Institutions involved in the } \\
\text { elaboration: leader and others }\end{array}$ & Institutes involved in the coordination & Examples of implementation \\
\hline $\begin{array}{l}\text { - M. of Health and Care Services } \\
\text { - Norwegian Directorate of Health, } \\
\text { Norwegian Food Safety Authority and } \\
\text { Ministries of } 12 \text { departments: Labour } \\
\text { and Social Inclusion, Children and } \\
\text { Equality, Finance, Health and Care } \\
\text { Services, Local Government and } \\
\text { Regional Development, Culture and } \\
\text { Church Affairs, Education and } \\
\text { Research, Agriculture and Food, } \\
\text { Environment, Fisheries and Costal } \\
\text { Affairs, Trade and Industry, Foreign } \\
\text { Affairs. } \\
\text { - Nutrition Council, Norwegian } \\
\text { Institute of Public Health, } \\
\text { Norwegian Scientific Committee for } \\
\text { Food Safety }\end{array}$ & - M of Health and Care Services & $\begin{array}{l}\text { - To change the diet in line with the } \\
\text { recommendations of the health } \\
\text { authorities } \\
\text { - To reduce social inequalities in } \\
\text { diet }\end{array}$ \\
\hline - Minister of Health & Minister of Health & $\begin{array}{l}\text { - Preparation of guidelines for } \\
\text { school meals - receipe book. }\end{array}$ \\
\hline $\begin{array}{l}\text { - National Institute of Food and Nutrition } \\
\text { - Human Nutrition Committee (Polish } \\
\text { Academy of Sciences) } \\
\text { - Institute of Cardiology } \\
\text { - Polish Society of Nutritional Sciences } \\
\text { - Child's Health Centre } \\
\text { - NGOs involved in health promotion }\end{array}$ & $\begin{array}{l}\text { Polish Diet, Physical Activity and Health } \\
\text { Council, coordinated by the Chief } \\
\text { Sanitary Inspector Members of the } \\
\text { Council represent public institutions, } \\
\text { academics, food chain participants } \\
\text { (private sector), consumer organizations, } \\
\text { NGOs linked to nutrition and physical } \\
\text { activity }\end{array}$ & $\begin{array}{l}\text { - Trzymaj Formę ('Keep fit') } \\
\text { educational programme for } \\
\text { adolescents } \\
\text { - establishment of Healthy Eating } \\
\text { and Physical Activity Promotion } \\
\text { Centre }\end{array}$ \\
\hline$-M$ of Health & $-M$ of Health & $\begin{array}{l}\text { - Assessment of overweight and } \\
\text { obesity in children }\end{array}$ \\
\hline \multicolumn{3}{|l|}{ - M of Education } \\
\hline $\begin{array}{l}\text { - Primary healthcare centres, } \\
\text { Municipalities, Schools }\end{array}$ & & $\begin{array}{l}\text { - Training of health staff about } \\
\text { obesity and food/nutrition related } \\
\text { issues }\end{array}$ \\
\hline
\end{tabular}


Table 9.1. Continued

\begin{tabular}{llll}
\hline Country & Name of the food and nutrition policy document & Year of elaboration & Year of implementation \\
\hline Romania & National Plan of Food for Nutrition & 2009 & $2009-2010$
\end{tabular}

\begin{tabular}{llll}
\hline Slovenia & $\begin{array}{l}\text { Resolution on the national nutritional policy } \\
\text { programme 2005-2010 }\end{array}$ & 2005 & 2005
\end{tabular}

programme 2005-2010

\begin{tabular}{llll}
\hline Spain & 1. NAOS (Spanish strategy for nutrition, physical & $1.2004-2005$ & 1.2005 \\
& activity and obesity prevention) & 2.2008 & 2.2008
\end{tabular}

2. Estrategia de Seguridad Alimentaria (Food safety and Nutrition Strategy ) 2008-2012 
Institutions involved in the elaboration: leader and others

$-\mathrm{M}$ of Health
Institutes involved in the coordination

$-M$ of Health

Examples of implementation

- Surveillance and monitoring the dietary intake and nutritional status of population

- Implementation of the programmes in order to correct micronutrient deficiencies and prevent their occurrence

- Dissemination of nutrition information, nutrition education in school and at national levels to provide information concerning the nutritional value, information on proper food preparation, information about risk factors that affect nutritional status

- M of Health, Institute for Public Health - M of Health, Institute for Public Health

- For Health

- New study programs for nutrition and dietetics

- New dietary surveys

- For Food Science and Nutrition

- National Consumer Organization

1. M of Health and Consumer Affairs and corresponding institutions: the Spanish Agency of Food Safety and Nutrition and the General Directorate of Public Health

2. Spanish Agency of Food Safety and Nutrition

1. M of Education and Science, $M$ of Agriculture, Fisheries and Food / Independent experts in food, nutrition and physical activity; professional associations, Spanish Society of Community Nutrition (PERSEO), Mediterranean Diet Foundation (Caterer programmes) / Consumer organizations, teachers associations, town planners, publicists./ Private sector (food and drink companies, distribution companies, restaurant chains)

2. M of Health and Consumer Affairs, M of Agriculture, Fisheries and Food
1. M of Health and Consumer Affairs and corresponding institutions: the Spanish Agency of Food Safety and Nutrition and the General Directorate of Public Health 2. Spanish Agency of Food Safety and Nutrition

1. Implementation and evaluation): Autonomous communities, city halls (FEMP). Food and drink industry (FIAB, ANEDA), Catering chains (ANGED, ASEDAS), teachers, urban planners, publicists, foundations, Parent associations, Catering industry (FEHR. FEHRCAREM), bakers associations (CEOPAN)

2. $M$ of Agriculture, Fisheries and Food; $M$ of Public Administration; M of the Treasury; M of Internal Affairs; $M$ of Industry, Tourism and Commerce; $M$ of Science and Innovation; M of Education, Social policy and Sports (Interinstitutional collaboration)
1. Involvement of the food industry: development of the publicity and marketing code PAOS. This is a code for the self-regulation of the food industry in their food publicity targeting minors to comply with obesity prevention and health promotion principles, implemented in 2005 / School based actions: Programa PERSEO. Model pilot obesity prevention programme on health and exercise targeting Spanish schoolchildren. Implemented in 2006 at 67 centers in six Spanish autonomous regions and Ceuta and Melilla

2. Legislation enacted in various areas of food safety, traceability and labelling. / Design and implementation of the 'Total Diet' study 
Table 9.1. Continued

\begin{tabular}{|c|c|c|c|}
\hline Country & Name of the food and nutrition policy document & Year of elaboration & Year of implementation \\
\hline Sweden & $\begin{array}{l}\text { A better life through diet and physical activity. Nordic } \\
\text { Plan of Action on better health and quality of life } \\
\text { through diet and physical activity }\end{array}$ & 2006 & \\
\hline $\begin{array}{l}\text { The } \\
\text { Netherlands }\end{array}$ & $\begin{array}{l}\text { 1. Healthy Nutrition from start to finish Policy } \\
\text { document on nutrition and health } \\
\text { 2. Nota Overgewicht. Uit balans: de last van } \\
\text { overgewicht } \\
\text { 3. Nota voedselveiligheid: Veilig voedsel voor } \\
\text { iedereen: een gezamenlijke } \\
\text { verantwoordelijkheid' (=food safety) }\end{array}$ & $\begin{array}{l}\text { 1. } 2008 \\
\text { 2. } 2009 \\
\text { 3. } 2005\end{array}$ & $\begin{array}{l}\text { 1. } 2008-2011 \\
\text { 2. from } 2009 \text { on } \\
\text { 3. } 2005-2009 \\
\text { (new 'Nota } \\
\text { voedselveiligheid' } \\
\text { expected this year) }\end{array}$ \\
\hline
\end{tabular}

in their development and coordination, as well as the implementation mechanisms undertaken. The main institutions responsible for developing policy documents were mostly ministries. In some countries (the Czech Republic, Denmark, Greece, Latvia, Lithuania, Norway, Portugal, Poland, Romania and Spain) the Ministry of Health was the lead institution. In Estonia the Ministry of Social Affairs was the one responsible for this task. The Health Department of the 'Ministry of Labour, Health and Welfare' was the governmental body in charge of delivering the National Health Plan in Italy. In Austria, Finland, The Netherlands and Germany, the FNP was developed by the Ministry of Health together with the Ministry of Agriculture. In Slovenia the Ministry of Health and the Institute for Public Health developed the documents. In France the Interministerial Nutrition Policy Committee (PNNS) and the Ministry of Health worked together to chair the policy document. In Hungary the National Institute for Food and Nutrition Science was the lead institution. Regarding The Nordic Action Plan on Health, Food and Physical Activity, the Nordic Council of Ministers were the lead institution, in collaboration with national institutions in the Nordic countries.

\section{FNP as an Intersectorial Approach}

Most of the countries worked within an intersectorial approach, with several organizations collaborating in the development of the FNP. Some partners reported the collaboration of other ministries (the Czech Republic, Denmark, Estonia, Finland, France, Greece, Hungary, Latvia, Lithuania, The Netherlands, Norway, Portugal, 


\begin{tabular}{|c|c|c|}
\hline $\begin{array}{l}\text { Institutions involved in the } \\
\text { elaboration: leader and others }\end{array}$ & Institutes involved in the coordination & Examples of implementation \\
\hline $\begin{array}{l}\text { - Nordic Council of Ministers } \\
\text { - National Food Administration (M of } \\
\text { Agriculture), National Public Health } \\
\text { Institute (M of Health and Social Affairs) }\end{array}$ & & - Common monitoring \\
\hline $\begin{array}{l}\text { 1. M of Health, Welfare and Sport }+M \text { of } \\
\text { Agriculture, Nature and Food Quality } \\
\text { 2. M of Health, Welfare and Sport } \\
\text { 3. M of Agriculture, Nature and Food } \\
\text { Quality }\end{array}$ & $\begin{array}{l}\text { - Institution that coordinates differs per } \\
\text { action, see policy documents } \\
\text { 1. Among others M of Health, Food and } \\
\text { Consumer Product Safety Authority, RIVM, } \\
\text { TNO, Health Council, ZonMw, FNLI } \\
\text { (industry), Nutrition Centre }\end{array}$ & $\begin{array}{l}\text { - Monitoring of food consumption; } \\
\text { consumer education on a healthy } \\
\text { dietary pattern } \\
\text { - Internet site for professionals by } \\
\text { 'Centrum Gezond Leven'; } \\
\text { 'Nationaal Actieplan Sport en }\end{array}$ \\
\hline $\begin{array}{l}\text {-M of Health, Welfare and Sport; M of } \\
\text { Agriculture, Nature and Food Quality; } \\
\text { M of Economic Affairs } \\
\text { - Food and Consumer Product Safety } \\
\text { Authority (VWA); Netherlands Nutrition } \\
\text { Centre }\end{array}$ & $\begin{array}{l}\text { 2. Among others Health Council, Nutrition } \\
\text { Centre, Consumer Organisation, NOC NSF, } \\
\text { partners in 'Convenant Overgewicht' } \\
\text { 3. Among others Food and Consumer } \\
\text { Product Safety Authority, industry }\end{array}$ & Bewegen' \\
\hline
\end{tabular}

Spain and Sweden), scientific societies (Austria, the Czech Republic, Estonia, Greece, Hungary, Latvia, Lithuania, Norway, Poland, Spain and Slovenia), consumer organizations (Austria, the Czech Republic, Estonia, Finland, France, Greece, Latvia, Lithuania, Portugal, Spain and Slovenia), and NGOs (Estonia, Greece and Lithuania). Other institutions, such as associations of producers or the food industry (Austria, the Czech Republic, Finland, France, Greece, Spain and Slovenia), local governments (Austria, Estonia, Germany, Italy and Spain), representatives from universities, schools, or hospitals (Estonia, Finland, Greece, Latvia and Portugal), primary healthcare centers (Portugal) and the Food and Consumer Product Safety Authority (Austria and The Netherlands), were also involved in developing the FNP.

\section{Examples for FNP Implementation Actions}

Most of the countries provided some examples that indicated ongoing implementation actions (table 9.1).

A coordination mechanism existed in all countries except for Hungary, Poland and Sweden. The Ministry of Health was responsible for the coordination in most of the countries. The food industry took part in the coordination mechanism for certain countries (Austria, Finland, France, Greece, Italy, The Netherlands, Spain and Poland).

Fig. 9.1 shows the priority areas addressed in the FNP of the participating countries. Most of them included the promotion of optimal healthy nutrition (all countries 


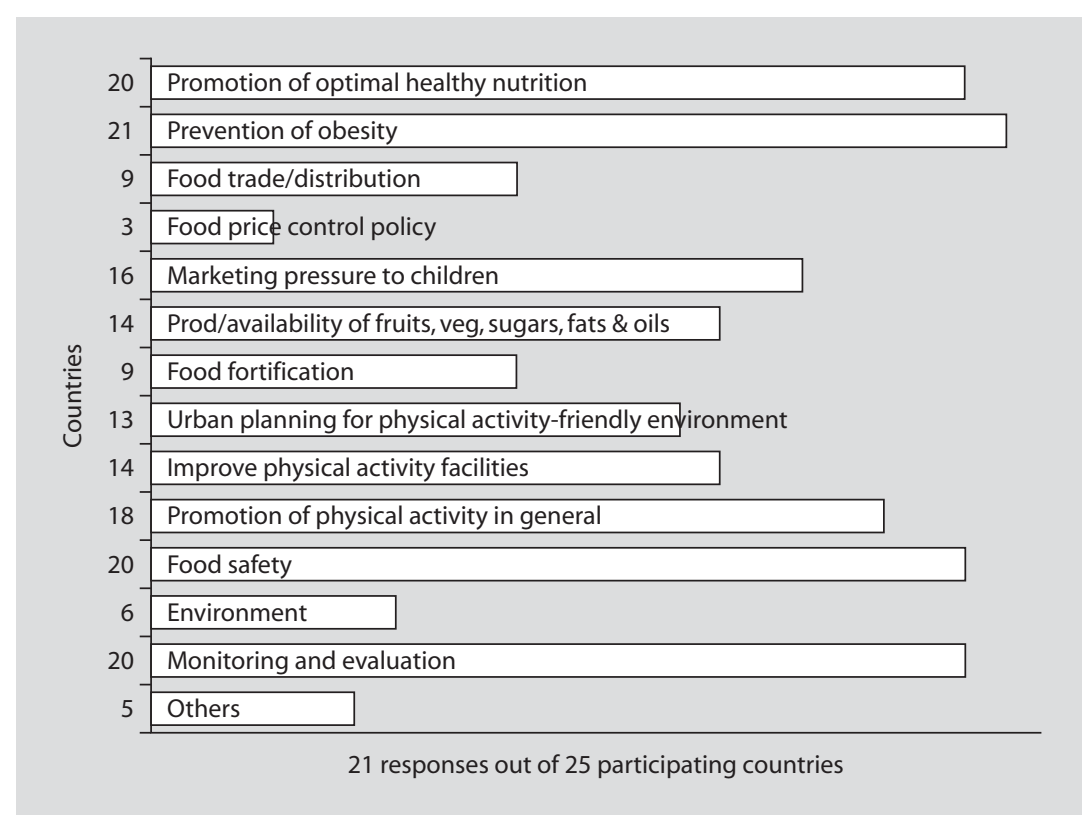

Fig. 9.1. Policy areas covered by the FNP.

except Italy), the prevention of obesity, the promotion of physical activity (PA) in general (except Finland and Slovenia), or through improving PA facilities (except Estonia, Finland, Hungary, Norway, Portugal and Romania), or through promoting an urbanfriendly PA environment (only in Austria, the Czech Republic, France, Greece, Italy, Latvia, The Netherlands and Poland). Certain economic and agricultural aspects were also included as a principal area covered by the FNP. For instance, food trade and distribution were included in the policy documents of Austria, Denmark, Finland, France, Hungary, Italy, Lithuania, Norway and Spain. Food price controls were included in the FNP of Finland, Hungary and Norway. The production and availability of certain products such as fruits, vegetables, sugars, fats or oils were also included in the documents of all the participating countries except for Estonia, Germany, Poland, Romania, Spain and Sweden. Food safety was also a main area of concern in all the FNP of all countries except Germany. Marketing pressure to children was regarded in all the documents except those from Denmark, Germany, Hungary, Lithuania and Poland. Certain aspects of the health consequences of environmental modification, such as regulation on genetically-modified organisms, were included in the FNP of Austria, the Czech Republic, Italy, Lithuania, Spain and Slovenia. Food fortification was included as an area of concern in the FNP of Austria, the Czech Republic, Italy, Lithuania, The Netherlands, Norway, Poland and Romania. Mandatory food fortification with iodine in salt was reported in Austria, the Czech Republic, Denmark (and 
also iodized salt used for making bread), Finland, Lithuania, Poland, Romania and Slovenia. In Finland and Sweden fortification of margarine with vitamins D and A, and vitamin D in milk products (only if liquid in Finland, only if $\leq 1.5 \%$ fat in Sweden) were mandatory. In Hungary, and Austria the fortification (vitamins and minerals) of baby fruit drinks, infant formulas for substituting breast milk, infant formulas after finishing breastfeeding, formulas for special medical treatment, special formulas for obesity treatment and some cereal-based baby foods had mandatory fortification requirements. It was not mandatory but rather recommended to fortify margarines with vitamins A and D in Hungary and The Netherlands, as well as for the addition of iodine to salt in The Netherlands and Spain.

\section{FNP, Quality Assurance, Objectives and Means}

Monitoring and evaluation was included as a principal policy area in all the participating countries except Slovenia.

Some countries included other areas of action. For instance, Austria included a National and European Alert Programme (food quality and safety). Denmark included other settings for promoting health (child-care institutions and schools, workplace and healthcare services). Greece included the application of food quality principles to improve mass catering services. Also Hungary included specific action areas for promoting healthy nutrition such as education in schools, improving the energy and nutrient content of foods and consumer information by improved food labeling as well as dietary surveys to check changes in energy and nutrient intake. Slovenia reported specific action areas that dealt with local products and organic food.

Only certain countries reported specific action areas related to agricultural policy, food fisheries and livestock production. Austria had incentives or subsidies for the production of milk and for the promotion of meat, meat products, sugar and organic foods. Finland had incentives or subsidies for the production of rapeseed oil and rye. Italy had incentives or subsidies for the production of organic foods and for the promotion of Italian products to be exported. Lithuania had incentives or subsidies for the production and promotion of vegetables that were ecological and sustainable. Certain types of incentives or subsidies for the promotion of fruits at school in Denmark were reported, as well as for the promotion of fruits and vegetables in Greece, the promotion of local food in Slovenia, and for the promotion of fruits and vegetables, fish and milk in Poland. Spain reported subsidies for the promotion of products having the category of Protected Designation of Origin. Austria, Denmark, Estonia, France, Hungary, Italy, The Netherlands, Norway, Romania and Spain had intersectorial collaborations between the parties responsible for food production, manufacturing and sales, control and legislation.

\section{FNP Addresses Health Issues}

Fig. 9.2 shows the main health challenges addressed by the FNP. Diet-related noncommunicable diseases and prevention of overweight and obesity were included in all the 
Fig. 9.2. Health challenges addressed by the FNP.

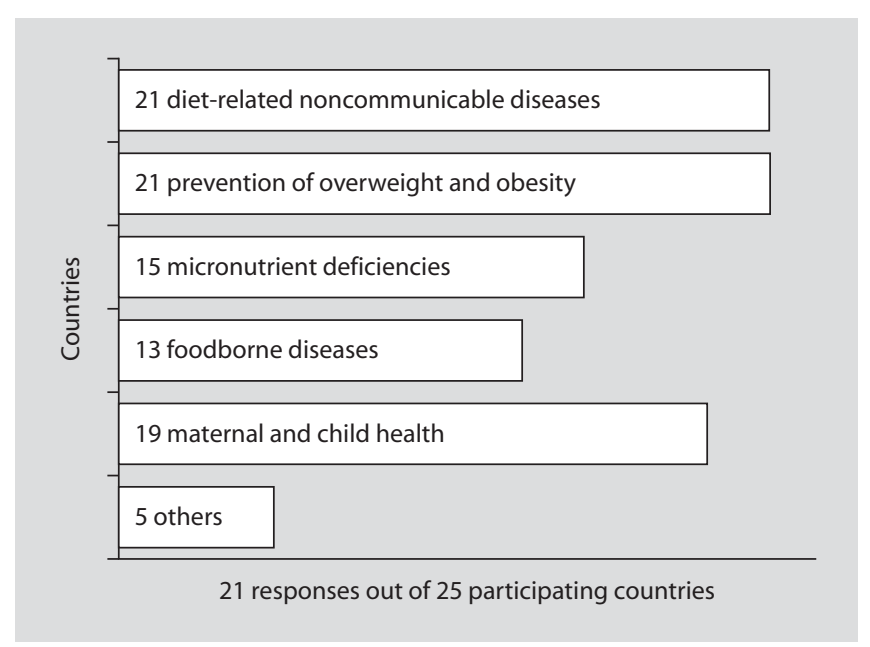

documents. All countries but Estonia, Germany, Portugal, Slovenia, Spain and Sweden included micronutrient deficiencies as a health concern of the FNP. Foodborne diseases were considered a main issue to address in all countries but Estonia, Finland, France, Germany, Norway, Romania and Sweden. Maternal and child health was not a health challenge cited in the FNP of Germany and Sweden. Some FNP addressed specific population groups: Hungary included the elderly, Austria and France the elderly and disadvantaged people and Lithuania included the elderly and hospitalized individuals, respectively, as target groups of interest in their health challenges. The promotion of adequate practices and the quality of ethnic foods were included in the Austrian FNP. Denmark included physical inactivity as a health challenge. Greece included action areas that dealt with nutritional disorders, oral health and adverse effects of alcohol consumption.

\section{FNP, National Dimension and the Global Action Plan}

Fig. 9.3 and 9.4 show the level of compliance of each country's FNP with the WHO Second Action Plan. In reference to the action area 'Supporting a healthy start', and the specific subareas cited in the WHO Second Action plan, only six countries, the Czech Republic, Denmark, Hungary, Latvia, The Netherlands and Romania, reported having a compulsory micronutrient supplementation program for pregnant women. Nevertheless, with the exception of Ireland, all the participating countries provided advice on diet and food safety to pregnant women (at the individual level by obstetricians in Greece). Pregnant women from low socioeconomic groups were specifically addressed in the FNP of Austria, the Czech Republic, France, Germany, Hungary, Italy, Lithuania, Norway and Slovenia, as well as in the documents from Ireland. Although breastfeeding was promoted in all the participating countries except for Estonia, the baby-friendly hospital initiative was not included in the FNP of Denmark 


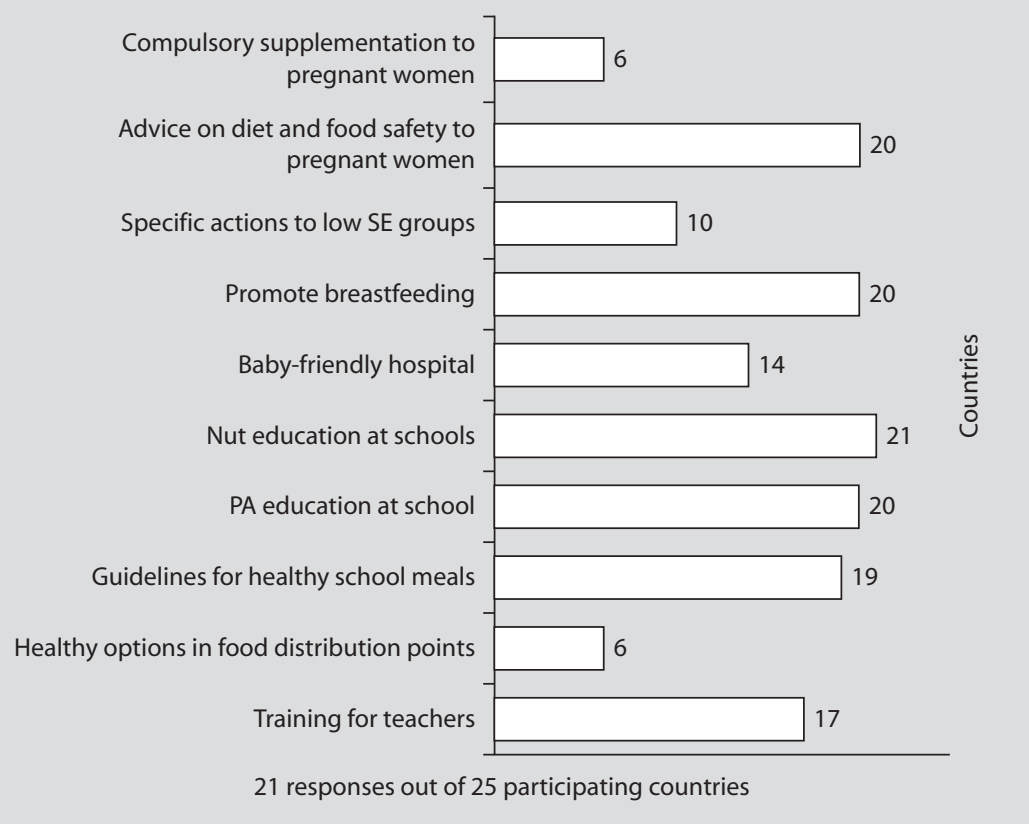

Fig. 9.3. Adherence to the WHO first action area 'Supporting a healthy start'.

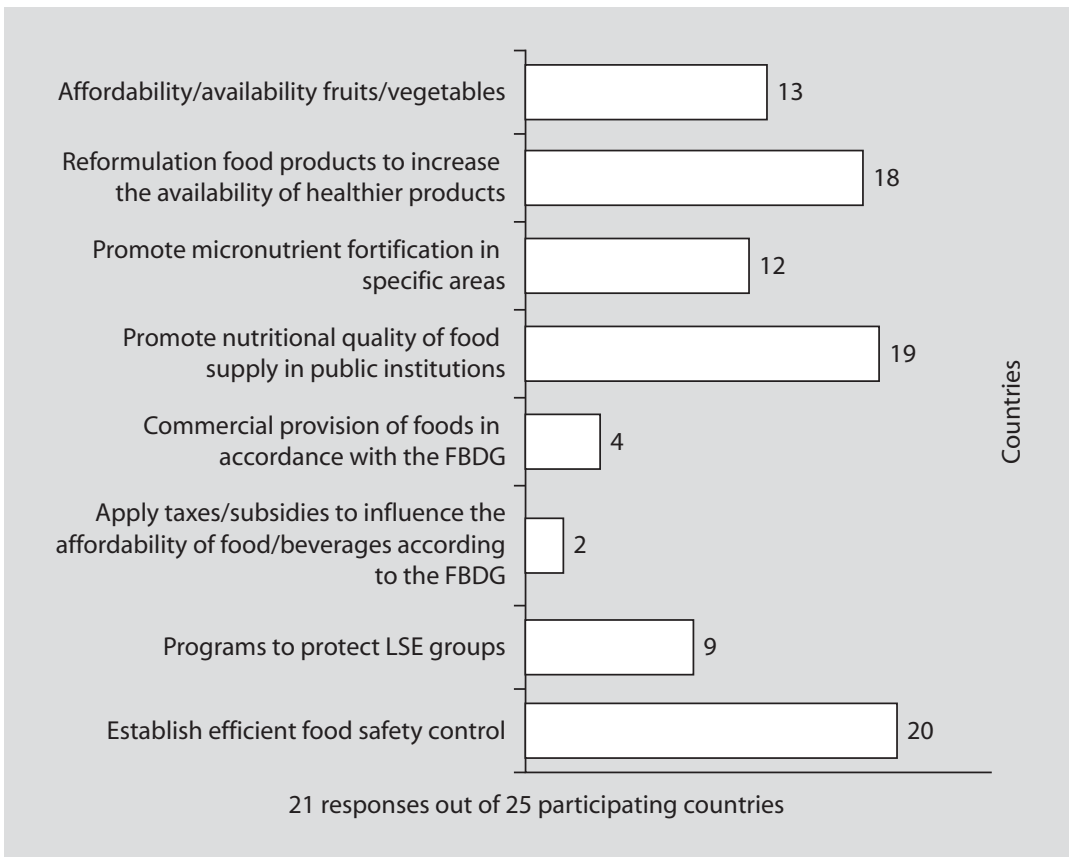

Fig. 9.4. Adherence to the WHO second action area 'Ensure a safe, healthy and sustainable food supply'. 
(although not included in the FNP they reported being adhered to the initiative), Finland, Germany, Greece (promoted, but not yet implemented), Italy, Norway and Spain (Estonia did not include this as well). The action areas addressing nutrition at schools showed that all countries, Italy (for PA), and Finland and Slovenia (for providing guidelines) included nutrition and PA education at schools, and provided guidance for developing guidelines for healthy school meals. In Italy, the catering enterprises are obliged to follow a protocol of best practice assuring food quality in order to be contracted to provide services to school canteens. In Greece, although not being mandatory, individual initiatives existed regarding nutrition-related topics at high schools. All countries but Finland, Hungary, Romania and Sweden provided training on nutrition for teachers and other school staff.

\section{FNP to Ensure Diet Quality}

Compliance with the second action area 'Ensure a safe, healthy and sustainable food supply' was as follows (fig. 9.4): all countries but Finland, Germany, Greece (planned but not yet implemented), Ireland, Norway, Romania, Spain and Sweden reported improving the affordability and availability of fruits and vegetables. In accordance with an EC recommendation, Denmark, Portugal and Italy reported including a fruit break at schools (in Portugal it was recently (2009) approved the provision of fruit to schoolchildren and should start next academic year). In Poland the action area will be implemented when the Common Agricultural Policy fruit strategy is undertaken. Certain types of food product reformulation were promoted to increase the availability of healthier products in all countries except Germany, Greece (planned but not yet implemented) and Lithuania. In Portugal and Spain a reduction of the salt content in bread was promoted. In certain countries, micronutrient fortification was promoted in areas where micronutrient deficiencies were a public health concern (Austria, the Czech Republic, Denmark, Finland, Ireland, Hungary, Lithuania, The

Netherlands, Poland, Romania, Spain and Sweden). Only Norway and Poland did not include a promotional action to assure the quality of the food supply in public institutions. Only Denmark, Hungary, Norway and Romania reported ensuring that the commercial provision of foods was in accordance with the FBDG, but only in Norway were taxes or subsidies applied to influence the affordability of foods recommended in their FBDGs. Low socioeconomic groups were target groups to be protected with certain subsidies or other types of support in Austria, France, Hungary, Ireland, Italy, Lithuania, The Netherlands, Poland and Slovenia. With respect to the specific action area to establish efficient food safety control, only Finland did not include actions for this area in its FNP.

The communication and information strategies developed to disseminate the FNP information/contents included FBDG (except Slovenia), consumer education campaigns, and the promotion of adequate food product labeling following the latest EU regulations (http://eur-lex.europa.eu/LexUriServ/LexUriServ.do?uri = COM:2008:0040:FIN:EN:PDF) (except Greece, Lithuania and Spain). Regarding food 
labeling, the information to be included on the package of the product included: the list of ingredients (except for Hungary and Poland), the best-before or use-by date of the product (except Hungary) and the presence of allergens (only in the Czech Republic, Denmark, Estonia, Finland, Germany, Greece, Hungary, Ireland, Lithuania, The Netherlands, Poland, Romania, Spain and Sweden). As for nutrition labeling, Denmark, Greece, Hungary, Ireland, Portugal and Sweden followed current EC regulations. Austria included information on the content of total fat, SFA, trans FA, energy and sugar; the Czech Republic included nutrition labeling for specified food products only, indicating the content of energy, protein, fat, saccharides, SFA, fiber and sodium; Estonia included information on energy, protein, fat, carbohydrates, fat, SFA, dietary fiber, and sodium; Germany only included information on the alcohol content of a product when it was over $1.2 \mathrm{vol} \%$; in Italy, Portugal and Lithuania nutrition labeling was mandatory only when there was a nutrition or health claim on the product; Norway included information on energy, carbohydrates, protein and fat.

\section{FNP for Health Promotion and Prevention of Chronic Diseases}

The action areas to prevent diet-related noncommunicable diseases included the promotion of PA, the reduction of alcohol consumption (not addressed in Germany and Spain, and only addressed to drivers and young people in Italy), and the reduction of soft drink consumption by assuring the provision of safe drinking water (in Austria, Denmark, France, Greece, Italy, Latvia, Lithuania, The Netherlands, Norway, Romania, Slovenia and Sweden).

Action areas to strengthen nutrition and food safety in health included the engagement of healthcare professionals in providing lifestyle counseling in Austria, the Czech Republic, Denmark, France, Germany, Greece, Ireland, Hungary, Italy, Lithuania, The Netherlands, Norway, Portugal, Romania, Slovenia and Spain. Also the quality of nutrition services and food safety in hospitals was addressed in the FNP of participating countries except for Estonia, Germany and Poland. Another area of action was to improve the process of prevention diagnosis and treatment of nutrition-related diseases, which was identified as an area of interest in all countries except Estonia, Italy and Slovenia.

Monitoring and evaluation was conducted in most of the participating countries albeit with different levels of application. There was a surveillance system on food availability in all countries except for Hungary, Ireland and Portugal. Surveillance of nutritional status was included in all but Denmark, Estonia, Greece, Hungary, Poland, Portugal, Slovenia, Spain and Sweden; food consumption monitoring occurred in all countries except for Greece, Hungary, Portugal and Slovenia. Estonia, Finland, Greece, Hungary, Poland, Portugal and Spain did not have a surveillance system for PA patterns. Excluding Ireland and Slovenia, all countries had a surveillance system for foodborne diseases and systems for monitoring microbial and chemical hazards. The level of evaluation carried out varied between countries: some of them evaluated impact measures (Austria, Denmark, Estonia, Greece, Italy, Latvia, The Netherlands, 
Poland, Romania and Spain) and others assessed outcomes (Denmark, Estonia, Greece, Hungary, Italy, Lithuania, The Netherlands, Norway, Romania, Spain and Sweden). Public and/or private research was supported in all countries except for Greece, Hungary, Ireland, Lithuania, Portugal and Slovenia.

\section{Forms and Implementation of FNP}

Food-based dietary guidelines were an implementation tool existing in all participating countries except for Ireland and Slovenia. Norway, Romania and Sweden were working on an updated version of their existing guidelines. The Dutch FBDG was the most recently updated (2009) and Greece had the oldest version (although published in 1999, the Greek guidelines are based on the traditional Mediterranean diet and the evidence for its beneficial effect is repeatedly and constantly confirmed). Most countries reported a periodical revision of their FBDG (except for the Czech Republic, Greece, Italy, Poland, Portugal, Spain and Sweden). The FBDGs were the result of intersectorial collaborations between several institutions in Austria (the Ministries of Health, Education, Agriculture, the University and the Foundation for a Healthy Austria, the Austria Agency for Health and Food Security), in the Czech Republic (the Ministry of Health and the Czech Nutrition Society), in Greece (the Ministry of Health and the Supreme Health Council of the Hellenic Ministry of Health), in Hungary (the Ministry of Health together with the National Institute for Food and Nutrition Science, and a Committee of Experts called up by this Institute), in Norway (the Ministry of Health and the National Nutrition Council), in Portugal (the Portuguese Consumers Institute and the Faculty of Nutrition and Food Sciences from Porto University) and in Spain (the Ministry of Health, Scientific Societies and Autonomous Communities). The channels used to disseminate and foster the implementation of the FBDG included: information at the point of purchase (Austria, Denmark, Germany, Greece, Hungary, Norway, Romania, Spain and Sweden), mass media campaigns (all except The Netherlands, Portugal and Sweden), educational tools for schools (all except for Romania) and primary care settings (all but Estonia and Germany). The sectors involved in the implementation of the guidelines were health professionals in all countries but Estonia, school teachers in all of them but Finland, The Netherlands, Romania and Spain, nutritionists (except Estonia), the industry (except in Estonia, France, Hungary, Latvia, Lithuania, The Netherlands, Portugal and Spain), and consumer organizations (except for Estonia, France, Latvia, Lithuania, The Netherlands, Poland and Spain). In Hungary the dissemination of FBDG booklets took place during health education conferences and meetings; in Poland academics collaborated in the dissemination of the information. The data used to develop the FBDG were mainly individual food consumption data (Austria, Denmark, Estonia, Finland, France, Greece, Hungary, Italy, Latvia, Lithuania, The Netherlands, Norway, Romania, Spain and Sweden), household food consumption data (the Czech Republic, Estonia, Finland, Greece, Hungary, Latvia, Norway, Poland, Portugal, Romania and Spain), and national food supply data (the Czech Republic, Estonia, Finland, France, Hungary, Latvia, Norway, Poland, Portugal and Romania). Only Austria and Spain 
included all the target population groups in their FBDG (general population, children, adolescents, pregnant women, adults, elderly and immigrants). Estonia, France, The Netherlands, Romania and Sweden included all the population groups except for immigrants, and Norway excluded the elderly and immigrants; the FBDG from Italy and Hungary included all target groups with the exception of adolescents and immigrants (the elderly were not included in the FBDG of Hungary). In Latvia, all the population groups but pregnant women and immigrants were included as target groups. The FBDG of the Czech Republic, Greece and Lithuania included information for the general population and adults, and those of Denmark for the general population and children. Germany, Poland and Portugal only targeted the general population (although Portugal indicated some information on nutrient needs for different age groups), and Finland focused on adults.

The pyramid was the graphic most countries applied to represent their FBDG (Austria, the Czech Republic, Estonia, Germany, Greece, Italy, Latvia, Lithuania, Poland, Romania and Spain). Some countries included specific written guidelines, tables, recommendations or other graphical representations such as the plate model (Austria, the Czech Republic, Germany, The Netherlands, Norway, Poland, Portugal, Romania and Sweden) or the food compass (Denmark). Most of the FBDG included information on food safety (except for those from Austria, Estonia, Finland, Germany, Greece, Lithuania, Poland, and Portugal) and all of them but Sweden included PA recommendations.

\section{Discussion}

The WHO Nutrition and Food Security Programme encourages and supports the WHO member states to develop sound and sustainable FNPs (http://www.euro.who. int/nutrition/20070808_1). For that purpose from 2000 to 2005 WHO assisted countries and their governments in establishing a FNP that followed guidelines provided by experts on food policies, emphasizing the necessity to include collaborative action amongst stakeholders in the development of the document. The present report summarizes information on FNPs from countries participating in ENHR 2009, with a focus on FBDG and food fortification. The WHO documents 'Comparative analysis on nutrition policies in the WHO European Region' published in 1998 and in 2006 [WHO, 1998, 2006] were used as tools for comparative reference. The present evaluation summarizes the results obtained from a research activity that was addressed within the framework of the ENHR 2009. Therefore, it is subject to certain limitations, such as those who answered the questionnaire may not have been those who were involved in FNP development in their country, and may not have had access to all the information needed to thoroughly complete the questionnaire.

All the participating countries had FNP strategies or action plans addressing the main areas of interest related to health and nutrition. However, in some cases these do not constitute a 'real' policy as they are not issued by a single body and actions are 
distributed throughout a variety of distinct documents. Twelve countries reported having new or updated FNP after the WHO 2006 compilation document: Estonia with the National Health development plan 2009-2020 and the National cancer strategy 2007-2015; Germany with their National Action Plan on the prevention of unhealthy eating, lack of exercise, overweight and related diseases titled 'IN FORM' - Germany's Initiative for a healthy diet and more exercise; Greece with two recently developed documents, the National Action Plan for nutrition and nutritional disorders 20082012 and the National Action Plan for the promotion of Breastfeeding; Hungary with the National Nutrition Policy of Hungary; Italy with the National Health Plan 2006-2008; Lithuania with the State Food and Nutrition Strategy and Action Plan for 2003-2010; The Netherlands with the Healthy Nutrition from start to finish Policy document on nutrition and health and the 'Nota Overgewicht. Uit balans: de last van overgewicht'; Norway with the Norwegian Action Plan on Nutrition (2007-2011) Recipe for a healthier diet, launched by the Government in January 2007; Poland with the National Programme for the prevention of overweight, obesity and noncommunicable diseases through diet, physical activity and the National Health Programme; Portugal with the 'Programa Nacional de Intervenção Integrada sobre Determinantes da Saúde Relacionados com Estilos de Vida'/National Programme on Health-Related Lifestyles Determinants (2003), the 'Programa de Saúde Escolar e Promoção de uma Alimentação Saudável em meio escolar'/School Health programme and Healthy food promotion within schools (2006), and the 'Plataforma contra a obesidade'/Platform against Obesity (2007); Romania with the National Plan of Food for Nutrition; and the Nordic Countries with the document 'A better life through diet and physical activity, Nordic Plan of Action on better health and quality of life through diet and physical activity. The Austrian Action Plan on nutrition is in the process of being developed.

An optimal FNP should comprehend issues related to nutrition and health, food safety and food production. Most of the FNP included in this report cover all these aspects. However, in certain countries, Food Safety was not included in the FNP although the inclusion of specific policies or legislative framework dealing with these issues was verbally confirmed by respective partners. Moreover, some countries classified FNPs as subcategories of their National Health Plan (e.g. Italy).

Several recommendations were set by WHO in its comparative analysis when working on country specific FNP: to improve healthy lifestyles in the population and specifically among disadvantaged groups (immigrants, low socioeconomic groups); to encourage collaboration between all the sectors involved in the food chain system to manage a sustainable food production and distribution; to assure the correct implementation of the action plans, and to establish a monitoring system to evaluate the effectiveness of the policy.

Regarding actions addressing the promotion of healthy lifestyles, governments have realized that the responsibility for adopting healthy food habits and practicing PA does not only rest on the individual. Most of the countries analyzed in the report referred to specific initiatives for improving the environment to enhance population- 
based efforts to adopt a healthier lifestyle. With reference to disadvantaged population groups, much work remains to be done as only a few countries included specific actions for these segments of the population so as to assure that everyone has the same possibilities for adopting adequate lifestyles.

Intersectorial collaborations were employed for the development of the FNP in almost all the participating countries, although with different levels of involvement in terms of the number of stakeholders representing the different institutions. The network of collaborations should include the private sector, including the agricultural and food manufacturing sector, the marketing and distribution sectors as well as the health sector, the educational sector and NGOs. This enhances the attainment of a comprehensive network that assures that no one from the food chain is excluded, and that all target groups are included in the process.

A monitoring system exists in all the participating countries. However, one of the aspects that need further discussion when addressing issues related to monitoring is the identification of indicators that should be used to monitor a FNP, which would reflect its adequate implementation and application. As previously stated, a FNP has three fundamental pillars: food safety, food production and nutrition and health. How each should be monitored, so as to facilitate a clear overview of how European countries are facing nutrition-related health challenges remains an unresolved question. For the time being, this report together with more detailed information obtained from other ongoing European projects such as the HOPE project (http://www.hopeproject.eu/) and the PREVOB project (see http://www.lshtm.ac.uk/hsru/prevob//) will serve as an overview of how European countries are currently facing the public health challenges related to nutrition and diet.

\section{Acknowledgements}

The authors would like to thank Rocio Ortiz (University of Alicante) and Trudy Wijnhoven (WHO Euro) for their advice on the development of the questionnaire, and the ENHR 2009 partners who responded and collaborated in compiling information and completing the questionnaire. 


\section{General Discussion}

\section{Introduction}

For an easier comparison of the 25 European countries participating in the present report, four regions were distinguished: North (Denmark, Estonia, Finland, Latvia, Lithuania, Norway, Sweden), West (Belgium-Luxembourg, France, Ireland, The Netherlands, the UK), Central and East (Austria, the Czech Republic, Germany, Hungary, Poland, Romania, Slovenia) and South (Cyprus, Greece, Italy, Portugal, Spain). However, it has to be kept in mind that some of these regions are made up of countries with rather different dietary patterns and food preferences despite a general trend to a modern so-called Westernized diet. This is particularly true for the Central and Eastern region of the European Union encompassing nations as different as Germany and Austria on the one hand and Romania and Bulgaria on the other. Besides cultural differences, the economic gap between these countries alone entails differences in food choice. The other regions are more homogeneous in this regard, although discrepancies between single countries exist as well.

\section{Nutrition Surveillance Methods - Potential and Limitations}

The ENHR 2009 contains data on nutrition surveillance derived from Food Balance Sheets (FBS), Household Budget Surveys (HBS), and specifically designed individual nutrition surveys. As a novelty in the recent report, a chapter on data from individual nutrition surveys re-codified as consumption of food categories was included.

FBS of the Food and Agriculture Organization of the United Nations (FAO) provide detailed information on the patterns of a country's food supply from 1961 on, up to 2003. The total food supply is calculated from production, imports, stock changes and exports. For the determination of per capita average food supply, feed, seed, losses through processing, storage and transportation as well as waste and other uses are considered [FAO, 2001]. On the one hand, FBS allow reliable comparisons within one country and amongst countries, as the methods for obtaining data are completely harmonized. On the other hand, several limitations do exist. Food supply is not equal 
to the real intake of food. Moreover, they do not give any indication of different population groups (age and ethnic groups), ecological zones, geographical areas, or seasonal variations. They are in general higher than the actual intake [Elmadfa and Weichselbaum, 2004].

HBS as evaluated through the EU-supported DATA Food Networking (DAFNE) initiative gathered data on food availability on household level. Like FBS, the HBS enable comparisons within one and amongst different countries. By describing results on the household level, they enable a description of population subgroups defined by their demographic and socioeconomic characteristics [Elmadfa and Weichselbaum, 2004]. Food consumed in restaurants, canteens, and comparable establishments is not consistently available in the DAFNE database.

The database on individual food consumption has been compiled by the European Food Safety Authority (EFSA). Participating countries re-codified their latest and most recent dietary survey according to harmonized guidelines. This evaluation enables a view on how much of different food types were effectively consumed. As a limitation, it has to be mentioned that the methods used, as well as age groups and duration of surveillance, are inhomogeneous in this compilation. Furthermore, these data are limited to adults and were available for only 16 countries with Italy being the only representative of the Southern region.

Table 10.1 shows a comparison of food supply, food availability, and food consumption using Portuguese data from the year 2000. All across the selected categories, FBS consistently presented higher values than HBS. On average, considering all food groups HBS generated data on food availability (g/capita/day), which were about half the calculated food supply based on the FBS. In general, values assessed with HBS were lower than those reported with Food Frequency Questionnaires (FFQ), but exceptions were noticed for oils and fats, as well as for meat, potatoes, and sugar.

HBS and FFQ yielded equal results for energy and carbohydrate intake. However, HBS lipids $(+29 \%)$ were above and protein $(-15 \%)$ as well as alcohol $(-27 \%)$ below the values from the FFQ. For energy and all macronutrients, FBS generated the higher values than HBS and FFQ (table 10.2) [Rodriguez et al., 2007].

\section{Food Supply, Availability and Consumption in European Regions}

Although the relation between supply, availability and consumption was not consistent across the described food categories, the three surveillance methods mostly agreed with differences between the ENHR 2009 regions. FBS, HBS and food consumption yielded highest values for fruits and vegetables in the South region and low values for the North region. For fruit and vegetable juices highest values appeared in the Central and East regions. Vegetable oils were most common in the South region. The recommendation to eat at least $400 \mathrm{~g}$ of vegetables and fruit per day was only achieved in four countries, Austria, Germany, Italy and Poland with the latter reporting the 
Table 10.1. Portuguese average food and beverages supply/consumption/availability derived from FBS, HBS and FFQ (g/capita/day) (\%E)

\begin{tabular}{llll}
\hline & FBS & HBS & FFQ \\
\hline Milk and diary products & $587(10.0)$ & $263(10.4)$ & $389(11.5)$ \\
Meat and offal & $266(11.4)$ & $161(13.2)$ & $146(16.1)$ \\
Fish and seafood & $153(2.2)$ & $83(4.5)$ & $91(6.3)$ \\
Eggs & $27(1.0)$ & $10(0.7)$ & $12(0.9)$ \\
Cereals & $369(27.9)$ & $221(34.6)$ & $260(26.3)$ \\
Potatoes and other starchy roots & $352(6.2)$ & $137(4.7)$ & $136(8.4)$ \\
Vegetables & $493(3.1)$ & $133(1.9)$ & $189(2.8)$ \\
Fruits & $341(4.2)$ & $202(4.8)$ & $297(7.4)$ \\
Oils and fats & $77(17.3)$ & $42(14.5)$ & $16(5.5)$ \\
Sugar and sweeteners & $94(8.4)$ & $26(4.2)$ & $23(3.8)$ \\
Alcoholic beverages & $338(3.6)$ & $112(3.4)$ & $192(5.1)$ \\
\hline
\end{tabular}

Table is limited to selected food groups which were comparable. Hence, the sum of $\% E$ from the food groups does not reach $100 \%$

Source: Rodriguez et al. [2007].

Table 10.2. Portuguese average supply/availability/consumption of energy and macronutrients derived from FBS, $\mathrm{HBS}$, and FFQ

\begin{tabular}{llll}
\hline & FBS & HBS & FFQ \\
\hline Energy, kcal & 3,751 & 2,249 & 2,199 \\
Protein, g (\%E) & $118(12.6)$ & $85(16.3)$ & $100(18.4)$ \\
Lipids, g (\%E) & $140(33.5)$ & $93(34.0)$ & $72(29.3)$ \\
Carbohydrates, g (\%E) & $468(46.8)$ & $266(46.7)$ & $268(48.9)$ \\
Alcohol, g (\%E) & $38(7.1)$ & $11(3.1)$ & $15(4.6)$ \\
\hline
\end{tabular}

Source: Rodriguez et al. [2007].

highest consumption according to the individual consumption data from the EFSA. When fruit and vegetable juices were included, Hungary and Belgium also reached the recommended amount, albeit only just. In this context, it is interesting to consider that programs to enhance the consumption of fruit and vegetables, namely the 5-aday campaign, are in action in some of these countries but also in those reporting low intakes such as Norway and France. Denmark, where fruit and vegetable intake was also rather low even has a 6-a-day campaign, while in Poland, so far, there is no program at all. 
Furthermore, FBS, HBS and consumption data agreed that for sugar and sugar products, a north-south gradient could be observed. In addition, results from the DAFNE database indicated that the availability of fish and seafood was related to costs and proximity to the sea. Highest values for milk and milk products were found in the North and West regions where milk traditionally plays an important role in nutrition. However, cheese consumption was lowest in the countries of the region North and highest in Italy followed by Germany. Overall, supply of milk and milk products based on FAO food balance sheets increased over the last four decades in all regions of the European Union but the North where it stayed quite constant. The largest rise in milk supply was seen in the South. This region also experienced the sharpest increase in meat supply that was less marked in the other regions. Over the same period, fish and seafood supply also increased in all regions albeit to different degrees. Highest levels were reached in the North and South regions. The latter showed a particular rise in minimum supply resulting in a narrowing of the gap between maximum and minimum levels. These trends led to a higher contribution of animal products to total energy supply in the South while in the other regions, no major changes in this regard were observed except for a sharp rise in Romania. This development mirrors the economic development in the concerned countries.

\section{Trends in Food Supply}

Concerning trends in food supply several changes could be observed. Since 1961, the average supply of fruits increased in the EU from 68 to $94 \mathrm{~kg} /$ capita/year. Likewise the supply of vegetables increased from 82 to $119 \mathrm{~kg} / \mathrm{capita} /$ year. Outstandingly high increases in the supply during the last four decades were observed for poultry meat (5-21 kg/capita/year), pork (22-42 kg/capita/year), and beer (41-80 kg/capita/year). Declines were assessed for potatoes (104-85 kg/capita/year), pulses (4-3 kg/capita/ year), and wine (28-23 kg/capita/year).

Based upon the FBS a trend for the proportion of macronutrients was calculated. During the past four decades the proportion of protein $(12 \% \mathrm{E})$ remained almost unchanged. However, the proportion of fat $(30-35 \% \mathrm{E})$ has increased and the proportion of carbohydrates $(58-53 \% \mathrm{E})$ has decreased.

\section{Reported Daily Energy and Macronutrient Intake in the EU Regions}

The reported daily intake levels of energy and nutrients show a wide variation across the 25 European countries having participated in this report and also within regions. Nevertheless, some regional characteristics appear across all age groups. The most consistent fact was that contribution of carbohydrate to reported total energy intake was highest in Northern as well as Central and Eastern Europe and lowest in Southern 
regions. In turn, in these latter, reported total fat intake was highest except for the elderly. The South also showed the highest intake of monounsaturated fatty acids (MUFA) in accordance with the predominant use of olive oil in the Mediterranean diet. In Northern, Central and Eastern Europe, bread and potatoes traditionally have an important place in nutrition possibly contributing to the reported comparatively high carbohydrate intake. This is supported by the observation that Northern countries also showed the lowest sucrose intake while it was highest in the South. The Central and Eastern region showed the highest average intake of saturated fatty acids (SFA) as well as total fat in the elderly. However, looking at the single countries, it becomes apparent that the very high SFA intake of Romanian subjects (26.3 and $24.8 \% \mathrm{E}$ in adult men and women, respectively) contributed the major part of this value, while the other countries had a SFA intake close to average. Moreover, the high total fat intake of the South was largely accounted for by the Greek collective while Portugal showed the lowest of all participants. This may exemplify the inhomogeneity of the regions in many a way.

\section{Selected Micronutrients}

However, average folate equivalents intake was below the recommended level in all the participating countries, the only exception being seen for Estonian men between 19 and 64 years. Austria, Hungary and The Netherlands showed the lowest reported intake levels in all age groups while intake was best in the Baltic States (data for adults only), Italy, Portugal and Denmark. For children, the South showed the highest intake levels of most vitamins with exception of vitamin D. For all age groups, reported intake of this vitamin was markedly higher in the Northern region, especially in the Scandinavian countries characterized by a traditionally high consumption of fatty fish and fish liver. This is mirrored in a low prevalence of vitamin D deficiency and a good overall biochemical status in Northern populations [Ovesen et al., 2003; Meyer et al., 2004]. Mean supply of vitamins $B_{6}$ and $B_{12}$ was generally sufficient in all regions and countries with the latter even much above the reference level. In the Central and Eastern region, namely Austria, Germany, Hungary and Poland, intake of vitamin E was above average. Interestingly, a reported high intake does not necessarily entail a good biochemical status of a nutrient. Indeed, in elderly Austrians aged $\geq 70$ years, intake of folate equivalents was much below the recommended level while that for the vitamins $B_{6}$ and $B_{12}$ was closer to the references. Nevertheless, a higher percentage of people showed inadequate status of these latter two than of folate $(32,47$ and $10 \%$, respectively; see figure in the Austrian National Report).

Reported calcium intake was also generally high in the Northern region, albeit again mainly in the Scandinavian countries and particularly for men. The Central and Eastern region had lower intakes except for Germany situated on the upper end, with lowest levels found in Poland and the Czech Republic. The Northern countries 
also showed a rather high reported iodine intake although the paucity of data on this trace element does not allow an in-depth analysis of regional differences. Indeed, consumption of both, fish and milk was high in this region [see chapter on 'Food Availability' and Gil et al., 1999] and this diet pattern is mirrored in the micronutrient intake. In turn, the Central and East region had the highest iron intake that was lowest in the North. Here again, the very high intake levels of two countries, Romania and the Czech Republic, contributed the major part.

\section{Overweight/Obesity, Physical Activity and Health Indicators in the EU}

Concerning overweight and obesity, the Northern and Western regions showed the lowest average prevalence. Especially, obesity was lower. However, with Finland and the UK, each of both regions also contained a country with a very high percentage of overweight and obese subjects, once again suggesting a high degree of inhomogeneity within the regions. The Southern region had the highest overweight and obesity rates for both genders, although they were low in Italians and Spanish women.

For countries having participated in both European Nutrition and Health Reports and provided data on overweight and obesity, it was possible to compare the prevalence in both years 2004 and 2009. The development showed a gender-specific difference with an average increase of overweight and obesity rates in men and a slight decrease and a stagnation, respectively, in women. Despite great differences between countries, less of them showed increases in women than in men. Higher rates of obesity were more common than of overweight. Considering that data are available for two years only and do not come from the same collective, it is difficult to draw conclusions from these results. Generally, prevalence of overweight is lower in women than in men, but this difference is less apparent for obesity.

The most likely cause for overweight are imbalances in energy metabolism resulting from an excessive intake and/or an insufficient expenditure. However, as the data for body weight and energy intake used in this report do not necessarily come from the same surveys and are therefore from different samples, they cannot be put in relationship to each other. Physical activity (PA) was assessed in completely separate studies. Thus, only careful assumptions could be made. Previous surveys on PA in Europe show a comparatively high level in the Nordic countries and The Netherlands [European Commission, 2004, 2006; Rütten and Abu-Omar, 2004]. Interestingly, in the current report, many of these countries also had lower percentages of persons with excessive body weight whereas energy intake was not lower. However, a comparable association cannot be seen for all countries.

Concerning the data on health indicators, many of the limitations encountered previously still exist in the current report. Data on blood lipids in adults were available for only eight of the participating countries and for only six of them in elderly. Besides, Finland, Romania and the UK did not supply the full range of parameters. 
Information on alcohol consumption and smoking is also not universally available. Again, differences in survey design limit the comparability.

Data on total mortality in 2007 from the WHO's European Health for All database were available for ten countries with additional information about cardiovascular and cancer-related death cases for nine participating countries. In turn, data on incidence of some common malignant neoplasms in 2002 as well as diabetes mellitus in 2007 exist for all countries. These were obtained from the IARC's (International Agency for Research on Cancer) GLOBOCAN database and the International Diabetes Federation (IDF), respectively.

Overall, the European Nutrition and Health Report 2009 gives an overview of the nutritional situation and some important health indicators. However, comparability of these data is limited and there is still a need for harmonization in study design and methodologies. 
Annex

Annex table 1. Classification of body weight in children and adolescents of the European countries according to Cole et al. [2000] and Cole et al. [2007], body height and body weight self-reported; $\mathrm{n}=23,517$

\begin{tabular}{|c|c|c|c|c|c|}
\hline Country & Age years & Underweight \% & Normal range \% & Overweight \% & Obese \% \\
\hline \multicolumn{6}{|l|}{ Female } \\
\hline \multirow[t]{2}{*}{ Austria } & $10-14$ & 15 & 64 & 20 & 2 \\
\hline & $15-18$ & n.a. & n.a. & 9 & 2 \\
\hline Belgium & $15-18$ & 12 & 88 & 0 & 0 \\
\hline \multirow[t]{4}{*}{ Denmark } & $4-6$ & 0 & 84 & 14 & 2 \\
\hline & $7-9$ & 0 & 79 & 17 & 4 \\
\hline & $10-14$ & 0 & 87 & 11 & 2 \\
\hline & $15-18$ & 5 & 86 & 8 & 1 \\
\hline \multirow[t]{2}{*}{ Estonia } & $10-14$ & 59 & 39 & 2 & 0 \\
\hline & $15-18$ & 25 & 72 & 3 & 1 \\
\hline Italy & $11-15$ & n.a. & n.a. & 8 & 2 \\
\hline \multirow[t]{3}{*}{ Latvia } & 11 & n.a. & n.a. & 5 & 1 \\
\hline & 13 & n.a. & n.a. & 4 & 1 \\
\hline & 15 & n.a. & n.a. & 6 & 0 \\
\hline \multirow[t]{3}{*}{ Lithuania } & 11 & 21 & 73 & 6 & 1 \\
\hline & 13 & 23 & 74 & 3 & 1 \\
\hline & 15 & 21 & 75 & 3 & 1 \\
\hline \multirow[t]{3}{*}{ Norway } & $4-6$ & 13 & 75 & 9 & 4 \\
\hline & $7-9$ & 11 & 71 & 12 & 4 \\
\hline & $10-14$ & 12 & 77 & 10 & 1 \\
\hline \multirow[t]{3}{*}{ Sweden } & $4-6$ & n.a. & 82 & 16 & 2 \\
\hline & $7-9$ & n.a. & 80 & 17 & 3 \\
\hline & $10-14$ & n.a. & 83 & 16 & 1 \\
\hline \multicolumn{6}{|l|}{ Male } \\
\hline \multirow[t]{2}{*}{ Austria } & $10-14$ & 13 & 76 & 10 & 1 \\
\hline & $15-18$ & n.a. & n.a & 14 & 3 \\
\hline Belgium & $15-18$ & 6 & 83 & 10 & 1 \\
\hline
\end{tabular}


Annex table 1. Continued

\begin{tabular}{|c|c|c|c|c|c|}
\hline Country & Age years & Underweight \% & Normal range \% & Overweight \% & Obese \% \\
\hline \multirow[t]{4}{*}{ Denmark } & $4-6$ & 0 & 87 & 11 & 3 \\
\hline & $7-9$ & 0 & 85 & 10 & 4 \\
\hline & $10-14$ & 0 & 88 & 10 & 2 \\
\hline & $15-18$ & 0 & 85 & 14 & 1 \\
\hline \multirow[t]{2}{*}{ Estonia } & $10-14$ & 50 & 44 & 5 & 1 \\
\hline & $15-18$ & 18 & 76 & 5 & 1 \\
\hline Germany & $15-18$ & 5 & 72 & 14 & 9 \\
\hline Italy & $11-15$ & n.a. & n.a. & 23 & 4 \\
\hline \multirow[t]{3}{*}{ Latvia } & 11 & n.a. & n.a. & 9 & 2 \\
\hline & 13 & n.a. & n.a. & 10 & 1 \\
\hline & 15 & n.a. & n.a. & 7 & 1 \\
\hline \multirow[t]{3}{*}{ Lithuania } & 11 & 10 & 75 & 14 & 1 \\
\hline & 13 & 12 & 79 & 8 & 1 \\
\hline & 15 & 8 & 83 & 8 & 1 \\
\hline \multirow[t]{3}{*}{ Norway } & $4-6$ & 16 & 76 & 5 & 3 \\
\hline & $7-9$ & 8 & 74 & 15 & 3 \\
\hline & $10-14$ & 8 & 80 & 9 & 3 \\
\hline \multirow[t]{3}{*}{ Sweden } & $4-6$ & n.a. & 81 & 15 & 4 \\
\hline & $7-9$ & n.a. & 81 & 16 & 3 \\
\hline & $10-14$ & n.a. & 85 & 13 & 2 \\
\hline
\end{tabular}

n.a. = Not available. 
Annex table 2. Classification of body weight in female children and adolescents of the European countries according to Cole et al. [2000] and Cole et al. [2007], body height and body weight measured; $\mathrm{n}=59,401$

\begin{tabular}{|c|c|c|c|c|c|}
\hline Country & Age years & Underweight \% & Normal range \% & Overweight \% & Obese \% \\
\hline \multicolumn{6}{|l|}{ Female } \\
\hline \multirow[t]{2}{*}{ Austria } & $7-9$ & 4 & 71 & 17 & 7 \\
\hline & $10-14$ & 10 & 66 & 20 & 4 \\
\hline \multirow[t]{4}{*}{ Belgium } & $4-6$ & 9 & 78 & 9 & 4 \\
\hline & $7-9$ & 10 & 73 & 13 & 4 \\
\hline & $10-14$ & 13 & 72 & 13 & 3 \\
\hline & $15-18$ & 10 & 74 & 12 & 3 \\
\hline \multirow[t]{4}{*}{ France } & $4-6$ & 9 & 69 & 19 & 4 \\
\hline & $7-9$ & 10 & 69 & 19 & 2 \\
\hline & $10-14$ & 5 & 79 & 12 & 5 \\
\hline & $15-17$ & 13 & 72 & 11 & 5 \\
\hline \multirow[t]{4}{*}{ Germany } & $3-6$ & 5 & 86 & 6 & 3 \\
\hline & $7-10$ & 9 & 77 & 9 & 6 \\
\hline & $11-13$ & 8 & 73 & 12 & 7 \\
\hline & $14-17$ & 6 & 77 & 8 & 9 \\
\hline \multirow[t]{6}{*}{ Greece } & 12 & n.a. & 91 & 8 & 1 \\
\hline & 13 & n.a. & 87 & 11 & 2 \\
\hline & 14 & n.a. & 87 & 11 & 2 \\
\hline & 15 & n.a. & 84 & 15 & 1 \\
\hline & 16 & n.a. & 84 & 14 & 2 \\
\hline & 17 & n.a. & 77 & 21 & 2 \\
\hline \multirow[t]{2}{*}{ Hungary } & $7-14$ & 7 & 67 & 20 & 6 \\
\hline & 15-19 & 7 & 83 & 8 & 3 \\
\hline Italy & $8-9$ & n.a. & n.a. & 24 & 11 \\
\hline \multirow[t]{3}{*}{ Latvia } & $7-9$ & 9 & 78 & 11 & 2 \\
\hline & $10-14$ & 7 & 82 & 9 & 2 \\
\hline & $15-17$ & 10 & 81 & 9 & 1 \\
\hline
\end{tabular}


Annex table 2. Continued

\begin{tabular}{|c|c|c|c|c|c|}
\hline Country & Age years & Underweight \% & Normal range \% & Overweight \% & Obese \% \\
\hline \multirow[t]{4}{*}{ Poland } & $3-6$ & 20 & 63 & 10 & 7 \\
\hline & $7-9$ & 19 & 64 & 15 & 3 \\
\hline & $10-14$ & 15 & 73 & 10 & 2 \\
\hline & $15-18$ & 11 & 80 & 8 & 1 \\
\hline \multirow[t]{3}{*}{ Portugal } & $3-6$ & n.a. & n.a. & 20 & 7 \\
\hline & $7-9$ & n.a. & n.a. & 21 & 12 \\
\hline & 13 & 4 & 71 & 19 & 6 \\
\hline Slovenia & $15-18$ & 10.0 & 73.6 & 16 & 4 \\
\hline \multirow[t]{2}{*}{ Spain } & $10-14$ & 0 & 64 & 30 & 6 \\
\hline & $15-18$ & 8 & 74 & 19 & 0 \\
\hline $\begin{array}{l}\text { The } \\
\text { Netherlands }\end{array}$ & $4-6$ & 8 & 78 & 10 & 4 \\
\hline \multirow{2}{*}{$\begin{array}{l}\text { United } \\
\text { Kingdom }\end{array}$} & $2-10$ & n.a. & n.a. & 13 & 13 \\
\hline & $11-15$ & n.a. & n.a. & 16 & 17 \\
\hline
\end{tabular}

${ }^{1}$ BMI P3, P10, P90, P97: sex and age group-specific percentile according to Kromeyer-Hauschild et al. [2001]; n.a. = not available. 
Annex table 3. Classification of body weight in male children and adolescents of the European countries according to Cole et al. [2000] and Cole et al. [2007], body height and body weight measured; $\mathrm{n}=60,268$

\begin{tabular}{|c|c|c|c|c|c|}
\hline Country & Age years & Underweight \% & Normal range \% & Overweight \% & Obese \% \\
\hline \multicolumn{6}{|l|}{ Male } \\
\hline \multirow[t]{2}{*}{ Austria } & 7-9 & 4 & 66 & 23 & 7 \\
\hline & $10-14$ & 5 & 71 & 16 & 7 \\
\hline \multirow[t]{4}{*}{ Belgium } & $4-6$ & 12 & 79 & 7 & 2 \\
\hline & $7-9$ & 9 & 78 & 9 & 3 \\
\hline & $10-14$ & 12 & 74 & 12 & 3 \\
\hline & $15-18$ & 9 & 78 & 9 & 3 \\
\hline \multirow[t]{4}{*}{ France } & $4-6$ & 8 & 80 & 8 & 5 \\
\hline & $7-9$ & 9 & 79 & 9 & 3 \\
\hline & $10-14$ & 10 & 70 & 18 & 2 \\
\hline & $15-17$ & 8 & 76 & 13 & 4 \\
\hline \multirow[t]{4}{*}{ Germany } & $3-6$ & 5 & 86 & 6 & 3 \\
\hline & $7-10$ & 7 & 77 & 9 & 7 \\
\hline & $11-13$ & 10 & 72 & 11 & 7 \\
\hline & $14-17$ & 7 & 76 & 9 & 8 \\
\hline \multirow[t]{6}{*}{ Greece } & 12 & n.a. & 86 & 11 & 3 \\
\hline & 13 & n.a. & 81 & 16 & 3 \\
\hline & 14 & n.a. & 82 & 14 & 4 \\
\hline & 15 & n.a. & 74 & 20 & 6 \\
\hline & 16 & n.a. & 66 & 29 & 5 \\
\hline & 17 & n.a. & 59 & 34 & 8 \\
\hline \multirow[t]{2}{*}{ Hungary } & $7-14$ & 5 & 69 & 18 & 7 \\
\hline & 15-19 & 7 & 73 & 16 & 4 \\
\hline Italy & $8-9$ & n.a. & n.a. & 23 & 13 \\
\hline \multirow[t]{3}{*}{ Latvia } & $7-9$ & 14 & 75 & 9 & 2 \\
\hline & $10-14$ & 13 & 76 & 9 & 2 \\
\hline & $15-17$ & 14 & 80 & 4 & 2 \\
\hline
\end{tabular}


Annex table 3. Continued

\begin{tabular}{|c|c|c|c|c|c|}
\hline Country & Age years & Underweight \% & Normal range \% & Overweight \% & Obese \% \\
\hline \multirow[t]{4}{*}{ Poland } & $3-6$ & 20 & 59 & 13 & 9 \\
\hline & $7-9$ & 8 & 70 & 16 & 5 \\
\hline & $10-14$ & 12 & 69 & 17 & 2 \\
\hline & $15-18$ & 5 & 78 & 14 & 3 \\
\hline \multirow[t]{3}{*}{ Portugal } & $3-6$ & n.a. & n.a. & 17 & 7 \\
\hline & $7-9$ & n.a. & n.a. & 19 & 10 \\
\hline & 13 & 5 & 68 & 21 & 7 \\
\hline Slovenia & $15-18$ & 4.1 & 77 & 19 & 4 \\
\hline \multirow[t]{2}{*}{ Spain } & $10-14$ & 6 & 60 & 31 & 3 \\
\hline & $15-18$ & 0 & 88 & 10 & 2 \\
\hline $\begin{array}{l}\text { The } \\
\text { Netherlands }\end{array}$ & $4-6$ & 9 & 80 & 8 & 3 \\
\hline \multirow{2}{*}{$\begin{array}{l}\text { United } \\
\text { Kingdom }\end{array}$} & $2-10$ & n.a. & n.a. & 12 & 17 \\
\hline & $11-15$ & n.a. & n.a. & 15 & 18 \\
\hline
\end{tabular}

${ }^{1}$ BMI P3, P10, P90, P97 sex and age group-specific percentile according to Kromeyer-Hauschild et al. [2001]; n.a. = not available. 
Annex table 4. Classification of body weight in men and women, body height and body weight self-reported; $n=86,936$

\begin{tabular}{llllll}
\hline Country & Age years & Underweight $\%$ & Normal range $\%$ & Overweight $\%$ & Obese \% \\
\cline { 2 - 5 } & $<18.50 \mathrm{~kg} / \mathrm{m}^{2}$ & $18.50-24.99 \mathrm{~kg} / \mathrm{m}^{2}$ & $25.00-29.99 \mathrm{~kg} / \mathrm{m}^{2}$ & $\geq 30.00 \mathrm{~kg} / \mathrm{m}^{2}$ \\
\hline
\end{tabular}

\section{Female}

\begin{tabular}{|c|c|c|c|c|c|}
\hline Austria & $19-64$ & 4 & 66 & 22 & 9 \\
\hline Belgium & $19-63$ & 4 & 62 & 21 & 13 \\
\hline Denmark & $19-64$ & 4 & 64 & 22 & 10 \\
\hline Estonia & $19-63$ & 5 & 52 & 24 & 18 \\
\hline \multirow[t]{3}{*}{ Germany ${ }^{1}$} & $18-29$ & 7 & 72 & 16 & 4 \\
\hline & $30-39$ & 2 & 56 & 28 & 11 \\
\hline & $40-65$ & 1 & 30 & 40 & 27 \\
\hline \multirow[t]{2}{*}{ Hungary } & $18-34$ & 9 & 69 & 14 & 9 \\
\hline & $35-59$ & 2 & 43 & 35 & 20 \\
\hline Ireland & $18-64$ & 3 & 57 & 27 & 13 \\
\hline Italy & $18+$ & n.a. & n.a. & 28 & 9 \\
\hline Lithuania & $19-63$ & 3 & 48 & 30 & 19 \\
\hline Norway & $19-63$ & 3 & 66 & 24 & 7 \\
\hline Poland & $19-63$ & 4 & 61 & 28 & 7 \\
\hline Portugal & $20-64$ & 3 & 51 & 30 & 15 \\
\hline Sweden & $19-64$ & 3 & 56 & 27 & 14 \\
\hline \multirow{3}{*}{$\begin{array}{l}\text { The } \\
\text { Netherlands }\end{array}$} & $20-25$ & 7 & 74 & 14 & 5 \\
\hline & $25-45$ & 3 & 64 & 23 & 10 \\
\hline & $45-65$ & 1 & 54 & 30 & 14 \\
\hline \multicolumn{6}{|l|}{ Male } \\
\hline Austria & $19-64$ & 1 & 47 & 39 & 13 \\
\hline Belgium & $19-63$ & 2 & 45 & 41 & 13 \\
\hline Denmark & $19-64$ & 1 & 50 & 39 & 11 \\
\hline Estonia & $19-63$ & 1 & 43 & 40 & 17 \\
\hline \multirow[t]{3}{*}{ Germany ${ }^{1}$} & $18-29$ & 2 & 65 & 29 & 4 \\
\hline & $30-39$ & 0 & 36 & 48 & 15 \\
\hline & $40-65$ & 0 & 19 & 56 & 24 \\
\hline
\end{tabular}


Annex table 4. Continued

\begin{tabular}{|c|c|c|c|c|c|}
\hline \multirow[t]{2}{*}{ Country } & \multirow{2}{*}{ Age years } & \multirow{2}{*}{$\frac{\text { Underweight \% }}{<18.50 \mathrm{~kg} / \mathrm{m}^{2}}$} & \multirow{2}{*}{$\begin{array}{l}\text { Normal range } \% \\
18.50-24.99 \mathrm{~kg} / \mathrm{m}^{2}\end{array}$} & \multirow{2}{*}{$\begin{array}{l}\text { Overweight } \% \\
25.00-29.99 \mathrm{~kg} / \mathrm{m}^{2}\end{array}$} & \multirow{2}{*}{$\begin{array}{l}\text { Obese } \% \\
\geq 30.00 \mathrm{~kg} / \mathrm{m}^{2}\end{array}$} \\
\hline & & & & & \\
\hline \multirow[t]{2}{*}{ Hungary } & $18-34$ & 2 & 58 & 33 & 7 \\
\hline & $35-59$ & n.a. & 40 & 39 & 21 \\
\hline Ireland & $18-64$ & 2 & 41 & 42 & 15 \\
\hline Italy & $18+$ & n.a. & n.a. & 44 & 11 \\
\hline Lithuania & $19-63$ & 1 & 42 & 36 & 21 \\
\hline Norway & $19-63$ & 0 & 54 & 40 & 6 \\
\hline Poland & $19-63$ & 0 & 53 & 38 & 10 \\
\hline Portugal & $20-64$ & 1 & 44 & 42 & 14 \\
\hline Sweden & $19-64$ & 0 & 43 & 45 & 12 \\
\hline \multirow{3}{*}{$\begin{array}{l}\text { The } \\
\text { Netherlands }\end{array}$} & $20-25$ & 3 & 78 & 18 & 1 \\
\hline & $25-45$ & 1 & 53 & 38 & 9 \\
\hline & $45-65$ & 1 & 39 & 48 & 13 \\
\hline
\end{tabular}

${ }^{1}$ Underweight: $<19.00 \mathrm{~kg} / \mathrm{m}^{2}$, normal range: $19.00-24.99 \mathrm{~kg} / \mathrm{m}^{2}$, overweight $25.00-<27.50 \mathrm{~kg} / \mathrm{m}^{2}$, obese: $27.5-<30 \mathrm{~kg} / \mathrm{m}^{2}$; n.a. = not available. 
Annex table 5. Classification of body weight in men and women, body height and body weight measured; $\mathrm{n}=73,926$

\begin{tabular}{llllll}
\hline Country & Age years & Underweight \% & Normal range \% & Overweight $\%$ & Obese $\%$ \\
\cline { 2 - 5 } & $<18.50 \mathrm{~kg} / \mathrm{m}^{2}$ & $18.50-24.99 \mathrm{~kg} / \mathrm{m}^{2}$ & $25.00-29.99 \mathrm{~kg} / \mathrm{m}^{2}$ & $\geq 30.00 \mathrm{~kg} / \mathrm{m}^{2}$ \\
\hline
\end{tabular}

Female

\begin{tabular}{|c|c|c|c|c|c|}
\hline Cyprus & $19-64$ & 10 & 38 & 26 & 26 \\
\hline $\begin{array}{l}\text { Czech } \\
\text { Republic }\end{array}$ & $19-64$ & 1 & 38 & 33 & 28 \\
\hline Finland & $25-64$ & & 43 & 33 & 23 \\
\hline France & $19-64$ & 5 & 55 & 23 & 17 \\
\hline Germany & 19-64 & 2 & 53 & 27 & 18 \\
\hline Greece & 19-64 & 0 & 27 & 37 & 36 \\
\hline Ireland & 19-64 & 1 & 50 & 33 & 16 \\
\hline Latvia & $19-64$ & 3 & 47 & 33 & 17 \\
\hline Lithuania & $19-64$ & 3 & 51 & 31 & 15 \\
\hline Poland & $19-64$ & 4 & 52 & 27 & 17 \\
\hline Portugal & $18-64$ & 3 & 49 & 34 & 15 \\
\hline Romania & $19-64$ & n.a. & n.a. & 35 & 24 \\
\hline Spain & 19-64 & 3 & 56 & 28 & 13 \\
\hline \multirow{5}{*}{$\begin{array}{l}\text { United } \\
\text { Kingdom }\end{array}$} & $16-24$ & 7 & 62 & 20 & 12 \\
\hline & $25-34$ & 2 & 50 & 29 & 18 \\
\hline & $35-44$ & 1 & 45 & 30 & 24 \\
\hline & $45-54$ & 1 & 37 & 35 & 27 \\
\hline & $55-64$ & 1 & 33 & 36 & 30 \\
\hline \multicolumn{6}{|l|}{ Male } \\
\hline Cyprus & $19-64$ & 1 & 17 & 54 & 29 \\
\hline $\begin{array}{l}\text { Czech } \\
\text { Republic }\end{array}$ & $19-64$ & 0 & 25 & 47 & 28 \\
\hline Finland & $25-64$ & & 30 & 48 & 22 \\
\hline France & $19-64$ & 1 & 43 & 40 & 16 \\
\hline Germany & $19-64$ & 1 & 36 & 45 & 19 \\
\hline Greece & $19-64$ & 0 & 19 & 53 & 28 \\
\hline Ireland & $19-64$ & 0 & 33 & 46 & 20 \\
\hline
\end{tabular}


Annex table 5. Continued

\begin{tabular}{|c|c|c|c|c|c|}
\hline \multirow[t]{2}{*}{ Country } & \multirow{2}{*}{ Age years } & \multirow{2}{*}{$\frac{\text { Underweight \% }}{<18.50 \mathrm{~kg} / \mathrm{m}^{2}}$} & \multirow{2}{*}{$\begin{array}{l}\text { Normal range } \% \\
18.50-24.99 \mathrm{~kg} / \mathrm{m}^{2}\end{array}$} & \multirow{2}{*}{$\begin{array}{l}\text { Overweight \% } \\
25.00-29.99 \mathrm{~kg} / \mathrm{m}^{2}\end{array}$} & \multirow{2}{*}{$\begin{array}{l}\text { Obese } \% \\
\geq 30.00 \mathrm{~kg} / \mathrm{m}^{2}\end{array}$} \\
\hline & & & & & \\
\hline Latvia & 19-64 & 1 & 49 & 41 & 10 \\
\hline Lithuania & 19-64 & 1 & 40 & 49 & 11 \\
\hline Poland & 19-64 & 1 & 43 & 40 & 15 \\
\hline Portugal & 18-64 & 1 & 39 & 45 & 15 \\
\hline Romania & 19-64 & n.a. & n.a. & 22 & 26 \\
\hline Spain & 19-64 & 1 & 40 & 45 & 14 \\
\hline \multirow{5}{*}{$\begin{array}{l}\text { United } \\
\text { Kingdom }\end{array}$} & $16-24$ & 6 & 61 & 25 & 9 \\
\hline & $25-34$ & 0 & 38 & 41 & 21 \\
\hline & $35-44$ & 0 & 27 & 48 & 25 \\
\hline & $45-54$ & 0 & 24 & 48 & 28 \\
\hline & $55-64$ & 1 & 20 & 47 & 33 \\
\hline
\end{tabular}

n.a. = Not available. 
Annex table 6. Classification of body weight in elderly, body height and body weight self-reported; $n=27,874$

\begin{tabular}{llllll}
\hline Country Age years & Underweight $\%$ & Normal range $\%$ & Overweight $\%$ & Obese $\%$ \\
\cline { 2 - 5 } & $<18.50 \mathrm{~kg} / \mathrm{m}^{2}$ & $18.50-24.99 \mathrm{~kg} / \mathrm{m}^{2}$ & $25.00-29.99 \mathrm{~kg} / \mathrm{m}^{2}$ & $\geq 30.00 \mathrm{~kg} / \mathrm{m}^{2}$ \\
\hline
\end{tabular}

Female

\begin{tabular}{|c|c|c|c|c|c|}
\hline Austria & $65+$ & 3 & 40 & 35 & 22 \\
\hline Belgium & $65+$ & 4 & 44 & 38 & 15 \\
\hline Denmark & $65+$ & 4 & 49 & 35 & 12 \\
\hline Estonia & $65+$ & 2 & 30 & 39 & 30 \\
\hline Germany¹ & $65+$ & 1 & 19 & 44 & 29 \\
\hline Hungary & $60+$ & 0 & 36 & 40 & 23 \\
\hline Ireland & $65+$ & 4 & 49 & 35 & 12 \\
\hline Italy & $65+$ & n.a. & n.a. & 41 & 13 \\
\hline \multirow[t]{3}{*}{ Lithuania } & $65-74$ & 1 & 25 & 41 & 32 \\
\hline & $75-84$ & 1 & 39 & 40 & 20 \\
\hline & $85+$ & 4 & 56 & 29 & 11 \\
\hline Norway & $65+$ & 2 & 48 & 39 & 11 \\
\hline Poland & $65+$ & 0 & 46 & 46 & 8 \\
\hline Portugal & $65+$ & 3 & 38 & 38 & 21 \\
\hline Sweden & $65+$ & 0 & 48 & 41 & 11 \\
\hline $\begin{array}{l}\text { The } \\
\text { Netherlands }\end{array}$ & $65+$ & 2 & 45 & 37 & 16 \\
\hline \multirow{2}{*}{$\begin{array}{l}\text { United } \\
\text { Kingdom }\end{array}$} & $65-74$ & n.a. & n.a. & 38 & 35 \\
\hline & $75+$ & n.a. & n.a. & 42 & 27 \\
\hline
\end{tabular}

\section{Male}

\begin{tabular}{|c|c|c|c|c|c|}
\hline Austria & $65+$ & 0 & 22 & 60 & 18 \\
\hline Belgium & $65+$ & 1 & 42 & 45 & 12 \\
\hline Denmark & $65+$ & 1 & 40 & 47 & 12 \\
\hline Estonia & $65+$ & 1 & 40 & 43 & 17 \\
\hline Germany ${ }^{1}$ & $65+$ & 0 & 17 & 58 & 23 \\
\hline Hungary & $60+$ & n.a. & 24 & 55 & 33 \\
\hline Ireland & $65+$ & 1 & 37 & 45 & 17 \\
\hline Italy & $65+$ & n.a. & n.a. & 52 & 13 \\
\hline
\end{tabular}


Annex table 6. Continued

\begin{tabular}{|c|c|c|c|c|c|}
\hline \multirow[t]{2}{*}{ Country } & \multirow{2}{*}{ Age years } & \multirow{2}{*}{$\frac{\text { Underweight \% }}{<18.50 \mathrm{~kg} / \mathrm{m}^{2}}$} & \multirow{2}{*}{$\begin{array}{l}\text { Normal range } \% \\
18.50-24.99 \mathrm{~kg} / \mathrm{m}^{2}\end{array}$} & \multirow{2}{*}{$\begin{array}{l}\text { Overweight \% } \\
25.00-29.99 \mathrm{~kg} / \mathrm{m}^{2}\end{array}$} & \multirow{2}{*}{$\begin{array}{l}\text { Obese } \% \\
\geq 30.00 \mathrm{~kg} / \mathrm{m}^{2}\end{array}$} \\
\hline & & & & & \\
\hline \multirow[t]{3}{*}{ Lithuania } & $65-74$ & 0 & 39 & 46 & 15 \\
\hline & $75-84$ & 1 & 37 & 52 & 10 \\
\hline & $85+$ & 0 & 48 & 45 & 7 \\
\hline Norway & $65+$ & 1 & 42 & 45 & 12 \\
\hline Poland & $65+$ & 0 & 29 & 43 & 29 \\
\hline Portugal & $65+$ & 2 & 35 & 46 & 18 \\
\hline Sweden & $65+$ & 0 & 32 & 59 & 9 \\
\hline $\begin{array}{l}\text { The } \\
\text { Netherlands }\end{array}$ & $65+$ & 1 & 42 & 33 & 12 \\
\hline \multirow{2}{*}{$\begin{array}{l}\text { United } \\
\text { Kingdom }\end{array}$} & $65-74$ & n.a. & n.a. & 49 & 31 \\
\hline & $75+$ & n.a. & n.a. & 51 & 18 \\
\hline
\end{tabular}

${ }^{1}$ Underweight: $<19.00 \mathrm{~kg} / \mathrm{m}^{2}$, normal range: $19.00-24.99 \mathrm{~kg} / \mathrm{m}^{2}$, overweight $25.00-<27.50 \mathrm{~kg} / \mathrm{m}^{2}$, obese: $27.5-<30 \mathrm{~kg} / \mathrm{m}^{2} ;$ n.a. $=$ not available. 
Annex table 7. Classification of body weight in elderly, body height and body weight measured; $n=25,805$

\begin{tabular}{llllll}
\hline \multirow{2}{*}{ Country } & Age years & Underweight \% & Normal range \% & Overweight \% & Obese \% \\
\cline { 2 - 5 } & $<18.50$ & $18.50-24.99$ & $25.00-29.99$ & $\geq 30.00$ \\
\hline
\end{tabular}

\section{Female}

\begin{tabular}{|c|c|c|c|c|c|}
\hline Austria & $65+$ & 1 & 22 & 41 & 36 \\
\hline Cyprus & $65+$ & 10 & 38 & 26 & 26 \\
\hline Finland & $65+$ & n.a. & 25 & 40 & 34 \\
\hline France & $65-74$ & 3 & 41 & 35 & 22 \\
\hline Germany & $65+$ & 0 & 26 & 40 & 34 \\
\hline Greece & $65+$ & 0 & 11 & 37 & 52 \\
\hline Ireland & $65+$ & 0 & 30 & 37 & 33 \\
\hline Poland & $65+$ & 2 & 27 & 37 & 35 \\
\hline Portugal & $65+$ & 1 & 29 & 42 & 29 \\
\hline Spain & $65+$ & 1 & 19 & 47 & 33 \\
\hline \multicolumn{6}{|l|}{ Male } \\
\hline Austria & $65+$ & 0 & 8 & 52 & 40 \\
\hline Cyprus & $65+$ & 1 & 17 & 54 & 29 \\
\hline Finland & $65+$ & n.a. & 20 & 55 & 25 \\
\hline France & $65-74$ & 0 & 19 & 53 & 28 \\
\hline Germany & $65+$ & 0 & 17 & 54 & 29 \\
\hline Greece & $65+$ & 0 & 20 & 49 & 30 \\
\hline Ireland & $65+$ & 1 & 27 & 45 & 27 \\
\hline Poland & $65+$ & 2 & 35 & 45 & 18 \\
\hline Portugal & $65+$ & 2 & 27 & 52 & 19 \\
\hline Spain & $65+$ & 0 & 31 & 41 & 28 \\
\hline
\end{tabular}

n.a. $=$ Not available. 


\section{National Reports}

The responsibility for the information contained in this document lies with the authors of the respective National Report.

\subsection{Austria ${ }^{1}$}

Data on energy and nutrient intake in Austria were obtained from the Austrian Study on Nutritional Status that has been conducted on a regular basis since 1991. It includes several representative substudies focusing on different population groups or special aspects such as physical activity and fluid intake. Follow-up studies in 2007/2008 provided information about long-term trends of food and nutrient intake. The results of these surveys were published in March 2009 in the Austrian Nutrition Report 2008. An overview of the collectives and methods of the partial surveys can be seen in table 11.1. Slight differences from the respective data in the main report may arise from the use of diverging age groups in the present overview.

\section{Prevalence of Overweight and Other Health Indicators}

Overweight is a common health issue in Austria. Thus, in 19\% of schoolchildren aged 6 to 15 years, body weight exceeds the recommended ranges according to KromeyerHauschild et al. [2001]. Eight percent are obese. In high school students, prevalence is slightly lower (6\%), but reaches $16 \%$ in adolescents. Overweight becomes more frequent with increasing age: $31 \%$ of 18 - to 64 -year-olds are overweight and $11 \%$ obese. As many as $40 \%$ of the elderly aged between 65 and 100 years have a body mass index (BMI) above the reference level. However, in this latter group, 10\% are underweight. $26 \%$ of pregnant women are overweight or obese (see table 11.2).

A trend of increasing overweight can be observed across all age groups and for both genders. The number of obese male adults has doubled since 2003 (from 6 to $13 \%)$. Men and boys of all ages are more often overweight than women and girls. The previously observed West-to-East upward gradient is still apparent.

\footnotetext{
${ }^{1}$ Meyer A, Elmadfa I; University of Vienna, Vienna, Austria. E-Mail alexa.leonie.meyer@univie.ac.at
} 
Table 11.1. Studies and methods used for the assessment of nutritional status of the Austrian population (sample size is shown)

\begin{tabular}{lllll}
\hline & $\begin{array}{l}\text { Nutrition } \\
\text { surveys }\end{array}$ & $\begin{array}{l}\text { Anthropo- } \\
\text { metry }\end{array}$ & $\begin{array}{l}\text { Biochemical } \\
\text { analyses }\end{array}$ & $\begin{array}{l}\text { Eating } \\
\text { behavior }\end{array}$ \\
\hline $\begin{array}{l}\text { Schoolchildren } \\
(6-15 \text { years })\end{array}$ & $780^{\mathrm{a}}$ & $984^{\mathrm{c}}$ & - & - \\
\hline $\begin{array}{l}\text { High school attendants } \\
(14-19 \text { years) }\end{array}$ & $208^{\mathrm{b}}$ & $398^{\mathrm{d}}$ & - & - \\
\hline $\begin{array}{l}\text { Apprentices } \\
(14-19 \text { years })^{*}\end{array}$ & $1,990^{\mathrm{b}}$ & $2,823^{\mathrm{d}}$ & $143^{*}$ & - \\
\hline $\begin{array}{l}\text { Adults } \\
(18-65 \text { years })\end{array}$ & $2,123^{\mathrm{b}}$ & $2,310^{\mathrm{d}}$ & $133^{*}$ & - \\
\hline $\begin{array}{l}\text { Adults } \\
(18-65 \text { years })\end{array}$ & $459^{\mathrm{e}}$ & - & - & - \\
\hline $\begin{array}{l}\text { Adults } \\
(18-65 \text { years })\end{array}$ & $288^{\mathrm{f}}$ & - & - & - \\
\hline $\begin{array}{l}\text { Adults } \\
(18-65 \text { years })\end{array}$ & $719^{\mathrm{g}}$ & - & - & - \\
\hline $\begin{array}{l}\text { Adults } \\
(>55 \text { years })\end{array}$ & $423^{\mathrm{a}}$ & $816^{\mathrm{c}}$ & $102^{\mathrm{h}}$ & - \\
\hline \begin{tabular}{l} 
Pregnant women \\
\hline
\end{tabular} & $426^{\mathrm{b}}$ & $479^{\mathrm{d}}$ & $133^{*}$ & - \\
\hline
\end{tabular}

* Viennese sample (does not represent all of Austria).

a 3-day food protocol (estimate of food intake).

b 24 -hour recall.

' Measured data of body height and weight.

d Self-reported body height and weight (pooled data from the Adults' subsamples 18-65 years).

e 1-day-protocol of fluid intake (ÖSES.aqa07).

${ }^{f}$ Questionnaire for the assessment of nutrient intake from supplements (ÖSES.nem07).

${ }^{g}$ Questionnaire for the assessment of physical activity (ÖSES.pal07).

${ }^{\mathrm{h}}$ Sample from the Burgenland (does not represent all of Austria).

The analysis of plasma lipid profiles in a sample of 102 elderly aged $>70$ years from the eastern region of Austria (Burgenland) of Burgenland showed that most values were within the reference levels with the exception of total cholesterol in men and 70to 75 -year-old women as well as LDL cholesterol.

Hypertension is another common health risk. The Austrian Health Survey based on personal interviews revealed that $21.3 \%$ of Austrians over 15 years have a high blood pressure and as expected, prevalence increases with age. As for overweight, occurrence is higher in the eastern than in the western regions [Klimont et al., 2007]. 
Table 11.2. Body weight distribution in the Austrian elderly and pregnant women

\begin{tabular}{|c|c|c|c|c|}
\hline & \multicolumn{4}{|c|}{ BMI categories according to WHO 2003 (prevalence in \%) } \\
\hline & underweight & normal weight & overweight & obesity \\
\hline \multicolumn{3}{|l|}{ Elderly ${ }^{a, c}$} & \multicolumn{2}{|c|}{ overweight } \\
\hline Men & 7 & 54 & \multicolumn{2}{|c|}{40} \\
\hline Women & 12 & 48 & \multicolumn{2}{|c|}{40} \\
\hline $65-74$ years & 9 & 51 & \multicolumn{2}{|c|}{40} \\
\hline $75-84$ years & 10 & 57 & \multicolumn{2}{|c|}{33} \\
\hline$>84$ years & 18 & 51 & \multicolumn{2}{|c|}{31} \\
\hline Pregnant women ${ }^{\mathrm{b}}$ & 8 & 66 & 16 & 10 \\
\hline$<25$ years & 15 & 62 & 16 & 7 \\
\hline $25-35$ years & 6 & 67 & 16 & 11 \\
\hline$>35$ years & 9 & 64 & 17 & 10 \\
\hline \multicolumn{5}{|c|}{$\begin{array}{l}\text { a Calculated from measured data. } \\
\text { b Calculated from self-reported data. } \\
\text { c Categories for subjects aged }>65 \text { years according to National Research Council [NRC, 1989]: under- } \\
\text { weight: BMI }<24 \mathrm{~kg} / \mathrm{m}^{2} \text {, normal weight BMI } 24-29 \mathrm{~kg} / \mathrm{m}^{2} \text {, overweight BMI }>29 \mathrm{~kg} / \mathrm{m}^{2} \text {. }\end{array}$} \\
\hline
\end{tabular}

Frequently associated with overweight, diabetes mellitus affects $5.9 \%$ of Austrians. Again, the prevalence rises with the age of the subjects, reaching 18.6 and $22.6 \%$ in men and women over 75 years, respectively [Klimont et al., 2007]. Physical activity contributes significantly to health and the prevention and elimination of overweight. Using the International Physical Activity Questionnaire (IPAQ) on a sample of 719 Austrian adults, an average physical activity level (PAL) of 1.64 was found. Men reached a mean PAL of 1.71, women of 1.57. Especially young women showed lower levels and thus are at a higher risk for diseases associated with a sedentary lifestyle. A PAL of 1.7, as recommended by the World Health Organization, was achieved by $53 \%$ of the participating men, but only $23 \%$ of the women.

\section{Energy and Nutrient Intake}

In all age groups but women aged $>84$ years, mean energy intake of Austrians was below the recommended level based on a moderate PAL [D-A-CH, 2000]. However, with regard to the low PAL found in adults and the high prevalence of overweight, energy intake must be considered sufficient or even excessive.

This can also be said of protein intake which was within or slightly above the recommended levels in all groups. Thus, in schoolchildren it accounted for $14-15 \%$ of total energy intake (E\%), 14-16\% $\mathrm{E}$ in adolescents, 15 and 17\% $\mathrm{E}$ in adult women and men, respectively, and $15 \% \mathrm{E}$ in elderly of both genders. Recommended levels for 
Table 11.3. Mean daily intake of PUFA in Austrians by gender and age

\begin{tabular}{|c|c|c|c|c|c|c|c|}
\hline & \multicolumn{2}{|c|}{$\begin{array}{l}\text { Schoolchildren } \\
\text { (6-15 years) }\end{array}$} & \multicolumn{2}{|c|}{$\begin{array}{l}\text { Younger adults } \\
18-64 \text { years }\end{array}$} & \multicolumn{2}{|c|}{$\begin{array}{l}\text { Elderly } \\
>64 \text { years }\end{array}$} & \multirow[t]{2}{*}{$\begin{array}{l}\text { D-A-CH, } \\
2000\end{array}$} \\
\hline & $\mathrm{m}$ & $f$ & $\mathrm{~m}$ & $f$ & $\mathrm{~m}$ & $f$ & \\
\hline $\mathrm{LA}(\mathrm{n}-6-\mathrm{FA}), \mathrm{E} \%$ & 5.4 & 5.6 & 7 & 7 & 7 & 6 & 2.5 \\
\hline ALA (n-3-FA), E\% & 0.5 & 0.5 & 0.6 & 0.6 & 0.6 & 0.6 & 0.5 \\
\hline$A A(n-6), m g$ & 159 & 132 & 276 & 216 & 196 & 179 & - \\
\hline $\operatorname{EPA}(n-3), m g$ & 35 & 28 & 90 & 71 & 62 & 62 & - \\
\hline $\mathrm{DHA}(\mathrm{n}-6), \mathrm{mg}$ & 98 & 82 & 190 & 180 & 135 & 157 & - \\
\hline
\end{tabular}

$\mathrm{LA}=$ Linoleic acid; $\mathrm{ALA}=$ a-linolenic acid; $\mathrm{AA}=$ arachidonic acid; $\mathrm{EPA}=$ eicosapentaenoic acid; DHA = docosahexaenoic acid.

carbohydrate intake $(\geq 50 \% \mathrm{E})$ were only achieved by schoolchildren, in whom they supplied $50-51$ and $51-52 \% \mathrm{E}$ for boys and girls, respectively. In all other groups, carbohydrate intake was too low $(46-48 \% \mathrm{E}$ in adolescents, 46 and $43 \% \mathrm{E}$ in adult women and men, 46 and $41 \% \mathrm{E}$ in elderly women and men, respectively). The contribution of added sugar was generally rather high with highest levels found in children (16$18 \% \mathrm{E})$. In elderly, sucrose consumption was higher in women and in the eldest group of men. While values also encompass natural sources of monosaccharides like fruit, a high consumption of isolated sugar can nevertheless be assumed from the rather low intake of fruit in Austrian children. Dietary fiber intake was below the recommended levels as well.

In turn, fat intake was too high. Only in schoolchildren was it within the national D-A-CH recommendations for all age subgroups (up to 35\%E), supplying 33-35\%E. In the other age groups, it was $\geq 37 \% \mathrm{E}$ with the highest level reached by men $>84$ years $(43 \% \mathrm{E})$. Fat quality was not optimal either, with saturated fatty acids providing more than the allotted $10 \% \mathrm{E}$ (from $14 \% \mathrm{E}$ in schoolchildren and adult men to $20 \% \mathrm{E}$ in male adolescents). Intake of polyunsaturated ( $5-8 \% \mathrm{E})$ and monounsaturated fatty acids (11-14\%E) did not reach the levels recommended for cardiovascular health and therefore higher intake should be encouraged. Furthermore, while D-A-CH reference values were reached for both fatty acid classes, this was largely accounted for by $n-6$ fatty acids (mainly LA) that exceeded the recommendation of $2.5 \mathrm{E} \%$, while $\mathrm{n}-3$ fatty acids (ALA) were consumed to a lesser extent (table 11.3). Elderly men between 65 and 84 years showed the highest alcohol consumption compared to the other age groups. Cholesterol intake exceeded the allotted level in male adolescents and adults as well as elderly of both genders. It was highest in men $>84$ years $(433 \mathrm{mg}$ ). 
Table 11.4. Overview of critical micronutrients in the Austrian population

\begin{tabular}{|c|c|}
\hline Critical in: & Micronutrients \\
\hline Whole population & $\begin{array}{l}\text { folate equivalents, vitamin D, calcium, too high intake of } \\
\text { sodium }\end{array}$ \\
\hline School children (6-15 years) & $\begin{array}{l}\text { vitamin } A \text {, vitamin } B_{1} \text {, vitamin } B_{2}{ }^{a} \text {, vitamin } B_{6}{ }^{b} \text {, iodine, iron, } \\
\text { magnesium }{ }^{\mathrm{a}} \text {, potassium }{ }^{\mathrm{b} \neq} \text {, zinc }{ }^{*+}\end{array}$ \\
\hline Women before menopause & iron \\
\hline Pregnant women & vitamin $B_{1}^{*}$, vitamin $B_{2}{ }^{*}$, vitamin $B_{6}$, iodine, zinc ${ }^{*}$ \\
\hline Elderly (55-84 years) & vitamin $A^{\dagger}$, vitamin $B_{1}^{\neq c}$, vitamin $B_{6}^{* \dagger}$, magnesium \\
\hline \multicolumn{2}{|c|}{$\begin{array}{l}{ }^{*} \text { Marginal; }{ }^{*} \text { female population only; }{ }^{\dagger} \text { male population only. } \\
{ }^{a} 10-15 \text { years only; }{ }^{\text {b }} 13-15 \text { years only; }{ }^{c} 75-84 \text { years only }\end{array}$} \\
\hline
\end{tabular}

\section{Vitamins}

Vitamin D and folate equivalents must be considered the most critical vitamins in Austrians. Considering the sun intensity in Austria, the low vitamin D intake is probably insufficient to assure an optimal status particularly during wintertime. Folate equivalent intake was below the recommended $400 \mu \mathrm{g} /$ day in all age groups. With the important role of folate in fetal development, this is of particular concern in women of child-bearing age, but also in elderly in whom folate deficiency has been associated with cognitive deterioration [Moretti et al., 2004].

Overall, intake of the other vitamins was satisfying in adults. The average diet of Austrian children aged $>10$ years did not provide the estimated average requirements of vitamin E. Suboptimal supplies were also observed for vitamin A, thiamine, riboflavin and vitamin $B_{6}$ for both genders in the older age groups. Vitamin $\mathrm{E}$ intake was also suboptimal in adolescents. Adolescents (apprentices) showed a low intake of $\beta$-carotene and a suboptimal supply of vitamin $C$, suggesting a modest consumption of fruit and vegetables, also supported by the rather low potassium intake of female apprentices. In elderly, intake of vitamin A, thiamine, riboflavin and vitamin $\mathrm{B}_{6}$ did not meet the recommendations in some age groups (men or women; table 11.4).

\section{Minerals}

Across all age groups, calcium intake was below the, albeit high, D-A-CH recommendations. In adolescent girls and adult premenopausal women, iron intakes were too low to meet the higher requirements due to menstrual blood loss. Magnesium intake was below the recommended levels in children $>10$ years, in adolescents, adult women $<25$ years and elderly of all age groups and both genders. The intake should 
thus be improved to assure an optimal supply. A tendency to low zinc intakes was apparent in men between 75 and 84 years. However, intake of other minerals was satisfying in adult and elderly Austrians, but too low in children aged $>10$ years. Intake of iodine, a critical nutrient in Alpine regions, did not reach the national recommendation despite a certain improvement since the last report. Use of iodized salt was assumed for the calculation of the intake levels.

\section{Trends in Nutrition}

Compared to the previous report, intakes of energy declined in all age groups, while macronutrients showed no major changes. In schoolchildren, cholesterol intake was lower, as were intakes of many vitamins and minerals, particularly in the older age subgroups. In adults, a slight increase was seen for dietary fiber intake, slight decreases for the intake of total fat, saturated fatty acids and cholesterol. Intake of vitamin $\mathrm{E}$ improved, but decreases occurred for most other vitamins. This was particularly the case for vitamin D and folate equivalents. Declines were also observed for calcium and iron in particular and, to a lesser degree, for magnesium, zinc and, in men, for potassium. Elderly showed lower intakes of dietary fiber, but higher ones of cholesterol and alcohol. Intake of most vitamins and minerals was also lower with the exception of vitamin $\mathrm{C}, \mathrm{E}$ and iodine. Thus, despite some favorable trends, average nutrition of Austrian adults leaves room for improvement.

A survey on supplement use in 282 Austrian adults shows that supplements can contribute significantly to nutrient supply. They were regularly consumed by $39 \%$ of the participants and, in the case of vitamin $\mathrm{A}, \mathrm{D}$, folic acid and niacin, provided on average $100 \%$ of the daily recommendation. Although the upper level of tolerable intake was not exceeded in any of the subjects, a tighter control might be advisable, at least for micronutrients with a narrow safety margin. Another point is the gratuitous supplementation of micronutrients already contained in sufficient amounts in the diet as exemplified by vitamin $\mathrm{C}$ being the most commonly supplemented nutrient.

\section{Nutritional Status of Some Austrian Population Groups}

Nutritional status analyses performed in a subsample of vocational school attendants confirmed the low intake of folate, $\beta$-carotene, vitamin $\mathrm{D}$ and vitamin $\mathrm{E}$ as well as riboflavin. Deficiencies were also found for vitamin $B_{1}, B_{6}$ and $B_{12}$. Status of calcium, zinc and, for the female subjects, iron was also suboptimal. In turn, only $91 \%$ of male adolescents showed a sufficient magnesium status in contrast to $99 \%$ of their female counterparts (fig. 11.1).

Analysis of plasma levels of vitamin $\mathrm{B}_{6}, \mathrm{~B}_{12}$ and folate in a subsample of 102 elderly ( 45 men and 57 women) from the eastern region of Austria (Burgenland) showed a high prevalence of inadequate status for vitamin $\mathrm{B}_{6}$ (29\% marginal, 24\% markedly inadequate) and for $\mathrm{B}_{12}$ (32\% inadequate) and a lower one for folate (10\% inadequate) 


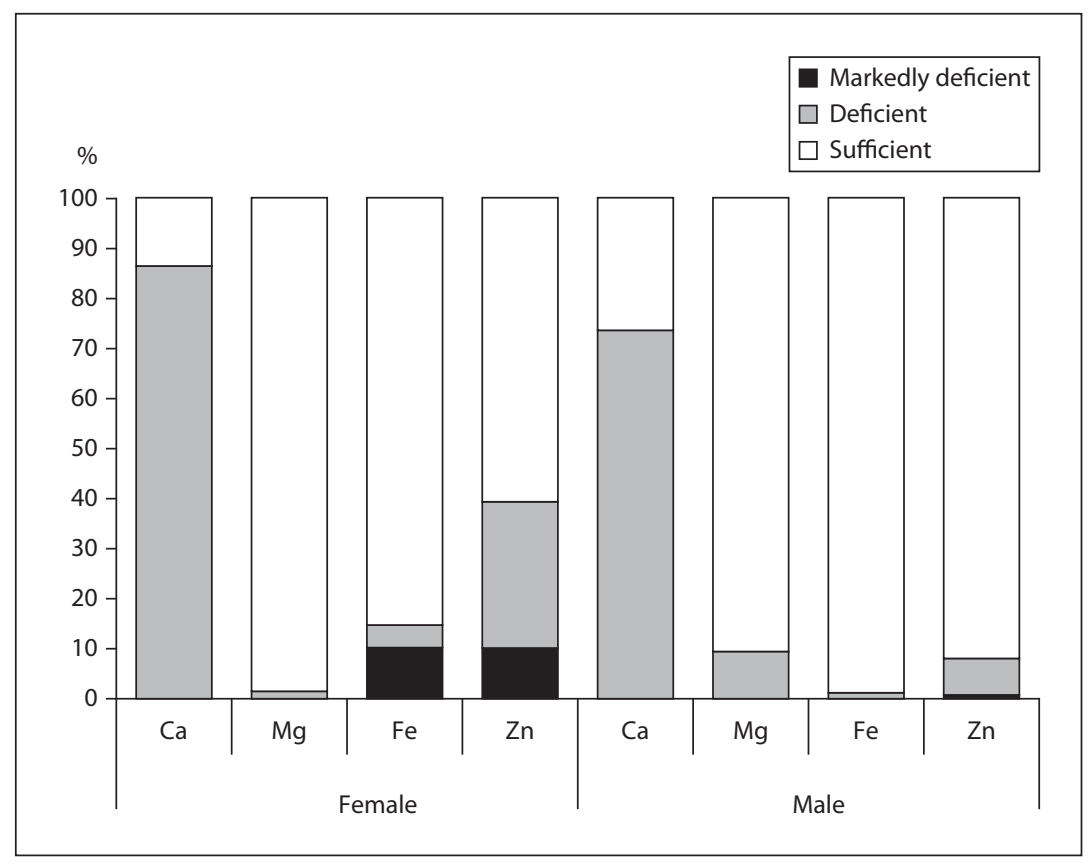

Fig. 11.1. Mineral status of Austrian adolescent vocational school attendants ( $n=143 ; 14-19$ years). Results are given as percentage of analyzed subjects with adequate, deficient, or markedly deficient status. Calcium and magnesium were determined in urine, using an ion-selective electrode; iron and zinc in serum using photometry and atomic absorption spectrometry, respectively.

(see fig. 11.2). Interestingly, intake was much more insufficient for the latter than for vitamin $\mathrm{B}_{6}$, and even above the recommended level for vitamin $\mathrm{B}_{12}$, implying that status not always correlates with intake.

\section{Focus on Pregnant Women}

Mean energy intake of Austrian pregnant women was below the recommended level. However, considering the BMI distribution of the sample and the average gestational weight gain, energy supply appears sufficient. Intake of protein and fat was slightly above the recommended levels ( 16 and $36 \% \mathrm{E}$, respectively), with saturated fatty acids accounting on average for $16 \% \mathrm{E}$ in all age groups. Intake of PUFA was within the recommended range, but in light of the role of $n-3$ fatty acids for fetal development, intake of these latter should be optimized. Carbohydrate intake was lower than recommended $(47 \% \mathrm{E})$, and consumption of starchy fiber-rich foods should be encouraged considering the already high sucrose and low dietary fiber consumption.

Mean intake of vitamin D and folate was below the recommendations (table 11.5), and especially the latter must be considered a risk nutrient in pregnant women. The 
Fig. 11.2. Status of vitamins $B_{6}, B_{12}$ and folate in elderly from the eastern region of Austria (Burgenland) (Eastern Austria) ( $n=102 ; 45$ men, mean age $75 \pm 4$; 57 women, mean age $76 \pm 4$ ). Percentage of subjects with adequate and inadequate status by age and gender. Status was evaluated through content in the erythrocytes by RIA and photometrically [Fabian et al., 2009].
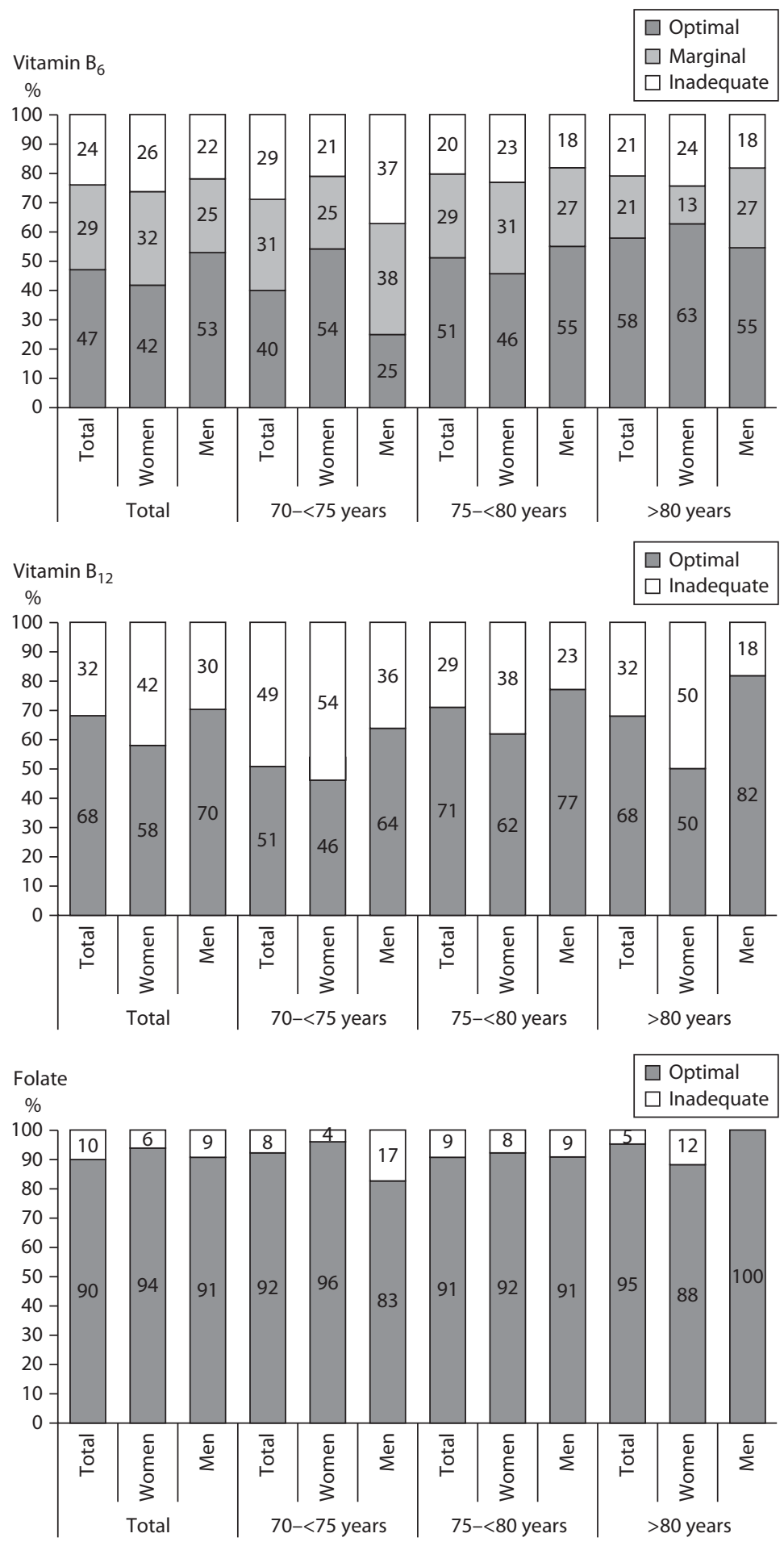
Table 11.5. Critical micronutrients in Austrian pregnant women: mean daily intake

\begin{tabular}{llllll}
\hline & Total & $<25$ years & $25-35$ years & $>35$ years & D-A-CH \\
\hline Vitamin $\mathrm{D}, \mu \mathrm{g}$ & 2.5 & 2.1 & 2.6 & 3.3 & 10 \\
\hline Thiamine, $\mathrm{mg}$ & 1.1 & 1.1 & 1.1 & 1.3 & 1.2 \\
\hline Riboflavin, $\mathrm{mg}$ & 1.4 & 1.3 & 1.5 & 1.6 & 1.5 \\
\hline Vitamin $_{6}, \mathrm{mg}$ & 1.5 & 1.4 & 1.5 & 1.7 & 1.9 \\
\hline Folate' ${ }^{1} \mu \mathrm{gg}$ & 234 & 209 & 232 & 270 & 600 \\
\hline Calcium, $\mathrm{mg}$ & 919 & 798 & 919 & 1,078 & 1000 \\
\hline Magnesium, $\mathrm{mg}$ & 322 & 273 & 321 & 374 & 310 \\
\hline Iron, $\mathrm{mg}$ & 12.6 & 11.3 & 12.5 & 15.0 & 30 \\
\hline lodine, $\mu \mathrm{mg}$ & 186 & 176 & 184 & 213 & 230
\end{tabular}

${ }^{1}$ Folate equivalent $(=1 \mu \mathrm{g}$ food folate $=0.5 \mu \mathrm{g}$ folic acid $(\mathrm{PGA})=0.6 \mu \mathrm{g}$ folic acid taken with meals).

$\mathrm{D}-\mathrm{A}-\mathrm{CH}$ recommendations were not met for thiamine and vitamin $\mathrm{B}_{6}$ as well as for riboflavin in women $<25$ years. Iron and, for younger women, calcium were the main critical minerals. Especially younger women showed magnesium and iodine intakes below the higher levels recommended during pregnancy.

Blood analyses in a Viennese sample of 113 pregnant women showed a high prevalence of vitamin $B_{6}$, folate and iron deficiency. Vitamin D levels were also low in many women albeit to a lesser degree. Zinc status was also suboptimal despite a mean intake within the recommended range.

\subsection{Belgium²}

\section{Introduction}

Worldwide non-communicable diseases (NCD) such as cardiovascular diseases (CVD), cancer, diabetes and obesity have been increasingly jeopardizing overall public health. In Belgium, deaths attributable to NCD make $88.7 \%$ of all deaths whereas in Europe this is 76.6\% [WHO, 2004]. In Belgium, the largest contributors to these deaths were malignant neoplasms and CVD, respectively 30.8 and $43.1 \%$ [WHO,

\footnotetext{
${ }^{2}$ Verstraeten R, Remaut-DeWinter AM, Kolsteren P; Faculty of BioScience Engineering, Department of Food Safety and Food Quality, Ghent, Belgium.

E-Mail roosmarijn.verstraeten@ugent.be, annemarie.dewinter@ugent.be
} 
Table 11.6. Studies from Belgium included in the ENHR 2009

\begin{tabular}{|c|c|c|c|c|c|}
\hline Survey & Study population & Age & $\mathrm{n}$ & $\begin{array}{l}\text { Year(s) of } \\
\text { the study }\end{array}$ & $\begin{array}{l}\text { Supplied nutrition and health } \\
\text { indicators }\end{array}$ \\
\hline \multicolumn{6}{|l|}{ National level } \\
\hline $\begin{array}{l}\text { Food Consumption } \\
\text { Survey }{ }^{1} \text { (FCS) }\end{array}$ & $\begin{array}{l}\text { Adolescents } \\
\text { and adults }\end{array}$ & $>15$ years & 3,249 & 2004 & $\begin{array}{l}\text { Health indicators } \\
\text { (self-reported anthropometry, } \\
\text { self-reported BMI) } \\
\text { Nutrition indicators } \\
\text { (energy and nutrient intake) }\end{array}$ \\
\hline $\begin{array}{l}\text { Health Interview } \\
\text { Survey }^{2}(\text { HIS) }\end{array}$ & $\begin{array}{l}\text { Belgian } \\
\text { population }\end{array}$ & All ages & 12,945 & 2004 & $\begin{array}{l}\text { Health indicators (smoking, } \\
\text { alcohol consumption, measured } \\
\text { BMI and measured anthropometry) }\end{array}$ \\
\hline \multicolumn{6}{|c|}{ Regional level: Flanders } \\
\hline $\begin{array}{l}\text { Flemish Growth } \\
\text { Study }^{3} \text { (FGS) }\end{array}$ & $\begin{array}{l}\text { Children and } \\
\text { adolescents }\end{array}$ & $0-20$ years & 18,000 & 2002-2004 & $\begin{array}{l}\text { Health indicators (measured } \\
\text { anthropometry and measured BMI) }\end{array}$ \\
\hline \multicolumn{6}{|c|}{$\begin{array}{l}\text { ' De Vriese et al., } 2006 . \\
\text { 2 Bayingana et al., } 2006 . \\
{ }^{3} \text { Roelants et al.; } 2009 .\end{array}$} \\
\hline
\end{tabular}

2004]. In addition, NCD attribute $86.8 \%$ to the total loss of disability-adjusted life years in Belgium, which is similar to the prevalence seen in Europe, $85.7 \%$. The associated burden of NCD is consequently high in Belgium. As a large amount of these disability-adjusted life years are lost due to nutrition or unhealthy lifestyle [Murray and Lopez, 1996], nutritional information provides policymakers the opportunity to develop focused and efficient strategies for preventive actions. However, out of the first European Nutrition and Health Report 2004 (ENHR 2004) appeared that Belgium was one of the few countries where no such up-to-date national epidemiologic data were available [Elmadfa et al., 2004].

The Belgian government acknowledged this need and a first National Food Consumption Survey (FCS) was organized in 2004. It was conducted, amongst other reasons, to obtain more recent data regarding nutrient and energy intake of the Belgian population ( $>15$ years old) at the individual level and to enable the identification of subgroups at risk within the Belgian population. The FCS together with the Health Interview Survey (HIS) were the two national surveys that provided national data on the nutrition and health indicators identified within the current European Nutrition and Health Report 2009 (ENHR 2009). In addition to these national surveys, published research provided information on health indicators for children and adolescents of high quality at regional level, i.e. Flanders [Roelants et al., 2009]. In 
table 11.6 an overview is given of the studies that were reported in the ENHR 2009. This national report will focus specifically on the methodology of each study that was included for Belgium, since the main nutrition and health indicators are reported in the main part of the ENHR 2009.

\section{Methodology of Surveys in Belgium}

\section{Methodology of the Belgian Food Consumption Survey (FCS)}

The FCS provided nutrient intake and self-reported anthropometry and BMI for the Belgian population in the ENHR 2009 [De Vriese et al., 2006]. The FCS started in 2004 and the first results were presented in 2006. The overall study design of the FCS was mainly based on the recommendations of the European Food Consumption Survey Method group [Brussaard et al., 2002a, 2002b]. Dietary intake was assessed by a repeated non-consecutive 24-hour recall. An adapted Belgian version of EPIC soft was used to process the data [Slimani and Valsta, 2002]. The fieldwork was spread over 1 year and the 24-hour recalls were distributed equally over the 7 days of the week [De Vriese et al., 2005], in order to take into account the effect of the week day and the different season. To obtain an estimate of the habitual dietary intake at group level, the software package C-side was used [Dodd, 1996; Iowa State University, 1996]. The main food composition database used in the FCS was the NUBEL brands databank [2005] which was updated in April 2005. Since this databank was not supplying all the information needed for each food, additional information was retrieved from other sources, in particular the Belgian Food Composition Table of the Institut Paul Lambin (IPL), the Dutch NEVO-table and the British table of McCance and Widdowson's [De Vriese et al., 2006]. Underreporters were identified based on the cut-offs of Goldberg [Goldberg et al., 1991; Black, 2000]. Using these cut-offs, 20\% of the population were identified as underreporters. The number of underreporters was higher among females (26\%) than among males (14\%) [De Vriese et al., 2006]. When using the cut-off value of Goldberg for group means, 15\% were underreporting their energy intake. When compared between the different regions, it was found that underreporting was higher in Wallonia and Brussels (17\%) than in Flanders (14\%) [De Vriese et al., 2006].

The target population of the FCS was all individuals aged 15 years or older registered in the National Population Register in Belgium. People who are not incorporated in this register such as homeless people, people living in institutions or prisons, etc. were therefore automatically excluded from the survey. The sample was stratified (probability proportionate to size) per region, province and community and the households were used as sample unit. The sample was also stratified according to predefined age groups $(15-18,19-59,60-74,>75$ years $)$ and gender. In total, a sample of 3,249 people (1,623 females and 1,626 males) of all inhabitants of Belgium was selected [De Vriese et al., 2006]. The participation rate was 42\% [De Vriese et al., 
2005]. Differences in the sample size were addressed by using weighing factors when analyzing the results [De Vriese et al., 2005].

\section{Methodology of the Belgian Health Interview Survey}

The HIS provided the health indicators (smoking, alcohol consumption, measured BMI and anthropometry) for the Belgian population in the ENHR 2009 [Bayingana et al., 2006]. The HIS started in 2004 and results were presented in 2006. The objective of this survey was, amongst others, to estimate the prevalence and distribution of health indicators and to identify health problems among the Belgian population. The target population was the Belgian population registered in the National Register of Belgium and therefore homeless, illegal people, persons who were staying in an institution (except for homes for the elderly) or were part of a religious movement, or were in prison were excluded from the survey. The sample was stratified per region, province and community. The participants were selected probability proportionate to size. The sampling method was adapted so that 1,250 additional individuals of 65 years and older were considered. In total, 12,945 persons (of which 3,515 older than 65 years) took part in this study, of which 4,513 interviews were in Flanders, 4,992 interviews were in Wallonia and 3,440 interviews were in Brussels. The results provided a representative sample of the population. Out of all the households contacted, $61.4 \%$ agreed to participate in the questionnaire.

\section{Methodology of the Flemish Growth Study}

The Flemish Growth Study (FGS) was the first large-scale growth survey in Belgium [Roelants et al., 2009]. It is however only representative for Flanders, the northern region in Belgium. The study aimed characterization of Flemish growth curves. The results of this study provided the health indicators (BMI from measured data and measured anthropometry) of children and adolescents for Flanders in the ENHR 2009. An initial cross-sectional sample of 18,050 subjects aged 0-25 years was selected

and measured between September 2002 and June 2004. The following groups were excluded from analysis: (i) children of non-Belgian origin (having no parent of Belgian or north western European origin (France, Luxembourg, Germany, The Netherlands, Austria, the UK, Denmark, Sweden or Norway)), (ii) children with known growth disorders, severe chronic diseases or children that were on medication that might affect growth, and (iii) children with an uncertain origin or health status. In addition, children that were born earlier than 37 weeks of gestational age were excluded as well from the analysis; but low birth weight was not an exclusion criterion. After adapting these criteria to the total sample, 15,989 subjects (7,859 boys and 8,130 girls) were included in the analysis. The subjects were recruited in different settings according to age as shown in table 11.7. 
Table 11.7. Settings per age category for the Flemish Growth Study

\begin{tabular}{lll}
\hline Subjects & Time period & Setting \\
\hline $0-3$ years old & September 2002-July 2004 & $\begin{array}{l}49 \text { well-baby clinics } \\
25 \text { accredited day-care } \\
\text { centers }\end{array}$ \\
\hline $2.5-20$ years old & January 2002-June 2004 & 59 school health services \\
\hline $17-25$ years old & 2002-2004 & $\begin{array}{l}\text { Student health center of the VUB } \\
\text { (freshmen students) } \\
\text { Centre for occupational medicine } \\
\text { (young employees) }\end{array}$ \\
& &
\end{tabular}

\section{Discussion}

Belgium is a Federal State comprising three regions, i.e. the Flemish region (Flanders), the Walloon region (Wallonia) and the Brussels-capital region. In view of this complex structure, there is a need to report the results regarding the nutrition and health indicators specifically for each of these regions. A more detailed national report including the results from analysis by region and for different educational levels are reported elsewhere (http://www.univie.ac.at/enhr).

Two out of the three studies included in the current ENHR 2009 were conducted at national level; the other one provided only information at regional level. In general, up-to-date and high-quality information on health and nutrition indicators were available. However, it should be noted that data for certain age groups (especially young children and adolescents) and for certain regions were lacking. In addition, not all requested indicators could be provided on a national level nor was the formulation of these indicators as requested by the ENHR 2009. Based on the present data, there is a need for the Belgian government to develop national policies and interventions tailored for certain population groups (age, educational level, etc.) and for the different regions. Moreover, the government needs to address the identified needs by (i) collecting data on nutrition and health indicators that are lacking for specific age groups on a national level, and (ii) streamlining these indicators with European recommendations in order to facilitate comparison between European countries. Addressing these needs would contribute to a better evaluation of the health of the Belgian population and consequently to a more evidence-based and effective development of national policies. 


\subsection{Czech Republic ${ }^{3}$}

\section{6th Nationwide Anthropological Survey}

The anthropometric data of children presented in the ENHR 2009 are based on the 6th Nationwide Anthropological Survey 2001, Czech Republic. Data on 18,584 children (9,541 boys and 9,043 girls) under 6 years of age and 40,525 schoolchildren and adolescents (18,605 boys and 21,920 girls) were collected with the participation of pediatricians, nursery teachers, biology and physical education teachers from elementary and secondary schools, staff of the district public health service and regional public health service. The age of investigated children varied from 0 to 18 years. The basic anthropometrical method of Martin and Saller [1957] was used for the investigation. Waist circumference was measured at the umbilicus level with the measuring tape being placed in a horizontal plane around the trunk. The measurement was taken at the front side of the body with an accuracy of $0.5 \mathrm{~cm}$.

The Czech Republic has undergone rapid political, social, and economic transformation since the late 1980s. While obesity rates among children and adolescents in the Czech Republic have been previously relatively low, this has changed in recent years. Across the past 50 years, body weight, BMI for age, and adiposity rebound (the time when a child reaches the lowest BMI before their BMI gradually begins to increase until adulthood) have occured earlier. The most dramatic changes have been observed among school-aged children, where BMI values have increased at the 50th, 90th, and 97th percentiles. In contrast, adolescent girls appear to be thinner than in the past. Our findings suggest that the secular trend of increased height, accelerated growth, and earlier maturation is responsible for Czech children experiencing adiposity rebound at earlier ages compared to the past [Vignerová et al., 2008].

Using the IOTF reference standards, the prevalence of obesity among Czech children in 2001 thus exceeded the expected 3\% among 4- to 10-year-old boys and girls. In addition, only $0.8 \%$ of 15 - to 18 -year-old adolescent girls were identified as obese in 2001 based on the IOTF reference values. The prevalence of overweight exceeded the expected $10 \%$ among 4- 15-year-old boys only. In girls the prevalence of overweight was smaller than $10 \%$ in all age categories.

Although the prevalence of child overweight is clearly increasing among Czech children in certain age categories, it is important to note that the rates of overweight and obesity among 4- to 18-year-old children remain relatively low (13\% in 2001) compared to $32 \%$ in the USA and $19 \%$ in Europe for children in the same age category [Lobstein et al., 2004].

\footnotetext{
${ }^{3}$ Dostálová J ${ }^{\mathrm{a}}$, Dlouhý $\mathrm{P}^{\mathrm{b}}$, Tláskal $\mathrm{P}^{\mathrm{c}}$, Adámková $\mathrm{V}^{\mathrm{d}}$, Vignerová ${ }^{\mathrm{d}}$, Cífková $\mathrm{R}^{\mathrm{e}}, \mathrm{Krsková} \mathrm{A}^{\mathrm{e}}$, Skálová $\mathrm{L}^{\mathrm{e}}$, Mydlilová $\mathrm{A}^{\mathrm{f}}$, Hrnčírová $\mathrm{D}^{\mathrm{c}}$, Rambousková $\mathrm{J}^{\mathrm{c}}$; ${ }^{\mathrm{a} C z e c h}$ Nutrition Society, Prague, ${ }^{\mathrm{b}} 3 \mathrm{rd}$ Faculty of Medicine, Charles University, Prague, ' University Hospital Prague-Motol, Institute for Clinical and Experimental Medicine, Prague, e'National Institute of Public Health, Prague, and fThomayer University Hospital, Prague, Czech Republic. E-Mail jana.dostalova@vscht.cz, pavel.dlouhy@lf3.cuni.cz
} 
Table 11.8. Infant feeding in the Czech Republic at discharge from maternity hospital (\% of infants)

\begin{tabular}{clllll}
\hline & \multicolumn{1}{l}{ Year } & & & \\
\cline { 2 - 6 } & 2004 & 2005 & 2006 & 2007 & 2008 \\
\hline Total breastfeeding & 96.1 & 96.1 & 96.1 & 95.9 & 96.1 \\
\hline Exclusive breastfeeding & 90.6 & 90.3 & 89.5 & 88.6 & 88.1 \\
\hline Partially breastfeeding & 5.6 & 5.8 & 6.5 & 7.3 & 8.0 \\
\hline Non-breastfeeding & 2.8 & 2.9 & 2.9 & 2.9 & 2.9 \\
\hline
\end{tabular}

Table 11.9. Long-term breastfeeding in the Czech Republic (\% of infants)

\begin{tabular}{llllllll}
\hline & \multicolumn{1}{l}{ Year } & & & & & \\
\cline { 2 - 7 } & 2000 & 2001 & 2002 & 2003 & 2004 & 2005 & 2006 \\
\hline Total breastfeeding & & & & & & & \\
\hline 6 weeks & 69.2 & 73.5 & 74.9 & 76.5 & 76.9 & 78.4 & 79.3 \\
\hline 3 months & 47.9 & 53.6 & 56.7 & 59.2 & 59.8 & 62.2 & 64.2 \\
\hline 6 months & 23.8 & 28.4 & 31.7 & 35.1 & 35.8 & 38.3 & 40.9 \\
\hline Exclusive breastfeeding & & & & & & & \\
\hline 6 weeks & 41.8 & 47.0 & 48.1 & 49.0 & 48.8 & 51.0 & 50.5 \\
\hline 3 months & 26.1 & 31.8 & 34.2 & 35.8 & 35.9 & 39.0 & 39.4 \\
\hline 6 months & 10.5 & 14.5 & 16.7 & 18.6 & 18.6 & 21.4 & 22.8 \\
\hline
\end{tabular}

Infant Feeding in the Czech Republic

Data on infant feeding at discharge from maternity hospitals are based on the Report on the Newborn which is referred to the National Institute of Health Information and Statistics of the Czech Republic in Prague by the staff of neonatological departments. Newborns with parenteral feeding were excluded from this survey [Mydlilová et al., 2008, 2009] (table 11.8).

Data on long-term breastfeeding were obtained from the Annual Report on Activities of Pediatricians-Practitioners provided by every outpatient office of pediatrician-practitioner.

Data were obtained at regular preventive examinations at one year of child age. The sample of children evaluated in the period of 2000-2006 comprised 648,625 children [Mydlilová et al., 2006] (table 11.9). 
Fig. 11.3. Lipid intakes.

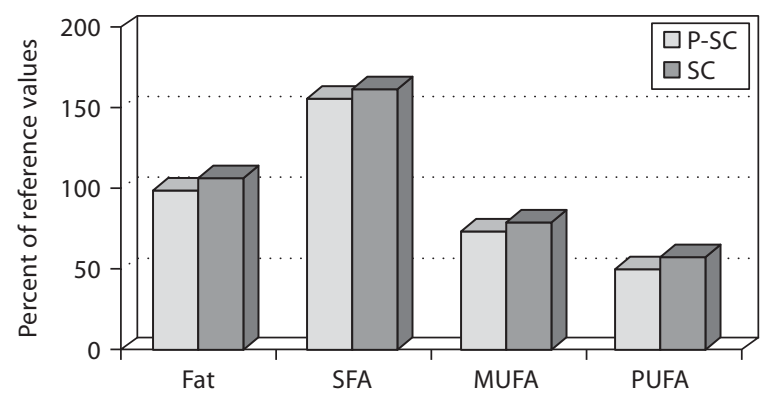

Nutrition Practices of the Czech Children before and at Early School Age

The nutrition and anthropometric data of 1,655 Czech children aged of 4-7 years and 1,137 Czech children aged 7-10 years were obtained in 2007. Individually, 48-hour food consumption data were gathered. Intake of over 30 nutrients from food was evaluated by the Nutridan Program. For the estimation of daily recommended intake of nutrients and other alimentary components tables of German, Austrian and Swiss Nutrition Societies (D-A-CH) reference values for nutrient intake were used [D-A-CH, 2002].

The nutrition status in monitored children (evaluated by means of BMI) corresponded to a reference file of Czech children. The file consisted of $4.6 \%$ pre-schoolchildren (P-SC) and 5.6\% schoolchildren (SC) being obese. A higher ( $>90$ percentile) daily energetic intake could be observed with $8.9 \%$ P-SC and 10.9\% SC. On average, the energy intake nevertheless amounted to $98 \%$ of the reference values. The proportion of the macronutrients fat, protein and carbohydrates in the total energy intake was in P-SC 30, 14 and 56\%E, and in the schoolchildren these proportions were 32.2, 14.5 and $53.4 \% \mathrm{E}$ of energy. The daily intake of proteins was higher than the reference values (183, resp. $123 \%$ of recommended intake in P-SC and SC). Fig. 11.3 shows the lipid intakes in proportions of individual components - saturated fatty acids (SFA), monounsaturated (MUFA) and polyunsaturated fatty acids (PUFA). The oversupply of saturated fatty acids as well as a shortage of polyunsaturated fatty acids was evident in both groups of children.

Above all, the excessive consumption of sodium $(\mathrm{Na})$ is evident with both groups of children. The iodine (I) intake corresponded to 76 and $75 \%$ of the recommended nutrition dose only. A low consumption of vitamin $\mathrm{D}$ has been found in both groups ( 46 and $55 \%$ of the recommended intake). A higher vitamin $B_{12}$ intake (557 and 515\% of recommended intake) showed a fair consumption of the animal food component and further that intake of vitamin C (620 and 560\% of recommended intake) represents a very good supply of the plant food component (fig. 11.4). 


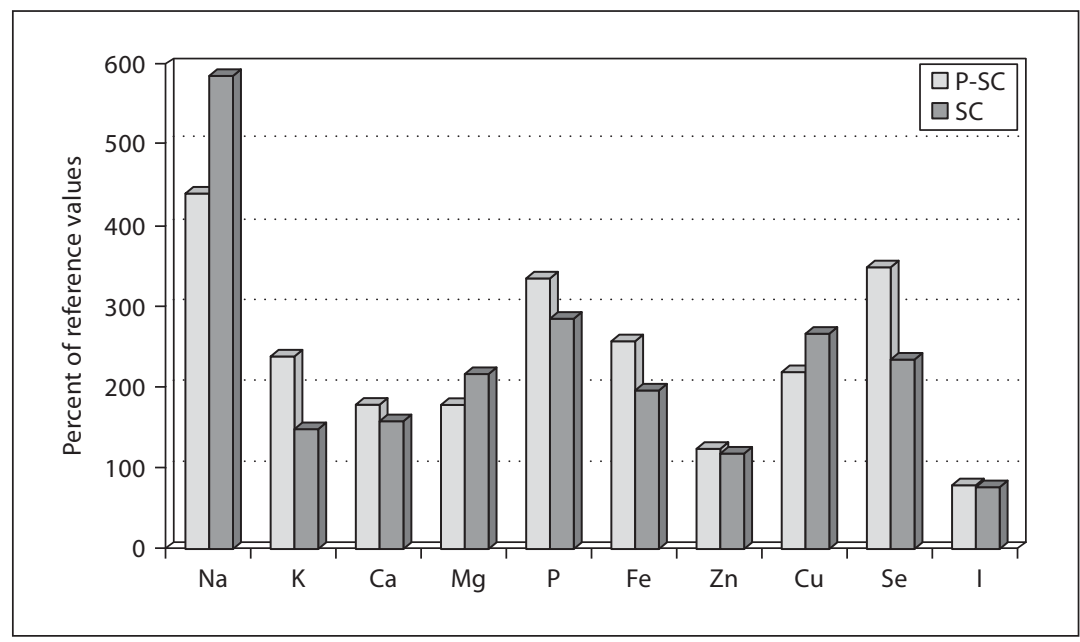

Fig. 11.4. Mineral and the trace element intakes.

\section{Czech Post-MONICA Study}

The nutrition and anthropometric data for the Czech population were obtained as a substudy of the Czech Post-MONICA Study in 2000/2001, which involved a representative $1 \%$ population random sample aged $25-64$ years (stratified by age, sex, and community size). A total of 1,627 males and 1,693 females were examined (response rate 63.8 and $64.8 \%$, respectively).

The nutrient intake data were obtained in 1,046 males and 1,094 females using individual 24-hour dietary recalls and food tables for the Czech Republic. Anthropometric data were calculated for the same individuals [Cífková et al., 2004a, 2004b; Cífková and Škodová, 2004].

\section{Longitudinal Follow-Up (15 Years) Study of the Trend of Nutrition of the 1\% Random} Sample of Population of a District of Central Bohemia

The trend of nutrition in a $1 \%$ random sample of population of one district of Central Bohemia was ascertained. Three surveys in 1992, 2000 and 2007 were compared. Dietary recalls of 804 persons (390 males, 412 females, age 25-64 years) were analyzed. The first survey was performed in 1990, the second in 2000 and the last one in 2007. Waist-hip ratio (WHR), calculated BMI, analyzed intake of energy, protein, fats, saccharides, vitamin C, fiber and cholesterol were measured.

BMI and intake of cholesterol increased continually from 1992 in both groups ( $\mathrm{p}<0.0001)$. WHR increased in females from 1992 to 2007 ( $\mathrm{p}<0.001)$, in males it increased only from 1992 to $2000(\mathrm{p}<0.001)$, now stagnates.

Intake of energy increased in 1992-2000 ( $p<0.01$ ), in 2000-2007 it decreased in both groups $(\mathrm{p}<0.001)$. Decreased intake of fat in both groups and decreased 
Table 11.10. BMI (\% of males, $n=996$ ) [National CINDY Health Monitor Report, 2002]

\begin{tabular}{llllll}
\hline BMI & Age group & & & \multicolumn{2}{c}{ Total } \\
\cline { 2 - 5 } & $25-34$ & $35-44$ & $45-54$ & $55-64$ & \\
\hline$<19.9$ & 2 & 1 & 0 & 1 & 1 \\
\hline $20.0-24.9$ & 52 & 44 & 28 & 19 & 37 \\
\hline $25.0-29.9$ & 39 & 48 & 59 & 64 & 52 \\
\hline$>30.0$ & 6 & 7 & 13 & 16 & 10 \\
\hline
\end{tabular}

Table 11.11. BMI (\% of females, $n=1,007$ ) [National CINDY Health Monitor Report, 2002]

\begin{tabular}{llllll}
\hline BMI & Age group & & & Total \\
\cline { 2 - 5 } & $25-34$ & $35-44$ & $45-54$ & $55-64$ & \\
\hline$<19.9$ & 23 & 14 & 6 & 4 & 12 \\
\hline $20.0-24.9$ & 61 & 58 & 48 & 26 & 49 \\
\hline $25.0-29.9$ & 12 & 23 & 32 & 41 & 26 \\
\hline$>30.0$ & 4 & 5 & 14 & 30 & 13 \\
\hline
\end{tabular}

intake of carbohydrates in males $(\mathrm{p}<0.001)$ were found. Intake of protein equal in both groups. Vitamin C increased between 1992 and 2000, from 2000 it decreased $(\mathrm{p}<0.01)$. Intake of fibers increased from 1992 to 2000 , and decreased from 2000 to 2007 it decreased $(\mathrm{p}<0.01)$.

\section{CINDI Health Monitor}

Self-reported data were gained at the national level as a representative sample of the adult population $(\mathrm{n}=2,003)$ by questionnaire of CINDI Health Monitor in 2002. BMI was calculated from self-reporting data of respondent's body height and weight (tables 11.10, 11.11).

\section{Biological Monitoring Project in the Czech Republic}

The project of biological monitoring in the Czech Republic includes the monitoring of toxic substances (lead, cadmium, mercury) and selected essential trace elements (copper, selenium, zinc) in body fluids of the Czech population.

Biological monitoring is in routine operation since 1994. In the first period (19962003), altogether 3,245 adults and 3,075 children were involved. The second period 
Table 11.12. Trace elements in blood and urine of the adult population $(2005,2007)$ [National Institute of Public Health, 2006, 2008)

\begin{tabular}{llllll}
\hline & & 2005 & 2007 & 2005 & 2007 \\
\cline { 3 - 6 } & $\begin{array}{l}\text { blood, } \mathrm{g} / \mathrm{l} \\
\text { (median) }\end{array}$ & & \multicolumn{2}{l}{$\begin{array}{l}\text { urine, } \mu \mathrm{g} / \mathrm{g} \\
\text { creatinine } \\
\text { (median) }\end{array}$} & \\
\hline Copper & men & 870 & 850 & 5.2 & 26.3 \\
\cline { 2 - 6 } & women & 1,020 & 1,000 & & 348 \\
\hline Zinc & 6,490 & 6,505 & 275 & 7 \\
\hline Selenium & 111 & 106 & 12 & 7 \\
\hline
\end{tabular}

Table 11.13. Trace elements in blood and urine of children (2006) [National Institute of Public Health, 2007)

\begin{tabular}{lll}
\hline & Blood, $\mu \mathrm{g} / \mathrm{l}$ (median) & Urine, $\mu \mathrm{g} / \mathrm{g}$ creatinine (median) \\
\hline Copper & 1,040 & 27.8 \\
\hline Zinc & 5,205 & 450 \\
\hline Selenium & 108 & 12 \\
\hline
\end{tabular}

was started in 2005. Until now, 821 adults (18-59 years) and 400 children (8-10 years) were monitored. Blood and urine samples were collected from March till June. A questionnaire is focused on age, weight, height, individual lifestyle (smoking habit in adults, passive smoking exposure, fish consumption, frequency of dietary supplement use, professional exposure, etc.) (tables 11.12, 11.13).

\section{Nutrition Survey in the Homeless Population}

This study was undertaken to define the prevalence of protein-energy malnutrition and main cardiovascular risk factors in the homeless population in Prague. Data of the homeless population was obtained from two cross-sectional studies carried out in 2003 (201 homeless, 174 males and 27 females, mean age males $42 \pm 11$, females 40 $\pm 9)$.

Mean BMI values were within the normal range, with only $3 \%$ of the men and $7 \%$ of the women below $18.5 \mathrm{~kg} / \mathrm{m}^{2}$. Wasted muscle mass was found in only $0.6 \%$ men. Decreased body fat (below 10\%) was described in $5 \%$ men. Serum protein levels were within normal limits. Lymphocytopenia was reported in $2 \%$ of the men. Results of the 
Table 11.14. Comparison of prevalence of the cardiovascular risk factors among the homeless and the general Czech population (\%) [Kubisová et al., 2008]

\begin{tabular}{|c|c|c|c|c|c|}
\hline \multirow[t]{2}{*}{ Cardiovascular risk factors } & \multicolumn{2}{|c|}{ Homeless population } & \multicolumn{2}{|c|}{ General population } & \multirow[t]{2}{*}{$\mathrm{p}$ value } \\
\hline & $\begin{array}{l}\text { men } \\
\mathrm{n}=162\end{array}$ & $\begin{array}{l}\text { women } \\
n=24\end{array}$ & $\begin{array}{l}\text { men } \\
n=1,408\end{array}$ & $\begin{array}{l}\text { women } \\
n=543\end{array}$ & \\
\hline $\mathrm{BMI} \geq 25 \mathrm{~kg} / \mathrm{m}^{2}$ & 41 & 33 & 75 & 60 & $<0.001$ \\
\hline $\begin{array}{l}\text { Abdominal obesity, men } \\
(W C>102 \mathrm{~cm})\end{array}$ & 6 & & 31 & & $<0.001$ \\
\hline $\begin{array}{l}\text { Abdominal obesity, women } \\
(W C>88 \mathrm{~cm})\end{array}$ & & 21 & & 31 & n.s. \\
\hline Total cholesterol $\geq 5 \mathrm{mmol} / \mathrm{l}$ & 33 & 38 & 80 & 72 & $<0.001$ \\
\hline Triacylglyceroles $\geq 1.7 \mathrm{mmol} / \mathrm{l}$ & 15 & 0 & 48 & 23 & $<0.001$ \\
\hline Active smokers & 93 & 83 & 38 & 35 & $<0.001$ \\
\hline
\end{tabular}

$p$ values hold after the adjustment for age and sex. Abdominal obesity was evaluated for each sex separately because of different cut-off points. In this case, $p$ values are age-adjusted only.

CAGE questionnaire gave a strong indication of alcoholism in $12 \%$ and alcoholism in $16 \%$ of the participants [Kubisová et al., 2008].

Mean values of BMI, waist circumference (WC), total cholesterol (TC) and triacylglycerols (TAG) in homeless men and women were within normal limits. Compared with the majority of the Czech population, the homeless had significantly lower mean levels of TC and TAG and lower BMI and WC values; the incidence of smoking among the homeless was significantly higher [Cífková et al., 2004a, 2004b; Cífková and Škodová, 2004; Kubisová et al., 2008] (table 11.14).

\subsection{Denmark ${ }^{4}$}

\section{Data Sources}

This report is mainly based on three sources: The Danish National Survey of Dietary Habits and Physical Activity, The National Health Interview Survey 1987-2005, and

\footnotetext{
${ }^{4}$ Trolle E, Fagt S, Biltoft-Jensen A, Mathiessen J, Groth MV; Technical University of Denmark, Denmark. E-Mail eltr@food.dtu.dk
} 
The Public Health Report of Denmark 2007 which compiles data from several other sources.

\section{The Danish National Survey of Dietary Habits and Physical Activity}

The National Food Institute conducts the dietary surveys in Denmark, since 2000, in collaboration with interviewers from The Danish National Centre of Social Research.

The first national dietary survey was conducted among adults in 1985, based on the dietary history method. In 1995 the method was changed to 7-day food records and since 2000 physical activity was included and data collected each year until 2008.

The dietary surveys are cross-sectional, representative, based on random samples from the central population register. The method combines face-to-face interviews at the participants' home, with self-administered food and physical activity records for seven consecutive days.

The interview gives information about age, gender, height, weight, employment status, occupational status, education, family status, smoking habits, health status and attitudes to and knowledge about food and physical activity.

The food records are precoded, including open-ended answering possibilities, and structured according to the common Danish meal pattern. For quantification, food photo series, household measurements and standard units are used. The participants get personal and written instructions in completing the records and receive a booklet, optional for registering foods eaten out of home.

In the precoded records, participants register physical activity at work, school or child care and in leisure time (a modified version of the International Physical Activity Questionnaire (IPAQ)). The first results on physical activity among adults will be published in 2009 .

At the National Food Institute the food and physical activity records are scanned, and food and nutrient intakes estimated by the General Intake Estimation System (GIES) and the Danish Food Composition Tables.

\section{Participants}

In 1995, in total 3,098 (1-80 years), in 2000-2002 4,120 (4-75 years), and in 2003-2006 3,247 (4-75 years) participants were included. The response rate was $66 \%$ in 1995 , 53\% in 2000-2002 and 52\% in 2003-2006 (preliminary). The decreasing response rate is a general tendency, also seen in other surveys. The National Food Institute has taken several initiatives to counteract further decrease in response rate.

The National Health Interview Survey 1987-2005

The surveys on health and morbidity are carried out by The National Institute of Public Health among adult Danes selected representatively. The survey program makes it possible to follow the populations' health status through a lifespan. Surveys 
have been conducted in 1987, 1994, 2000, and 2005. The 2005 survey was based on a sample of 21,832 Danish citizens ( $>16$ years). Data was collected through a personal interview in the respondent's home combined with a self-administered questionnaire after the interview. In total, 14,566 interviews (66.7\%) were obtained, while 11,238 (51.5\% of the sample) returned the self-administered questionnaire [Ekholm et al., 2006].

\section{Diet}

The macronutrient and food intake of children 4-14 years as well as for adolescents and adults 15-75 years are based on data from the National Dietary survey 1995, 2000-2002 and 2003-2006 [Fagt et al., 2008]. The food intake, the macronutrient content and the micronutrient content of the Danish diet 2000-2002 according to age groups and gender were published in 2005 [Lyhne et al., 2005].

\section{Food Intake}

\section{Sugar-Sweetened Beverages and Confectionary}

The main sources of added sugar in the diet were sugar-sweetened beverages and confectionary, cakes and similar. The content of added sugar in the diet of children decreased from 2000-2002 to 2003-2006, but for 60\% the diet still contained too much sugar compared to the Nordic Nutrition Recommendations [NNR, 2004]. Children with the highest amount of added sugar in their diet $(\geq 15 \% \mathrm{E})$ drank 4 times as much soft drinks and fruit syrup as the group of children whose diet contained the recommended level ( $\leq 10 \% \mathrm{E}$ added sugar) [cited and modified after Fagt et al., 2008].

\section{Fruit and Vegetables}

The recommended intake of fruit and vegetables in Denmark is $600 \mathrm{~g} /$ day (children 4-10 years: $300-500 \mathrm{~g} /$ day). The average intake of fruit and vegetables increased from 1995 to 2003-2006, but the intake of vegetables stagnated between 2000-2002 and 2003-2006. The mean intake in 2003-2006 among adults was $398 \mathrm{~g} /$ day, and among children 353 g/day. Especially the adolescents (11-24 years) had low intake. 16\% of adults and $33 \%$ of children met the 6 -a-day recommendation, whereas only $5 \%$ between 11 and 14 years met the recommendation [cited and modified after Fagt et al., 2008].

\section{Other Foods}

Intake of fish did not change considerably from 1995 to 2003-2006. The mean intake in 2000-2002 was only $12 \mathrm{~g}$ /day among children and $19 \mathrm{~g} /$ day among adults. Three out of 4 children and adults consumed too little fish compared with the Danish dietary guidelines (30-40 g/day) [Astrup et al., 2005]. 
Table 11.15. Development in intake of energy, macronutrients and energy distribution, and ratio between energy intake and basal metabolic rate (EI/BMR $\left.R_{\text {est }}\right)$, average for children (4-14 years) [modified from Fagt et al., 2008]

\begin{tabular}{|c|c|c|c|c|c|c|c|c|c|}
\hline & \multicolumn{3}{|l|}{ Male } & \multicolumn{3}{|c|}{ Female } & \multicolumn{3}{|l|}{ All } \\
\hline & 1995 & $\begin{array}{l}2000 / \\
2002\end{array}$ & $\begin{array}{l}2003- \\
2006\end{array}$ & 1995 & $\begin{array}{l}2000 / \\
2002\end{array}$ & $\begin{array}{l}2003- \\
2006\end{array}$ & 1995 & $\begin{array}{l}2000 / \\
2002\end{array}$ & $\begin{array}{l}2003- \\
2006\end{array}$ \\
\hline $\mathrm{n}$ & 483 & 428 & 329 & 500 & 395 & 340 & 983 & 823 & 669 \\
\hline Energy, MJ & 9.6 & 9.3 & 8.9 & 8.6 & 8.1 & 7.7 & 9.1 & 8.7 & 8.3 \\
\hline $\mathrm{El} / \mathrm{BMR}_{\mathrm{est}}$ & 1.87 & 1.78 & 1.65 & 1.85 & 1.72 & 1.58 & 1.86 & 1.76 & 1.62 \\
\hline $\mathrm{El} / \mathrm{BMR}_{\mathrm{est}}<1.1, \%$ & 2 & 8 & 13 & 3 & 11 & 19 & 2 & 9 & 16 \\
\hline Fat, $\% \mathrm{E}^{1}$ & 35 & 33 & 34 & 35 & 33 & 33 & 35 & 33 & 34 \\
\hline Carbohydrate, total, \% $\mathrm{E}^{1}$ & 52 & 53 & 53 & 52 & 54 & 54 & 52 & 53 & 54 \\
\hline Added sugar, $\% \mathrm{E}^{1}$ & 14 & 13 & 11 & 14 & 13 & 12 & 14 & 13 & 12 \\
\hline Dietary fiber, g/10 MJ & 20 & 20 & 21 & 20 & 20 & 22 & 20 & 20 & 21 \\
\hline Alcohol, \%E & 0 & 0 & 0 & 0 & 0 & 0 & $0^{2}$ & $0^{2}$ & $0^{2}$ \\
\hline
\end{tabular}

Atwater's energy factors used for energy intake, energy distribution.

${ }^{1}$ Excluding alcohol.

${ }^{2}$ Less than $0.5 \%$.

The mean intake of meat and meat products in 2000-2002 was $87 \mathrm{~g} /$ day for children and $109 \mathrm{~g} /$ day for adults ( $138 \mathrm{~g}$ for men and $81 \mathrm{~g}$ for women). In addition, the mean intake of poultry was $19 \mathrm{~g} /$ day for children and $25 \mathrm{~g} /$ day for adults. In 20032006 the intake was on the same level. The Danish dietary guidelines have no recommendation on meat, but compared to an amount of $100 \mathrm{~g} /$ day $(10 \mathrm{MJ})$, recognized as appropriate, the intake is too high especially for men.

The whole grain intake based on data from the dietary survey 2000-2004 showed a mean intake approximately half the recommended amount, and only $6 \%$ of the population had a dietary whole grain density of $75 \mathrm{~g} / 10 \mathrm{MJ}$. The typical content (median) was $30 \mathrm{~g} / 10 \mathrm{MJ} /$ day [Mejborn et al., 2008].

\section{Energy and Macronutrients}

Results in table 11.15 show a decrease in energy intake among children aged $4-14$ years, which probably is due to an increasing degree of underreporting. Excluding data of the underreporters $(\mathrm{EI} / \mathrm{BMR}<1.1)$ shows a decrease of energy intake per day of $0.4 \mathrm{MJ}$, corresponding to approximately $4 \%$ reduction from 1995 to $2003-2006$. Table 11.16 shows decrease in energy intake among adults. After exclusion of underreporters the decrease in daily energy intake is $0.7 \mathrm{MJ}$ for men and $0.5 \mathrm{MJ}$ for women. 
Table 11.16. Development in intake of energy, macronutrients and energy distribution, and ratio between energy intake and basal metabolic rate (EI/BMR est , average for adults (15-75 years) [modified from Fagt et al., 2008]

\begin{tabular}{|c|c|c|c|c|c|c|c|c|c|}
\hline & \multicolumn{3}{|l|}{ Male } & \multicolumn{3}{|c|}{ Female } & \multicolumn{3}{|l|}{ All } \\
\hline & 1995 & $\begin{array}{l}2000 / \\
2002\end{array}$ & $\begin{array}{l}2003- \\
2006\end{array}$ & 1995 & $\begin{array}{l}2000 / \\
2002\end{array}$ & $\begin{array}{l}2003- \\
2006\end{array}$ & 1995 & $\begin{array}{l}2000 / \\
2002\end{array}$ & $\begin{array}{l}2003- \\
2006\end{array}$ \\
\hline $\mathrm{n}$ & 849 & 1,526 & 1,184 & 897 & 1,771 & 1,394 & 1,746 & 3,297 & 2,578 \\
\hline Energy, MJ & 11.7 & 10.8 & 10.5 & 8.8 & 8.4 & 7.9 & 10.2 & 9.5 & 9.1 \\
\hline $\mathrm{El} \mathrm{BMR}_{\mathrm{est}}$ & 1.54 & 1.42 & 1.38 & 1.52 & 1.43 & 1.34 & 1.53 & 1.42 & 1.36 \\
\hline $\mathrm{El} / \mathrm{BMR}_{\mathrm{est}}<1.1, \%$ & 13 & 21 & 24 & 16 & 22 & 29 & 14 & 22 & 26 \\
\hline Fat, $\% \mathrm{E}^{1}$ & 37 & 33 & 34 & 37 & 31 & 32 & 37 & 32 & 33 \\
\hline Fat, $\% \mathrm{E}^{2}$ & 39 & 35 & 36 & 38 & 33 & 34 & 39 & 34 & 35 \\
\hline Carbohydrate, total, \% $\% \mathrm{E}^{2}$ & 45 & 50 & 50 & 47 & 53 & 53 & 46 & 51 & 52 \\
\hline Added sugar, \% $\mathrm{E}^{2}$ & 9 & 9 & 9 & 9 & 10 & 9 & 9 & 10 & 9 \\
\hline Dietary fiber, g/10 MJ & 20 & 20 & 21 & 21 & 23 & 25 & 20 & 21 & 23 \\
\hline Alcohol, \%E & 6 & 7 & 7 & 4 & 5 & 5 & 5 & 6 & 6 \\
\hline Alcohol, g/day & 24 & 25 & 24 & 11 & 13 & 12 & 17 & 18 & 17 \\
\hline
\end{tabular}

Atwater's energy factors used for energy intake, energy distribution.

${ }^{1}$ Including alcohol.

2 Excluding alcohol.

The decrease of total fat in the diet of both children and adults observed from 1995 to 2000-2002 was not seen between 2000-2002 and 2003-2006. The mean fat content of children's diet in $2003-2006$ is $34 \% \mathrm{E}$ and in adults' 35\% $\mathrm{E}$ (excl. alcohol), and still too high compared to NNR 2004 (30\%E on population level).

The sugar content in children's diet decreased between 2000-2002 and 2003-2006 which is very positive. However, on average, children still consume $20 \%$ more added sugar than recommended.

In 2000-2002 the mean alcohol intake of men was $25 \mathrm{~g} /$ day and for women $13 \mathrm{~g} /$ day. Analyses [Bøgh-Sørensen et al., 2009] show that $26 \%$ of men and $18 \%$ of women consumed more alcohol than recommended from the National Board of Health (21 units/week or 3 units/day for men and 14 units/week or 2 units/day for women $(1$ unit $=12 \mathrm{~g})$ ) during the week registration. 56\% of men and $38 \%$ of women had an energy intake from alcohol above the NNR 2004 of max. 5\%E. Only 8\% of men and $15 \%$ of women consumed no alcohol during the week of registration. According to The National Health Interview Survey 1987-2005 [Ekholm et al., 2006], 14.3\% of the 
adults have consumed more than recommended by the National Board of Health. $29.5 \%$ of women and $15 \%$ of men consumed no alcohol during the week before answering the questionnaire. This inconsistency in results reflects difficulties in measuring alcohol intake and use of different methods (prospective records and retrospective questionnaires).

The mean intake of dietary fiber for both children and adults was around $21 \mathrm{~g} / 10 \mathrm{MJ}$. Only $10 \%$ of men and $17 \%$ of women met the NNR 2004 for dietary fiber $(30 \mathrm{~g} / 10 \mathrm{MJ})$.

\section{Differences in Diet According to Gender}

Comparison of boys' and girls' diets only shows differences in the mean daily energy intake, which is $1.2 \mathrm{MJ}$ higher for boys than for girls. Additionally, $40 \%$ of boys but only $32 \%$ of girls have a diet containing max. $10 \% \mathrm{E}$ from added sugar.

Among adults, differences in diet between men and women are more pronounced. The mean daily energy intake is $2.6 \mathrm{MJ}$ higher for men than for women. The diet of women contains less fat (2\%E-point), less alcohol (2.3\%E-point), and more dietary fiber $(4 \mathrm{~g} / 10 \mathrm{MJ})$. The diet of women appears healthier than the diet of men, although the diet of women has tendency to contain more added sugar.

\section{Micronutrients}

Micronutrient intakes are estimated based on the dietary survey 2000-2002 without taking the use of supplements into account [Lyhne et al., 2005]. The content of micronutrients in the average diet is generally sufficient assessed from NNR 2004. For the vitamins $\mathrm{A}$, riboflavin, niacin and $\mathrm{B}_{12}$ and the minerals calcium, phosphorus and iodine the content is abundant. The content is on an acceptable level for the vitamins $\mathrm{E}$, thiamine, $\mathrm{B}_{6}$, folate and $\mathrm{C}$ and the minerals magnesium, zinc, selenium and potassium. The content of vitamin $\mathrm{D}$ is too low in all age groups, and the iron content is insufficient for many women in fertile age.

\section{Supplements}

Data from the dietary survey 2000-2004 (4-75 years) show that 54\% of males and 60\% of females were users of dietary supplements. The lowest percentage of users was found in the age group of $15-24$ years, where $39 \%$ of males and $44 \%$ of females were users.

\section{Physical Activity}

Data from the European Youth Heart Study [Ekelund et al., 2004, cited by Pedersen and Saltin, 2005] showed that all the Danish children 9 years of age adhered to the recommendation of a minimum of 60 minutes moderate to vigorous intensity physical activity each day, but this was the case for only $50-75 \%$ of adolescents 15 years of age. The National Health Interview Survey on Children from 2005 showed that $78 \%$ of the children 6-15 years of age attended sports activity of at least 2 hours a week (outside school) and $65 \%$ of the children have active transportation to school at least 4 times a week [Johansen et al., 2009]. 
In total, $27 \%$ of adults stated being moderately or intensively physically active for at least 4 hours a week in their leisure time, while 13\% indicated being physically inactive. Almost twice as many men as women were moderately or intensively physically active, while the proportion of physically inactive people was the same among men and women. Since 1987, there has been a marked rise in the proportion with a moderate or intensive level of physical activity during their leisure time.

In total, 59\% of adults practiced sport, collectively or in some self-organized form. Just below three quarters of those in active employment spent less than 15 minutes of their daily transport time walking or cycling, while slightly more than $10 \%$ spent half an hour or more. Most of those in active employment (39\%) have sedentary jobs [cited and modified from Kjøller et al., 2007].

\section{Smoking}

The proportion of smokers has fallen steadily in recent decades - for men to $31 \%$ in 2006 and for women to $25 \%$. In 2005, more than 50\% among male and $40 \%$ of female smokers were heavy smokers. There is a clear educational gradient in the smoking pattern: the higher the level of education, the fewer daily smokers and heavy smokers [cited and modified from Kjøller et al., 2007]. Data from the national dietary survey 2002-2002 indicated that smoking is more likely among young women than among young men.

\section{Overweight and Obesity}

Measured data of weight and height are not available on national representative level. Self-reported data in the dietary survey showed that mean BMI increased significantly between 1995 and 2000-2002 for both boys and girls of all age groups (4-6, $7-10,11-14$ and 15-18 years). Prevalence of overweight increased between survey years for boys and girls for all age groups, although statistical significance was not reached. When all children and adolescents (4-18 years) were analyzed, the prevalence of overweight rose significantly from 10.9 to $14.4 \%$ between 1995 and 20002002, whereas the increase in the prevalence of obesity (2.3 vs. $2.4 \%$ ) did not reach significance [Matthiessen et al., 2008].

The National Health Interview Survey 1987-2005 [Ekholm et al., 2006], based on self-reported data, showed that $41 \%$ among men and $26 \%$ among women were overweight, with 12 and 11\%, respectively, being obese. Session data, too, showed a marked increase in the prevalence of obesity. Data from the national dietary survey 2000-2002 showed that 39\% among men 19-65 years and 22\% among women 19-65 years were overweight, with 11 and $10 \%$, respectively, being obese.

\section{Life Expectancy and Lifestyle-Related Disorders}

Life expectancy continues to rise and in 2006 was 75.9 years of age for men and 80.4 for women, but Danish men ranked 18th among 20 OECD countries and Danish women ranked last. 


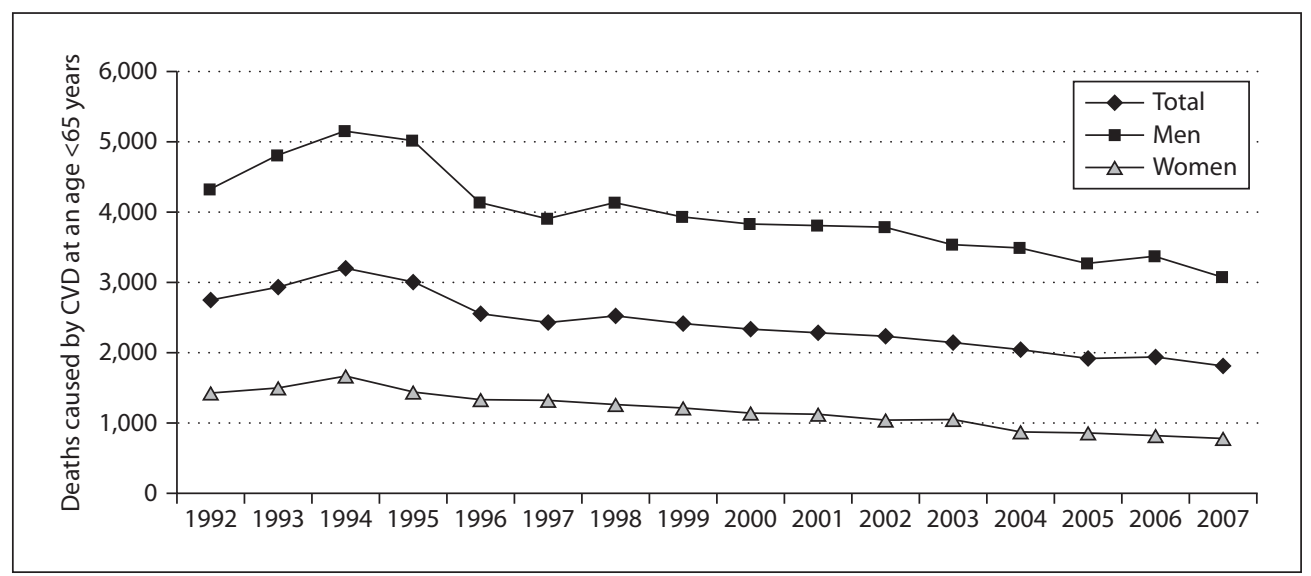

Fig. 11.5. CVD-caused deaths (at age <65 years) in the Estonian population (1992-2007).

Mortality is increasing for several lifestyle-related disorders. That applies to lung cancer, chronic obstructive pulmonary disease, cirrhosis of the liver among men and diabetes. For bowel cancer and breast cancer, mortality has been more or less constant, while mortality from heart diseases and stroke has generally declined. More than 300,000 adults state having a long-standing cardiovascular illness.

The number of new cancer cases rose to just below 33,000 in 2001. Approximately 216,000 adults $(4 \%)$ were living with a cancer diagnosis. The strongly age-related incidence is part of the explanation for the continued increase in the number of cancer cases.

Approximately 25,000 people have type 1 diabetes and an estimated 200,000 have type 2 diabetes in Denmark. A further 200,000 are presumed to have the illness without knowing it.

Around 500,000 Danes are estimated to have osteoporosis.

However, the majority (79\%) of adults rate their health to be really good or good [cited and modified from Kjøller et al., 2007].

\section{Social Inequality}

In Denmark virtually all manifestations of illness and all risk factors are unevenly distributed in social terms. A number of examples of social differences in relation to life expectancy and mortality, health and morbidity, lifestyle and health habits, living conditions and use of the health services are described [cited and modified from Kjøller et al., 2007]. For instance, education was shown to be related to healthier eating pattern [Groth et al., 2003] and in addition education is shown to be the dimension most consistently associated with BMI and obesity, and the gender-specific pattern showed a stronger social gradient for women, but not for men [Groth et al., 2009]. 
Table 11.17. Availability of ENHR 2009 indicators from nationally representative surveys in Estonia

\begin{tabular}{llllll}
\hline Age group & Body mass index & Nutrient intake & $\begin{array}{l}\text { Dietary } \\
\text { supplements }\end{array}$ & $\begin{array}{l}\text { Alcohol } \\
\text { consumption }\end{array}$ & Smoking \\
\hline Children (4-14) & - & - & - & - & - \\
\hline $\begin{array}{l}\text { Adolescents } \\
\text { (15-18) }\end{array}$ & (1) HB 2002-2008 & - & - & (1) HB 2002-2008 & (1) HB 2002-2008 \\
\hline Adults (19-64) & (2) EHIS 2006 & & & (2) EHIS 2006 & (2) EHIS 2006 \\
& HB 1997 & (1) BNS 1997 & (1) BNS 1997 & BNS 1997 & BNS1997 \\
& EHIS 2006 & & & HB 2002-2008 & HB 2002-2008 \\
\hline Elderly (65+) & (1) EHIS 2006 & - & - & (3) EHIS 2006 & (3) EHIS 2006 \\
\hline
\end{tabular}

HB 2002-2008 - Health Behavior among the Estonian Adult Population Survey 02/04/06/08.

BNS 1997 - Baltic Nutrition Survey 1997.

EHIS 2006 - Estonian Health Interview Survey 2006.

\subsection{Estonia ${ }^{5}$}

Cardiovascular diseases (CVD) are the main reason for early loss of work capacity and death (at age $<65$ ) in Estonia. Estonia is leading in CVD-caused deaths in Europe and the whole world. Each year, at least 250 men and 80 women out of every 100,000 inhabitants die of CVD before the age of 65. During Estonia's re-independence period in 1992-1994, premature deaths caused by CVD increased substantially. In recent years mortality has decreased to the same level as it was at the beginning of the 1990s, but a stable and constant decrease has still not been reached (fig. 11.5). The main factors in reducing CVD are: regular physical activity, balanced nutrition, abstinence from smoking, control of blood pressure and cholesterol level, valuing of health and psychosocial factors [National Institute for Health Development, 2005].

Cancer is a prevalent disease in Estonia, holding the second place among causes of death. In 2005, 20\% out of all death cases were caused by cancer. There are almost 3,500 malignant tumor deaths in Estonia every year and the mortality index has not decreased during the last decade [World Health Organization Cancer Mortality Database, 2005]. Men's cancer death coefficients in Estonia are one of the highest on a European scale [Bray et al., 2002; Ferlay et al., 2004]. Cancer prevention depends mostly on lifestyle and environment including balanced nutrition [National Institute for Health Development, 2007].

In this report, data originates from three nationally representative surveys conducted in Estonia (table 11.17). Data about nutritional indicators among adults are

\footnotetext{
${ }^{5}$ Liis Kambek, Natalja Gluškova; National Institute for Health Development, Tallinn, Estonia. E-Mail
} liis.kambek@tai.ee 
available from the Baltic Nutrition Survey (BNS) conducted in 1997. Nationally representative nutritional data are not available for children, adolescents or the elderly. There are no nationally representative data available about nutritional biomarkers among the Estonian population.

Data about diet-related health indicators such as anthropometric measures, alcohol consumption and smoking are available from two nationally representative surveys. The Estonian Health Interview Survey (EHIS) was conducted in 2006 and provides data for adolescents, adults and the elderly. Health Behavior among the Estonian Adult Population Survey (HB) has been conducted since 1990 after every 2 years and provides data for adolescents and adults. However, data for this report were chosen from the last four HB survey years - 2002, 2004, 2006 and 2008.

The BNS was conducted in 1997 by the World Health Organization [Pomerlau et al., 2000]. The survey was done with a simple random sample of 3,000 people aged 19-64. The sample was drawn from the National Population Register and stratified by age. The questionnaire was discussed and agreed within all Baltic countries. All subjects were interviewed once, using the 24-hour recall method.

24-hour recall was done in the first part of the interview. Common sets of household measures were used for assessing food portion sizes, as well as a standard book with photographs and drawings of commonly consumed foods. The second part of the interview was a questionnaire with questions about socioeconomic status and health behavior.

Data were collected in July and August 1997. The response rate was $67.3 \% ; 2,108$ people altogether participated in the survey.

Nutrient intake was calculated from 24-hour recalls using Micro-Nutrica Nutritional Analysis program (Estonian version 2, 1997, Food Processing Institute, Tallinn University of Technology).

The Health Behavior among the Estonian Adult Population Survey (HB) has been conducted since 1990 with a 2-year interval in cooperation with the Finnish National Health Institute (Helsinki). The research is done within the joint 'FinBalt Health Monitor' project. The survey sample is drawn from the National Population Register. The survey is conducted with a questionnaire sent by mail. The questionnaire includes a module on dietary habits that composes of a food frequency and preference questionnaire.

In 2002, the HB survey was conducted by the Estonian Centre for Health Education and Promotion [Kasmel et al., 2003]. The survey was done with a simple random sample of 2,000 residents of Estonia aged 16-64. The sample was stratified by place of residence, age and sex. The response rate was $67 \%$ with a final number of completed eligible questionnaires that were used for data entry being 1,338 .

In 2004, the HB survey was conducted by the National Institute for Health Development [Rahu et al., 2005]. The survey was done with a simple random sample of 5,000 residents of Estonia aged 16-64. The questionnaires were mailed during the time period between April and June 2004. The response rate was $63.4 \%$ with a 
final number of completed eligible questionnaires that were used for data entry being 3,074 .

In 2006, the HB survey was conducted by the National Institute for Health Development [Tekkel et al., 2007]. The survey was done with a simple random sample of 5,000 residents of Estonia aged 16-64. The questionnaires were mailed during the time period between March and May 2006. The response rate was 59.2\% with a final number of completed eligible questionnaires that were used for data entry being 2,867 .

In 2008, the HB survey was conducted by the National Institute for Health Development [Tekkel et al., 2009]. The survey was done with a simple random sample of 5,000 residents of Estonia aged 16-64. The questionnaires were mailed during the time period between March and May 2008. The response rate was $62.2 \%$ with a final number of completed eligible questionnaires that were used for data entry being 3,004 .

The EHIS was conducted for the second time in 2006 by the National Institute for Health Development [Oja et al., 2008]. The survey was conducted as a paper-assisted personal interview. The survey was done with a systematic stratified sample of 11,023 permanent residents of Estonia aged 15-84. The sample was drawn from the National Population Registry and stratified by region, age and sex group. Data was collected in a 1-year period, October 2006-2007. The response rate was 60.2\%, with 6,494 being the total number of completed eligible questionnaires that were used for data entry. The interview questionnaire consisted of 22 modules, including separate modules on smoking, use of alcohol, eating habits and physical activity. The eating habits module composed of a limited food frequency and preference questionnaire.

\section{Adolescents}

There are no nationally representative data available about nutrient intake among adolescents in Estonia (table 11.17).

Prevalence of overweight (including obesity) among male adolescents aged 16-18 has been on the rise. In 2002, prevalence of overweight (including obesity) was $6.7 \%$ and by 2008 it was $19.8 \%$. Prevalence of overweight (including obesity) is lower among female adolescents in the same age group, in 2002 there was no prevalence and by 2008 it was $8.9 \%$. Prevalence of underweight among female adolescents aged $16-18$ was $14.9 \%$ in 2008.

The proportion of current smokers among male adolescents aged 16-18 was 50\% in 2002 , by 2008 it was $32.2 \%$. The proportion of current smokers among female adolescents in the same age group was $37 \%$ in 2002 and by 2008 it was $23.1 \%$. The average number of cigarettes smoked daily among boys was 8.5 and 6.4 among girls in 2008 (fig. 11.6).

In 2002, the share of abstainers among male adolescents aged 16-18 was $12.9 \%$ and by 2008 it was $13.5 \%$ (table 11.18). Among female adolescents in the same age group the share of abstainers was $15.2 \%$ in 2002 and by 2008 it was $11.4 \%$. 
Fig. 11.6. Average number of cigarettes smoked daily among adolescents (current smokers) aged $\mathbf{1 6 - 1 8}$ years in Estonia (2004-2008).

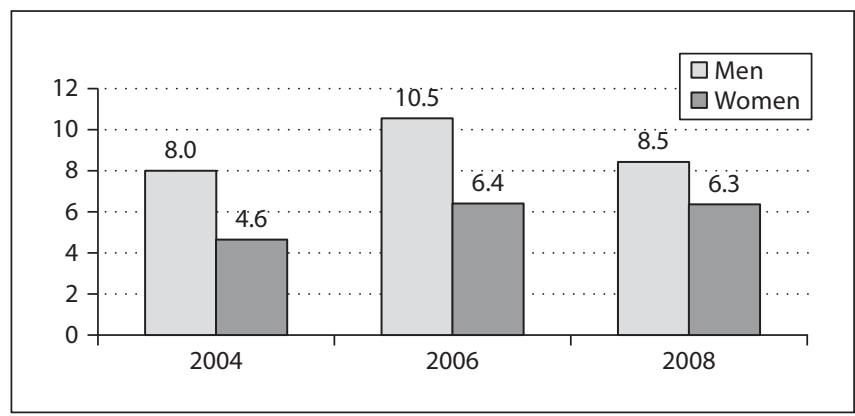

Table 11.18. Abstainers among adolescents aged 16-18 in Estonia, \% (2002-2008) [source: HB 02/04/06/08]

\begin{tabular}{lllll}
\hline & 2002 & 2004 & 2006 & 2008 \\
\hline Men & $\mathrm{n}=62$ & $\mathrm{n}=186$ & $\mathrm{n}=158$ & $\mathrm{n}=178$ \\
\hline Total & 12.9 & 12.9 & 11.4 & 13.5 \\
\hline Women & $\mathrm{n}=66$ & $\mathrm{n}=200$ & $\mathrm{n}=202$ & $\mathrm{n}=210$ \\
\hline Total & 15.2 & 13.0 & 11.9 & 11.4 \\
\hline
\end{tabular}

Abstainer - never drinks and no drinking during the last 12 months.

Adults

In 1997, average energy intake among male adults was normal up to the age of 34 years, but then showed a decrease under the recommended energy intake. Among female adults the average energy intake was lower than the recommendation in all age groups. Among both male and female adults the average proportion of protein in total energy intake was in a normal range. The proportion of carbohydrates in total energy intake was lower than the national recommendation [RDI, 2006] for both male and female adults in all age groups, with men's intake of carbohydrates being lower than women's. The intake of dietary fiber was also lower than the recommended minimal daily amount of $25 \mathrm{~g}$ for both men and women. The average daily intake of dietary fiber was $16.2 \mathrm{~g}$ for women and $20.7 \mathrm{~g}$ for men.

The proportion of fat and SFA in total energy intake was constantly higher than the maximum recommendation of $30 \%$ and $10 \%$, respectively, for both male and female adults in all age groups with the average proportion of energy from fat being $\sim 36 \% \mathrm{E}$. However, the average intake of cholesterol was within recommended limits for women. For men, the intake of cholesterol (348 mg/day) was slightly higher than 
Fig. 11.7. Consumption of dietary supplements at least once a week among adults (19-64 years) in Estonia, \% (1997).

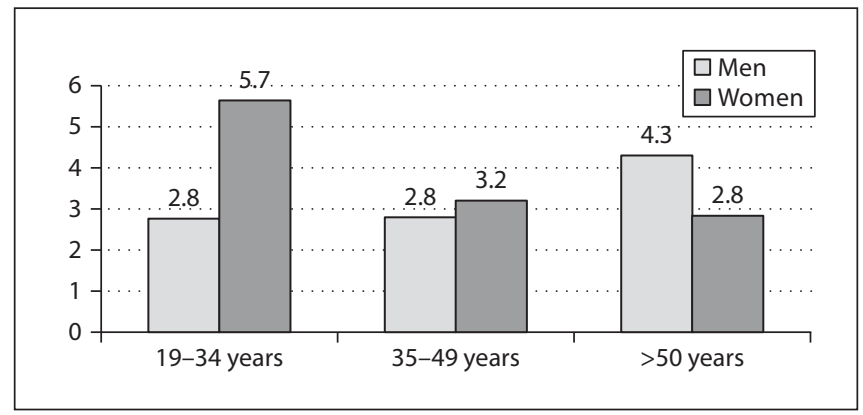

the recommended $300 \mathrm{mg} /$ day. For men, the proportion of alcohol in total energy intake was higher than the recommended maximum limit of $5 \%$.

The average daily intake of vitamin $\mathrm{C}$ was relatively constant up to the age of 50 years, but showed a considerable decrease for male and female adults $>50$ years of age. The intake of vitamin $\mathrm{D}$ for male and female adults in all age groups was remarkably low compared to the recommended minimal daily intake of $7.5 \mu \mathrm{g}$ [Vaask et al., 2006]. For men, the average daily intake of vitamin D was $3.6 \mu \mathrm{g}$ and for women $2.8 \mu \mathrm{g}$. The average daily intake of vitamin $\mathrm{E}$ was insufficient for both sexes in all age groups, except for men aged 19-34.

The average daily intake of sodium exceeded the national recommendation for both male and female adults. Intake of sodium was higher among men. Average calcium intakes were lower than the recommended $800 \mathrm{mg} /$ day for men and for women. For men, the average intake of calcium was $717 \mathrm{mg} /$ day and for women $590 \mathrm{mg} / \mathrm{day}$. For women aged 19-34, the average intake of iron was lower than the recommended $15 \mathrm{mg} /$ day.

The proportion of women using dietary supplements at least once a week decreased with age. For men the opposite trend applied (fig. 11.7).

The prevalence of normal weight among male adults in Estonia decreased from 2002 to 2008. In 2002, the proportion of men belonging to the normal weight category was $53.5 \%$, by 2008 the proportion was $40.9 \%$ (table 11.19). Simultaneously, the proportion of men belonging to the overweight and obese category has been on the rise. In 2002, the proportion of overweight and obese men was 33.1 and $12.1 \%$, respectively. By 2008, these proportions had risen to 40.5 and $18.1 \%$, respectively. The prevalence of obesity among female adults in Estonia slowly rose from 2002 to 2008. In 2002, the proportion of obese women was $14.5 \%$, by 2008 it was $19.5 \%$.

In 2008, the prevalence of overweight and obesity among male and female adults increased with age. The prevalence of overweight (including obesity) among men was $58.5 \%$, it was highest among men over 50 years old, with the proportion being $70.3 \%$. The prevalence of overweight (including obesity) was lower among women, $46.5 \%$, among women over 50 years, the proportion was $72.2 \%$. The prevalence of underweight was $8.8 \%$ among women aged 19-34. 
Table 11.19. BMI distribution among adults (19-64 years) in Estonia, \% (2002-2008), self-reported data [source: HB 02/04/06/08]

\begin{tabular}{lllll}
\hline & 2002 & 2004 & 2006 & 2008 \\
\hline Men & $\mathrm{n}=529$ & $\mathrm{n}=1,299$ & $\mathrm{n}=1,112$ & $\mathrm{n}=1,162$ \\
\hline Underweight & 1.3 & 2.1 & 1.2 & 0.6 \\
\hline Normal weight & 53.5 & 52.2 & 46.8 & 40.9 \\
\hline Overweight & 33.1 & 32.0 & 37.7 & 40.5 \\
\hline Obese & 12.1 & 13.7 & 14.4 & 18.1 \\
\hline Women & $\mathrm{n}=1,520$ & $\mathrm{n}=1,734$ & $\mathrm{n}=1,706$ & $\mathrm{n}=1,597$ \\
\hline Underweight & 6.3 & 4.5 & 5.1 & 4.1 \\
\hline Normal weight & 53.3 & 54.9 & 52.8 & 49.5 \\
\hline Overweight & 25.9 & 25.7 & 25.7 & 27.0 \\
\hline Obese & 14.5 & 14.9 & 16.4 & 19.5 \\
\hline
\end{tabular}

Underweight: $<18.49 \mathrm{~kg} / \mathrm{m}^{2}$; normal weight: $18.5-24.99 \mathrm{~kg} / \mathrm{m}^{2}$; overweight: $25-29.99 \mathrm{~kg} / \mathrm{m}^{2}$; obese: $>30 \mathrm{~kg} / \mathrm{m}^{2}$.

The proportion of current smokers has decreased among male and female adults in Estonia. In 2002, 57.4\% of men were smokers, by 2008, 47\% were smokers. Among women, in $2002,26.4 \%$ were smokers and by $2008,25.4 \%$ were smokers. The only group that has had an increase in smoking were women aged over 50 years, in 2002 $15.9 \%$ were smokers and by $200820.2 \%$ were smokers.

In 2008, the average number of cigarettes smoked daily among adult women was 9.3 (fig. 11.8). Among adult men, the average number of daily cigarettes was 16.6. Among both men and women, the number of daily cigarettes increased with age.

In 2008, the share of heavy drinkers among male adults was $1.3 \%$ (table 11.21). Among female adults the share of heavy drinkers was $0.1 \%$. In 2002, the share of abstainers among adult men was 3.8\% and by 2008 it was 7\% (table 11.20). Among female adults, the share of abstainers was $7.4 \%$ in 2002 and by 2008 it was $7.6 \%$.

\section{Elderly}

There are no nationally representative data available about nutrient intake among elderly (65+ years) in Estonia.

In 2006, prevalence of overweight (including obesity) among elderly men was $58.5 \%$ and among elderly women $68.3 \%$. Obesity is more prevalent among elderly women than elderly men in Estonia. 


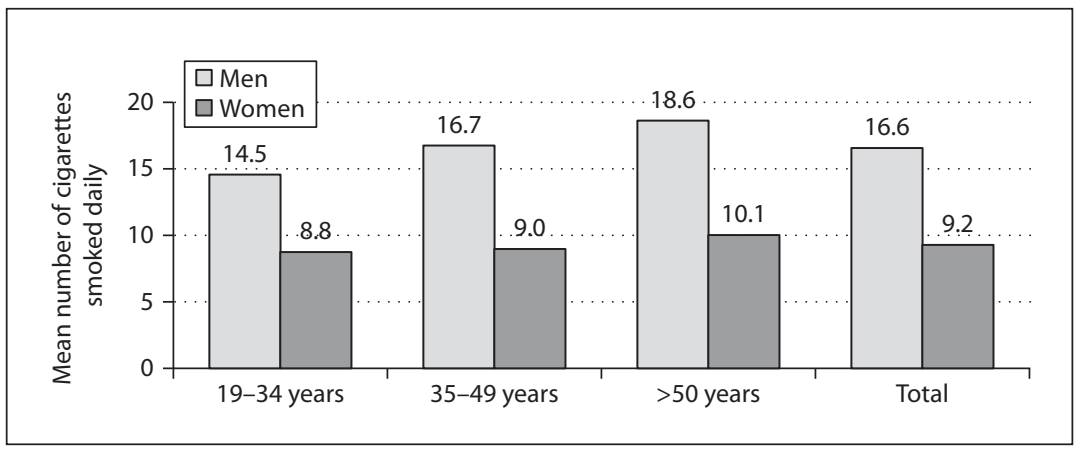

Fig. 11.8. Average number of cigarettes smoked daily among adults (19-64 years, current smokers) in Estonia (2008).

Table 11.20. Abstainers among adults (19-64 years) in Estonia, \% (2002-2008) [source: HB 02/04/06/08]

\begin{tabular}{lllll}
\hline & 2002 & 2004 & 2006 & 2008 \\
\hline Men & $\mathrm{n}=503$ & $\mathrm{n}=1,194$ & $\mathrm{n}=1,017$ & $\mathrm{n}=1,182$ \\
\hline $19-34$ & 2.7 & 4.8 & 5.9 & 6.8 \\
\hline $55-49$ & 4.2 & 7.2 & 3.1 & 6.1 \\
\hline Total & 4.6 & 6.0 & 7.7 & 8.3 \\
\hline Women & 3.8 & 5.9 & 5.6 & 7.0 \\
\hline $19-34$ & $\mathrm{n}=731$ & $\mathrm{n}=1,553$ & $\mathrm{n}=1,549$ & $\mathrm{n}=1,628$ \\
\hline $35-49$ & 6.8 & 4.2 & 5.8 & 7.0 \\
\hline $50+$ & 6.3 & 4.9 & 6.9 & 5.5 \\
\hline Total & 9.1 & 13.3 & 9.5 & 10.4 \\
\hline
\end{tabular}

Abstainer - never drinks and no drinking during the last 12 months.

In 2006, proportion of current smokers among elderly men was $65.4 \%$ and the average number of cigarettes smoked daily was 13.1. Among women, the proportion of current smokers was $10.8 \%$ and the average number of cigarettes smoked daily was 10.9.

The share of abstainers among elderly men was $8.8 \%$ and $32.2 \%$ among elderly women in 2006 (table 11.22).

To summarize, there is no nationally representative data about the nutrient intake of children or adolescents to give an overview of their dietary status. Prevalence of overweight (including obesity) among male adolescents aged 16-18 has been on the 
Table 11.21. Heavy drinkers among adults (19-64 years) in Estonia, \% (2002-2008) [source: HB 02/04/06/08]

\begin{tabular}{lllll}
\hline & 2002 & 2004 & 2006 & 2008 \\
\hline Men & $\mathrm{n}=503$ & $\mathrm{n}=1,194$ & $\mathrm{n}=1,017$ & $\mathrm{n}=1,144$ \\
\hline $19-34$ & 1.6 & 2.4 & 1.7 & 1.7 \\
\hline $35-49$ & 1.8 & 2.1 & 3.1 & 1.8 \\
\hline $50+$ & 0.7 & 0.6 & 0.3 & 0.3 \\
\hline Total & 1.4 & 1.8 & 1.7 & 1.3 \\
\hline Women & $\mathrm{n}=731$ & $\mathrm{n}=1,553$ & $\mathrm{n}=1,549$ & $\mathrm{n}=1,576$ \\
\hline $19-34$ & 0 & 0 & 0.4 & 0 \\
\hline $35-49$ & 0.4 & 0 & 0.6 & 0.2 \\
\hline $50+$ & 0.4 & 0 & 1.2 & 0 \\
\hline Total & 0.3 & 0 & 0.7 & 0.1 \\
\hline
\end{tabular}

Heavy drinker $->5$ units of alcohol per day. 1 alcohol unit $=8-12 \mathrm{~g}$ of absolute alcohol $(1$ shot of vodka $=1$ glass of wine $=10.5$-liter bottle of beer).

Table 11.22. Abstainers and heavy drinkers among elderly (65+ years) in Estonia, \% (2006) [source: EHIS 2006]

\begin{tabular}{lll}
\hline & Abstainers & Heavy drinkers \\
\hline Men & $\mathrm{n}=1,063$ & \\
\hline $65-70$ & 7.2 & 1.8 \\
\hline $70-75$ & 9.2 & 1.1 \\
\hline $75+$ & 9.8 & 0.4 \\
\hline Total & 8.8 & 1.0 \\
\hline Women & $\mathrm{n}=1,097$ & \\
\hline $65-70$ & 22.1 & 0 \\
\hline $70-75$ & 31.4 & 0 \\
\hline $75+$ & 39.9 & 0.2 \\
\hline Total & 32.2 & 0.1
\end{tabular}

Heavy drinker $->5$ units of alcohol per day. 1 alcohol unit $=8-12 \mathrm{~g}$ of absolute alcohol ( 1 shot of vodka $=1$ glass of wine $=10.5$-liter bottle of beer).

Abstainer - never drinks. 
rise during the last 6 years. The proportion of underweight female adolescents aged $16-18$ has been relatively high for the last 6 years (14.9\% in 2008). The proportion of current smokers among male and female adolescents aged 16-18 has been declining. The share of abstainers among female adolescents aged 16-18 has been declining.

Main nutritional problems among adults in 1997 included a high proportion of fat and SFA in total energy and a rather low carbohydrate intake. The intake of dietary fiber was low. For men, the proportion of alcohol in total energy intake was higher than the recommended amount. The intake of vitamin D was remarkably low for both male and female adults. The intake of vitamin $\mathrm{E}$ was insufficient and the intake of sodium was high. Calcium intakes were low for women, for women aged 19-34 the intake of iron was low.

Recent surveys show that the prevalence of overweight (including obesity) is on the rise among adult men in Estonia. The prevalence of obesity among female adults has been slowly rising for the past six years. The prevalence of overweight (including obesity) increases with age. In 2008, the prevalence of underweight was highest among women aged 19-34. The proportion of current smokers has decreased among male and female adults during the last six years. The share of abstainers among adult men has been on the rise. The proportion of heavy drinkers among adult men and women has not had a stable decline.

There are no nationally representative data about the nutrient intake of elderly to give an overview of their dietary status. In 2006, obesity was more prevalent among elderly women than elderly men in Estonia. The proportion of current smokers was very high among elderly men. Share of abstainers was higher among elderly women.

\subsection{Finland ${ }^{6}$}

Finland has a long tradition in actively supporting public nutrition in order to affect public health. For instance, free lunch has been offered to schoolchildren for over 60 years, and nutrition counseling has been a part of action at the maternity clinics free for all pregnant women in Finland. Many actions have been introduced to eliminate nutritional deficiencies: iodizing of salt and adding vitamins $\mathrm{A}$ and $\mathrm{D}$ to margarine began in the 1940s, adding selenium to multiple nutrient inorganic fertilizers began in 1985 , and the latest fortification of liquid milk products and fat spreads with vitamin D was revised in 2003.

Food supply data has been collected systematically in Finland since the 1950s (Food Balance Sheets, Ministry of Agriculture and Forestry). According to these sheets the Finnish diet has changed remarkably during the past decades. The supply of potatoes and fats has decreased, while the supply of vegetables has tripled since

\footnotetext{
${ }^{6}$ Voutilainen S, Erkkilä A; School of Public Health and Clinical Nutrition, Kuopio, Finland. E-Mail sari. voutilainen@uku.fi, arja.erkkila@uku.fi
} 


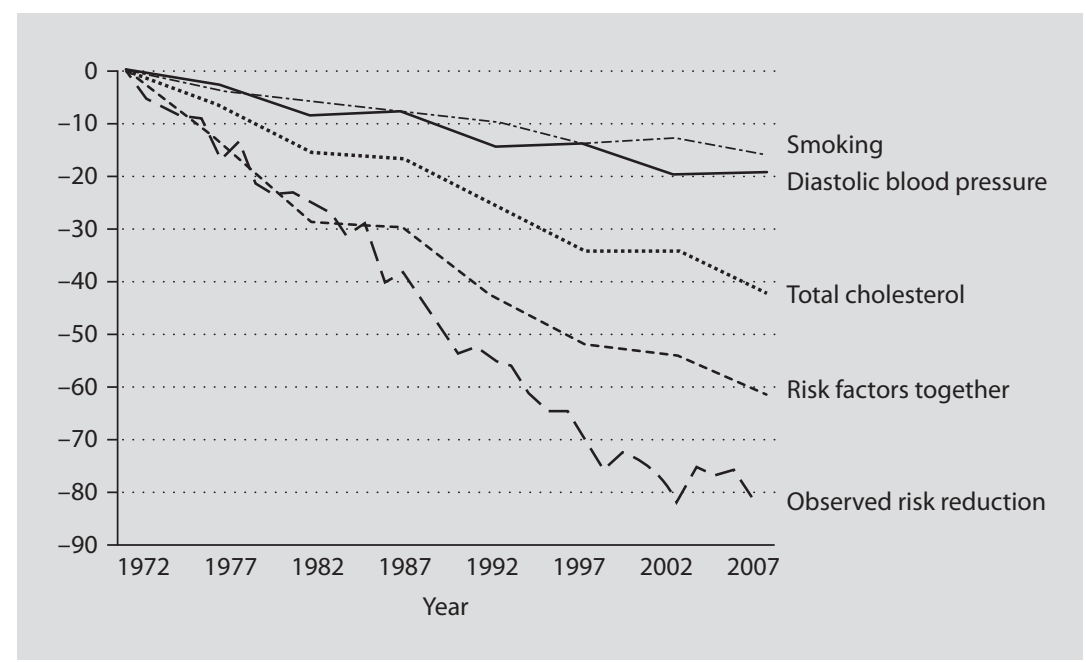

Fig. 11.9. Observed and risk factor-predicted reduction in CVD mortality in 36- to 64-year-old Finnish men. Figure adopted from Vartiainen et al. [2008].

the 1970s, being almost $70 \mathrm{~kg}$ per capita per year in 2007. Vegetable margarines and butter/oil mixtures have mostly replaced butter and low-fat and skimmed milk have replaced fatty milks. According to the latest publication, in the year 2007, skimmed milk accounted for one third of the total milk supply, being about 501 per capita per year, with increase of $3.6 \%$ from the previous year. In the 1980s the supply of butter was about $12 \mathrm{~kg}$ per capita per year as compared to $2.6 \mathrm{~kg}$ in 2007 . Fruit juices and juices with added sugar have also become more popular and supply of soft and alcohol drinks has increased. The meat that Finns eat most is pork. Pork supply amounts to almost $35 \mathrm{~kg}$ per capita, while beef and poultry meat supply were 18.7 and $17.6 \mathrm{~kg}$ per capita in 2007, respectively. The favorable trends in food intake are reflected to the intake of fats and salt. The intake of total fat has decreased from about $38 \%$ of energy $(\% \mathrm{E})$ to $31-32 \% \mathrm{E}$ and the proportion of saturated fat from 20 to $12-13 \% \mathrm{E}$ during 1980-2007. Trans-fatty acids are not the problem in Finnish diet, the daily intake of trans-fatty acids was $<1 \mathrm{~g}$ in all age groups. The small amount of trans-fatty acids in Finland is due to the fact that the Finnish margarine industry adopted interesterification as the hardening method for vegetable oils already in the 1990s, and this method does not produce trans-fatty acids. There has been about a $40 \%$ decrease in the intake of salt during the past 30 years being now $6.0 \mathrm{~g} /$ day in women and $8.3 \mathrm{~g} /$ day in men [Paturi et al., 2008]. These trends have favorably affected cardiovascular risk factors, and for instance cardiovascular mortality has decreased by $80 \%$ in the middle-aged population since the 1970s (fig. 11.9) [Vartiainen et al., 2008].

There are still many challenges in spite of these great achievements. In 2008, several national publications about nutrient intake and health indicators were published 
in Finland. The following chapters present collected information of these reports in infants, children, adolescents, adults and in the elderly Finnish population.

Infants and Children Under 7 Years of Age

A large population-based Type 1 Diabetes Prediction and Prevention (DIPP) Nutrition Study started in 1996-97. The aim of the study is to evaluate longitudinally the dietary factors in relation to the development of diabetic autoantibodies and clinical type 1 diabetes. Information on food consumption and food supplement usage was collected from children living in the regions of Tampere and northern Ostrobothnia. Table 11.23 offers information on the nutrition of 1- to 6-year-old Finnish children [Kyttälä et al., 2008] assessed as a part of DIPP Study. The information on food consumption was collected from 2,535 children between the years 2003-2005 using food diaries. The report by Kyttälä et al. describes differences in food consumption and nutrient intake between different sociodemographic groups, and between children cared at home and those in day care outside the family home. Food diary data was entered and processed using Finessi software, an application developed at the National Public Health Institute, Finland.

Based on the infant feeding records of 5,993 DIPP Nutrition Study children, the duration of breastfeeding does not currently meet the internationally recommended levels [Kyttälä et al., 2008]. The mean duration of exclusive breastfeeding was 1.4 months, while partial breastfeeding was continued, on average, up to the age of 7 months. Low educational level, young maternal age and smoking were associated with a shorter duration of breastfeeding. 1-year-old children were given industrial baby food frequently.

In 2005 the Ministry of Social Affairs and Health carried out a nationwide survey of breastfeeding and complementary feeding among the Finnish children under 1 year of age [Hasunen and Ryynänen, 2006]. The survey was a follow-on to the surveys carried out in 1995 and 2000. Altogether 74 primary healthcare centers with over 10,500 children participated. Nearly all newborn babies were breastfed. At the age of 1 month, $87 \%$ of babies were breastfed, at the age of 2 months $76 \%$ and at the age of 6 months $60 \% .36 \%$ of the children were receiving breast milk in addition to complementary feeding at the age of 11 months. There was a clear progress in exclusive breastfeeding at the age of 1-5 months compared to the situation in year 2000 [Hasunen and Ryynänen, 2006].

According to DIPP Nutrition Study, a quarter of the children studied had been on a special diet before the age of 3 years. The prevalence of food allergies was highest at the age of 1 year (14\%), falling to $6 \%$ by the age of 6 years. The most common allergens were milk, wheat, barley, rye, fish and eggs [Kyttälä et al., 2008].

Consumption of fresh vegetables, fruit and berries was low, along with the consumption of fish and use of fat spreads. The intake of sucrose and saturated fatty acids among 2- to 6-year-old children was higher than the levels suggested by the Finnish Nutrition Recommendations, whilst the intake of polyunsaturated fatty acids 
Table 11.23. Mean daily intake of energy and nutrients (mean $\pm S D$ ) in Finnish children aged 1, 4 and 6 years [source: Kyttälä P et al. 2008]

\begin{tabular}{|c|c|c|c|c|c|c|}
\hline \multirow[t]{2}{*}{ Nutrient } & \multicolumn{2}{|l|}{1 year old ${ }^{1}$} & \multicolumn{2}{|l|}{4 years old } & \multicolumn{2}{|l|}{6 years old } \\
\hline & girls $(n=198)$ & boys $(n=257)$ & girls $(n=247)$ & boys $(n=307)$ & girls $(n=349)$ & boys $(n=364)$ \\
\hline Energy, MJ & $3.6 \pm 0.6$ & $3.9 \pm 0.7$ & $5.5 \pm 1.0$ & $5.8 \pm 1.1$ & $6.0 \pm 1.1$ & $6.7 \pm 1.2$ \\
\hline Protein, \%E & 16 & 15 & 15 & 15 & 15 & 16 \\
\hline Carbohydrates, \%E & 55 & 55 & 53 & 53 & 53 & 53 \\
\hline Total, g & $90 \pm 29$ & $99 \pm 29$ & $170 \pm 30$ & $180 \pm 36$ & $185 \pm 37$ & $206 \pm 39$ \\
\hline Sucrose, $g$ & $11.7 \pm 8.0$ & $3.0 \pm 9.4$ & $43.6 \pm 16.0$ & $46.9 \pm 18.0$ & $43.6 \pm 16.0$ & $46.9 \pm 18.0$ \\
\hline Dietary fiber, $\mathrm{g}$ & $8.3 \pm 3.2$ & $8.8 \pm 3.2$ & $9.4 \pm 3.0$ & $9.6 \pm 3.2$ & $10.3 \pm 3.3$ & $11.4 \pm 3.7$ \\
\hline Fat, \%E & 29 & 29 & 31 & 31 & 31 & 31 \\
\hline SFA, \%E & 11.8 & 11.5 & 13.7 & 13.7 & 13.8 & 13.5 \\
\hline MUFA, \%E & 10.5 & 10.4 & 10.6 & 10.6 & 10.7 & 10.8 \\
\hline PUFA, \%E & 5.1 & 5.1 & 4.0 & 4.0 & 4.1 & 4.2 \\
\hline Trans-fatty acids, g & $0.2 \pm 0.1$ & $0.3 \pm 0.2$ & $0.7 \pm 0.3$ & $0.7 \pm 0.4$ & $0.8 \pm 0.3$ & $0.8 \pm 0.4$ \\
\hline$\% \mathrm{E}$ & 0.3 & 0.2 & 0.5 & 0.5 & 0.5 & 0.5 \\
\hline $\mathrm{n}-3$ fatty acids, $\mathrm{g}$ & $0.9 \pm 0.4$ & $1.0 \pm 0.5$ & $1.3 \pm 0.6$ & $1.4 \pm 0.6$ & $1.4 \pm 0.5$ & $1.7 \pm 0.7$ \\
\hline$\% \mathrm{E}$ & 1.0 & 1.0 & 0.9 & 0.9 & 0.9 & 0.9 \\
\hline Vitamin A, $\mu \mathrm{g}$ & $317 \pm 243$ & $313 \pm 286$ & $536 \pm 527$ & $606 \pm 706$ & $591 \pm 628$ & $676 \pm 689$ \\
\hline Vitamin D, $\mu \mathrm{g}$ & $12.2 \pm 4.6$ & $12.2 \pm 4.6$ & $6.1 \pm 4.1$ & $5.9 \pm 3.6$ & $5.5 \pm 3.2$ & $6.3 \pm 3.5$ \\
\hline Vitamin $E$, mg & $3.8 \pm 1.4$ & $4.1 \pm 1.7$ & $5.1 \pm 2.8$ & $5.3 \pm 2.5$ & $5.5 \pm 2.3$ & $6.1 \pm 2.5$ \\
\hline Thiamine, mg & $0.68 \pm 0.17$ & $0.74 \pm 0.19$ & $0.95 \pm 0.54$ & $1.00 \pm 0.57$ & $1.01 \pm 0.56$ & $1.13 \pm 0.65$ \\
\hline Riboflavin, mg & $1.29 \pm 0.37$ & $1.34 \pm 0.41$ & $1.73 \pm 0.62$ & $1.81 \pm 0.74$ & $1.81 \pm 0.69$ & $2.06 \pm 0.81$ \\
\hline Niacin, mg & $12.0 \pm 2.5$ & $12.8 \pm 3.3$ & $18.4 \pm 5.9$ & $19.5 \pm 6.1$ & $19.5 \pm 5.9$ & $22.2 \pm 6.5$ \\
\hline Vitamin $B_{6}, \mathrm{mg}$ & $0.98 \pm 0.27$ & $1.04 \pm 0.29$ & $1.47 \pm 0.68$ & $1.51 \pm 0.58$ & $1.47 \pm 0.58$ & $1.64 \pm 0.63$ \\
\hline Folate, $\mu \mathrm{g}$ & $107 \pm 28$ & $114 \pm 31$ & $132 \pm 49$ & $135 \pm 52$ & $144 \pm 51$ & $166 \pm 61$ \\
\hline Cobalamin, $\mu \mathrm{g}$ & $2.7 \pm 1.1$ & $2.8 \pm 1.2$ & $4.2 \pm 2.1$ & $4.6 \pm 2.6$ & $4.5 \pm 2.4$ & $5.3 \pm 2.8$ \\
\hline Ascorbic acid, mg & $75 \pm 31$ & $80 \pm 34$ & $61 \pm 41$ & $60 \pm 41$ & $61 \pm 36$ & $72 \pm 49$ \\
\hline Sodium, $\mathrm{g}$ & $0.73 \pm 0.37$ & $0.74 \pm 0.45$ & $1.84 \pm 0.47$ & $2.01 \pm 0.52$ & $2.03 \pm 0.56$ & $2.29 \pm 0.51$ \\
\hline Calcium, mg & $696 \pm 267$ & $670 \pm 293$ & $930 \pm 255$ & $983 \pm 306$ & $991 \pm 286$ & $1,103 \pm 302$ \\
\hline Magnesium, mg & $157 \pm 37$ & $168 \pm 42$ & $199 \pm 41$ & $211 \pm 52$ & $216 \pm 50$ & $246 \pm 67$ \\
\hline Iron, mg & $6.2 \pm 2.8$ & $6.6 \pm 2.3$ & $7.0 \pm 2.7$ & $8.0 \pm 3.6$ & $7.7 \pm 3.3$ & $8.6 \pm 2.8$ \\
\hline Zinc, mg & $5.7 \pm 1.4$ & $6.3 \pm 1.6$ & $7.2 \pm 2.0$ & $8.0 \pm 2.7$ & $8.2 \pm 3.1$ & $9.1 \pm 2.8$ \\
\hline lodine, $\mu \mathrm{g}$ & $121 \pm 39$ & $123 \pm 44$ & $183 \pm 47$ & $196 \pm 57$ & $197 \pm 55$ & $223 \pm 53$ \\
\hline Selenium, $\mu \mathrm{g}$ & $23.2 \pm 6.6$ & $24.5 \pm 8.2$ & $35.3 \pm 9.5$ & $39.2 \pm 12.1$ & $39.1 \pm 11.4$ & $44.5 \pm 12.9$ \\
\hline
\end{tabular}

${ }^{1}$ Breast fed 1-year-old children are not included.

$\% \mathrm{E}=\%$ of total energy; SFA = saturated fatty acids; MUFA = monounsaturated fatty acids; PUFA = polyunsaturated fatty acids. 
was below the recommended level. The majority of the children in that age group had inadequate intake of vitamin $\mathrm{D}$. The intakes of vitamin $\mathrm{E}$ and iron were also below the recommendations. Use of supplements, apart from vitamin D, was uncommon among the children of this study [Kyttälä et al., 2008].

\section{Adolescents}

The report of nutrition and wellbeing of secondary school pupils was published in 2008 [Hoppu et al., 2008]. The objective of that study was to evaluate the eating habits, especially eating at school, in 7th and 8th grade school pupils (mean age 13.8 years). The study included 48-hour dietary interviews of 300 pupils. The dietary interview method was the same as in the National FINDIET Survey [Paturi et al., 2008].

Table 11.24 presents energy and nutrient intake of the study subjects. $40 \%$ of the girls and $28 \%$ of the boys consumed fresh vegetables daily. The proportion of energy derived from sucrose was higher and the intake of fiber, vitamin D, vitamin A and folate were below the recommendations. Around $20 \%$ of the daily energy intake was derived from the school lunch, compared to the recommended level of $33 \%$. Approximately 25\% of the girls and 33\% of the boys reported eating all parts of the school lunch, which in addition to the main course included salad, bread and milk. In this study population, $20 \%$ of the boys and $28 \%$ of the girls reported using supplements, of which multivitamin tablets were the most common [Hoppu et al., 2008].

\section{Adults and Elderly}

The National FINDIET Surveys conducted by the National Public Health Institute of Finland have followed the dietary habits and nutrient intake of the adult Finnish population since 1982. The FINDIET Survey is a part of the National FINRISK Study that monitors chronic disease risk factors in Finland every fifth year. The first surveys were conducted in eastern Finland in the North Karelia and Kuopio provinces aiming to evaluate the effectiveness of the North Karelia Project. Later the surveys were also carried out in other parts of Finland. The basic study design has been the same during the follow-up period of 35 years [Peltonen et al., 2008; Paturi et al., 2008].

The latest FINRISK Study was conducted in 2007 . The sample size was 11,953 persons aged $25-74$ years, the response rate being $67 \%$. The sample was a random sample from the Finnish Population Information System, stratified according to sex, 10-year age groups, and six geographical areas. From the participants in the FINRISK 2007, $33 \%$ were invited to participate in the FINDIET 2007 Survey. The 48-hour dietary recall data was collected by dietary interviewers. Every second interviewee was also asked to fill in a 3-day food record twice during the following year. A picture book of food portions was used to estimate portion sizes. The final number of the FINDIET 2007 Survey participants was 2,039 [Peltonen et al., 2008; Paturi et al., 2008].

Table 11.25 presents the daily intake of energy and nutrients of the study participants separately in two age groups aged 25-64 and 65-74 years. There were no major differences in the nutrient intake between the adults at working age and the older age 
Table 11.24. Mean daily intake of energy and nutrients (mean \pm SD) of Finnish 7th and 8th grade secondary school pupils [source: Hoppu et al., 2008]

\begin{tabular}{|c|c|c|c|}
\hline Nutrient & Girls $(n=170)$ & Boys $(n=136)$ & Recommendation ${ }^{1}$ \\
\hline Age, years & 13.8 & 13.8 & \\
\hline Energy, MJ & $6.7 \pm 2.0$ & $8.3 \pm 2.3$ & $9.5 / 10.8 \mathrm{MJ}$ \\
\hline Protein, \%E & $16.0 \pm 3.0$ & $16.7 \pm 3.2$ & $10-20 \% \mathrm{E}$ \\
\hline Carbohydrates, \%E & $54.1 \pm 7.1$ & $53.2 \pm 7.0$ & $50-60 \% \mathrm{E}$ \\
\hline Sucrose, \%E & $12.3 \pm 6.4$ & $13.0 \pm 6.4$ & $\leq 10 \% \mathrm{E}$ \\
\hline Dietary fiber, g & $16.6 \pm 7.6$ & $16.9 \pm 7.7$ & $3 \mathrm{~g} / \mathrm{MJ}$ \\
\hline Fat, \%E & $29.8 \pm 6.9$ & $30.0 \pm 6.5$ & $25-35 \% \mathrm{E}$ \\
\hline SFA, \%E & $11.5 \pm 3.5$ & $11.8 \pm 3.4$ & $\begin{array}{l}10 \% \mathrm{E} \\
\text { (incl. trans-fatty acids) }\end{array}$ \\
\hline MUFA, \%E & $10.9 \pm 3.0$ & $10.9 \pm 2.6$ & $10-15 \% \mathrm{E}$ \\
\hline PUFA, \%E & $5.3 \pm 1.7$ & $5.2 \pm 1.5$ & $5-10 \% \mathrm{E}$ \\
\hline Trans-fatty acids, $g$ & $0.68 \pm 0.44$ & $0.81 \pm 0.40$ & \\
\hline$\% \mathrm{E}$ & $0.37 \pm 0.18$ & $0.36 \pm 0.13$ & $<1 \% \mathrm{E}$ \\
\hline n-3 fatty acids, $g$ & $2.1 \pm 0.97$ & $2.5 \pm 1.2$ & \\
\hline$\% \mathrm{E}$ & $1.1 \pm 0.44$ & $1.1 \pm 0.38$ & $1 \% \mathrm{E}$ \\
\hline Vitamin $A, \mu g$ & $537 \pm 472$ & $524 \pm 370$ & $700 / 900 \mu \mathrm{g}$ \\
\hline Vitamin $D, \mu g$ & $5.0 \pm 2.8$ & $6.6 \pm 3.8$ & $7.5 \mu \mathrm{g}$ \\
\hline Vitamin $\mathrm{E}, \mathrm{mg}$ & $7.1 \pm 2.7$ & $8.1 \pm 3.1$ & $8 / 10 \mathrm{mg}$ \\
\hline Thiamine, mg & $1.1 \pm 0.43$ & $1.3 \pm 0.58$ & $1.2 / 1.5 \mathrm{mg}$ \\
\hline Riboflavin, mg & $1.7 \pm 0.67$ & $2.2 \pm 0.89$ & $1.3 / 1.7 \mathrm{mg}$ \\
\hline Niacin, mg & $22 \pm 6.7$ & $28 \pm 10$ & $15 / 20 \mathrm{mg}$ \\
\hline Vitamin $\mathrm{B}_{6}, \mathrm{mg}$ & $1.6 \pm 0.59$ & $2.1 \pm 1.3$ & $1.3 / 1.6 \mathrm{mg}$ \\
\hline Folate, $\mu \mathrm{g}$ & $190 \pm 73$ & $203 \pm 69$ & $300 \mu \mathrm{g}$ \\
\hline Cobalamin, $\mu \mathrm{g}$ & $3.9 \pm 2.4$ & $5.3 \pm 2.7$ & $2 \mu g$ \\
\hline Ascorbic acid, mg & $93 \pm 60$ & $87 \pm 59$ & $75 \mathrm{mg}$ \\
\hline Sodium, g & $2.2 \pm 0.70$ & $2.8 \pm 0.86$ & $2.4 / 2.8 \mathrm{~g}$ \\
\hline Calcium, mg & $1,032 \pm 428$ & $1,273 \pm 553$ & $900 \mathrm{mg}$ \\
\hline Magnesium, mg & $278 \pm 89$ & $325 \pm 99$ & $280 / 350 \mathrm{mg}$ \\
\hline Iron, mg & $8.9 \pm 3.1$ & $10.4 \pm 3.3$ & $15 / 11 \mathrm{mg}$ \\
\hline Zinc, mg & $9.4 \pm 3.0$ & $12.0 \pm 3.8$ & $9 / 12 \mathrm{mg}$ \\
\hline lodine, $\mu \mathrm{g}$ & $190 \pm 67$ & $248 \pm 86$ & $150 \mu \mathrm{g}$ \\
\hline Selenium, $\mu \mathrm{g}$ ) & $51 \pm 16$ & $67 \pm 21$ & $40 / 50 \mu \mathrm{g}$ \\
\hline
\end{tabular}

${ }^{1}$ National recommendation for girls/boys [Finnish Nutrition Recommendations, 2005, Finnish Nutrition Recommendations for School Nutrition, 2008].

$\% \mathrm{E}=\%$ of total energy; $\mathrm{SFA}=$ saturated fatty acids; MUFA = monounsaturated fatty acids; $\mathrm{PUFA}=$ polyunsaturated fatty acids. 
Table 11.25. Mean daily intake of energy and nutrients (mean \pm SD) of Finnish adults aged 25-64 and 65-74 years [source: Paturi et al., 2008]

\begin{tabular}{|c|c|c|c|c|c|}
\hline Nutrient & $\begin{array}{l}\text { Women } \\
25-64 \text { years } \\
(n=846)\end{array}$ & $\begin{array}{l}\text { Men } \\
25-64 \text { years } \\
(\mathrm{n}=730)\end{array}$ & $\begin{array}{l}\text { Women } \\
65-74 \text { years } \\
(n=234)\end{array}$ & $\begin{array}{l}\text { Men } \\
65-74 \text { years } \\
(\mathrm{n}=229)\end{array}$ & Recommendation $^{1}$ \\
\hline Age, years & 45.2 & 46.2 & 68.9 & 68.9 & \\
\hline Energy, MJ & $6.8 \pm 2.0$ & $9.2 \pm 3.0$ & $5.9 \pm 1.7$ & $7.7 \pm 2.3$ & $8.1-10.7 / 10.4-13.8 \mathrm{MJ}$ \\
\hline Protein, \%E & $17.2 \pm 4.1$ & $16.8 \pm 3.7$ & $17.6 \pm 3.4$ & $17.4 \pm 3.8$ & $10-20 \% \mathrm{E}$ \\
\hline Carbohydrates, \%E & $50.2 \pm 8.3$ & $47.1 \pm 8.8$ & $51.2 \pm 8.0$ & $49.7 \pm 8.9$ & $50-60 \% \mathrm{E}$ \\
\hline Sucrose, \%E & $10.5 \pm 5.1$ & $9.7 \pm 5.9$ & $9.3 \pm 5.1$ & $9.3 \pm 5.6$ & $\leq 10 \% \mathrm{E}$ \\
\hline Dietary fiber, $\mathrm{g}$ & $16.6 \pm 7.6$ & $16.9 \pm 7.7$ & $21.0 \pm 9.0$ & $24.0 \pm 10.0$ & $3 \mathrm{~g} / \mathrm{MJ}$ \\
\hline Fat, \%E & $31.2 \pm 7.4$ & $33.1 \pm 7.9$ & $30.4 \pm 7.3$ & $31.4 \pm 7.9$ & $25-35 \% \mathrm{E}$ \\
\hline SFA, \%E & $12.0 \pm 3.9$ & $12.9 \pm 4.1$ & $11.4 \pm 3.9$ & $12.0 \pm 4.5$ & $\begin{array}{l}10 \% \mathrm{E} \\
\text { (incl. trans-fatty acids) }\end{array}$ \\
\hline MUFA, \%E & $10.9 \pm 3.2$ & $12.0 \pm 3.9$ & $10.4 \pm 3.2$ & $11.1 \pm 3.5$ & $10-15 E$ \\
\hline PUFA, \%E & $5.7 \pm 2.2$ & $5.9 \pm 2.2$ & $5.6 \pm 1.9$ & $5.7 \pm 2.0$ & $5-10 \% \mathrm{E}$ \\
\hline $\begin{array}{l}\text { Trans-fatty acids, } g \\
\% \mathrm{E}\end{array}$ & $\begin{array}{l}0.7 \pm 0.5 \\
0.4 \pm 0.2\end{array}$ & $\begin{array}{l}1.0 \pm 0.6 \\
0.4 \pm 0.2\end{array}$ & $\begin{array}{l}0.6 \pm 0.5 \\
0.4 \pm 0.2\end{array}$ & $\begin{array}{l}0.8 \pm 0.5 \\
0.4 \pm 0.2\end{array}$ & $<1 \% \mathrm{E}$ \\
\hline $\begin{array}{l}\text { n-3 fatty acids, } g \\
\% \mathrm{E}\end{array}$ & $\begin{array}{l}2.2 \pm 1.2 \\
1.2 \pm 0.5\end{array}$ & $\begin{array}{l}3.1 \pm 1.8 \\
1.2 \pm 0.6\end{array}$ & $\begin{array}{l}2.7 \pm 1.7 \\
1.2 \pm 0.5\end{array}$ & $\begin{array}{l}2.1 \pm 1.1 \\
1.2 \pm 0.6\end{array}$ & $1 \% \mathrm{E}$ \\
\hline Vitamin $A, \mu g$ & $690 \pm 692$ & $963 \pm 1,397$ & $770 \pm 1,150$ & $836 \pm 1097$ & $700 / 900 \mu \mathrm{g}$ \\
\hline Vitamin $D, \mu \mathrm{g}$ & $5.2 \pm 4.2$ & $7.1 \pm 5.7$ & $6.5 \pm 4.6$ & $9.0 \pm 8.2$ & $7.5 \mu \mathrm{g}$ \\
\hline Vitamin E, mg & $8.1 \pm 3.7$ & $10.3 \pm 4.8$ & $7.3 \pm 3.2$ & $8.7 \pm 3.9$ & $8 / 10 \mathrm{mg}$ \\
\hline Thiamine, mg & $1.1 \pm 0.4$ & $1.5 \pm 0.6$ & $1.0 \pm 0.4$ & $1.3 \pm 0.5$ & $1.1 / 1.5 \mathrm{mg}$ \\
\hline Riboflavin, mg & $1.6 \pm 0.6$ & $2.1 \pm 0.9$ & $1.5 \pm 0.6$ & $1.8 \pm 0.8$ & $1.3 / 1.7 \mathrm{mg}$ \\
\hline Niacin, mg & $26 \pm 8$ & $35 \pm 12$ & $23 \pm 7$ & $30 \pm 11$ & $15 / 20 \mathrm{mg}$ \\
\hline Vitamin $\mathrm{B}_{6}, \mathrm{mg}$ & $1.7 \pm 0.6$ & $2.1 \pm 0.9$ & $1.5 \pm 0.7$ & $2.0 \pm 1.2$ & $1.2 / 1.6 \mathrm{mg}$ \\
\hline Folate, $\mu \mathrm{g}$ & $226 \pm 88$ & $270 \pm 120$ & $210 \pm 96$ & $234 \pm 102$ & $300 \mu \mathrm{g}$ \\
\hline Cobalamin, $\mu \mathrm{g}$ & $4.5 \pm 3.4$ & $6.6 \pm 6.5$ & $5.2 \pm 4.8$ & $6.5 \pm 6.0$ & $2 \mu \mathrm{g}$ \\
\hline Ascorbic acid, mg & $118 \pm 82$ & $98 \pm 88$ & $97 \pm 68$ & $92 \pm 72$ & $75 \mathrm{mg}$ \\
\hline Sodium, $\mathrm{g}$ & $2.4 \pm 0.8$ & $3.3 \pm 1.2$ & $2.3 \pm 0.7$ & $3.1 \pm 1.3$ & $2.4 / 2.8 \mathrm{~g}$ \\
\hline Calcium, mg & $1,007 \pm 450$ & $1,202 \pm 592$ & $900 \pm 426$ & $1,032 \pm 521$ & $800 \mathrm{mg}$ \\
\hline Magnesium, mg & $340 \pm 102$ & $428 \pm 134$ & $313 \pm 95$ & $379 \pm 115$ & $280 / 350 \mathrm{mg}$ \\
\hline Iron, mg & $10.3 \pm 3.4$ & $13.6 \pm 5.7$ & $9.9 \pm 3.6$ & $12.6 \pm 5.6$ & $15 / 9 \mathrm{mg}$ \\
\hline Zinc, mg & $10.0 \pm 3.3$ & $13.6 \pm 4.8$ & $9.4 \pm 2.9$ & $12.3 \pm 4.3$ & $7 / 9 \mathrm{mg}$ \\
\hline lodine, $\mu \mathrm{g}$ & $194 \pm 121$ & $253 \pm 220$ & $182 \pm 62$ & $226 \pm 94$ & $150 \mu \mathrm{g}$ \\
\hline Selenium, $\mu \mathrm{g}$ & $54 \pm 19$ & $73 \pm 27$ & $49 \pm 17$ & $66 \pm 26$ & $40 / 50 \mu g$ \\
\hline
\end{tabular}

${ }^{1}$ National recommendation for 18-60 years old women and men [Valtion ravitsemusneuvottelukunta, 2005].

$\% \mathrm{E}=\%$ of total energy; SFA = saturated fatty acids; MUFA = monounsaturated fatty acids; PUFA = polyunsaturated fatty acids. 
group. The total intake of energy from fat was 33\% in men and $31 \%$ in women. As in adolescents, the intake of vitamin $\mathrm{D}$ and folate were below the recommendations.

Although salt intake has decreased in the last decades, it is still higher than recommended. Among the whole sample, $33 \%$ of men and $52 \%$ of women used supplements. The use of supplements was poorly targeted; those who took supplements had already a higher intake of vitamins and minerals from foods as compared to those who did not use supplements. According to this survey, the dietary habits of the adult Finnish population have headed in a positive direction overall. The most important issues researchers of the FINDIET 2007 Survey highlighted were quality of fat, high intake of salt, and intake of folate and vitamin $\mathrm{D}$, which did not meet recommendations. The authors concluded that a diet comprised of more whole grains, fish, vegetables, fruit, and berries, and less of foods containing high levels of sucrose and saturated fats would also help to increase the intake fiber and cut down the intake of sucrose [Paturi et al., 2008].

Use of alcohol has increased considerably in Finland over the last four decades, and the consumed amount has about quadrupled since the early 1960s. The alcohol consumption is unevenly distributed [Mustonen et al., 1999]. A small part of the population does not drink at all, a large part drinks, but most of them moderately, and only a small part excessively. Over the past three decades the distribution of alcohol consumption has only slightly evened out. The top $10 \%$ of males and females drink the most, accounting for about $40-50 \%$ of all alcohol consumed by the population. According to the latest statistics, the overall alcohol consumption per inhabitant in 2008 was approximately 10.4 liters and decreased for the first time since 1970s [Valvira, 2009].

\section{Vitamin D fortification in Finland}

Fortification of fluid milks and margarines with vitamin D was made mandatory in Finland in 2003-2004. The researchers from the Turku University Central Hospital investigated the effect of fortification on dietary intake and on blood levels of 25-hydroxyvitamin $\mathrm{D}\left(25-\mathrm{OHD}_{3}\right)$, the inactive storage form of the vitamin, in Finnish 4-year-old children [Piirainen et al., 2007]. They found that average vitamin $\mathrm{D}$ intake was higher in the group studied after the introduction of fortification than in the children before mandatory fortification ( $4.5 \mathrm{vs.} 2.1 \mu \mathrm{g} /$ day). Two other Finnish studies examined the impact of vitamin $\mathrm{D}$ fortification on serum $25-\mathrm{OHD}_{3}$ concentrations among 12- to 15-year-old females [Lehtonen-Veromaa et al., 2008] and 18- to 21-year-old males [Välimäki et al., 2007]. Both studies concluded that fortification of milk products slightly but insufficiently improved the poor vitamin D status of young Finnish adolescents during winter. Among 18- to 28-year-old men, Laaksi et al. [2006] found that the mean serum $25-\mathrm{OHD}_{3}$ concentrations during the wintertime increased by $50 \%$ after implementation of the vitamin $\mathrm{D}$ fortification of dairy products and the prevalence of vitamin $\mathrm{D}$ insufficiency $(<40 \mathrm{nmol} / \mathrm{l})$ decreased from $78 \%$ in January 2003 to 35\% in January 2004. Lamberg-Allardt et al. [2006] evaluated the effect of expanded fortification by comparing vitamin $\mathrm{D}$ intake and status in the 
Finnish population in 2002 to the situation in 2004, one year after the fortification had started. The subjects studied were 4 to 76 years of age and $70 \%$ of them were female $(\mathrm{n}=630)$. They found that fortification improved vitamin D status, as in 2004 $21 \%$ had inadequate $(<50 \mathrm{nmol} / \mathrm{l}), 37 \%$ had adequate, and $42 \%$ had optimal vitamin D status, whereas in 2002 the corresponding distribution was 38,38 and $24 \%$, respectively. They concluded that a diet based on the current dietary guidelines is sufficient to provide enough vitamin $\mathrm{D}$, but if the consumption of vitaminized milk products is $<500 \mathrm{~g} /$ day and fish intake fewer than twice a week, the use of supplements should be encouraged at least during the winter season.

\section{Health Indicators and Status in Finnish Population}

According to the FINRISK 2007 Study, serum total cholesterol levels have declined since 1972. There was a leveling-off period between 1997 and 2002, but in the past 5 years serum cholesterol concentrations have again declined by about $5 \%$ now being $5.39 \mathrm{mmol} / \mathrm{l}$ in 30 - to 59 -year-old men and $5.18 \mathrm{mmol} / \mathrm{l}$ in women. Blood pressure has declined since 1972, but the decline seems to have leveled off during the past 5 years. Possible reasons for that are increases in obesity level and alcohol consumption. The prevalence of smoking among men has declined for decades, and this has now been shown also for women. Mean BMI and the prevalence of obesity have increased both in men and women in recent decades. In 2007, mean BMI was $27.2 \mathrm{~kg} / \mathrm{m}^{2}$ in men and $26.5 \mathrm{~kg} / \mathrm{m}^{2}$ in women. The prevalence of obesity (BMI $\geq 30 \mathrm{~kg} / \mathrm{m}^{2}$ ) among men and women was $21 \%$ in 2007 . There has been also a steady increase in the waist circumference both in men and women. In contrast, favorable changes in self-reported health status, leisure-time physical activity, physical fitness and quality of life in the general population have been observed [Peltonen et al., 2008].

\section{Conclusions}

According to the FINDIET 2007 Survey, the dietary habits of the adult Finnish population have headed in a positive direction overall [Paturi et al., 2008]. In 2008, the total alcohol consumption per inhabitant decreased for the first time since the 1970s [Valvira, 2009]. Despite these positive findings there is still room for improvement. The intake of saturated fat is still more than recommended, and there is a need to increase intake of folate and vitamin $\mathrm{D}$, which are below the recommendations.

Vitamin D is synthesized in the body on exposure to sunlight and only small amounts can be obtained from food. However, studies have shown that sunshine levels in northern countries are so weak during the winter months that the body makes no vitamin D at all, leading some researchers to estimate that $30-50 \%$ of the population in such countries have insufficient or deficient levels of the vitamin [Holick and Chen, 2008]. Results of published Finnish studies show that at the population level, national fortification safely improved vitamin D levels [Laaksi et al., 2006; LambergAllardt et al., 2006; Piirainen et al., 2007; Välimäki et al., 2007; Lehtonen-Veromaa et al., 2008]. There is increasing evidence that vitamin $\mathrm{D}$ may be protective against 
several other diseases besides those related to bone health. Therefore, new innovative and feasible ways of improving vitamin D nutrition among those who are not consuming enough milk products and fish are urged also in Finland.

More attention should also be paid to the constantly growing obesity problem in Finland. It is the fundamental question in public healthcare how to decrease prevalence of obesity and type 2 diabetes in Finland. The Development Program for the Prevention and Care of Diabetes (DEHKO 2000-2010) is Finland's national diabetes program (http://www.diabetes.fi/sivu.php?artikkeli_id $=831$ ). It aims to prevent type 2 diabetes and diabetes-related complications, to improve the quality of diabetes care, and to support the self-care of people with diabetes. Such a program has been adopted as a model for creating and implementing national programs in some other European countries. It is evident that at the population level, excess energy intake will be the most important dietary challenge during the following years also in Finland.

\subsection{France $^{7}$}

Adherence to Nutritional Guidelines in France: The French Nutrition and Health Survey 2006-2007

\section{Background}

As most industrialized countries, France is affected by increasing prevalence of overweight and obesity [Charles et al., 2008] and obesity-related diseases. In 2005, over 155,000 people died from cancer and 149,000 from cardiovascular diseases (CVD) [Belot et al., 2008]. In 2001, the French Ministry of Health implemented the National Nutrition and Health Program (PNNS) [Hercberg et al., 2008]. Its main objective is to improve the state of health of the population by acting on nutrition. The PNNS is based on nine priority health objectives which focus on food consumption (increase consumption of fruits and vegetables, calcium, etc.), nutritional status (reduce average adult LDL cholesterol levels, average adult systolic blood pressure and the prevalence of overweight and obesity among adults and stabilize the increase of childhood overweight prevalence) and physical activity (increase daily physical activity). Besides, ten specific nutritional objectives focus on special population groups (elderly, children, pregnant and lactating women, and socially disadvantaged people).

Prior to the establishment of the PNNS, existing data on French population nutritional status were rarely comparable: they usually came from specific surveys (clinical trials, longitudinal studies, etc.) or one-time studies. Moreover, these surveys were often regional and used heterogeneous data collection methods. Therefore, in

\footnotetext{
${ }^{7}$ Vernay M; Nutritional Surveillance and Epidemiology Unit (USEN), France. E-Mail michel.vernay@ univ-paris13.fr
} 
the framework of the PNNS, a national monitoring system has been implemented in order to evaluate the nutritional status of the population living in France. The French Institute for Public Health Surveillance (InVS), in partnership with the Conservatoire National des Arts et Métiers (CNAM) and the University of Paris 13, have created a Surveillance and Nutritional Epidemiology Unit (USEN) which is intended to (1) conduct the monitoring of nutritional status of the population, (2) evaluate the effectiveness of adopted measures with regard to the PNNS objectives, and (3) support the standardization of data collection methods. The monitoring system primarily focuses on a large general population study, the National Nutrition and Health Survey (ENNS), first carried out in 2006/2007 and planned to be repeated. The surveillance plan is completed by other studies carried out in specific populations: infants $<1$ year old, populations receiving food aid, elderly persons living in institutions.

\section{The French Nutrition and Health Survey 2006/2007 (ENNS)}

The ENNS included a food consumption survey and a health examination realized in a health center of the French National Health Insurance System (CNAM-TS) or at home. Methods have been described elsewhere [Castetbon et al., 2009]. Briefly, ENNS was based on a three-degree sampling plan: selection of 190 geographical zones on the whole French continental territory, random selection of households, and finally random selection of one adult or child. Food intake was assessed by trained dieticians using three 24-hour recalls, randomly distributed within a period of 2 weeks. Sociodemographic and behavioral characteristics were collected through a faceto-face questionnaire and health examinations were carried out by trained physicians, nurses or dieticians. Physical activity level was assessed using the International Physical Activity Questionnaire (IPAQ) (15-74 years) and questionnaires adapted to the age (3-15 years). Weight and height were measured in children. In adults, the health examination included anthropometry (weight, height, waist and hip circumferences) and blood pressure measurements. A venous blood sample was drawn. Among subjects who were contacted and eligible, $62.3 \%$ accepted to participate in the survey (adults 59.7\%, children 67.9\%). Analyses were carried out on 3,115 adults 18-74 years and 1,675 children 3-17 years with at least two 24-hour recalls. In addition, $96.7 \%$ of children were measured for weight and height. Among adults, $77.5 \%$ underwent anthropometry measurements and for $67.5 \%$, a blood sample was drawn.

Food intake (in g) proportions of adherence to nutritional guidelines disseminated through the PNNS since 2001 were assessed. In adults, food consumption was described in 2,734 subjects after exclusion of diet underreporting subjects (12.2\%), using the method and the equations proposed by Black [2000] and Schofield [1985]. Probabilities of inclusion and potential participation bias have been taken into account by calibration on age, diploma, whether household includes or not at least 1 child and period of collection data, using French census data. Analyses were carried out using STATA V V.10 (Stata Corp., College Station, Tex., USA). 
Table 11.26. Proportion of inadequate nutrient intake according to the PNNS objectives in children aged 3-17 years. French Nutrition and Health Survey (ENNS) 2006-2007

\begin{tabular}{|c|c|c|c|c|}
\hline \multirow[t]{2}{*}{ Nutrient intake } & \multicolumn{2}{|l|}{ Boys } & \multicolumn{2}{|l|}{ Girls } \\
\hline & $\%$ & $\mathrm{Cl}_{95}$ & $\%$ & $\mathrm{Cl}_{95}$ \\
\hline Total carbohydrate, $\% \leq 50 \% \mathrm{EI}$ & 55.9 & $51.5-60.3$ & 57.9 & $53.5-62.3$ \\
\hline Complex carbohydrate, $\%<27.5 \% \mathrm{EI}$ & 66.4 & $62.1-70.7$ & 73.8 & $69.9-77.6$ \\
\hline Simple sugar, $\% \geq 12.5 \% \mathrm{EI}$ & 58.5 & $54.2-62.8$ & 54.5 & $50.2-58.9$ \\
\hline Total fat, $\% \geq 35 \%$ El & 55.1 & $50.7-59.5$ & 57.5 & $53.1-61.8$ \\
\hline Saturated fatty acids, $\% \geq 35 \%$ total lipids & 89.8 & $86.7-92.9$ & 90.0 & $87.3-92.7$ \\
\hline Calcium, $\%<2 / 3$ RDA & 22.6 & $18.9-26.3$ & 35.3 & $31.1-39.5$ \\
\hline
\end{tabular}

$\mathrm{El}=$ Energy intake without alcohol, $\mathrm{RDA}=$ recommended dietary allowance.

\section{Nutritional Status of Children}

In children, food consumption was characterized by rather high lipid intake, especially saturated fatty acids (table 11.26): $90.0 \%$ of children were above the recommendation regarding SFA intake. Children food consumption was also characterized by low fruit and vegetable intake (table 11.27): $79.6 \%$ consumed $<5$ daily servings of fruits and vegetables, and $57.6 \%$ were 'low consumers' $(<3.5$ servings of fruits and vegetables). Low fruit and vegetable consumption was associated with low education level of parents, occupation status and household income. Some food groups, like sweetened foods ( $35.8 \%$ of children consumed $>125 \mathrm{ml}$ of sweetened beverages daily), will probably deserve more attention during the years to come.

\section{Nutritional Status of Adults}

In adults, food consumption was characterized by an insufficient carbohydrate intake (particularly complex carbohydrates) and high fat intake, especially saturated fat intake (table 11.28). Intake of fiber was low: $86.2 \%$ of adults consuming less than the recommended $25 \mathrm{~g} /$ day. Regarding bread, cereal, legume and potato consumption (table 11.29), $42.3 \%$ of adults consumed $<3$ servings a day which could contribute to explain the low intake of complex carbohydrates and fiber. In women, the low intake of calcium (31.6\% of women did not reach the recommendation) was related to low intake of dairy products ( $56.3 \%$ of women consumed $<2.5$ servings a day). Seafood intakes were also insufficient: $70.1 \%$ of adults consume $<2$ weekly servings. Food consumption was also characterized by rather low fruit and vegetable intake: $56.2 \%$ of adults consumed $<5$ daily servings recommended and $34.1 \%<3.5$ daily servings. However, some food consumption characteristics are positive such as mean salt 
Table 11.27. Proportion of inadequate nutrient intake according to the PNNS dietary recommendations in children aged 3-17 years. French Nutrition and Health Survey (ENNS) 2006-2007

\begin{tabular}{|c|c|c|c|c|}
\hline \multirow[t]{2}{*}{ Nutrient intake } & \multicolumn{2}{|l|}{ Boys } & \multicolumn{2}{|l|}{ Girls } \\
\hline & $\%$ & $\mathrm{Cl}_{95}$ & $\%$ & $\mathrm{Cl}_{95}$ \\
\hline Fruits and vegetables, $\%<400 \mathrm{~g} /$ day & 79.0 & $75.8-82.2$ & 80.2 & 76.7-83.7 \\
\hline \multicolumn{5}{|l|}{ Dairy products ${ }^{2}$} \\
\hline$\%<2.5$ servings/day & 41.4 & $37.0-45.7$ & 52.3 & $47.9-56.7$ \\
\hline$\%>3.5$ servings/day & 11.5 & $9.1-14.0$ & 7.3 & $5.2-9.5$ \\
\hline \multicolumn{5}{|l|}{ Meat, poultry, seafood and eggs } \\
\hline$\%<1$ serving/day & 19.1 & $15.5-22.8$ & 30.9 & $26.8-35.0$ \\
\hline$\%>2$ servings/day & 32.1 & $28.0-36.2$ & 23.5 & $19.5-27.4$ \\
\hline$\%$ seafood $<2$ servings/week & 69.6 & $65.4-73.8$ & 74.1 & $70.2-77.9$ \\
\hline \multicolumn{5}{|l|}{ Bread, cereals, legumes, potatoes } \\
\hline$\%<3$ servings/day & 57.3 & $53.0-61.6$ & 72.2 & $68.4-76.1$ \\
\hline$\% \geq 6$ servings/day & 3.8 & $2.2-5.3$ & 0.6 & $0.0-1.3$ \\
\hline $\begin{array}{l}\text { Home-added fat (butter, oils, margarine), } \\
\% \geq 16 \% \mathrm{El}\end{array}$ & 3.6 & $1.5-5.6$ & 3.8 & $2.3-5.3$ \\
\hline Sweetened beverage, $\%>125 \mathrm{ml} /$ day & 38.7 & $34.4-43.1$ & 32.7 & $28.4-36.9$ \\
\hline Salt $^{3}, \geq 8 \mathrm{~g} /$ day & 26.4 & $22.8-30.0$ & 13.9 & $11.0-16.8$ \\
\hline \multicolumn{5}{|c|}{$\begin{array}{l}{ }^{1} \text { Including } 100 \% \text { juices, processed fruits and vegetables, excluding legumes, beans, starchy food } \\
\text { and potatoes. } \\
{ }^{2} \text { Excluding desserts with high contents of sugar and fat. } \\
{ }^{3} \text { Including salt added when cooking and during meals. }\end{array}$} \\
\hline
\end{tabular}

Table 11.28. Proportion of inadequate nutrient intake according to the PNNS objectives in adults aged 18-74 years. French Nutrition and Health Survey (ENNS) 2006-2007

\begin{tabular}{|c|c|c|c|c|}
\hline \multirow[t]{2}{*}{ Nutrient intake } & \multicolumn{2}{|l|}{ Men } & \multicolumn{2}{|c|}{ Women } \\
\hline & $\%$ & $\mathrm{Cl}_{95}$ & $\%$ & $\mathrm{Cl}_{95}$ \\
\hline Total carbohydrate, $\% \leq 50 \% \mathrm{El}$ & 71.9 & $68.0-75.7$ & 75.3 & $72.5-78.0$ \\
\hline Complex carbohydrate, $\%<27.5 \% \mathrm{EI}$ & 55.9 & $51.7-60.0$ & 72.2 & $69.4-75.0$ \\
\hline Simple sugar, $\% \geq 12.5 \% \mathrm{EI}$ & 25.9 & $22.1-29.6$ & 25.1 & $22.2-28.1$ \\
\hline Total dietary fiber, $\% \leq 25 \mathrm{~g} /$ day & 80.7 & $77.6-83.8$ & 91.6 & $90.1-93.2$ \\
\hline Total fat, $\% \geq 35 \% \mathrm{El}$ & 64.3 & $60.3-68.2$ & 62.3 & $59.2-65.4$ \\
\hline Saturated fatty acids, $\% \geq 35 \%$ total lipids & 83.0 & $79.8-86.2$ & 80.0 & $77.5-82.4$ \\
\hline Calcium, $\%<2 / 3$ RDA & 15.4 & $12.3-18.4$ & 31.6 & $28.6-34.5$ \\
\hline
\end{tabular}

$\mathrm{El}=$ Energy intake without alcohol, $\mathrm{RDA}=$ recommended dietary allowance. 
Table 11.29. Proportion of inadequate food intake according to the PNNS dietary recommendations in French adults aged 18-74 years. French Nutrition and Health Survey (ENNS) 2006-2007

\begin{tabular}{|c|c|c|c|c|}
\hline \multirow[t]{2}{*}{ Nutrient intake } & \multicolumn{2}{|l|}{ Men } & \multicolumn{2}{|c|}{ Women } \\
\hline & $\%$ & $\mathrm{Cl}_{95}$ & $\%$ & $\mathrm{Cl}_{95}$ \\
\hline Fruits and vegetables $1, \%<400 \mathrm{~g} /$ day & 56.3 & $52.3-60.4$ & 56.1 & $53.0-59.1$ \\
\hline \multicolumn{5}{|l|}{ Dairy products ${ }^{2}$} \\
\hline$\%<2.5$ servings/day & 43.9 & $39.8-48.0$ & 56.3 & $53.2-59.4$ \\
\hline$\%>3.5$ servings $/$ day $^{3}$ & 20.9 & $18.7-23.0$ & 8.5 & $6.9-10.0$ \\
\hline \multicolumn{5}{|l|}{ Meat, poultry, seafood and eggs } \\
\hline$\%<1$ serving/day & 19.7 & $16.3-23.2$ & 37.1 & $34.1-40.2$ \\
\hline$\%>2$ servings/day & 29.1 & $25.4-32.8$ & 9.3 & $7.5-11.1$ \\
\hline$\%$ seafood $<2$ servings/week & 72.0 & $68.5-75.5$ & 67.5 & $64.8-70.5$ \\
\hline \multicolumn{5}{|l|}{ Bread, cereals, legumes, potatoes } \\
\hline$\%<3$ servings/day & 24.3 & $20.7-28.0$ & 60.0 & $57.0-63.0$ \\
\hline$\% \geq 6$ servings/day & 15.6 & $12.5-18.6$ & 2.0 & $1.1-3.0$ \\
\hline \multicolumn{5}{|l|}{ Home added fat (butter, oils, margarine) } \\
\hline$\% \geq 16 \%$ El & 13.0 & $10.2-15.9$ & 18.1 & $15.8-20.5$ \\
\hline Sweetened beverage, $\%>250 \mathrm{ml} /$ day & 15.1 & $11.7-18.6$ & 7.7 & $5.5-9.9$ \\
\hline \multicolumn{5}{|l|}{ Alcoholic beverage } \\
\hline$\%$ consumers & 86.8 & $83.6-90.0$ & 72.7 & $69.7-75.6$ \\
\hline $\begin{array}{l}\% \text { consumers }{ }^{4} \geq 20 \mathrm{~g} / \text { day (women) and } \\
30 \mathrm{~g} / \text { day (men) }\end{array}$ & 23.0 & $19.2-26.8$ & 8.4 & $6.6-10.3$ \\
\hline Salt $^{5}, \geq 8 \mathrm{~g} /$ day & 70.2 & $66.3-74.1$ & 30.2 & $27.4-32.9$ \\
\hline $\begin{array}{l}{ }^{1} \text { Including } 100 \% \text { juices, processed fruits an } \\
\text { and potatoes. } \\
{ }^{2} \text { Excluding desserts with high contents of s } \\
3 \%<2.5 \text { or }>4.5 \text { servings/day in } 55 \text {-t o } 74 \text {-ye } \\
{ }^{4} \text { Non-consumers excluded. } \\
5 \text { Including salt added when cooking and du }\end{array}$ & $\begin{array}{l}\text { etable } \\
\text { and fa } \\
\text { d adul }\end{array}$ & Iuding legu & beans, & hy food \\
\hline
\end{tabular}

intake in the general population ( $50.1 \%$ of adults consuming $<8 \mathrm{~g} /$ day) and especially in women (30.2\% of them consuming $>8 \mathrm{~g} /$ day).

\section{Conclusion}

The French nutritional situation would appear to be intermediate between a protective Mediterranean diet in southern Europe and a northern European diet, with positive elements and less favorable ones. In French adults, the prevalence of overweight and obesity is low compared to that in the UK and eastern European countries [Vernay et al., 2009]. A stabilization of the prevalence of overweight and obesity has 
been observed in 7- to 9-year-old children between 2000 and 2007 [Salanave et al., 2009]. Lifestyle, culinary habits and healthy attitudes in general, mainly depending on socioeconomic status, appear to play a major role in the prevalence of obesity [Pettinger et al., 2004]. Therefore, it is of importance to pursue a public health plan such as PNNS and a continuous monitoring system on nutrition.

\subsection{Germany $^{8}$}

\section{Introduction}

Two national surveys were conducted in Germany to assess current data on energy and nutrient intake as well as other health-related aspects of the German population. The German Health Interview and Examination Survey for Children and Adolescents is specialized in children and adolescents aged 0-17 years and the German National Nutrition Survey II (NVS II) provides comprehensive data about adults aged 14-80 years. The essential characteristics of each survey will be presented in the subsequent sections.

The German Health Interview and Examination Survey for Children and Adolescents

The German Health Interview and Examination Survey for Children and Adolescents (KiGGS) is a nationwide, representative survey which was conducted by the Robert Koch Institute, Berlin. The aim was to collect comprehensive data about the health status of children and adolescents in Germany aged 0-17 years [Kamtsarius et al., 2007].

Altogether 17,641 children and adolescents, among them 8,656 girls and 8,985 boys, were medically examined and interviewed together with their parents from May 2003 to May 2006 at 167 study locations. The participants were randomly selected from the official registers of local resident' registration offices. The participation rate was $66.6 \%$. The survey was financed by the Federal Ministry of Health (BMG) and the Federal Ministry of Education and Research (BMBF) [Kamtsarius et al., 2007].

The examination program was adapted according to age groups and consisted of a questionnaire filled in by parents and additionally questionnaires for children (11 years and older), physical examinations and tests, a computer-assisted personal interview as well as a collection of blood and urine samples [Hölling et al., 2007].

\footnotetext{
${ }^{8}$ Krems C, Vasquez-Caicedo AL, Urban C; Max Rubner-Institut, Karlsruhe, Germany. E-Mail carolin. krems@mri.bund.de
} 
The core survey was completed by three other modules which investigated specific topics using subsamples and focus on mental health, motor fitness and physical activity as well as environmental exposures [Kurth et al., 2002].

In a further module of KiGGS the dietary behavior of a randomized subsample of children and adolescents of 6- to 17-year-olds was assessed between January and December 2006. The study, named EsKiMo (Eating Study as a KiGGS Module), was performed by the Robert Koch Institute together with the Division of Nutrition and Consumer Education at the University of Paderborn. It was funded by the Federal Ministry of Food, Agriculture and Consumer Protection. Parents of participants $<12$ years were asked to conduct a 3 -day dietary record. Participants of $\geq 12$ years were personally interviewed about their eating behavior during the last 4 weeks using DISHES (Dietary Interview Software for Health Examination Studies). Furthermore, they were asked to fill in a food frequency questionnaire which was already used in the core module. Additional data were collected for sociodemographic background, leisure-time activities, supplement use, meals at school, body weight and height of all participants [Mensink et al., 2007a].

The German Nutrient Database version II.3 (BLS II.3) was used for data analysis of energy and nutrient intake. To analyze food consumption data as detailed as possible further data regarding fortification of vitamins and minerals were needed. Therefore, nutrient facts of special food groups (e.g. confectionary, dairy products, breakfast cereals and fast food) were collected and a new database filled with 1,225 food items was built [Mensink et al., 2007b].

\section{The German National Nutrition Survey II}

NVS II is a nationwide representative study. The Federal Ministry for Food, Agriculture and Consumer Protection commissioned the Max Rubner Institute for this purpose aiming at providing current, reliable and representative data of food consumption of the German population for nutrition policymaking. The first National Nutrition Survey was carried out 20 years ago and at that time only the western part of Germany was considered. Furthermore, major changes have occurred in the German society with respect to food availability, nutritional habits as well as diversification of working conditions, leisure-time activities and consumer behavior since the first survey was carried out.

The objectives of the NVS II were: (1) to collect current representative data on food consumption, energy and nutrient intake as well as nutritional behavior of the German population; (2) to improve existing dietary assessment methods; (3) to provide a revised version of the German Nutrient Database (BLS), and (4) to implement a National Nutrition Monitoring in Germany.

About 20,000 German-speaking women and men between 14 and 80 years of age living in private households were interviewed between November 2005 and January 2007. Interviews were conducted in 500 nationwide sample points, which were selected 
considering the proportion of the population living in each Federal State. The participants were randomly chosen by age and sex with the aid of the local register offices of these 500 municipalities. To cover seasonal variations, the study was divided into four survey waves.

Several methods were applied to obtain a comprehensive description of food consumption and nutritional behavior. A Computer-Assisted Personal Interview (CAPI) and a questionnaire were applied to obtain data about sociodemographic variables, nutritional and buying behavior as well as health aspects (e.g. smoking behavior, physical activity, diseases).

Food and beverage consumption data were collected by use of a dietary history interview. The DISHES program (Diet Interview Software for Health Examination Studies), developed by the Robert Koch Institute in Berlin [Mensink et al., 1998, 2001], was updated and adapted for the purpose of the NVS II. With this method, frequency of the meals as well as kind and amount of foods and beverages consumed over the last 4 weeks were obtained.

In the following weeks after conducting the dietary history interviews the participants were twice asked about their nutrition by phone using 24-hour recalls $(\mathrm{n}=$ 13,926). For this purpose the program EPIC-SOFT was used. EPIC-SOFT was developed within the scope of the cohort study 'European Prospective Investigation into Cancer and Nutrition' (EPIC) [Slimani et al., 1999, 2000].

A subsample of the participants $(n=1,021)$ completed a 4-day dietary weighing record twice. The participants weighed all foods they ate and drank during the protocol time. The dietary weighing records allow determining precise consumption quantities to define up-to-date portion sizes.

Body weight and body height of 14,331 participants could be measured. Participants were weighed wearing light clothes and without shoes. A calibrated scale (Seca Vogel \& Halke, Hamburg, Germany) with a precision of $0.1 \mathrm{~kg}$ was used. Accounting for the clothes, $1 \mathrm{~kg}$ was subtracted from the measurement. Body height was measured at upright standing participants without shoes using a portable Harpenden Stadiometer (Holtain Ltd, Crymych, UK) with a precision of $0.1 \mathrm{~cm}$.

The energy and nutrient intake was calculated by linking food and beverage consumption data with the German Nutrient Database (BLS). Food items especially needed for the analysis of the NVS II were reviewed and updated or newly incorporated in the BLS. Therefore, fortified and energy-reduced food items were incorporated, recipes were reviewed for fat and salt contents and names were adapted. For analysis of energy and nutrient intake out of the dietary history interviews, a preliminary version of the BLS II.4 was used [Max Rubner Institute, 2008b].

Data were weighted with regard to the data of the official representative statistics for different aspects like gender, age, region, employment, school education and month of interview to correct for selection bias [Max Rubner Institute, 2008a].

From the total number of subjects approached $(\mathrm{n}=46,587) 19,329$ women and men agreed to participate. This corresponds to a response rate of $42 \%$. 
Table 11.30. Prevalence of underweight, normal weight, overweight and obesity according to socioeconomic status, 6,117 men 18-80 years (weighted data)

\begin{tabular}{|c|c|c|c|c|c|}
\hline & & Underweight & Normal weight & Overweight & Obesity \\
\hline \multirow[t]{3}{*}{ Lower } & $\mathrm{n}$ & 7 & 96 & 126 & 89 \\
\hline & $\%$ & 2.6 & 37.0 & 36.4 & 24.0 \\
\hline & $\mathrm{Cl}$ & $1.4-4.3$ & $32.3-41.3$ & $31.7-40.6$ & $20.1-27.9$ \\
\hline \multirow[t]{3}{*}{ Lower middle } & $\mathrm{n}$ & 5 & 209 & 350 & 228 \\
\hline & $\%$ & 1.0 & 29.8 & 43.8 & 25.3 \\
\hline & $\mathrm{Cl}$ & $0.5-1.8$ & $26.8-32.6$ & $40.3-46.8$ & $22.5-28.0$ \\
\hline \multirow[t]{3}{*}{ Middle } & $\mathrm{n}$ & 14 & 506 & 834 & 389 \\
\hline & $\%$ & 0.7 & 30.7 & 47.2 & 21.4 \\
\hline & $\mathrm{Cl}$ & $0.4-1.2$ & $28.5-32.7$ & $44.6-49.3$ & $19.5-23.2$ \\
\hline \multirow[t]{3}{*}{ Upper middle } & $\mathrm{n}$ & 10 & 607 & 812 & 365 \\
\hline & $\%$ & 0.6 & 34.2 & 45.0 & 20.3 \\
\hline & $\mathrm{Cl}$ & $0.3-1.0$ & $31.7-36.4$ & $42.2-47.3$ & $18.3-22.2$ \\
\hline \multirow[t]{3}{*}{ Upper } & $\mathrm{n}$ & 6 & 525 & 722 & 217 \\
\hline & $\%$ & 0.5 & 37.6 & 48.9 & 13.1 \\
\hline & $\mathrm{Cl}$ & $0.2-1.1$ & $34.3-40.5$ & $45.3-51.8$ & $11.1-15.2$ \\
\hline
\end{tabular}

Selected Results from the German National Nutrition Survey II

Anthropometric Measurements According to Socioeconomic Status

Within the NVS II education, occupation of the main earner and household income were used to define socioeconomic status (SES) [Max Rubner Institute, 2008c].

In Germany, $66.0 \%$ of men and $50.6 \%$ of women are overweight or obese and even every fifth German citizen is obese (20.5\% of men and $21.2 \%$ of women) [Max Rubner Institute, 2008a].

The prevalence of overweight and obesity among 6,117 men and 6,790 women (18 to 80 years) according to SES is shown in tables 11.30 and 11.31 . The two lower socioeconomic groups show the highest proportion of obesity for men (24.0 and 25.3\%, respectively) and women (35.3 and $28.0 \%$, respectively). With increasing SES, obesity strongly decreases, reaching levels in the upper SES group of $13.1 \%$ in men and 10.0\% in women. Regression analysis shows a significant reduction of BMI with increasing SES in men and women $(\mathrm{p}<0.001)$. 
Table 11.31. Prevalence of underweight, normal weight, overweight and obesity according to socioeconomic status, 6,790 women $\mathbf{1 8 - 8 0}$ years (weighted data)

\begin{tabular}{|c|c|c|c|c|c|}
\hline & & Underweight & Normal weight & Overweight & Obesity \\
\hline \multirow[t]{3}{*}{ Lower } & $\mathrm{n}$ & 11 & 155 & 196 & 204 \\
\hline & $\%$ & 2.0 & 29.4 & 33.3 & 35.3 \\
\hline & $\mathrm{Cl}$ & $1.1-3.3$ & $25.8-32.8$ & $29.5-36.8$ & $31.4-38.8$ \\
\hline \multirow[t]{3}{*}{ Lower middle } & $\mathrm{n}$ & 18 & 338 & 334 & 299 \\
\hline & $\%$ & 1.7 & 37.7 & 32.6 & 28.0 \\
\hline & $\mathrm{Cl}$ & $1.0-2.6$ & $34.5-40.5$ & $29.6-35.4$ & $25.2-30.7$ \\
\hline \multirow[t]{3}{*}{ Middle } & $\mathrm{n}$ & 29 & 924 & 663 & 477 \\
\hline & $\%$ & 1.4 & 44.6 & 31.9 & 22.1 \\
\hline & $\mathrm{Cl}$ & $0.9-2.0$ & $42.2-46.7$ & $29.7-33.9$ & $20.2-23.9$ \\
\hline \multirow[t]{3}{*}{ Upper middle } & $\mathrm{n}$ & 50 & 1219 & 626 & 335 \\
\hline & $\%$ & 2.4 & 56.5 & 26.9 & 14.2 \\
\hline & $\mathrm{Cl}$ & $1.8-3.2$ & $53.6-58.8$ & $24.6-29.0$ & $12.6-15.9$ \\
\hline \multirow[t]{3}{*}{ Upper } & $\mathrm{n}$ & 23 & 800 & 264 & 125 \\
\hline & $\%$ & 2.3 & 67.0 & 20.6 & 10.0 \\
\hline & $\mathrm{Cl}$ & $1.4-3.6$ & $62.8-70.3$ & $17.7-23.6$ & $7.9-12.3$ \\
\hline
\end{tabular}

\section{Energy and Nutrient Intake}

The following results are based on 15,371 participants from whom dietary history data are available. In contrast, results presented in the chapter Energy and Nutrient Intake relate to 15,162 participants between 15 and 80 years of age, because data of the NVS II was recalculated according to the European Nutrition and Health Report 2009 age groups to enable comparisons between the different European countries.

The median energy intake of adolescents, adults and elderly corresponds to the guiding value for energy intake valid for low physical activity of the German, Austrian and Swiss (D-A-CH) reference values for nutrient intake [D-A-CH, 2000]. The percentage of energy intake from fat is still too high for both women and men (women $35 \%$ and men 36\%). In general, the median intake of most of the vitamins and minerals corresponds to the reference values or is above. However, intake levels of folate and vitamin $\mathrm{D}$ are below the recommendations for all age groups. In addition, the median intake of calcium of elderly women and men as well as female adolescents is clearly below the recommended intake level. In contrast, the median calcium intake of male adolescents corresponds to the recommendations. Adult men even exceed 
the recommended intake of calcium, whereas the median intake of calcium of adult women corresponds to the recommendations. There are no differences in the median iron intake for women of all age groups. But the recommended intake level of iron for women up to the age of 50 is higher in comparison to that for older women (15 and $10 \mathrm{mg} /$ day). Therefore, women up to the age of 50 are below the recommended iron intake whereas elderly women reach the recommendations. The median iron intake of men is above the recommended intake in all age groups.

\section{Intake of Dietary Supplements}

In the NVS II, $27.6 \%$ of the participants reported taking dietary supplements. This represents 4,261 people, 1,696 men (24.2\%) and 2,565 women (30.9\%). At ages between 25 and 80 years, women take supplements more frequently than men, whereas male adolescents between 14 and 18 years of age take supplements more frequently than female adolescents. In general, dietary supplements are taken more often with increasing age. Elderly men and women (65+ years) take the highest amount of dietary supplements (30.2\% for men and $43.2 \%$ for women) [Max Rubner Institute, 2008a]. In Germany, the intake of dietary supplements shows a relation to SES. There is an increase of the intake of dietary supplements from middle SES to the upper SES group in women and men, respectively (30.1-33.7\% for women and 18.8-28.4\% for men) [Max Rubner Institute, 2008c].

\section{Summary}

NVS II provides current information on food consumption, energy and nutrient intake as well as on lifestyle and eating behavior of the German population. In total, 19,329 women and men aged 14 to 80 years participated in the study. Women and men with lower SES show a higher percentage of obesity in comparison to the other groups. In general, the intake of most of the vitamins and minerals of the German population corresponds or is above the German reference values, but critical nutrients are folate and vitamin $\mathrm{D}$ for all age groups as well as calcium and iron for some age groups.

\subsection{Greece $^{9}$}

\section{Preamble}

The present report summarizes current evidence on the dietary intake and health status of the Greek population with the aim to supplement the data presented for Greece in the European Nutrition and Health Report 2004 (http://www.univie.ac.at/enhr) [Elmadfa et al., 2004]. A single study examining dietary intake and health among representative samples of the population has not yet been undertaken. Information can

\footnotetext{
${ }^{9}$ Naska A, Efstathopoulou E, Trichopoulou A; Hellenic Health Foundation, Athens, Greece and Department of Hygiene, Epidemiology and Medical Statistics, School of Medicine, National and Kapodistrian University of Athens, Greece. E-Mail anaska@nut.uoa.gr
} 
be derived from the nationally representative household budget survey data available in the DAFNE databank (http://www.nut.uoa.gr/dafnesoftweb/), from studies undertaken in localized settings and limited population samples and from larger studies usually within the context of Greek participation in multicenter international projects.

The information presented in this report is based on studies: (a) published in PubMed from 2004 to 2009, (b) generally involving $>200$ participants and (c) referring to apparently healthy individuals. Several studies were retrieved and reported to the ENHR 2009 Coordinators, who made the final selection. The report is largely based on these selected studies, which are presented collectively, but grouped according to the age groups they refer to as these were defined in the European Nutrition and Health Report 2009.

The reader is reminded that evidence from these sources should be considered with caution, as the choice of study groups and measurement methods may differ between studies. Furthermore, the present document consists of the concise version of a more comprehensive Greek national report, available online (www.univie.ac.at/enhr).

\section{Children 1-7 Years Old}

According to the Growth, Exercise and Nutrition Epidemiological - GENESIS study, conducted in 2003-2004 among 2,374 children 1 to 5 years old, the mean energy intake (EI) was $17 \%$ higher than the estimated energy requirements of the total population [Institute of Medicine, 2005]. Overweight and 'at risk of being overweight' children, when compared to normal-weight ones, reported significantly higher EI overall. When compared with the estimated average requirements, 22 and $20 \%$ of children reported inadequate vitamin $\mathrm{E}$ and folate intake, respectively. Light to vigorous physical activity was limited among the very young children [Manios et al., 2008].

\section{Children and Adolescents 10-15 Years Old}

In their longitudinal study among peri-adolescent Greek schoolchildren, Koutedakis et al. [2005] reported age of being significantly positively associated with EI (both genders) and negatively associated with physical activity (boys). In the Physical Activity, Nutrition and Allergies in Children Examined in Athens (PANACEA) study survey of 700 children aged 10 to 12 years, Priftis et al. [2007] reported that 16\% of boys and $17 \%$ of girls had very low physical activity. The average hours/day of 'watching TV or playing video games' was 2.3 for boys and 2.1 for girls.

In the study of Hassapidou et al. [2006], among 502 adolescents aged 11 to 14 years, overweight adolescents reported greater negative energy balance, lower energy and macronutrient intakes per kilogram of body weight and lower iron and niacin intakes compared to non-overweight adolescents. Papandreou et al. [2006], in their study among 6- to 15-year-old children, noted that boys generally had higher intakes of dietary fat, sugar, fiber, folate and vitamin $B_{12}$ than girls. According to their 2006 study in 276 randomly selected children, 8 to 12 years old, Hassapidou et al. [2009] reported that the prevalence of overweight and obesity was higher among boys than girls. Furthermore, overweight and obesity rates were significantly lower, whereas 
Table 11.32. Dietary intake, BMI (mean \pm SD) and distribution of smoking habits among males in Greece

\begin{tabular}{|c|c|c|c|c|c|}
\hline & \multicolumn{5}{|l|}{ Name of the study } \\
\hline & $\begin{array}{l}\text { Students of } \\
\text { Crete University }\end{array}$ & $\begin{array}{l}\text { ATTICA } \\
\text { study }^{2}\end{array}$ & $\begin{array}{l}\text { EPIC study (adults) } \\
\text { (unpubl. } \\
\text { EPIC results) }\end{array}$ & $\begin{array}{l}\text { National survey on } \\
\text { prevalence of obesity } \\
\text { and abdominal } \\
\text { fat distribution }\end{array}$ & $\begin{array}{l}\text { EPIC study } \\
\text { (elderly) } \\
\text { (unpubl. } \\
\text { EPIC results) }\end{array}$ \\
\hline Year of data collection & 1989-2001 & $2001-2002$ & 1994-1999 & 2003 & 1994-1999 \\
\hline$n$ & 500 & 1,514 & 8,365 & 8,234 & 2,508 \\
\hline Age, years & $22 \pm 2$ & $46 \pm 13$ & $47.2 \pm 9.8$ & $44.9 \pm 25$ & $69.7 \pm 3.5$ \\
\hline \multicolumn{6}{|l|}{ Dietary data } \\
\hline Energy, kcal & $2,493 \pm 992$ & $2,596 \pm 337$ & $2,490.8 \pm 726.6$ & & $2,018.2 \pm 600.6$ \\
\hline Protein, \%E & $14.2 \pm 4$ & 15 & $14.1 \pm 1.7$ & & $14.1 \pm 1.7$ \\
\hline Carbohydrates, \%E & $44.8 \pm 9.7$ & 36 & $37.9 \pm 5.9$ & & $39.8 \pm 6.0$ \\
\hline Fat, \%E & $40.2 \pm 8.5$ & 46 & $45.0 \pm 5.5$ & & $43.9 \pm 5.4$ \\
\hline SFA, \%E & $12.9 \pm 3.8$ & 11 & $12.7 \pm 2.5$ & & $12.1 \pm 2.6$ \\
\hline MUFA, \%E & $17.1 \pm 5.6$ & 23 & $21.9 \pm 4.0$ & & $21 \pm 4.2$ \\
\hline PUFA, \%E & $5.1 \pm 2.7$ & 7 & $6.4 \pm 2.6$ & & $6.7 \pm 3.1$ \\
\hline Alcohol, \%E & & & $5.3 \pm 5.9$ & & $4.5 \pm 5.7$ \\
\hline Cholesterol, mg & $300.3 \pm 221.6$ & & $282.9 \pm 116.2$ & & $214.8 \pm 96.4$ \\
\hline \multicolumn{6}{|c|}{ Anthropometry and lifestyle choices } \\
\hline BMI & $24.5 \pm 3.1$ & $27.4 \pm 4$ & $28.2 \pm 3.8$ & $27.3 \pm 4.8$ & $28.2 \pm 4.0$ \\
\hline \multicolumn{6}{|l|}{ Smoking habits, \% } \\
\hline Non-smokers & 61 & 34 & $\begin{array}{l}19 \text { to < } 42 \text { years: } 24 \\
42 \text { to <65 years: } 24\end{array}$ & & 31 \\
\hline Former smokers & 7 & 15 & $\begin{array}{l}19 \text { to }<42 \text { years: } 18 \\
42 \text { to }<65 \text { years: } 33\end{array}$ & & 46 \\
\hline Current smokers & 32 & 51 & $\begin{array}{l}19 \text { to }<42 \text { years: } 58 \\
42 \text { to }<65 \text { years: } 43\end{array}$ & & 23 \\
\hline $\begin{array}{l}\text { SFA = Saturated; MUFA } \\
{ }^{1} \text { Mammas et al., } 2004 . \\
{ }^{2} \text { Pitsavos et al., 2003; Pa } \\
{ }^{3} \text { Kapantais et al., } 2006 .\end{array}$ & $\begin{array}{l}=\text { monounsaturated } \\
\text { nagiotakos et al., } 20\end{array}$ & $\begin{array}{l}\text { PUFA = polyu } \\
\text { 06; Arvaniti et }\end{array}$ & $\begin{array}{l}\text { aturated fatty acids. } \\
2006 .\end{array}$ & & \\
\hline
\end{tabular}


energy, carbohydrate intakes and exercise were significantly higher among immigrant children compared to Greek ones.

\section{Adolescents 15-19 Years Old}

The VYRONAS cross-sectional survey of 2004-2005, among 2008 volunteers aged 12-17 years, reported that age was positively associated with the risk of being overweight or obese in both genders and obesity was more prevalent among adolescent boys than girls in all age groups [Kosti et al., 2007].

In the study of Tzotzas et al. [2008], among 14,456 Greeks aged 13 to 19 years, the prevalence of overweight and obesity was higher among males, but abdominal obesity was higher among females (21.7\% for females, $13.5 \%$ for males). In agreement with previous findings, the prevalence of obesity, overweight and abdominal obesity were significantly lower among immigrant male adolescents than Greek ones.

\section{Adults and Elderly}

Unpublished results on nutrient intakes and lifestyle characteristics from the Greek EPIC study are jointly presented with results from the study among university students in Crete, the National Survey on Prevalence of Obesity and Abdominal Fat Distribution and the ATTICA study (tables 11.32, 11.33). The latter used the EPIC food frequency questionnaire for dietary data collection increasing thus the feasibility of comparisons. Furthermore, in the Greek EPIC cohort the prevalence of hypertension (measured twice on a single occasion and age-adjusted to the adult Greek population of 2001) was for men $40.2 \%$ and for women $38.9 \%$; increased with age and was higher in rural areas and among individuals of lower education [Psaltopoulou et al., 2004]. In a subsample of the EPIC study, men had lower levels of total and HDL cholesterol but higher total/HDL cholesterol ratio (5.42), compared to women (4.58) [Benetou et al., 2000]. In the ATTICA study, $46 \%$ of male and $40 \%$ of female participants were hypercholesterolemic and $8 \%$ of males and $6 \%$ of females reported history of diabetes mellitus [Pitsavos, 2003].

Kapantais et al. [2006], based on self-reported nationwide data from 1,734 Greeks 20 to 70 years old, reported a higher prevalence of obesity and overweight among men, and abdominal obesity among women and in particular those $>50$ years. Among women, the prevalence of overweight and obesity increased with age.

\section{Pregnancy and Breastfeeding}

In their 2003 study of 200 pregnant women, Petrakos et al. [2006] concluded that total micronutrient intake was generally adequate, except for vitamin $\mathrm{E}$ and zinc. Nevertheless, diet alone was insufficient for the recommended intakes of folic acid, magnesium and iron.

Bakoula et al. [2007] in their 2000 study of 4,310 mothers reported that the full breastfeeding initiation rate was $23 \%$ and any breastfeeding initiation rate was $85 \%$. A month postpartum, these percentages were 61 and $79 \%$ respectively. 
Table 11.33. Dietary intake, BMI (mean \pm SD) and distribution of smoking habits among females in Greece

\begin{tabular}{|c|c|c|c|c|c|}
\hline & \multicolumn{5}{|l|}{ Name of the study } \\
\hline & $\begin{array}{l}\text { Students of } \\
\text { Crete University }{ }^{1}\end{array}$ & $\begin{array}{l}\text { ATTICA } \\
\text { study }^{2}\end{array}$ & $\begin{array}{l}\text { EPIC study } \\
\text { (adults) } \\
\text { (unpubl. } \\
\text { EPIC results) }\end{array}$ & $\begin{array}{l}\text { National survey on } \\
\text { prevalence of obesity } \\
\text { and abdominal } \\
\text { fat distribution }{ }^{3}\end{array}$ & $\begin{array}{l}\text { EPIC study } \\
\text { (elderly) } \\
\text { (unpubl. } \\
\text { EPIC results) }\end{array}$ \\
\hline $\begin{array}{l}\text { Year of data } \\
\text { collection }\end{array}$ & 1989-2001 & 2001-2002 & 1994-1999 & 2003 & 1994-1999 \\
\hline $\mathrm{n}$ & 451 & 1,528 & 12,034 & 9,107 & 3,600 \\
\hline Age, years & $22 \pm 2$ & $45 \pm 13$ & $48.5 \pm 10$ & $41.5 \pm 11$ & $69.3 \pm 3.3$ \\
\hline \multicolumn{6}{|l|}{ Dietary data } \\
\hline Energy, kcal & $1675 \pm 673$ & $2059 \pm 300$ & $1972.4 \pm 581.4$ & & $1620.1 \pm 491.7$ \\
\hline Protein, \%E & $14.1 \pm 4.8$ & 14 & $14.39 \pm 1.65$ & & $14.39 \pm 1.75$ \\
\hline Carbohydrates \%E & $46.7 \pm 11.2$ & 38 & $39.5 \pm 5.4$ & & $41.8 \pm 5.6$ \\
\hline Fat, \%E & $39.4 \pm 10.1$ & 47 & $47.2 \pm 4.7$ & & $45.3 \pm 5.0$ \\
\hline SFA, \%E & $12.9 \pm 4.4$ & 13 & $13.2 \pm 2.6$ & & $12.3 \pm 2.5$ \\
\hline MUFA, \%E & $16.9 \pm 6.6$ & 23 & $22.9 \pm 4.1$ & & $21.7 \pm 4.4$ \\
\hline PUFA, \%E & $4.5 \pm 2.4$ & 6 & $6.9 \pm 2.9$ & & $7.0 \pm 3.3$ \\
\hline Alcohol, \%E & & & $1.24 \pm 2.18$ & & $0.94 \pm 2.05$ \\
\hline Cholesterol, mg & $205.3 \pm 158.1$ & & $227.6 \pm 92.3$ & & $173.4 \pm 76.1$ \\
\hline \multicolumn{6}{|c|}{ Anthropometry and lifestyle choices } \\
\hline $\mathrm{BMI}, \mathrm{kg} / \mathrm{m}^{2}$ & $22.7 \pm 3.2$ & $25.3 \pm 5$ & $28.6 \pm 5.3$ & $25.7 \pm 5.2$ & $30.6 \pm 4.9$ \\
\hline \multicolumn{6}{|l|}{ Smoking habits, \% } \\
\hline Non-smokers & 64 & 54 & $\begin{array}{l}19 \text { to }<42 \text { years: } 49 \\
42 \text { to }<65 \text { years: } 79\end{array}$ & & 95 \\
\hline Former smokers & 6 & 7 & $\begin{array}{l}19 \text { to }<42 \text { years: } 9 \\
42 \text { to }<65 \text { years: } 6\end{array}$ & & 3 \\
\hline Current smokers & 30 & 39 & $\begin{array}{l}19 \text { to }<42 \text { years: } 42 \\
42 \text { to }<65 \text { years: } 15\end{array}$ & & 2 \\
\hline $\begin{array}{l}\text { SFA = Saturated; ML } \\
{ }^{1} \text { Mammas et al., } 200 \\
{ }^{2} \text { Pitsavos et al., } 200 \\
{ }^{3} \text { Kapantais et al., } 20\end{array}$ & $\begin{array}{l}\text { JFA = monounsatur } \\
4 . \\
\text {; Panagiotakos et a } \\
06 \text {. }\end{array}$ & $\begin{array}{l}\text { ated; PUFA = } \\
\text { I., 2006; Arvan }\end{array}$ & $\begin{array}{l}\text { polyunsaturated fatty } \\
\text { iti et al., } 2006 .\end{array}$ & acids. & \\
\hline
\end{tabular}


Pechlivani et al. [2005] in their study of 1,603 mothers in Athens reported that $96.1 \%$ of women initiated breastfeeding. Exclusive breastfeeding was initiated in 19.1\%, predominant breastfeeding in $7.2 \%$ and complementary breastfeeding in $69.8 \%$ of the cases.

Theofilogiannakou et al. [2006] in their study of 312 women reported that $90 \%$ of newborns were given breast milk substitutes one or more times within the first 2 days postpartum, while exclusive breastfeeding on the last day of hospital stay reached $85 \%$. Breastfeeding was practiced by $55 \%$ and exclusive breastfeeding by $35 \%$ of women 40 days postpartum and 16 and $12 \%$ respectively at 6 months.

Ladomenou et al. [2007], in their study of 1,049 mothers in Heraklion, reported overall breastfeeding rates of $60.7,35.3,16.7$ and $4.5 \%$, at months $1,3,6$ and 12 , respectively, including $24.6,17.2,10.2$ and $3.5 \%$ of exclusive breastfeeding at the same time points [Ladomenou, 2007].

\section{Conclusion}

In general, all research groups in Greece agree that contemporary food choices gradually deviate from the traditional Mediterranean ones. The health status of the Greek population is generally adequate, but the previously reported Greeks' advantage tends to obliterate, because of the smoking and obesity epidemics, the abandoning of regular physical activity, the inadequate control of hypertension and diabetes and the gradual depart from traditional food choices.

Within this context, in 1999 the Supreme Health Council formulated dietary guidelines for Greek adults [Ministry of Health and Welfare, 1999]; in 2005, the Ministry of Health and Social Solidarity established the 2nd National Breastfeeding Committee, and previously in 2002, the National Nutrition Policy Committee was established with the following priorities: (1) reduce childhood obesity; (2) increase the consumption of pulses and vegetables; (3) reduce meat and increase fish consumption; (4) increase consumers' awareness on food safety and quality, and (5) improve mass catering services, with the application of food quality principles.

\section{Acknowledgements}

The authors would like to thank Vassiliki Bountziouka for her assistance in the studies' search and Mr. Michael Katsoulis for producing the requested EPIC results.

\subsection{Hungary ${ }^{10}$}

\section{National Dietary Surveys in Hungary}

During the last 20 years three national dietary surveys of adult population have been performed in Hungary. The 1st Hungarian Representative Nutrition Survey (HRNS)

${ }^{10}$ Gábor Zajkás, Viktória Kovács, Éva Martos; National Institute for Food and Nutrition Science, Budapest, Hungary. E-Mail martos.eva@oeti.antsz.hu 
[Bíró, 1992] was carried out in 1985-1988 and the 2nd Hungarian Nutrition Survey (HNS) [Bíró, 1996] in 1992-1994. Methods and results were published in the ENHR 2004 [Elmadfa et al., 2004]. The 3rd Hungarian Nutrition Survey (HNS) was performed in 2003-2004 [Rodler et al., 2005; Bíró et al., 2007; Zajkás et al., 2007]. Data collection of this study was done in November/December 2003, analysis and evaluation of data were carried out in 2004. Of the interviewed 5,071 people, 1,362 sent back the completed 3-day dietary record of which 1,179 could be evaluated.

Besides there was a national representative survey on nutrition and lifestyle of secondary school students (National Representative Survey in Secondary Schools (NRSecS); $\mathrm{n}=6,347 ; 15$ to 18 years old) in 1997-2000 [Antal et al., 2003]. Apart from these studies a representative survey in metropolitan primary and secondary schools was carried out in 2005/2006 (Representative Survey in Metropolitan Primary and Secondary Schools (RSMPSS); $\mathrm{n}=1,9307$ to 14 years old and $\mathrm{n}=1,27315$ to 19 years old) [Péter et al., 2007; Bíró et al., 2007].

Two additional smaller surveys were performed, one to collect data on nutrition, lifestyle, serum vitamin D level and bone density in adolescents (Vitamin D status of Adolescents (VDSA); $\mathrm{n}=326$ students, 11 to 14 years old) in 2002/2003 [Antal et al., 2006]. In the other survey, cardiovascular risk factors were measured in 264 medical university students (Cardiovascular Risk Factors in University Students (CVRFUS); mean age $21.4 \pm 2.6$ years) in 2003 [Antal et al., 2006].

The above-mentioned surveys were carried out, analyzed, evaluated and published by the National Institute for Food and Nutrition Science (NIFNS), Hungary. The methods of the surveys are summarized in table 11.34.

\section{Energy and Nutrient Intake}

Children, Adolescents. The energy intake of 11- to 14-year-old boys was close to the recommended value, but it was insufficient in girls [Bíró et al., 2007]. The carbohydrate energy \% (\%E) was less, but the saccharose, fat and protein $\mathrm{E} \%$, and the cholesterol intake were higher than the Hungarian RDI. The dietary fiber intake was below the recommended value. The intake of vitamin $\mathrm{A}, \mathrm{D}$, riboflavin and folate was also insufficient, while sodium intake was 2.5 times higher, and the intake of phosphorus was higher by $25 \%$ (girls) and by $30 \%$ (boys) than the Hungarian RDI (for more detailed data, see chapter 7 in the main part of the ENHR 2009.

In the VDSA study it was revealed that the vitamin D intake of adolescents was far below the RDI. In addition, $33.3 \%$ of boys and $43.2 \%$ of girls had a calcium intake below $70 \%$ of RDI. Moreover, the bone density was insufficient in $12.9 \%$ of boys and $10.4 \%$ of girls.

Adults. Compared to the results of the two earlier nutrition surveys, the energy intake and the total fat $\% \mathrm{E}$ in the $3 \mathrm{rd} \mathrm{HNS}$ was smaller than in the two previous ones [Rodler et al., 2005] and much less than the estimated value of the food balance sheet (FBS) 2003 [Central Statistics Office, 2005]. There were only small differences in the $\% \mathrm{E}$ of macronutrients, but $\% \mathrm{E}$ of the added sugar and the SFA was much less in the 
Table 11.34. Overview of the Hungarian nutrition surveys

\begin{tabular}{lllll}
\hline Survey & Age group & Method & Year & Reference \\
\hline 1st HRNS & $14+$ & 24-hour recall & 1985-1988 & Bíró, 1992 \\
\hline 2nd HNS & $18+$ & 3-day dietary record & 1992-1994 & Bíró et al., 1996 \\
\hline 3rd HNS & $18+$ & 3-day dietary record & 2003-2004 & Rodler et al., 2005 \\
\hline NRSecS & $14-18$ & FFQ & 1997-2000 & Antal et al., 2003 \\
\hline RSMPSS & 7-14 and & 3-day dietary record' & $2005-2006$ & Bíró et al., 2007, \\
\hline VDSA & $15-19$ & & & Péter et al., 2007 \\
\hline CVRFUS & Mean age 21.3 \pm 2.6 & 3-day dietary record & $2002-2003$ & Antal et al., 2006 \\
\hline
\end{tabular}

${ }^{1}$ Only in 11- to 14 - and 15 - to 19 -year-old children.

Fig. 11.10. Trend in PUFA \%E in the 3rd Hungarian Nutrition Survey.

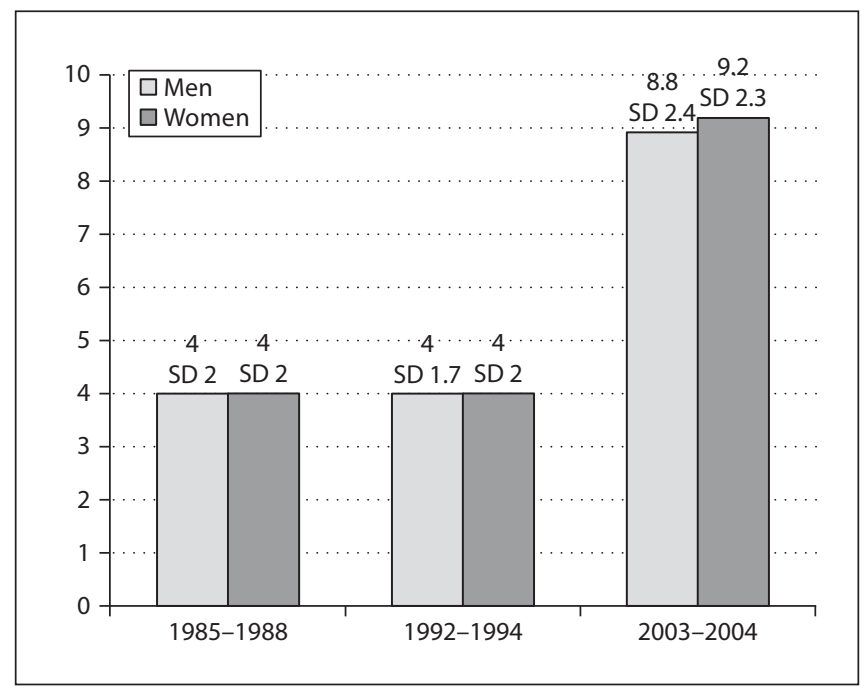

3rd HNS than previously. The \%E of PUFA was higher in the 3rd HNS. These results are confirmed by the FBS data - the purchase of vegetable oils has increased by 3 times during the last 20 years, and the buying of animal fats has decreased by $40 \%$ (fig. 11.10-11.12). The n-3 fatty acid intake was very low, 1.1 and $1.0 \mathrm{~g} /$ day in men and women, respectively.

The cholesterol intake was much less than in the earlier studies, but still it was 50 and $10 \%$ higher than the recommended value in case of men and women (fig. 11.13). The alcohol E\% was low 3.8, and 2.1 in case of men and women, respectively. 


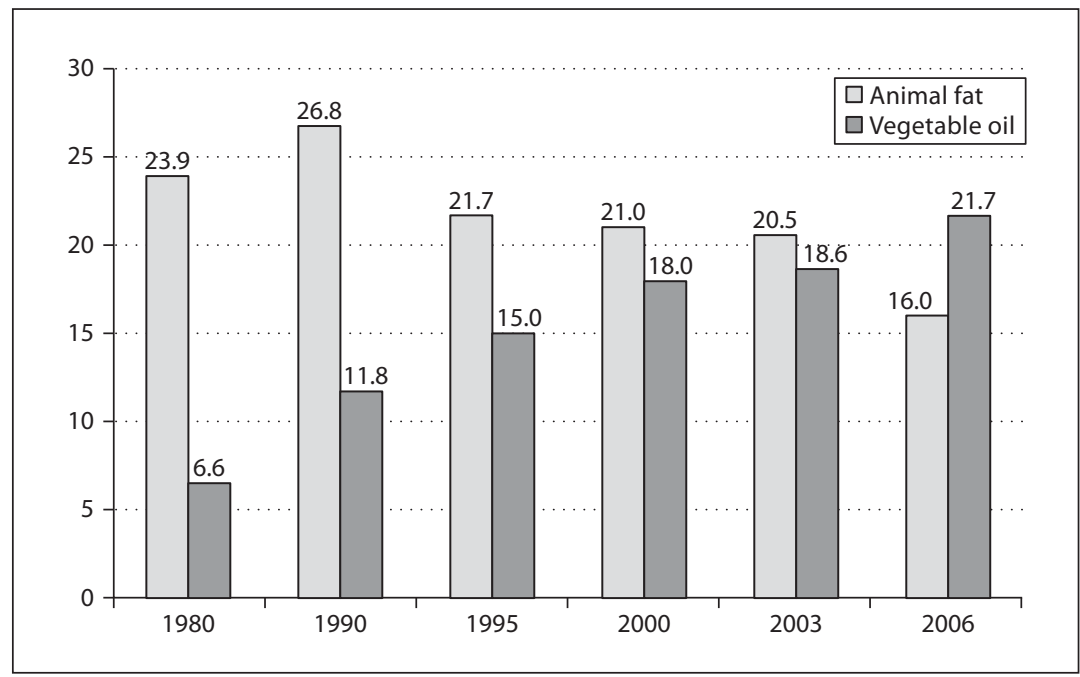

Fig. 11.11. Fat and vegetable oil supply in Hungary between 1980 and 2006 based on the FBS. Data are $\mathrm{kg} / \mathrm{capita} / \mathrm{year}$.

Fig. 11.12. Trend in added sugar \%E in the 3rd Hungarian Nutrition Survey.

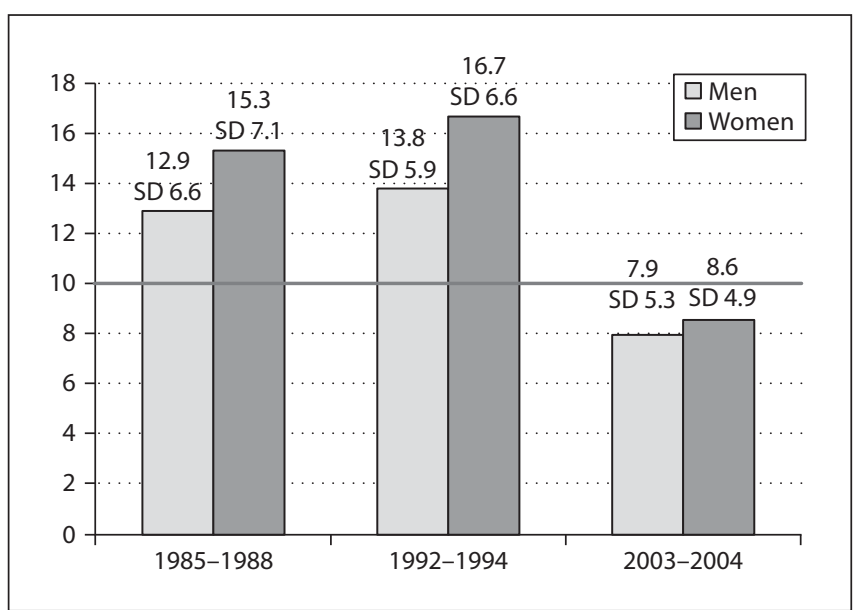

There was a favorable trend in the intakes of vitamin $A$, thiamine, vitamin $B_{6}, B_{12}$, and niacin, as well as biotin was sufficient in both gender, together with adequate vitamin $\mathrm{E}$ intake of men. However, the intake of vitamins $\mathrm{B}_{2}, \mathrm{C}, \mathrm{D}$ and folate of men and women did not meet the Hungarian recommendation, besides vitamin E intake of women was insufficient as well.

Magnesium intake was adequate in both sexes, and iron, zinc and copper intakes also met the RDI in case of men, but did not in women. In contrast, there was an insufficient calcium and an excessive phosphorus and sodium intake in both sexes. 
Fig. 11.13. Trend in cholesterol intake in the $3 \mathrm{rd}$ Hungarian Nutrition Survey. Data are mg/day.
Fig. 11.14. Trend in sodium intake in the 3rd Hungarian Nutrition Survey. Data are g/day.
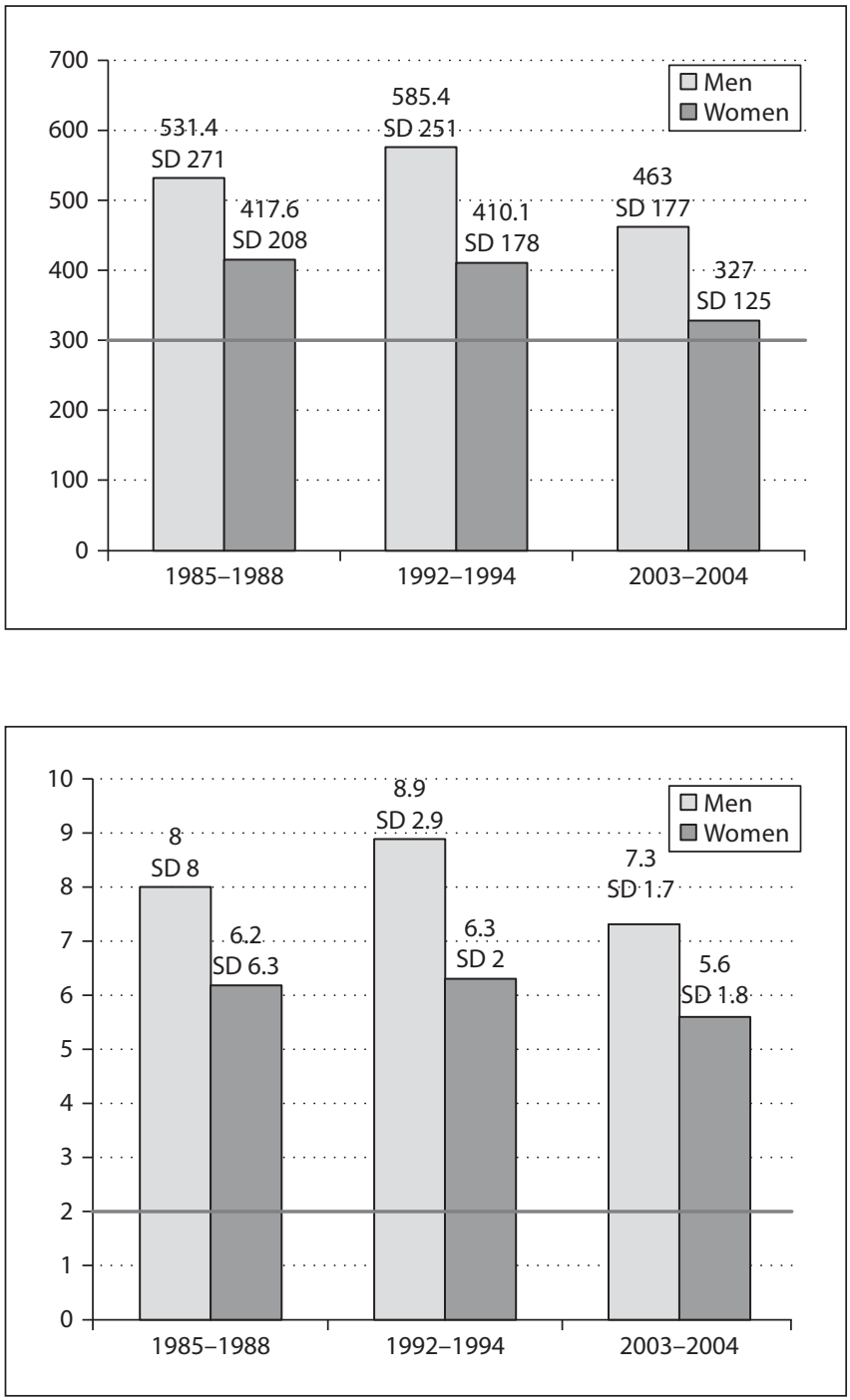

The sodium intake, however, showed a decreasing trend (fig. 11.14) (for more detailed data, see chapter 7 in the main report).

In addition, in the 3rd HNS the average amount of foods ( $n=77$ types) consumed daily was also evaluated. The most important foods and drinks are summarized in table 11.35 .

\section{Nutritional Biomarkers}

Biomarkers were checked only in two of the above-mentioned surveys. In the CVRFUS study, elevated total cholesterol levels were detected in $16.6 \%$ of men and $30.1 \%$ of women, and increased LDL-C levels in $9.2 \%$ of men and $7.6 \%$ of women, 
Table 11.35. Food consumption according to the 3rd Hungarian Nutrition Survey, 2003-2004. Some examples of 77 foods, drinks evaluated in the survey

\begin{tabular}{lll}
\hline & Men $(\mathrm{n}=470)$ & Women $(\mathrm{n}=702)$ \\
\hline White bread, g/day & $135.5 \pm 86.1$ & $86.1 \pm 61.0$ \\
\hline Whole grain bread, g/day & $6.7 \pm 26.1$ & $5.3 \pm 19.8$ \\
\hline Milk, milk drinks, ml/day & $206.2 \pm 209.4$ & $180.2 \pm 158.6$ \\
\hline Meat products, g/day & $89.6 \pm 65.0$ & $47.4 \pm 39.3$ \\
\hline Pig meat, g/day & $61.5 \pm 51.4$ & $46.6 \pm 41.3$ \\
\hline Animal fat, g/day & $15.6 \pm 22.4$ & $10.1 \pm 13.2$ \\
\hline Vegetable oil, g/day & $38.7 \pm 16.5$ & $33.8 \pm 14.3$ \\
\hline Fresh and deep-frozen vegetables, g/day & $122.0 \pm 72.9$ & $123.9 \pm 143.5$ \\
\hline Fresh and deep frozen fruits, g/day & $151.9 \pm 148.7$ & $185.2 \pm 149.1$ \\
\hline Confectionary products, g/day & $18.2 \pm 34.1$ & $19.0 \pm 30.6$ \\
\hline
\end{tabular}

and low level of HDL-C in $10.2 \%$ of men, and $1.1 \%$ of women (table 11.36). These results indicate an increased cardiovascular risk in this group of young adults [Antal et al., 2006].

In the VDSA study, serum vitamin D level after winter was $<40 \mathrm{nmol} / \mathrm{l}$ in 13 and $27 \%$ of boys and girls, respectively. No vitamin D insufficiency was observed after summer, and $24 \%$ of boys, and $21 \%$ of girls even had serum $25(\mathrm{OH}) \mathrm{D}$ higher than the upper limit of reference range.

\section{Dietary Supplements}

The use was investigated only in children and adolescents [Antal et al., 2003; Péter et al., 2007], but not in adults. Overall, $34 \%$ of 11 - to 14 -year-old boys and $37.2 \%$ of girls consumed vitamin products. In the secondary school students, $40.7 \%$ of boys and $41.1 \%$ of girls consumed vitamin products. Among the 11- to 14-year-old population, mineral products were taken by $6.4 \%$ of boys and $7.3 \%$ of girls, and combined mineral/ vitamin products were consumed by $21.1 \%$ of boys and 16.6 of girls, respectively.

\section{Anthropometric Data}

Children, Adolescents. In Hungary, all 5-year-old children together with the students in the 1st, 3rd, 5th and 7th grade are measured in the framework of annual school health examination. The collected and evaluated data are sent online to the Central Statistics Office. Based on these datasets, the prevalence of obesity is increasing year by year in all age groups. 
Table 11.36. Cardiovascular risk factors in Hungarian University students, Budapest (2003)

\begin{tabular}{lll}
\hline & Males $(\mathrm{n}=78), \%$ & Females $(\mathrm{n}=186), \%$ \\
\hline Total cholesterol, $\mathrm{mmol} / \mathrm{l}$ & & \\
\hline Average & $4.6 \pm 0.88$ & $4.75 \pm 0.84$ \\
\hline$<5.2$ & 74.4 & 69.9 \\
\hline $5.2-6.2$ & 10.5 & 24.2 \\
\hline$>6.2$ & 5.1 & 5.9 \\
\hline LDL-C, $\mathrm{mmol} / \mathrm{l}$ & & \\
\hline Average & $2.61 \pm 0.70$ & $2.46 \pm 0.64$ \\
\hline$<3.4$ & 89.7 & 92.5 \\
\hline $3.4-4.1$ & 5.4 & 6.5 \\
\hline$>4.1$ & 3.8 & 1.1 \\
\hline HDL-C, $\mathrm{mmol} / \mathrm{l}$ & & \\
\hline Average & $1.29 \pm 0.25$ & $1.54 \pm 0.30$ \\
\hline$>1$ & 89.8 & 98.9 \\
\hline$<1$ & 10.2 & 1.1 \\
\hline Triacylglycerol, $\mathrm{mmol} / \mathrm{l}$ & & 9.8 \\
\hline Average & $0.88 \pm 0.44$ & $0.87 \pm 0.38$ \\
\hline$\leq 1.7$ & 94.9 & 9.1 \\
\hline$>1.7$ & 5.1 & \\
\hline & & \\
\hline
\end{tabular}

According to the RSMPSS study, the prevalence of overweight (according to the Cole criteria) in 7- to 14-year-old boys and girls was 18.1 and $19.6 \%$, while the rate of obesity was 7.4 and $6.3 \%$, respectively. In the same study, $15.8 \%$ of 15 - to 19 -year-old boys and $7.9 \%$ of girls were overweight, and $4.1 \%$ of boys and $2.9 \%$ of girls were obese (fig. 11.15).

Adults. In the 3rd HNS, body weight and height were self-reported. The average BMI of men was $26.4 \pm 4.0 \mathrm{~kg} / \mathrm{m}^{2}$, and it was $25.6 \pm 4.7 \mathrm{~kg} / \mathrm{m}^{2}$ in women. The proportion of underweight in men and women was 0.4 , and $3.0 \%$, of normal weight 40.7, and $47.5 \%$, of overweight 41.8 , and $31.3 \%$, and of obesity 17.1 , and $18.2 \%$, respectively. It means that almost two thirds of men and half of the women are at increased risk because of overweight (table 11.37). In the CVRFUS study, anthropometric data were measured. The rate of overweight was $27 \%$ in men and $11.3 \%$ in women, and 

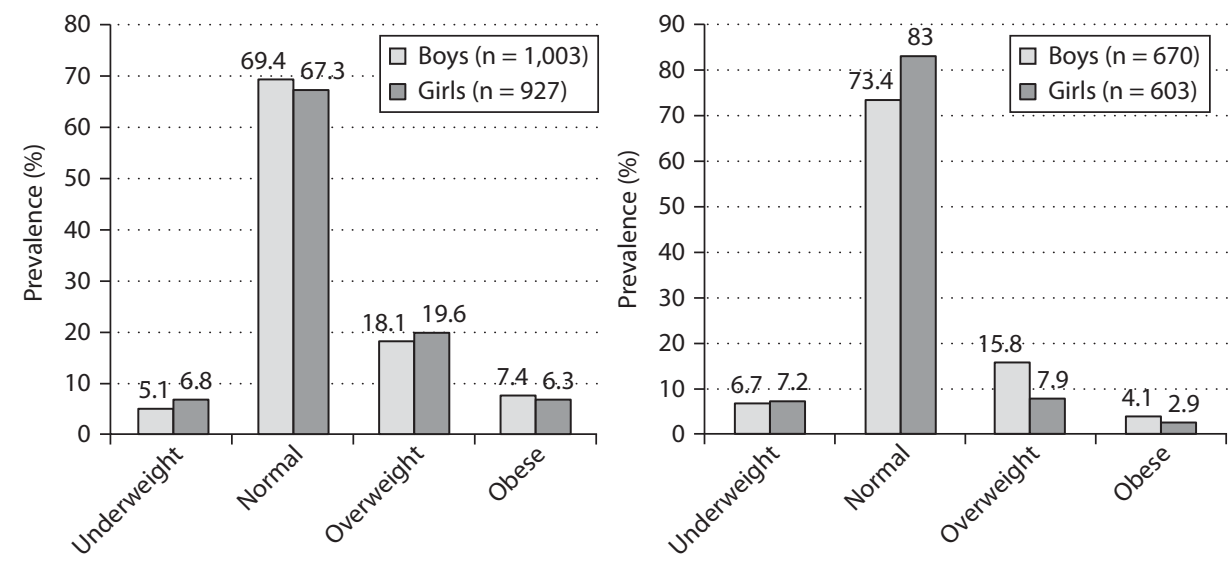

Fig. 11.15. Prevalence of overweight and obesity among primary $(n=1,930)$ and secondary $(n=$ 1,273 ) schoolchildren in $2005 / 2006$. Body weight and height were measured and prevalences were calculated according to the Cole criteria.

19.2 and $6.5 \%$ of men and women had $>90$ and $>80 \mathrm{~cm}$ waist circumference, respectively (table 11.38).

\section{Alcohol (Ethanol) Intake}

In the 3rd HNS, alcohol intake was $12.5 \pm 12.1 \mathrm{~g} /$ day, but the FBS data in the same year showed $30.4 \mathrm{~g} /$ day availability. In the National Health Survey of the Population (OLEF), 2003 [Boros, 2005], the proportion of heavy drinkers was 17.7\% in men and $2.5 \%$ in women.

\section{Data Available from Other Databases}

Food Balance Sheets (FBS)

According to the FBS data there was a decreasing trend in the supply of meat (mostly pork and beef, but not chicken), milk and milk products, flour and rice, sugar and honey between 1980 and 2006, while the supply of total fat, potatoes, and vegetables increased in the same time. Within the fat balance, the supply of vegetable oil has increased by $300 \%$, and the supply of animal fats has decreased by $40 \%$ in the period above.

\section{Household Budget Survey (HBS)}

The results are similar, but the availability of rice and fish has moderately increased, and the availability of alcoholic beverages has increased, after a small decrease. 
Table 11.37. Body mass indices (BMI) in the 3rd National Nutrition Survey, 2003-2004

\begin{tabular}{|c|c|c|c|c|}
\hline & BMI & & & \\
\hline & Total & $18-34$ years & $35-59$ years & $\geq 60$ years \\
\hline Men & $26.39 \pm 4.01$ & $26.77 \pm 3.89$ & $27.55 \pm 3.92$ & $26.39 \pm 4.01$ \\
\hline Women & $25.58 \pm 4.69$ & $26.07 \pm 4.61$ & $26.98 \pm 4.10$ & $25.58 \pm 4.69$ \\
\hline
\end{tabular}

Body height and body weight were self-reported.

Table 11.38. Anthropometric parameters in Hungarian university students, 2003

\begin{tabular}{cll}
\hline & Men $(n=78)$ & Women $(n=186)$ \\
\hline $\mathrm{BMI}, \mathrm{kg} / \mathrm{m}^{2}$ & $23.4 \pm 3.18$ & $21.37 \pm 2.84$ \\
\hline $\mathrm{BMI}<18.50, \%$ & 3 & 11.3 \\
\hline $18.50-24.99, \%$ & 69.2 & 77.4 \\
\hline $25.00-29.99, \%$ & 24.4 & 9.1 \\
\hline$\geq 30.00, \%$ & 2.6 & 2.2 \\
\hline Waist circumference, $\mathrm{cm}$ & $83.56 \pm 8.60$ & $69.40 \pm 6.14$ \\
\hline
\end{tabular}

Body height, body weight and waist circumference were measured

\section{Health Indicators}

Morbidity data in Hungary show that trachea/bronchus/lung cancer has the highest prevalence; it is followed by female breast cancer, colon cancer, and then rectum/anus tumors. The high prevalence of trachea/bronchus/lung cancer is obviously related to the high proportion of smoking, and the other cancers mentioned can be partly connected to the high rate of obesity.

Mortality. The Hungarian total mortality showed a decreasing trend between 1990 and 2007. Half of the total mortality was caused by cardiovascular diseases (decreasing trend), and $24.6 \%$ were caused by neoplasms (stagnant trend) in 2006 (table 11.39).

Smoking. In the NRSecS study, 30 and $25 \%$ of 15 to 16 years and 41 and $35 \%$ of 17 to 18 years boys and girls were smokers [Antal et al., 2003]. In the OLEF study the proportion of smokers in adults is high, and the rate of men and women smoking $>20$ cigarettes/day was rather high too (table 11.40) [Boros, 2005].

Breastfeeding. The proportion of breastfed babies at $0-3$ months of age is $63.0 \%$, and at $4-5$ months of age $43.8 \%$ [Central Statistics Office, 2009]. It can be the result of the health promotion, and the activity of midwives. 
Table 11.39. Death rates standardized for the age structure of the European population by selected main groups of causes of death (deaths per 100,000 population) in 2007 [source: Hungarian Central Statistics Office, 2008]

\begin{tabular}{ll}
\hline Total & $1,322.0$ \\
\hline Malignant neoplasms & 325.7 \\
\hline CVD (I100-I99) & 661.8 \\
\hline Cerebrovascular disease & 144.4 \\
\hline (Chronic) liver disease (K70-K76) & 55.7 \\
\hline
\end{tabular}

Table 11.40. Rate of smoking and proportion of heavy smokers in Hungarian adults in 2003

\begin{tabular}{|c|c|c|c|c|c|c|}
\hline & \multicolumn{2}{|c|}{ Never smoked } & \multicolumn{2}{|c|}{ Recent smoker } & \multicolumn{2}{|c|}{ Gave up smoking } \\
\hline & male & female & male & female & male & female \\
\hline \multicolumn{7}{|l|}{ Smoking rate, $\%$} \\
\hline $18-34$ years & 41.4 & 52 & 43.10 & 32.5 & 9.2 & 10.4 \\
\hline $35-64$ years & 30.9 & 52.3 & 39.00 & 28.50 & 27.3 & 15.7 \\
\hline $65+$ years & 34.2 & 86.6 & 15.90 & 5.30 & 49.6 & 7.6 \\
\hline Total & 35 & 59.8 & 36.90 & 24.6 & 24.5 & 12.4 \\
\hline Smoking >20 cigarettes/day, \% & & male & & female & & \\
\hline $18-34$ years & & 24.30 & & 13.9 & & \\
\hline $35-64$ years & & 26.9 & & 11.0 & & \\
\hline $65+$ years & & 6.9 & & 2.5 & & \\
\hline Total & & 23.0 & & 10.0 & & \\
\hline
\end{tabular}

In 2008, there was a representative study on breastfeeding in Budapest and in three other towns. 400 mothers were asked by interviewers [Szabó and Zagyva, 2009]. This survey revealed that $75 \%$ of the 3 -month-old babies and $60 \%$ of 6 -month-old babies were breastfed.

Physical Activity. In a representative sample of 11,000 primary school students (10 to 15 years), two thirds of the children did no sports [Kiss, 2003]. Similarly, in the RSMPSS study, only half of the primary school students and one fifth of the secondary school students were regularly physically active. In addition, only $23 \%$ of the secondary school students participated in extracurricular sports. The situation was even 
worse in adults. Based on the Eurobarometer database, $60 \%$ of the adult population had never done any sports. Similarly, the average time spent walking and/or exercising was 14 minutes/day for men and 8 minutes/day for women in 2002 .

\section{Food and Nutrition Policies}

The First Hungarian Food and Nutrition Policy (FNP) was elaborated and published in 2004. This FNP was worked out on the directives of WHO Regional Office for Europe: The First Action Plan for Food and Nutrition Policy, 2001.

The First FNP [Zajkás, 2004] contained an analysis of actual dietary risk factors in the Hungarian population - high energy, total fat, saturated fatty acids, cholesterol, sugar, sodium, but insufficient intake of some micronutrients (vitamin E, potassium, calcium, and in case of women iron and zinc). There were similar problems in the nutrition of children too. The FNP targeted the main objectives (decrease the risk of chronic diet-related diseases, and decrease of mortality caused by these diseases within 5-10 years) and indicated the strategies to realize objectives of FNP, besides outlined the Hungarian Dietary Guidelines, as well.

A new, revised draft of FNP 2nd Action Plan was elaborated by the experts of the National Institute for Food and Nutrition Science at the beginning of this year, but further consultations with professionals of other institutes, ministries, the food industry and catering, and civil organizations are necessary to compile the final version.

\subsection{Ireland ${ }^{11}$}

\section{Introduction}

In the past 20 years, attitudes and approaches to food in Ireland have altered dramatically. In Ireland, several national publications regarding nutrient intake and health indicators in children, adolescents, adults and the elderly have been published which has created a clearer picture which illustrates the significant changes in our dietary and lifestyle habits. Irish Universities Nutrition Alliance (IUNA) is made up of four academic nutrition units from University College Cork, University of Ulster and University College Dublin. Since 1990, IUNA has completed a number of comprehensive national dietary surveys, focusing on adults (The North South Ireland Food Consumption Survey), children (The National Children's Food Survey) and teenagers (The National Teen's Food Survey) which are presented in this report.

Results from the SLÁN survey are also presented in this report. SLÁN is a national survey of the lifestyle, attitudes and nutrition of people living in Ireland.

\footnotetext{
${ }^{11}$ Heavey P, Kelleher C; School of Public Health and Population Science, University College, Dublin, Ireland. E-Mail patricia.heavey@ucd.ie
} 
To date, surveys have been carried out in 1998, 2002 and 2007. SLÁN 2007 is the largest survey to date. A scientifically representative random sample of over 10,000 people aged 18+ were interviewed in their own homes, by experienced researchers from the Economic and Social Research Institute (ESRI). The survey covers general health, behaviors relating to health (e.g. exercise, nutrition) and the use of certain health services. The food frequency data was analyzed using a specially designed computer program called FFQ_Software, Version 1.0, developed by Juzer Lotya of the National Nutrition Surveillance Centre, School of Public Health and Population Science, University College, Dublin. The program converts the dietary information provided to food quantities and subsequently to food nutrient values, based on data from the Food Standards Agency (2002) and from McCance and Widdowson's Food Composition Tables (1997). In addition, over 1,200 people who participated in the survey also participated in a detailed medical examination.

\section{Dietary Habits}

Adults. The North/South Ireland Food Consumption Survey included a sample of 1,379 adults between the ages of 18 and 64 years, including 662 men and 717 women and excluding pregnant and lactating mothers, from all over the island of Ireland. The participants were randomly selected using the electoral register as the sampling frame. The response rate was $63 \%$. The survey sample was representative of the population of the island of Ireland with respect to age, sex, geographical location (urban/ rural), marital status, social class and socioeconomic group. Data collection took place between 1997 and 1999 and was seasonally balanced. Food intake was determined using a 7-day estimated food record. Self-administered questionnaires were used to collect information on employment status, social and demographic variables, lifestyle factors, habitual physical activity levels, attitudes, health status, medication, supplement use and dieting habits. Researchers also carried out anthropometric measurements.

The mean daily intakes of fat in men and women (37\% of food energy) exceeded current recommendations for fat (for a maximum intake of $35 \%$ of food energy) and the mean daily intakes of carbohydrate (46\%) were lower than current recommendations for $\mathrm{CHO}$ (for a minimum intake of $50 \%$ of food energy). When compared to existing recommendations, protein intake in adults was found to be more than adequate.

The mean daily intake of dietary fiber in the total sample was $20.2 \mathrm{~g}$ and on average, men consumed larger quantities of fiber (23.2 g) than women (17.4 g). Expressed as non-starch polysaccharide (NSP), i.e. dietary fiber excluding lignin and resistant starch, the mean daily intake in the total sample was $14.8 \mathrm{~g}(16.7 \mathrm{~g}$ in men and $13.0 \mathrm{~g}$ in women). Women aged 18 to 35 years had the lowest mean daily dietary fiber intake (16.1 g).

Intakes were adequate in the population for most vitamins. However, a significant prevalence of inadequate intakes was observed for vitamin A (20\% of men 
and $17 \%$ of women) and riboflavin (13\% of men and $20 \%$ of women). The proportions of men (16\%) and women (11\%) with mean daily vitamin $\mathrm{E}$ intakes less than the lowest threshold intake indicate that the prevalence of vitamin $\mathrm{E}$ inadequacy may be significant. A substantial proportion of the population had low vitamin $\mathrm{D}$ intakes and was largely dependent on sunlight to maintain adequate vitamin $\mathrm{D}$ status.

Only $2 \%$ of women aged 18 to 35 years and $5 \%$ of women aged 36 to 50 years achieved the recommended folate intake of $600 \mathrm{mg} /$ day for women of reproductive years for the prevention of neural tube defects. All of the women who met the recommendation were using folate containing supplements. A significant prevalence of intakes below the average requirement (AR) was observed for iron, calcium, zinc and copper, particularly in women. $48 \%$ of women aged 18 to 50 years (whose iron requirements are high due to menstrual losses) had inadequate iron intakes, exposing them to increased risk of depletion of iron stores and iron deficiency anemia. Calcium intakes were below the AR in $23 \%$ of women. Approximately $33 \%$ of men and $32 \%$ of women were current smokers.

Children. The National Children's Food Survey investigated habitual food and drink consumption, health and lifestyle characteristics and assessed body weight status in children from the Republic of Ireland. This was a cross-sectional study that was carried out between March 2003 and March 2004. Children aged 5 to 12 years (293 boys, 301 girls) were selected from 28 primary schools throughout the Republic of Ireland from a database of primary schools obtained from the Department of Education and Science. All schools in the database that contained at least 8 students in each of the eight classes ( $\mathrm{n}=1,194$ schools) were classified according to size, gender served, disadvantaged/not disadvantaged and location (urban or rural). A 7-day weighed food diary was used to collect food and beverage intake data. The researcher made four visits to the respondent and his/her parent/guardian during the 7-day period: a training visit to show how to keep the food diary and how to use the weighing scales; a second visit 24-36 hours into the recording period to review the diary, check for completeness and clarify details regarding specific food descriptors and quantities; a third visit 4 or 5 days into the recording period to check the previous 2 or 3 days and to encourage completion, and a final visit 1 or 2 days after the recording period to check the last days and to collect the diary.

The average intake of energy was 7.0 MJ/day. The current recommendation for dietary fat in Irish children is that it should be less than $35 \%$ of their overall energy intake. In Irish 5- to 12-year-old children, fat intakes are high, with $40 \%$ of children not meeting the fat recommendation and having diets that are $>35 \%$ of daily energy coming from fat. One fifth of calories $(18 \%)$ and fat $(21 \%)$ in 5 - to 12 -year-olds diets comes from cakes, biscuits, savory snacks, chocolate and confectionary.

Dietary fiber intakes were low with $61 \%$ of children consuming less than the minimum requirement of 'age of child $+5 \mathrm{~g} /$ day'. The Food Standards Agency of Ireland (2005) recommendations for daily salt intake are $3 \mathrm{~g} /$ day for 4 - to 6-year-olds, $5 \mathrm{~g} /$ 
day for 7 - to 10 -year-olds and $6 \mathrm{~g} /$ day for 11 - to 14 -year-olds. However, Irish children are currently taking in 4.6, 5.3 and $5.9 \mathrm{~g}$ daily from food alone (5-6, 7-10 and 11-12 years, respectively). The average vitamin $\mathrm{D}$ intakes were very low at $2.3 \mu \mathrm{g} / \mathrm{day}$. There was no effect of age, sex or season on vitamin $\mathrm{D}$ intake and the main determinant of intake was nutritional supplement use. $18 \%$ of children took supplements containing vitamin $\mathrm{D}$ and the average intake in these children was $5.6 \mu \mathrm{g} /$ day. This was almost 4 times higher than the intake of $1.5 \mu \mathrm{g}$ in those who did not use supplements.

Teenagers. The National Teens' Food Survey investigated habitual food and drink consumption, health and lifestyle characteristics and assessed body weight status in 441 teenagers, from the Republic of Ireland. This was a cross-sectional study that was carried out between September 2005 and September 2006. Teenagers aged 13 to 17 years (224 males, 217 females) were selected from 32 secondary schools throughout the Republic of Ireland. Schools were selected from a database of secondary schools available from the Department of Education and Science and were classified into secondary $(56 \%)$, vocational $(31 \%)$ or comprehensive/community schools (13\%). Schools were further classified according to gender served, whether disadvantaged or not disadvantaged and location (urban or rural). A 7-day semi-weighed food diary was used to collect food and beverage intake data.

The average intake of energy was $8.3 \mathrm{MJ} /$ day. The current recommendation for dietary fat in Irish teenagers is that it should be less than $35 \%$ of their overall energy intake. Irish teenagers obtained $36 \%$ of their energy from fat. The findings show that $42 \%$ of teenage girls and $23 \%$ of teenage boys are not getting enough calcium in their diet. Furthermore, Irish teenagers are consuming a higher energy and fat intake from biscuits, confectionary and snacks than from milk, cheese or yoghurt.

Elderly. The Irish population is ageing. In 1996, 11.4\% of the population was over 65 years and this figure is expected to rise to $14.1 \%$ by 2011 [Central Statistics Office 1997]. There is an important relationship between nutritional status, diet and health status of the population. The Third National Survey of Lifestyle, Attitudes and Nutrition (SLÁN) was conducted in 2007, following previous surveys in 1998 and 2002. The 2007 SLÁN survey involved a nationally representative sample of 10,364 respondents (62\% response rate), of whom 9,223 (89\%) completed a standard Willett Food Frequency Questionnaire adapted for use in the Irish population. This survey also included males and females over the age of 65 years. The food frequency questionnaire was completed by 742 females (median age 74 years) and 580 males (median age 72 years).

The median daily energy intake for females was 7.5 MJ and for males 7.9 MJ. The percentage contribution to daily food energy intake from protein and fat was above the recommended guidelines for both men and women and was below that recommended for carbohydrates. In SLAN 2007, 19\% of food energy was derived from protein for females and males, 33 and $34 \%$ from fat for females and males, respectively and 50 and $49 \%$ for carbohydrates for females and males, respectively. 


\subsection{Italy $^{12}$}

\section{Dietary Profile}

The present national report aims to provide information on food- and nutritionrelated issues by presenting tendency analyses and recent Italian dietary trends in order to widen the current nutritional scenario of our country. Moreover, some of the most sensitive issues are examined, such as child obesity as well as alcohol consumption and smoking habits among young individuals and elderly. A section dedicated to breastfeeding is also included because of its generally known importance for child health and prevention among the population. This report also deals with specific topics so far studied in limited geographical areas or in special groups of people.

Some focused studies are included here to represent the background information which may lead to wider and detailed studies in larger areas or population groups.

The total dietary intake variability was estimated from the nationwide dietary survey data [Turrini et al., 2001] collected during the 1990s (7-day-weighed food records; 0-94 age range; response rate was 48\%; improper reporters were deleted; Italian food composition table [Carnovale and Marletta, 1997] using a combination of cluster and correspondence analyses). Three different quantitative diets were identified and tagged as 'min diet', 'mid diet' and 'max diet' [SAS, 1990]. Total proteins, total lipids and their fractions, and 'carbohydrates + alcohol' accounted for the same percentage contribution to the per-capita daily energy intake in all reported diet choices. The three diet boundaries were linked to the energy intakes, e.g. all nutrient intakes were proportional to the total energy intakes (fig. 11.16).

The contingency analysis performed on the dietary choices highlighted a tendency to adopt a 'min diet' profile among females, children, elderly individuals and underweight adults; on the contrary, a tendency to choose mid or max diet profiles was found among males and obese people; an almost balanced frequency for adults and normal weight individuals was registered, while the most divergent group was represented by adolescents showing min and max diet choices both at observed frequencies higher than the expected ones.

The estimated average per-capita daily energy intake during the three last decades falls within the range estimated in the '90s (fig. 11.17). Overall, energy intake showed decreasing values over time [Saba et al., 1990; Turrini et al., 2001; Leclercq et al., 2009]. Such decreasing values occur in spite of an increasing availability of food supplies together with an average annual rate of available energy of $0.62 \%$, this latter value being due to an average annual protein and fat supply increase of 0.94 and $2.5 \%$, respectively [FAO, 2007b].

The sociodemographic structure of the population, particularly the age class distribution, the workforce sector of employment [ISTAT, 2009a], and the sedentary

\footnotetext{
12 Turrini A; INRAN, Rome, Italy. E-Mail turrini@inran.it
} 


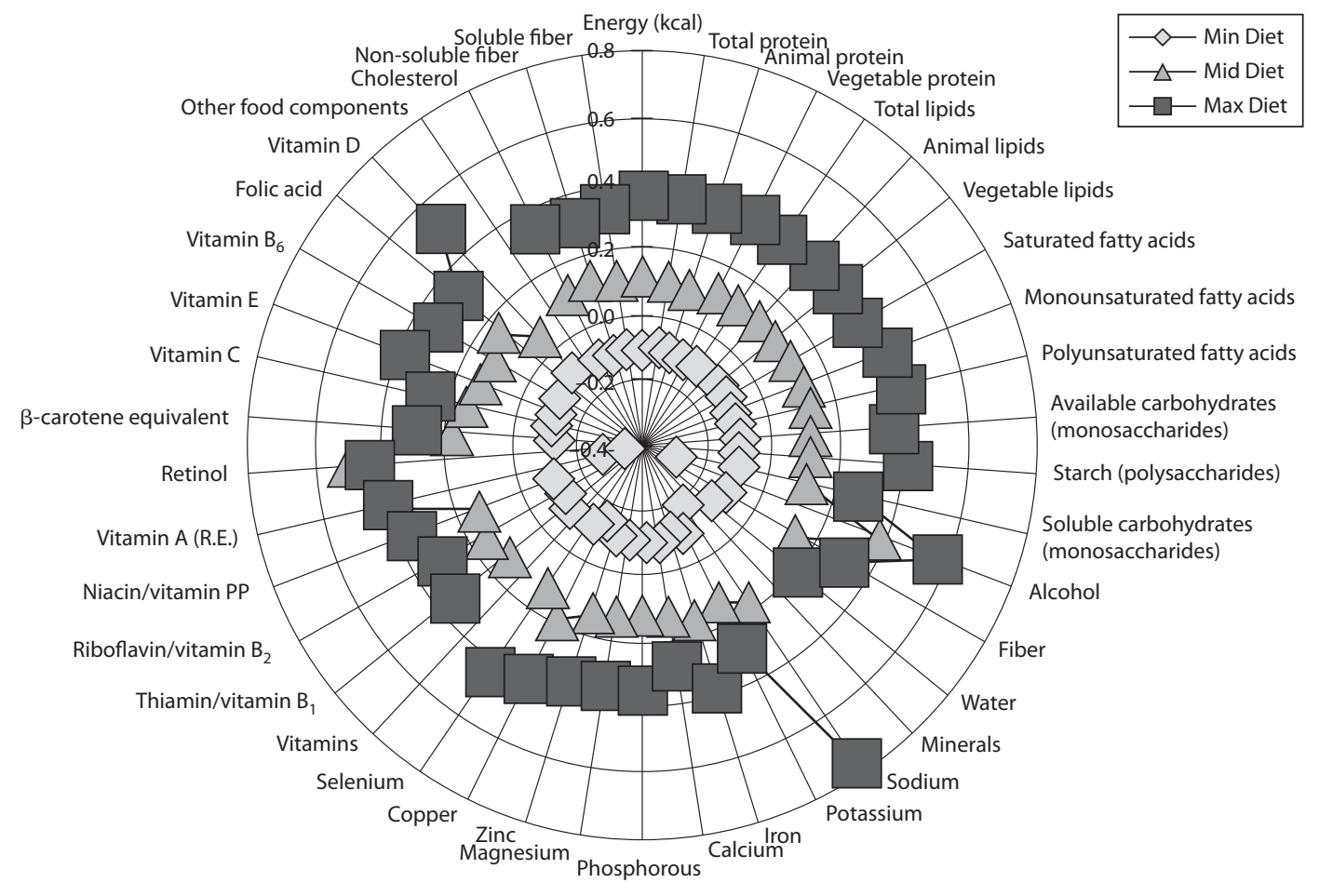

Fig. 11.16. Energy and nutrient average daily intakes in Italy. Relative \% difference of overall profiles in the three groups - Min Diet, Mid Diet and Max Diet vs. total sample profile (source: INN-CA Study 1994-1996, INRAN, Rome, Italy).

lifestyle [ISTAT, 2009a], showed a decreasing energy requirement trend (ageing) as well as a reduction of the energy expenditure, both for work and physical activities. The average annual growth rates of $3+$ year-old people by physical activity levels are shown in table 11.41.

The observed decrease of young age groups (0-24) (negative growth rates) and the increase of the number of $25+$ aged people (positive growth rates) made the overall population ageing index to vary from 62.5 to 142.2 through 25 years (1982-2007) [ISTAT, 2009a].

The workforce has moved from agriculture and industry to other activities.

Physical activity - sport or everyday activities - shows only a moderate to slightly increase of people percentage, while the number of people practicing sport occasionally was reduced. The overall percentage of individuals not doing any kind of physical activity remained almost constant over 7 years (2001-2007), even though it should be noted that a decrease of women's percentage was observed during that period [ISTAT, 2009b]. 


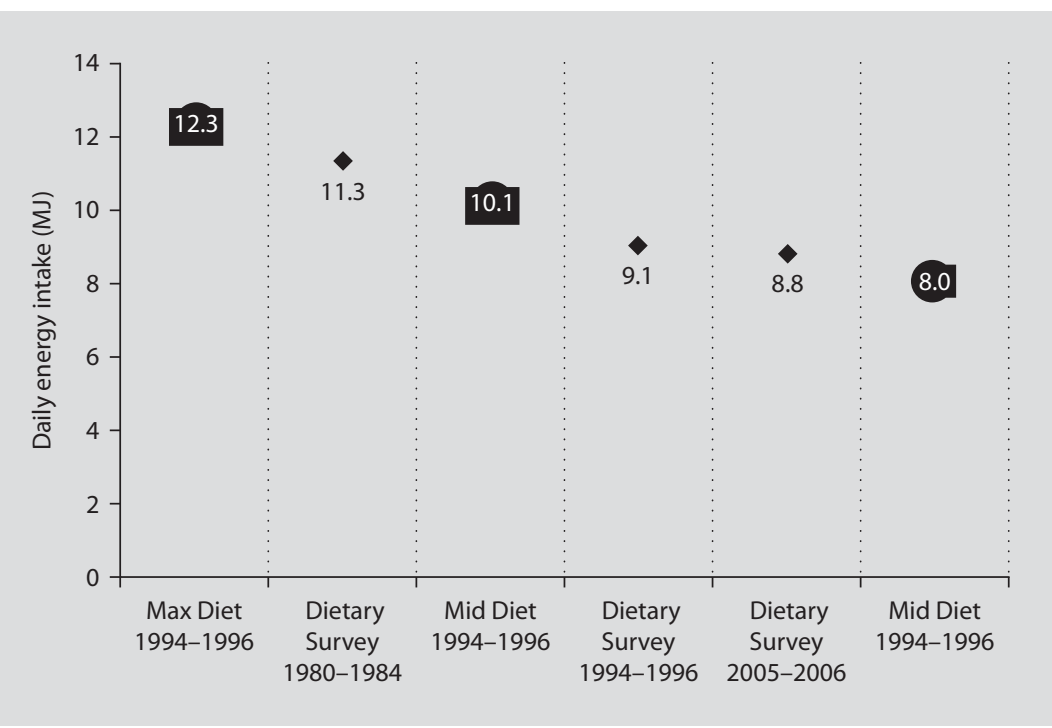

Fig. 11.17. Italian nationwide dietary surveys about daily energy intake (MJ) vs. dietary boundaries (1994-96). Source: INRAN nationwide individual food consumption survey: INN Study 1980-1984, INN-CA Study 1994-1996, INRAN-SCAI 2005-2006, Rome, Italy [Saba et al., 1990; Turrini et al., 2001; Leclercq et al., 2009].

Regarding the national average energy requirement, it is progressively decreasing considering the age-gender distribution of the population and the levels of sport/ physical activity observed (fig. 11.18) [D’Amicis, 2008]. This could likely have determined the observed trend in the prevalence of overweight and obesity, increasing +2.8 and $+2.6 \%$ in the period $1994-2007$, respectively. These two similar values are actually related to different starting points: in 1994 the overweight value was $32.8 \%$, while the obesity value accounted for $7.3 \%$ of the total population, increasing to 35.6 and $9.9 \%$, respectively, in 2007 [ISTAT, 2009b].

Comprehensively, data indicated a decreased physical activity on a daily basis probably because of the more frequent sedentary jobs beside which a limited increase of a regular exercise versus the occasional one was observed (table 11.41). This is probably affecting the overall population energy requirement (fig. 11.18) steadily increasing the share overweight and obese in the population.

The overall picture of energy and nutrient intakes, the percentage contribution of proteins, fats, carbohydrates and alcohol to the mean daily energy intake, and the mean of the individual 'actual intake/recommended intake' ratios (I\%RDA) - calculated considering a sedentary activity - were assessed.

Generally, almost all the energy and nutrient intakes provided more than the recommended dietary allowance, even though the inferior extreme of the mean minus the standard deviation was below $100 \%$ in all cases, except for total proteins, 
Table 11.41. Average growth rate percent of 3+ year-old people by physical activity levels; Italy, 2001-2007. Source: ISTAT, Multi-purpose survey - daily life in 2007 [ISTAT, 2009b]

\begin{tabular}{|c|c|c|c|c|c|c|}
\hline \multirow[t]{2}{*}{ Years } & \multicolumn{2}{|l|}{ Sport } & \multirow{2}{*}{$\begin{array}{l}\text { Physical } \\
\text { activities }\end{array}$} & \multirow[t]{2}{*}{ Sedentary lifestyle } & \multirow[t]{2}{*}{ Not specified } & \multirow[t]{2}{*}{ Total } \\
\hline & $\begin{array}{l}\text { regular } \\
\text { exercise }\end{array}$ & $\begin{array}{l}\text { occasional } \\
\text { exercise }\end{array}$ & & & & \\
\hline
\end{tabular}

\section{Males}

\begin{tabular}{lllllll}
\hline 2001 & 23.1 & 13.2 & 28.8 & 34.2 & 0.7 & 100.0 \\
\hline 2002 & 23.9 & 12.4 & 27.9 & 35.1 & 0.6 & 100.0 \\
\hline 2003 & 25.1 & 12.3 & 26.9 & 35.1 & 0.6 & 100.0 \\
\hline 2005 & 25.2 & 12.9 & 26.4 & 34.7 & 0.8 & 100.0 \\
\hline 2006 & 24.4 & 12.7 & 26.1 & 36.0 & 0.9 & 100.0 \\
\hline 2007 & 24.8 & 11.9 & 28.1 & 34.5 & 0.7 & 100.0
\end{tabular}

Females

\begin{tabular}{lllllll}
\hline 2001 & 15.3 & 8.0 & 29.9 & 46.1 & 0.6 & 100.0 \\
\hline 2002 & 15.7 & 7.6 & 29.3 & 46.9 & 0.6 & 100.0 \\
\hline 2003 & 16.3 & 7.9 & 28.1 & 47.0 & 0.7 & 100.0 \\
\hline 2005 & 16.9 & 7.9 & 29.8 & 44.5 & 0.8 & 100.0 \\
\hline 2006 & 16.8 & 8.1 & 28.4 & 45.9 & 0.8 & 100.0 \\
\hline 2007 & 16.7 & 7.4 & 31.0 & 44.2 & 0.6 & 100.0 \\
\hline Males and females & 19.1 & 10.6 & 29.4 & 40.3 & & \\
\hline 2001 & 19.7 & 9.9 & 28.6 & 41.2 & 0.6 & 100.0 \\
\hline 2002 & 20.6 & 10.1 & 27.5 & 41.2 & 0.6 & 100.0 \\
\hline 2003 & 20.9 & 10.3 & 28.2 & 39.8 & 0.6 & 100.0 \\
\hline 2005 & 20.5 & 10.3 & 27.3 & 41.1 & 0.8 & 100.0 \\
\hline 2006 & 20.6 & 9.6 & 29.6 & 39.5 & 0.8 & 100.0 \\
\hline 2007 & & & & 0.7 & 100.0
\end{tabular}

phosphorus, zinc, and vitamin $\mathrm{B}_{6}$. The maximum level of sodium intake was exceeded by all groups except for the children records.

The free (added) sugar threshold (10\% of the total energy) was exceeded by all population groups and even if the first ten main sources of energy (65.3\% in total) in the Italian average diet - bread and pizza (19.2\%) followed by olive oil (8.3\%), pasta (7.7\%), sweets (7.5\%), cheese (6.4\%), milk (3.5\%), meat (3.5\%), first course (3.2\%), fruit 
Fig. 11.18. Energy daily requirement $(\mathrm{kcal})$ of the Italian population, 1961-2001. Source: ISTAT data elaborated by INRAN [D'Amicis, 2008].

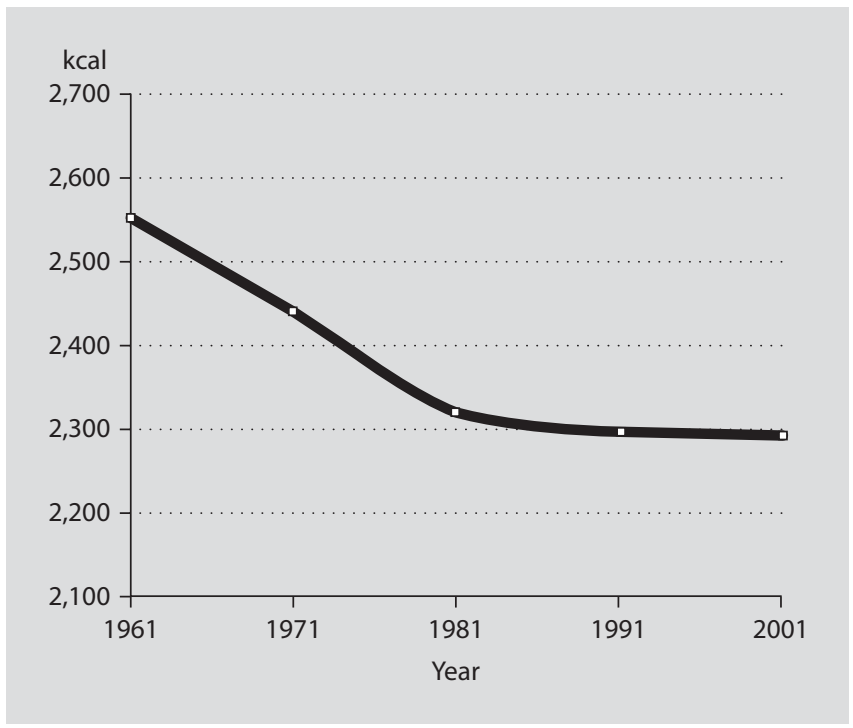

(3.2\%) and wine and sparkling wine (2.9\%) - include several sources of carbohydrates, the recommended daily allowance was not achieved. Females generally showed lower nutrient intakes, except for lipids and soluble carbohydrates in adolescents, and sodium levels in elderly. Many nutrient intakes had values below the recommended ones also among males, such as fiber and magnesium, while starch and available carbohydrates were below $100 \%$ for all groups.

\section{Focus on Specific Issues}

Child Obesity. The study OKkio alla salute is the most recent survey measuring anthropometric variables among 8-year-old children [CNESPS, 2008]. The prevalence of overweight and obesity values in this population group [Cole et al., 2000] is reported in the main report.

A comparison of these survey data with the results of the previous survey carried out in 2000-2001 [Bevilacqua et al., 2003] showed a decreasing trend of Tuscany percentages, both for overweight and obesity values (overweight 22.0 vs. $22.7 \%$; obesity 7.0 vs. $9.0 \%$ ). Although the extension of the present and past studies did not completely correspond, the most different overweight values were observed in Emilia-Romagna (20.0 vs. $25.9 \%$ ), while for obesity the Calabria percentage appeared quite increased (16.0 vs. $27.3 \%)$; other values did not show significant differences.

These data are also consistent with the overweight and obesity percentages estimated for children aged 7 to 9 within the INRAN-SCAI 2005-2006 study; this is quite evident especially in relation to the obesity rate at a national level, being $12.2 \%$ [Leclercq et al., 2009]. 
Breastfeeding. The percentage of women breastfeeding their children remained around the $81.1 \%$ of the total women in labor in 2004-2005, almost the same value observed in 1999-2000. The duration of the lactating period increased from 6.2 months in 1999-2000 to 7.3 [ISTAT, 2006].

The percentage of breastfeeding women was lower in the insular regions (74.2\%) compared to the North-East areas (86.1\%). The lactating period was shorter in the Islands, as only $26.6 \%$ of women breastfed for longer than 6 months, while $36.8 \%$ breastfed over 7 months in North-East zones. A similar distribution was registered for predominant or exclusive breastfeeding (53.5 vs. $73.8 \%$ in Islands and North-East regions, respectively).

Higher education levels seemed to promote breastfeeding (86.4 vs. $76.1 \%)$ as well as its predominant or exclusive use (71.2 vs. $53.2 \%)$.

Cesarean sections were associated with a lower percentage of lactating women ( 75.9 vs. $83.9 \%$ ), while geographical differences were observed in relation to breastfeeding start immediately after the delivery: $38.3 \%$ in the South and $59.9 \%$ in the North.

When women breastfed their first child they were more likely to also breastfeed the new baby ( 92.1 vs. $32.7 \%$ ), preferring their own milk as the exclusive or predominant feeding (74.3\%).

Alcohol. Data collected during 9 years (1998-2008) [ISTAT, 2009c] showed that there were no significant changes in alcohol consumption among Italians (70\% of $14+$ year-old people consumed alcohol in the previous year), except for young women, aged 18 to 19 and 20 to 24 , showing an increase in consumption (53.7-58.0\% and $58.4-63.6 \%$, respectively).

In 2008, the proportion of adolescents aged 11 to 15 showing an increase of drinking behavior was quite worrying (19.7\%); similarly, the percentage of young individuals, aged 14 to 17 , consuming alcohol at least once during the previous year was definitely increasing over the same period (12.6-18.7\%), showing a different trend in relation to the gender: $9.7-14.4 \%$ and $15.2-22.7 \%$, for females and males, respectively.

When at least one of the parents consumed alcohol consumption excessively, a proportion of $22.7 \%$ of young individuals, aged 11 to 17 , showed a similar drinking behavior too, while such a proportion decreased to $15.0 \%$ if parents did not drink or consumed alcohol moderately.

People aged 20 to 24 represented a proportion of $16.0 \%$ showing at least one unhealthy drinking, in particular high daily consumption in general (2.2\%), during the meals (29.7\%), or getting drunk (14.9\%).

Beside this dangerous behavior, the binge drinking phenomenon ( $>6$ glasses at once) was also observed: in $2008,7.3 \%$ of $11+$ year-old people severely exceeded in alcohol consumption in a single occasion; in addition, people aged 20 to 24 and 25 to 29 widely represented the phenomenon ( 14.9 and $14.0 \%$, respectively), the percentage value in men being higher than in women (12.1 vs. $2.8 \%)$. The binge drinking habit was mainly observed in northern Italy, especially north-eastern men (15.5\%). 
Geographical differences were quite marked as north-eastern regions accounted for $73.2 \%$ of $11+$ aged individuals drinking alcohol at least once a last year, Veneto being even higher (74.5\%), while Trentino-Alto Adige and Friuli-Venezia Giulia were just below the area rate: 71.3 and $72.0 \%$ respectively.

In 2008 , the general trend showed that $55.0 \%$ of $14+$ year-old people used to drink wine, while beer was preferred by $46.3 \%$. Among these results, wine represented an everyday drink in $37.3 \%$ of men vs. $14.2 \%$ of women in relation to daily alcohol consumption.

Concerning alcohol quantities, a consumption of 3 units/day was observed among $13.4 \%$ of men aged 55 to 64 versus 2 units in $3.2 \%$ of women, the percentages being even higher when education levels were lower.

Elderly Italians usually drink wine during meals and this is considered a less unhealthy habit even when their consumption exceeds 1 unit of alcohol, the threshold for 65-years-old subjects.

The most recent data [ISTAT, 2009c] have substantially confirmed the long-term trend evidenced since 2007 [ISTAT, 2008]. The most important signal is the decreasing percentage of 14- to 17-year-old drinkers (defined as people drinking alcohol at least once during meals in the last year): $18.7 \%$ in 2008 versus $20.5 \%$ in 2006 . Overall, less $14+$ aged people drink every day (28.3\% in 1998 vs. $33.5 \%$ in 2008$)$ and more people drink occasionally (37.0\% in 1998 and 41.7\% in 2008).

Smoking. In 2007 the percentage of $14+$ year-old smokers (cigarette, cigar, pipe) was $28.2 \%$, higher than the results in 2006 (22.7\%). 22.5\% gave up smoking (22.8\% in 2006), and the 53.2\% declared they no longer smoked (52.6\% in 2006) [ISTAT, 2009b].

A quite significant percentage of Italians already started smoking habits around 18 to 19 years of age (23.1\%), smoking up to 10 cigarettes/day, but the percentage of smokers, as well as the daily cigarette number, increased to around 20 to 24 years (29.0\%). The main age classes are 25 to 34 years (39.3\%) and 35 to 44 years $(25.0 \%)$ for men and women, respectively.

Smokers are slightly predominant in big towns and their peripheries as compared with the rest of Italy (24.2 and 23.8\%). In southern and insular regions, people smoke 13.9 and 14.5 cigarettes/day, respectively, while in north-eastern regions smoking decreases to 12.2 cigarettes/day. Cigarettes are definitely preferred to cigars or pipes (98.3\%) and data showed that $42.8 \%$ of the Italians smoked 11-20 cigarettes/day, this percentage being different according to gender: $48.6 \%$ in men, with a number of 14.8 cigarettes/day, and $33.7 \%$ in women smoking a mean of 11.1 cigarettes/day.

The global trend is a slight but constant reduction of smokers: $22.1 \%$ in 2007 versus $23.7 \%$ in 2001 , and the opposite trend of ex-smokers: $22.5 \%$ in 2007 versus $20.2 \%$ in 2001 .

Research Team and Acknowledgements

The Italian nationwide individual food consumption surveys carried out by the INRAN research teams in 1980-1984, 1994-1996, and 2005-2006 were supported by the Italian Ministry of 
Agricultural, Food and Forestry Policy. Aida Turrini coordinated the present research work, elaborated and revised all drafts and the final content of this paper. Paola D'Acapito, Fabrizia Maccati and Alessandro Vienna gathered information and materials on Italian data sources. Laura D'Addezio performed statistical data processing of the Italian dietary profiles. Alessandro Vienna organized the information database and revised paper drafts. Sabina Bietolini translated and reviewed the paper content.

\subsection{Latvia $^{13}$}

\section{Dietary Habits}

A nationwide survey about nutrition and lifestyle in the Baltic Republic was carried out in the summer of 1997 [Pomerlau et al., 1999], using a cross-sectional survey and a 24-hour recall of dietary intake questionnaire. The samples included 2,308 adult persons, $46.4 \%$ male and $53.6 \%$ female, between the age of 16 and 65 , the area of residence being $68.8 \%$ urban and $33.4 \%$ rural.

The average daily energy intake in all age groups was $2,583 \mathrm{kcal} /$ day in men and $1,791 \mathrm{kcal} /$ day in women.

The dietary fat intake was $42.7 \% \mathrm{E}$ on average in men and $41.1 \% \mathrm{E}$ in women, which was higher than the recommended range (table 11.42). In men, the highest fat consumption was in the age group 19 to 34 years, $44.2 \% \mathrm{E}$, in women respondents the age group $>50$ years showed the lower fat consumption $-39.8 \% \mathrm{E}$.

The dietary carbohydrate proportion in total energy intake was $42.4 \% \mathrm{E}$ on average in men and $44.6 \% \mathrm{E}$ in women.

The dietary protein intake was $13.7 \% \mathrm{E}$ on average. Alcohol intake amounted to $1.2 \% \mathrm{E}$ in men and $0.5 \% \mathrm{E}$ in women.

The average vitamin and mineral intakes in both sexes and in all age groups were lower than recommended. Vitamin $\mathrm{C}$ intake was low in men $64 \pm 63 \mathrm{mg}$ (mean \pm $\mathrm{SD})$, and in women $70 \pm 65 \mathrm{mg}$.

Mean daily intakes of minerals (excluding supplements) in women and men were generally close to the recommended daily intakes. Mean iron level in men was about $45 \%$ higher than recommended in all age groups.

The mean consumption of vegetables (excluding potatoes) and fruits was low in men (268 g/day) and in women as well (265 g/day), and decreased with ageing. In the age group older than 50 years, the mean intakes of vegetables (excluding potatoes) and fruits were $237 \mathrm{~g} /$ day in men and $233 \mathrm{~g} /$ day in women, which is less than $60 \%$ of the WHO recommendation of at least $400 \mathrm{~g}$ of vegetables and fruits per day.

\section{Body Mass Index}

Adults. Based on measurements [Pomerleau et al., 1999] and analyzing BMI, 33\% of women aged 19 to 65 years were overweight $\left(\mathrm{BMI}>25 \mathrm{~kg} / \mathrm{m}^{2}\right)$ and $17.4 \%$ were obese

\footnotetext{
${ }^{13}$ Selga G; Sports Medicine State Agency, Riga, Latvia. E-Mail selga@sveiks.lv
} 
Table 11.42. BMI groups by sex and age (\%) based on self-reported weight and height [Pudule et al., 2003, 2005, 2007]

\begin{tabular}{|c|c|c|c|c|c|c|c|c|c|c|c|c|}
\hline \multirow[t]{3}{*}{ Parameters } & \multicolumn{6}{|l|}{ Men } & \multicolumn{6}{|c|}{ Women } \\
\hline & \multicolumn{5}{|c|}{ age groups } & \multirow[t]{2}{*}{ total } & \multicolumn{5}{|c|}{ age groups } & \multirow[t]{2}{*}{ total } \\
\hline & $15-24$ & $25-34$ & $35-44$ & $45-54$ & $55-64$ & & $15-24$ & $25-34$ & $35-44$ & $45-54$ & $55-64$ & \\
\hline \multicolumn{13}{|l|}{ Underweight } \\
\hline 2006 & 4.5 & - & 0.7 & - & 0.8 & 1.2 & 17.8 & 5.9 & 1.2 & 1.2 & 1.0 & 5.3 \\
\hline 2004 & 8.8 & - & 0.7 & 0.8 & - & 2.6 & 18.4 & 6.3 & 3.8 & 0.5 & 0.9 & 4.5 \\
\hline 2002 & 5.5 & 1.1 & 0.5 & - & - & 1.6 & 13.4 & 2.8 & - & 0.9 & - & 3.4 \\
\hline \multicolumn{13}{|l|}{ Normal } \\
\hline 2006 & 82.8 & 56.8 & 52.1 & 37.6 & 38.9 & 54.1 & 73.0 & 68.0 & 57.1 & 30.6 & 24.8 & 49.1 \\
\hline 2004 & 80.8 & 59.3 & 49.7 & 39.4 & 37.7 & 55.5 & 75.7 & 67.6 & 52.4 & 36.4 & 25.2 & 50.7 \\
\hline 2002 & 84.9 & 60.9 & 48.4 & 48.9 & 42.5 & 58.6 & 81.5 & 71.3 & 60.4 & 37.7 & 24.4 & 54.9 \\
\hline \multicolumn{13}{|l|}{ Overweight } \\
\hline 2006 & 11.9 & 36.7 & 32.6 & 35.0 & 45.8 & 32.3 & 8.6 & 18.3 & 25.2 & 43.4 & 38.6 & 27.5 \\
\hline 2004 & 8.8 & 34.1 & 31.5 & 40.9 & 44.2 & 30.1 & 2.9 & 17 & 26.4 & 33.5 & 37.6 & 23.9 \\
\hline 2002 & 9.0 & 29.9 & 40.2 & 38.1 & 39.2 & 30.3 & 3.7 & 19.4 & 30.2 & 37.3 & 35.1 & 25.2 \\
\hline \multicolumn{13}{|l|}{ Obesity } \\
\hline 2006 & 0.7 & 6.5 & 14.6 & 27.4 & 14.5 & 12.3 & 0.6 & 7.8 & 16.6 & 24.9 & 35.7 & 18.1 \\
\hline 2004 & 1.6 & 6.7 & 18.1 & 18.9 & 18.1 & 11.9 & 2.9 & 9.1 & 17.3 & 29.6 & 36.2 & 19.5 \\
\hline 2002 & 0.5 & 8.2 & 10.9 & 12.9 & 18.3 & 9.5 & 1.4 & 6.5 & 9.5 & 24.1 & 40.4 & 16.5 \\
\hline
\end{tabular}

$\left(\mathrm{BMI}>30 \mathrm{~kg} / \mathrm{m}^{2}\right) ; 41 \%$ of men were overweight and $9.5 \%$ were obese. $2.7 \%$ of women and $0.9 \%$ of men were classified as underweight $\left(\mathrm{BMI}<18.50 \mathrm{~kg} / \mathrm{m}^{2}\right)$.

Self-reported data from the Latvian population (table 11.42 ) showed that $32.3 \%$ of men and $27.5 \%$ of women were overweight and $12.3 \%$ of men and $18.1 \%$ of women were obese at age 15 to 64 years in 2006 [Pudule et al., 2007].

Children. Based on self-reported data, $6.7 \%$ of adolescents (age 11, 13, 15 years old) were overweight and $0.9 \%$ were obese in 2005/2006 [Gobina et al., 2007]. Based on measured data from the Sports Medicine State agency, $12.4 \%$ of boys and $11.7 \%$ of girls were overweight or obese in the period 2004-2006 and 13.9\% of girls and 9\% of boys at age 7 to 17 were underweight.

Data from the Sports Medicine State Agency by age and gender from the period 2004-2008 are represented in tables 11.43 and 11.44 [Lide, 2009]. 
Table 11.43. Overweight and obesity (\%) among boys, 2004-2008 [Lide, 2009]

\begin{tabular}{lllllll}
\hline Age & $\mathrm{n}$ & $\begin{array}{l}\text { IsoBMl } \\
<16.1 \mathrm{~kg} / \mathrm{m}^{2}\end{array}$ & $\begin{array}{l}\text { IsoBMl } \\
<18.5 \mathrm{~kg} / \mathrm{m}^{2}\end{array}$ & Normal & $\begin{array}{l}\text { IsoBMl } \\
>25 \mathrm{~kg} / \mathrm{m}^{2}\end{array}$ & $\begin{array}{l}\text { IsoBMI } \\
>30 \mathrm{~kg} / \mathrm{m}^{2}\end{array}$ \\
\hline 7 & 508 & 1.2 & 6.7 & 77 & 11 & 4.1 \\
\hline 8 & 572 & 0.9 & 6.5 & 79.5 & 11 & 2.1 \\
\hline 9 & 588 & 1.2 & 7.8 & 77.9 & 10.5 & 2.6 \\
\hline 10 & 669 & 1 & 6.7 & 78.3 & 11.5 & 2.4 \\
\hline 11 & 567 & 1.9 & 7.1 & 82.5 & 6.5 & 1.9 \\
\hline 13 & 457 & 1.3 & 9 & 78.1 & 9.4 & 2.2 \\
\hline 14 & 453 & 1.1 & 10.2 & 77.3 & 9.6 & 1.7 \\
\hline 15 & 327 & 1.8 & 9 & 78.5 & 8.3 & 2.4 \\
\hline 16 & 304 & 0.7 & 8.3 & 81.7 & 6.4 & 1.8 \\
\hline 17 & 119 & 3.4 & 7.2 & 81.9 & 8.6 & 1.6 \\
\hline Total & 4434 & 3.2 & 5.9 & 84 & 5 & 1.7 \\
\hline & & 10.7 & 73.5 & 10.4 & 2 \\
\hline
\end{tabular}

Table 11.44. Overweight and obesity (\%) among girls (2004-2008) based on measurements [Lide, 2009]

\begin{tabular}{lllllll}
\hline Age & $\mathrm{n}$ & $\begin{array}{l}\text { IsoBMl } \\
<16.1 \mathrm{~kg} / \mathrm{m}^{2}\end{array}$ & $\begin{array}{l}\text { IsoBMl } \\
<18.5 \mathrm{~kg} / \mathrm{m}^{2}\end{array}$ & Normal & $\begin{array}{l}\text { IsoBMI } \\
>25 \mathrm{~kg} / \mathrm{m}^{2}\end{array}$ & $\begin{array}{l}\text { IsoBMI } \\
>30 \mathrm{~kg} / \mathrm{m}^{2}\end{array}$ \\
\hline 7 & 466 & 3 & 9.7 & 74.9 & 9.9 & 2.6 \\
\hline 8 & 550 & 4 & 10.2 & 71.6 & 11.3 & 2.9 \\
\hline 9 & 554 & 1.8 & 7.6 & 76.4 & 11.9 & 2.3 \\
\hline 10 & 594 & 2.5 & 11.1 & 72.4 & 10.6 & 3.4 \\
\hline 11 & 456 & 3.1 & 13.8 & 70.6 & 11.2 & 1.3 \\
\hline 13 & 372 & 4.6 & 15.3 & 67.7 & 10.5 & 1.9 \\
\hline 14 & 328 & 4.9 & 10.7 & 73.8 & 8.8 & 1.8 \\
\hline 15 & 281 & 4.6 & 10.3 & 75.3 & 9.7 & 1.7 \\
\hline 16 & 275 & 2.2 & 11.7 & 74 & 9.3 & 0.4 \\
\hline 17 & 128 & 1.6 & 9.8 & 78.5 & 9.1 & 0.4 \\
\hline Total & 5141 & 1.3 & 9.4 & 80.5 & 7.8 & 0.8 \\
\hline
\end{tabular}

BMI Classification according to Cole et al. [2000, 2007]. 
Table 11.45. Smoking trends among adult men and women: 1998-2006 (\%)

\begin{tabular}{|c|c|c|c|c|c|c|c|c|c|c|}
\hline & \multicolumn{2}{|l|}{1998} & \multicolumn{2}{|l|}{2000} & \multicolumn{2}{|l|}{2002} & \multicolumn{2}{|l|}{2004} & \multicolumn{2}{|l|}{2006} \\
\hline & men & women & men & women & men & women & men & women & men & women \\
\hline Daily smokers & 51.3 & 19.2 & 51.3 & 18.2 & 51.1 & 19.2 & 47.3 & 17.8 & 46.6 & 18.2 \\
\hline $\begin{array}{l}\text { Occasional } \\
\text { smokers }\end{array}$ & 6.0 & 4.9 & 4.9 & 5.2 & 4.4 & 6.4 & 5.7 & 5.9 & 4.0 & 5.5 \\
\hline $\begin{array}{l}\text { Quitter: } \\
1-12 \text { months }\end{array}$ & 2.4 & 1.6 & 2.9 & 1.7 & 2.5 & 1.9 & 3.1 & 1.6 & 3.4 & 1.9 \\
\hline $\begin{array}{l}\text { Quitter: } \\
\geq 1 \text { year }\end{array}$ & 10.4 & 3.7 & 10.7 & 4.5 & 11.4 & 4.0 & 13.4 & 4.4 & 12.8 & 7.5 \\
\hline Non-smokers & 23.8 & 64.9 & 26.4 & 67.5 & 27.7 & 66.9 & 29.6 & 69.5 & 29.3 & 64.0 \\
\hline $\begin{array}{l}\text { Incomplete } \\
\text { information }\end{array}$ & 6.1 & 5.7 & 3.8 & 2.9 & 2.9 & 1.6 & 0.9 & 0.8 & 4.0 & 2.9 \\
\hline
\end{tabular}

According to a statement from the 2nd International Congress on Physical Activity and Public Health (Amsterdam, April 13-16, 2008), there is no need to spend money on studies to assess the level of overweight and obesity; the problem exists. There is a need to conduct studies on which kind of intervention can help to motivate people and how to increase physical activity in the population. The authors of this report would like to add that we cannot forget those with underweight, and weight control has to cover public concern for those with underweight as well as overweight.

\section{Smoking}

Adults. The results from the 2006 FinBalt Health Monitoring Survey (see table 11.45) showed that $46.6 \%$ of men and $18.2 \%$ of women at age 15 to 64 were daily smokers [Pudule et al., 2007]. The percentage of daily smokers did not decrease in the period 1998-2002 (51\% of men and 19\% of women), but slowly decreased in the period between 2004 and 2006 (47\% in men and 18\% in women).

Children. The proportion of Latvian adolescents who smoke regularly (see table 11.46) increased, especially among girls the years 1991-2006 [Gobina et al., 2006]. $30 \%$ of boys and $23 \%$ of girls smoked regularly at the age of 15 in 2006.

\section{Physical Activity}

Adults. For substantial health benefits, adults should do at least 150 minutes of moderate-intense aerobic physical activity a week [Donnelly et al., 2009]. Women are less active compared to men. The level of physical activity is more than insufficient for both genders [Pudule et al., 2003, 2005, 2007] (see table 11.47). 
Table 11.46. Smoking trends among adolescent men and women: 1998-2006 (\%)

\begin{tabular}{|c|c|c|c|c|c|c|}
\hline \multirow[t]{2}{*}{ Years } & \multicolumn{3}{|l|}{ Men } & \multicolumn{3}{|l|}{ Women } \\
\hline & 11 years & 13 years & 15 years & 11 years & 13 years & 15 years \\
\hline 1991 & 4.8 & 9.3 & 18.5 & 1 & 1.3 & 5.3 \\
\hline 1994 & 1.5 & 12.4 & 32.7 & 0.6 & 2.6 & 13.3 \\
\hline 1998 & 3.1 & 15.1 & 36.4 & 0.3 & 6.9 & 18.8 \\
\hline 2002 & 1.8 & 16.4 & 28.9 & 0.5 & 5.9 & 21.1 \\
\hline 2006 & 2.7 & 11.2 & 29.9 & 1.1 & 10 & 22.7 \\
\hline
\end{tabular}

Children. Physical fitness can be measured more accurately than physical activity [Suni et al., 1996]. The Sports Medicine State Agency (SMVA) measured physical fitness of 2,188 school-aged children and adolescents in 2008 using a set of EUROFIT tests. $75.2 \%$ of children and adolescents at age 7 to 17 years showed unsatisfactory results at cardiorespiratory fitness (under the 40th percentile); 67.9\% of participants showed muscular endurance, and in $60.1 \%$ the muscular strength was unsatisfactory; flexibility was unsatisfactory in $61.7 \%$ of children and adolescents [Sports Medicine State Agency report to the Latvian Parliament, 2009].

\subsection{Lithuania ${ }^{14}$}

Since regaining independence in 1990, Lithuania has been undergoing substantial political, economic, and social changes. These changes have influenced people's lifestyles and health. The most remarkable changes have occurred in nutrition habits. Food globalization, aggressive advertising, and changes in food prices have had an impact on the diet of Lithuanians.

Nutrition plays a major role in the development of chronic non-communicable diseases that are widespread in Lithuania. Cardiovascular mortality in the country is considerably higher than in the old EU member states (744.9/100,000 among men and 419.6/100,000 among women in 2007). The incidence and mortality of some cancers related to diet are also high. In 2007, the incidence of all cancers among men was $578.1 / 100,000$ and among women 399.6/100,000. Cancer mortality in the same year was 305.6/100,000 among men and 133.3/100,000 among women. Type 2 diabetes is a growing health problem in Lithuania.

In 2003, the Lithuanian government approved the National Food and Nutrition Strategy and Action Plan for 2003-2010. Monitoring of diet and nutritional status of

\footnotetext{
${ }^{14}$ Petkeviciene J, Klumbiene J; Institute for Biomedical Research for Kaunas, University of Medicine, Kaunas, Lithuania. E-Mail janpet@vector.kmu.lt
} 
Table 11.47. Leisure-time physical activity of at least 30 min 4-6 times a week or more by sex and age (\%)

\begin{tabular}{|c|c|c|c|c|c|c|c|c|c|c|c|c|}
\hline \multirow[t]{3}{*}{ Year } & \multicolumn{6}{|l|}{ Men } & \multicolumn{6}{|l|}{ Women } \\
\hline & \multicolumn{5}{|c|}{ age groups } & \multirow[t]{2}{*}{ total } & \multicolumn{5}{|c|}{ age groups } & \multirow[t]{2}{*}{ total } \\
\hline & $15-24$ & $25-34$ & $35-44$ & $45-54$ & $55-64$ & & $15-24$ & $25-34$ & $35-44$ & $45-54$ & $55-64$ & \\
\hline 2006 & 29.4 & 17.3 & 20.6 & 18.6 & 17.6 & 20.8 & 18.2 & 12.3 & 14.2 & 13.3 & 14.2 & 14.5 \\
\hline 2004 & 34.2 & 16.8 & 19 & 18.3 & 27.1 & 23.9 & 16.2 & 14.1 & 10.4 & 13.2 & 19.4 & 14.7 \\
\hline 2002 & 34.4 & 18.9 & 20.5 & 23.8 & 15.8 & 23.3 & 15.8 & 12.2 & 12.8 & 10.5 & 15.4 & 13.4 \\
\hline
\end{tabular}

the Lithuanian population is one of the goals of the strategy. Some studies on food consumption and nutrient intake as well as nutrition status carried out in Lithuania are reviewed in the report.

\section{National Nutrition Survey}

National Nutrition surveys were conducted in 1997, 2002, and 2007. The aim of the surveys was to measure average food and nutrient intake in adults. For every survey, a national, random sample of 3,000 inhabitants aged 19 to 65 was taken from the National Population Register. Response rates were 72.7, 64.4, and 64.5\% respectively. For dietary assessment, the participants were interviewed at home using the 24-hour recall method. Height and weight of the respondents were measured. In 2007, the Lithuanian Food Database was used for the nutrient intake calculation.

According to the data of the 2007 survey, Lithuanian diet was characterized by high fat content. The proportion of fat in total energy intake was $44.9 \%$ in men and $41.9 \%$ in women. Also, the proportion of saturated fatty acids (SFA) was too high in both men and women (13.5\% of total energy and $12.9 \%$ of total energy, respectively), indicating a high consumption of fatty milk and meat products. Consequently, the content of cholesterol in the diet was higher than recommended (477.9 $\mathrm{mg}$ in men and $318.8 \mathrm{mg}$ in women). The proportion of polyunsaturated fatty acids (PUFA) corresponded with the requirements ( $8.8 \%$ of total energy). Vegetable oil was mostly used for cooking. The intake of carbohydrates was below the recommended level. Carbohydrates constituted only $38.9 \%$ of total energy in men and $42.9 \%$ in women. In contrast, the proportion of mono- and disaccharides was higher than recommended (10.8\% of total energy in men and $14 \%$ of total energy in women). The intake of dietary fiber was higher than the recommended level in men $(20.9 \mathrm{~g})$ rather than in women $(15.6 \mathrm{~g})$. Supply of protein was slightly higher than the recommendation (16.6\% of total energy).

Concerning most vitamins and minerals, the average supply was sufficient. The sodium intake was too high, especially in men ( 4.3 vs. $2.8 \mathrm{~g} /$ day in women) and might 
contribute to the high prevalence of hypertension in the country. The content of calcium was too low in the diet of both men and women $(812.3$ and $673.9 \mathrm{mg} /$ day, respectively). The mean daily intake of iron in women $(9.9 \mathrm{mg} /$ day $)$ as well as the intake of iodine in men $(67.3 \mu \mathrm{g} /$ day $)$ and women ( $48 \mu \mathrm{g} /$ day $)$ was below the recommended level. The content of folate was too low in the diet of men $(343.5 \mu \mathrm{g} /$ day $)$ and particularly of women $(237.9 \mu \mathrm{g} /$ day $)$. The supply of vitamin D was insufficient in the diet of women $(3.9 \mu \mathrm{g} /$ day $)$. Because of low calcium and vitamin D intake, a large proportion of Lithuanian women are at high risk of osteoporosis.

The prevalence of obesity (BMI $\geq 30 \mathrm{~kg} / \mathrm{m}^{2}$ ) was $11.3 \%$ among men and $15.2 \%$ among women. The proportion of overweight persons (BMI $\geq 25 \mathrm{~kg} / \mathrm{m}^{2}$ ) was 59.8 and $46.1 \%$, respectively.

\section{CINDI Program}

The Countrywide Integrated Non-Communicable Disease (CINDI) program aims to reduce mortality and morbidity from non-communicable chronic diseases by reducing common risk factors. In 1987, 1993, 1999, and 2007, CINDI cross-sectional random sample surveys were carried out in five regions of Lithuania. Almost 8,000 men and women aged 25 to 64 participated in the surveys. 24-hour recalls and food frequency questionnaires were used for dietary assessment. Height, weight, waist circumference, and level of serum lipids were measured.

Over the period of 20 years, the diet of Lithuanians has shown some positive trends. The intake of animal fat decreased. In 1987, the majority of the population (91\%) spread butter on bread. In 2007, only $30 \%$ of the respondents stated that they used butter. Animal fat (lard and butter) was replaced by vegetable oil in cooking. The proportion of respondents using fresh vegetables at least on 3 days in a week increased, especially among women. However, the intake of fat, especially saturated fat and cholesterol, is still too high.

Since 1987, the prevalence of hypercholesterolemia ( $>5 \mathrm{mmol} / \mathrm{l}$ ) decreased from 85 to $52 \%$ in men and from 83 to $51 \%$ in women. In 2007 , the mean value of total cholesterol was $5.4 \mathrm{mmol} / \mathrm{l}$ in men and $5.1 \mathrm{mmol} / \mathrm{l}$ in women. The proportion of men with a high level of low density lipoprotein cholesterol $(>3 \mathrm{mmol} / \mathrm{l})$ decreased from 88 to $53 \%$, the same proportion of women from 86 to $48 \%$. The most remarkable decrease in the prevalence of lipids disorders was observed in the youngest age group (25 to 34 years). The prevalence of overweight (BMI $\geq 25 \mathrm{~kg} / \mathrm{m}^{2}$ ) decreased among both men and women.

\section{Health Behavior Survey among Lithuanian Adult Population}

Since 1994, Lithuania - together with Finland, Estonia, and Latvia - has been participating in the international FinBalt Health Monitor project. The aim of this study is to assess health behavior, including nutrition habits, of different sociodemographic groups and to monitor time trends in health behavior. In 1994-2008, eight cross-sectional surveys were conducted among the population aged 20 to 64 . An independent 
national random sample of 3,000 inhabitants of Lithuania was drawn from the National Population Register for every survey. The data were collected through mailed questionnaires covering sociodemographic characteristics, health behaviors, and selfreported height and weight. Food frequency questionnaires were used for assessment of nutrition habits. The response rate varied from 59.2 to $74.4 \%$. Altogether, 15,161 respondents participated in the surveys.

Over a period of 14 years, the diet of Lithuanians has shown some positive trends. The usage of vegetable oil for cooking increased considerably from 31\% in 1994 to $85 \%$ in 2008 among men, and from 48 to $94 \%$ among women. The intake of animal fat decreased. In 1994, 71\% of men and $66 \%$ of women reported the usage of butter on bread. In the year 2008, the proportion of men spreading butter on bread was $34 \%$, the proportion of women $36 \%$. The consumption of high-fat milk decreased over the observational period. The survey data show that it was consumed by $35 \%$ of men and $28 \%$ of women, while in the year 1994 almost a half of the respondents (47\%) reported whole milk consumption. The frequency of fresh vegetables and fruits consumption increased. In 1996, $18 \%$ of men and $25 \%$ of women consumed fresh vegetables on at least 3 days during the week before questioning while in the year 2008 this proportion was $63 \%$ in men and $68 \%$ in women. In 2008 , fresh fruits and berries were eaten at least 3 days a week by $38 \%$ of men and $54 \%$ of women. Highly educated people and those living in urban areas had a healthier diet compared to persons with lower education and the rural population.

In 2008 , the prevalence of obesity (BMI $\geq 30 \mathrm{~kg} / \mathrm{m}^{2}$ ) calculated using self-reported data was $16.9 \%$ among men and $19.7 \%$ among women. The proportion of overweight persons (BMI $\geq 25 \mathrm{~kg} / \mathrm{m}^{2}$ ) was 61.4 and $46.1 \%$, respectively. Since 1994, both indicators have increased in men and have not changed in women. Overweight was inversely related to educational achievement in women.

\section{Health Behavior Survey in School-Aged Children}

The Health Behavior Survey in Lithuanian school-aged children collects information about some nutrition habits and self-reported weight and height. This study is carried out within the international HBSC study. Lithuania joined this project in 1994. Four surveys in 4-year intervals were conducted (the latest survey in 2006). The target population is schoolchildren 11, 13, and 15 years of age. Clustered sampling design was used. The sampling unit was the class. About 6,000 children were selected for every study. Response rate was about $95 \%$. The food frequency questionnaire was used for assessment of nutrition habits. Self-reported weight and height were used for calculation of BMI. Age- and gender-specific BMI international cut-off points recommended by the International Obesity Task Force [Cole et al., 2000] were used to calculate the prevalence of overweight and obesity.

In 2006, the proportion of children eating breakfast every day was the highest among 11-year-old girls (70\%) and boys (68\%). It decreased among teenagers, especially among girls (to $51 \%$ ). Daily fruit and vegetable consumption was more often 
reported by girls than boys. The proportion of girls consuming vegetables daily was $27 \%$, that of boys $22 \%$. Fruits were eaten daily by $26 \%$ of girls and $21 \%$ of boys. The rates declined with age. The proportion of girls drinking coke or other soft drinks every day was $13 \%$, that of boys $16 \%$.

The prevalence of overweight and obesity was one of the lowest among all countries participating in the HBSC survey. Only $4.5 \%$ of girls and $10.5 \%$ of boys were overweight. The proportion of obese girls was $0.5-0.7 \%$ and the proportion of obese boys $0.8-1.3 \%$. The highest prevalence of overweight was in 11-year-old children.

\section{Childhood Obesity Surveillance}

In 2008, Lithuania joined the WHO European Childhood obesity surveillance initiative. A cross-sectional survey was conducted in 156 randomly selected Lithuanian schools. Weight and height of 4,902 first-grade schoolchildren aged 7 to 8 years were measured, using electronic scales. BMI international cut-off points [Cole et al., 2000] were used for evaluation of body weight. The proportion of overweight children was $11.6 \%$, the prevalence of obesity $5.7 \%$. No differences were found between girls and boys. The proportion of underweight boys was $6.7 \%$ and that of girls $10.2 \%$.

\section{Anthropometry of Children and Adolescents}

In 2000-2002, more than 9,000 schoolchildren, 7 to 18 years old, participated in a crosssectional survey that was carried out in the five largest cities of Lithuania: Vilnius (the capital), Kaunas, Klaipeda, Siauliai, and Panevezys. The prevalence of overweight was estimated using BMI international cut-off points [Cole et al., 2000]. Compared to older adolescents, the prevalence of overweight was lower among younger schoolchildren. The proportion of overweight girls varied from 1.5 to $10.5 \%$, that of boys from 2.6 to $9.9 \%$. The prevalence of obesity varied from 0 to $2.9 \%$ in girls and from 0 to $4.4 \%$ in boys. Overweight was more common among older boys if compared with older girls.

\section{Conclusions}

In Lithuania, the majority of dietary surveys were carried out in the adult population. Dietary habits, nutrient content of diet, data on height and weight, as well as the trends of those indicators over the past decade are available at a national level. Since 1990, the nutritional habits of the Lithuanian adult population have changed considerably. The diet of Lithuanians tends to get closer to the recommendations of healthy nutrition because of a decrease in animal fat consumption and an increase in vegetable fat as well as vegetable and fruit consumption. However, high intake of fat, especially saturated, as well as sugars, low consumption of vegetables and fruits still are the main problems of Lithuanian diet. Consequently, the prevalence of overweight and lipid disorders is high in Lithuanian population. Unhealthy diet, high prevalence of diet related as well as other risk factors (smoking, low physical activity) might be responsible for high morbidity and mortality from cardiovascular diseases, cancers, and other chronic non-communicable diseases in the country. 
The dietary data on children and adolescents as well as the elderly are very limited. Data are not available for macronutrients, micronutrients, and energy intake. The prevalence of overweight and obesity among children and adolescents is not very high in Lithuania; however, the rates are increasing, especially among young children.

In 2005, dietary guidelines for the Lithuanian population were issued by the Ministry of Health. Those guidelines are focused on the limitation of the consumption of fat, especially animal fat, free sugars, and salt; promotion of vegetables, fruits, and whole grain products consumption; balanced energy intake and increase of physical activity in order to decrease the prevalence of obesity. The promotion of healthy nutrition through the life cycle as well as ensuring healthy and sustainable food supply for all population groups are very important for successful implementation of the Lithuanian Food and Nutrition Strategy and Action Plan for 2003-2010.

\subsection{Norway ${ }^{15}$}

\section{Dietary Surveys in Norway}

National dietary surveys in Norway were carried out among adults (16 to 79 years of age) in 1997 and among children and adolescents (4, 9 and 13 years of age) in 2000-2001. Moreover, national dietary surveys were conducted among 6-month-olds in 2006 and among 12-month and 2-year-olds in 2007. The national dietary surveys were carried out in collaboration between the Norwegian Directorate of Health, the Norwegian Food Safety Authority and the University of Oslo, Department of Nutrition. A new national dietary survey among adults is planned for 2009/2010.

\section{Children 4 Years and at 4th and 8th Grade of School (9 and 13 Years)}

\section{Subjects}

Four-year-olds were recruited to participate in the national dietary survey through child health centers. Statistics Norway established a sample of 11 urban and 22 rural municipalities. Child health centers in these municipalities were invited to participate. A total of 38 child health centers from 9 urban and 18 rural municipalities decided to participate in the study. The child health centers included in the analysis were 25 from rural and 13 from urban municipalities. Invitation letters were sent to 746 4-year-olds; of these, 126 children did not meet at the health control at the child health center. Information about the survey was given to 511 parents of 4 -year-olds, whereof 394 completed the survey. Three children were excluded, 2 because they were

15 Totland TH, Andersen LF; Universitetet i Oslo, Norway. E-Mail l.f.andersen@medisin.uio.no 
ill during recording and 1 because the diaries were not filled in correctly. The final sample consisted of 3914 -year-olds, which represents a participation rate of 52\%.

For 4th and 8th graders, a random, nationally representative sample of 106 schools ( 53 primary and 53 secondary schools) from 53 municipalities was drawn by Statistics Norway to participate in the national dietary survey. Nine schools refused to participate, and seven randomly chosen schools in the same municipality were included. One school completed their recording too late to be included in the survey. In total, 103 of 106 schools and 1,835 of 2,214 invited students responded, which represents a participation rate of $83 \%$. A total of 20 students were excluded, 14 because they only recorded 3 days and 6 because they were 3rd graders. The final sample consisted of 1,815 children, 810 in the 4 th grade ( 9 years old) and 1,005 in the 8 th grade (13 years old), with a participation rate of 81 and $84 \%$ for the 4 th and 8 th grade, respectively.

The regional ethical committee approved the study, and informed consent was obtained from the parents [Øverby et al., 2003].

\section{Design}

Data collection among the 4-year-olds was carried out from August to the first week of December 2001. The child health centers included an invitation letter to the present survey in the invitation for the general health control at 4 years of age. Parents who wanted their child to participate returned a short questionnaire and a signed informed consent at the child health center when they came for health control, and they met a nutritionist for instructions on how the diet should be recorded for 4 days. The parents of the participants were phoned by a nutritionist on the second day of their recording period, to encourage and answer questions. Participating parents could call the nutritionists at any time with questions [Øverby et al., 2003].

Data collection among students in the 4th and 8th grades was carried out from September to the first week of December 2000. The students received an invitation letter and a short frequency questionnaire at school approximately 2 weeks before food recording started. Children and parents returned the questionnaire and the informed consent to the University of Oslo. A nutritionist visited each school and gave oral and written instructions to the pupils on how the diet should be recorded. The participants received four food diaries, a booklet regarding portion sizes and written instructions [Øverby et al., 2003].

\section{Food Diary}

The parents of the 4-year-olds and students in the 4th grade recorded the participating child's entire food intake for 4 consecutive days. Participants in the 8th grade recorded their diet for 4 consecutive days themselves. The participants recorded their entire food intake in an 18-page precoded food diary. The 4-day period consisted of 3 weekdays and 1 weekend day.

The diary had lists of 277 drinks, food items and dishes grouped together according to a typical Norwegian diet. Each food group was supplemented with open-ended 
alternatives. The design of the precoded food diary was similar to a cross-table, with food listed on the left and time span on top. Food amounts were presented in predefined household units (e.g. beverage is recorded in glasses, bread in slices) or as portions estimated from photographs. Along with the diary, each participant received a photographic booklet which embodied 13 color photograph series, each with four different portion sizes ranging from small to large. The participants indicated an eating event by filling in how many units they had eaten of each food item in the correct time span. The day was divided into five time spans (from 06:00 to 10:00 h, from 10:00 to 14:00 h, etc.) [Øverby et al., 2003]. The photographic booklet has been evaluated and the results are published elsewhere [Lillegaard et al., 2005]. The food diary has also been validated among 9- and 13-year-olds [Lillegaard et al., 2007; Andersen et al., 2005; Lillegaard and Andersen, 2005].

\section{Calculations of Dietary Intake}

Data were entered by scanning, using the Teleform program (6.0) (Datascan, Oslo, Norway). Daily intakes of foods, energy and nutrients were computed using a food database and software system developed at the Department of Nutrition, University of Oslo. The database is mainly based on the official food composition table, and is continuously supplemented with data on new food items and nutrients. Corrections for losses in cooking are done when calculating nutrient contents [ $\varnothing$ verby et al., 2003].

\section{Short Questionnaire}

A short questionnaire containing questions about height, weight, physical activity, time spent watching television (TV)/using a personal computer (PC), and parents' education were filled in. Data on weight and height were given by $74 \%$ of the 4 -yearolds and $664(74 \%)$ and $825(82 \%)$ of the 4th and 8th graders included in the survey, respectively [Øverby et al., 2003].

\section{Adults (16-79 Years)}

The second national dietary survey among adults (Norkost 2) was carried out in 1997, based on a self-administered quantitative food frequency questionnaire (QFFQ). Distribution and collection of the dietary questionnaires were coordinated with Statistics Norway's Omnibus Survey performed during March, June, September and November of 1997 [Johansson and Solvoll, 1997].

\section{Subjects}

A nationwide, representative and randomly selected sample of 2,500 Norwegians aged 16 to 79 years of age was drawn to participate in each Omnibus survey. A random half of each Omnibus sample (some 1,250 subjects in each period) was drawn to participate in the dietary survey. The distribution of subjects in different groups of socioeconomic status and location of residence was similar in Norkost 2 compared with the general population. However, the amount of subjects with higher level of education 
was somewhat overrepresented and the amount of subjects with shorter duration of schooling and the amount of subjects living in cities was somewhat underrepresented, compared with the general population.

Of the original 5,000 subjects drawn to participate in the survey, 40 had died or emigrated and were excluded from the sample. Of the reminding 4,960 subjects, a total of 2,803 returned their QFFQ. The completed forms were checked before optical mark reading. Double marks for frequency, as well as portion size, were corrected to the highest alternative of adjacent marks or to the mean of the two marks. A total of 131 forms were rejected, mainly because (a) the whole questionnaire or at least two pages were blank, (b) the questionnaire was impossible to read, or (c) identification was insufficient. Rejected forms were included in non-respondents and information from 2,672 questionnaires (54\%) was used in our analysis. There were only small differences between respondents and the total random sample regarding sex, age group, regions of Norway and education level.

For each round of the Omnibus survey, the Norwegian Data Inspectorate was notified according to standard procedures [Johansson and Solvoll, 1997].

\section{Design}

The QFFQ was mailed to the subjects together with information letters about the Omnibus and Norkost 2 surveys. Based on experiences from the Norkost 1 survey respondents were offered to participate in a lottery of four cheques for USD 150-700. After 1-3 weeks the QFFQ was collected by personnel from Statistics Norway during a visit in the home of each subject in connection with the Omnibus survey. The last round of the Omnibus survey was mainly carried out by interviewing by phone. In these cases the subjects received a return envelope with their questionnaire, to return their QFFQ by mail. Non-respondents received one reminder by mail after 4 weeks.

The questionnaire included a short written instruction and the subjects received no further guidance. On average it took about 1 hour to complete the QFFQ [Johansson and Solvoll, 1997].

\section{Questionnaire}

A self-administered, optical mark readable QFFQ was used in the survey. The questionnaire was designed to cover the whole diet and included about 180 food items grouped according to a Norwegian meal pattern. The QFFQ was phrased to obtain information about the 'usual' food intake during the last year. The frequency of consumption was given per day, per week or per month depending on the food item. The portion sizes were units such as slices, glasses, cups, pieces, deciliters and spoons. The portion sizes of the different food items were converted to weights on the basis of standard portions estimated from previous Norwegian dietary studies.

Questions about weight, height, physical activity, smoking habits, meal patterns and attitudes towards diet and body weight were included. Statistics Norway provided 
information about the subject's level of education and several other demographic and geographical variables from their registers.

The questionnaire asked about the frequency use of and portion size of 7 kinds of dietary supplements (cod liver oil, cod liver oil capsules, fish oil capsules, multivita$\mathrm{min} /$ mineral mixtures, vitamin $\mathrm{C}$, vitamin $\mathrm{E}$ and iron supplements). Use of cod liver oil has traditionally been recommended during the dark period of the year. Thus, for cod liver oil and cod liver oil capsules, the questionnaire differentiated between the whole year and use during winter only. Reported frequency for use during winter was divided by a factor of 2 [Johansson et al., 1997].

\section{Calculations of Dietary Intake}

Software systems for optical mark reading were developed in collaboration with the Norwegian Government Computer Centre. The daily intake of energy and nutrients was computed using a food composition database [AKF, 1995] and software developed at the Institute for Nutrition Research (now Department of Nutrition) at the University of Oslo. Calculations of fatty acids were based on analytical data from a composition of margarine program in 1997 [Johansson and Solvoll, 1997].

\section{Intake of Selected Nutrients and Food Groups in Norway}

Intake of Fruit and Vegetables among Norwegian Children and Adolescents

The intake of fruit and vegetables was on average 225-250 g/day among the 4-yearolds, 4th and 8th graders participating in the national dietary survey among children and adolescents in Norway (2000). The survey further showed a positively association between fruit and vegetable consumption and the intake of dietary fiber, vitamins and minerals, especially vitamin C. A negative association was found between fruit and vegetable consumption and the intake of added sugar and saturated fatty acids.

The amount of fruit and vegetable consumption increased with age, as well as the percentage of children fulfilling the recommended intake of $500 \mathrm{~g} /$ day which was 5 , 11 and 19\% respectively. Hence, the data showed that Norwegian children and adolescents consume less than half the amount of fruit and vegetables recommended by the government [Andersen et al., 2004].

\section{Intake of Sugar among Norwegian Children and Adolescents}

The national dietary survey of children and adolescents (2000) showed an increased intake of energy from added sugar from 4-year-old children to students in the 8th grade $(\mathrm{p}<0.001)$. On average, 4 -year-olds and students in the 4 th and 8 th grades had a mean added sugar intake of 55, 80 and $95 \mathrm{~g}$ /day respectively, accounting for 15.1, 16.8 and $18.4 \%$ of energy intake [Øverby et al., 2003].

The intakes of all nutrients, except $\alpha$-tocopherol among 4 -year-olds and vitamin $\mathrm{C}$ among 4-year-olds and 4th grades, decreased with increasing content of added sugar 
Table 11.48. Intake of cod liver oil (CLO) and cod liver oil capsules (CLC) among Norwegian children measured in percentage (\%) of times per week or day

\begin{tabular}{|c|c|c|c|c|c|c|c|c|}
\hline Age group & $\begin{array}{l}\text { Toddlers } \\
2 \text { years }\end{array}$ & & $\begin{array}{l}\text { Children } \\
4 \text { years }\end{array}$ & & $\begin{array}{l}\text { Children } \\
9 \text { years }\end{array}$ & & $\begin{array}{l}\text { Adolescents } \\
13 \text { years }\end{array}$ & \\
\hline Subjects, n & 1,674 & & 391 & & 810 & & 1,005 & \\
\hline Product & CLO & $\mathrm{CLC}$ & $\mathrm{CLO}$ & CLC & CLO & $\mathrm{CLC}$ & $\mathrm{CLO}$ & CLC \\
\hline Do not use & 62 & - & 73 & 99 & 81 & 94 & 92 & 90 \\
\hline 1-2 times/week & $7^{a}$ & - & 7 & 1 & 4 & 1 & 3 & 3 \\
\hline 3-4 times/week & - & - & 4 & 0 & 4 & 2 & 1 & 2 \\
\hline 5-6 times/week & $11^{b}$ & - & 7 & 0 & 5 & 1 & 2 & 2 \\
\hline Every day & 20 & - & 9 & 0 & 6 & 2 & 2 & 3 \\
\hline$>1$ times/day & 0 & - & 0 & 0 & n.a. & n.a. & n.a. & n.a. \\
\hline
\end{tabular}

n.a. $=$ No data available.

a 1-3 times/week. ${ }^{\text {b }}$ 4-6 times/week.

in the diet. Among 4-year-olds with low added sugar intake, a higher percentage of mothers had long education (61\%). Moreover, high consumers of added sugar had $30-40 \%$ lower intake of fruit and vegetables than did low consumers [Øverby et al., 2003].

A negative association was observed between consumption of added sugar and BMI among girls in the 8 th grade $(\mathrm{p}=0.0013)$, whereas a positive association was observed among 4-year-old boys ( $\mathrm{p}=0.055)$ [Øverby et al., 2003].

\section{Intake of Cod Liver Oil and Vitamin D among Norwegians}

Cod liver oil is recommended as a supplement for toddlers in Norway (0-2 years) because of its contribution of vitamin D. Groups of the Norwegian population are partly deficient in vitamin D during the dark period of the year. Increased intake of fatty fish or supplementation of cod liver oil is therefore recommended during winter months for the whole population. Table 11.48 shows the intake of cod liver oil among children in Norway (2000-2007).

Data from 2007 showed that $38 \%$ of the 2 -year-olds use cod liver oil as a supplement. The frequency of users declined as the children get older. $27 \%$ of the 4 -yearolds reported the use of cod liver oil in comparison to $8 \%$ of the 13 -year-olds. Cod liver oil consumers tended to change their supplement to cod liver oil capsules when they get older as $1 \%$ of the 4 -year-olds and $10 \%$ of the 13 -year-olds reported the use of cod liver oil capsules. 
Table 11.49. Average vitamin $D$ intake among Norwegian children measured with and without supplements ${ }^{1}$ and with the intake of cod liver oil supplements (CLO) only

\begin{tabular}{|c|c|c|c|c|}
\hline $\begin{array}{l}\text { Age group } \\
\text { measurements }\end{array}$ & $\begin{array}{l}\text { Subjects } \\
n\end{array}$ & $\begin{array}{l}\text { Vitamin D intake } \\
\text { without } \\
\text { supplements } \\
\mu \mathrm{g} / \text { day (SD) }\end{array}$ & $\begin{array}{l}\text { Vitamin D intake } \\
\text { including CLO } \\
\mu \mathrm{g} / \text { day (SD) }\end{array}$ & $\begin{array}{l}\text { Vitamin D intake } \\
\text { with all } \\
\text { supplements } \\
\mu \mathrm{g} / \text { day (SD) }\end{array}$ \\
\hline Toddlers 2 years & 1674 & $2.8(1.8)$ & $6.1(5.4)$ & $7.6(6.1)$ \\
\hline Children 4 years & 391 & $2.6(1.9)$ & $4.9(4.5)$ & $6.9(5.4)$ \\
\hline Children 9 years & 810 & $2.8(2.3)$ & $4.3(4.1)$ & $5.7(5.0)$ \\
\hline Adolescents 13 years & 1005 & $2.5(2.7)$ & $3.4(3.9)$ & $4.2(5.0)$ \\
\hline
\end{tabular}

${ }^{1}$ Supplements of vitamin D including cod liver oil, other fish oils, all multivitamin and mineral supplements.

The influence of cod liver oil and other supplements on children's average vitamin D intake in Norway is shown in table 11.49. In Norway the daily recommended intake of vitamin $\mathrm{D}$ is $7.5 \mu \mathrm{g}$ among 2- to 60-year-olds [Nordic Nutrition Recommendations, 2004]. The average intake of vitamin $D$ without including supplementation was not sufficient among Norwegian 2-, 4-, 9- and 13-year-olds, the average intake without supplementation was $2.8,2.6,2.8$ and $2.5 \mu \mathrm{g}$ /day respectively in the different age groups. Cod liver oil was the main contributor to vitamin D intake from supplements in all age groups shown. The 2-year-olds had the largest proportion of cod liver oil consumers, and were the only age group that meets the nutrition recommendations when supplements are included.

\subsection{Poland ${ }^{16}$}

The data from Poland on energy and nutrient intake was prepared with the use of two main sources of information. The first one - 'Food consumption and anthropometric survey 2000' - was based on data collected from randomly selected individuals reflecting the demographic characteristics of the whole population. The second survey - 'Social stratification in food consumption' - focused on low-income consumers. In both cases the data was collected with the use of 24-hour recall method. To assure the best practice of the interviews, a photographic album of food products and dishes was used to help respondents give a precise description of the amount of food consumed. Additional data concerning the nutritional and health status were introduced in the

\footnotetext{
${ }^{16}$ Halicka E, Rejman K, Kowrygo B; Warsaw University of Life Sciences WULS-SGGW, Warsaw, Poland.
} E-Mail ewa_halicka@sggw.pl 
report from the National Multicenter Health Survey [WOBASZ, 2003-2005] and Health Behaviour in School-Aged Children [HBSC, 2006] WHO collaborative study.

Polish Food Consumption and Anthropometric Survey 2000

\section{Methodology}

The institution responsible for the 'Food consumption and anthropometric survey project' carried out in Poland in 2000 with the FAO's financial support was the National Food and Nutrition Institute (IŻŻ). In cooperation with the Polish Central Statistical Office, a sample of 1,362 households was selected [Szponar et al., 2003]. Out of 4,310 members of these households, 4,200 people took part in 24-hour recalls on individual food consumption and anthropometric measurements. Due to administrative mistakes, inadequate age of individuals (under the age of 1 ) and overestimation of intake levels, ultimately 4,134 individual diets were analyzed and the nutritional status of 4,153 persons based on anthropometric measurements was assessed. The information gathered included data on the location and time of meal eaten (also purchased and consumed away from home), meal composition and its recipe.

\section{Survey Results}

The randomly collected data showed that the diet composition of a considerable part of the Polish population was highly unsatisfactory in many aspects, but mainly due to the fact that it was imbalanced [Szponar et al., 2003]. In the light of this study the average energy content of diet was calculated at 2,427 kcal (10.15 MJ). The reported daily energy intake of surveyed individuals was either complied with (1- to 9-yearold children and women 16 and older) or exceeded national recommendations. The highest energy value characterized the diets of 19- to 25-year-old males, and on average it was $3,657 \mathrm{kcal}(15.30 \mathrm{MJ})$. According to the data the daily energy intake of $20 \%$ of men in this age group surpassed 4,495 kcal. Overall the average level of energy content in the male population diets reached $2,919 \mathrm{kcal}(12.21 \mathrm{MJ})$ and more than $70 \%$ of the surveyed men had a higher than recommended energy value of diet. In the female subpopulation the average daily energy intake was 2,003 kcal (8.38 MJ). In the majority of the sample a high percent of energy from fat (36-37\% adult males, $33-35 \%$ adult females) was reported.

The daily protein content of individual diets was also assessed as relatively high, especially of older boys and adult males. It was shown that in all studied age groups the daily protein intake was higher in the case of males (average $60 \mathrm{~g} /$ day), while protein deficiencies most frequently occurred in diets of $15+$ girls. Additionally the study demonstrated that in the adult female subpopulation protein intake depended on the place of living and it was on average $3 \mathrm{~g} /$ day higher among women living in urban households. In general, dietary protein was predominantly (two thirds) of animal origin in the surveyed Polish population. 
The average daily fat intake was $100 \mathrm{~g} /$ person $(121 \mathrm{~g} / \mathrm{male}$ and $80 \mathrm{~g} / \mathrm{female})$ and reached its highest level in the group of young men. Fat content of studied diets was estimated as too high (with the exception of 1- to 9-year-olds), especially in youth and adult male subpopulations. The fatty acid composition of the diets was disadvantageous due to too high share of saturated fats providing $12 \%$ of energy on average. Polyunsaturated fats provided above $5 \%$ of energy, the average P:S ratio was 0.53 . The recorded average daily cholesterol level in the diets of males was $489 \mathrm{mg}$, compared to $395 \mathrm{mg}$ in diets of females.

In contrast, data on carbohydrate content of individual diets indicated that in many age groups it was quite low, especially in adolescent boys. Statistically higher levels of total carbohydrates and dietary fiber were noted in diets of individuals living in rural areas. Data also proved that a relatively low percentage of energy was derived from carbohydrates (for example in adult males less than 50\%). Dietary fiber intake was assessed as low, on average $22.4 \mathrm{~g} /$ person, slightly higher in the case of males.

The food consumption survey carried out in 2000 by IZZZ was also an important source of information on the content of minerals (sodium, potassium, calcium, phosphorus, magnesium, iron, zinc, copper, and manganese) and vitamins $\left(\mathrm{A}, \mathrm{E}, \mathrm{B}_{1}, \mathrm{~B}_{2}\right.$, $\mathrm{B}_{6}$, Niacin and C). Szponar et al. [2003] concluded that in the case of most minerals and vitamins the reported intake was not conform with recommendations. Excessive intake of vitamin A, sodium, potassium and phosphorus was frequently found while too low levels of niacin, calcium, zinc, copper as well as thiamine and iron (in female population) were also reported. Additionally, a very high variability of vitamin $\mathrm{C}$ intake was noticed.

Special attention should be drawn to excessive sodium intake which was shown in $99 \%$ of reported diets. The average content of sodium in daily diet was $4,170 \mathrm{mg}$ in the sample, slightly higher in males (5,183 mg), and the highest in men aged 19-25 (6,472 $\mathrm{mg})$. It was also noticed that the dietary sodium intake was lower in the urban population compared to consumers living in rural areas (4,119 and 4,252 $\mathrm{mg}$, respectively).

The assessment of the nutritional status of a representative sample of children and adults was also conducted in the 2000 Polish study. In the case of the children's sample, which comprised 1,216 individuals (609 boys and 607 girls), the National Research Institute of Mother and Child percentile charts of body weight to height were applied. About $75 \%$ of the children had adequate body weight (table 11.50). Overweight was identified in $8 \%$ of the surveyed boys and $7.2 \%$ of girls, while obesity in $4.6 \%$ in both groups. Underweight was recorded in $14 \%$ of girls and $12 \%$ of boys, however declined with age, accounting for only $6-7 \%$ in older children.

The presented results are similar to data collected during the WHO Health Behaviour in School-Aged Children (HBSC) study, conducted in Poland in 20052006 with the use of the questionnaire method among 2,287 adolescents. According to HBSC calculations based on self-reports, $11 \%$ of 15 -year-olds were overweight and obese [Oblacińska, 2006]. Statistically a larger number of boys were overweight (12.9\%) in comparison to girls (8.7\%). Underweight concerned $4.4 \%$ of 15 -year-olds 
Table 11.50. The share (\%) of undernutrition and excess weight in studied population samples, according to gender [source: Szponar et al., 2003]

\begin{tabular}{lllll}
\hline & Boys & Girls & Males & Females \\
\hline Underweight & 11.8 & 14.2 & 1.0 & 3.3 \\
\hline Body weight adequate to height $^{\mathrm{a}}$ & 75.5 & 74.0 & 42.3 & 48.2 \\
\hline Overweight $^{\mathrm{b}}$ & 8.0 & 7.2 & 41.0 & 28.7 \\
\hline Obesity & 4.6 & 4.6 & 15.7 & 19.9 \\
\hline
\end{tabular}

a In case of children: slimness, body weight adequate to height and overweight tendency.

b $90-97$ th percentile.

and was more common among girls (5.0\%) than boys (3.7\%). In 2005, 'Keep Fit', a nationwide educational program, was launched and aimed at promoting balanced diet principles and physical activity among secondary school students [Halicka et al., 2008].

In reference to table 11.50 , the WOBASZ adult sample, consisting of 2,825 people, including 1,255 men, measurements showed that in general $56.7 \%$ of men and $48.6 \%$ of women were overweight or obese. The male population was characterized by higher risk of overweight ( $41 \%$ compared to $29 \%$ ), however more women were obese. It must be emphasized that in the light of earlier representative nationwide surveys and the POL-MONICA project, the frequency of overweight and obesity in both male and female populations in Poland had increased markedly in the 1990s.

Another source of information on the nutritional and health status of the Polish population based on individual data was the WOBASZ survey which was conducted by five Polish research institutions in the years 2003-2005 and coordinated by the Institute of Cardiology in Warsaw. For the purpose of the research, a sample of 13,545 adults (20 to 74 years old) was randomly selected and individual interviews were carried out. Food frequency questionnaires and a 24 -hour recall method were utilized as basic tools for evaluating intake [Rywik et al., 2005].

Nutritional data from 6,806 adults (including 3,215 males) showed that the average energy content of daily diet was $2,473 \mathrm{kcal}$ in males and 1,681 kcal in females [Waśkiewicz et al., 2005]. Fats were the source of $37 \%$ of energy of diet in the case of males and $35 \%$ in females. Anthropometric measurements showed that the prevalence of overweight among 20- to 74-year-old men in Poland was higher comparing to the female subpopulation and reached $40 \%$ (almost $28 \%$ among women), while the percentage of obese persons was similar for both genders and amounted to 22\% (table 11.51) [Biela et al., 2005]. BMI calculations were made with the use of data from 13,408 individuals, including 6,320 men and 7,088 women. 
Table 11.51. BMI groups in WOBASZ study (\%) [source: Biela et al., 2005]

\begin{tabular}{lll}
\hline & Males & Females \\
\hline Body weight adequate to height $\mathrm{BMI}<25$ & 38.4 & 49.7 \\
\hline Overweight $25 \leq \mathrm{BMI}<30$ & 40.4 & 27.9 \\
\hline Obesity $30 \leq \mathrm{BMI}$ & 21.2 & 22.4 \\
\hline
\end{tabular}

\section{Low-Income Consumers Study}

\section{Methodology}

Food consumption survey and nutritional evaluation of the daily diet of low-income consumers in Poland was a part of a project entitled 'Social stratification in food consumption in Poland and Belgium' carried out at University of Gent and Warsaw University of Life Sciences during the period 2004-2006 in the frame of bilateral scientific and technological cooperation with Flanders.

The Polish research based on interviews with 215 low-income respondents, was conducted in a medium-size city of central Poland (38,000 inhabitants) during the first quarter of 2005. In order to select the target group of consumers, the city was chosen taking into consideration high indices of unemployment and decreasing standard of living due to economic transition. The sample selection factor was the monthly income per person which varied depending on household size. For a single person, the income limit was set at 1,100 PLN per month $(1 \mathrm{EUR}=$ ca. 4 PLN $)$ and for the households with 2 or more persons the limit was $800 \mathrm{PLN} /$ person. The studied group of low-income consumers consisted mainly of adults $\leq 50$ years of age (32\% of 18 to 35 year olds, $47 \%$ of 36 to 50 year olds) with a medium (54\%), vocational or elementary (22\%) level of education and one fourth of the group with a higher/ university degree. In the sample, $83 \%$ were women and $17 \%$ were men and they lived mostly (58\%) in 3- to 4-person households [Rejman, 2007].

\section{Survey Results}

The economical factors, especially household's income and the cost of food, are the decisive factors determining the access to food products and possibility of purchase for low-income consumers, implying a greater tendency to consume unbalanced diets resulting in undernutrition (micronutrient deficiency) or overnutrition (overweight and obesity). An increasing number of studies show that the poor in affluent societies do not suffer from dramatic energy deficiencies, in fact high-energy density of the diet and food poverty are more common showing that overweight and obesity are the socioeconomic issue. 
The average daily diet of the low-income respondent consisted of $1,944 \mathrm{kcal}$, coming from $66 \mathrm{~g}$ protein, $73 \mathrm{~g}$ fat and $269 \mathrm{~g}$ carbohydrates. High standard deviation noticed for most of the nutrients and energy value of the diet indicated big differences among the consumers. Energy intake on average was below the recommendation $(82 \%)$, however the share of total fat and saturated fat in energy supply exceeded the WHO recommendations (it was 31.9 and $12.2 \%$, respectively). The average daily diet showed deficiency of minerals: copper (48\% of RDA), calcium (71\%), iron (79\%), zinc (84\%), potassium (82\%) and vitamins: thiamine (72\%), niacin (76\%), riboflavin $(85 \%)$ and vitamin $\mathrm{B}_{6}(85 \%)$, while the intake of sodium exceeded the recommendation 6 times.

The only two consumers' features significantly influencing nutrients and energy intake were BMI and gender (intakes were not linked to age, education level and the number of persons living in the respondent household). Between the group of underweight and normal BMI consumers and the overweight individuals, significant differences occurred in the case of: energy, protein, fat, SFA, MUFA, PUFA, sodium, vitamin $\mathrm{E}$, vitamin $\mathrm{B}_{6}$, and dietary fiber intakes. Gender influenced the nutritional value of the diet to a higher degree than BMI. Differences between men and women did not occur only in the case of three vitamins $A, C$, and $B_{12}$ as well as with respect to the share of protein and fat in daily energy structure.

The study also showed that the most important factors determining food choice and everyday nutrition were: preferences and habits, economical factors and convenience of preparing meals. This order suggests that presumably the food consumption choice of low-income consumers will evolve to worsening of the diet implicating the changes which are observed in more affluent countries. For this population group it is essential to improve their knowledge on food, nutrition and health linkages with the aim to create proper food choice and behavior.

\section{Health Status of Polish Population}

The most important change in health, concerning all Poles, is a significant decline of morbidity and mortality from cardiovascular diseases [Zatoński, 2008]. In 2002 these rates were 192/100,000 (men) and 58/100,000 (women). Life expectancy at birth in 2002 for Polish women was 78.8 years, and 70.3 years for men. Vascular diseases are still the major cause of death of Polish men and second (after cancer) in women. In terms of risk factors, $53 \%$ of the difference in life expectancy between EU15 countries in men and 35\% in women aged 35 to 64 results from tobacco-related mortality. In 2002, smoking prevalence in Poland was one of the highest in Europe (46\% of men aged 20 to 64 and 31\% in women aged 20 to 64). In 1995, Poland introduced a comprehensive tobacco control program, followed by a significant decline in smoking prevalence in men and young women. According to the WOBASZ study, $42 \%$ of men and 1 out of 4 Polish women smoked regularly. The average number of smoked cigarettes reached 
18 (men) and 14 (women). Some $46 \%$ of men and 67\% of women never smoked and $80 \%$ declared that they want to quit smoking [Polakowska et al., 2005].

\section{Conclusions}

The nutritional and health status of the Polish population is determined by dietary habits strongly influenced by market transformation. Due to changes in consumer habits and low level of physical activity, the prevalence of overweight and obesity is increasing. The diet of Polish consumers, including low-income population, in general is improperly balanced. In the case of minerals' consumption, excessive daily intake of sodium can be seen as a big challenge for public health nutrition.

The most important change in health status is the decrease of morbidity and mortality ratios from cardiovascular diseases. The prevalence of another cause of premature mortality - smoking - is one of the highest in Europe, however in men and young adult women it is showing a decline.

The Polish population of 38.8 million is strongly differentiated by social, demographical and regional factors. This implies the need to conduct further research and monitoring of food intake in different subpopulation groups as well as to implement education and information programs aimed at changing food consumption patterns.

\subsection{Portugal ${ }^{17}$}

\section{Introduction}

The present report compiles the most relevant data published between 2000 and 2008 which were not included in the ENHR 2009 main text.

\section{Household Food and Nutrient Availability}

Following the household food and beverages availability data computed under the DAFNE project, the household availability of energy, nutrient and other food components retrieved from the 2000 Portuguese Household Budget Survey (HBS) are shown in table 11.52 [Rodrigues, 2007].

Two diet-quality scores (compliance with the WHO population dietary goals and adherence to the Mediterranean food pattern) were computed in order to compare Portuguese regions [Rodrigues et al., 2007] (table 11.53).

\section{Food and Nutrient Intake}

In the absence of a National Dietary Survey (the only one was carried out in 1980), the food and nutrient intake information was retrieved from several different studies

\footnotetext{
${ }^{17}$ Rodrigues S, Pinhão S, Silva Ferreira L, Lopes C, Ramos E, Vaz Almeida MD; Faculty of Food Science
} and Nutrition from Porto University, Portugal. E-Mail saraspr.@fcna.up.pt 
Table 11.52. Household energy, nutrient and other food component availability (per person/day) in Portugal, 2000 [source: Rodrigues, 2007]

\begin{tabular}{ll}
\hline Energy, kcal & 2,249 \\
\hline Protein, \%E & 16 \\
\hline Lipid, \%E & 34 \\
\hline Carbohydrates, \%E & 47 \\
\hline Alcohol, \%E & 3 \\
\hline MUFA, \%E & 13 \\
\hline PUFA, \%E & 8 \\
\hline SFA, \%E & 9 \\
\hline Cholesterol, mg & 196 \\
\hline Sugars, \%E & 16 \\
\hline Fiber, g & 20 \\
\hline Sodium, mg & 6,573 \\
\hline From food/beverages, mg & 2,638 \\
\hline From table/cooking salt, mg & 3,935 \\
\hline
\end{tabular}

that have been conducted in the context of particular scientific research but mostly in restricted geographical areas and rarely at the national level.

Children and Adolescents. In the ENHR 2009 main text, data on children's food intake was retrieved from Moreira et al. [2005] and the EpiTeen Study [Ramos, 2008]. Moreira et al. [2007] evaluated the diet of 4,845 children (6 to 12 years old) using a 24-hour recall and compared groups according to BMI distribution (non-overweight vs. overweight; table 11.54). The Health Behaviour in School-Aged Children Study [Matos et al., 2006] evaluated a national representative sample of 4,877 children (11 to 16 years old) by asking participants how much fruit, vegetables, soft drinks and sugar products they usually eat (table 11.55).

Adults and Elderly. In the main text of the ENHR 2009, data on this subject comes from the EPI Porto study [Lopes et al., 2006], a study on the health and nutrition situation in the city of Porto that evaluated 18- to 92-year-old subjects from 1999 to 2003 using a validated semiquantitative food frequency questionnaire. The whole report of this study can be found at http://higiene.med.up.pt/consumoalimentarporto/home.

\section{Nutritional Status}

Children and Adolescents. Self-reported weight and height data collected within the National Health Survey 2005/2006 [INE/INSA, 2008] allowed the computation of 
Table 11.53. Diet-quality scores (based on per person per day household availability) by Portuguese region in 2000 [source: Rodrigues et al., 2007]

\begin{tabular}{|c|c|c|c|c|c|c|c|c|}
\hline $\begin{array}{l}\text { Household } \\
\text { diet quality }\end{array}$ & North & Center & $\begin{array}{l}\text { Lisbon } \\
\text { area }\end{array}$ & Alentejo & Algarve & Azores & Madeira & Portugal \\
\hline MAI $^{1}$ (median) & 2.26 & 2.34 & 1.77 & 2.37 & 2.21 & 1.67 & 2.79 & 2.09 \\
\hline \multicolumn{9}{|l|}{$\%$ families with } \\
\hline Low $\leq 2$ & 42.0 & 41.6 & 57.3 & 38.6 & 43.7 & 62.0 & 28.5 & 47.1 \\
\hline Intermediate 2-4 & 39.1 & 38.8 & 32.0 & 41.2 & 37.9 & 30.9 & 43.2 & 36.6 \\
\hline High $\geq 4$ & 18.8 & 19.7 & 10.7 & 20.1 & 18.4 & 7.1 & 28.3 & 16.2 \\
\hline $\mathrm{HDIr}^{2}$ (median) & 4.00 & 4.00 & 4.00 & 4.00 & 4.00 & 4.00 & 5.00 & 4.00 \\
\hline \multicolumn{9}{|l|}{$\%$ families with } \\
\hline Low $\leq 4$ & 61.3 & 56.6 & 65.6 & 57.2 & 63.8 & 66.1 & 42.0 & 61.4 \\
\hline Intermediate 5-6 & 33.2 & 36.5 & 31.5 & 37.1 & 30.5 & 30.4 & 48.3 & 33.6 \\
\hline High $\geq 7$ & 5.5 & 6.9 & 2.9 & 5.7 & 5.7 & 3.5 & 9.8 & 4.9 \\
\hline
\end{tabular}

Table 11.54. Nutrient intake in Portuguese children (6-12 years old) by gender and presence of overweight [source: Moreira et al., 2007]

\begin{tabular}{|c|c|c|c|c|}
\hline & \multicolumn{2}{|l|}{ Girls } & \multicolumn{2}{|l|}{ Boys } \\
\hline & \multicolumn{2}{|l|}{ overweight } & \multicolumn{2}{|l|}{ overweight } \\
\hline & no & yes & no & yes \\
\hline Energy, kcal & $2,316 \pm 699$ & $2,325 \pm 802$ & $2,525 \pm 938$ & $2,518 \pm 825$ \\
\hline Total fat, \%E & $35.3 \pm 6.7$ & $35.4 \pm 6.9$ & $35.1 \pm 6.9$ & $35.3 \pm 6.8$ \\
\hline SFA, \%E & $12.8 \pm 3.4$ & $12.8 \pm 3.1$ & $12.7 \pm 3.3$ & $12.8 \pm 3.6$ \\
\hline MUFA, \%E & $14.6 \pm 3.5$ & $14.7 \pm 3.6$ & $14.6 \pm 3.5$ & $14.7 \pm 3.4$ \\
\hline PUFA, \%E & $5.0 \pm 1.7$ & $5.0 \pm 1.6$ & $4.9 \pm 1.7$ & $5.0 \pm 1.7$ \\
\hline Proteins, \%E & $16.6 \pm 3.7$ & $17.0 \pm 3.8$ & $16.6 \pm 3.8$ & $16.9 \pm 4.0$ \\
\hline Carbohydrates, \%E & $49.2 \pm 7.8$ & $48.7 \pm 8.1$ & $49.4 \pm 8.0$ & $48.8 \pm 8.0$ \\
\hline Sugars, \%E & $22.1 \pm 7.0$ & $22.4 \pm 7.3$ & $22.7 \pm 7.2$ & $22.3 \pm 7.4$ \\
\hline Fiber, $g$ & $18.1 \pm 8.7$ & $17.7 \pm 7.9$ & $19.4 \pm 10.6$ & $18.5 \pm 8.3$ \\
\hline Calcium, mg & $1,065 \pm 450$ & $1,053 \pm 447$ & $1,129 \pm 506$ & $1,144 \pm 633$ \\
\hline
\end{tabular}


Table 11.55. Food intake in Portuguese children (\% eating at least one portion/day) by gender and age [source: Matos et al., 2006]

\begin{tabular}{|c|c|c|c|c|c|c|}
\hline & Gend & & Age, & & & \\
\hline & boys & girls & 11 & 13 & 15 & 16 \\
\hline Fruit & 49.7 & 47.6 & 51.9 & 45.4 & 38.2 & 34.1 \\
\hline Vegetables & 22.0 & 28.2 & 29.7 & 27.5 & 23.7 & 17.2 \\
\hline Soft drinks & 31.3 & 23.8 & 25.9 & 25.4 & 27.4 & 34.1 \\
\hline Chocolates/cakes/sweets & 22.0 & 23.9 & 23.0 & 23.7 & 22.8 & 21.9 \\
\hline
\end{tabular}

BMI distribution among Portuguese children and adolescents (table 11.56). Several other studies have also reported data on the BMI of Portuguese children and adolescents (table 11.57). Biochemical data for Portuguese children is very scarce. Pedrosa et al. [2008] have evaluated 61 children in order to identify cardiovascular disease risk (table 11.58).

Adults and Elderly. Self-reported weight and height data collected for the last National Health Survey of 2005/2006 [INE/INSA, 2008] allowed the computation of the Body Mass Index group's proportion among Portuguese adults and elderly (table 11.59). Some other studies have also reported data on the body mass index of Portuguese adults and elderly (table 11.60). Adults' biochemical data for adults is shown in the ENHR 2009 main text [source: Instituto de Alimentação Becel, 2001].

\section{Length of Breastfeeding}

To complement data on breastfeeding which is included in the ENHR 2009 main text, two additional tables are included. Length of breastfeeding within the NHS 2005/2006 [INE/INSA, 2008] by women (aged 15+ years) with children $<5$ years old in 2006 is shown in tables 11.61 and 11.62.

\section{Conclusion}

Portugal still lacks nutrition-related data collected at the national level and therefore most information is supplied by Household Budget Surveys and Food Balance Sheets. This is a strong limitation to identify associations between food intake, nutritional and health status and undermines national policies in this field. 
Table 11.56. BMI of Portuguese children and adolescents by age groups (using the IOTF criteria)

\begin{tabular}{|c|c|c|c|c|c|c|c|c|c|c|c|c|}
\hline \multirow[t]{2}{*}{$\begin{array}{l}\text { Age group } \\
\text { years }\end{array}$} & \multicolumn{3}{|c|}{$\begin{array}{l}\text { Underweight, \% } \\
\left(\mathrm{BMI}<18.5 \mathrm{~kg} / \mathrm{m}^{2}\right)\end{array}$} & \multicolumn{3}{|c|}{$\begin{array}{l}\text { Normal range, } \% \\
\left(\mathrm{BMI} \geq 18.5 \text { and }<25 \mathrm{~kg} / \mathrm{m}^{2}\right)\end{array}$} & \multicolumn{3}{|c|}{$\begin{array}{l}\text { Overweight } \% \\
\left(\mathrm{BMI} \geq 25 \text { and }<30 \mathrm{~kg} / \mathrm{m}^{2}\right)\end{array}$} & \multicolumn{3}{|c|}{$\begin{array}{l}\text { Obese, } \% \\
\left(\mathrm{BMI} \geq 30 \mathrm{~kg} / \mathrm{m}^{2}\right)\end{array}$} \\
\hline & total & men & women & total & men & women & total & men & women & total & men & women \\
\hline $6-9$ & 9.70 & 10.32 & 9.09 & 49.01 & 50.07 & 47.99 & 24.30 & 25.03 & 23.60 & 16.99 & 14.58 & 19.32 \\
\hline $10-14$ & 7.45 & 7.75 & 7.13 & 65.03 & 61.49 & 68.70 & 20.85 & 24.40 & 17.19 & 6.66 & 6.36 & 6.98 \\
\hline $15-17$ & 5.62 & 4.52 & 6.68 & 76.21 & 72.22 & 80.02 & 15.30 & 18.91 & 11.84 & 2.87 & 4.35 & 1.46 \\
\hline
\end{tabular}

Source: Data computed on purpose for this report by the Portuguese National Statistics Institute (INE) from the database of the National Health Survey 2005/2006. INE, INSA.

Table 11.57. BMI of Portuguese children and adolescents by age groups (all studies using the Cole criteria, with the exception of Ramos e Barros 2007 who have used the IOTF criteria)

\begin{tabular}{|c|c|c|c|c|c|c|c|c|c|}
\hline \multirow[t]{2}{*}{ Age years } & \multirow{2}{*}{$\begin{array}{l}\mathrm{n} \text { and method } \\
\text { of data } \\
\text { collection }\end{array}$} & \multirow{2}{*}{$\begin{array}{l}\text { Place and year of } \\
\text { data collection }\end{array}$} & \multicolumn{3}{|c|}{ Overweight, \% } & \multicolumn{3}{|c|}{ Obesity, \% } & \multirow[t]{2}{*}{ Reference } \\
\hline & & & total & female & male & total & female & male & \\
\hline $3-6$ & $\begin{array}{l}2,400 \\
\text { measured }\end{array}$ & Coimbra (2001) & 16.9 & 20.4 & 13.6 & 6.7 & 6.9 & 6.5 & Rito, 2004 \\
\hline $6-10$ & $\begin{array}{l}1,225 \\
\text { measured }\end{array}$ & Sintra (2004) & 23.0 & - & - & 12.6 & - & - & $\begin{array}{l}\text { Ferreira and } \\
\text { Marques-Vidal, } \\
2008\end{array}$ \\
\hline $6-10$ & $\begin{array}{l}2,384 \\
\text { measured }\end{array}$ & Madeira (2004/2005) & 19.2 & 22.6 & 16.1 & 11.1 & 10.7 & 11.4 & $\begin{array}{l}\text { Sousa et al., } \\
2006\end{array}$ \\
\hline $6-12$ & $\begin{array}{l}4,845 \\
\text { measured }\end{array}$ & National (2002/2003) & - & 20.9 & 18.5 & - & 11.9 & 10.3 & Moreira et al., 2007 \\
\hline $7-9$ & $\begin{array}{l}905 \\
\text { measured }\end{array}$ & Aveiro (2006) & 20.0 & 21.4 & 18.6 & 8.1 & 9.6 & 6.6 & Pedrosa et al., 2006 \\
\hline $7-9$ & $\begin{array}{l}3,044 \\
\text { measured }\end{array}$ & National (2002/2003) & - & 24.1 & 22.2 & - & - & - & Moreira et al., 2005 \\
\hline $10-15$ & $\begin{array}{l}819 \\
\text { measured }\end{array}$ & Porto & - & 23.8 & 30.9 & - & - & - & Ribeiro et al., 2006 \\
\hline 11 & $\begin{array}{l}1,176 \\
\text { self-reported }\end{array}$ & National (2003) & - & 17.7 & 26.5 & - & 2.2 & 6.2 & Yngve et al., 2007 \\
\hline 13 & $\begin{array}{l}2,161 \\
\text { measured }\end{array}$ & Porto & - & 18.8 & 20.8 & - & 5.7 & 6.6 & $\begin{array}{l}\text { Ramos and Barros, } \\
2007\end{array}$ \\
\hline $11-16$ & $\begin{array}{l}7,400 \\
\text { self-reported }\end{array}$ & National (2005/2006) & 15.2 & 14.9 & 15.6 & 2.8 & 2.7 & 2.8 & Matos et al., 2006 \\
\hline $10-18$ & $\begin{array}{l}3,013 \\
\text { measured }\end{array}$ & $\begin{array}{l}\text { Lisbon and region } \\
(2000 / 2002)\end{array}$ & - & 19.7 & 17.4 & - & 4.7 & 5.3 & $\begin{array}{l}\text { Marques-Vidal } \\
\text { et al., } 2008\end{array}$ \\
\hline
\end{tabular}


Table 11.58. Biochemical markers of Portuguese children (7-9 years old) [source: Pedrosa et al., 2008]

\begin{tabular}{ll}
\hline Biochemical markers & Mean \pm SD \\
\hline Total cholesterol, $\mathrm{mg} / \mathrm{dl}$ & $167.4 \pm 26.9$ \\
\hline $\mathrm{LDL}, \mathrm{mg} / \mathrm{dl}$ & $105.6 \pm 23.7$ \\
\hline $\mathrm{HDL}, \mathrm{mg} / \mathrm{dl}$ & $50.4 \pm 9.9$ \\
\hline Triglycerides, $\mathrm{mg} / \mathrm{dl}$ & $71.7 \pm 39.5$ \\
\hline
\end{tabular}

\subsection{Romania ${ }^{18}$}

\section{Demographics}

Romania's population was 21.58 million inhabitants in 2006 (51.3\% females). People aged $0-14$ years made up $15.4 \%$, while those aged $\geq 65$ years made up $14.7 \%$ of the total population. A decline in fertility (from 1.5 to 1.3 births per woman), a decline in birth rate (from 11.4 to 10.2/1,000 inhabitants), and an increase in the death rate (from 11.6 to 12.0/1,000) also occurred between 1992 and 2006 [NIS, 2006].

\section{Life Expectancy}

The average life expectancy in Romania increased from 71.25 years in 2000 to 72.7 years in 2006 (69.2 years for men and 76.2 years for women), 6 years below the EU average (78.5 years in 2005) and 7 years below the average for the EU countries prior to May 2004 (79.7 years in 2005) [Vladescu et al., 2008].

\section{Infant, Child, and Maternal Mortality}

Infant and maternal mortalities are among the highest in the European Region despite a large decline in maternal mortality since 1990 . The infant death rate decreased from 26.9 (in 1990) to 13.9 infant deaths per 1,000 live births (in 2006) respectively [NIS, 2006]. The mortality rate under 5 years of age decreased from 34.3 per 1,000 live births (in 1990) to 16.5 per 1,000 live births (in 2006). Moreover, maternal mortality rates also decreased dramatically from 83.6 per 100,000 live births in 1990 to 32.8 and 15.5 in 2000 and 2006 respectively.

\section{Health Status and Main Causes of Death}

During the past decades, the morbidity and mortality patterns have changed, an increased prevalence of chronic diseases being observed. Lately, in 2006, the main causes of death [WHO Regional Office for Europe, 2007] were cardiovascular diseases

\footnotetext{
${ }^{18}$ Vlad M; Institute of Public Health Cluj-Napoca, Cluj-Napoca, Romania. E-Mail mvlad1212@yahoo.
} com 
Table 11.59. BMI of Portuguese adults and elderly by gender and age groups (computed from self-reported height and weight)

\begin{tabular}{|c|c|c|c|c|c|c|c|c|c|}
\hline \multirow[t]{2}{*}{ Age group } & \multicolumn{3}{|c|}{$\begin{array}{l}\text { Underweight, \% } \\
\left(\mathrm{BMI}<18.5 \mathrm{~kg} / \mathrm{m}^{2}\right)\end{array}$} & \multicolumn{3}{|c|}{$\begin{array}{l}\text { Overweight, } \% \\
\left(\text { BMI } \geq 25 \mathrm{~kg} / \mathrm{m}^{2}<30 \mathrm{~kg} / \mathrm{m}^{2}\right)\end{array}$} & \multicolumn{3}{|c|}{$\begin{array}{l}\text { Obese, } \% \\
\left(\mathrm{BMI} \geq 30 \mathrm{~kg} / \mathrm{m}^{2}\right)\end{array}$} \\
\hline & total & men & women & total & men & women & total & men & women \\
\hline Total & 2.22 & 0.99 & 3.34 & 35.72 & 40.64 & 31.22 & 15.20 & 14.33 & 16.00 \\
\hline $18-24$ & 5.55 & 3.17 & 8.09 & 15.36 & 17.93 & 12.62 & 3.88 & 4.23 & 3.51 \\
\hline $25-34$ & 3.39 & 0.99 & 5.82 & 30.71 & 39.36 & 21.94 & 8.61 & 7.88 & 9.35 \\
\hline $35-44$ & 1.33 & 0.24 & 2.40 & 36.18 & 43.67 & 28.86 & 12.84 & 12.81 & 12.87 \\
\hline $45-54$ & 0.76 & 0.08 & 1.40 & 41.68 & 45.94 & 37.64 & 21.00 & 21.69 & 20.35 \\
\hline $55-64$ & 0.98 & 0.46 & 1.46 & 44.15 & 45.65 & 42.82 & 23.21 & 21.81 & 24.46 \\
\hline $65-74$ & 1.02 & 0.59 & 1.37 & 43.45 & 46.25 & 41.18 & 21.87 & 19.55 & 23.73 \\
\hline $75-84$ & 2.65 & 2.38 & 2.82 & 39.37 & 45.33 & 35.47 & 16.84 & 13.43 & 19.08 \\
\hline $85+$ & 7.12 & 6.48 & 7.43 & 25.58 & 35.16 & 21.03 & 14.11 & 16.35 & 13.04 \\
\hline
\end{tabular}

Source: Data computed on purpose for this report by the Portuguese National Statistics Institute (INE) from the database of the National Health Survey 2005/2006. INE, INSA.

Table 11.60. BMI of Portuguese adults and elderly by age groups

\begin{tabular}{|c|c|c|c|c|c|c|c|c|c|}
\hline \multirow[t]{2}{*}{ Age years } & \multirow{2}{*}{$\begin{array}{l}\mathrm{n} \text { and method } \\
\text { of data } \\
\text { collection }\end{array}$} & \multirow{2}{*}{$\begin{array}{l}\text { Place and } \\
\text { year of data } \\
\text { collection }\end{array}$} & \multicolumn{3}{|c|}{ Overweight, \% } & \multicolumn{3}{|c|}{ Obesity, \% } & \multirow[t]{2}{*}{ Reference } \\
\hline & & & total & female & male & total & female & male & \\
\hline $18-64$ & $\begin{array}{l}8,116 \\
\text { measured }\end{array}$ & $\begin{array}{l}\text { National } \\
(2003 / 2005)\end{array}$ & 39.4 & 34.4 & 45.2 & 14.2 & 13.4 & 15.0 & Carmo et al., 2008 \\
\hline $18-65$ & $\begin{array}{l}9,991 \\
\text { self-reported }\end{array}$ & $\begin{array}{l}\text { Azores } \\
(2004)\end{array}$ & - & 35.5 & 45.8 & - & 18.8 & 16.0 & Santos et al., 2008 \\
\hline $65+$ & $\begin{array}{l}307 \\
\text { self-reported }\end{array}$ & National (2004) & 44.0 & 41.8 & 45.7 & 18.6 & 19.4 & 17.9 & Afonso, 2008 \\
\hline
\end{tabular}

(62.1\%), cancer (17.6\%), digestive diseases (5.5\%), accidents, injuries, and poisoning (4.9\%), and respiratory diseases $(4.9 \%)$.

Cardiovascular Diseases. Ischemic heart disease is the single biggest killer in Romania, responsible for about 20\% of all yearly deaths during the 2000-2006 period. 
Table 11.61. Portugal, length of breastfeeding (of children $<5$ years old in 2006)

\begin{tabular}{llc}
\hline Length of breastfeed & $\mathrm{n}=657,888$ & $\%$ \\
\hline$\leq 1$ week & 52,152 & 7.9 \\
\hline$\leq 1$ month & 91,058 & 13.8 \\
\hline$\leq 2$ months & 87,012 & 13.2 \\
\hline$\leq 3$ months & 112,968 & 17.2 \\
\hline$\leq 6$ or more months & 257,623 & 39.2 \\
\hline Did not breastfeed & 57,075 & 8.7 \\
\hline
\end{tabular}

Source: Computed on purpose for this report from the 4th INS (2005/2006) database.

Cancer. Cancer accounted for $16 \%$ of all deaths in Romania, which is much less than the Eur- $\mathrm{A}^{19}$ average (28\%) (referring to WHO epidemiological subregions). The prevalence of localizations varied, with no particular pattern related to age or gender. At least until 2006, the general standard death rates due to malignant tumors presented a slow, constant increase with time. Among cancer-related deaths, mortality from cervical cancer is twice as high in Romania than the EU average; however, mortality from trachea/bronchus/lung cancers and breast cancer is below the EU average [Vladescu et al., 2008].

Digestive Tract Diseases. During the past decades, mortality from diseases of the digestive tract increased and decreased successively. Over $70 \%$ of the deaths from digestive diseases in Romania are caused by chronic liver disease and cirrhosis, the Eur- $\mathrm{B}+\mathrm{C}^{20}$ and Eur- $\mathrm{A}^{21}$ average rates being about 60 and $40 \%$ respectively [WHO Regional Office for Europe, 2005].

\section{Food Intake, Feeding Habits, and Nutritional Status}

Infants. Data about feeding practices and nutritional status concerning infants and children were essentially derived from an extensive project [Stanescu et al., 2005] that started in April 2004 and conducted by the Alfred Rusescu Institute for Mother and Child Care in Bucharest, with technical and financial support from UNICEF (United Nations International Children's Emergency Fund). Cross-sectional and retrospective studies were performed and a main consideration was given to surveys, questionnaires, body measurements, and laboratory analysis data collected during past periods, which involved: a national representative sample of 83,000 children aged under 5 [NPNS

${ }^{19}$ WHO epidemiological subregions

${ }^{20} \mathrm{WHO}$ epidemiological subregions

${ }^{21}$ WHO epidemiological subregions 
Table 11.62. Portugal, length of breastfeeding (of children $<5$ years old in 2006) by mothers' age groups

\begin{tabular}{|c|c|c|c|c|c|c|c|}
\hline \multirow{2}{*}{$\begin{array}{l}\text { Age } \\
\text { group } \\
\text { years }\end{array}$} & \multirow[t]{2}{*}{$\mathrm{n}$} & \multicolumn{6}{|c|}{ Length of breastfeeding } \\
\hline & & $\leq 1$ week & $\leq 1$ month & $\leq 2$ months & $\leq 3$ months & $\begin{array}{l}\leq 6 \text { or more } \\
\text { months }\end{array}$ & $\begin{array}{l}\text { Did not } \\
\text { breastfed }\end{array}$ \\
\hline $15-19$ & 10,013 & 14.8 & 27.5 & 2.6 & 13.5 & 38.1 & 3.6 \\
\hline $20-24$ & 82,300 & 9.6 & 12.1 & 21.8 & 15.0 & 28.7 & 12.8 \\
\hline $25-29$ & 171,854 & 8.2 & 12.3 & 15.8 & 17.4 & 36.2 & 10.0 \\
\hline $30-34$ & 228,447 & 8.1 & 14.0 & 10.0 & 18.6 & 43.1 & 6.1 \\
\hline $35-39$ & 113,869 & 5.0 & 15.1 & 11.5 & 16.8 & 43.1 & 8.5 \\
\hline $40-44$ & 44,393 & 8.7 & 14.2 & 11.1 & 16.6 & 40.9 & 8.5 \\
\hline $45-49$ & 6,807 & 5.5 & 23.8 & 11.6 & 6.6 & 30.2 & 22.2 \\
\hline $50-54$ & 205 & 40.6 & 18.8 & - & - & 40.7 & - \\
\hline
\end{tabular}

Source: Computed on purpose for this report from the 4th INS (2005/2006) database.

Project, 1993-2003]; a sample of 2,120 pupils aged 7 to 8 (2002-2003); a pilot survey on the iodine deficiency in 1,387 pregnant women (2003-2004), and a reproductive health survey (2004).

Overall, 88.0-92.2\% of the surveyed children were breastfed for various periods of time and only $7.7-12.0 \%$ of them were never breastfed. These data rank Romania among the countries with good breastfeeding practices. The average age of exclusively breastfed children (with no other solid or liquid foods) was 3.9 months, with a median of 3.2 months. Data collected in 2004 indicate that only $16 \%$ of infants aged between 0 and 5 months were exclusively breastfed.

The average age for introducing bottle-feeding was 4.2 months, irrespective of gender, area of residence, or maternal educational level. At the age of 3 months, $50 \%$ of infants began to be fed on the bottle while $36.8 \%$ of infants received complementary feeding and at 4 months their share increased to $70.9 \%$. Fruit puree was the first complementary food given to infants by most families (65\%), followed by vegetables (17.8\%), and cereals.

Children. Anemia is more frequent in children under 5 years of age, especially in rural areas, where two thirds of the children between 1 and 2 years of age have a hemoglobinemia $<11 \mathrm{~g} / \mathrm{dl}$. Thus, in infants, the average level of $10.5 \mathrm{~g} / \mathrm{dl}$ blood hemoglobin was lower in 2004 than the lowest mean (of $11 \mathrm{~g} / \mathrm{dl}$ ) recorded in the 2000-2003 period [Stanescu et al., 2005].

Anemia is also frequent in schoolchildren. Between 2002 and 2004, its mean prevalence increased up to $23.4 \%$, and even higher in rural areas. 
The low weight-for-height indicator (under $-2 \mathrm{SD}$ ) had an overall prevalence of $4.4 \%$ in children under 5 years, with values above $5 \%$ in rural areas. Low weight-forheight was also recorded in about $5 \%$ of children aged 6 to 7 years, with a maximum of $7.7 \%$ in girls aged 7 and $6.1 \%$ in children aged 6 years living in urban areas [Stanescu et al., 2005]. On average, the prevalence of low weight-for-age was $5.5 \%$ in children under 5 , a value above the reference sample. The prevalence of high weight-for-height was systematically higher than in the reference population for all age groups $(4.2 \%$ of children aged under 5 are overweight).

The prevalence of low height-for-age of children under 5 years of age is above the reference values, ranging between 5 and 10\%, with lower values in 2004. The persistence of a mild height deficiency, close to the reference values, was also reported in children aged 6 to 7 [Stanescu et al., 2005].

Adults and Elderly. Between 2000 and 2008, several cross-sectional nutritional and health status surveys were carried out by the Institute of Public Health Cluj-Napoca in various regions of Romania and with national representative samples. The methods used were the 24-hour recall for dietary intake, and 24-hour recall and 7-day estimated record for food intake. In 2003 and 2008, two national studies were conducted in 614 and 473 adults of both genders [Vlad et al., 2003, 2008]. In a 2006 study, the daily food and nutrient intake in 641 adults ( 315 men and 326 women) from 10 counties of the Transylvania area was also assessed [Zapirtan, 2006]. In all studies, the subjects were grouped by age ( 20 to 45,45 to 62 , and $>60$ or 62 years of age). Data from subjects recruited from regions all over Romania, aged 55 to 85 and living in their own households, were also reported and analyzed in the EU Ageing Nutrition Project [Stehle and Volkert, 2006].

In these studies, the average energy intake was below the recommended level in all adults $(2,772 \mathrm{kcal} /$ day in men and 2,391 kcal/day in women). Due to lower energetic needs at older ages, the energetic deficit decreases from $15 \%$ in young adults to $6 \%$ in the elderly. The relative share of protein in total energy intake was similar in men and women (18 and 17\%E, respectively), with no significant differences between age groups defined by the WHO [2003]. The mean proportion of carbohydrates in total energy intake was low both in men $(45 \% \mathrm{E})$ and women $(48 \% \mathrm{E})$. The proportion of fat in total energy intake was high, far above the maximum level of 30\%E recommended by Eurodiet [2000], with $36 \%$ in men and $37 \% \mathrm{E}$ in women, respectively. Dietary fat intake was high, especially in women. The average cholesterol intake was very high in all subjects (and particularly in the elderly), being $520 \mathrm{mg} /$ day in women and $>640 \mathrm{mg} /$ day in men.

Mean calcium and magnesium intake was lower than the population reference intake of Eurodiet [2000] in both genders. The average magnesium intake in men (303.32 mg/day) was up to the recommendations, decreasing slightly in elderly, while in women it was $263.46 \mathrm{mg} /$ day, representing $89.94 \%$ of the RDA.

Iron intake was above the recommendation of the Scientific Committee for Food. On average, the intake was relatively high in both genders $(21.04 \mathrm{mg}$ Fe/day in men and $18.09 \mathrm{mg} \mathrm{Fe} /$ day in women). In elderly only, where the needs are lower, the intake was higher than the RDA by $45 \%$. 
In both genders, vitamin A intake exceeds the recommendation of SCF, with the exception of elderly men, where it was close to the recommendations. The average intake in all adults was $2 \mathrm{mg} /$ day. Vitamin $\mathrm{B}_{1}$ intake covered the recommendations, with mean values of $1.74 \mathrm{mg} /$ day in men and $1.45 \mathrm{mg} /$ day in women. The mean intake of vitamin $\mathrm{B}_{2}$ was above the recommended level by more than $50 \%$, having a mean value of $3.66 \mathrm{mg} /$ day in men and $3.16 \mathrm{mg} /$ day in women. All subjects had a vitamin $\mathrm{C}$ intake higher than the recommended level, $76 \mathrm{mg} /$ day in men and $75 \mathrm{mg} /$ day in women, representing $159 \%$ of the RDA and decreasing with age.

The anthropometric and nutritional status data show that the average height was $1.73 \mathrm{~m}$ in adult men and $1.62 \mathrm{~m}$ in women, while in elderly it was lower than the EU average, ranging between $1.64-1.71 \mathrm{~m}$ in men and $1.57-1.60 \mathrm{~m}$ in women. In adults, the highest percentage of overweight (43\%) was found in men, while only $35 \%$ was found in women. BMI showed that the percentage of obesity was rather high in both genders, almost $25 \%$ in women and above $19 \%$ in men, declining with age. Systolic and diastolic blood pressures were higher than the normal values $(140 / 90 \mathrm{~mm} \mathrm{Hg})$ in both genders in about $15 \%$ of the subjects for systolic and in $6 \%$ for diastolic pressure.

Serum lipid values $>800 \mathrm{mg} / \mathrm{dl}$ in $18 \%$ of the men and in $11 \%$ of the women are related to an increased cardiovascular risk. A high cholesterolemia ( $>240 \mathrm{mg} / \mathrm{dl}$ ) present in $32 \%$ of men and $26 \%$ of women - was also associated with a high cholesterol intake. About $16 \%$ of men and $11 \%$ of women had an HDL cholesterol level below the protective limit of $40 \mathrm{mg} / \mathrm{dl}$. Referring to the elderly, $64 \%$ of men and $43 \%$ of women had healthy cholesterol levels with high HDL cholesterol levels. Hypertriglyceridemia ( $>150 \mathrm{mg} / \mathrm{dl}$ ) was found in $40 \%$ of the men and in $26 \%$ of the women.

Serum levels lower than the reference values were found for $\mathrm{Ca}$ (23 and 29\%), $\mathrm{Mg}$ (27 and 32\%), and Fe (22 and 27\%) in men and women, respectively. Low hemoglobin values were found in $24 \%$ of the men and in $21 \%$ of the women. Although only $4.4 \%$ of the investigated subjects declared themselves as suffering from diabetes, $9.6 \%$ of the men and $11.0 \%$ of the women were found with blood sugar levels above $120 \mathrm{mg} / \mathrm{dl}$. Other nutritional disorders were also self-reported: hypertension (15.0\%), dyslipidemia (8.5\%), and gastrointestinal diseases (5.3\% of the cases).

The 2000-2008 average food intake assessments showed that the common Romanian diet contains too much fat and fatty meat [Vlad, 2003, 2008; Zapirtan, 2006]. The meat consumption rate was consistent with the experts' recommendations, but red meat and especially meat products are consumed at a higher rate than necessary. Conversely, extremely low fish consumption was recorded: $61 \%$ of the surveyed subjects seldom eat fish, $31 \%$ eat fish only once per week, while only about $8 \%$ of the subjects eat fish several times per week, as recommended.

The alcohol consumption is moderate in our studied samples, with the exception of $8-9 \%$ of men and $5-6 \%$ of women who drink it daily. In elderly, drinking beer or wine is not popular, but $11 \%$ of men and $3 \%$ of women still drink wine daily. During the past 5 years, cross-sectional studies on representative samples reported a high prevalence of tobacco current use (mainly cigarette smoking), generally between 33.0 
Table 11.63. Prevalence (\%) of current tobacco use in representative samples of the general population of Romania (2003-2007)

\begin{tabular}{lllll}
\hline Study & $\begin{array}{l}\text { 1. Smoking and } \\
\text { public health } \\
\text { age 14-60 (2003) }\end{array}$ & $\begin{array}{l}\text { 2. Prevalence of } \\
\text { drug use age } \\
15-64(2005)\end{array}$ & $\begin{array}{l}\text { 3. Knowledge, attitude, } \\
\text { and practices } \\
\text { age 15-59 (2007) }\end{array}$ & $\begin{array}{l}\text { 4. Romania GYTS } \\
\text { age 13-15 (2003) }\end{array}$ \\
\hline Males, \% & 40.0 & 48.7 & 33.0 & 27.7 \\
\hline Females, \% & 19.5 & 25.3 & 27.1 & 20.1 \\
\hline Total, \% & 29.6 & 36.5 & 30.0 & 23.8 \\
\hline $\mathrm{n}$ & 1,209 & 3,500 & 2,434 & 4,129 \\
\hline
\end{tabular}

and $48.7 \%$ in men and 19.5 and $27.1 \%$ in women (table 11.63 ), with variations related to age [CHPS 2007; Romanian National Antidrug Agency, 2005].

Using interviewer-administered questionnaires, peak values of smoking prevalence (up to $61 \%$ in men and $42 \%$ in women) were mostly noticed in young and middleaged adults (between 25 and 44 years of age). In schoolchildren aged 13 to 15, at least one use of a tobacco product during the past 30 days was reported by $28 \%$ of the boys and $20 \%$ of the girls, while daily use was mentioned by $6.9 \%$ of the boys and $5.9 \%$ of the girls [CDC, 2004]. Response rates varied between 58 and 87\%. Several campaigns and programs for discouraging smoking and encouraging the cessation resulted in a mild lowering of the tobacco consumption prevalence in adults lately.

Nutritional failure leading to different forms of nutrition-related chronic diseases therefore is a major public health concern. Developing and implementing intervention programs towards healthy behavior is urgently needed. The data presented in this report could be useful in formulating such nutrition intervention for Romania.

\subsection{Slovenia 22}

\section{Introduction}

In Slovenia, the Ministry of Health [2005] introduced the National nutritional policy program 2005-2010 to emphasize the importance of the pubic sector in the implementation of national food nutrition policy and healthy lifestyle. The National Institute of Public Health of the Republic of Slovenia is a major research center for epidemiology, prevention of disease and promotion of health in Slovenia. It provides national surveys covering areas such as, socioeconomic conditions, dietary habits, physical activ-

\footnotetext{
${ }^{22}$ Simčič M, Podgrajšek K; University of Ljubljana, Biotechnical Faculty, Slovenia. E-Mail marjan.sim-
} cic@bf.uni-lj.si 
Table 11.64. Mean daily intake of energy, macronutrients and dietary fiber among Slovenian adults [Koch, 1997]

\begin{tabular}{llll}
\hline Nutrients & Average & Women & Men \\
\hline Energy, MJ/day & 11.4 & 10.7 & 12.1 \\
\hline Protein, \%E & 13 & 13 & 13 \\
\hline Fat, \%E & 44 & 45 & 44 \\
\hline SFA, \%E & 15 & 15 & 15 \\
\hline MUFA, \%E & 13 & 13 & 13 \\
\hline PUFA, \%E & 4 & 4 & 4 \\
\hline Carbohydrates & 39 & & 38 \\
\hline \multicolumn{1}{c}{ Total, \%E } & 20 & 40 & 20 \\
\hline \multicolumn{1}{c}{ Sucrose, \%E } & 20 & 20 & 20 \\
\hline Dietary fiber, g/day & 1.7 & 20 & 1.8 \\
\hline Dietary fiber, g/MJ & 3 & 1.6 & 4 \\
\hline Alcohol, \%E & 1.5 & \\
\hline
\end{tabular}

ity and health. Food consumption surveys are also part of projects conducted at the Biotechnical Faculty and the Medical Faculty, University of Ljubljana. Also, the WHO countrywide integrated noncommunicable disease intervention (CINDI) and other programmes make important information available.

In the next sections, some highlights of the studies conducted in Slovenia over the past decade will be presented.

\section{Dietary Habits Adults}

The first large national survey on dietary habits, with a representative sample of 2,185 subjects in Slovenia, was conducted in 1997 by Koch [1997]. The nutritional habits of adults, aged 18 to 65, in Slovenia were assessed using food frequency questionnaire and the 24-hour recall method: frequency and time of daily meals, nutrition and energy intake of average daily consumed food, frequency of daily consumed food (milk and dairy products, fruits, vegetables, meat, cereals and drinks), smoking habits and physical activity regarding to sociodemographic variables were recorded.

The most important finding of this survey was higher energy intake compared to the recommended values (table 11.64), which together with inadequate physical activity leads to disproportionate energy balance and weight gain. The average daily energy intake was $12.1 \mathrm{MJ}$ for men and 10.7 MJ for women. The dietary protein content was $13 \% \mathrm{E}$, with a surprisingly high fat content of $44 \% \mathrm{E}$, which consequently 
Table 11.65. BMI among Slovenian adults [Koch, 1997]

\begin{tabular}{lll}
\hline BMI & Male, \% & Female, \% \\
\hline$<20$ & 1.2 & 13.4 \\
\hline $20-25$ & 43.1 & 51.8 \\
\hline $25-27$ & 25.8 & 12.0 \\
\hline$>27$ & 29.9 & 22.8 \\
\hline
\end{tabular}

Table 11.66. Mean daily intake of energy, macronutrients and dietary fiber among Slovenian adolescents [Fidler Mis, 2005]

\begin{tabular}{lll}
\hline & Female & Male \\
\hline $\mathrm{n}$ & 1,214 & 1,010 \\
\hline Age, years & 15.6 & 15.7 \\
\hline Energy, MJ & 9.7 & 12.7 \\
\hline Protein, \%E & 14 & 15 \\
\hline Carbohydrates & & \\
\hline \multicolumn{1}{c}{ Total, \%E } & 57 & 57 \\
\hline \multicolumn{1}{c}{ Sucrose, \%E } & 13 & 11 \\
\hline Dietary fiber, g & 27 & 33 \\
\hline Fat, \%E & 29 & 28 \\
\hline SFA, \%E & 13 & 13 \\
\hline MUFA, \%E & 10 & 10 \\
\hline PUFA, \%E & 6 & 5 \\
\hline
\end{tabular}

reduced carbohydrate content (39\%E). On average, alcohol provided 4 and $1.5 \% \mathrm{E}$ for men and women, respectively. The average portion of saturated fatty acids was $15 \% \mathrm{E}$, which is considerably higher than recommended. In contrast, the intake of average dietary fiber was $20 \mathrm{~g} /$ day, corresponding to 1.6 and $1.8 \mathrm{~g} / \mathrm{MJ}$ for men and women, respectively, significantly insufficient compared to the recommended values.

A major percentage of investigated subjects had normal BMI (between 20 and 25), $43 \%$ for women and $52 \%$ for men (table 11.65). $26 \%$ of the male and $12 \%$ of the female subjects had BMI between 25 and 27 . Moreover, $30 \%$ of the male subjects had BMI higher than 27 , compared to $23 \%$ of the female subjects [Koch, 1997]. 
Table 11.67. BMI among Slovenian adolescents [Fidler Mis, 2005]

\begin{tabular}{lll}
\hline BMI $^{1}$ & Male & Female \\
\hline$<18.5$ & 4.1 & 10 \\
\hline $18.5-25$ & 77.3 & 73.6 \\
\hline $25-30$ & 18.6 & 16.4 \\
\hline$>30$ & 4.1 & 3.5 \\
\hline${ }^{1}$ Extrapolated BMI at age 18 years & \\
\hline
\end{tabular}

Covering the period from 2007 to 2008, a second nationwide survey on dietary habits with 1,123 participants was conducted by The National Institute of Public Health of the Republic of Slovenia, using a similar method as Koch [1997]. In May 2009 , the preliminary results on fat content and BMI were presented. These findings showed that dietary fat intake dropped from $44 \% \mathrm{E}$ in 1997 to $39 \% \mathrm{E}$ in 2008 . On average, $42 \%$ of female and $60 \%$ of male participants, aged 19 to 65 , were overweight or obese with a BMI $>25$. Full details are provided in table 11.68.

\section{Dietary Habits Adolescents}

In adolescent subjects in Slovenia, the most important dietary habits survey covered the period from 2003 to 2005 using a food frequency questionnaire [Fidler Mis, 2005; Štimec et al., 2007]. A representative sample of 2,813 people, aged 14 to 17 years (representing $10 \%$ of the population), were asked to complete a questionnaire on body weight, height, physical activity and other lifestyle factors. 2,224 persons (1,214 female, 1,010 male) responded to the questionnaire (participation rate 83.6\%).

The average energy intake was 9.7 and $12.7 \mathrm{MJ}$ for female and male subjects, respectively. The distribution of energy between protein $(14-15 \% \mathrm{E})$, carbohydrates $(57 \% \mathrm{E})$ and fat $(28-29 \% \mathrm{E})$ was in accordance to the recommendations (table 11.66). In addition, average dietary fiber intake was 27 and $33 \mathrm{~g} /$ day, corresponding to 2.8 and $3.3 \mathrm{~g} / \mathrm{MJ}$ for female and male subjects, respectively. BMI values are presented in table 11.67 [Fidler Mis, 2005].

\section{General Comments}

Data on food consumption patterns in Slovenia are still scarcely available. Some master theses studies are reported, unfortunately with limited representation of the population and limited targets of study. The general trend in the data described above is in fact similar as the general trend observed in the whole of the European Union. Because of application of slightly different methodologies, it is hard to compare both studies reported [Koch, 1997; Gabrijelčič Blenkuš et al., 2009]. Nevertheless, the 
Table 11.68. BMI among Slovenian adults [Gabrijelčič Blenkuš, 2009]

\begin{tabular}{lllll}
\hline BMI & $\begin{array}{l}\text { Male } \\
(19-65 \text { years })\end{array}$ & $\begin{array}{l}\text { Female } \\
(19-65 \text { years })\end{array}$ & $\begin{array}{l}\text { Male } \\
(>65 \text { years })\end{array}$ & $\begin{array}{l}\text { Female } \\
(>65 \text { years })\end{array}$ \\
\hline$<18.5 \mathrm{~kg} / \mathrm{m}^{2}$ & 1 & 3 & 0 & 0 \\
\hline $18.5-25 \mathrm{~kg} / \mathrm{m}^{2}$ & 38.6 & 54.8 & 50 & 14.3 \\
\hline $25-30 \mathrm{~kg} / \mathrm{m}^{2}$ & 41.8 & 30 & 37.5 & 57.1 \\
\hline$>30 \mathrm{~kg} / \mathrm{m}^{2}$ & 18.7 & 12.3 & 12.5 & 28.6 \\
\hline
\end{tabular}

results indicate that it is necessary to thoroughly standardize the procedures used in order to generate data that can be compared.

Recently, more efforts were conducted to obtain data on food consumption behavior with appropriate research methodology. Unfortunately, the results have not yet been published. An extended review was published [Ostan et al., 2009] that elucidates the deeper reasons of food choice and feeding behavior and indicates that the observed pattern is based on a complex genetic drive. The existence of this drive is one of the reasons why studies on nutritional behavior and food consumption are so difficult to conduct and interpret.

\subsection{Spain ${ }^{23}$}

The Catalan Nutrition Survey was realized in 2002-2003 (ENCAT 2002-2003) [SerraMajem et al., 2007a; Ribas-Barba et al., 2007] on behalf of the Health Department of the Catalan Government. Catalonia, located in northeastern Spain, has a population of more than 7 million inhabitants.

\section{Study Methodology}

Sample: The study included a representative sample of 2,160 individuals aged 10 to 80 . The sample was stratified according to household size and randomized into subgroups, with Catalan municipalities being the primary sample units and individuals within these municipalities comprising the final sample units. Samples were selected by considering the proportion of the number of inhabitants and the specific weight of each municipality in the sample and were obtained from the census registers of the selected municipalities [Serra-Majem et al., 2007a; Ribas-Barba et al., 2007]. The response rate was $65 \%$.

\footnotetext{
${ }^{23}$ Serra-Majem L, Román-Viñas B, Ribas-Barba L; Nutrition Research Foundation, Barcelona, Spain. E-Mail fin@pcb.ub.es
} 
Methods: Data were collected at the individual's home via personal interviews carried out by dietitians who had previously undergone a rigorous selection and training process. All subjects were administered a general questionnaire compiling information about socioeconomic variables (profession, educational level, etc.) as well as the following: food habits, chronic disease control, smoking, physical activity, and opinions and knowledge about nutrition. Furthermore, several anthropometric measurements were taken under standardized conditions [Serra-Majem et al., 2007a; Ribas-Barba et al., 2007].

The dietary assessment method consisted of combining two 24-hour recalls and a food frequency questionnaire. All individuals completed one 24-hour recall, and $62 \%$ of the sample completed a second 24-hour recall 8-30 days later, conducted on a different weekday from the first interview. Interviews were conducted on all days of the week. In order to estimate volumes and portion sizes, the household measures found in the subjects' own homes were used. In subjects with disabilities or memory defects the primary caretaker was interviewed. Children $<13$ years were assisted by a parent. Food data coding was carried out by the interviewers [Serra-Majem et al., 2007a; Ribas-Barba et al., 2007]. The food frequency questionnaire was qualitative and included 80 food items.

Analysis: To convert food into nutrients, the Spanish tables developed by CESNID were applied. Data were adjusted for intraindividual variability using the method originally described by Beaton et al. [1983]. Underreporters were evaluated according to the Goldberg method [Goldberg et al., 1991]. In order to evaluate inadequate or deficient energy and nutrient intakes, the updated version of the Spanish recommended nutrient intake (RNI) values were used. They included energy and 14 nutrients, of which the recommendation for folate in females aged 13 to 49 was doubled, resulting in a value of $400 \mu \mathrm{g} /$ day. The results are shown according to age group and gender.

Statistical analysis was carried out using the program SPSS v12. Food consumption was analyzed by age group and gender. Student's $t$ test was used to compare means between two groups, and one-way analysis of variance was used for comparison between more than two groups. For non-parametric variables the Mann-Whitney and Kruskal-Wallis tests were conducted. The $\chi^{2}$ statistics were used for comparison of proportions.

\section{Risk of Inadequate Intakes}

Nutritional intake adequacy in the Catalan population was assessed by applying the probabilistic approach method. Table 11.69 shows the risk of inadequate nutrient intakes by gender and age group in ENCAT 2002-2003. Vitamins A, D and E in males and females; zinc, magnesium, and riboflavin in males and iron and folate in females were the micronutrients with higher risk of inadequate intakes among the Spanish population. Certain population groups showed greater levels of nutrient intake inadequacy for given micronutrients. For instance, iron, magnesium and vitamin $\mathrm{C}$ were 
Table 11.69. Risk of inadequate nutrient intake in Spain by gender and age groups (\%) [ENCAT 2002-2003]

\begin{tabular}{|c|c|c|c|c|c|c|c|c|c|c|c|c|c|}
\hline $\begin{array}{l}\text { Age } \\
\text { group }\end{array}$ & Iron & Zinc & $\begin{array}{l}\text { Magne- } \\
\text { sium }\end{array}$ & Thiamine & Riboflavin & Niacin & $\begin{array}{l}\text { Vitamin } \\
\mathrm{B}_{6}\end{array}$ & Folate & $\begin{array}{l}\text { Vitamin } \\
\mathrm{B}_{12}\end{array}$ & $\begin{array}{l}\text { Vitamin } \\
\text { C }\end{array}$ & $\begin{array}{l}\text { Vitamin } \\
\text { A }\end{array}$ & $\begin{array}{l}\text { Vitamin } \\
\text { D }\end{array}$ & $\begin{array}{l}\text { Vitamin } \\
\text { E }\end{array}$ \\
\hline \multicolumn{14}{|l|}{ Male } \\
\hline $\begin{array}{l}10-17 \\
\text { years }\end{array}$ & 16.7 & 70.0 & 47.5 & 0.1 & 7.5 & 3.3 & 9.2 & 1.1 & 0.0 & 19.3 & 92.1 & 99.6 & 46.8 \\
\hline $\begin{array}{l}18-24 \\
\text { years }\end{array}$ & 8.4 & 69.2 & 39.4 & 1.8 & 20.4 & 5.9 & 13.4 & 11.2 & 0.2 & 5.9 & 89.7 & 97.6 & 51.0 \\
\hline $\begin{array}{l}25-44 \\
\text { years }\end{array}$ & 0.6 & 79.4 & 27.6 & 4.3 & 25.0 & 3.4 & 5.9 & 5.7 & 0.0 & 5.5 & 92.7 & 95.5 & 47.3 \\
\hline $\begin{array}{l}45-64 \\
\text { years }\end{array}$ & 0.1 & 91.7 & 21.4 & 2.6 & 14.0 & 0.6 & 1.6 & 0.4 & 0.0 & 1.7 & 89.2 & 99.9 & 37.9 \\
\hline $\begin{array}{l}65-75 \\
\text { years }\end{array}$ & 1.4 & 97.4 & 40.2 & 7.1 & 15.1 & 6.2 & 18.4 & 4.4 & 0.0 & 2.7 & 97.1 & 100.0 & 65.5 \\
\hline Total & 3.1 & 83.1 & 31.0 & 3.5 & 18.4 & 3.3 & 7.6 & 4.3 & 0.0 & 5.5 & 92.0 & 97.9 & 47.6 \\
\hline \multicolumn{14}{|c|}{ Female } \\
\hline $\begin{array}{l}10-17 \\
\text { years }\end{array}$ & 84.0 & 91.0 & 33.8 & 0.1 & 4.7 & 3.8 & 14.4 & 60.7 & 0.1 & 22.4 & 85.2 & 99.8 & 65.9 \\
\hline $\begin{array}{l}18-24 \\
\text { years }\end{array}$ & 89.5 & 94.9 & 48.1 & 0.3 & 7.2 & 4.1 & 7.0 & 96.6 & 0.9 & 7.7 & 86.7 & 99.9 & 66.2 \\
\hline $\begin{array}{l}25-44 \\
\text { years }\end{array}$ & 89.4 & 95.6 & 45.6 & 1.3 & 12.3 & 1.4 & 7.0 & 91.1 & 0.3 & 0.7 & 87.2 & 98.3 & 67.1 \\
\hline $\begin{array}{l}45-64 \\
\text { years }\end{array}$ & 30.6 & 97.7 & 25.4 & 1.4 & 3.5 & 0.7 & 4.1 & 28.5 & 0.3 & 0.6 & 70.6 & 100.0 & 60.8 \\
\hline $\begin{array}{l}65-75 \\
\text { years }\end{array}$ & 11.5 & 99.7 & 32.6 & 2.4 & 1.6 & 0.0 & 12.5 & 5.1 & 0.8 & 0.5 & 98.7 & 100.0 & 79.9 \\
\hline Total & 59.9 & 96.5 & 37.5 & 1.3 & 6.9 & 1.5 & 7.6 & 58.3 & 0.5 & 3.0 & 84.5 & 99.3 & 67.4 \\
\hline
\end{tabular}

nutrients with higher levels of inadequate intake among males aged 10 to 17 . Females aged 10 to 17 had a higher prevalence of inadequate intakes in vitamin $B_{6}$ and vitamin C. The prevalence of inadequacy for folate was high in females of childbearing age (from 10 to 44). The elderly showed high levels of inadequacy for certain micronutrients such as thiamine and vitamin $\mathrm{B}_{6}$.

Three points should be borne in mind when evaluating these results. First of all, the fact that the analysis included the entire sample without excluding underreporters, whose food underreporting contributed to the underestimation of mean energy and nutrient intakes and thus to increased percentages of inadequate intake. In ENCAT 
2002-2003, 41\% of individuals were underreporters, according to the Goldberg method. Additionally, this analysis did not take into account vitamin-mineral supplement intake, which, despite the fact that in Catalonia the consumption $(8.3 \%)$ is not as high as in other countries, their consumption would also have contributed to an increase of certain nutrient values [Serra-Majem et al., 2002b]. Finally, it should also be kept in mind that fortified foods have not totally been considered. The consumption of these foods is relatively 'modern' and the food composition tables used had scarce information on their composition. All this can contribute to results that overestimate low intakes of certain nutrients and thus, the 'problem', in reality, is smaller in dimension.

\section{Overweight and Obesity}

There is evidence that overweight and obesity have related socioeconomic components. In order to analyze the influence of socioeconomic determinants on overweight and obesity prevalence based on BMI and waist circumference (WC), the following variables were used and rearranged according to the following categories [Aranceta et al., 2001; García-Álvarez et al., 2007]: (1) socioeconomic level (SEL): occupation of the subject with low, medium and high classifications; (2) educational level of the subject (ELS) (a) low: primary school incomplete or illiterate ( $<6$ years at school); (b) medium: primary school completed, secondary school or further education (6-12 years of education); (c) high: high school, college or university degree (>12 years of education), and (3) population of residence size $(<10,000$ inhabitants; 10,000-100,000 inhabitants, and $>100,000$ inhabitants). With respect to SEL, significant differences were observed only in females. An inverse association was observed in this group for BMI overweight and WC obesity (table 11.70). ELS showed an inverse relationship with BMI overweight and obesity prevalence, but with differences among ELS groups only being significant in females; this inverse relationship was also observed between ELS and male and female WC obesity (note the high male and female WC obesity rates in the lowest ELS group, 34.3 and 69.5\% respectively) (table 11.70). Regarding the size of the population of residence, the significant difference observed was in female WC obesity.

\section{Adherence to the Mediterranean Diet}

Although a Mediterranean country, Spain has been undergoing a continuous and progressive globalization in its dietary pattern, being of a similar magnitude to other countries in the region. To evaluate the quality of the Catalan diet, an index of adherence to the Mediterranean Diet (MD) was created for the study, which has been explained elsewhere [Serra-Majem et al. $2007 \mathrm{c}$ ]. This new variable was recoded as 0 to $<50$ indicating poor adherence; 50 to $<75$ indicating good adherence, and 75 to 100 , indicating excellent adherence to the Mediterranean Diet. The results showed that $48 \%$ of the Catalan population had a poor MD adherence score (being higher among females, with 52\% showing good adherence versus $46 \%$ among males). Only $3 \%$ of the sample had the highest score (excellent) and the prevalence increased with age (fig. 11.19). In individuals aged 65 to 80 , adhesion to the MD was classified as good in 
Table 11.70. Overweight and obesity prevalence (BMI and WC) in Spanish population by gender and socioeconomic and demographic characteristics (ENCAT 2002-2003)

\begin{tabular}{|c|c|c|c|c|c|c|}
\hline & \multicolumn{2}{|c|}{ BMI overweight ${ }^{a}$} & \multicolumn{2}{|c|}{$\mathrm{BMI}$ overweight ${ }^{\mathrm{a}}$} & \multicolumn{2}{|c|}{ WC obesityc } \\
\hline & $\begin{array}{l}\text { males } \\
\%\end{array}$ & $\begin{array}{l}\text { females } \\
\%\end{array}$ & $\begin{array}{l}\text { males } \\
\%\end{array}$ & $\begin{array}{l}\text { females } \\
\%\end{array}$ & $\begin{array}{l}\text { males } \\
\%\end{array}$ & $\begin{array}{l}\text { females } \\
\%\end{array}$ \\
\hline Overall prevalence & 43.7 & 30.1 & 16.6 & 15.2 & 24.4 & 31.1 \\
\hline \multicolumn{7}{|l|}{ Socioeconomic level } \\
\hline Low & 44.0 & 33.3 & 16.5 & 19.9 & 25.8 & 39.0 \\
\hline Medium & 45.0 & 28.2 & 16.4 & 11.2 & 21.5 & 26.0 \\
\hline High & 42.6 & 25.2 & 16.5 & 11.2 & 26.3 & 19.3 \\
\hline Differences & ns & s & ns & s & ns & s \\
\hline \multicolumn{7}{|c|}{ Education level of subject } \\
\hline Low & 50.5 & 45.5 & 21.2 & 36.6 & 34.3 & 69.5 \\
\hline Medium & 43.9 & 32.5 & 17.6 & 15.7 & 25.7 & 34.1 \\
\hline High & 41.6 & 20.5 & 13.6 & 5.7 & 19.0 & 11.6 \\
\hline Differences & ns & s & ns & s & $\mathrm{s}$ & s \\
\hline \multicolumn{7}{|c|}{ Population size (inhabitants) } \\
\hline$<10,000$ & 46.6 & 27.8 & 16.7 & 18.0 & 26.2 & 38.6 \\
\hline $10,000-100,000$ & 44.6 & 33.6 & 15.7 & 15.1 & 24.2 & 32.1 \\
\hline$>100,000$ & 41.8 & 28.8 & 17.1 & 13.8 & 23.7 & 26.8 \\
\hline Differences & ns & ns & ns & ns & ns & $s$ \\
\hline $\begin{array}{l}\mathrm{BMI}=\text { body mass inc } \\
{ }^{\mathrm{a}} \mathrm{BMI} \geq 25 \text { to }<30 \mathrm{~kg} / \\
\mathrm{s}=\mathrm{X}^{2} \text { test significant }\end{array}$ & $\begin{array}{l}N C=W \\
\text { BMI } \geq 3 \\
\text { erences }\end{array}$ & $\begin{array}{l}\text { cumferen } \\
n^{2} ;{ }^{d} W C \geq \\
{ }^{2} \text { test sig }\end{array}$ & $\begin{array}{l}\mathrm{cm} f \mathrm{C} \\
\text { ant di }\end{array}$ & Ind $\geq 88$ & r femal & \\
\hline
\end{tabular}

around $70 \%$ of the sample. On the other hand, less than $30 \%$ of individuals younger than age 24 had a good score. Referring to the effect that socioeconomic level had on $\mathrm{MD}$ adherence, no clear differences were seen between high and low socioeconomic levels. The high number of individuals with poor adherence to a MD pattern, especially amongst the youngest age groups, shows the urgency for developing nutritional education campaigns that educate and incite the population to adopt and maintain dietary patterns that are as close as possible to the traditional MD. Apart from interventions to improve knowledge about the benefits of the MD on health and the relationship between healthy habits and the quality of life, it is also necessary to develop 


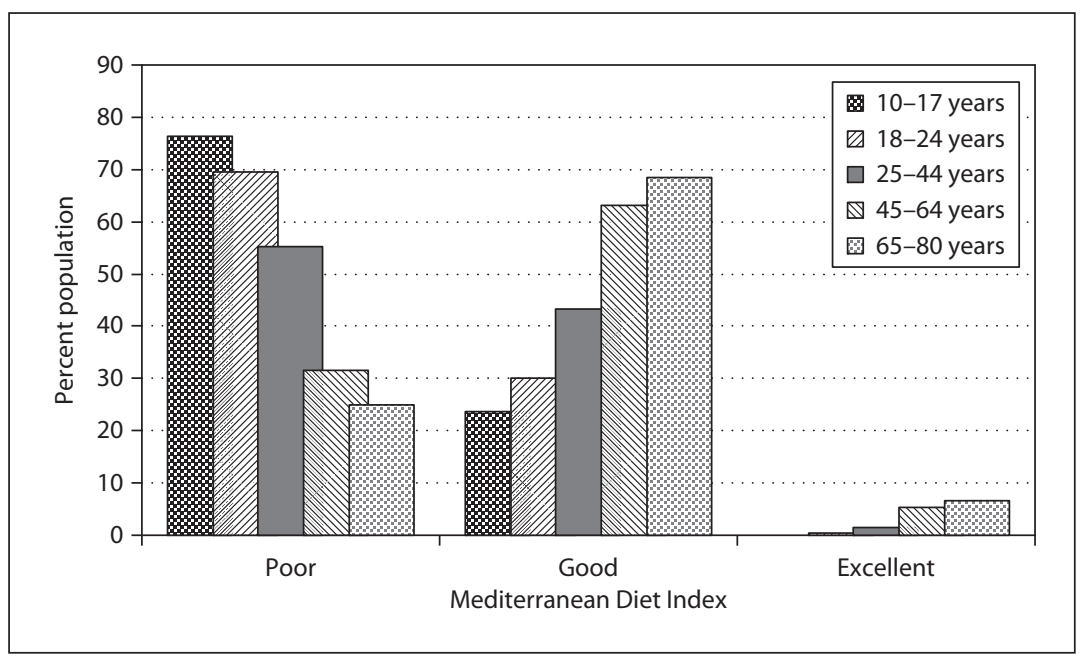

Fig. 11.19. Adherence to the Mediterranean diet pattern according to age group.

tools to motivate and provide the means for the population to adopt such healthy patterns. Otherwise the numerous benefits associated to the MD will not be reflected in the health of individuals comprising future generations of the Spanish population.

\subsection{Sweden ${ }^{24}$}

\section{Introduction}

In Sweden the National Food Administration is responsible for the nutrition recommendations, food-based dietary guidelines and also conducts food consumption surveys. The Swedish National Public Health Institute is responsible for monitoring and coordinating the implementation of the national public health policy, e.g. through national surveys covering areas such as living conditions, physical and mental health, socioeconomic conditions, dietary habits, and physical activity.

\section{Dietary Habits}

\section{Adults}

The second nationwide food consumption survey, Riksmaten 1997-98 [Becker and Pearson, 2002], was a follow-up of the previous survey (Hulk) carried out in 1989

\footnotetext{
${ }^{24}$ Becker W; National Food Administration, Uppsala, Sweden. E-Mail wulf.becker@slv.se
} 
Table 11.71. Mean daily intake of energy, macronutrients and dietary fiber among Swedish adults 18-74 years in 1997-1998

\begin{tabular}{llll}
\hline & $\begin{array}{l}\text { Women } \\
\mathrm{n}=626\end{array}$ & $\begin{array}{l}\text { Men } \\
\mathrm{n}=589\end{array}$ & NNR 2004 \\
\hline Energy, MJ/day & 7.8 & 9.9 & - \\
\hline Protein, \%E & 16 & 15.5 & $10-20$ \\
\hline Fat, \%E & 34 & 34 & $25-35$ \\
\hline SFA, \%E & 14.2 & 14.6 & $\sim 10^{\mathrm{b}}$ \\
\hline MUFA, \%E & 12.3 & 12.6 & $10-15$ \\
\hline PUFA, \%E & 4.7 & 4.6 & $5-10$ \\
\hline Carbohydratesc, \%E & 47.5 & 46 & $50-60$ \\
\hline Sucrose, \%E & 9.0 & 8.5 & $\leq 10^{\mathrm{d}}$ \\
\hline Dietary fiber, g/day & 16.4 & 18.1 & $25-35$ \\
\hline g/MJ & 2.1 & 1.8 & $\sim 3$ \\
\hline Alcohol, \%E & 2.9 & 3.8 & $\leq 5$ \\
\hline Alcohol, g/day & 7.6 & 12.7 & \\
\hline
\end{tabular}

${ }^{a}$ Nordic Nutrition Recommendations. ${ }^{b}$ Including trans-fatty acids. ${ }^{c}$ Excluding dietary fiber.

${ }^{\mathrm{d}}$ As added sugars.

[Becker, 1994], using similar design and methods. In Riksmaten a representative sample of 2,000 subjects aged 18 to 74 years were asked to fill in a precoded 7-day record book and a questionnaire with information on body weight, height, education, occupation, physical activity, smoking habits and other lifestyle factors. About 1,200 persons completed the study with a participation rate of approximately $60 \%$. Participation was lower in larger cities and surrounding areas than in rural areas and lower among young as well as older households.

The dietary fat content was on average $34 \% \mathrm{E}$, which was in the close to the upper bound of the recommended range (table 11.71). The differences among sex and age groups were generally small. The proportion of saturated fatty acids was $14 \% \mathrm{E}$, and together with about $1 \% \mathrm{E}$ trans-fatty acids [Hulshof et al., 1999] intake is considerably higher than the recommended level of about $10 \% \mathrm{E}$. Carbohydrates provided on average 47 and $46 \% \mathrm{E}$ among women and men, respectively. The proportion of sucrose in the diet was higher among women than among men. The average intake of dietary fiber was $17-18 \mathrm{~g} /$ day, corresponding to $1.8 \mathrm{~g} / \mathrm{MJ}$ among men and $2.0 \mathrm{~g} / \mathrm{MJ}$ among women, well below the recommended level of about $3 \mathrm{~g} / \mathrm{MJ}$. Alcohol provided on average 4 and 3\%E among men and women, respectively. 
The average daily energy intake was 7.8 MJ/day in women and 9.9 MJ/day in men. An evaluation of underreporting using the Goldberg technique showed that around $20 \%$ of the subjects reported an energy intake below cut-off. Underreporters had a somewhat lower fat proportion and somewhat higher carbohydrate proportion compared to acceptable reporters.

The average intakes of vitamins and minerals were generally close to or above the recommended daily intakes (RI). The intake of vitamin $\mathrm{D}$ was lower than recommended for older persons, while the intake of folate corresponds to about $75 \%$ of the RI. The intake of iron among women in reproductive ages was only two thirds of the RI. The iron intake has decreased due to the termination of iron fortification of flour in 1995 . The selenium intake was about $75 \%$ of the RI. The intake of sodium (excl. salt added at the table) was 2.8 and $3.6 \mathrm{~g} /$ day among women and men respectively. This corresponds to 7 and $9 \mathrm{~g}$ salt per day, respectively, or about $9 \mathrm{~g} / 10 \mathrm{MJ}$. Intake was higher compared to recommended intakes.

Pronounced age differences in dietary habits were seen. Older people ate more of 'traditional' foods like potatoes, root vegetables, fish, offal and blood dishes, porridge and sweet bakery products, while young people eat and drink more of 'modern' foods, e.g. pasta, rice, pizza, sweets, nuts and snacks, and soft drinks. Older people also ate more fruit and vegetables. Women ate more fruit and vegetables than men did. There were generally small differences in the nutrient composition of the diet between men and women.

Low education was among men associated with a lower consumption of fruit and vegetables and a higher intake of fat spreads. Men with short education consumed a diet with higher fat content compared to high-educated men (35 and 33\%E, respectively). No such difference was observed for women. High education was linked to a higher alcohol intake in both sexes.

Smokers, especially men, had poorer dietary habits than non-smokers, with e.g. a lower consumption of fruit and vegetables. Among men smoking was associated with a higher fat content of the diet.

\section{Children}

A national food survey was carried out in 2003 comprising 4-year-old children and grade 2 and grade 5 schoolchildren. The method was an open-ended and estimated food diary over 4 consecutive days. All days were evenly represented in the survey. The food diary was filled in by the children or with the help of parents or carer. Portion sizes and amounts of all food and drink consumed by the children were estimated with the help of a picture book. It was also noted when and where food was eaten as well as the fat content, mode of preparation, certain additives and brand name. Intake of supplements and level of physical activity were also recorded. In addition, an optically readable questionnaire contained questions about weight, height, parents' education and profession, ethnic background and regional residence. 
Table 11.72. Intake of energy, macronutrients (as \%E) and dietary fiber (per MJ) for 4-year-old children and in 2 nd and 5 th grade

\begin{tabular}{|c|c|c|c|c|c|}
\hline & $\begin{array}{l}4 \text { years } \\
n=590\end{array}$ & $\begin{array}{l}\text { Grade } 2 \\
n=889\end{array}$ & $\begin{array}{l}\text { Grade } 5 \\
\mathrm{n}=1,016\end{array}$ & All & $\begin{array}{l}\text { NNR } \\
2004\end{array}$ \\
\hline Energy, MJ/day & 6.3 & 7.6 & 7.4 & & \\
\hline Protein, \%E & 14 & 15 & 16 & 15 & $10-20$ \\
\hline Fat, \%E & 32 & 31 & 32 & 32 & $25-35$ \\
\hline SFA, \%E & 14 & 14 & 14 & 14 & $10^{\mathrm{a}}$ \\
\hline Trans-fatty acids, \%E & 0,9 & 1.0 & 1.0 & 0.9 & \\
\hline MUFA, \%E & 11 & 11 & 12 & 11 & $10-15$ \\
\hline PUFA, \%E & 3.7 & 3.6 & 3.7 & 3.7 & $5-10$ \\
\hline $\mathrm{n}-3$ fatty acids, \%E & 0.7 & 0.6 & 0.7 & 0.7 & 1 \\
\hline n-6 fatty acids, \%E & 3.0 & 2.9 & 3.0 & 2.9 & 4 \\
\hline Carbohydrates $^{\mathrm{b}}, \% \mathrm{E}$ & 54 & 53 & 53 & 53 & $50-60$ \\
\hline Monosaccharides, \%E & 8.0 & 6 & 6 & 6 & \\
\hline Disaccharides, \%E & 21 & 19 & 18 & 19 & \\
\hline Sucrose, \%E & 14 & 13 & 12 & 13 & $\leqq 10^{c}$ \\
\hline Fiber, g/MJ & 1.8 & 1.8 & 1.7 & 1.8 & \\
\hline
\end{tabular}

${ }^{\mathrm{a}}$ Including trans-fatty acids. ${ }^{\mathrm{b}}$ Excluding dietary fiber. ${ }^{\mathrm{c}}$ As added sugars.

Four-year-old children were randomly sampled from a stratified sample of municipalities representative for Sweden. Schoolchildren were sampled on the basis of school classes. The survey took place during spring and autumn 2003. The sample size of 4-year-old children was 924 of which 590 fulfilled the survey. 1,209 children in grade 2 were sampled of which 889 fulfilled the survey and 1,290 children in grade 5 were sampled of which 1,016 fulfilled the survey. 4-year-old children dropped out due to time constraints or that day-care institutions did not want to participate. Schoolchildren dropped out because it was too difficult, they forgot or did not have time.

The average intake of energy was $6.3 \mathrm{MJ} /$ day $(1,500 \mathrm{kcal}), 7.6 \mathrm{MJ}(1,820 \mathrm{kcal})$ and $7.4 \mathrm{MJ}(1,760 \mathrm{kcal})$ for 4 -year-old children, grade 2 and grade 5 children, respectively (table 11.72). The reported average energy intake for 4 -year-old children was 108\% of the calculated reference value for that age and the average reference value for grade 2 children was $81 \%$. For grade 5 children, the reported average energy intake was 76 and $86 \%$ respectively of the calculated reference value for a moderate and for a low physical activity level. 
Table 11.73. BMI, overweight and obesity among adults in 2007

\begin{tabular}{lllllll}
\hline & \multicolumn{1}{l}{$19-64$ years } & & & $65-84$ years & \\
& women & men & & women & men \\
\hline Height, $\mathrm{cm}$ & 166.3 & 180.0 & & 162.9 & 176.2 \\
\hline Weight, $\mathrm{kg}$ & 68.3 & 84.1 & 69.4 & 81.4 \\
\hline $\mathrm{BMI}$ & 24.7 & 26.0 & 26.2 & 26.2 \\
\hline $\mathrm{BMI} \geq 25, \%$ & 24.8 & 43.5 & 34.9 & 51.1 \\
\hline $\mathrm{BMI} \geq 30, \%$ & 12.7 & 12.1 & 18.0 & 10.4 \\
\hline
\end{tabular}

The children's diet had a satisfactory distribution of energy between protein (1416 energy percent, $\% \mathrm{E})$, fat $(31-32 \% \mathrm{E})$ and carbohydrates $(53-54 \% \mathrm{E})$ but the quality of fat and carbohydrates were not (table 11.72). Generally, children consumed too much added sugars $(13-15 \% \mathrm{E})$, saturated fatty acids $(14 \% \mathrm{E})$ but not enough of polyunsaturated fatty acids $(3.6-3.7 \% \mathrm{E})$ and dietary fiber $(1.8 \mathrm{~g} / \mathrm{MJ})$. Trans-fatty acids contributed to $0.9 \% \mathrm{E}$.

Intake of vitamin $\mathrm{D}$ was low compared to recommended intakes. Sodium intake corresponded to 5-7 g salt per day, and was close to the intake of adults when expressed per $10 \mathrm{MJ}(8.1-9.4 \mathrm{~g} / 10 \mathrm{MJ})$. Children received most of the salt from meals with meat and meat products.

The fruit and vegetable intake was on average 225, 239 and $193 \mathrm{~g} /$ day for 4-yearold children, grade 2 and grade 5, respectively. This is about half of the recommended amount of $400 \mathrm{~g} /$ day fruit and vegetables for children aged 4 to 10 years. The children ate fish 1-2 times per week and sausages 2-3 times per week.

Approximately $25 \%$ of the consumed daily energy originated from foodstuffs like soft drinks, sweets, crisps, ice cream, desserts, and cakes and biscuits. The children consumed on average 100-200 g of sweets per week distributed on 3-5 occasions. $13 \%$ of the children ate sweets daily. The children ate buns, cakes and biscuits 3-5 times per week. 4-year-old children ate ice cream 2-3 times per week and schoolchildren 1-2 times per week. The children drank on average $200 \mathrm{ml}$ soft drinks every day but $10 \%$ of the schoolchildren drank daily $500 \mathrm{ml}$ or more. Altogether, these foods provide the largest sources of added sugars, fat, saturated fatty acids and trans-fatty acids.

No large differences were seen in food choice or nutrient intake between children from different socioeconomic groups. Children from parents with a university education consumed more fruits and vegetables and had a diet with a slightly higher nutrient density. Children from parents with a non-Swedish background ate more fruits and vegetables but drank less milk. 
Table 11.74. Mean height, weight, BMI, and proportion of overweight and obesity among children in different age groups, 2003 [source: from Enghardt Barbieri et al., 2006]

\begin{tabular}{|c|c|c|c|c|c|c|}
\hline & \multicolumn{2}{|c|}{4 years ( 4.2 years) } & \multicolumn{2}{|c|}{ Grade 2 (8.6 years) } & \multicolumn{2}{|c|}{ Grade 5 (11.7 years) } \\
\hline & girls & boys & girls & boys & girls & boys \\
\hline Height, cm (SD) & $106(5.6)$ & $106(4.7)$ & $135(6.8)$ & $135(6.2)$ & $152(8.2)$ & $151(7.6)$ \\
\hline Weight, kg (SD) & $18(2.4)$ & $18(2.7)$ & $31(6.2)$ & $32(5.7)$ & $42(9.0)$ & $42(8.7)$ \\
\hline BMI (SD) & $16(1.8)$ & $16(1.7)$ & $17(2.5)$ & $17(2.2)$ & $18(2.8)$ & $18(2.6)$ \\
\hline IsoBMI $\geq 25, \%$ & 18 & 19 & 20 & 19 & 17 & 15 \\
\hline IsoBMI $\geq 30, \%$ & 2 & 4 & 3 & 3 & 1 & 2 \\
\hline
\end{tabular}

\section{Overweight and Obesity}

\section{Adults}

The proportion of overweight increased in all socioeconomic groups from the beginning of the 1980s. The trend has leveled off somewhat after year 2000 and a decrease has been seen among well-educated adults. Social differences are seen for both sexes. Data on self-reported weight and height among Swedish adults in 2007 are given in table 11.73. A higher proportion of overweight/obesity was seen among women with short education, with no differences among men.

\section{Children}

Overweight and obesity increased among children aged 10 years during 1970-2000 (National Institute of Public Health, Sweden) and among adolescents (16 to 19 years) during 1988-2000/1 [Statistics Sweden, 2007]. The trend seems to have leveled off after year 2000. There are no time trends for younger children.

Between 17 and $23 \%$ of the children participating in the national food consumption survey 2003 were classified as overweight (IsoBMI >25), of which $1-4 \%$ were obese (IsoBMI >30) (see table 11.74).

\section{Smoking}

Smoking has declined in all socioeconomic groups and both sexes since the beginning of the 1980s. For men, the decline has been more rapid than for women. For women, the proportion of smokers started to decline considerably later than for men. Data on smoking habits for 2007 are shown in figure 11.20. Daily smoking was more common among women than men 16 to 29 and 45 to 64 years, among men and women with short education, and among men and women born in other European countries (except Nordic) compared to Swedish-born. 
Fig. 11.20. Smoking habits among Swedish adults (16-84 years). Data from 2007.

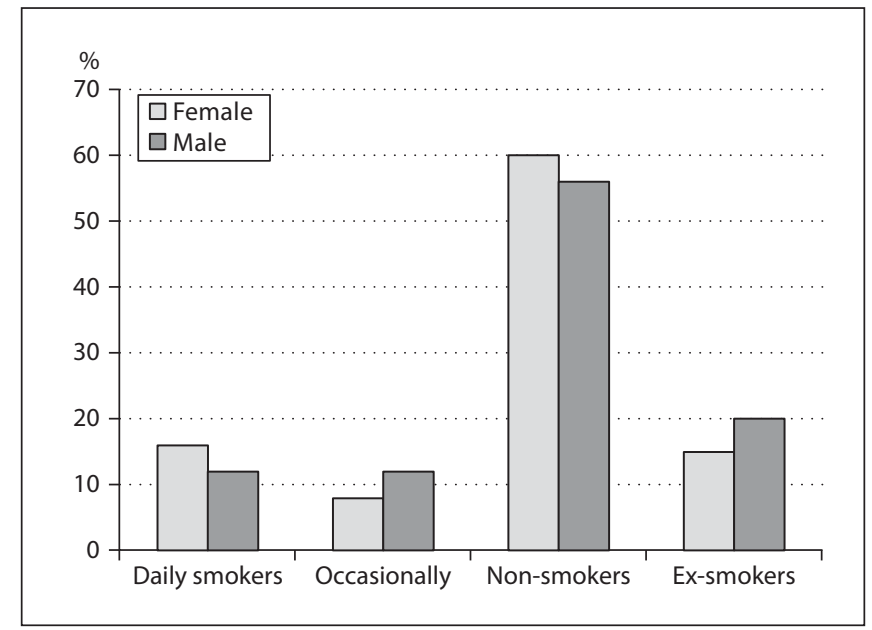

\section{Alcohol Habits}

Data from food consumption surveys indicate that alcohol intake increased among adults during 1990s. In the national public health survey alcohol habits were assessed using the AUDIT test. Risk consumption was assessed based on how often alcohol is consumed, how many glasses on a normal occasion, and how often a fairly large amount drunk on one and the same occasion. In addition, those who have been drunk 2-3 times a month or more are included in the risk consumption group. Results from 2007 show that the majority of the adult population has acceptable alcohol habits, or never or seldom drinks alcohol (abstainers) (fig. 11.21). However, about 10\% of women and $17 \%$ of men were characterized as 'heavy drinkers', i.e. frequent alcohol use and/or intoxication. A larger proportion of young women and men (16 to 29 years) have risky alcohol habits compared to older age groups.

\section{General Comments}

Certain improvements in the dietary pattern in the Swedish population have occurred since the late 1980s, including an increased intake of fruit and vegetables and an improved fat quality among adults. However, the proportion of saturated fat is still above recommended levels and intake of polyunsaturated below, especially among children. Intake of refined (added) sugars is high among children, while intake of dietary fibers is in general low. Intake of trans-fatty acids has decreased during the last decade [Becker et al., 2008]. Factors like smoking and low education are associated with poorer dietary habits, especially among men, while a certain equalization seems to have occurred among women in this respect. 
Fig. 11.21. Alcohol habits among Swedish adults (16-84 years). Data from 2007.

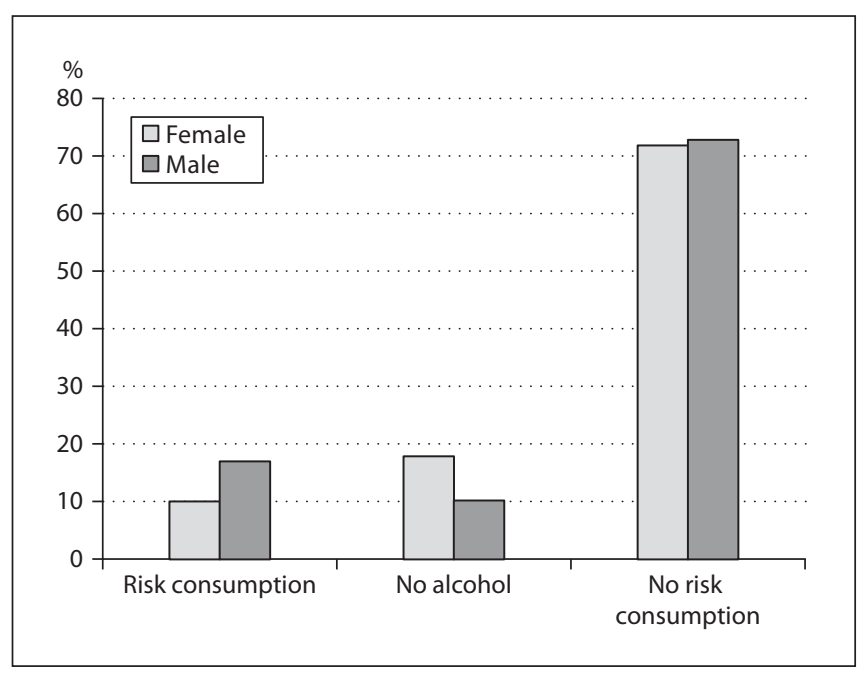

Desirable dietary changes include increased consumption of fruit, berries, vegetables, whole grain cereals, and fish. Lower-fat alternatives of meat and dairy products should be prioritized, while high-fat and sugary foods like bakery products, soft drinks and sweets should be consumed in moderation. Soft or fluid edible fats should replace solid, hard fats to a larger degree.

The nutrient intake of children is similar to that of adults and therefore the food based dietary recommendations aimed at adults are also largely valid for children: especially to reduce intake of soft drinks, sweets, crisps, cakes and biscuits and increase intake of fruits and vegetables and fish.

An adequate physical activity in the population is equally important to facilitate weight balance. While smoking has decreased in most age groups the trend among young girls should be addressed. Problematic alcohol habits are also more common in teenagers and young adults.

\subsection{The Netherlands ${ }^{25}$}

\section{Methods and Results of National Surveys on Nutrition and Health: The Netherlands}

\section{Dutch National Food Consumption Surveys}

Collection of periodic data on food consumption and nutritional status of the Dutch population has been carried out in 1987/1988, 1992 and 1997/1998. Due to

\footnotetext{
${ }^{25}$ Fransen H, van Rossum C, Ocké M; National Institute for Public Health and the Environment,
} Bilthoven, The Netherlands. E-Mail heidi.fransen@rivm.nl 
the changing policy needs, sociodemographic developments, trends in food habits as well as developments in research methods the need for a new method for food and nutrition surveys has arisen. Since 2003, the Dutch National Food Consumption Surveys (DNFCS) have been redesigned into a modular system, consisting of a core module for the Dutch population and other modules for specific groups within the population, such as ethnic groups and young children. The new method was piloted among young adults in 2003 and young children as a specific group were surveyed (2005/2006). From 2007 onwards the DNFCS core survey is ongoing, obtaining data on the food consumption of men and women in The Netherlands, aged 7 to 69 years. General information on the DNFCS and its specific surveys, including the objectives, methods, planning and main results as well as detailed information on the food consumption surveys conducted from 2003 onwards are available (www.rivm. nl/vcp_en).

\section{Survey Methodology}

Nutrient and energy intake in this ENHR 2009 report was calculated for the three most recent food consumption surveys in The Netherlands, DNFCS-3 (1997-1998), DNFCS-young adults (2003) and DNFCS-young children (2005-2006). A brief overview of these studies is given in table 11.75 .

For a correct evaluation of nutrient intake with regard to nutrition standards, habitual intake was calculated. Habitual intake is estimated from the actual intake on two independent days, using the ISU method [Nusser et al., 1996]. Median intake is compared with adequate intake (AI) or recommended dietary allowances (RDA) for a qualitative evaluation of intake or with EAR, if available, for prevalence of inadequate intakes.

\section{Nutrition Indicators}

In this chapter, the main nutritional issues in The Netherlands will be discussed based on the two most recent food consumption surveys [Ocké et al., 2005, 2008]. If available, information on the food sources of the specific nutrients is given.

\section{Vitamins and Minerals}

When comparing intake of nutrients from foods with nutritional guidelines (table 11.76) intake of most vitamins and minerals were adequate in young children, except for vitamin D (children 2-6 years) and folate in children of 4-6 years. For these vitamins no statement could be given on prevalence of inadequate intakes, because the median intake was below AI (adequate intake). A low vitamin D and folate intake in Europe was also reported in the first ENHR 2004 [Elmadfa et al., 2004] and in the current ENHR 2009 report. Vitamin D intake in European children was generally low and folate intake was low in most children. 
Table 11.75. Dutch National Food Consumption Surveys (DNFCS) reported in ENHR 2009

\begin{tabular}{|c|c|c|c|}
\hline Survey & DNFCS-3 & DNFCS-young adults & DNFCS-young children \\
\hline Reference & $\begin{array}{l}\text { [Hulshof and van Staveren, } \\
\text { 1991] }\end{array}$ & [Ocké et al., 2005] & [Ocké et al., 2008] \\
\hline Year of data collection & 1997-1998 & 2003 & $2005-2006$ \\
\hline Age group & $1+$ years & 19-30 years & $2-6$ years \\
\hline Sampling frame & $\begin{array}{l}\text { Households in consumer's } \\
\text { panel }\end{array}$ & $\begin{array}{l}\text { Individuals in consumer's } \\
\text { panel }\end{array}$ & $\begin{array}{l}\text { Individuals in consumer's } \\
\text { panel }\end{array}$ \\
\hline Sampling method & Stratified random & Stratified random & Stratified random \\
\hline Number & 5,958 & 750 & 1,279 \\
\hline Response rate & $71 \%$ & $42 \%$ & $78 \%$ \\
\hline $\begin{array}{l}\text { Dietary assessment } \\
\text { method }\end{array}$ & $\begin{array}{l}\text { 2-day diet record } \\
\text { (consecutive days) +FFQ }\end{array}$ & $\begin{array}{l}2 \cdot 24 \mathrm{~h} \text { diet recall by } \\
\text { trained dieticians } \\
\text { (non-consecutive days) }+ \\
\text { general questionnaire }\end{array}$ & $\begin{array}{l}\text { Two 1-day diet records } \\
\text { (non-consecutive days), + } \\
\text { general questionnaire }\end{array}$ \\
\hline $\begin{array}{l}\text { Dietary assessment } \\
\text { software }\end{array}$ & - & EPIC-Soft [Slimani et al., 2000] & $\begin{array}{l}\text { Data entry with EPIC-Soft by } \\
\text { dietician [Slimani et al., 2000] }\end{array}$ \\
\hline $\begin{array}{l}\text { Exclusions based on } \\
\text { misreporting }\end{array}$ & None & None & None \\
\hline $\begin{array}{l}\text { Food composition } \\
\text { database }\end{array}$ & $\begin{array}{l}\text { NEVO } 1996 \text { [Stichting NEVO, } \\
\text { 1996] }\end{array}$ & $\begin{array}{l}\text { NEVO } 2001 \text { [Stichting NEVO, } \\
\text { 2001] }\end{array}$ & $\begin{array}{l}\text { NEVO } 2006 \text { [Stichting NEVO, } \\
\text { 2006] }\end{array}$ \\
\hline $\begin{array}{l}\text { Dietary supplement } \\
\text { database }\end{array}$ & none & $\begin{array}{l}\text { NES } 2004 \text { [Buurma-Rethans } \\
\text { et al., 2008] }\end{array}$ & $\begin{array}{l}\text { NES } 2006 \text { [Buurma-Rethans } \\
\text { et al., 2008] }\end{array}$ \\
\hline $\begin{array}{l}\text { Software to estimate } \\
\text { habitual intake }\end{array}$ & - & $\begin{array}{l}\text { ISU method [Nusser et al., } \\
\text { 1996] }\end{array}$ & $\begin{array}{l}\text { ISU method [Nusser et al., } \\
\text { 1996] }\end{array}$ \\
\hline
\end{tabular}

Because for vitamin E the Dutch guidelines were set in 1989 [Voedingsraad, 1992], the more recent American EAR (estimated average requirement) was used to estimate a prevalence of inadequate intake, which varied between $16 \%$ for 2 - to 3 -yearold boys to $26 \%$ for 4 - to 6-year-old girls. Also for iron, if the EAR was used, $1-2 \%$ of young children had an inadequate intake.

For young adult men in The Netherlands, intake of most vitamins and minerals was adequate, except for folate and vitamin E. 35\% of young men had an inadequate folate intake when compared with the EAR of $200 \mu \mathrm{g} /$ day. In women, $85 \%$ had an inadequate intake, median folate intake in women was $154 \mu \mathrm{g} /$ day. In chapter 7 of this report it is shown that folate recommendations were not met by adults in many countries. For comparison of vitamin E intake, US guidelines were used. Vitamin E intake 
Table 11.76. Nutritional guidelines: Al (adequate intake), RDA (recommended dietary allowances) or EAR (estimated average requirement)

\begin{tabular}{|c|c|c|c|c|c|}
\hline & $\begin{array}{l}\text { Folate }^{1} \\
\mu \mathrm{g} / \text { day }\end{array}$ & $\begin{array}{l}\text { Vitamin } D^{2} \\
\mu \mathrm{g} / \text { day }\end{array}$ & $\begin{array}{l}\text { Vitamin } \mathrm{E}^{3} \\
\mathrm{mg} / \mathrm{day}\end{array}$ & $\begin{array}{l}\text { Calcium }^{2} \\
\mathrm{mg} / \text { day }\end{array}$ & $\begin{array}{l}\text { Iron }{ }^{4} \\
\mathrm{mg} / \text { day }\end{array}$ \\
\hline Children $2-3$ years & $85(\mathrm{Al})$ & $5-10(\mathrm{Al})$ & 5 (EAR) & $500(\mathrm{Al})$ & 3 (EAR) \\
\hline Children 4-6 years & 150 (Al) & $2.5-5(\mathrm{Al})$ & 6 (EAR) & 700 (Al) & 4.1 (EAR) \\
\hline Adults $19-30$ years & 200 (EAR) & $2.5-5(\mathrm{Al})$ & 12 (EAR) & $1,000(\mathrm{Al})$ & $\begin{array}{l}6 \text { (men) } \\
8.1 \text { (women) } \\
\text { (EAR) }\end{array}$ \\
\hline
\end{tabular}

${ }^{1}$ Health Council of the Netherlands, 2003. ${ }^{2}$ Health Council of the Netherlands, 2000. ${ }^{3}$ IOM, 2000. ${ }^{4}$ IOM, 2001.

was inadequate in $35 \%$ of young men and $73 \%$ of young women. Calcium intake was slightly below AI for women: median intake was $960 \mathrm{mg} /$ day while the AI is $1,000 \mathrm{mg} /$ day. Also, iron intake was inadequate for $23 \%$ of the young women. Most important sources of iron were cereal products and meat products. Calcium was mostly provided by dairy products and folate by cereals, vegetables and milk products.

\section{Role of Supplements}

In total, $43 \%$ of the children used a kind of dietary supplement on at least one of both recording days. The use in 2- to 3-year-olds (62\%) was twice as high as in 4- to 6-yearolds (30\%). Vitamin D supplements were the most used in 2- to 3-year-olds, and 1 out of 5 children used multivitamins/mineral supplements. In The Netherlands, supplementation with vitamin $D$ containing supplements is advised for children until the age of 4 . However, only 3 in 5 children received a supplement containing vitamin D [Ocké, 2008].

The contribution of supplements to nutrient intake in children varied from 0 to $24 \%$ and was highest for vitamin D (24\%) and folic acid (12\%). Dietary supplement use increased the mean habitual micronutrient intake. Median habitual intake of vitamin D was still below AI for 2- to 6-year-old children and intake of folate was still low for the 4- to 6-year-old children when nutrient intake from dietary supplements was included.

Intake of zinc and folic acid exceeded UL in some children when looked at intake from food and dietary supplements. Fortified foods were also an important source of nutrients, especially for vitamin $B_{6}(19 \%)$ and vitamin C (29\%). In young adults, $27 \%$ used dietary supplements on one or both survey days. Mean calcium intake increased in female supplement users, reaching a total intake above the recommended 1,000 $\mathrm{mg} /$ day [Ocké et al., 2005]. 


\section{Macronutrients}

The diet of children is adequate in terms of the proportions of total fat, carbohydrates and protein. Over half of the men and women 19 to 30 years of age had a food pattern with less than $35 \% \mathrm{E}$ fat. The fatty acid composition of the diet of young children as well as the young adults was unfavorable. Intake of saturated fatty acids was higher than recommended $(10 \% \mathrm{E})$ in a majority of the 4 - to 6 -year-old children $(85 \%$ of boys and $92 \%$ of girls) and intake of fish fatty acids (based on fish consumption) was low. Among the young adults intake of saturated fatty acids was above recommendations in both men and women, only $11 \%$ of men and $6 \%$ of women met the recommendation $(<10 \% \mathrm{E})$. The main sources of saturated fatty acids were dairy products, meat products, fat and cakes for both the children and the young adults.

Almost two thirds of the women and half of the men had a trans-fatty acid intake above the recommended maximum of $1 \% \mathrm{E}$. This was also seen in the 4- to 6-year-old children (10\%). The main sources were dairy products (including cheese) and cakes in children and snacks (cakes, pies and salty biscuits) and dairy products in young adults. In addition, as was the case for many European children, fiber intake was low in most of the Dutch children, median intake was below AI. Cereal products, fruits, nuts and olives, and potatoes and other tubers were the most important fiber sources for these children. In line with the low fiber intake, bread, potatoes and fruit intake was below recommended amounts (table 11.77).

\section{Vegetables and Fruit}

In children, intake of vegetables and fruit was low. Only 1 in 4 children met the fruit recommendations, while none of the 4- to 6-year-olds, and 1 in 5 2- to 3-year-olds met the vegetable recommendations. Also at the older ages the recommendation was not met by many persons; less than $10 \%$ of young adults met the dietary recommendations for vegetables and fruit. None of the women met the recommended daily vegetable intake of $150 \mathrm{~g}$.

\section{Energy Balance}

Obesity is an increasing problem around the world; in The Netherlands the problem is also increasing. In $2007,14-30 \%$ of female adults in The Netherlands were overweight or obese and $18-48 \%$ of the males [CBS Statline, 2007]. The recent survey on young children indicated that 1 in 7 children of 2 to 6 years was overweight or obese [Ocké, 2008].

\section{Discussion}

The studies among the young children and young adults have shown that the intake of most micronutrients were adequate. However, in The Netherlands the intake of folate is low [Health Council of The Netherlands, 2008]. In children also intake of vitamin D 
Table 11.77. Food-based dietary guidelines in The Netherlands [Voedingscentrum, 2009]

\begin{tabular}{|c|c|c|c|c|}
\hline Food group & $\begin{array}{l}\text { Children } \\
1-3 \text { years }\end{array}$ & $\begin{array}{l}\text { Children } \\
4-8 \text { years }\end{array}$ & $\begin{array}{l}\text { Men } \\
19-50 \text { years }\end{array}$ & $\begin{array}{l}\text { Women } \\
19-50 \text { years }\end{array}$ \\
\hline Bread, number of slices (g) & $2-3(70-105)$ & $3-4(105-140)$ & $7(245)$ & $6(210)$ \\
\hline $\begin{array}{l}\text { Potatoes (or rice, pasta or } \\
\text { legumes), g }\end{array}$ & $50-100$ & $100-150$ & 250 & 200 \\
\hline Vegetables, g & $50-100$ & $100-150$ & 200 & 200 \\
\hline Fruits, $\mathrm{g}$ & 150 & 150 & 200 & 200 \\
\hline Dairy products, ml & 300 & 400 & 450 & 450 \\
\hline Cheese, $\mathrm{g}$ & 10 & 10 & 30 & 30 \\
\hline $\begin{array}{l}\text { Meat (products), fish, chicken, } \\
\text { egg or other meat replacement } \\
\text { products, g }\end{array}$ & $50-60$ & $60-80$ & $100-125$ & $100-125$ \\
\hline Low-fat spread, g & $10-15$ & $15-20$ & 35 & 30 \\
\hline Cooking fat, $\mathrm{g}$ & 15 & 15 & 15 & 15 \\
\hline Drinks (including milk), liters & 0.75 & 1 & $1.5-5$ & $1.5-2$ \\
\hline
\end{tabular}

from food is low. This intake is not sufficiently compensated by dietary supplements, as many young children do not receive the advised vitamin $\mathrm{D}$ supplementation.

Dutch young women do not meet the iron intake recommendations. The implications of this low iron intake should be further investigated. Furthermore, intake of vitamin E was below recommendations in both children and adults in The Netherlands. US guidelines were used for evaluation of vitamin $\mathrm{E}$ intake because the Dutch dietary reference intakes for vitamin E were set in 1989 [Voedingsraad, 1992]. Therefore, the Health Council advises an assessment of the Dutch micronutrient dietary reference values to values applied in the USA, Australia and New Zealand. From this assessment it was concluded that the vitamin $\mathrm{E}$ intake of young adults is in order. It is however advised to investigate the low intake of vitamin $\mathrm{E}$ in children [Health Council of The Netherlands, 2009].

Intake of trans-fatty acids has improved in The Netherlands, but intake of saturated fatty acids is still too high and should be reduced. These results are in line with previous DNFCS among the whole population [Hulshof and van Staveren, 1991] and the results for other European populations in chapter 7 of the present report. This is also the case for the low consumption of fruit and vegetables in both children and adults.

Obesity is an increasing problem in The Netherlands, already at younger ages. Attention should be given to maintaining a good energy balance. 
Methodologically, to get a full insight into nutrient intake it is important to include intake from dietary supplements and fortified foods. Furthermore, it is important to estimate the habitual intake distribution to evaluate nutrient adequacy.

\section{Conclusion}

Thus, these studies showed that the fatty acid composition of the diet is not optimal in the diet of young children and young adults in The Netherlands, although the trans-fatty acid intake has improved during the last decades. Also, the vegetable and fruit consumption is far from the recommendations. Policy measures need to focus on achieving optimal body weight, increasing intakes of vegetables, fruit, fish and fiber, and improving the fatty acid composition.

\subsection{United Kingdom ${ }^{26}$}

In 2008 The Scientific Advisory Committee on Nutrition (SACN) published a report summarizing the findings from all previous National Diet and Nutrition Surveys in Britain (SACN, 2008; and see: www.sacn.gov.uk/pdfs/nutritional_health_of_the_ population_final_oct_08.pdf). The only specific survey conducted since the last EU Monitoring Report was the Low-Income Diet and Nutrition Survey; details are described further below.

In addition to the specific nutritional survey there continue to be annual health surveys that include some aspects of nutrition, the annual compilation of food and expenditure data, and the annual feeding (breast) survey. In addition, there are adhoc surveys of consumer attitudes.

\section{Low-Income Diet and Nutrition Survey}

\section{Sample Selection}

The aim of the survey was to select the bottom $15 \%$ of the population based on material deprivation. In order to identify and recruit this sample, a material deprivation screening questionnaire was developed and covered whether subjects were in receipt of benefits, household composition, car ownership and employment status.

The sample was selected using a five-stage clustered design:

- 528 wards were selected, with deprived wards oversampled relative to other wards. Scotland, Wales and Northern Ireland were oversampled relative to England, with the final dataset weighted to take this into account.

\footnotetext{
${ }^{26}$ Margetts B; University of Southampton, UK. E-Mail B.M.Margetts@soton.ac.uk
} 
- A fixed sample of addresses was selected in each ward, according to the level of deprivation. The final issued sample consisted of 25,818 addresses.

- A doorstep screening questionnaire was used to establish eligibility for inclusion in the survey, based on markers of deprivation. It also included questions that were used to assess non-response bias.

- At each address, one household was selected.

- In eligible households with two or more residents, two respondents were then randomly selected. If children were present, one adult and one child were selected; otherwise two adults were selected.

As well as oversampling wards in Scotland, Wales and Northern Ireland and in more deprived areas, there was differential non-response to the survey (e.g. by age, sex, country/region). In order to ensure that the correct population proportions were used in the analysis, the final dataset was weighted. All results are based on these weighted data so that the reported findings reflect the demographic characteristics of the UK low-income population as a whole. The overall response rate for fully productive individuals was 55\% (59\% for Scotland and Northern Ireland, 55\% for Wales, and $52 \%$ for England), giving a sample size of 3,728 fully productive individuals. Of these, $73 \%$ were visited by a nurse and a blood sample was obtained from $46 \%$ of fully productive individuals. Response rates for blood samples were lower for children, with blood samples obtained from $18 \%$ of boys and $22 \%$ of girls who were fully productive.

\section{Survey Components}

The key stages of the survey, which were administered by trained interviewers and nurses, were as follows:

- A face-to-face interview and self-completion questionnaire.

- Four 24-hour recalls of diet on random days (including at least 1 weekend day) within a 10-day period.

- Physical measurements. These differed by age group but included, where appropriate, height, weight, waist and hip circumferences, demi-span, mid-upper arm circumference and blood pressure.

- Blood sample collection (in respondents aged 8 years and over) to measure indices of nutritional status.

Information collected in the interview and self-completion questionnaire included, where, appropriate: food shopping habits (e.g. main type of shop used, distance to shop); access to food storage and cooking facilities; usual food preparation methods; cooking skills; access to food at school and to free food; eating habits, including use of dietary supplements; general health and dental health, including use of prescribed medicines; weight change; drinking and smoking; physical activity; education; income; attitudes and barriers to healthy eating; and food security and coping strategies.

Further details and results are available (http://www.food.gov.uk/science/ dietarysurveys/lidnsbranch). 


\section{Health Survey for England}

The Health Survey for England provides annual data about the nation's health; to estimate the proportion of the population with specific health conditions; to estimate the prevalence of risk factors associated with those conditions; to assess the frequency with which combinations of risk factors occur; to examine differences between population subgroups; to monitor targets in the health strategy; and from 1995 to measure the height of children at different ages, replacing the national study of health and growth. The Health Survey for England contains a 'core' which is repeated each year and each survey year has one or more modules on subjects of special interest. The 'core' includes: questions on general health and psychosocial indicators; smoking; alcohol; demographic and socioeconomic indicators; questions about use of health services and prescribed medicines - the focus for these may vary from year to year to suit the modular content of the survey; blood pressure; and measurements of height, weight and blood pressure. Over recent years the survey has also included questions about diet and physical activity.

From 1994 onwards the survey has been carried out by the Joint Survey Unit of the National Centre of Social Research and the Department of Epidemiology and Public Health at University College London. The HSE 2007 included a general population sample of adults and children, representative of the whole population at both national and regional level, and a boost sample of children aged 2 to 15 . For the general population sample, 7,200 addresses were randomly selected in 720 postcode sectors, issued over 12 months from January to December 2007. Interviews were held in 4,200 households with 6,882 adults aged 16 or over, and 1,727 children from the general population. The boost sample resulted in an additional 5,777 children aged 2 to 15 being interviewed, giving a total child sample of 7,504. Among the general population sample, 4998 adults and 1,233 children had a nurse visit. Response to the survey can be calculated in two ways: at a household level and at an individual level. Interviews were carried out at $66 \%$ of sampled eligible households in the general population (after removing vacant addresses, etc.), and at $75 \%$ of known eligible boost sample households. Within the general population sample, interviews were obtained with $88 \%$ of adults and $95 \%$ of (sampled) children in 'cooperating' households (where at least one person was interviewed). Assuming that households where the number of adults and children was not known, the individual response rate for the general population sample, based on all eligible households, was estimated to be $58 \%$ among adults and $65 \%$ among (sampled) children.

Diet and activity were assessed by questionnaire. For Diet the general form of questions was 'How often on average do you eat...' With options of: 6 or more times a week, 3-5 times a week, 1-2 times a week, $<1$ once a week, rarely or never. There were further questions on the types of foods eaten (such as what type of bread), and what kind of fruit, salad, etc, were eaten yesterday, and for fruits and vegetables how much was eaten. For physical activity, parents were asked how long their child spent doing various activities (including sitting). 
Fig. 11.22. Trends in obesity from 1993 to 2007 for adults and children based on Health Survey for England (\%).

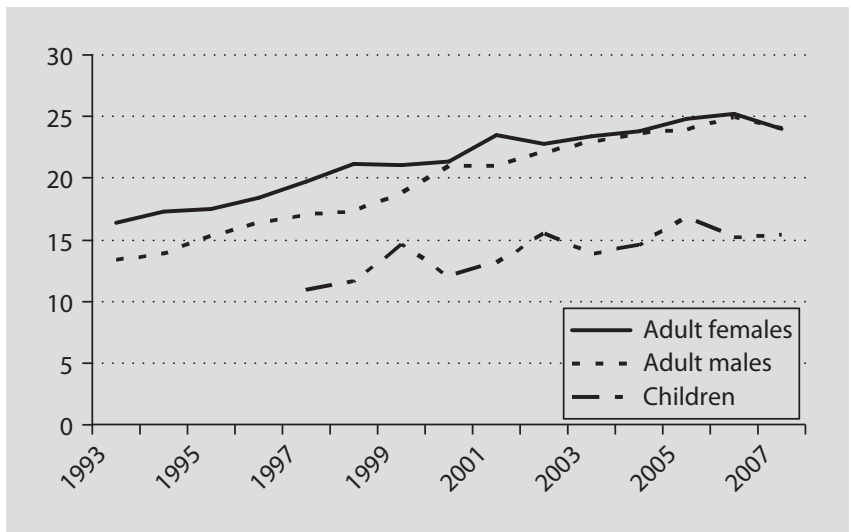

Further details and copies of all reports are available from The NHS Information Centre (http://www.ic.nhs.uk/); for surveys prior to 2005 see to the Department of Health (http://www.dh.gov.uk/en/Publicationsandstatistics/PublishedSurvey/ HealthSurveyForEngland/Healthsurveyresults/index.htm). Trends in levels of obesity are presented in figure 11.22.

\section{Main Findings from HSE 2007 for England}

\section{Obesity}

- In 2007, 24\% of adults (aged 16 or over) in England were classified as obese (BMI $30 \mathrm{~kg} / \mathrm{m}^{2}$ or over); an overall increase from $15 \%$ in 1993 .

- Men and women were equally likely to be obese, however men were more likely than women (41 compared to 32\%) to be overweight (BMI 25 to $<30 \mathrm{~kg} / \mathrm{m}^{2}$ ).

- 37\% of adults had a raised waist circumference in 2007 compared to $23 \%$ in 1993. Women were more likely then men (41 and 33\% respectively) to have a raised waist circumference ( $>88 \mathrm{~cm}$ in women, $>102 \mathrm{~cm}$ in men).

- Using both BMI and waist circumference to assess risk of health problems, for men: $19 \%$ were estimated to be at increased risk; $13 \%$ at high risk and $21 \%$ at very high risk. Equivalent figures for women were: 15\% at increased risk; $16 \%$ at high risk and $23 \%$ at very high risk.

- In 2007, $17 \%$ of boys aged 2 to 15 , and $16 \%$ of girls were classed as obese, an increase from 11 and $12 \%$ respectively in 1995 . Indications suggest that the trend in obesity may be flattening out and the next couple of years' data will be important in confirming whether this is a continuing pattern.

- Boys were slightly more likely than girls to be overweight or obese (31\% compared to $30 \%)$. 
- Overall, physical activity has increased among both men and women since 1997, with $40 \%$ of men and $28 \%$ of women meeting the recommended levels in 2006 (at least 30 minutes of at least moderate intensity activity at least 5 times a week) compared with $32 \%$ and $21 \%$ respectively in 1997.

- In 2007, approximately one third of adults had not heard of the government guidelines for physical activity (34\% of men and $29 \%$ of women).

- When asked how much physical activity adults thought people their age should do, $16 \%$ of men and $9 \%$ of women specified a level equivalent to the Chief Medical Officer's minimum recommended target. A further $25 \%$ of men and $23 \%$ of women specified a level of physical activity greater than the minimum recommendations, while most adults (69\% of men and $68 \%$ of women) either underestimated how much physical activity adults should do or did not know.

- Over two thirds of adults (66\% of men and $69 \%$ of women) said they would like to do more physical activity.

- Among men, the most common reason given which stopped them doing more physical activity was work commitments (45\%), followed by lack of leisure time (38\%). Women were most likely to report a lack of leisure time $(37 \%)$, with work commitments almost as frequently mentioned (34\%).

- In 2007, boys aged 2 to 15 were more likely than girls to meet the recommended levels of physical activity with $72 \%$ of boys and $63 \%$ of girls reporting taking part in 60 minutes or more of physical activity on each of the 7 days in the previous week.

- During the 2007/08 academic year, 90\% of pupils took part in at least 2 hours of high-quality physical education (PE) and sport at school a week, a gradual increase since $2003 / 04$ when the figure was $62 \%$.

- Only a small proportion of children aged 11 to 15 said they thought they should be doing physical activity at the level of the current minimum recommendation (10\%). A further $8 \%$ of boys and $3 \%$ of girls overestimated the minimum recommendation.

- Girls were more likely than boys to want to do more physical activity, $74 \%$ of girls aged $11-15$ compared to $61 \%$ of boys.

Diet

- In 2007, 27\% of men and $31 \%$ of women consumed five or more portions of fruits and vegetables a day, up from 22 and 25\% respectively in 2001.

- Women were more likely than men to know the recommended number of portions per day (78\% of women compared to $62 \%$ of men).

- Among children aged 5-15, in 2007, 21\% of both boys and girls consumed five or more portions of fruit and vegetables a day, up from $11 \%$ of both boys and girls in 2001.

- $63 \%$ of boys and $73 \%$ of girls knew that five portions of fruit and vegetables a day was the recommendation. 
Fig. 11.23. Regional variation in rates of obesity in children (\%) (based on National Child Measurement Program) and adults (based on Health Survey for England).

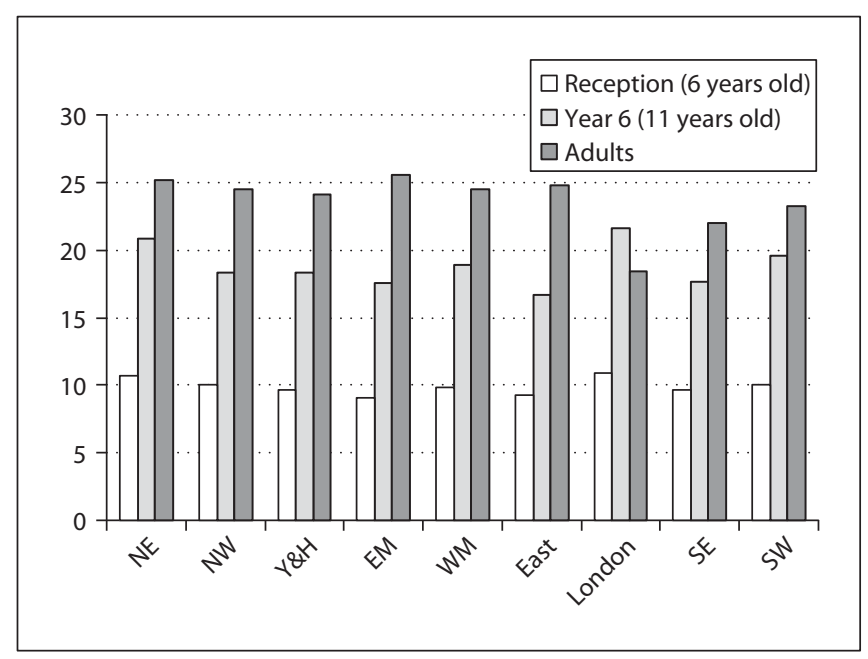

\section{Health Literacy and Health Improvement}

Over half of England's adult population has literacy skills below Level 2 - the level of skills needed to discuss a condition interactively with a doctor or specialist. Only $25 \%$ were able to calculate BMI with a formula or estimate food groups needed for a balanced diet. UK research has indicated that low health literacy has been associated with poorer health outcomes where each incremental increase towards higher health literacy is associated with a greater likelihood of: eating at least five portions of fruits and vegetables a day; being a non-smoker [von Wagner et al., 2007].

\section{National Child Measurement Program}

In addition to the HSE, every 6- and 11-year-old child in England is measured annually (see for report http://www.ncmp.ic.nhs.uk). The report presents the prevalence of underweight, healthy weight, overweight and obese children, in Reception (aged 4 to 5 years) and Year 6 (aged 10 to 11 years), measured in the school year 2007/08. Where possible, comparisons have also been made with the 2006/07 NCMP results. Figure 11.23 presents the results from the NCMP compared with the HSE 2007 for adults, broken down by regions.

\section{Summary Findings}

- 973,073 valid measurements were received for children, in England, in Reception and Year 6 - about $88 \%$ of those eligible.

- In Reception, almost 1 in 4 of the children measured was either overweight or obese. In Year 6, this rate was nearly 1 in 3.

- The percentage of children who are obese is almost twice as high in Year 6 than in Reception. 
- The percentage of children who are overweight is only slightly higher in Year 6 than in Reception.

- The overall percentage of children who are underweight is similar for both years.

- The prevalence of underweight, overweight and obese children by NCMP year in England for 2006/07 and 2007/08 does not differ significantly.

- Obesity prevalence is significantly higher than the national average in the North East, West Midlands and London SHAs for both age groups as well as North West SHA for Reception children and Yorkshire and Humber SHA for Year 6 children.

- Obesity prevalence is significantly below the national average in the South East Coast, South Central, South West and East Midlands SHAs for children in both school years, as well as for Year 6 children in the East of England SHA.

- As in the 2006/07 NCMP findings, a strong positive relationship exists between deprivation and obesity prevalence for children in Reception and Year 6.

- Obesity prevalence is significantly higher in urban areas than in rural areas, as was the case in NCMP 2006/07

\section{Local and Regional Health Profiles}

Based largely on the HSE (similar surveys are also undertaken in Scotland and Wales) there are reports now that are produced as part of an annual Health Profile for England (and separately Scotland and Wales) that includes estimates broken down by regions. The UK health observatories (http://www.apho.org.uk) compile an annual health profile for each city/local area that allows further disaggregation of data by local wards. It is thus now possible to disaggregate key indicators at national, regional and local levels, that reveals considerable heterogeneity in measures within the UK.

There have been significant improvements in the health of the population over the last 10 years, an improvement almost wholly shared by disadvantaged groups and areas, as measured by life expectancy and infant mortality [Department of Health, 2008].

Average life expectancy for all groups in England has increased significantly - for males by an extra 3.1 years and for females by an extra 2.1 years - between 1995-1997 and 2005-2007.

For disadvantaged areas (the spearhead group of 70 local authority areas with the worst health and deprivation indicators) there has been an increase in life expectancy of only very slightly less than for the whole population -2.9 years for men and 1.9 for females.

Infant mortality rates have fallen to an historic low level over the last 10 years having fallen from 5.6 infant deaths per 1,000 live births in 1995-1997 to 4.7 per 1,000 in 2005-2007 for all those in England with a valid socioeconomic group, with rates in the routine and manual (target) group having fallen from 6.3 to 5.4 per 1,000 live births over the same period.

Note: All web addresses were correct at the time of preparing this report in June 2009. 


\section{References}

Abu-Omar K, Rütten A: Relation of leisure time, occupational, domestic, and commuting physical activity to health indicators in Europe. Prev Med 2008;47: 319-323.

Adamkova V, Suchanek P, Lanska V, Stochlova J, Cifkova R: Longitudinal follow-up (15 years) study of the trend of nutrition of the $1 \%$ random sample of population of a district of Central Bohemia. J Clin Lipidol 2008;2:109.

Afonso C: Personal data from a PhD study under development, 2008.

Andersen LF, Øverby NC, Lillegaard ITL: Intake of fruit and vegetables among Norwegian children and adolescents (in Norwegian). Tidsskr Nor Lægeforen 2004;124:1396-1398.

Andersen LF, Pollestad ML, Jacobs DR Jr, Lovo A, Hustvedt BE: Validation of a pre-coded food diary used among 13-year-olds - comparison of energy intake with energy expenditure. Public Health Nutr 2005;8:1315-1321.

Antal M, Nagy K, Bíró L, et al.: Hazai reprezentatív felmérés a középiskolás fiatalok táplálkozási és életmódbeli szokásairól [National representative survey on he nutritional and life-style habits of secondary school students]. Orvosi Hetilap [Hung Med J] 2003;144:1631-1636.

Antal M, Nagy K, Regöly-Mérei A, et al.: Assessment of cardiovascular risk factors among Hungarian university students in Budapest. Ann Nutr Metab 2006;50:103-107.

Antal M, Regöly-Mérei A, Bíró L, et al.: Nutrition. Lifestyle practice, serum vitamin $\mathrm{D}$ concentration and bone density in Hungarian adolescents. Acta Alimentaria 2006;35:53-61.

Aranceta J, Pérez Rodrigo C, Serra Majem LI, Ribas L, Quiles-Izquierdo, Vioque J, Foz M, and Spanish Collaborative Group for the Study of Obesity: Influence of sociodemographic factors in the prevalence of obesity in Spain. The SEEDO'97 Study. Eur J Clin Nutr 2001;55:430-435.
Armstrong T, Bull F: Development of the World Health Organization Global Physical Activity Questionnaire (GPAQ). J Public Health 2006;14:66-70.

Arvaniti F, Panagiotakos DB, Pitsavos C, Zampelas A, Stefanadis C: Dietary habits in a Greek sample of men and women: the ATTICA study. Cent Eur J Public Health 2006;14:74-78.

Astrup A, Andersen L, Stender S, Trolle E: Kostrådene 2005 [Dietary Guidelines 2005]. Søborg, Danmarks Fødevareforskning og Ernæringsrådet, 2005.

Bakoula C, Nicolaidou P, Veltsista A, Prezerakou A, Moustaki M, Kavadias G, Lazaris D, Fretzayas A, Krikos X, Karpathios T, Matsaniotis N: Does exclusive breastfeeding increase after hospital discharge? A Greek study. J Hum Lact 2007;23:165-173.

Balance Sheets for Food Commodities. Information Centre of the Ministry of Agriculture and Forestry (Tike), Helsinki, Finland.

Barzda A, Bartkeviciute R, Abaravicius A, Stukas R, Satkute R: Food consumption survey in adult Lithuanian population (in Lithuanian). Medicinos teorija ir praktika 2009;15:53-58.

Bayingana K, Demarest S, Gisle L, Hesse E, Miermans PJ, Tafforeau J, van der Heyden J: Gezondheidsenquête door middel van Interview, België, 2004. Depot No. D/2006/2505/4, IPH/EPI Report No. 2006-035. Brussels, Scientific Institute of Public Health/ Epidemiology Unit, 2006.

Beaton GH, Milner J, McGuire V, Feather T, Little JA: Sources of variance in 24-hour dietary recall data: implications for nutrition study design and interpretation. Carbohydrate sources, vitamins and minerals. Am J Clin Nutr 1983;37:986-995.

Becker W: Dietary Habits and Nutrient Intake in Sweden 1989 (in Swedish/English summary). Uppsala, Statens Livsmedelsverk, 1994.

Becker W, Pearson M: Riksmaten 1997-98. Dietary Habits and Nutrient Intake in Sweden 1997-98 (in Swedish/English summary). Uppsala, Livsmedelsverket, 2002. 
Becker W, Haglund M, Wretling S: Fat and Fatty Acids in the Swedish Diet - Analyses of Market Baskets Purchased in 2005 (in Swedish). Rapport 17. Uppsala, Livsmedelsverket, 2008.

Belot A, Grosclaude P, Bossard N, Jougla E, Benhamou E, Delafosse P, et al.: Cancer incidence and mortality in France over the period 1980-2005. Rev Epidemiol Sante Publique 2008;56:159-175.

Benetou V, Chloptios Y, Zavitsanos X, Karalis D, Naska A, Trichopoulou A: Total cholesterol and HDL cholesterol in relation to socioeconomic status in a sample of 11,645 Greek adults: The EPIC study in Greece. European Prospective Investigation into Nutrition and Cancer. Scand J Public Health 2000;28:260-265.

Bevilacqua N, Branca F, Cairella G, Censi L, D’Addesa D, D’Amicis A, Leclercq C, Rossi L, Saba A, Sette S, Tabacchi G, Turrini, A: Manuale di sorveglianza nutrizionale [Nutritional Surveillance Handbook]. Rome, INRAN, 2003.

Biela U, Pająk A, Kaczmarczyk-Chałas K, Głuszek J, Tendera M, Wawrzyńska M, Kuriata P, Wyrzykowski B: Częstość występowania nadwagi i otyłości u kobiet i mężczyzn w wieku 20-74 lat. Wyniki programu WOBASZ. Kardiol Polska Supl 2005;63;4:1-4.

Bíró G: First Hungarian Representative Nutrition Survey 1985-1988 Results, vol 1. Budapest, 1992.

Bíró G, Antal M, Zajkás G: Nutrition Survey of the Hungarian population in a randomized trial between 1992-1994. Eur J Clin Nutr 1996;50:201208.

Bíró L, Regöly-Mérei A, Nagy K, et al.: Dietary habits of schoolchildren: representative survey in metropolitan elementary schools: Part 2. Ann Nutr Metab 2007;51:454-460.

Bíró L, Zajkás G, Greiner E, et al: Táplálkozási vizsgálat Magyarországon,2003-2004.Mikro-tápanyagbevitel: ásványi sók [Dietary survey in Hungary, 2003-2004. Micro-nutrient intake: minerals]. Orvosi Hetilap [Hung Med J] 2007;148:703-708.

Black AE: Critical evaluation of energy intake using the Goldberg cut-off for energy intake: basal metabolic rate. A practical guide to its calculation, use and limitations. Int J Obes 2000;24:1119-1130.

Blair SN, Kohl HW, Paffenbarger RS: How much physical activity is good for health? Annu Rev Public Health 1992;13:99-126.

Blair SN, Connelly JC: How much physical activity should we do? The case for moderate amounts and intensities of physical activity. Res Q Exerc Sport 1996;67:193-205.

Bøgh-Sørensen L, Biltoft-Jensen A, Groth MV, Matthiessen J, Fagt S, Hels O: Sammenhængen mellem alkoholindtag og kostkvalitet [Associations between alcohol intake and quality of diet]. Ugeskr Læger 2009;3/2:1-5.
Boros J: Research Report: National Health Survey of Population, 2003 (OLEF). National Centre of Epidemiology, Hungary 2005.

Brussaard JH, Johansson L, Kearney J: Rationale and methods of the EFCOSUM project. Eur J Clin Nutr 2002a;56:4-7.

Brussaard JH, Lowik MRH, Steingrimsdottir L, Moller A, Kearney J, de Henauw S, Becker W: A European food consumption survey method - conclusions and recommendations. Eur J Clin Nutr 2002b;56:8994.

Buurma-Rethans E, Fransen H, Ghameshlou Z, de Jong $\mathrm{N}$ : Een supplementendatabestand: behoeftes en acties. Voeding Nu 2008;10:21-24.

Carmo I, Santos J, Camolas J, Vieira J, Carreira M, Medina L, Reis L, Myatt J, Galvão-Teles A: Overweight and obesity in Portugal: national prevalence in 2003-2005. Obes Rev 2008;9:11-19.

Carnovale E, Marletta L (eds): Tabelle di composizione degli alimenti. Milan, Edra, 1997.

Castetbon K, Vernay M, Malon A, Salanave B, Deschamps V, Roudier C, et al: Dietary intake, physical activity and nutritional status in adults: the French nutrition and health survey (ENNS, 2006-2007). Br J Nutr 2009; doi:10.1017/S0007114509274745.

CBS Statline (http://statline.cbs.nl/statweb/) (accessed 2007).

Center for Disease Control and Prevention (CDC): Romania Global Youth Tobacco Survey (GYTS) fact sheet, 2004 (http://www.who.int/infobase/reportviewer.aspx?rptcode $=$ ALL\&uncode $=642 \& \mathrm{dm}=$ 8\&surveycode $=102292 \mathrm{a} 1)($ accessed April 15, 2009)

Central Statistics Office: Food Balance Sheets in 2003. Hungary, 2005.

Central Statistics Office: Yearbook of Health Statistics, 2007. Hungary, 2009.

Central Statistics Office: Irish Census 96. Principle demographic results. 1997.

Centre for Health Policies and Services (CHPS): Knowledge, attitudes and practices of the general Romanian population regarding tobacco use and the legal provisions, Bucharest, Romania, 2007 (http://www.who.int/infobase/reportviewer. aspx? rptcode $=$ ALL\&uncode $=642 \& \mathrm{dm}=8 \&$ surveycode $=102715 \mathrm{a} 1)($ accessed April 15, 2009).

Centre for Health Policies and Services (CHPS): Smoking and public health in Romania: knowledge, attitudes and practices about tobacco use in the Romanian general population, Bucharest, Romania, 2004 (http://www.who.int/infobase/reportviewer. aspx? rptcode $=$ ALL\&uncode $=642 \& \mathrm{dm}=8 \&$ surveycode $=102399 \mathrm{a} 1$ ).

CESNID: Tablas de Composición de Alimentos. Barcelona: Edicions Universitat de Barcelona-Centre d'Ensenyament Superior de Nutrició i Dietètica, 2002. 
Charles MA, Eschwege E, Basdevant A: Monitoring the obesity epidemic in France: the Obepi surveys 1997-2006. Obesity (Silver Spring) 2008;16:21822186.

Cífková R, Škodová Z: Dlouhodobé trendy hlavních rizikových faktorů kardiovaskulárních onemocnění v české populaci [Longitudinal trends in major cardiovascular risk factors in the Czech population]. Čas Lék Čes 2004;143:219-226.

Cífková R, Škodová Z, Lánská V, Adámková V, Novozámská E, Jozífová M, Plášková M, Hejl Z, Petržílková Z, Galovcová M, Palouš D: Prevalence, awareness, treatment and control of hypertension in the Czech Republic. Results of two nationwide cross-sectional surveys in 1997/98 and 2000/01, Czech Post-MONICA Study. J Hum Hypertens 2004a;18:571-579.

Cífková R, Škodová Z, Lánská V, Adámková V, Novozámská E, Pitha J, Jozífová M, Plášková M, Šraierová D, Hauserová G, Dušková A: Trends in blood pressure levels, prevalence, awareness and control of hypertension in the Czech population from 1985 to 2000/01. J Hypertens 2004b;22:14791485.

CNESPS - Centro Nazionale di Epidemiologia, Sorveglianza e Promozione della Salute [National Centre for Epidemiology, Surveillance and Health Promotion]: OKkio alla salute [Mind your health] (https://www.okkioallasalute.it/), 2008.

Cole TJ, Bellizzi MC, Flegal KM, Dietz WH: Establishing a standard definition for child overweight and obesity worldwide: international survey. BMJ 2000;320: 1240-1243.

Cole TJ, Flegal KM, Nicholls D, Jackson AA: Body mass index cut offs to define thinness in children and adolescents: international survey. BMJ 2007;335: 166-167.

Craig CL, Marshall AL, Sjöström M, Bauman AE, Booth ML, Ainsworth BE, Pratt M, Ekelund U, Yngve A, Sallis J, Oja P: International Physical Activity Questionnaire: 12-country reliability and validity. Med Sci Sports Exerc 2003;35:1381-1395.

D-A-CH, Deutsche Gesellschaft für Ernährung (DGE), Österreichische Gesellschaft für Ernährung (ÖGE), Schweizerische Gesellschaft für Ernährung (SGE), Schweizerische Vereinigung für Ernährung (SVE): D-A-CH Referenzwerte für die Nährstoffzufuhr. Frankfurt/Main, Umschau Braus, 2000 [D-A-CH, German Nutrition Society (DGE), Austrian Nutrition Society (ÖGE), Swiss Society for Nutrition Research (SGE), Swiss Nutrition Association (SVE): Reference values for nutrient intake. Frankfurt/ Main, Umschau Braus, 2002].

D’Amicis A: Il quadro nutrizionale della popolazione in Italia. Riv Sci Alim 2000;3:7-11.
D’Amicis A: Alimentazione, stile di vita e salute. Conference 'Nutrizione, stili di vita: rendere facili le scelte salutari', Lucca, October 11, 2008 (www.usl2. toscana.it/sup/modulistica/alimenti/20081011 DAmicis.pdf).

De Almeida MDV, Graça P, Afonso C, D’Amicis A, Lappalainen R, Damkjaer S: Physical activity levels and body weight in a nationally representative sample in the European Union. Public Health Nutr 1999;2:105-113.

De Vriese S, de Backer G, de Henauw S, Huybrechts I, Kornitzer M, Leveque A, Moreau M, van Oyen H: The Belgian food consumption survey: aims, design and methods. Arch Public Health 2005;63:1-16.

De Vriese S, Huybrechts I, Moreau M, van Oyen H: De Belgische Voedselconsumptiepeiling 1 - 2004. Depot No. D/2006/2505/17, IPH/EPI Reports No. 2006-016. Brussels, Scientific Institute of Public Health/Epidemiology Unit, 2006.

Demarest S, Roskam AJ, Cox B, Regidor E, van Oyen H, Mackenbach JP, Kunst A: Socioeconomic inequalities in leisure time physical activity; in The Eurothine Project (2007). Tackling Health Inequalities in Europe: an integrated approach EUROTHINE (http:// survey.erasmusmc.nl/eurothine/index.php?ind).

Department of Health. Tackling Health Inequalities: 2007 Status Report on the Programme for Action, 2008.

Dodd KW: A technical guide to C-SIDE. Software for Intake Distribution Estimation. Tech. Rep. 96-TR 32, 1-68. Department of Statistics and Center for Agricultural and Rural Development. Des Moines, Iowa State University, 1996.

Donnelly JE, Blair SN, Jakicic JM, Manore MM, Rankin JW, Smith BK: American College of Sports Medicine Position Stand. Appropriate physical activity intervention strategies for weight loss and prevention of weight regain for adults. Med Sci Sports Exerc 2009;41:459-471.

ECHI: Annex 5 to the ECHI-2 Report of June 20, 2005 - The ECHI comprehensive indicator list (long list) Version of July 7, 2005. Internet: http://www.healthindicators.eu/healthindicators/object_binary/ o2713_ECHI-2\%20Final\%20report\%20June.pdf (accessed July 2009).

EFCOSUM: European food consumption survey method. Eur J Clin Nutr 2002;56(suppl 2).

EFSA (European Food Safety Authority): Guidance Document for the Use of the Concise European Food Consumption Database in Exposure Assessment. Parma, 2008a (http://www.efsa.europa. eu/cs/BlobServer/General/Coincise_database_ guidance_document_and_annexes.pdf?ssbinary = true) (accessed May 2009). 
EFSA (European Food Safety Authority): Concise Database Summary Statistics - Total Population, 2008b (http://www.efsa.europa.eu/cs/BlobServer/ General/DATEX_ConciseDB_All_subjects,3. $\mathrm{xls}$ ?ssbinary $=$ true $)($ accessed May 2009).

Ekholm O, Kjøller M, Davidsen M, Hesse U, Eriksen L, Christensen AI, Grønbæk M: Sundhed og sygelighed i Danmark 2005 \& udviklingen siden 1987 [Health and Morbidity in Denmark 2005 and development since 1987]. Copenhagen, Statens Institut for Folkesundhed, 2006.

Elmadfa I, Weichselbaum E, König J, de Winter AMR, Trolle E, Haapala I, Uusitalo U, Mennen L, Hercberg S, Wolfram G, Trichopoulou A, Naska A, Benetou V, Kritsellis E, Rodler I, Zajkás G, Branca F, D’Acapito P, Klepp KI, Ali-Madar A, De Almeida MD, Alves E, Rodrigues S, Serra-Majem L, Roman B, Sjöström M, Poortvliet E, Margetts B: European Nutrition and Health Report 2004. Forum Nutr 2005;58:1-220.

Elmadfa I, Freisling H, Nowak V, Hofstädter D, et al: Österreichischer Ernährungsbericht 2008, ed 1. Wien, 2009.

Enghardt Barbieri H, Pearson M, Becker W: Dietary habits and nutrient intake in Swedish 4-year-old children and school children in grade 2 and 5 . Riksmaten - children 2003 (in Swedish/English summary). Uppsala, Livsmedelsverket, 2006.

EUFIC (European Food Information Council) (2006): Food allergy and food intolerance. 2006. (http:// www.eufic.org/jarticle/en/food-nutrition-guide/ food-allergy/expid/basics-food-allergy-intolerance) (accessed May 2009).

Eurodiet core report: Nutrition \& Diet for Healthy Lifestyles in Europe. Science and Policy Implications, University of Crete School of Medicine (coord.), Greece, 2000.

European Commission: The citizens of the European Union and Sport. Special Eurobarometer 213/Wave 62.0. 2004, Directorate General Education and Culture.

European Commission: Health and Food: Special Eurobarometer 246/Wave 64.3. TNS Opinion and Social. 2006.

European Communities: Household budget surveys in the EU Methodology and recommendations for harmonization. Luxembourg, Office for Official Publications of the European Communities, 2003.

Eurostat (2009): Population statistics. European Communities, 2009. (http://epp.eurostat.ec.europa. $\mathrm{eu} / \mathrm{portal} / \mathrm{page} / \mathrm{portal} / \mathrm{population/data/main}$ tables) (accessed July 2009).
Fabian E, Kickinger A, Wagner KH, Elmadfa I: Folsäure, Vitamin B12 und B6 bei älteren Menschen in Ostösterreich - Diskrepanz zwischen Zufuhr und tatsächlichem Status. [Folic acid, vitamin B12 and B6 in elderly in Eastern Austria - Discrepancy between intake and actual status.] Proceedings of the German Nutrition Society Abstracts zum 46. Wissenschaftlichen Kongress der DGE Volume 13, 2009.

Fagt S, Biltoft-Jensen A, Matthiessen J, Groth MV, Christensen T, Trolle E: Danskernes kostvaner 1995-2006. Status og udvikling med fokus på frugt og grønt samt sukker [Dietary Habits of Denmark 1995-2006. Status and development with focus on fruits, vegetables and added sugar]. Søborg, Fødevareinstituttet, Danmarks Tekniske Universitet, 2008.

Ferlay J, Bray F, Pisani P, Parkin DM: GLOBOCAN 2002. Cancer Incidence, Mortality and Prevalence Worldwide. IARC Cancer Base No. 5, Vers. 2.0. Lyon, IARC Press, 2004. Internet: http://www-dep. iarc.fr/globocan/database.htm. (accessed: June 2008).

Ferreira RJ, Marques-Vidal PM: Prevalence and determinants of obesity in children in public schools of Sintra, Portugal. Obesity 2008;16:497-500.

FFQ_Software, Version 1.0. National Nutrition Surveillance Centre, School of Public Health and Population Science, University College, Dublin, 2007.

Fidler Mis N: Nutrition of Slovenian adolescents. Project supported by Ministry of Health, Ljubljana, 2005.

Fidler Mis N, et al.: Dietary intake of Slovenian adolescents (submitted).

Food and Agriculture Organization of the United Nations (FAO): Food Balance Sheets - A Handbook. Rome, FAO, 2001.

Food and Agriculture Organization of the United Nations (FAO): FAOSTAT. Food Balance Sheets. Portugal 2003 (http://faostat.fao.org/site/368/DesktopDefault. aspx? PageID = 368) (accessed October 2008).

Food and Agriculture Organization of the United Nations (FAO): Workshop on supply utilization accounts and food balance sheets. Food Balance Sheets: History, Sources, Concepts and Definitions, 2007a (http://www.foodsec.org/tr/stat/tr_taj_02_ fbs.pdf) (accessed June 2009).

Food and Agriculture Organization of the United Nations (FAO): FAOSTAT data. 2007b. Internet: http://www. fao.org/documents/show_cdr.asp?url_file=/ docrep/003/x9892e/X9892E00.htm (acessed: March 2007).

Food and Agriculture Organization of the United Nations (FAO): FAOSTAT data 2009 (http://faostat.fao.org/ site/368/default.aspx\#ancor) (accessed January 2009). 
Food and Agriculture Organization of the United Nations (FAO): Food Balance Sheets (http://www.fao.org/ documents/show_cdr.asp?url_file $=$ /docrep $/ 003 /$ x9892e/X9892E00.htm) (accessed March 2007b).

Food Standards Agency: Food Portion Sizes, ed 3. London, HMSO, 2002.

Freisling H, Elmadfa I: Prevalence of overweight and obesity among Austrian children (3-15 years): using three different sets of reference values for body mass index. Ernährung/Nutrition 2007;31:6-11.

Friel S, Nic Gabhainn S, Kelleher C: The National Health and Lifestyles Surveys: Survey of Lifestyle, Attitudes and Nutrition (SLÁN) and the Irish Health Behaviour in Schoolaged Children (HBSC) Survey 1998, Department of Health and Children. Dublin, The Stationery Office, 1999.

Friel S, Nelson M, McCormack K, Kelleher C, Thriskos $\mathrm{P}$ : Methodological issues using household budget survey expenditure data for individual food availability estimation: Irish experience in the DAFNE pan-European project. DAta Food NEtworking. Public Health Nutr 2001;4:1143-1147.

Gabrijelčič Blenkuš $\mathrm{M}$, Gregorič M: Nutritional habits of the adult population of Slovenia from the aspect of the health protection II, 2009. Project supported by Slovenian Research Agency.

García-Alvarez A, Serra-Majem L, Ribas-Barba L, Castell C, Foz M, Uauy R, Plasencia A, Salleras L: Obesity and overweight trends in Catalonia, Spain (19922003): gender and socioeconomic determinants. Public Health Nutr 2007;10:1368-1378.

Gil JM, Gracia A, Angulo AM: Trends in the consumption of animal food products in Mediterranean countries; in Brufau J, Tacon A (eds): Feed Manufacturing in the Mediterranean Region: Recent Advances in Research and Technology. Zaragoza, CIHEAM-IAMZ, 1999, pp 11-31.

Gobiņa I, Pudule I, Grīnberga D, Velika B, Tilgale N, Villeruša A, Teibe U: Uztura paradumi un ķermeņa masa skolas vecuma bērniem Latvijā/Nutritional habits and body mass of school-age children in Latvia, Rīga, 2007.

Gobiṇa I, Pudule I, Grīnberga D, Velika B, Tilgale N, Villeruša A, Teibe U: Smēḳēšnas un alkohola lietošanas izplatība skolas vecuma bērniem Latvijā 1991-2006 [Incidence of smoking and alcohol consumption in school-age children in Latvia 19912006], Riga, 2008.

Goldberg GR, Black A, Jebb SA, Cole TJ, Murgatroyd PR, Coward WA, Prentice AM: Critical evaluation of energy intake data using fundamental principles of energy physiology. 1. Derivation of cut-off limits to identify underrecording. Eur J Clin Nutr 1991;45:569-581.
Grabauskas V, Klumbiene J, Petkeviciene J, Petrauskiene A, Tamosiunas A, Kriaucioniene V, et al.: Risk factors for non-communicable diseases in Lithuanian rural population: CINDI survey in 2007. Medicina 2008;44:633-639.

Grabauskas V, Klumbiene J, Petkeviciene J, Sakyte E, Kriaucioniene V, Veryga A, Prattala R: Suaugusiu Lietuvos žmonių gyvensenos tyrimas, 2008. Health behaviour among Lithuanian adult population (2008), Kaunas, 2009.

Graham I, Atar D, Borch-Johnson K, Boysen G, Burell G, Cifkova R, Dallongeville J, De Backer G, Ebrahim S, Gjelsvik B, Herrmann-Lingen C, Hoes A, Humphries S, Knapton M, Perk J, Priori SG, Pyorala K, Reiner Z, Ruilope L, Sans-Menendez S, Scholte op Reimer W, Weissberg P, Wood D, Yarnell J, Zamorano JL: European guidelines on cardiovascular disease prevention in clinical practice: executive summary. EHJ 2007;28:2375-2414.

Greenfield H, Southgate DAT: Food Composition Data - Production, Management and Use. Rome, FAO, 2003.

Groth MV, Fagt S, Brønsted L: Social determinants of dietary habits in Denmark. Eur J Clin Nutr 2001;55:959-966.

Groth MV, Fagt S, Stockmarr A, Matthiessen J, BiltoftJensen A: Dimension of socioeconomic position related to body mass index and obesity among Danish women and men. Scand J Public Health 2009;37:418-426.

Halicka E, Rejman K, Kowrygo B: European food and drink industry activities aimed at encouraging consumer healthy choice; in Kowrygo B (ed): Challenges and Perspectives for the European Food Market. Warsaw, WULS Press, 2008, pp 25-37.

Haskell WL, Lee I, Pate RR, Powell KE, Blair SN, Franklin BA, Macera CA, Heath GW, Thompson PD, Bauman A: Physical activity and public health: updated recommendation for adults from the American College of Sports Medicine and the American Heart Association. Med Sci Sports Exerc 2007;39:14231434.

Hassapidou M, Fotiadou E, Maglara E, Papadopoulou SK: Energy intake, diet composition, energy expenditure, and body fatness of adolescents in northern Greece. Obesity 2006;14:855-862.

Hassapidou M, Papadopoulou SK, Frossinis A, Kaklamanos I, Tzotzas T: Sociodemographic, ethnic and dietary factors associated with childhood obesity in Thessaloniki, Northern Greece. Hormones (Athens) 2009;8:53-59.

Hasunen K, Ryynänen S: Imeväisikäisten ruokinta Suomessa vuonna 2005. Helsinki, Sosiaali-ja terveysministeriön selvityksiä, 2006, p 19. 
Health and Consumer Protection Directorate General: The DAFNE Food Classification System. Operationalization in 16 European Countries. Luxemburg, European Commission, 2005.

Health Council of the Netherlands: Dietary Reference Intakes: Calcium, Vitamin D, Thiamin, Riboflavin, Niacin, Pantothenic Acid, and Biotin. The Hague, Health Council of the Netherlands, 2000, Publ. No. 2000/12.

Health Council of the Netherlands: Towards an Optimal Use of Folic Acid. The Hague, Health Council of the Netherlands, 2008, Publ. No. 2008/02.

Health Council of the Netherlands: Towards an Adequate Intake of Vitamins and Minerals. The Hague, Health Council of the Netherlands, 2009, Publ. No. 2009/ 06.

Hercberg S, Chat-Yung S, Chauliac M: The French National Nutrition and Health Program: 20012006-2010. Int J Public Health 2008;68-77.

Holick MF, Chen TC: Vitamin D deficiency: a worldwide problem with health consequences. Am J Clin Nutr 2008;87:1080S-1086S.

Hölling H, Kamtsirius P, Thierfelder W, Thamm M, Schlack R: Der Kinder- und Jugendgesundheitssurvey (KiGGS): Studienmanagement und Durchführung der Feldarbeit. Bundesgesundheitsbl Gesundheitsforsch Gesundheitsschutz 2007;50:5557-566.

Hoppu U, Kujala J, Lehtisalo J, Tapanainen H, Pietinen P (eds): Nutrition and wellbeing of secondary school pupils. Situation at baseline and results of the intervention study during academic year 2007-2008.

Helsinki, National Public Health Institute, 2008, B30/ 2008.

Hulshof KFAM, van Staveren WA: The Dutch National Food Consumption Survey: design, methods and first results. Food Policy 1991;16:257-260.

Hulshof KFAM, van Erp-Baart MA, Anttolainen M, Becker W, Church SM, Couet C, et al: Intake of fatty acids in Western Europe with emphasis on transfatty acids: the TRANSFAIR study. Eur J Clin Nutr 1999;53:143-157.

Hungarian Centre for Healthcare Audit and Inspection: Annual Report of the Hungarian Network of Midwives on Infant Feeling, 2008.

INE/INSA: Database of the Portuguese 4th National Health Survey 2005-2006. 2008.

Inequalities in Young People's Health; in Currie C, Gabhainn SN, Godeau E, Roberts C, Smith R, Currie D, et al (eds): HBSC International Report from the 2005/2006 Survey. Geneva, WHO, 2008.

Institute of Medicine: Dietary Reference Intakes for Energy, Carbohydrate, Fiber, Fat, Fatty Acids, Cholesterol, Protein, and Amino Acids. Washington, National Academies Press, 2005.
Instituto de Alimentação Becel: Estudo epidemiológico de caracterização do perfil lipídico da população portuguesa. Lisboa, Instituto de Alimentação Becel, 2001.

Internubel (http://www.internubel.be) (accessed 2005).

International Diabetes Federation (IDF): Prevalence of diabetes mellitus. Diabetes Atlas 3rd edition. 2006. (http://www.eatlas.idf.org/downloadables/Data\%20 Tables/index.html) (accessed July 14, 2009).

IOM (Institute of Medicine): Reference Levels of Nutrients and Interpretation and Uses of DRIs, Standing Committee on the Scientific Evaluation of Dietary Reference Intakes, Food and Nutrition Board, Institute of Medicine. Washington, The National Academies Press, 2000.

IOM (Institute of Medicine): Dietary reference intakes for vitamin C, vitamin E, selenium, and carotenoids. Panel on Dietary Antioxidants and Related Compounds, Subcommittees on Upper Reference Levels of Nutrients and Interpretation and Uses of DRIs, Standing Committee on the Scientific Evaluation of Dietary Reference Intakes, Food and Nutrition Board, Institute of Medicine. Washington, The National Academies Press, 2000.

IOM (Institute of Medicine): Dietary reference intakes for vitamin $\mathrm{A}$, vitamin $\mathrm{K}$, arsenic, boron, chromium, copper, iodine, iron, manganese, molybdenum, nickel, silicon, vanadium, and zinc. Panel on Dietary Antioxidants and Related Compounds, Subcommittees on Upper Reference Levels of Nutrients and Interpretation and Uses of DRIs, Standing Committee on the Scientific Evaluation of Dietary Reference Intakes, Food and Nutrition Board, 2001.

Irish Universities Nutrition Alliance: North/South Ireland Food Consumption Survey 2001 (http:// www.iuna.net) (accessed June 2009).

ISTAT (Istituto Nazionale di Statistica) [Italian National Statistics Bureau]: Stili di vita e condizioni di salute, 2003 [Lifestyle and health conditions]. Released November 18, 2005.

ISTAT (Istituto Nazionale di Statistica) [Italian National Statistics Bureau]: Allattamento al seno [Breastfeeding]; in Gravidanza, parto, allattamento al seno, 2004-2005 [Pregnancy, childbirth, breastfeeding]. Released June 5, 2006.

ISTAT (Istituto Nazionale di Statistica) [Italian National Statistics Bureau]: L'uso e l'abuso di alcol in Italia, 2007 [The use and abuse of alcohol in Italy]. Released April 17, 2008.

ISTAT (Istituto Nazionale di Statistica) [Italian National Statistics Bureau]: La vita quotidiana nel 2006 [Daily life in 2006]. Released November 6, 2007.

ISTAT (Istituto Nazionale di Statistica) [Italian National Statistics Bureau]: Health-for-All. 2009a. Last update January 27, 2009 (www.istat.it/sanita/Health/). 
ISTAT (Istituto Nazionale di Statistica) [Italian National Statistics Bureau]: La vita quotidiana nel 2007 [Daily life in 2007]. Released March 12, 2009b.

ISTAT (Istituto Nazionale di Statistica) [Italian National Statistics Bureau]: Uso e abuso di alcool in Italia. Anno 2008 [Use and over-indulgence of alcohol in Italy, 2008]. Released April 23, 2009c.

ISTAT (Istituto Nazionale di Statistica) [Italian National Statistics Bureau]: La vita quotidiana nel 2008 [Daily life in 2008]. Released April 23, 2009.

Johansson L, Thelle DS, Solvoll K, Bjørneboe GEA; Drevon CA: Healthy dietary habits in relation to social determinants and lifestyle factors. Br J Nutr 1999;81:211-220.

Johansson L, Solvoll K, Bjørneboe G-EA, Drevon CA: Dietary habits among Norwegian men and women. Scand J Nutr 1997;41:63-70.

Johansson L, Solvoll K: Norkost 1997 - Landsomfattende kostholdsundersøkelse blant menn og kvinner i alderen 16-79 år. National Council on Nutrition and Physical Activity, Report 1999;2.

Johansen A, Jespersen LN, Davidsen M, Michelsen SI, Morgen CS, Helweg-Larsen K, Andersen AMN, Mortensen L, Juhl M, Due P: Danske børns sundhed og sygelighed [The National Health Interview Survey on Children 2005]. København, Statens Institut for Folkesundhed, Syddansk Universitet, 2009.

Kadziauskiene K, Abaravicius A, Bartkeviciute R: Nutrition and Lifestyle in the Baltic Republics. Geneva, WHO, 1999.

Kafatos A, Diacatou A, Voukiklaris G, Nikolakakis N, Vlachonikolis J, Kounali D, Mamalakis G, Dontas AS: Heart disease risk-factor status and dietary changes in the Cretan population over the past 30 years: the Seven Countries Study. Am J Clin Nutr 1997;65:1882-1886.

Kamtsiuris P, Lange M, Schaffrath Rosario A: Der Kinder- und Jugendgesundheitssurvey (KiGGS): Stichprobendesign, Response und NonresponseAnalyse. Bundesgesundheitsbl Gesundheitsforsch Gesundheitsschutz 2007;50:547-556.

Kapantais E, Tzotzas T, Ioannidis I, Mortoglou A, Bakatselos S, Kaklamanou M, Lanaras L, Kaklamanos I: First national epidemiological survey on the prevalence of obesity and abdominal fat distribution in Greek adults. Ann Nutr Metab 2006;50:330-338.

Kasmel A, Lipand A, Markina A: Health Behavior among the Estonian Adult Population, 2002. Tallinn, Estonian Centre for Health Education and Promotion, 2003.
Kelleher C, Nic Gabhainn S, Friel S, Corrigan H, Nolan G, Sixsmith J, Walsh O, Cooke M: The National Health and Lifestyles Surveys. Survey of Lifestyle, Attitudes and Nutrition (SLÁN) and the Irish Health Behaviour in School-Aged Children (HBSC) Survey 2002, Department of Health and Children. Dublin, The Stationery Office, 2003.

Kiss É: Physical activity - fitness - prevention. Családorvosi Fórum, 2003;4 (in Hungarian).

Kjøller M, Juel K, Kamper-Jørgensen F (eds): Folkesundhedsrapporten, Danmark 2007 [The Report on Public Health, Denmark 2007]. Copenhagen, Statens Institut for Folkesundhed, 2007.

Klimont J, Kytir J, Leitner B: Österreichische Gesundheitsbefragung 2006/2007, Hauptergebnisse und methodische Dokumentation. Wien, Statistik Austria, 2007.

Koch M: Nutritional Habits of Slovenian Adults in Health Protection Aspect, PhD Diss Thesis, Ljubljana, Biotechnical Faculty, Department of Food Science and Technology, 1997.

Kosti RI, Panagiotakos DB, Mihas CC, Alevizos A, Zampelas A, Mariolis A, Tountas Y: Dietary habits, physical activity and prevalence of overweight/obesity among adolescents in Greece: the Vyronas Study. Med Sci Monit 2007;13:437-444.

Koutedakis Y, Bouziotas C, Flouris AD, Nelson PN: Longitudinal modeling of adiposity in periadolescent Greek schoolchildren. Med Sci Sports Exerc 2005;37:2070-2074.

Kromeyer-Hauschild K, Wabitsch M, Kunze D, Geller F, Geiss HC, Hesse V, von Hippel A, Jaeger U, Johnsen D, Korte W, Menner K, Müller G, Müller JM, Niemann-Pilatus A, Remer T, Schaefer F, Wittchen HU, Zabransky S, Zellner K, Ziegler A, Hebebrand J: Perzentile für den Body-Mass-Index für das Kindes- und Jugendalter unter Heranziehung verschiedener deutscher Stichproben. Monatsschr Kinderheilkd 2001;149:807-818.

Kubisová D, Adamkova V, Lanska V, Dlouhý P, Rambouskova J, Anděl M: Higher prevalence of smoking and lower BMI, waist circumference, cholesterol and triacylglyceride levels in Prague's homeless compared to a majority of the Czech population. BMC Public Health 2007;7:51.

Kubisová D, Dlouhý P, Rambousková J, Anděl M: Absence of protein-energy malnutrition in Prague Homeless. Int J Public Health 2008;53:57-63.

Kunachowicz H, Nadolna I, Przygoda B: Tabele składu i wartości odżywczej żywności [Food composition tables]. Warszawa, PZWL, 2005.

Kurth BM, Bergmann KE, Hölling H, Kahl H, Kamtsiuris P, Thefeld W: Der bundesweite Kinder- und Jugendgesundheitssurvey. Gesundheitswesen 2002; 64(suppl 1):3-11. 
Kushi LH, Byers T, Doyle C, Bandera EV, McCullough M, Gansler T, Andrews KS, Thun MJ and The American Cancer Society 2006: Nutrition and Physical Activity Guidelines Advisory Committee: American Cancer Society Guidelines on Nutrition and Physical Activity for Cancer Prevention: Reducing the risk of cancer with healthy food choices and physical activity. CA Cancer J Clin 2006;56:254-281.

Kyttälä P, Ovaskainen M, Kronberg-Kippilä C, Erkkola M, Tapanainen H, Tuokkola J, Veijola R, Simell O, Knip M, Virtanen SM: The Diet of Finnish Preschoolers. Helsinki, National Public Health Institute, 2008, B32/2008.

Laaksi IT, Ruohola JPS, Ylikomi TJ, Auvinen A, Haataja RI, Pihlajamäki HK, Tuohimaa PJ: Vitamin D fortification as public health policy: significant improvement in vitamin D status in young Finnish men. Eur J Clin Nutr 2006;60:1035-1038.

Ladomenou F, Kafatos A, Galanakis E: Risk factors related to intention to breastfeed, early weaning and suboptimal duration of breastfeeding. Acta Paediatr 2007;96:1441-1444.

Lamberg-Allardt C, Viljakainen H and a Working Group: Follow-up study on the vitamin D status in the Finnish population 2002 and 2004. Helsinki, Reports of the Ministry of Social Affairs and Health, 2006.

Layte R, Tully N, van Lente E, Ward M, Lutomski J, Conroy R, Brugha R: SLÁN 2007: Survey of Lifestyle, Attitudes and Nutrition in Ireland. Main Report. Dublin, Department of Health and Children, 2008.

Leclercq C, Arcella D, Piccinelli R, Sette S, Le Donne C, Turrini A, on behalf of the INRAN SCAI 2005-06 Study Group: The Italian National Food Consumption Survey INRAN-SCAI 2005-06. Main results in terms of food consumption. Public Health Nutr, 2009.

Lehtonen-Veromaa M, Möttönen T, Leino A, Heinonen OJ, Rautava E, Viikari J: Prospective study on food fortification with vitamin D among adolescent females in Finland: minor effects. Br J Nutr 2008; 100:418-423.

Līde Z: Antropometrisko rādītāju izvērtējums Latvijas skolēniem [Assessment of anthropometric data among Latvian school-age children], Master Thesis, University of Latvia, Riga, 2009.

Lillegaard ITL, Andersen LF: Validation of a pre-coded food diary with energy expenditure, comparison of underreporters vs. acceptable reporters. Br J Nutr 2005;94:998-1003.

Lillegaard ITL, Øverby NC, Andersen LF: Can children and adolescents use photographs of food to estimate portion sizes? Eur J Clin Nutr 2005;59:611-617.
Lillegaard ITL, Løken EB, Andersen LF: Relative validation of a pre-coded food diary among children, under-reporting varies with reporting day and time of the day. Eur J Clin Nutr 2007;61:61-68.

Lobstein T, Baur L, Uauy R: Obesity in children and young people: a crisis in public health. Obes Rev 2004;5(suppl 1):4-9.

Lopes C, Oliveira A, Santos AC, Ramos E, Gaio AR, Severo M, Barros H: Consumo alimentar no Porto. Faculdade de Medicina da Universidade do Porto, 2006 (http://higiene.med.up.pt/consumoalimentarporto/home.php?var = intro_rel $)($ accessed December 2008).

Lyhne N, Christensen T, Groth MV, Fagt S, Biltoft-Jensen A, Hartkopp H, Hinsch HJ, Matthiessen J, Møller A, Saxholt E, Trolle E: Danskernes kostvaner 2000-2002 - Hovedresultater [Dietary habits of Denmark 20002002]. Søborg, Danmarks Fødevareforskning, 2005.

Mammas I, Bertsias G, Linardakis M, Moschandreas J, Kafatos A: Nutrient intake and food consumption among medical students in Greece assessed during a clinical nutrition course. Int J Food Sci Nutr 2004;55:17-26.

Manios Y, Grammatikaki E, Papoutsou S, Liarigkovinos T, Kondaki K, Moschonis G: Nutrient intakes of toddlers and preschoolers in Greece: the GENESIS Study. J Am Diet Assoc 2008;108:357-361.

Marques-Vidal P, Ferreira R, Oliveira JM, Paccaud F: Is thinness more prevalent than obesity in Portuguese adolescents? Clin Nutr 2008;27:531-536.

Martin R, Saller K: Lehrbuch der Anthropologie und systematischer Darstellung. Stuttgart, 1957, p 661.

Martinez-Gonzalez MA, Varo JJ, Santos JL, Irala J, Gibney M, Kearney J, Martinez A: Prevalence of physical activity in the European Union. Med Sci Sports Exerc 2001;33:1142-1146.

Matos MG, Simões C, Tomé G, Gaspar T, Camacho I, Diniz JÁ: Equipa do Projecto Aventura Social: Aventura social e saúde: a saúde dos adolescentes portugueses - hoje e em 8 anos. Relatório preliminar do estudo HSBC 2006 (http://www.fmh.utl.pt/ aventurasocial/pdf/Relatorio_nacional_2006.pdf) (accessed September 2008).

Matthiessen J, Groth MV, Fagt S, Biltoft-Jensen A, Stockmarr A, Andersen JS, Trolle E: Prevalence and trends in overweight and obesity among children and adolescents in Denmark. Scand J Public Health 2008;36:153-160.

Max Rubner-Institut (eds): Nationale Verzehrsstudie II. Ergebnisbericht Teil 1. Die bundesweite Befragung zur Ernährung von Jugendlichen und Erwachsenen. Karlsruhe, 2008a.

Max Rubner-Institut (eds): Nationale Verzehrsstudie II. Ergebnisbericht Teil 2. Die bundesweite Befragung zur Ernährung von Jugendlichen und Erwachsenen. Karlsruhe, 2008b. 
Max Rubner-Institut (eds): Nationale Verzehrsstudie II. Ergänzungsband zum Ergebnisbericht Teil 1. Karlsruhe, 2008c.

McCance RA, Widdowson EM: The Composition of Foods, ed 5. London, HMSO, 1997.

Mejborn H, Biltoft-Jensen A, Trolle E, Tetens I (eds): Fuldkorn - Definition og vidensgrundlag for anbefaling af fuldkornsindtag i Danmark [Wholegrain definition and scientific background for recommendations of wholegrain intake in Denmark]. Søborg, Fødevareinstituttet, Danmarks Tekniske Universitet, 2008.

Mensink GBM, Hermann-Kunz E, Thamm M: Der Ernährungssurvey. Gesundheitswesen 1998;60:8386.

Mensink GBM, Haftenberger M, Thamm M: Validity of DISHES 98, a computerized dietary history interview: energy and macronutrient intake. Eur J Clin Nutr 2001;55:409-417.

Mensink GBM, Richter A, Vohmann C, Stahl A, Six J, Kohler S, Fischer J, Heseker H: EsKiMo - Das Ernährungsmodul des Kinder- und Jugendgesundheitssurveys (KiGGS). Ernährung 2007a;1:225-229.

Mensink GBM, Heseker H, Richter A, Stahl A, Vohmann C: Forschungsbericht. Ernährungsstudie als KiGGSModul (EsKiMo). Robert Koch-Institut, Universität Paderborn (eds); Berlin, Paderborn, 2007b.

Mensink, GBM, Heseker H, Richter A, Stahl A, Vohmann C: Ernährungsstudie als KiGGS-Modul (EsKiMo) (Forschungsbericht i. A. Bundesministerium für Ernährung, Landwirtschaft und Verbraucherschutz, 2007, pp 91-94.

Meyer HE, Falch JA, Søgaard AJ, Haug E: Vitamin D deficiency and secondary hyperparathyroidism and the association with bone mineral density in persons with Pakistani and Norwegian background living in Oslo, Norway: the Oslo Health Study. Bone 2004;35:412-417.

Ministry of Health 2005; in Maučec Zakotnik, Poličnik R, Mičović E, Hlastan Ribič C (eds): Slovene National Nutrition Policy Programme for 20052010. Ljubljana, Ministry of Health, 2005.

Ministry of Health and Welfare, Supreme Scientific Health Council. Dietary guidelines for adults in Greece. Arch Hellenic Med 1999;16:516-524.

Mohd Hairi F, Avendano M, Kunst A, Mackenbach J: Changes in health-behaviour-related determinants; in Börsch-Supan A, Brugiavini A, Jürges $\mathrm{H}$, et al. (eds): First Results from the Survey of Health, Ageing and Retirement in Europe (2004-2007). Mannheim, Mannheim Research Institute for Economics of Aging, 2008.

Moreira P, Padez C, Mourão I, Rosado V: Dietary calcium and body mass index in Portuguese children. Eur J Clin Nutr 2005;59:861-867.
Moreira P, Padez C, Mourão-Carvalhal I, Rosado V: Maternal weight gain during pregnancy and overweight in Portuguese children. Int $\mathrm{J}$ Obes 2007;31:608-614.

Moretti R, Torre P, Antonello RM, Cattaruzza T, Cazzato G, Bava A: Vitamin $B_{12}$ and folate depletion in cognition: a review. Neurol India 2004;52:310-318.

Morgan K, McGee H, Watson D, Perry I, Barry M, Shelley E, Harrington J, Molcho M, Layte R, Tully $\mathrm{N}$, van Lente E, Ward M, Lutomski J, Conroy R, Brugha R: SLÁN 2007: Survey of Lifestyle, Attitudes and Nutrition in Ireland. Main Report. Dublin, Department of Health and Children, 2008.

Murray CJL, Lopez AD: The Global Burden of Disease: A Comprehensive Assessment of Mortality and Disability from Diseases, Injuries and Risk Factors in 1990 and Projected to 2020. Cambridge, Harvard School of Public Health on behalf of the WHO and the World Bank, 1996.

Mustonen H, Metso L, Paakkanen P, Simpura J, Kaivonurmi M: Finnish Drinking Habits in 1968, 1976, 1984, 1992 and 1996. Tables and Publications Based on Finnish Drinking Habit Surveys. Themes 7/1999. Helsinki, National Research and Development Centre for Welfare and Health, 1999.

Mydlilová A: Standardní praktické pokyny pro kojení v ČR. MZ ČR, 2006, p 60.

Mydlilová A, Šípek A, Wiesnerová J: Vliv některých faktorů na počet kojených dětí při propuštění z porodnice v letech 2000-2004 v ČR. ČeskoSlovenská Pediatrie 2008;63:182-191.

Mydlilová A, Šípek A, Vignerová J: Breastfeeding rates in baby-friendly and non-baby-friendly hospitals in the Czech Republic from 2000 to 2006. J Hum Lact 2009;25:73-78.

Naska A, Vasdekis VG, Trichopoulou A, Friel S, Leonhauser IU, Moreiras O: Fruit and vegetable availability among ten European countries: how does it compare with the 'five-a-day' recommendation? DAFNE I and II projects of the European Commission. Br J Nutr 2000;84:549-556.

Naska A, Fouskakis D, Oikonomou E, Almeida MD, Berg MA: Dietary patterns and their socio-demographic determinants in 10 European countries: data from the DAFNE databank. Eur J Clin Nutr 2006;60:181-190.

Naska A, Oikonomou E, Trichopoulou A, Wagner K, Gedrich K: Estimations of daily energy and nutrient availability based on nationally representative household budget survey data. The Data Food Networking (DAFNE) project. Public Health Nutr 2007;10:1422-1429.

Naska A, Berg MA, Cuadrado C, Freisling H, Gedrich K, Gregorič K, et al: Food balance sheet and household budget survey dietary data and mortality patterns in Europe. Br J Nutr 2009;102:166-171. 
National Children's Food Survey: 2005 (www.iuna.net) (accessed June 2009).

National Institute for Health Development. National Strategy for Prevention of Cardiovascular Diseases 2005-2020. 2005 (http://www.tai.ee/failid/Heart Strategy.pdf) (accessed July 30, 2009).

National Institute for Health Development. National Cancer Strategy 2007-2015. 2007 (http://www2.tai. ee/teated/arenduskeskus/Vahistrateegia/National_ Cancer_strategy_2007_2015eng.pdf) (accessed July 30, 2009).

National Institute for Statistics (NIS). Anuarul statistic al României [Yearbook]. Bucharest, National Institute for Statistics, 2006 (http://www.insse.ro/).

National Institute of Public Health, Sweden. National public health survey 2007. Health on equal terms? (http://www.fhi.se/en/News/News/Results-of-theNational-Public-Health-Survey-now-in-English/).

National Institute of Public Health: Obesity development, children (in Swedish) (http://www.fhi.se/sv/ Handbocker/Uppslagsverk-barn-och-unga/Fetmautveckling-over-tid/).

National Institute of Public Health: Environmental Health Monitoring System in the Czech Republic. Summary Report - 2005. Prague, 2006, p 126.

National Institute of Public Health: Environmental Health Monitoring System in the Czech Republic. Summary Report - 2006. Prague, 2007, p 106.

National Institute of Public Health: Environmental Health Monitoring System in the Czech Republic. Summary Report - 2007. Prague, 2008, p 116.

National Institute of Public Health: National CINDY Health Monitor Report 2002. Prague, p 47.

National Research Council: Diet and Health: Implication for Reducing Chronic Disease Risk. Washington, National Academy Press, 1989, pp 563-565.

Navone C, Mannheimer R, Salvi E, Tuè P, Di Pietro P: Indagine nazionale della Società Italiana di Pediatria sulla promozione dellallattamento materno [National survey of the Italian Pediatric Association on promotion of breastfeeding]. Proc 64th National Congress, Genoa, October 15-18, 2008. Minerva Pediatr 2008;60:504-525.

NNR 2004: Nordic Nutrition Recommendations 2004 integrating nutrition and physical activity. Copenhagen, Nordic Council of Ministers, 2005.

Nordic Nutrition Recommendations 2004: Integrating nutrition and physical activity: Nord 2004:013. Nordic Council of Ministers, Copenhagen (http:// www.norden.org/pub/sk/showpub.asp?pubnr = 2004:013).

Nusser SM, Carriquiry AL, Dodd KW, Fuller WA: A semiparametric transformation approach to estimating usual daily intake distributions. J Am Stat Assoc 1996;91:1440-1449.
Oblacińska A: Zaburzenia stanu odżywienia; in Mazur J (ed): Status materialny rodziny i otoczenia a samopoczucie i styl życia młodzieży 15-letniej. Warszawa, Instytut Matki i Dziecka, 2006, pp 50-58.

Ocké MC, Hulshof KFAM, van Rossum CTM: The Dutch National Food Consumption Survey, 2003. Methodological issues. Arch Public Health 2005;63: 227-241.

Ocké MC, Buurma-Rethans EJM, Fransen HP: Dietary supplement use in the Netherlands: current data and recommendations for future assessment. RIVM report 350100001. Bilthoven, RIVM, 2005.

Ocké MC, van Rossum CTM, Fransen HP, BuurmaRethans EJM, de Boer EJ, Brants HAM, Niekerk EM, van der Laan JD, Drijvers JJMM, Ghameshlou Z: Dutch National Food Consumption Survey Young Children 2005/2006. RIVM report 350030002. Bilthoven, RIVM, 2008.

Oja L, Matsi A, Leinsalu M: Estonian Health Interview Survey 2006. Methodological report. Tallinn, National Institute for Health Development, 2008.

Oppert JM, Thomas F, Charles MA, Benetos A, Basdevant A, Simon C: Leisure time and occupational physical activity in relation to cardiovascular risk factors and eating habits in French adults. Public Health Nutr 2006;9:746-754.

Orfanos P, Naska A, Trichopoulos D, Slimani N, Ferrari P, van Bakel M, et al: Eating out of home and its correlates in 10 European countries. The European Prospective Investigation into Cancer and Nutrition (EPIC) study. Public Health Nutr 2007;10:15151525.

Ostan I, Poljšak B, Simčič M, Tijskens LMM: Nutrition for the Selfish Gene. Trends Food Sci Technol 2009 (in press). DOI:10.1016/j.tifs.2009.04.002.

Øverby NC, Lillegaard ITL, Johansson L, Andersen LF: High intake of added sugar among Norwegian children and adolescents. Public Health Nutr 2003;7: 285-293.

Øverby N, Kristiansen AL, Andersen LF, Lande B: Spedkost 6 months - a national dietary survey among 6-month-old infants. Helsedirektoratet, Mattilsynet og Universitetet i Oslo, 2008a.

Øverby N, Kristiansen AL, Andersen LF, Lande B: Spedkost 12 months - a national dietary survey among 12-month-old infants. Helsedirektoratet, Mattilsynet og Universitetet i Oslo, 2008b.

Ovesen L, Andersen R, Jakobsen J: Geographical differences in vitamin D status, with particular reference to European countries. Proc Nutr Soc 2003;62:813821.

Paffenbarger RS, Wing AL, Hyde RT: Paffenbarger physical activity questionnaire. Am J Epidemiol 1978;108:161-175. 
Papandreou D, Mavromichalis I, Makedou A, Rousso I, Arvanitidou M: Reference range of total serum homocysteine level and dietary indexes in healthy Greek schoolchildren aged 6-15 years. Br J Nutr 2006;96:719-724.

Paturi M, Tapanainen H, Reinivuo H, Pietinen P: The National FINDIET 2007 Survey. Helsinki, National Public Health Institute B23/2008, 2008.

Pechlivani F, Vassilakou T, Sarafidou J, Zachou TH, Anastasiou C, Sidossis S: Prevalence and determinants of exclusive breastfeeding during hospital stay in the area of Athens, Greece. Acta Paediatr 2005;94:928-934.

Pedersen BK, Saltin B: Børn og unge - fysisk aktivitet, fitness og sundhed [Children and adolescents physical activity, fitness and health]. København, Sundhedsstyrelsen, 2005.

Pedrosa C, Lebreiro A, Sebra D, Simões-Pereira C, Correia F, Almeida MDV: Avaliação da prevalência de obesidade em crianças dos 7 aos 9 anos de idade do Concelho de Aveiros (Resumo). Endocrinol Metabol Nutr 2006;15:275-276.

Pedrosa C, Simões-Pereira C, Correia F, Almeida MDV: Improvement in cardiovascular risk factors in obese children treated with lifestyle intervention. Eur Heart J 2008;29(suppl 1):228.

Peltonen M, Harald K, Männistö S, Saarikoski L, Peltomäki P, Lund L, Sundvall J, Juolevi A, Laatikainen $T$, Aldén-Nieminen $H$, Luoto $R$, Jousilahti P, Salomaa V, Taimi M, Vartiainen E: The National FINRISK 2007 Study. Helsinki, National Public Health Institute, 2008, B34/2008.

Péter SZ, Regöly-Mérei A, Bíró L, et al: Lifestyle of schoolchildren: representative survey in metropolitan elementary schools: Part 1. Ann Nutr Metab 2007;51:448-453.

Petrakos G, Panagopoulos P, Koutras I, Kazis A, Panagiotakos D, Economou A, Kanellopoulos N, Salamalekis E, Zabelas A: A comparison of the dietary and total intake of micronutrients in a group of pregnant Greek women with the dietary reference intakes. Eur J Obstet Gynecol Reprod Biol 2006;127:166-171.

Pettinger C, Holdsworth M, Gerber M: Psychosocial influences on food choice in Southern France and Central England. Appetite 2004;42:307-316.

Piirainen T, Laitinen K, Isolauri E: Impact of national fortification of fluid milks and margarines with vitamin $\mathrm{D}$ on dietary intake and serum 25-hydroxyvitamin D concentration in 4-year-old children. Eur J Clin Nutr 2007;61:123-128.

Pitsavos C, Panagiotakos D.B, Chrysohoou C, Stefanadis C: Epidemiology of cardiovascular risk factors in Greece: aims, design and baseline characteristics of the ATTICA study. BMC Public Health 2003;3:32.
Polakowska M, Piotrowski W, Tykarski A, Drygas W, Wyrzykowski B, Pająk A, Kozakiewicz K, Rywik S: Nałóg palenia tytoniu w populacji polskiej. Wyniki programu WOBASZ. Kardiol Polska Supl 2005;63;4:S1-S6.

Pomerleau J., McKee M. Robertson A., Vaask S., Pudule I., Grinberga D., Kadziauskiene K., Abaravicius A., Bartkeviciute R: Nutrition and lifestyle in the Baltic Republics. European Centre on Health of Societies in Transition and World Health Organisation Regional Office for Europe, WHO; 1999.

Pomerlau J, McKee M, Roberston A, Vaask S, Pudule I, Gringberga D, Kamelija K, Abaravicius A, Bartkeviciute R: Nutrition and lifestyle in the Baltic Republics 1997. Summary report. London: London School of Hygiene and Tropical Medicine, WHO; 2000.

Priftis KN, Panagiotakos DB, Anthracopoulos MB, Papadimitriou A, Nicolaidou P: Aims, methods and preliminary findings of the Physical Activity, Nutrition and Allergies in Children Examined in Athens (PANACEA) epidemiological study. BMC Public Health 2007;7:140.

Prochaska JJ, Sallis JF, Long B: A physical activity screening measure for use with adolescents in primary care. Arch Pediatr Adol Med 2001;155:554-559.

Project WOBASZ Report: Warsaw, Department of CVD Epidemiology, Prevention and Health Promotion, National Institute of Cardiology, 2005.

Psaltopoulou T, Orfanos P, Naska A, Lenas D, Trichopoulos D, Trichopoulou A: Prevalence, awareness, treatment and control of hypertension in a general population sample of 26,913 adults in the Greek EPIC study. Int J Epidemiol 2004;33:13451352.

Pudule I, Grīnberga D, Rituma A, Villeruša A, Zīle S, Prättälä R, Helasoja V, Puska P: Health Behaviour among Latvian Adult Population, 2000. Helsinki, National Public Health Institute, 2001.

Pudule I, Grīnberga D, Villeruša A, Dzērve V, Zīle S, Helasoja V, Vähäsarja K, Prättälä R: Health Behaviour among Latvian Adult Population, 2002. Helsinki, National Public Health Institute, 2003.

Pudule I, Jece I, Velika B, Grīnberga D, Villeruša A, Dzērve V, Zīle S, Helasoja V, Paalanen L, Prättälä R: Health Behaviour among Latvian Adult Population, 2004. Helsinki, National Public Health Institute, 2005.

Pudule I, Villeruša I, Grīnberga D, Velika B, Tilgale N, Dzērve V, Zile S, Konttinen H, Prättälä R: Health Behaviour among Latvian Adult Population, 2006. Helsinki, National Public Health Institute, 2007.

Rahu M, Tekkel M, Veideman T: Health Behavior among Estonian Adult Population 2004. Tallinn, National Institute for Health Development, 2005. 
Ramos E: Data collected under the EPITeen project from the Epidemiology Department - Medical School Oporto University (unpubl. pers. data, 2008)

Ramos E, Barros H: Family and school determinants of overweight in 13-year-old Portuguese adolescents. Acta Pediatr 2007;96:281-286.

Rejman K: Analysis of food consumption and nutritional behaviour of low-income consumers in Poland; in Januszewska R, Rejman K, Viaene J (eds): Food Consumption of Low-Income Groups in Poland and Belgium. Warsaw, WULS Press, 2007, pp 62-87.

Ribas-Barba L, Serra-Majem L, Salvador G, Castell C, Cabezas C, Salleras L, Plasencia A: Trends in dietary habits and food consumption in Catalonia, Spain (1992-2003). Public Health Nutr 2007;10:13401353.

Ribeiro J, Santos P, Duarte J, Mota J: Association between overweight and early sexual maturation in Portuguese boys and girls. Ann Hum Biol 2006; 33:55-63.

Rito A: Estado nutricional de crianças e oferta alimentar do pré-escolar do município de Coimbra, Portugal 2001. Tese de doutorado apresentada à Escola Nacional de Saúde Pública Sérgio Arouca da Fundação Oswaldo Cruz, Rio de Janeiro, 2004.

Rodler I, Bíró L, Greiner E, et al: Táplálkozási vizsgálat Magyarországon, 2003-2004. Energia- és makrotápanyagbevitel [Dietary survey in Hungary, 20032004. Energy and macro-nutrient intake]. Orvosi Hetilap [Hung Med J] 2005;146:1781-1789.

Rodrigues SSP: PhD dissertation. Faculty of Nutrition and Food Science, Porto University, 2007.

Rodrigues SSP, Lopes C, Naska A, Trichopoulou A, de Almeida MDV: Comparison of national food supply, household food availability and individual food consumption data in Portugal. J Public Health 2007;S10389-007-0102-8.

Rodrigues SSP, Trichopoulou A, de Almeida MDV: Regional food availability in representative samples of Portuguese households from 1990 to 2000; in Hanson KV, Larsen S, Edwards JSA (eds): Culinary Arts and Sciences. VI. Global, National and Local Perspectives. The Worshipful Company of Cooks and Norwegian School of Hotel Management, 2008, pp 137-145. University of Stavanger.

Roelants M, Hauspie R, Hoppenbrouwers K: References for growth and pubertal development from birth to 21 years in Flanders (Belgium). Ann Hum Biol 2009.

Romanian National Antidrug Agency: Prevalence of drug use in Romania, Bucharest, Romania, 2005 (http://www.who.int/infobase/reportviewer. aspx? rptcode $=$ ALL\&uncode $=642 \& \mathrm{dm}=8 \&$ surveycode $=102400 \mathrm{a} 1)($ accessed April 14, 2009).
Rütten A, Abu-Omar K: Prevalence of physical activity in the European Union. Soz Praventiv Med 2004;49:281-289.

Rütten A, Ziemainz H, Schena F, et al: Using different physical activity measurements in eight European countries. Results of the European Physical Activity Surveillance System (EUPASS) time series study. Public Health Nutr 2003;6:371-376.

Rywik S, Kupić W, Piotrowski W, Broda G, Piwoński J, Kuriata P, Waśkiewicz A, Gaździk D: Projekt WOBASZ. Założenia metodyczne oraz logistyka. Kardiol Polska Supl 2005;63;4:1-9.

Saba A, Turrini A, Mistura G, Cialfa E, Vichi M: Indagine nazionale sui consumi alimentari delle famiglie 1980-1984: alcuni principali risultati. J It Soc Food Sci 1990;19:53-65.

SACN: The Nutritional Wellbeing of the British Population. TSO, 2008.

Salanave B, Peneau S, Rolland-Cachera MF, Hercberg S, Castetbon K: Stabilization of overweight prevalence in French children between 2000 and 2007. Int J Pediatr Obes 2009;4:66-72.

Salt and Health: Review of the Scientific Evidence and Recommendations for Public Policy in Ireland. Food Standards Agency of Ireland, 2005.

Santos R, Aires L, Santos P, Ribeiro JC, Mota J: Prevalence of overweight and obesity in a Portuguese sample of adults: results from the Azorean Physical Activity and Health Study. Am J Hum Biol 2008;20:78-85.

SAS: SAS Procedure Guide. Cary, SAS Institute Inc, 1990.

Schofield WN: Predicting basal metabolic rate, new standards and review of previous work. Hum Nutr Clin Nutr 1985;39(suppl 1):5-41.

Scientific Committee for Food (SCF): Report of the Scientific Committee for Food. Nutrition and energy intakes for the European Community. Commission of the European Communities, Directorate General, 1993.

Scientific Committee for Food (SCF): Opinion of the Scientific Committee on Food on the revision of reference values for nutrition labelling. SCF/CS/ NUT/GEN/18, 2003.

Sekuła W, Nelson M, Figurska K, Ołtarzewski M, Weisel R, Szponar L: Comparison between household budget survey and 24-hour recall data in a nationally representative sample of Polish households. Public Health Nutr 2005;8:430-439.

Selga G, Lāriņš V, Sauka M: Liekās ķermeņa masas un aptaukošanās problēma Latvijas skolēniem [Overweight and obesity problem in Latvian school age children]. Doctus 2008;7:20-24.

Sempos CT, Johnson NE, Smith EL, Gilligan C: Effects of intraindividual and interindividual variation in repeated dietary recalls. Am J Epidemiol 1985;121: 120-130. 
Serra Majem L, Ribas Barba L, Salvador Castell G, Castell Abat C, Román Viñas B, Serra Farró J, et al: Avaluació de l'estat nutricional de la població catalana 2002-2003. Evolució dels hàbits alimentaris i dels consum d'aliments i nutrients a Catalunya (1992-2003). Barcelona, Departament de Salut, Generalitat de Catalunya, 2006 (www.gencat.net).

Serra Majem L, Ribas Barba L (eds): Trends in Nutrition Status in Catalonia, Spain (1992-2003). Public Health Nutr 2007;10:1339-1414.

Serra Majem L, Ribas Barba L, Salvador Castell G, Roman Viñas B, Castell Abat C, Cabezas Peña C, Pastor Ferrer MC, Raidó Quintana B, Ngo de la Cruz J, García Alvarez A, Serra Farró J, Salleras Sanmartí L, Taradach Antoni P: Trends in the nutritional status of the Spanish population: results from the Catalan nutrition monitoring system (19922003). Rev Esp Salud Publica 2007;81:559-570.

Serra-Majem L, Ribas-Barba L, Salvador G, Jover L, Raidó B, Ngo J, Plasencia A: Trends in energy and nutrient intake and risk of inadequate intakes in Catalonia, Spain (1992-2003). Public Health Nutr 2007a;10:1354-1367.

Serra-Majem L, Román-Viñas B, Salvador G, RibasBarba L, Ngo J, Castell C, Cabezas C: Knowledge, opinions and behaviours related to food and nutrition in Catalonia, Spain (1992-2003). Public Health Nutr 2007b;10:1396-1405.

Serra-Majem L, Ribas-Barba L, Salvador G, Serra J, Castell C, Cabezas C, Plasencia A: Compliance with dietary guidelines in the Catalan population: basis for a nutrition policy at the regional level (the PAAS strategy). Public Health Nutr 2007c;10:1406-1414.

Sierosławski J: Substancje psychoaktywne - postawy i zachowania Polaków. Raport z ogólnopolskich badań ankietowych 2002; in Postawy i zachowania Polaków wobec alkoholu i problemów alkoholowych. Warszawa, Wydawnictwo Edukacyjne PARPA, 2004, pp 11-74.

Sierosławski J: Używanie alkoholu i narkotyków przez młodzież szkolną. Raport z ogólnopolskich badań ankietowych 2007. Warszawa, PARPA/Instytut Psychiatrii i Neurologii, 2007.

Simila M, Fagt S, Vaask S, Thorgeirsdottir H, Pudule I, Petkeviciene J, et al.: The Norbagreen 2002 study: consumption of vegetables, potatoes, fruit, bread and fish in the Nordic and Baltic countries. Copenhagen, 2003.

SINU - Società Italiana di Nutrizione Umana [Italian Society of Human Nutrition]: Livelli di Assunzione Raccomandata in energia e nutrienti (LARN) [Recommended Daily Allowances of Energy and Nutrient Intakes for the Italian Population]. Rome, Istituto Nazionale della Nutrizione, 1996.
Slimani N, Deharveng G, Charrondière RU, van Kappel AL, Ocké MC, Welch A, Lagiou A, van Liere M, Agudo A, Pala V, Brandstetter B, Andrén C, Stripp C, van Staveren WA, Riboli E: Structure of the standardized computerized 24-hour diet recall interview used as reference method in 22 centres participating in the EPIC project. Comp Meth Prog Biomed 1999;58:251-266.

Slimani N, Ferrari P, Ocké M, Welch A, Boeing H, van Liere M, Pala V, Amiano P, Lagiou A, Mattisson I, Stripp C, Engeset D, Charrondière R, Buzzard M, van Staveren W, Riboli E: Standardization of the 24-hour diet recall calibration method used in the European Prospective Investigation into Cancer and Nutrition (EPIC): general concepts and preliminary results. Eur J Clin Nutr 2000;54:900-917.

SlimaniI N, Valsta L: Perspectives of using the EPICSOFT programme in the context of pan-European nutritional monitoring surveys: methodological and practical implications. Eur J Clin Nutr 2002;56:6374.

Sousa B, Oliveira BMPM, Almeida MDV: Assessment of nutritional status in 6- to 10-year-olds of autonomous region of Madeira, Portugal. Public Health Nutr 2006;9:109.

Spagner C: The Contribution of Micronutrients from Dietary Supplements, Master Thesis, University of Copenhagen, 2007.

Stanescu A, et al: Nutritional status of pregnant women, children under 5 years old, and schoolchildren aged 6-7 years. Bucharest, Alfred Rusescu Institute for Mother and Child Care, 2005, pp 1-20.

Statistics Estonia 2009 (www.stat.ee). Statistics Sweden: Välfärd No. 4, 2007 (http://www.scb.se/Grupp/valfard/BE0801_2007K04_TI_02_A05ST0704.pdf).

Stehle P, Volkert D (coord.): Comparative analysis of existing data on nutrition and lifestyle of the ageing population in Europe, especially in the 'New' Baltic, Central and Eastern regions of the Community: Ageing Nutrition, EU-Project, 2006 (http://ec. europa.eu/health/ph_projects/2003/action 1/docs/ ageing_nutrition_final_report.pdf).

Stichting NEVO: NEVO-tabel. Nederlandse Voedingsstoffenbestand 1996. Den Haag, Voedingscentrum, 1996.

Stichting NEVO: NEVO-tabel. Nederlandse Voedingsstoffenbestand 2001. Den Haag, Voedingscentrum, 2001.

Stichting NEVO: NEVO-tabel. Nederlandse Voedingsstoffenbestand 2006. Den Haag, Voedingscentrum, 2006.

Štimec M, Fidler Mis N, Smole K, Širca-Čampa A, Kotnik P, Zupančič M, Battelino T, Križišnik C: Iodine intake of Slovenian adolescents. Ann Nutr Metab 2007;51:439-447. 
Suni J, Oja P, Laukkanen R, Miïlunpalo SI, Pasanen ME, Vuori IM, Vartiainen TM, Bos K: Health-related fitness test battery for adults: aspects of reliability. Arch Phys Med Rehabil 1996;77:399-405.

Szabó L, Zagyva E: Breastfeeding in Hungary, 2008. 53rd Congress of the Hungarian Society of Paediatricians, Eger, June 18-20, 2009.

Szponar L, Sekuła W, Rychlik E, et al: Household Food Consumption and Anthropometric Survey 2000, National Food and Nutrition Institute (unpubl. data).

Szponar L, Sekuła W, Rychlik E, Ołtarzewski M, Figurska $\mathrm{K}$ : Badania indywidualnego spożycia żywności i stanu odżywienia $\mathrm{w}$ gospodarstwach domowych [Household food consumption and anthropometric survey]. Warszawa, IŻŻ, 2003.

Tekkel M, Veideman T, Rahu M: Health Behavior among the Estonian Adult Population 2006. Tallinn, National Institute for Health Development, 2007.

Tekkel M, Veideman T, Rahu M: Health Behavior among the Estonian Adult Population 2008. Tallinn, National Institute for Health Development, 2009.

The National Teen's Food Survey: 2008 (www.iuna.net) (accessed June 2009).

The National Findiet 2007 Survey (http://www.ktl.fi/ a t t a c h m ent s/s u o m i / julkais u t/ julkaisusarja_b/2008/2008b23.pdf).

Theofilogiannakou M, Skouroliakou M, Gounaris A, Panagiotakos D, Markantonis SL: Breastfeeding in Athens, Greece: factors associated with its initiation and duration. J Pediatr Gastroenterol Nutr 2006;43: 379-384.

Trichopoulou A, DAFNE Contributors: The DAFNE databank as a simple tool for nutrition policy. Data Food Networking. Public Health Nutr 2001;4:1187_ 1198.

Trichopoulou A (ed): Methodology and public health aspects of dietary surveillance in Europe, the use of Household Budget Surveys. Eur J Clin Nutr 1992; 46(suppl 5).

Trichopoulou A, Naska A (eds): The DAFNE Initiative: assessment of dietary patterns across Europe using household budget survey data. A European Commission-supported project. Public Health Nutr 2001;4.

Trichopoulou A, Naska A and the DAFNE III Group: European food availability databank based on household budget surveys - the Data Food Networking initiative. Eur J Public Health 2003; 13(suppl):24-28.

Trichopoulou A, Naska A, Costacou T and DAFNE III Group: Disparities in food habits across Europe. Proc Nutr Soc 2002;61:553-558.

Turrini A, Saba A, Perrone D, Cialfa E, D’Amicis A: Food consumption patterns in Italy: the INN-CA Study 1994-1996. Eur J Clin Nutr 2001;55:571-588.
Tutkuviene J: Body mass index, prevalence of overweight and obesity in Lithuanian children and adolescents, 1985-2002. Coll Antropol 2007;31:109-121.

Tzotzas T, Kapantais E, Tziomalos K, Ioannidis I, Mortoglou A, Bakatselos S, Kaklamanou M, Lanaras L, Kaklamanos I: Epidemiological survey for the prevalence of overweight and abdominal obesity in Greek adolescents. Obesity 2008;16:1718-1722.

US Department of Health and Human Services: Physical activity; in Dietary Guidelines for Americans, 2005. Washington, US Government Printing Office, 2005, pp 19-22.

UNICEF (2009): Overview of breastfeeding patterns (http://www.childinfo.org/breastfeeding_overview. html) (accessed June 2009).

Vaask S, Liebert T, Maser M, Pappel K, Pitsi T, Saava M, Sooba E, Vihalemm T, Villa I: Estonian National Dietary Recommendations (RDI 2006). Tallinn, Estonian Society of Nutrition Science, 2006.

Välimäki VV, Löyttyniemi E, Välimäki MJ: Vitamin D fortification of milk products does not resolve hypovitaminosis D in young Finnish men. Eur J Clin Nutr 2007;61:493-497.

Vasdekis VG, Stylianou S, Naska A: Estimation of age and gender-specific food availability from household budget survey data. Public Health Nutr 2001; 4:1149-1151.

Valvira THL: Tilastokeskus: Alkoholin kulutus 2008. 2009.

Vartiainen E, Peltonen M, Laatikainen T, Sundvall J, Salomaa V, Jousilahti P, et al: FINRISKI-tutkimus: Sekä miesten että naisten sydän- ja verisuonisairauksien kokonaisriski pieneni viime vuosina. Suom Lääkäril 2008;15:1375-1381.

Veiga P: Out-of-pocket healthcare expenditures due to excess of body weight in Portugal. Econ Hum Biol 2008;6:127-142.

Vernay M, Malon A, Oleko A, Salanave B, Roudier C, Szego E, et al: Association of socioeconomic status with overall overweight and central obesity in men and women: the French Nutrition and Health Survey 2006. BMC Public Health 2009;9:215.

Vignerová J, Humeníková L, Brabec M, Riedlová J, Bláha $\mathrm{P}$ : Long-term changes in body weight, BMI, and adiposity rebound among children and adolescents in the Czech Republic. Econ Hum Biol 2007;5:409-425.

Vlad M, Zapirtan H, Creteanu E: Nutritional status in adult population in relation to the food intake. Annual Report of IPH Cluj-Napoca, Romania, 2003.

Vlad M, Zapirtan H, Creteanu E: Food Intake and Nutritional Status of the Population, Annual National Syntheses, Institute of Public Health ClujNapoca, Romania, 2008. 
Vladescu C, Scintee G, Olsavszky V, Allin S, Mladovsky P: Romania: Health system review, Health Systems in Transition 2008;10:1-172.

Voedingscentrum. Richtlijnen voedselkeuze, Januari 2009 (http://www.voedingscentrum.nl/NR/rdonlyres/ A 21 A A 80 A - 2 D 87 - 45 A 4 - 8333 F7F01CEA3492/0/RICHTLIJNENVOEDS ELKEUZE09.pdf).

Voedingsraad: Nederlandse voedingsnormen 1989. Den Haag, Voorlichtingsbureau voor de Voeding, 1992.

Von Wagner C, Knight K, Steptoe A, Wardle J: Functional health literacy and health promoting behaviour in a national sample of British adults. J Epidemiol Commun Health 2007;61:1.

Waśkiewicz A, Sygnowska E, Jasiński B, Kozakiewicz K, Biela U, Kwaśniewska M, Głuszek J, Zdrojewski T: Wartość energetyczna i odżywcza diety dorosłych mieszkańców Polski. Wyniki programu WOBASZ. Kardiol Polska Supl 2005;63;4:S1-S7.

WCRF (World Cancer Research Fund)/AICR (American Institute for Cancer Research): Food, Nutrition Physical Activity, and the Prevention of Cancer: a Global Perspective. Washington, AIRC, 2007 (http:// www.dietandcancerreport.org/downloads/chapters/chapter_12.pdf) (accessed May 2009).

WHO Regional Office for Europe: Comparative analysis of nutrition policies in WHO European Member States, Copenhagen 1998 (http://www.euro.who.int/ Document/E60770.pdf) (accessed June 2009).

WHO Regional Office for Europe: The First Action Plan for Food and Nutrition Policy, WHO European Region 2000-2005, Copenhagen 2000 (http://www. euro.who.int/Document/E72199.pdf) (accessed June 2009).

WHO Regional Office for Europe. Highlights on health in Romania, 2005, EUR/05/5046415O (http://www. euro.who.int/highlights).

WHO Regional Office for Europe: Comparative analysis of nutrition policies in WHO European Member States, Copenhagen 2006 (http://www.euro.who.int/ document/Nut/instanbul_conf_\%20ebd02.pdf (accessed May 2009).

WHO Regional Office for Europe: Health-for-All Database (HFA-DB). Copenhagen, WHO Regional Office for Europe, 2007 (http://www.euro.who.int/ hfadb).

WHO Regional Office for Europe: European action plan for food and nutrition policy 2007-2012, Copenhagen 2008 (http://www.euro.who.int/Document/ E91153. pdf) (accessed June 2009).

World Health Organization (WHO): Obesity: preventing and managing the global epidemic. Report of a WHO consultation. World Health Organ Tech Rep Ser 2000;894:i-xii, 1-253.
World Health Organization (WHO): The optimal duration of exclusive breastfeeding. Results of a WHO systematic review. Note for the Press, No. 7, April 2, 2001 (http://www.who.int/inf-pr-2001/en/note200107.html). Geneva, WHO (accessed July 2009).

World Health Organization (WHO), Food and Agriculture Organization (FAO): Diet, Nutrition and the Prevention of Chronic Diseases. Report of a Joint WHO/FAO Expert Consultation. World Health Organ Tech Rep Ser 2003;916:i-vii, 1-149.

World Health Organization (WHO): The Global Burden of Disease: 2004 Update. Geneva, WHO, 2004.

World Health Organization (WHO): Joint FAO/WHO Scientific update on carbohydrates in human nutrition. Eur J Clin Nutr 2007;61(suppl 1).

World Health Organization (WHO): Inequalities in Young People's Health. HBSC International Report from the 2005/2006 Survey. Geneva, WHO, 2008a.

World Health Organization (WHO): WHO Global Strategy on Diet Physical Activity and Health: A Framework for Countries to Monitor and Evaluate Implementation. Geneva, WHO, 2008b.

WHO Regional Office for Europe: Health for All database (HFA-DB). Copenhagen, WHO Regional Office for Europe, 2009a. Internet: http://www.euro. who.int/hfadb. (accessed: June 2009)

World Health Organization (WHO): Joint FAO/WHO Expert Consultation on fats and fatty acids in human nutrition. Ann Nutr Metab 2009b;548(suppl 3).

Yngve A, De Bourdeaudhuij I, Wolf A, Gribovski A, Brug J, Due P, Ehrenblad B, et al: Differences in prevalence of overweight and stunting in 11-yearolds across Europe: the Pro Children Study. Eur J Pubic Health 2007;1-5.

Zajkás G: National Food and Nutrition Policy of Hungary, 2004.

Zajkás G, Bíró L, Greiner E, et al.: Táplálkozási vizsgálat Magyarországon, 2003-2004. Mikro-tápanyagbevitel: vitaminok [Dietary survey in Hungary, 20032004. Micro-nutrient intake: vitamins]. Orvosi Hetilap [Hung Med J] 2007;148:1593-1600.

Zapirtan H: Nutritional Habits in Transylvania, a Risk Factor in Nutritional and Cardiovascular Diseases, Doctoral Thesis, University of Medicine and Pharmacy Cluj-Napoca, Romania, 2006.

Zatoński W (ed): Closing the Health Gap in European Union. Warsaw, Cancer Center and Institute of Oncology, 2008. 


\section{Subject Index}

Adolescents

energy and nutrient intake assessment country comparison $99,110-112$

methodology 98, 99, 108

region comparison 99, 112, 113

mineral intake 116-121

obesity/overweight prevalence 157-160

physical activity 191-193

vitamin intake $107,114-116$

Adults

dyslipidemia 171-173

energy and nutrient intake assessment country comparison $120,121,124-127$

methodology 120, 122, 123

region comparison 121, 123, 134, 135

mineral intake $134-140$

obesity/overweight prevalence 161,162 , 164-169

physical activity 188-191

reference values and comparison of recommendations for adults $68-73$

vitamin intake 123, 130-134

Alcohol, see also Beer, Wine

consumption

by country $182,185,186$

trends $3,10,30-33,42$

European Food Safety Authority surveys of consumption 65

Animal fat

European Food Safety Authority surveys of consumption 64

supply trends $24,25,42$

Ascorbic acid, intake

adolescents 115

adults 131,133

children

4-6 years 91, 97
7-9 years 93

10-14 years 95

elderly 149,151

Austria

energy and nutrient intake 252, 253

health indicators 250-252

mineral intake 254, 255

nutritional status

adolescents 255, 256

pregnancy $256-258$

nutrition trends 255

vitamin intake 254

Baltic Nutrition Survey 278

Beef, see Red meat

Beer, supply trends 30,31

Belgium

Flemish Growth Study 261, 262

Food Consumption Survey 259-261

Health Interview Survey 259, 261

Breast cancer, incidence by country 179

Breastfeeding recommendations 196

trends 10, 196-201

Calcium, intake

adolescents 118, 121

adults $136,138,140$

children

4-6 years 100, 106

7-9 years 102, 106

$10-14$ years 104,107

elderly $152,154,156$

overview 234, 235

Cancer

epidemiology 9, 184

incidence by country 175,181 
incidence by type $175-181$

mortality by country 176

Cardiovascular disease

epidemiology 9

mortality by country 176,177

$\beta$-Carotene, intake

adolescents 114

adults 130,132

children

4-6 years 90

7-9 years 92

10-14 years 94,96

elderly 148,150

Cereals

European Food Safety Authority surveys of consumption 64

supply trends 19,21, 33

Children

energy and nutrient intake assessment

$4-6$ years $69,74,78,79,84,85$

$7-9$ years $69,75,80,81,85$

$10-14$ years $69,73,76,82-84,86$

comparison of regions and countries $77-86$

mineral intake $88,89,98,100-107$

obesity/overweight prevalence 157-160

physical activity 191-193

vitamin intake $76,86-98$

Cholesterol, intake

adolescents 111, 113

adults $125,127,129$

children

$4-6$ years 79,85

7-9 years 81

10-14 years 83

dyslipidemia, see Dyslipidemia

elderly 143

Cobalamin, intake

adolescents 115, 116

adults 131, 133, 134

children

4-6 years 91, 96, 97

7-9 years 93

10-14 years 95,98

elderly 149,151

Coffee, European Food Safety Authority surveys of consumption 65

Colorectal cancer, incidence by country 178

Copper, intake

adolescents 119

adults 137,139 children

4-6 years 101

7-9 years 103

10-14 years 105

elderly 153,155

Countrywide Integrated Non-Communicable Disease

Czech Republic 267

Lithuania 336

Czech Republic

biological monitoring project 267, 268

CINDI Health Monitor 267

infant feeding data 264

Nationwide Anthropological Survey 263

nutritional status

children 265

homeless 268, 269

Post-MONICA Study 266

Data Food Networking

application tool 47

database goals 45

data collection 45,46

expense conversion to food quantities 46

food availability by household income

Central and Eastern European countries

49,52

educational attainment effects 53-57

mean availability per person per day 48-53

North European countries 51, 56, 57

overview 3,4

South European countries 48, 54, 55

West European countries 50, 56, 57

household budget survey data

incorporation $46,47,231,232$

Denmark

food intake by type 271-274

life expectancy 275,276

National Health Interview Survey 269-271

National Survey of Dietary Habits and

Physical Activity 269, 270

obesity/overweight 275

physical activity 274,275

sex differences in diet 274

smoking behavior 275

social inequality 276

Diabetes, epidemiology 9, 10, 181, 182

Dietary surveys, food consumption in

European adults 5, 6 
Dutch National Food Consumption Surveys 378, 379

Dyslipidemia adults $171-173$

elderly 174

epidemiology 8, 9

Eggs

European Food Safety Authority surveys of consumption 65,66

supply trends 30,31

Elderly

dyslipidemia 174

energy and nutrient intake assessment country comparison 142-146

methodology 135, 140, 141

region comparison 146,147

mineral intake $152-156$

obesity/overweight prevalence 163,164 , 170

physical activity 193

vitamin intake 147-151, 154

Energy, intake

adolescents 110, 112

adults $124,126,128$

children

$4-6$ years 78,84

$7-9$ years 80,87

$10-14$ years 82

elderly $142,144,146$

intake by region 233,234

Estonia

Baltic Nutrition Survey 278

Health Behavior among the Estonian Adult Population Survey 278, 279

morbidity and mortality 277

nutritional status

adolescents 279

adults $280-282$

elderly $282,283,285$

smoking and drinking behavior 282-284

European Food Safety Authority, dietary

surveys of food consumption

adolescents 7

adults $5-8$

children 6,7

elderly 8

food categories $60,61,64-67$

highest and lowest countries 63

methodology and survey years 60,62

European Nutrition and Health Report 2009 implementation strategy 14-16

objectives $1,2,12,14$

outlook 12, 13

Exercise, see Physical activity

Fat, see Animal fat

Fatty acids, intake

adolescents 111-113

adults 125,129

children

$4-6$ years $79,85,87$

$7-9$ years 81,88

$10-14$ years 83,89

elderly $143,145,147$

Fiber, intake

adolescents 111, 112

adults 127,128

children

$4-6$ years 84,85

$7-9$ years 85,88

10-14 years 8

elderly $143,145,146$

Finland

food supply data 285,286

health indicators and status 293

nutritional status

adolescents 289, 290

adults and elderly 289, 291, 292

infants and children 287-289

vitamin D fortification 292, 293

Fish and seafood

European Food Safety Authority surveys of consumption 65

supply trends 28, 29

Flemish Growth Study 261, 262

Folate

adolescents 115, 116

adults $131,133,134$

children

4-6 years 91, 96, 97

7-9 years 93,97

10-14 years 95,98

elderly 149,151

Food and nutrition policy

assessment 203-218

diet quality ensuring 224, 225

forms and implementation 226, 227

global action plan 222, 223, 227-229

health issue addressing 221, 222

health promotion and chronic disease prevention 225, 226 
implementation actions 219-221

intersectorial approach 218, 219

overview 202, 203

quality assurance 221

Food balance sheet, food supply data 17-19, $45,59,230,232$

Food supply

comparison of selected food groups 32 , $35-42$

proportion in total energy supply

animal and vegetable products 32,34

macronutrients 32,33

trends

animal fat $24,25,42$

beer 31,33

cereals $19,21,33$

eggs 30,31

fish and seafood 28,29

food balance sheet data $17-19$

fruits 18,20

milk 30, 42

mutton and goat meat 28

oil crops 22, 23, 42

olive oil 24,25

overview 2, 3, 18, 231-233

pork 26,27

potatoes $18,21,33$

poultry 28,29

pulses 22,23

red meat 26

sugar and sweeteners 22, 42

vegetable oils 24

vegetables 18,20

wine 32,33

France

National Nutrition and Health Program 294, 295, 299

nutritional status

adults 296-298

children 296, 297

Nutrition and Health Survey 295

Fruits

European Food Safety Authority surveys of consumption 64,65

supply trends 18,20

\section{Germany}

anthropometric measurements by

socioeconomic status 302,303

dietary supplement use 304

energy and nutrient intake 303, 304
Health Interview and Examination Survey for Children and Adolescents 299, 300

National Nutrition Survey II 300-304

Goat, see Mutton and goat meat

Greece

nutritional status

adolescents 305,307

adults and elderly 307

children 305

pregnancy and breastfeeding 307, 309

smoking behavior 306,308

Household budget survey, see Data Food

Networking

Hungary

alcohol intake 316

anthropometric data 314-316

databases 316

dietary supplement use 314

food and nutrition policies 319

health indicators 317-319

national dietary surveys 309,310

nutritional status

adults $310-313$

biomarkers 313,314

children and adolescents 310

Income, see Data Food Networking

Iodine

adolescents 119, 121

adults 137,139

children

4-6 years 101, 106

7-9 years 103,106

$10-14$ years 105, 107

elderly 153, 155, 156

Ireland

data sources 319,320

dietary habits

adolescents 322

adults 320,321

children 321,322

elderly 322

Iron, intake

adolescents 119, 121

adults $137,139,140$

children

4-6 years 101, 106

7-9 years 103, 106

$10-14$ years 105,107

elderly $153,155,156$ 
Italy

alcohol use 328,329

breastfeeding 328

dietary profile $323-327$

obesity in children 327

smoking behavior 329,330

Juices, European Food Safety Authority surveys of consumption 65

Latvia

dietary habits 330

obesity/overweight 330-333

physical activity 333,334

smoking behavior 333

Lithuania

anthropometry 338

CINDI program 336

dietary guidelines 339

health behavior surveys

adults 336,337

children 337,338

morbidity and mortality 334

national nutrition surveys 335,336

Low-density lipoprotein, see Dyslipidemia

Lung cancer, incidence by country 179

Magnesium, intake

adolescents 119

adults 137, 139

children

4-6 years 101

7-9 years 103

$10-14$ years 105

elderly 153,155

Malignant neoplasms, see Cancer

Manganese, intake

adolescents 119

adults 137,139

children

4-6 years 101

7-9 years 103

$10-14$ years 105

elderly 153,155

Meat, see also Fish and seafood, Mutton and goat meat, Pork, Poultry, Red meat

European Food Safety Authority surveys of consumption 65

Milk

European Food Safety Authority surveys of consumption 66 supply trends 30,42

Minerals, intake

adolescents 116-121

adults $134-140$

children $88,89,98,100-107$

elderly 152-156

Mortality rates $171,173,175-181$

Mutton and goat meat, supply trends 28

Netherlands

dietary supplement use 381

Dutch National Food Consumption Surveys 378, 379

food intake by type $381-384$

Niacin, intake

adolescents 115

adults 131,133

children

4-6 years 91, 97

7-9 years 93

$10-14$ years 95

elderly 149,151

Norway

dietary surveys 339

food intake by type $343-345$

nutritional status

adults $341-343$

children 339-341

Obesity/overweight

body weight classification by country 237 , 238, 243-246

epidemiology 8

prevalence

adults $161,162,165-169$

children and adolescents 157-160, 239-241

elderly $163,164,170,247-249$

study design 157

Oil crops, supply trends 22, 23, 42

Olive oil, supply trends 24,25

Overweight, see Obesity/overweight

Phosphorus, intake

adolescents 118, 121

adults 136, 138, 140

children

4-6 years 100, 106

7-9 years 102, 106

10-14 years 104, 107

elderly $152,154,156$ 
Physical activity

adults 188-191

assessment limitations 187

children and adolescents 191-193

elderly 193

national surveillance systems in Europe

187,188

outlook 201-203

recommendations 186, 187, 193-196

Poland

Food Consumption and Anthropometric Survey 346-348

health status 350,351

Social Stratification in Food Consumption study 349,350

Policy, see Food and nutrition policy

Pork, supply trends 26, 27

Portugal

body mass index 354,355

breastfeeding 354

food and nutrient intake 351-354

household food and nutrient availability 351

nutritional status 352,354

Potassium, intake

adolescents 118

adults 136,138

children

4-6 years 100

7-9 years 102

10-14 years 104

elderly 152,154

Potatoes

European Food Safety Authority surveys of consumption 64

supply trends $18,21,33$

Poultry, supply trends 28,29

Prostate cancer, incidence by country 179

Protein, intake

adolescents 110,112

adults $124,126,128$

children

$4-6$ years 78,84

$7-9$ years 80,87

$10-14$ years 82

elderly $142,144,146$

Pulses

European Food Safety Authority surveys of consumption 64

supply trends 22,23
Red meat, supply trends $26-28$

Reference values, comparison of recommendations for adults $68-73$

Riboflavin, intake adolescents 115

adults 131, 133

children

$4-6$ years 91,97

7-9 years 93

10-14 years 95

elderly 149,151

Romania

cancer 358

cardiovascular disease 357

demographics 356

life expectancy 356

mortality 356,357

nutritional status

adults and elderly 360,361

children 359,360

infants 358,359

obesity/overweight 357

smoking behavior 362

Seafood, see Fish and seafood

Selenium

adolescents 119, 121

adults 137, 139, 140

children

$$
\begin{gathered}
4-6 \text { years } 101,106 \\
7-9 \text { years } 103,106 \\
10-14 \text { years } 105,107 \\
\text { elderly } 153,155,156
\end{gathered}
$$

Slovenia

data sources 362,363

dietary habits

adolescents 365

adults 363-365

obesity/overweight 365,366

Smoking, behavior in Europe 180, 182

Sodium

adolescents 118

adults 136,138

children

4-6 years 100

7-9 years 102

10-14 years 104

elderly 152,154

Spain

Catalan National Survey 366, 367

inadequate intake risks $367-369$ 
Mediterranean diet adherence 369-371

obesity/overweight 369, 370

Stomach cancer, incidence by country 178

Sugar and sweeteners

European Food Safety Authority surveys of consumption 64

supply trends 22,42

Sweden

alcohol use 377

dietary habits

adults $371-373$

children $373-375$

trends 377,378

obesity/overweight 376

smoking behavior 376

Tap water, European Food Safety Authority surveys of consumption 67

Tea, European Food Safety Authority surveys of consumption 65

Thiamine, intake

adolescents 115

adults 131

children

4-6 years 91, 97

7-9 years 93

$10-14$ years 95

elderly 149,151

United Kingdom

health literacy and health improvement 389, 390

Health Survey for England 386-388

local and regional health profiles 390,391

Low-Income Diet and Nutrition Survey 384-385

obesity/overweight 387

physical activity 388

Vegetable oils

European Food Safety Authority surveys of consumption 64

supply trends 24

Vegetables

European Food Safety Authority surveys of consumption 64

supply trends 18,20

Vitamin A, intake

adolescents 114

adults 130,132 children

4-6 years 90

7-9 years 92

10-14 years 94, 96

elderly 148,150

Vitamin $\mathrm{B}_{6}$, intake

adolescents 115, 116

adults $131,133,134$

children

4-6 years 91, 96, 97

7-9 years 93,97

10-14 years 95, 98

elderly 149,151

Vitamin C, see Ascorbic acid

Vitamin D, intake

adolescents 114, 116

adults 130, 132, 134

children

4-6 years 90, 96, 97

7-9 years 92

$10-14$ years $94,96,98$

elderly $148,150,151$

fortification in Finland 292, 293

Vitamin E, intake

adolescents 114

adults 130, 132

children

$4-6$ years 90

7-9 years 92

10-14 years 94, 96

elderly 148,150

Vitamins, see also specific vitamins adolescent intake 107, 114-116

adult intake $123,130-134$

child intake $76,86-98$

elderly intake 147-151, 154

overview 234

Wine, supply trends 32,33

Zinc, intake

adolescents 119, 121

adults $137,139,140$

children

4-6 years 101, 106

7-9 years 103

10-14 years 105

elderly 153, 155, 156 